\section{New clinical trials I}

\section{New clinical trials I}

THE STROKE PREVENTION BY AGGRESSIVE REDUCTION IN CHOLESTEROL LEVELS (SPARCL) STUDY

The SPARCL Investigators

Background: Treatment with statins significantly reduces the risk of coronary heart disease (CHD) and stroke in persons with CHD and other defined risk factors. Whether statins reduce the risk of recurrent stroke in patients with recent stroke and no history of CHD remain uncertain. SPARCL is the first large-scale, multi-center, double-blind, randomized, placebo-controlled statin trial designed to answer this question.

Methods: The aim of SPARCL was to assess whether atorvastatin $80 \mathrm{mg}$ reduces the incidence of recurrent strokes in patients without a history of CHD who have had a recent stroke or transient ischemic attack (TIA). 4732 men and women aged $\geq 18$ years with a stroke or TIA within the previous 1-6 months who have a baseline low-density lipoprotein cholesterol between $100-190 \mathrm{mg} / \mathrm{dL}(2.6$ $\mathrm{mmol} / \mathrm{L}-4.9 \mathrm{mmol} / \mathrm{L}$ ) and a modified Rankin score of $\leq 3$ were randomized to receive atorvastatin $80 \mathrm{mg}$ or placebo. Randomized patients were followed for a median of 4.9 years (maximum 6.6 years). The primary endpoint was the time from randomization to the first occurrence of a fatal or non-fatal stroke. It was expected that approximately 540 patients would experience a primary endpoint over the course of the trial, thereby providing $90 \%$ power to detect a $25 \%$ hazard reduction for atorvastatin $80 \mathrm{mg}$ compared with placebo using a 2 -sided test (alpha $=0.05$ ). Secondary endpoints include composites of cerebrovascular (stroke+TIA) and cardiovascular endpoints and all-cause mortality.

Results: The target number of patients with a primary endpoint has been reached and SPARCL is in the final stages of closeout. Results will be available for the European Stroke Conference.

Conclusions: Results of SPARCL are anticipated to have a significant impact on the treatment of patients with a recent stroke.

\section{New clinical trials I}

\section{THE MAIN RESULTS OF THE CHARISMA TRIAL}

The CHARISMA Investigators

Background: Aspirin is the established antiplatelet agent across a variety of atherothrombotic disease states. The adenosine diphosphate receptor antagonist clopidogrel when added to aspirin reduces ischemic events in patients with both ST-segment and non-ST-segment elevation acute coronary syndromes. Whether the combination of aspirin plus clopidogrel is superior to aspirin alone in high-risk patients with stable cardiovascular disease is unknown.

Methods: The CHARISMA trial randomized over 15,000 high-risk patients with stable cardiovascular disease (coronary artery disease, cerebrovascular disease, peripheral arterial disease), or multiple risk factors, to receive either clopidogrel or placebo, on top of low-dose aspirin. The rate of cardiovascular death, myocardial infarction, or stroke was examined.

Results: Patients were followed for a median of 2.3 years and a maximum of 3.0 years. The blinded rate of cardiovascular death, myocardial infarction, or stroke in the overall population was $3.27 \%$ per year. The unblinded event rates will be presented, along with bleeding complications and any adverse effects.

Conclusions: Long term antiplatelet therapy with clopidogrel on top of low-dose aspirin has been evaluated in a geographically diverse population of high-risk patients with either symptomatic or asymptomatic (primary prevention) atherothrombosis. Potentially, long term use of clopidogrel plus aspirin may lead to improvement in patient irrevocable adverse endpoints compared with aspirin alone.

\section{New clinical trials I}

CHANT: A DOUBLE-BLIND, MULTICENTER, PLACEBO-CONTROLLED STUDY TO ASSESS THE SAFETY AND TOLERABILITY OF NXY-059 IN PATIENTS WITH ACUTE INTRACEREBRAL HEMORRHAGE The CHANT Investigators

Effective treatment of acute ischemic stroke (AIS) requires early initiation ideally prior to neuroimaging, which requires demonstration of efficacy in AIS and safety in intracerebral hemorrhage (ICH). Efficacy in ICH would be a bonus.

NXY-059 is a novel free-radical trapping neuroprotectant in development for AIS. SAINT I demonstrated significant reduction of global disability in AIS patients treated with NXY-059. The safety and tolerability of NXY-059 in ICH are being studied in the Cerebral Hemorrhage And NXY-059 Treatment (CHANT) trial; a double-blind, multicenter, placebo-controlled, parallel-group Phase IIb study.

Patients with a CT/MR-confirmed ICH were eligible for CHANT if they presented within 6 hours of stroke onset, alert, with NIHSS score $\geq 6$, including limb weakness. Randomization was stratified for country and total ICH score $(0,1$, 2 and $\geq 3$ ), based on location and size of hematoma, ventricular involvement, age and Glasgow Coma Scale. NXY-059 was administered by infusion over $72 \mathrm{~h}$. The target unbound plasma concentration was $260 \mu \mathrm{M}$. Scans were repeated post-infusion. Safety and tolerability assessments include mortality (overall and by cause), adverse events, and change in ICH volume. Stroke outcomes included modified Rankin scale (distribution of scores analyzed using the Cochran Mantel Haenzel test, supplemented by dichotomizations), Barthel Index and NIHSS up to 90 days. Recruitment into the trial is complete: $\mathrm{n}=603$ (607 patients randomized; 4 not treated) from 150 centers. Preliminary mean baseline demographic data are age 66 years, NIHSS score 14, ICH volume $26 \mathrm{~mL}$, and ICH score 0.9. Further results will be presented.

\section{New clinical trials I}

RECURRENT STROKE IS NOT ASSOCIATED WITH MASSIVE RIGHT-TO-LEFT SHUNT: PRELIMINARY RESULTS FROM THE 3-YEAR PROSPECTIVE SPANISH MULTICENTRE STUDY (CODICE STUDY) The CODICE Study Group

Few studies have prospectively examined the risk of recurrent stroke associated with PFO (patent foramen ovale). We present the preliminary results of the Spanish right-to-left shunt (RLSh) multicentre study set up in 2002.

Methods: 500 patients with cryptogenic stoke (CS) (TOAST criteria) were included in 20 participating hospitals. For analysis we considered the first 379 patients (Group A) and, as a separate group, the 168 patients younger than 55 y (Group A1). Patients were examined by contrast transcranial Doppler (c-TCD) and followed for 3 years. The magnitude of RLSh was quantified by counting the number of signals in the MCA during Valsalva maneuver. Transthoracic and/or transesophageal echocardiography, CT scan or MRI were performed. Age, vascular risk factors, treatment, stroke severity and Rankin Scale on follow-up were recorded. Statistical analysis was performed in both groups. The relationship between RLSh magnitude and stroke recurrence was determined through Cox regression analysis.

Results: Massive RLSh (m-RLSh) was detected in 139 patients $(36.7 \%)$. The mean follow-up was $733 \pm 367$ days. Stroke recurrence was low $(5.3 \%, n=20)$ and lower in patients with m-RLSh in comparison with patients with minor or no RLSh (Group A, $2.9 \%$ vs. 6.7\%; p=0.07; Group A1, 2.4\% vs. $3.5 \%$; $=0.52$ ). Migraine was more prevalent in m-RLSh patients in both Groups $(20.7$ vs. $9.4 ; p=0.03)$ as was atrial septal aneurysm (37.7 vs. $4.8, \mathrm{p}<0.001)$. Diabetes and hypertension were significantly more frequent in Group A patients without m-RLSh.

Cox regression found no association between $\mathrm{m}$-RLSh and recurrent stroke in both groups (OR 1.53, 95\% CI 0.42-3.83). These results were similar when ASA and $\mathrm{m}$-RLSh were analysed together, and when treatment and concomitant stroke risk factors were included.

Conclusions: These results suggest that neither m-RLSh nor m-RLSh together with ASA are independent risk factors for recurrent stroke, both in the general and in the younger stroke population.

\section{KARGER}

Fax +4161306 1234 E-Mail karger@karger.ch www.karger.ch
(C) 2006 S. Karger AG, Basel

1015-9770/06/2110-001\$23.50/0

Accessible online at: www.karger.com/ced 


\section{New clinical trials I}

SAFETY AND EFFICACY OF EXTENDED EXPOSURE TO SEVERAL DOSES OF A NEW ORAL DIRECT THROMBIN INHIBITOR DABIGATRAN ETEXILATE IN ATRIAL FIBRILLATION

The Petro-ex Investigators

Background: Vitamin $\mathrm{K}$ antagonists are the only effective oral drugs to prevent stroke in high risk patients with chronic atrial fibrillation (AF). A fixed dose, orally active, direct thrombin inhibitor was tested as an alternative.

Methods: A 12 week randomized trial of dabigatran etexilate (D) 50,150 or 300 mg b.i.d. (doses blind), with 0,81 or $325 \mathrm{mg}$ acetylsalicylic acid (ASA) q.d. in a factorial design, compared to adjusted dose warfarin (INR 2.0 to 3.0) was conducted in $502 \mathrm{AF}$ patients at high risk for stroke (S) or systemic embolism (SE). This was followed by open-label observation of D patients up to 18 months. Liver function tests were monitored every 2 - 4 weeks for the first 6 months and quarterly thereafter. S, SE, myocardial infarctions (MI) and major bleeds (MB) were documented. A DSMB monitored events and recommended discontinuation of $\mathrm{D}$ or transition to alternative regimens.

Results: 464 patients completed 12 weeks treatment, 29 discontinued for adverse events and 9 for other causes; 361 patients (age 69 years, $16 \%$ female, duration of AF 4 years, a median of 3 additional risk factors for stroke) were rolled over to the open-label follow-up phase for a total of 338 patient years of exposure to D. There were $11 \mathrm{~S}$ or SE, $10 \mathrm{MB}$ and $3 \mathrm{MIs}$. D $150 \mathrm{mg}$ /day or less yielded a S/SE rate of $8.2 \%$ per year with $0 \% \mathrm{MB}$. At $150 \mathrm{mg}$ bid of D, $1 \mathrm{SE}$ occurred (S/SE rate $0.7 \%$ per year) with $4 \mathrm{MB}(2.9 \% /$ year) and $3 \mathrm{MIs}(2.2 \% / \mathrm{y}) .300 \mathrm{mg}$ q.d. had a S/SE rate of $9.5 \% /$ year and no MB. At $300 \mathrm{mg}$ bid of D, no S or SE occurred $(0 \% /$ year) but $6 \mathrm{MB}$ (7.1\%/year). ASA increased the chance of bleeding, especially at high doses of D. Transaminase elevations were $1.5 \%$ at 1 year.There were no S or MB on warfarin. Conclusion: D $150 \mathrm{mg}$ bid and similar doses should be tested in larger trials.

\section{New clinical trials I}

THROMBOLYSIS FOR ACUTE ISCHEMIC STROKE: MAIN OUTCOME RESULTS FOR 2003-2005 OF SITS-MOST (SAFE IMPLEMENTATION OF THROMBOLYSIS IN STROKE-MONITORING STUDY) IN EUROPE

The SITS Collaborators

Background and Purpose: SITS-MOST is required by the European Union (EU) regulatory authority for temporary licensing of rt-PA in acute ischemic stroke for EU member states $2002(\mathrm{n}=15)$, Norway and Iceland. SITS-MOST is embedded within SITS-ISTR (International Stroke Thrombolysis register). SITS-MOST was designed to control for symptomatic intracerebral hemorrhage (SICH), mortality and independence when compared to prior Randomized Controlled Trials (RCTs). The results presented in this report constitute pivotal data for regulatory decision on permanent license in EU

Methods: 4961 patients entered SITS-MOST from 265 centers in 14 countries up to 31 December 2005. Data included: at baseline demographics, timing of procedures, NIHSS (National Institute of Health stroke score) and imaging results before treatment, during follow-up NIHSS at $2 \mathrm{~h}, 24 \mathrm{~h}$ and 7 days, control imaging within 22-36h after treatment, global outcome at 24 hours and 7 days, modified Rankin Score (mRS) at 3 months and adverse drug reactions. Definitions: SICH per protocol: primary ICH type 2 plus NIHSS worsening $\geq 4$ points at $24 \mathrm{~h}$ follow up. SICH per RCTs: any hemorrhage plus NIHSS worsening $\geq 1$ points within 7 days. Proportion of deaths and independency (mRS 0-2) were calculated to 3 months.

Results: Results are SITS-MOST vs. RCTs where relevant $(95 \%$ confidence intervals). Mean age 65.8 vs. 65.8 years; females $40 \%$ vs. $40 \%$, mean NIHSS 12.8 vs. 12.6 , mean stroke onset to treatment time 136 vs. 229 minutes. SICH per protocol: $1.5 \%$ (1.2-1.9), SICH per RCTs: $7.3 \%$ (6.6-8.1) vs. $8.6 \%$ (6.1-11.1), mortality within 3 months: $12.8 \%(11.8-13.9)$ vs. $17 \%(13.9-20.7)$, independence at 3 months: $53.3 \%(51.8-54.9)$ vs. $50 \%(45.6-54.6)$.

Conclusions: The first official presentation of the main results of SITS-MOST indicates that, overall, routine clinical use of rt-PA within $3 \mathrm{~h}$ of stroke onset is as safe as previously reported in RCTs.

\section{New clinical trials I}

SAFETY AND EFFICACY OF TICLOPIDINE AND ASPIRIN COMBINATION VERSUS ASPIRIN ALONE IN THE SECONDARY PREVENTION OF STROKE The TACS Study Group

Background: Data on the combination of thienopyridines with aspirin for secondary prevention in stroke patients are inconclusive. We evaluated whether combination therapy is safe and more effective than aspirin alone to prevent vascular events after TIA or stroke.

Methods: A randomised, parallel groups, double blind, placebo controlled study was conducted in 21 Italian centres. Patients were allocated to either aspirin (100 $\mathrm{mg}$ die) plus ticlopidine $(250 \mathrm{mg}$ bid $)$ or aspirin plus placebo through centralised randomisation, stratifying for TIA/stroke and clinical centre. The first occurrence of any stroke, myocardial infarction, or vascular death, and that of adverse events were compared between treatment groups by means of the chi-square test.

Results: 218 patients $(59.6 \%$ men) with a mean age of $69.9 \pm 11.2$ years, were followed for an average of 20.1 months (range 12 to 60) after a previous ischaemic stroke $(n=172)$ or a TIA $(n=46)$. With respect to patients treated with aspirin alone $(n=109)$, those on combination therapy $(n=109)$ tended to have a lower occurrence of the primary end-point $(7.3 \%$ vs $11.0 \% ; \mathrm{P}=0.48)$, vascular death $(1.8 \%$ vs $4.6 \%$; $\mathrm{P}=0.44)$, any stroke $(4.6 \%$ vs $5.5 \% ; \mathrm{P}=1.00)$, and myocardial infarction $(1.8 \%$ vs $4.6 \%$; $\mathrm{P}=0.44)$, while no difference was observed in overall mortality $(7.3 \%)$ Patients on combination therapy had a higher risk of bleeding $(16.5 \%$ vs $10.1 \%$; $\mathrm{P}=0.23)$, skin rash $(5.5 \%$ vs $0.9 \% ; \mathrm{p}=0.12)$, epigastralgia $(8.3 \%$ vs $4.6 \% ; \mathrm{P}=0.41)$, diarrhoea $(2.8 \%$ vs $0.9 \%$; $\mathrm{P}=0.61)$, and liver enzyme elevation $(28.4 \%$ vs $15.6 \%$; $\mathrm{P}=0.03)$, but less serious adverse events $(8.3 \%$ vs $11.0 \% ; \mathrm{P}=0.65)$

Discussion: Although this pilot study failed to show any significant difference between the two treatment regimens, it suggests that the combination of ticlopidine and aspirin in patients with previous TIA and stroke has an acceptable risk and a favourable outcome trend as compared to aspirin alone. A large clinical trial would be warranted.

\section{Recovery and rehabilitation}

\section{Recovery and rehabilitation}

IS THE PRIMARY MOTOR CORTEX (M1) INVOLVED DURING MENTAL MOTOR IMAGERY (MMI) IN STROKE PATIENTS: A FUNCTIONAL MAGNETIC RESONANCE IMAGING (FMRI) STUDY

N. Sharma, L.H. Simmons, P.S. Jones, D.J. Day, T.A. Carpenter, E.A. Warburton, J.C. Baron

University of Cambridge, Cambridge, United Kingdom

Background: Understanding brain plasticity mechanisms after stroke is important in predicting recovery and developing rehabilitation strategies. Active movement therapies that involve voluntary drive can improve recovery and influence cortical re-organisation, but depend upon motor performance. MMI is widely used by athletes as a training method to improve performance, raising the possibility of applying it to stroke patients to promote recovery and access the motor network, especially M1, independently of performance. However there are conflicting views as to the involvement of M1 in MMI, this may be due, in part, to the concealed nature of MMI; subjects may surreptitiously employ alternative cognitive strategies or fail to suppress movement. We have developed a unique screening protocol that has allowed us to use block design fMRI to map MMI, specifically M1, after stroke whilst providing objective evidence of compliance before \& during the fMRI. Methods: 12 first ever sub cortical stroke patients ( 8 M 4 F; mean age 63.6yrs; Avg NIHSS 2; $3 \mathrm{w}-3 \mathrm{yr}$ post stroke) and 15 aged matched Controls (7 M 8 F; mean Age $52.7 \mathrm{yrs}$ ) were prospectively recruited. Exclusion criteria; non compliance, M1 destroyed, critical stenosis, left handedness. Subjects performed MMI \& Executed Movement (EM) of finger thumb opposition during fMRI ( 2 patients could not perform EM). Images were processed in SPM2; lesions were flipped so all were in the left hemisphere $(n=3)$.

Results: In controls both MMI \& EM of each hand activated the contra-lateral M1 but more so during EM then MMI. In stroke patients this relationship was preserved during MMI \& EM of the non-affected hand. MMI \& EM of the affected hand also activated contra-lateral M1, however there was no difference in the degree of activation between tasks $(n=10)$. This implies ipsilesional M1 may be over activated during MMI after stroke.

Conclusion: This early data supports MMI as a means to access the motor network in stroke and supports the role of M1 during MMI training. The possible over activation of M1 is intriguing \& warrants further investigation. 


\section{Recovery and rehabilitation}

VALIDITY OF THE TORONTO BEDSIDE SWALLOWING SCREENING TEST (TOR-BSST) IN THE REHABILITATION SETTING

R. Martino, G. Nicholson, M. Bayley, R. Teasell, F. Silver, N.E. Diamant

University of Toronto, Toronto Western Research Institute, Toronto, Canada

Background: A bedside dysphagia screening tool (TOR-BSSTC) was developed for stroke patients. The tool includes the best available evidence. It identifies patients at greatest risk for dysphagia and prioritizes them for earlier assessment.

Objective: To assess the validity of trained nurses in their administration of the TOR-BSSTC in the rehabilitation setting.

Methods: Over 3 years, trained nurses from two rehabilitation facilities in Ontario prospectively administered dysphagia screenings to consecutively admitted eligible stroke inpatients. A positive screen identified patients at high risk for dysphagia. Twenty percent of enrolled patients were randomly allocated to gold standard clinical and videofluoroscopic assessments administered by separate blinded expert raters. Patients with a positive screen but not randomized were assessed clinically by a blind expert rater.

Results: A total of 208 stroke rehabilitation inpatients were enrolled (male, 59.1\%; mean age, $69.1 \mathrm{y}$; mean days post stroke, 31.9; mean NIHSS, 7.2; mean FIM, 76.3; left cortical stroke, $37.5 \%$; right cortical stroke, $34.6 \%$; brainstem stroke, $15.4 \%$; cerebellar stroke, $5.3 \%$ ). The proportion of positive first screenings was $37.5 \%$. Comparison of screening with clinical judgments $(n=115)$ derived a sensitivity of $82 \%$; specificity of $39 \%$; positive likelihood ratio of 1.34 ; positive predictive value of $24 \%$; and negative predictive value of $90 \%$. Comparison of screening with videofluoroscopic judgments $(n=42)$ derived a sensitivity of $100 \%$; specificity of $61 \%$; positive likelihood ratio of 2.6; positive predictive value of $21 \%$; and negative predictive value of $100 \%$.

Conclusion: The TOR-BSST $($ C offers an accurate method by which to identify stroke patients with dysphagia in the rehabilitation setting. There is confidence that stroke patients with a negative screen will not have dysphagia.

\section{Recovery and rehabilitation}

\section{MODULATION OF MOTOR CORTICAL ACTIVITY IN CHRONIC STROKE} PATIENTS BY A SINGLE DOSE OF DONEPEZIL

D. Tombari, D. Lenzi, G. Sirimarco, N. Bonaffini, M. Pasquini, M. Altieri,

G.L. Lenzi, P. Pantano, V. Di Piero

University of Rome "La Sapienza", Rome, Italy

Background: Recovery after stroke involves several mechanisms aimed to reorganize brain networks and restore impaired functions. These mechanisms might be influenced by drugs. Several studies pointed to the effects of monoaminergic substances whereas less is known about the influence of cholinergic system. At cortical level, acetylcholine may act by increasing neuronal plasticity and improving attention.

The aim of this study was to assess the effect of a single dose of donepezil, a cholinesterase inhibitor, on motor performances and fMRI cerebral motor activation in chronic stroke patients.

Methods: We conducted a randomized, crossover, double-blind study on 10 patients with a chronic motor hemiparesis due to a single subcortical ischemic infarct localized on the pyramidal tract. Patients were randomly assigned in two counterbalanced groups and underwent two fMRI examinations a week apart, after donepezil and after placebo. During each fMRI session, patients performed an active motor task consisting of a finger flexion-extension of the paretic hand and underwent a passive motor task, consisting of a flexion-extension of the paretic wrist performed by an examiner. Motor performance was evaluated by finger tapping test performed with the paretic hand after a single dose of placebo and donepezil (at peak plasma concentration) immediately before fMRI examination. Data were analyzed with SPM2.

Results: Donepezil significantly improved motor skill of the affected hand. At fMRI, a single dose of donepezil induced an activation in sensorimotor areas that was significantly inferior to the activation achieved after placebo.

Discussion: Improvement in motor performances and general hypoactivation in sensorimotor areas, observed after a single dose of donepezil, suggest more efficiency in cerebral motor processing.

\section{Recovery and rehabilitation}

HOW MUCH THERAPY OCCURS IN ACUTE STROKE CARE UNITS? RESULTS FROM A MULTICENTRE OBSERVATIONAL STUDY J. Bernhardt, J. Chan, I. Nicola, J.M. Collier National Stroke Research Institute, Heidelberg Heights, Melbourne, VIC, Australia

Background: Information about the contribution of the multidisciplinary team to acute stroke unit care is sparse. In Melbourne, acute stroke patients spend more than $50 \%$ of the day in bed (Bernhardt, 2004). In this paper we examine: 1) the amount and content of therapy provided; and 2) who was with the patient when engaged in activity likely to promote recovery of mobility.

Methods: Patients less than 14 days post stroke (infarct or haemorrhage) were recruited from five Melbourne metropolitan acute stroke units. We excluded those receiving palliative care. Participants were observed at 10 -minute intervals from $0800-1700$ over 2 consecutive days. The proportion of the day patients spent with nurses, physiotherapist (PTs), occupational therapists (OTs) and speech therapists (SPs) and the physical activity of patients was derived. PTs, OTs and SPs detailed intensity and frequency of therapy (including upper limb) provided. We defined moderate to high functional activities (transfers, standing and walking) as those most likely to promote recovery of mobility.

Results: Fifty eight patients were observed. Twenty five therapists (12 PTs, 8 OTs and 5 SPs) participated. Nurses spent $14 \%$ of the day with patients and therapists $5 \%$. When patients were seen by therapists, average session time was 24 (PT), 23 (OT) and 33 (SP) minutes. However, not all patients received therapy and those that did, were rarely seen on consecutive days. The affected upper limb was treated for 5-13 minutes. When averaged over the 9 hour day, patients with a nurse, PT, OT or SP, spent 9, 7, 1 and 0 minutes respectively engaged in moderate to high activity. Conclusion: Multidisciplinary teams are considered important to quality stroke unit care. There is currently no evidence on which to base decisions about optimal scheduling and content of therapy to promote recovery in the acute stroke phase. This is the first study to detail current therapy practices in acute stroke care units.

\section{Recovery and rehabilitation}

\section{A MULTI-CENTRE RANDOMISED CONTROLLED STUDY OF EARLY TASK-RELATED TRAINING OF THE UPPER LIMB FOLLOWING STROKE M. Galea, K. Miller, B. Phillips}

The University of Melbourne, School of Physiotherapy, Parkville, Victoria, Australia

Our objective was to evaluate the efficacy of an early, intensive task-related training program designed to improve motor and sensory function of the hemiplegic upper limb following stroke. Subjects were recruited within six weeks of their first cortical stroke and randomly allocated to Treatment $(\mathrm{T})$ or Control $(\mathrm{C})$ interventions. All subjects received three weeks of daily intervention including assigned independent practice activities in addition to their standard therapy program. The $\mathrm{T}$ group received task-related training of the upper limb, emphasising uni-manual and bimanual functional activities. The $\mathrm{C}$ group received exercises to improve postural control and concentration. Assessments were conducted pre-and post-intervention and at three months post-intervention. Outcome measures were: motor recovery of the affected upper limb, assessed using the Chedoke-McMaster Stroke Assessment (CMSA), manual dexterity, grip and pinch forces, and hand sensation. Perceived quality of life was recorded using the Stroke-Adapted 30-Item Sickness Impact Profile at three-months post-intervention. Thirty subjects (16T and 14C) completed the study. While there were no statistically significant differences between the groups initially, the $\mathrm{T}$ group showed significantly greater gains in motor recovery of the arm on the CMSA $(\mathrm{p}<0.001)$, in pinch forces between affected thumb and index finger $(\mathrm{p}=0.04)$, and in hand sensation $(\mathrm{p}=0.01)$ at three months post-intervention. The $\mathrm{T}$ group also reported significantly better quality of life $(\mathrm{p}<0.001)$. The results of this study support implementation of early task-related training to improve motor and sensory function of the upper limb following stroke. Early intervention may be a critical factor in functional recovery. 


\section{Recovery and rehabilitation}

GRANULOCYTE-COLONY STIMULATING FACTOR MOBILISES BONE MARROW STEM CELLS IN SUB-ACUTE STROKE: THE 'STEM CELL TRIAL OF RECOVERY ENHANCEMENT AFTER STROKE'

N. Sprigg, M. Willmot, L. Zhao, M. Walker, M. Dennis, N. Russell, P.M.W. Bath

Institute of Neuroscience, University of Nottingham, Nottingham, United Kingdom

Background: Stem cells are capable of self renewal and of differentiating into multiple cell types, and transplantation of bone marrow stem cells in experimental stroke improves motor recovery. Granulocyte-colony stimulating factor (G-CSF) mobilises bone marrow stem cells in humans and improves outcome in experimental stroke. We assessed G-CSF in sub-acute clinical stroke.

Methods: A two centre, dose-escalation double blind, randomised placebocontrolled trial was performed in 36 patients with recent ischaemic stroke. Each dose block comprised 6 patients ( 4 active, 2 placebo): 1 dose $1 \times 10^{5}, 3 \times 10^{5}, 1 \times 10^{6}$ $\mathrm{u} / \mathrm{kg} ; 5$ once daily doses $1 \times 10^{5}, 3 \times 10^{5}, 1 \times 10^{6} \mathrm{u} / \mathrm{kg}$. The primary outcome was peripheral blood CD34+ count (measured by flow cytometry). Secondary outcomes included: white cell count (WCC), safety, tolerability and functional outcome.

Results: 36 patients, mean age 75 (standard deviation SD 8.5), male 18, median SNSS 25 (interquartile range IQR 17-35) were randomised to receive one of six doses of G-CSF $(n=24)$ or placebo $(n=12)$. G-CSF increased mean CD34+ count in a dose-dependent manner (area under curve AUC, $\mathrm{p}=0.005$ ), increasing from 2.5 cells $\times 10^{6} / 1$ to 37.7 cells $\times 10^{6} / 1$ at day $5\left(5\right.$ doses of $\left.1 \times 10^{6} \mathrm{u} / \mathrm{kg}\right)$. A dose-dependent rise in WCC (AUC, $\mathrm{p}<0.001$ ) was also seen. No significant change in platelet or red count was seen. The number of serious adverse events (G-CSF 7/24 vs. placebo $3 / 12$ ) and patients achieving independence (median modified Rankin Scale 3 in both groups) did not differ significantly between treatment groups.

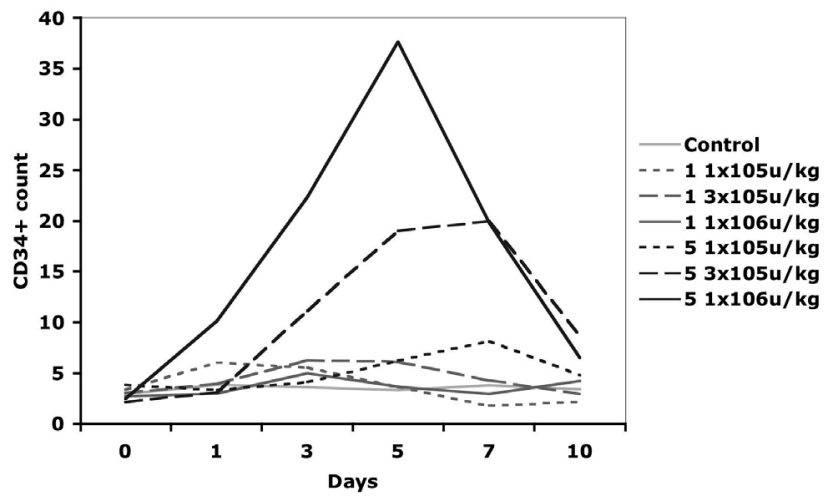

Conclusions: G-CSF is effective at mobilising circulating stems cells in patients with recent ischaemic stroke. Administration appears safe, feasible and is well tolerated. Further studies assessing G-CSF after stroke are warranted.

\section{Recovery and rehabilitation}

FUNCTIONAL MRI CORRELATES OF MOTOR RECOVERY AFTER TREADMILL TRAINING PROVIDE EVIDENCE OF TREATMENT INDUCED FUNCTIONAL PLASTICITY IN THE BRAIN IN SUBJECTS WITH ISCHEMIC STROKE

C. Enzinger, H. Johansen-Berg, H. Dawes, M. Bogdanovic, S. Ropele,

J. Collett, D. Wade, U. Kischka, F. Fazekas, P.M. Matthews

Medical University Graz, Graz, Austria

Background: There is increasing evidence that specific forms of motor rehabilitation therapy of the lower limbs may be effective in re-establishing function after ischemic stroke. However, treatment response is highly variable and also little is known about the changes in brain activities that are associated with performance gains. We therefore set out to perform serial functional MRI (fMRI) to characterise functional changes in the brain that correlate with behavioural improvements.

Methods: We studied 18 subjects with a residual walking impairment due to a singular subcortical ischemic stroke at least 6 months prior to study entry $(8 \mathrm{~F} / 10 \mathrm{M}$; mean age 59.9 \pm 13.5 , range 32-74 yrs). Participants had a mean Functional Ambulation Category of $4.4 \pm 0.6$ (3-5), a mean Rivermead Disability Index of $12.9 \pm 1.9$ (8-15), a modified Barthel Index of $18.7 \pm 1.6(15-20)$, and the Motricity Scores of the affected arm and leg were $73 \pm 23$ (9-99) and $78.3 \pm 10.4$ (58-91), respectively. They underwent 12 sessions of treadmill training with partial body weight support over 4 weeks. fMRI scans at 3.0T while active and passive ankle dorsiflexion movement were obtained twice before and twice after physiotherapy (EPI TR $=3000 \mathrm{~ms}, \mathrm{TE}=30 \mathrm{~ms}, \mathrm{FOV}=256 \times 256$, matrix $=64 \times 64)$. Behavioural testing included gait kinematicographics (GAITRite; SMS Technologies, Harlow). The control group comprised of 18 age- and sexed-matched healthy volunteers (HCs). fMRI data were analysed using FSL (FEAT 5.4).

Results: Movement of the hemiparetic limb elicited a brain response in a widespread supraspinal network including the contralateral primary motor and premotor cortex, bilateral secondary somatosensory cortices, the vermis cerebelli and regions of the cerebellar hemispheres bilaterally. Regarding the entire cohort of patients, there were no significant differences in activation patterns compared to HCs. However, compared to HCs, patients with worse motricity (cut-off: median of 77) of the affected limb demonstrated significantly increased activation in ipsilateral primary motor and premotor regions. No such differences were observed for movement of the unaffected limb. The brain response to passive movement mirrored that observed with active movement both in patients and HCs. There was no evidence of significant intersession variance between two baseline scans before treatment. Therapy related improvements in walking speed correlated with a distinct pattern of increase in fMRI activity, involving the primary sensorimotor and premotor cortices contralateral to the affected lower limb.

Conclusion: This study corroborates the utility of ankle dorsiflexion as an indirect probe of the supraspinal sensorimotor network for the neural control of walking. Importantly, the paradigm used proved both stable and feasible in a disabled patient group which bodes well for its future clinical applicability. Furthermore our results suggest that stroke victims might benefit from a dedicated gait therapy even in chronic stages, and that performance gains can be correlated with distinct activity changes in sensorimotor regions.

\section{Recovery and rehabilitation}

\section{RESIDUAL VISUAL PROCESSING AND THE EFFECT OF A VISUAL REHABILITATION PROGRAMME IN STROKE PATIENTS WITH CORTICAL BLINDNESS}

M.M. Macleod, C. Trevethan, A. Sahraie

University of Aberdeen, Aberdeen, United Kingdom

Background: Lesions of the occipital cortex may lead to areas of cortical blindness affecting the corresponding regions of the patient's visual field. Although the traditional view suggested that following a limited spontaneous recovery in acute stages, such areas of blindness are absolute and permanent, some residual visual capacities may persist. Such residual visual capacities are termed blindsight. To date, a systematic investigation of the spatial properties of any residual vision in a cohort of cortically blind patients has not been carried out.

Methods: We have devised a protocol to systematically study the properties of residual vision within the field defects of a group of patients with areas of cortically blind visual fields $(n=16)$ using psychophysical techniques. We have demonstrated the presence of a spatial channel of processing in 12 of the cases investigated. Detailed knowledge of the characteristics of residual visual processing provided crucial information for the development of a novel visual rehabilitation protocol based on repeated stimulation within the visually blind areas.

Results: In a group of 12 patients suggest that the visual sensitivity within the field defect can be increased following the training. There is also evidence for a modest, but significant shrinkage of the field defect as measured using standard perimetry. Discussion: These findings suggest that (a) blindsight in cases of cortical blindness may not be a rare phenomenon and (b) repeated stimulation based on appropriate visual stimuli may result in improvements in visual sensitivities.

\section{Recovery and rehabilitation}

\section{EFFECTS OF CORTICAL STIMULATION ON VISUOMOTOR INTEGRATION AND FORCE IN STROKE}

F. Hummel, B. Voller, P. Celnik, A. Floel, P. Giraux, C. Gerloff, L.G. Cohen Clinical Brain Research, Eberhard-Karls University, Tuebingen, Germany

Background: Brain polarization by anodal transcranial DC stimulation (tDCS) applied to the primary motor cortex of the affected hemisphere (M1-affected) enhances beneficial training effects on complex activities of daily living (ADL) [1]. Which mechanisms underlie these tDCS-induced improvements? One possible mechanism involves upregulation of activity within M1-affected which could conceivably improve sensorimotor integration and/or force production as basic components of skilled motor functions that rely on M1 activity. Alternatively, tDCS could modulate nonspecific functions such as motivation or attention. These questions has been addressed in the present study. 
Methods: We investigated the effects of anodal tDCS applied to M1-affected on pinch force (PF), a function that relies predominantly on M1 activity, and on visuomotor integration determined by simple reaction times (RT) in patients with chronic stroke in a double-blind cross-over sham-controlled study design.

Results: Anodal tDCS shortened reaction times (RT-Post-tDCS $=256.6 \pm 13.9 \mathrm{msec}$ ) and improved pinch force (PF-Post-tDCS $=124.8 \pm 24.0 \mathrm{~N}$ ) in the paretic hand relative to baseline (RT-Base-tDCS=267.5 $\pm 15.4 \mathrm{msec}$; PF-Base-tDCS $=118.8 \pm 23.0 \mathrm{~N}$ ) and to Sham (RT-Post-Sham=277.7 $\pm 11.6 \mathrm{msec}$; PF-Post-Sham=111.2 $\pm 19.8 \mathrm{~N}$ ) in the absence of measurable changes in attention, fatigue and discomfort.

Discussion: These findings suggest that the mechanisms underlying anodal tDCSinduced improvement in ADLs previously reported [1] are more likely related to direct effects on primary functions mediated by M1-affected than on nonspecific attentional or motivational influences. Further, they propose that tDCS, through modulation of activity in M1-affected, could benefit more impaired patients who cannot or have substantial difficulties in training complex ADLs but are able to train simpler motor tasks.

Reference: [1] Hummel et al. Brain (2005) 128:490-9

\section{Recovery and rehabilitation}

TRUNK PERFORMANCE ON ADMISSION TO THE REHABILITATION SETTING IS A SIGNIFICANT PREDICTOR OF BARTHEL INDEX SCORE AT 6 MONTHS AFTER STROKE

G. Verheyden, A. Nieuwboer, L. De Wit, H. Feys, B. Schuback, I. Baert, W. Jenni, W. Schupp, V. Thijs, W. De Weerdt

Katholieke Universiteit Leuven, Leuven, Belgium

Background: Trunk performance is an important predictor of functional recovery after stroke. However the explained variance reported in different studies varies considerably. This could be partly due to a ceiling effect of the scales used. The Trunk Impairment Scale (TIS) evaluates static and dynamic sitting balance and trunk coordination. The TIS has no ceiling effect. It was the aim of this study to evaluate the prognostic value of the TIS and its sub-scales when predicting Barthel index scores at six months after stroke.

Methods: A total of 102 subjects participated from three European rehabilitation centers. Participants were assessed on admission and at six months after stroke. General characteristics and measures of motor and functional performance on admission were included as variables in the prediction model. Stepwise multiple regression analysis was used to model outcome.

Results: The first predictors retained from the model were total TIS score (partial $\mathrm{R}^{2}=0.52, \mathrm{p}<.0001$ ) and static sitting balance sub-scale score (partial $\mathrm{R}^{2}=0.50$ $\mathrm{p}<.0001)$ if the sub-scales of the TIS were included in the model. Both were stronger predictors than Barthel index score on admission itself.

Discussion: This study emphasizes the importance of trunk performance when predicting functional outcome after stroke. The TIS and its static sitting balance sub-scale are quick and easy to perform clinical tools and recommended in the rehabilitation setting when considering the prognosis of stroke patients. Rehabilitation targeted at improving trunk performance should be studied in clinical trials.

\section{Recovery and rehabilitation}

\section{REPETITIVE TRANSCRANIAL STIMULATION (RTMS) AND/OR VOLUNTARY MOTOR CONTRACTION (VMC) TO ENHANCE UPPER LIMB} RECOVERY AFTER STROKE. A FEASIBILITY STUDY

V. Pomeroy, S. Miller, G. Cloud, C. Donaldson, V. Nayak, R. Tallis St George's University of London, Geriatric Medicine University of Manchester, United Kingdom

Aim: To test the efficacy of rTMS and VMC to improve corticospinal transmission, muscle function and purposeful movement. Design Factorial $2 \times 2$ randomised single-blind trial.

Subjects: $n=27$, mean age 75 years, mean 27 days after MCA infarct, a voluntary twitch of paretic arm muscle, able to follow a 1-stage command, no visuospatial neglect or contraindication to rTMS

Procedure: After giving written informed consent and undergoing baseline measurement subjects were randomised to 1 of 4 groups with a concealed computergenerated order stratified into lacunar or non-lacunar lesions. Treatment was given for the next 8 working days. Outcome measures were made 2 weeks after baseline. Interventions: Real-rTMS + RealVMC; Real-rTMS + PlaceboVMC; PlaceborTMS + RealVMC; Placebo-rTMS + PlaceboVMC. Real-rTMS consisted of 200 $1 \mathrm{~Hz}$ stimuli at $120 \%$ motor threshold in 5 blocks of 40 separated by $3 \mathrm{~min}$. Placebo-rTMS used a dummy coil. In Real-VMC the elbow flexed/extended for $5 \mathrm{~min}$. In Placebo-VMC subjects viewed pairs of drawings of upper limbs and reported likeness or not. Each subject then trained the paretic upper limb by turning a cranked wheel. Outcomes Primary, frequency of MEPs in Biceps and Triceps (1Hz rTMS). Secondary, muscle function (torque around elbow) and functional ability (Action Research Arm Test).

Results: Mean changes, outcome-baseline. In the Real-rTMS + RealVMC group MEP frequency increased $14 \%$ for Biceps and $20 \%$ for Triceps. Placebo-rTMS + PlaceboVMC group MEP frequency decreased 12\% for Biceps and $6 \%$ for Triceps. For other groups there were changes of intermediate values. No meaningful differences were found for secondary outcomes.

Conclusion: The positive results found for Real-rTMS + RealVMC justify further research into the neurofacilitatory effects of these interventions.

\section{Recovery and rehabilitation}

MEASURING AGREEMENT IN VIDEOFLUOROSCOPIC RATINGS OF SWALLOWING IMPAIRMENT IN STROKE PATIENTS

R. Martino, M. Kowgier, F. Silver, N.E. Diamant, G. Tomlinson

University of Toronto, Toronto Western Research Institute, Toronto, Canada

Background: Poor reliability has been reported for videofluoroscopic swallowing assessment (VFS). Hence there is no 'gold-standard' for the diagnosis of dysphagia; instead we derive a diagnosis from a combination of diagnostic items.

Purpose: To use a Bayesian model to infer the unobserved (latent) dysphagia status given the results of three widely used diagnostic tests Penetration-Aspiration Scale (PAS), MASA dysphagia sub-score (MASA-DYS), and MASA aspiration sub-score (MASA-ASP).

Methods: The three diagnostic tests (dichotomized as 'adequate' and 'inadequate') were each evaluated on 43 rehabilitation stroke patients by four raters. Raters were two acute and two rehabilitation speech language pathologists all with expertise in dysphagia. A latent class model was fitted that allowed for differences between the raters and between the effectiveness of the three diagnostic items with respect to classification as dysphagic.

Results: Regarding the latent dysphagia status as the 'true' diagnosis, the overall prevalence of dysphagia was 0.35 . The prevalences varied between raters: for rehabilitation clinicians they were 0.44 and 0.44 , whereas for acute clinicians they were 0.32 and 0.23 . One of the acute clinicians was less likely to classify a patient as having dysphagia compared to the both the rehabilitation clinicians $(\mathrm{OR}=0.36$, 95\% CR: 0.13-0.97; OR=0.42, 95\% CR: 0.13-0.98). For each diagnostic item, the rating of inadequate was independently predictive of dysphagia. Estimates of sensitivity for the diagnostic items PAS and MASA-DYS, were near $50 \%$ for all raters, while the MASA-ASP item attained a higher sensitivity near $94 \%$ for all raters. A gold standard may be suggested by evaluation of all three items as 'inadequate' for which the probability of dysphagia is approximately 1 for each rater.

Conclusions: A latent class model may be useful as a formal way of combining results from several diagnostic tests in the absence of a dysphagia gold standard.

\section{Recovery and rehabilitation}

\section{ICF CORE SETS IN STROKE}

K. Stibrant Sunnerhagen

Institute of Neuroscience and Physiology, Rehabiliation Medicine, Sweden

Background: International Classification of Functioning (ICF) is WHO's framework for measuring health and disability at both individual and population levels. While the International Classification of Diseases (ICD) classifies diseases, ICF classifies health. ICF is a multipurpose classification aiming to provide a scientific base for understanding and studying health and health-related states, to establish a common language for describing health, to permit comparison of data and to provide a systematic coding scheme.

Method: ICF organizes information in two parts. Part 1 deals with Functioning and Disability comprising body functions and structures as well as activities and participation. Part 2 covers Contextual Factors comprising environmental and personal factors. In cooperation with WHO and in international work ICF Core Sets for stroke has been developed. The development was based on evidence (preliminary studies as multicentric study of patients data, delphi exercises and literature review) and a consensus process. An ICF Core Set is a list with ICF categories that are relevant to most patients with a specific health condition.

Material: The first version of the developed ICF Core Sets is now tested in a multicenter international validation study in 49 countries. Göteborg coordinates the testing of the Stroke ICF Core Set in Sweden. The patient answers questions on co-morbidity, SF-36 and SIS, while health professionals complete the Stroke ICF Core Set, which contains 59 ICF categories of body functions, 11 of body structures, 59 of activities and participation and 37 of environmental factors.

Results: In Sweden, data from more than 100 persons with stroke had been collected. Most commonly affected were memory, sleep and energy drive func- 
tions, structures of brain, handling stress and other psychosocial demand, making decision, moving around. Family and friends as well as their attitudes were the main facilitators for the patients.

\section{Recovery and rehabilitation}

STROKE SEVERITY, EARLY RECOVERY AND OUTCOME ARE ALL RELATED WITH AETIOLOGICAL STROKE SUBTYPE: DATA FROM THE TINZAPARIN IN ACUTE ISCHAEMIC STROKE TRIAL' (TAIST)

N. Sprigg, L.J. Gray, P.M.W. Bath, on behalf of the Tinzaparin in Acute Ischaemic Stroke Trial' (TAIST) Trialists

Institute of Neuroscience, University of Nottingham, Nottingham, United Kingdom

Introduction: Baseline severity and aetiological subtype are predictors of outcome in stroke. We used data from the 'Tinzaparin in Acute Ischaemic Stroke Trial' (TAIST) to further assess the relationship between stroke severity, early recovery, outcome and stroke subtype.

Methods: TAIST was a randomised controlled trial assessing the safety and efficacy of tinzaparin versus aspirin in 1,484 patients with acute ischaemic stroke Severity was measured as the Scandinavian Neurological Stroke Scale (SNSS) at baseline and days 4, 7 and 10. Aetiological subtype was assigned after standard investigations. The relationship between SNSS at days 4 and 10 and stroke subtype was assessed using ANOVA. Recovery was calculated as change in SNSS from baseline at day 4 and day 10 . The relationship between poor outcome (modified Rankin scale $>2$ ) and subtype was assessed using logistic regression.

Results: Stroke severity at both 4 and 10 days was significantly different between all aetiological subgroups (both $\mathrm{p}<0.0001$ ) with cardio-embolic $(\mathrm{CE})$ the most severe and small vessel disease (SVD) the mildest.

Early recovery was greater in SVD than in both large artery disease (LAD) and CE at day 4 (mean improvement in SVD 4.4, LAD 1.8, CD 2.4) and day 10 (SVD 8.0, LAD 4.9, CE 5.7). The odds of having a poor outcome at 90 days were reduced in SVD (odds ratio (OR) $0.20,95 \%$ confidence interval (CI) 0.14-0.27) and LAD (OR $0.53,95 \%$ CI $0.38-0.76$ ) compared to $\mathrm{CE}$

Conclusions: Severity in the first 10 days following stroke is significantly related to aetiological subtype, with CE the most severe. Early recovery is greatest in SVD and does not differ between LAD and CE stroke. Poor outcome is increased in CE compared to both LAD and SVD.

\section{Recovery and rehabilitation}

\section{ROBOTIC IN HAND REHABILITATION - AN INNOVATION IN HAND \\ THERAPY}

P. Grieshofer, M. Scarpatetti, R. Scherer, D. Ram

Klinik Judendorf Strassengel, Austria

Background: The "Klinik Judendorf Strassengel" developed in cooperation with the technical university of Graz this technical device

Methods: The development of the hand robotic System after 4 years of research and 3 years of engineering has produced a crucial improvement in hand rehabilitation Therapy. Our robotic hand and finger movement orthosis is being used successfully in our clinic as a prototype of the institute of neurorehabilitation and research.

This hand robotic system is the first driven orthosis that assists finger movements of upper limb impaired patients. The theoretical background is the experience of the locomotion therapy. This automated process relieves therapists of the manual labour; therefore, the training sessions can be longer and more repeatable. The therapy is more efficient and the patients achieve their goals faster. Even in the early phases of the rehabilitation, one therapist can accomplish the training. We are using this hand robotic system as a prototype about half a year and our clinic practice shows after viewing patients that rehabilitation of the hand could be accelerated.

Results: Our first experience shows, that the rehabilitation of the hand could be accelerated and the spasticity is going down.

Conclusion: We know we need more experience and data but we want to present this technology as an new opportunity for hand rehabilitation.

\section{Risk factors of stroke}

\section{Risk factors of stroke}

HOW WELL CAN PRIMARY CARE PHYSICIANS, NURSES AND COMPUTER SOFTWARE DETECT ATRIAL FIBRILLATION ON ECG?

J. Mant, D. Fitzmaurice, R. Hobbs, S. Jowett, E. Murray, H. Roger, D. Michael, L. Greg

University of Birmingham, Birmingham, United Kingdom

Background: Atrial fibrillation (AF) is an important risk factor for stroke present in about $5 \%$ of people over the age of 64 , but may be asymptomatic. It can be diagnosed with a simple electro-cardiograph (ECG) which can be performed in primary care, but there are few data on the ability of primary care physicians (GPs) to read ECGs and so correctly diagnose AF. The aim of this study was to assess the accuracy with which GPs, primary care nurses (PNs) and computer software can diagnose $\mathrm{AF}$

Methods: 50 general practices in England were recruited to participate in a trial of screening for AF. During the study, 2,592 ECGs were performed on patients aged over 64 in these practices - 631 in people with an irregular pulse and the rest following a screening invitation. The ECGs were all read by interpretation software and independently by two cardiologists (the reference standard). 1,696 ECGs were randomly selected and batches of 33 or 34 were sent to the 50 participating GPs and PNs, who were asked to record whether or not AF was present.

Results: Prevalence of AF (from cardiologist interpretation) was $8.4 \%$. GPs correctly diagnosed 79 of 99 cases of AF (see table), but of 193 GP diagnoses of AF, 114 were in sinus rhythm (SR). PNs detected a similar proportion of the cases of $\mathrm{AF}$, but had a lower positive predictive value. The software was significantly more accurate than GP or PN ECG interpretation, but missed 26 cases of AF (13\%), and out of 200 software diagnoses of AF, $21(10 \%)$ showed SR.

Interpretation of ECGs by reade

\begin{tabular}{lcccc}
\hline & Sensitivity & Specificity & $\begin{array}{c}\text { Positive predictive } \\
\text { value } 40.9\end{array}$ & $\begin{array}{c}\text { Negative predictive } \\
\text { value } 98.4\end{array}$ \\
\hline General Practitioner & 79.8 & 91.6 & 40.9 & 98.4 \\
Practice nurse & 77.1 & 85.1 & 27.2 & 98.1 \\
\hline
\end{tabular}

Discussion: Many GPs cannot detect AF reliably on an ECG, and interpretative software is not sufficiently accurate to circumvent this problem. Programmes for community screening for AF need to factor in the reading of the ECG by appropriately trained individuals.

\section{Risk factors of stroke}

THE EARLY RISK OF STROKE AFTER VERTEBROBASILAR TIA IS HIGHER THAN AFTER CAROTID TIA

E. Flossmann, E. Touze, M.F. Giles, C.E. Lovelock, P.M. Rothwell

University Radcliffe Infirmary, Oxford, Oxford, United Kingdom

Background: Several small studies in the 1960s and 70s suggested that vertebrobasilar (VB) TIAs had a lower risk of stroke than carotid events, and so patients are often managed less aggressively. In contrast, in a recent systematic review of all studies, we found that VB TIA appeared to be associated with a higher early risk than carotid TIA.

Methods: To confirm or refute this observation, we studied the risk of stroke after TIA by vascular territory of the presenting event (excluding stroke on the same day) in a population based incidence study (Oxford Vascular Study 2002-2005). Vascular territory was classified by two independent neurologists, with adjudication by a third.

Results: Of 256 patients with TIA, $212(82.5 \%)$ had a carotid event and $44(17.1 \%)$ had a VB event. Interobserver agreement for vascular territory was very good (kappa $=0.87, \mathrm{p}<0.001)$. There were 50 ischaemic strokes on follow-up. The early risk of stroke was higher after VB than after carotid events (7-days: $15.9 \%$ vs $9.4 \%$ $\mathrm{p}=0.22$, 30-days: $20.5 \%$ vs $12.3 \% \mathrm{p}=0.16$ ) with a significant difference at 1 -year $(31.8 \%$ vs $17.0 \%$, hazard ratio $=2.0,95 \% \mathrm{CI} 1.1-3.7, \mathrm{p}=0.03)$. The 1 -year risk after VB TIA was slightly higher than that after carotid TIA even in patients with $>50 \%$ ipsilateral carotid stenosis (HR 1.3, 0.5-3.3, p=0.54). Vascular risk factors did not differ significantly between patients with VB vs carotid TIA

Discussion: Patients with VB TIA have a very high risk of ischaemic stroke, comparable to that in patients with symptomatic carotid stenosis, suggesting that the underlying pathology may often also be large artery atherosclerosis and highlighting the need for urgent investigation and treatment and for trials of interventions such as vertebral artery stenting in appropriate cases. 


\section{Risk factors of stroke}

THE COMBINED MEASUREMENT OF IMT, ABI AND HSCRP IMPROVES THE PREDICTION OF NEW CEREBROVASCULAR EVENTS COMPARED TO TRADITIONAL RISK FACTORS. THE INVADE STUDY

D. Sander, C. Schulze-Horn, H. Gnahn, K. Sander, B. Conrad

Technical University of Munich, München, Germany

Background: In addition to conventional risk factors, several new risk indicators like intima-media-thickness (IMT), ankle-brachial-index (ABI) and high-sensitivity (hs)-CRP have been used to define highh-risk patients. Up to now, limited information exists regarding the usefulness of these indicators combined for risk stratification. We investigated the joint impact of these parameters compared to conventional risk factors in predicting vascular events (a composite of myocardial infarction, stroke, and vascular death) and particular stroke.

Methods: We analyzed the data of INVADE (Intervention project on cerebrovascular diseases and dementia in the community of Ebersberg, Bavaria), an ongoing, prospective and population-based study conducted in 3532 subjects older than 55 years (mean, 68 years, $41 \%$ male). In addition to several common risk factors, measurements of carotid IMT, ABI, homocysteine, and hsCRP were performed at baseline. All subjects were followed up for at least 36 months (mean $44 \pm 3$ ).

Results: During follow-up, 246 vascular events including 102 strokes occurred. We observed a significant association between IMT (Hazard ratio (HR) 1.6), hsCRP (HR 2.5), ABI (HR 1.7), as well as homocysteine (HR 1.3) and new vascular events even after adjustment for conventional risk factors using Cox proportional hazard regression. The combination of IMT or ABI with hsCRP (HR $4.3[2.3 ; 6.6]$ and $5.1[2.7 ; 7.1])$ significantly improved the predictive power of the risk indicators, whereas the combination with homocysteine did not alter the predicitve power in the Cox model. The highest HR to predict vascular events as well as stroke (HR $5.4[2.5 ; 9.7]$ was observed for the combination of ABI and hsCRP.

Discussion: The combined measurement of hSCRP with $\mathrm{ABI}$ and/or IMT is a strong predictor for new cerebrovascular events and adds prognostic information at all levels of calculated Framingham risk.

\section{Risk factors of stroke}

THE STROKE OFFSPRING STUDY (SOS): STROKE RISK FACTORS IN THE OFFSPRING OF STROKE PATIENTS

N.D. Hart, M.E. Cupples, M.I. Wiggam, J.W. Yarnell

Queen's University Belfast, Belfast, United Kingdom

Background: It is known that parental history is a risk factor for stroke. However it is unclear whether the offspring of stroke patients have a higher prevalence of modifiable stroke risk factors than others in the general population. This knowledge would be an important precursor to the planning of population screening programmes.

Methods: Participants were recruited from 12 general practices across Northern Ireland. Practices were chosen to provide a mix of practice size, socioeconomic, ethnic, religious and urban/rural characteristics. Questionnaires, asking about parental history of stroke, were sent to 300 randomly selected individuals aged 40-64 years from each practice. From the returns, those with a parental history of stroke (cases) were matched on age, gender and socioeconomic status to those with no parental history of stroke (controls). The matched pairs were invited to meet the researcher at their practice address. Attendees answered questions about smoking, alcohol and medical history and completed diet and exercise questionnaires. Height, weight, blood pressure and serum lipids and glucose were measured.

Results: 433 individuals were screened and yielded 199 case-control pairs (398 individuals). Systolic and diastolic blood pressures were significantly higher in the cases than in paired controls (systolic 146.2 vs $140.6 \mathrm{mmHg}(\mathrm{p}<0.01)$; diastolic 87.7 vs $85.0 \mathrm{mmHg}(\mathrm{p}=0.014))$. Cases consumed more alcohol than their paired controls ( 13.7 vs 10.0 units/wk $(\mathrm{p}<0.01)$ ) but did not differ significantly from them in respect of BMI, lipids, diabetes, diet or exercise.

Discussion: Given the importance of blood pressure as a risk factor for stroke these results support the early screening of the offspring of stroke patients for raised blood pressure.

\section{Risk factors of stroke}

LIPOPROTEIN (A) AND RISK OF ISCHEMIC STROKE IN YOUNG ADULTS M. Rigal, J.B. Ruidavets, A. Viguier, R. Petit, B. Perret, J. Ferrieres, V. Larrue University of Toulouse, Toulouse, France

Lipoprotein (a) [Lp(a)] is composed of LDL-cholesterol particles linked to apoprotein (a) [apo(a)]. High Lp(a) plasma level is a risk factor for coronary heart disease and, in older men, for ischemic stroke. The role of $\operatorname{Lp}(\mathrm{a})$ as a risk factor for ischemic stroke in young adults is uncertain.

Methods: $\mathrm{Lp}$ (a) concentration was prospectively measured in 100 consecutive patients with acute ischemic stroke (58 men and 42 women) aged 18-55 years, and in 100 controls matched for age and gender.

Results: The distribution of $\mathrm{Lp}$ (a) concentration was skewed toward the highest and median tertiles in male patients. In multivariate logistic regression analyses adjusting on classical risk factors for ischemic stroke and lipid variables, $\mathrm{Lp}(\mathrm{a})$ concentration in the highest or median tertiles compared with the lowest tertile was significantly associated with ischemic stroke in men (OR 4.64, 95\% CI 1.58-13.64, $\mathrm{p}=0.006$ ), but was not in women (OR 0.40, 95\% CI 0.13-1.21, $\mathrm{p}=0.11$ ). Although large vessel atherosclerosis was more common in men than in women, there were no differences in $\mathrm{Lp}$ (a) concentration according to the cause of ischemic stroke.

Conclusion: Among subjects aged 18-55 years, a slightly elevated Lp(a) concentration was strongly and independently associated with ischemic stroke in men, but not in women. Further studies are required to elucidate the mechanisms underlying this gender specific association.

\section{Risk factors of stroke}

LIPOPROTEIN (A) AND STROKE: A META-ANALYSIS OF OBSERVATIONAL STUDIES

V. Thijs, B. Smolders

University Hospitals Leuven, Leuven, Belgium

Background: The relationship between elevated lipoprotein (a) levels and stroke is controversial. We systematically reviewed the literature to determine whether lipoprotein (a) is a risk factor for stroke.

Methods: Studies published from January 1960 through December 2005 were identified from electronic databases (MEDLINE), bibliographies of pertinent review articles and pertinent original articles, textbooks. From 261 potentially relevant English language references retrieved, we excluded studies lacking adequate controls, studies of children with stroke, and studies investigating carotid atherosclerosis and studies lacking adequate data to determine odds ratios (OR) or relative risks (RRs) and confidence intervals (CIs). Twenty seven studies with more than 24000 subjects met all inclusion criteria and were included in the meta-analysis.

Results: In case control studies ( $\mathrm{n}=19$ ) elevated $\mathrm{Lp}(\mathrm{a})$ was a risk factor for stroke (OR 2.3,95\% CI 1.8-3.0, $\mathrm{p}=0.001$ ). Sensitivity analysis did not find an influence of age, sex, measurement period of $\mathrm{Lp}(\mathrm{a})$ in relationship to stroke episode and whether adjustment for confounding variables was performed. There was no evidence of publication bias. In nested case control studies ( $\mathrm{n}=4$ with 1218 subjects) elevated $\mathrm{Lp}(\mathrm{a})$ was not a major risk factor for incident stroke (OR 1.1, 95\% CI $0.8-1.4$, $\mathrm{p}=0.75$ ). In prospective cohort studies ( $\mathrm{n}=4$ with 21232 subjects), subjects with the highest $\mathrm{Lp}$ (a) levels had a higher risk of incident stroke (RR 1.3, 95\% CI 1.0-1.7, $\mathrm{p}=0.032$ ).

Conclusion: This meta-analysis suggests that elevated lipoprotein (a) is a modest risk factor for incident stroke.

\section{Risk factors of stroke}

\section{ACTIVE HUMAN CAROTID ARTERY PLAQUES EXPRESS ENDOGENOUS} C-REACTIVE PROTEIN

J. Krupinski, M. Miguel Turu, J. Martinez Gonzalez, A. Carvajal Collado, O. Juan-Babot, E. Iborra, M.A. Slevin, F. Rubio, L. Badimon

University Hospital of Bellvitge (HUB), Barcelona, L'Hospitalet de Llobregat (Barcelona), Spain

Background: There is growing evidence suggesting that C-reactive protein (CRP) is an effecter molecule able to induce and promote atherothrombosis. The presence of CRP in atherosclerotic plaques may reflect local production and/or infiltration from circulating CRP increased in general inflammatory responses. Our aim was to analyze the presence of CRP in human advanced complicated and non complicated carotid artery plaques.

Methods: Human carotid artery specimens from 38 patients undergoing scheduled endarterectomy were classified into 3 groups: ulcerated (non-complicated) (UNC, $\mathrm{n}=19$ ), fibrous $(\mathrm{F}, \mathrm{n}=12)$ and ulcerated (complicated/hemorrhagic) plaques (UC, $\mathrm{n}=7$ ). The presence of CRP was evaluated by immunohistochemistry and plasma samples were screened for circulating high-sensitivity C-reactive protein (hs-CRP). TaqMan Low-density Arrays were used for study of CRP gen.

Results: CRP mRNA levels were predominantly detected in UNC-high risk plaques but not in UC ( $\mathrm{P}=0.001)$. Plaque CRP mRNA levels correlated with immunohistochemical findings but were independent of plasma hs-CRP. In UNC plaques endothelial cells and inflammatory cells were strongly positive for CRP around areas of newly formed microvessels.

Risk factors of stroke 
Conclusions: In human high-risk carotid artery plaques (UNC) CRP expression reflects an active pro-inflammatory stage. Local synthesis of CRP could be involved in plaque neovascularization and increased risk of hemorrhagic transformation.

\section{Risk factors of stroke}

\section{STROKE IN PREGNANCY}

P. Finet, S. Jeangette, C. Cuvelier, M. Pandolfo, S. Blecic

Erasme Hospital, Brussels, Belgium

Background: Pregnancy and puerperium increase the risk of stroke in women by 13 folds. Different causes can be found but both relationships with pregnancy and time distribution around childbirth remain unsettled

Objective: To assess the causes of stroke in pregnancy and puerperium

Methods: From 1991 to 2005 out of the 5048 patients (pts) admitted to the stroke Unit, 51 women $(1.0 \%)$ with a mean age of $33.0 \pm 7.2$ years, were referred for stroke during pregnancy or puerperium. 50/51 have had a normal gestation in their past history. They were either admitted via our obstetric department or referred from other hospitals. All pts had a complete stroke work-up, including in all urine and blood tests,MRI,MRA, trans-thoracic and trans-oesophageal echographies, transcranial Doppler, and coagulation tests.Lumbar puncture and catheter angiography (only after delivery) were performed in selected cases only. In all pts coagulation test were controlled 3 months after childbirth.

Results: 14 pts had venous stroke and 37 had arterial stroke. In pts with cerebral venous thrombosis (CVT), a coagulation disorder (CD) was found in 8: $1 \mathrm{pt}$ had congenital deficit in protein S, 2 pts had congenital deficit in protein $\mathrm{C}$ and 5 had resistance to activated protein $\mathrm{C}$ all with mutation of the factor $\mathrm{V}$ of Leyden. In the remaining 4 pts no cause other than pregnancy it self was found. In pts with arterial stroke, the following causes were found: idiopathic vasoconstriction (IV) in 16, CD in 7 (in whom $1 \mathrm{pt}$ had protein S deficiency, 2 had protein C deficiency, 1 had APC resistance without mutation of the factor $\mathrm{V}$ of Leyden, 1 had antithronmbin III deficiency and 2 had cardiolipine antibodies). 5 pts had a cardioembolic (CE) cause of stroke ( 2 had transient episods of atrial fibrillation and 3 had severe cardiac akinesia), 2 had amniotic fluid embolism and 1 had a choriocarcinoma complicating pregnancy. 6 pts had no cause of stroke in the arterial stroke group. 45 pts had stroke during the postpartum period, in which CVT occurred more frequently during the first fifteen day, while both IV and other causes of stroke were found after the fifteenth day after delivery. All pts with $C D$ were treated with anticoagulants for 3 months. Complete recovery was observed in 10/14 CVT and in 31/37 with arterial stroke. 3 pts died, 1 in the CVT group and 2 in the arterial group ( 1 with severe heart failure, and pt who had choriocharcinoma).

14 pts experienced a new pregnancy. No complication was encountered.

Conclusions: In our series,stroke in pregnacy remains a rare event, since it represents $1 \%$ of all admissions. The vast majority is found during the pospartum period. IV and $\mathrm{CD}$ are the most frequent etiologies of stroke. Complete recovery was found in most of pts but in this small series, death occurred in roughly $6 \%$ of the cases and was always found before childbirth.

\section{Risk factors of stroke}

\section{STROKE MORBIDITY AND MORTALITY CAUSED BY PASSIVE SMOKING} IN GERMANY

J. Heidrich, U. Keil, J. Wellmann, K. Kraywinkel, P.U. Heuschmann

Institute of Epidemiology and Social Medicine, University of Muenster,

Muenster, Germany

Background: Passive smoking is an established risk factor for coronary heart disease and increasing evidence suggest that passive smoking is also associated with ischemic and haemorrhagic stroke. The magnitude of the association and the impact of passive smoking on stroke morbidity and mortality, however, are currently unclear. Therefore, we estimated the burden of stroke caused by passive smoking in Germany.

Methods: Frequency of passive smoking was derived from the national German health survey. Non-smokers who reported exposure to environmental tobacco smoke (ETS) at home were considered for analyses. The relative risk for stroke and passive smoking was calculated from available cohort studies. Attributable risk for passive smoking was computed and mortality due to passive smoking was estimated using data from official German mortality statistics. Attributable stroke morbidity was calculated using the WHO Global Burden of Disease approach.

Results: Relative risk of combined fatal and non-fatal stroke due to passive smoking was 1.18 . In total, $1.2 \%$ of strokes among non-smoking males and $1.8 \%$ of strokes among non-smoking females are caused by exposure to ETS at home. In absolute numbers, 1,837 fatal and non-fatal first in a lifetime strokes in Germany occur every year due to passive smoking. More strokes occur among women (1248) than among men (589). The majority of strokes caused by ETS happen in age group 65-84 years. Regarding fatal strokes (first ever and recurrent events) only, 774 deaths each year in Germany are caused by passive smoking.

Conclusions: Passive smoking has an important public health impact on stroke morbidity and mortality in Germany. More people in Germany die of strokes caused by passive smoking than for example of HIV infections. More education on ETS hazards and implementation of smoke-free policies are needed to reduce the burden of passive smoking related strokes.

\section{Risk factors of stroke}

\section{ETHNIC DIFFERENCES IN STROKE RISK FACTORS AND STROKE} SUBTYPE - FIRST RESULTS FROM THE SOUTH LONDON ETHNICITY AND STROKE STUDY

U. Khan, P. Jerrard-Dunne, J. Birns, I. Burger, A. Evans, R. McGovern, C. Wolfe, A. Rudd, L. Kalra, H. Markus

St George's University of London and Guy's, King's, and St Thomas' School of Medicine, London, United Kingdom

Background: A more than two-fold increase in stroke incidence has been documented in the UK black population compared to whites. Stroke subtype and vascular risk factor differences with increased cerebral small vessel disease (SVD) in blacks may account for this increased incidence. The South London Ethnicity and Stroke Study has been set up to address the causes of increased stroke incidence in this ethnic group by the study of a well-phenotyped case control series of stroke.

Methods: Consecutive, prospective recruitment of black and consecutive recruitment of white stroke cases presenting to three district hospitals in South London was carried out. All cases underwent standardized clinical assessment were subtyped by one individual.

Results: 600 black and white stroke cases were recruited. The black stroke cohort had increased hypertension (OR $2.83(1.91-4.20) \mathrm{p}<0.001)$ and diabetes (OR 2.60 $(1.81-3.74) \mathrm{p}<0.001)$ and was younger (OR $0.96(0.95-0.98) \mathrm{p}<0.001)$, had less smoking (OR $0.33(0.23-0.47) \mathrm{p}<0.001$ ), atrial fibrillation (OR $0.27(0.18-0.42)$ $\mathrm{p}<0.001)$ and myocardial infarction $(\mathrm{OR} 0.55(0.31-0.98) \mathrm{p}<0.041)$ compared to whites. The black stroke cohort had increased SVD (OR $3.05(2.26-4.11) \mathrm{p}<0.001)$ but less large vessel atherosclerotic disease (OR $0.46(0.31-0.68) \mathrm{p}<0.001)$ an cardio-embolic disease (OR $0.55(0.41-0.73) \mathrm{p}<0.001)$ compared to whites. The black stroke cohort comprised 418 African-Caribbeans and 182 Africans. AfricanCaribbean strokes were older (OR $1.07(1.05-1.09) \quad \mathrm{p}<0.001)$ with increased male sex (OR $2.75(1.65-4.57) \mathrm{p}<0.001)$ and smoking (OR $6.98(4.01-12.13$ $\mathrm{p}<0.001)$ compared to Africans. The African stroke cohort had increased primary intracerebral haemorrhage $(\mathrm{PICH})(\mathrm{OR} 1.98(1.17-3.34) \mathrm{p}=0.010)$ compared to African-Caribbeans.

Discussion: Increased hypertension, diabetes and SVD may contribute to the increased incidence of stroke in blacks. Africans are younger and have increased PICH compared to African-Caribbeans.

\section{Risk factors of stroke}

\section{ASSOCIATION OF TWO APOLIPOPROTEIN E POLYMORPHISMS WITH CAROTID PLAQUES AND INTIMA-MEDIA THICKNESS}

S. Debette, J.C. Lambert, J. Gariépy, C. Tzourio, J.F. Dartigues, K. Ritchie, A. Alperovitch, P. Ducimetière, P. Amouyel, M. Zureik

University Hospital of Lille, Lille, France

Background: Carotid plaques and elevated carotid artery intima-media thickness (IMT) are major predictors of cardiovascular morbidity and mortality. Our aim was to test their association with two polymorphisms of the apolipoprotein E (apoE) gene, the epsilon and the $-219 \mathrm{G} / \mathrm{T}$ polymorphism.

Methods: The study was performed on 5856 subjects aged $>65$ years who were recruited in the French population for the Three-City Study. Carotid ultrasound examination included a measurement of IMT in the common carotid arteries (CCA) and an assessment of atherosclerotic plaques in the extracranial carotid arteries. The genetic association was tested using genotype and haplotype analyses.

Results: No association was found between the -219 polymorphism and either carotid plaques or CCA-IMT. In a multivariable analysis including both polymorphisms and vascular risk factors, carotid plaques were more frequent in $\varepsilon 4$ homozygotes (adjusted odds ratio $=2.12,95 \%$ CI $[1.27-3.53], \mathrm{p}=0.004$ ) and less frequent in $\varepsilon 2$ carriers (adjusted odds ratio $=0.79$ [0.66-0.95], $\mathrm{p}=0.01$ ) compared to $\varepsilon 3$ homozygotes. The haplotype study confirmed the association of carotid plaques with the $\varepsilon 4$ and $\varepsilon 2$ alleles. In a multiple regression an association was observed between CCA-IMT and both the $\varepsilon 34$ genotype (mean CCA-IMT $=0.744 \mathrm{~mm}$ vs. $0.732 \mathrm{~mm}$ for the $\varepsilon 33$ genotype, $\mathrm{p}=0.002$ ) and the GCC [G- $\varepsilon 4$ ] haplotype (mean 
difference in CCA-IMT compared to the GTC [G- $\varepsilon 3]$ reference haplotype $=+0.017$ $\mathrm{mm}, \mathrm{p}=0.02$ ).

Discussion: This study, conducted on a large population cohort of French elderly, demonstrated that carotid atherosclerosis was significantly associated with the apoE epsilon polymorphism, independently of the -219 polymorphism and vascular risk factors, including lipid levels.

\section{Risk factors of stroke}

RISK FACTORS OF HIGH CAROTID ARTERY INTIMA-MEDIA THICKNESS IN YOUNG WOMEN RESULTS FROM THE ARFY (ATHEROSCLEROSIS RISK-FACTORS IN FEMALE YOUNGSTERS) STUDY

M. Knoflach, S. Kiechl, A. Zangerle, D. Penz, A. Rossmann, P. Robatscher, A. Griesmacher, C. Schmidauer, J. Willeit, G. Wick

Innsbruck Medical Center, Innsbruck, Austria

Background: There is now ample evidence that atherosclerosis already starts in childhood. Relevance of classic and novel vascular risk factors - as extensively studied in the elderly - is not well established in young adults, especially in women. Methods and Results: In the ARFY study 205 18- to 22-year-old clinically healthy white females from the Innsbruck School of Nursing were enrolled. Classic vascular risk factors, lifestyle behaviours and family history were assessed by validated and standardized procedures. Laboratory blood examinations had a focus on inflammation and metabolism. Intima-media thickness (IMT), a well defined surrogate of early atherosclerotic vessel disease, was quantified in 6 well-defined segments of the left and right carotid arteries. In multivariate analysis, insulin resistance as estimated by the homeostatic model assessment and other components of the metabolic syndrome, hsCRP levels higher than $1 \mathrm{mg} / \mathrm{L}$, diastolic and systolic blood pressure assessed by multiple ambulatory pressure recordings and presence of common allergic disorders qualified as significant and independent risk predictors of high IMT. In addition, family history of premature cardiovascular disease was strongly associated with IMT indicating a prominent role of genetic factors in this age range. Prevalence of smoking and hypercholesterolemia, however, was low in our population. No relations between these factors and IMT emerged.

Discussion: Classic and novel vascular risk factors are associated with high IMT in clinically healthy young females but the impact of the individual factors differs from what is known from adult populations. Inflammation, insulin resistance and family history (genetic predisposition) were of particular relevance. Our findings may help to design tailored prevention programs in young individuals.

\section{Risk factors of stroke}

INTRACRANEAL ARTERIAL STENOSIS AND PULSATILITY INDEX IN COHORT AIRVAG. 2 YEAR OF FOLLOW-UP

C. Sánchez, C. Guijarro, B. Herreros, J.C. Belinchón, E. Puras, J. Jiménez, F.J. Barriga, L. Lopez Bescos

FHA, Alcorcon, Madrid, Spain

Background: Atherosclerosis is a systemic disease. Is unusual to evaluate intracraneal vessels in patients with extracranial clinical atherosclerosis.

Objective: To describe frequency of intracranial arterial estenosis (IAE) and high pulsatility index (HPI) in AIRVAG cohort and its relationship with vascular risk factors, clinical aspects and prognostic implications.

Patients and Methods: AIRVAG is a cohort of 269 consecutives patients $<70$ years with acute atherotrombotic event (coronary, peripheral or cerebral). Sex, age, classical vascular risk factors, microalbuminury, carotid intima-media thickness (IMT) and ankle-brachial index (ABI) were analized. Transcranial Doppler (TCD) was performed to detect IAE and HPI $(>1,2)$. After average of 2 years of follow-up were compared patients with new isquemic events by the chi 2 , Student's $t$ test, and multivariate logistic regression.

Results: Patients with TCD 185; mean age 59,6+8.1. Male 79.9\%. Normal TCD 127(75\%); IAE 18(10\%); HPI 28(16\%). First event and TCD: cardiac HPI $17 \%$, IAE5\%; cerebral HPI16\%, IAE12\%; peripheral ischemic HPI 13\%, IAE13\%. IAE was more frequent in patients hypertensive ( 83 vs $59 \%$ ), diabetic (67 vs $26 \%$ ) and poorly controlled ( $\mathrm{HbA} 1 \mathrm{C}>7,28$ vs $7 \%$ ), previous stroke (18\% vs $6 \%$ ), higher systolic blood pressure ( $146 \pm 27$ vs $125 \pm 18 \mathrm{mmHg})$, and IMT $(1.0 \pm 0.3$ vs $0.9 \pm 0.3 \mathrm{~mm})$. Microalbuminury was associated with HPI $(\mathrm{p}<0.007)$. Average follow-up of 2 years: 18 new events. IAE was associated with new ischemic events of any territory in univariate analysis (OR 4,1 95\% CI 1,3-13,4), and in multivariate analysis (OR 3,8 95\% CI 1,1-13,7). ICS remained as independent predictor of new events even after adjusting for the above, IMT, and ABI (OR 5,1 95\% CI 1,2-21,9). Conclusion: HPI might be an marker of microalbuminury. IAE is associated with systolic hypertension, previous stroke and diabetes and is an independent predictor of new ischemic events in patients with clinical atherosclerosis in any territory.

\section{Risk factors of stroke}

SYSTEMIC RISK SCORE EVALUATION IN ISCHAEMIC STROKE PATIENTS (SCALA): PROSPECTIVE CROSS SECTIONAL STUDY IN 85 STROKE UNITS J. Roether, H. Darius, I.-H. Kim, M. Goertler, E.B. Ringelstein, C. Weimar, H.C. Diener

Hospital Minden, Germany, Minden, Germany

Patients with transient ischaemic attacks (TIA) or ischaemic stroke (IS) carry a high risk of stroke recurrence. Stratification of patients according to their risk profiles, e.g. with the Essen Stroke Risk Score (ESRS) can contribute to optimised secondary prophylaxis of such patients. The risk factors included in this algorithm are: age, systolic blood pressure, antihypertensive therapy, diabetes mellitus, cigarette smoking, prior cardiovascular disease, atrial fibrillation, and left ventricular hypertrophy. We aimed (1) to describe stroke severity and the risk factor profiles of hospitalised patients with TIA/IS, (2) to stratify these patients in low versus high risk groups according to the ESRS, and (3) to correlate peripheral arterial disease (PAD) as a marker of generalised atherosclerosis with the ESRS.

In this cross-sectional observational study, 85 neurological stroke units throughout Germany documented consecutive TIA/IS patients on standardised questionnaires. Screening on PAD was done with Doppler ultrasonography to calculate the ankle brachial index (ABI). An $\mathrm{ABI} \leq 9$ was indicative for PAD.

A total of 852 patients $(57 \%$ men) with a mean age of $67 \pm 12.4$ years were included of whom $82.9 \%$ had IS. The median National Institutes of Health stroke sum score was 4 (sum score for TIA: 1). Arterial hypertension was reported in $71 \%$, diabetes mellitus in 26\%, clinical PAD in 10\%, and an ABI $\leq 9$ in $51 \%$. An ESRS $\geq 3$ was observed in $69 \%$, corresponding to a recurrent stroke risk of $>4 \% / \mathrm{year}$. The correlation between the ESRS and the ABI according to Pearson product moment coefficient was moderate $(\mathrm{r}=0.397)$.

While most documented patients had TIA or IS of low severity, a high proportion had a considerable risk of recurrent stroke according to the ESRS or ABI category. Concurrent risk factors, particularly those related to atherothrombosis, should therefore be considered in the choice of antiplatelet agents.

\section{Risk factors of stroke}

\section{PROGNOSTIC VALUE OF TRANSCRANIAL DOPPLER ULTRASONOGRAPHY IN PATIENTS WITH A RECENT TIA OR MINOR ISCHEMIC STROKE}

A.D. Wijnhoud, P.J. Koudstaal, D.W.J. Dippel

Erasmus MC, University Medical Center Rotterdam, Rotterdam, The Netherlands

Background: Vascular risk factors have a disappointing prognostic value regarding major vascular events after TIA or minor stroke. We assessed the prognostic value of TCD findings for recurrent stroke and major vascular events in patients with a recent TIA or minor stroke.

Methods: 598 patients with a TIA or non-disabling ischemic stroke underwent TCD-investigation. We studied mean, peak-systolic and end-diastolic flow velocity (EDV), as well as cerebrovascular CO2-reactivity and microembolic signals (MES) The primary outcome was the composite event of non-fatal stroke, non-fatal myocardial infarction or vascular death, whichever occurred first. Secondary outcome events were fatal or non-fatal stroke. Tertiary outcome events were fatal or non-fatal ischemic stroke, or TIA.

Results: TCD registration was successful in 490 patients. Mean follow-up was 2.1 years. The cumulative incidence of the primary and tertiary outcome was $12 \%$ (95\% CI: 9 to $15 \%$ ) and 14\% (95\% CI: 11 to $17 \%$ ), respectively. Flow velocity parameters were predictors for all outcome events after adjustment for age, sex, and cardiovascular risk factors. The strongest prognostic factor was the EDV: for every $10 \mathrm{~cm} / \mathrm{sec}$, the relative risk for a major vascular event was 1.4 (95\% CI: 1.2 to 1.7) and for TIA or stroke it was 1.2 (95\% CI: 1.0 to 1.5$)$. Cerebral CO2 reactivity and the occurrence of MES were not related to an increased risk of major vascular events in this study.

Conclusion: TCD flow velocity parameters are potent predictors of recurrent stroke or TIA and other major vascular events. 


\section{Stroke and diabetes}

\section{Stroke and diabetes}

TYPE 1 DIABETES AS A RISK FACTOR FOR STROKE IN MEN AND WOMEN AGED 15-49: A NATIONWIDE STUDY FROM SWEDEN

X. Li, J. Sundquist, S.E. Johansson, K. Sundquist

Karolinska Institute, Stockholm, Sweden

Background: Previous studies have made important contributions to a growing body of knowledge about type 1 diabetes and stroke risk; however, long-term longitudinal studies that control for concomitant risk factors like socioeconomic status are less common. The objective was to determine incident premature stroke risk by age among men and women in Sweden with and without type 1 diabetes, after accounting for socioeconomic status and geographic region.

Methods: The Swedish longitudinal neurological database at the Karolinska Institute, Stockholm, was used to identify all persons in Sweden aged 15 to 34 at onset of type 1 diabetes and aged 15 to 49 at time of incident premature nonfatal or fatal stroke during the study period (1987 to 2001). Standardized incidence ratios (SIRs) of incident premature nonfatal or fatal, ischemic or hemorrhagic stroke excluding subarachnoid hemorrhage were calculated and compared for persons with and without type 1 diabetes.

Results: Of the 12299 persons whose records were followed after first hospitalization for type 1 diabetes during the study period, 106 experienced a stroke event. The overall SIR of premature stroke in persons with type 1 diabetes was 9.14 (95\% CI, 7.48-11.06). In persons with type 1 diabetes, premature stroke was most common between age 30 and 39, and stroke incidence was higher in women than men throughout the whole period. Risk of stroke was increased by a factor of 51 among those with diabetic nephropathy. There was an upward trend in stroke incidence in both men and women with type 1 diabetes between 1987 and 2001. Conclusion: Our data indicate that young to middle-aged persons with type 1 diabetes had a considerably higher risk of developing premature stroke than those without type 1 diabetes. Physicians should be aware that young to middle-aged persons with type 1 diabetes need specific attention to reduce the risk of premature stroke.

\section{Stroke and diabetes}

\section{PATHOLOGICAL FEATURES OF SYMPTOMATIC CAROTID PLAQUES IN} PATIENTS WITH IMPAIRED GLUCOSE TOLERANCE AND DIABETES

J. Redgrave, J. Lovett, P. Rothwell

University of Oxford, Oxford, United Kingdom

Background: Diabetes is independently associated with increased risks of incident stroke and with both early and late recurrent stroke after TIA. The mechanism(s) of the increased risk of acute vascular events in diabetes is poorly understood. Some small studies have suggested that atherosclerotic plaques from diabetics have a higher prevalence of unstable features.

Method: We made detailed, reproducible histological assessments of 526 plaques from consecutive patients undergoing carotid endarterectomy for symptomatic stenosis and related these to the presence of impaired glucose tolerance (IGT) and diabetes.

Results: $53(10.1 \%)$ patients had diabetes, $26(5.0 \%)$ had IGT, and $447(84.9 \%)$ had neither. There were no overall differences in the prevalence of unstable plaque between the groups. Marked plaque inflammation $(\mathrm{OR}=0.94,0.5-1.76)$, large lipid core $(\mathrm{OR}=1.12,0.60-2.07)$, marked vascularity $(\mathrm{OR}=1.20,0.65-2.20)$, and overall plaque instability $(\mathrm{OR}=0.89,0.48-1.64)$ were no more prevalent in diabetics than non-diabetics. However, whereas plaques removed more than four months after the last symptomatic event were less unstable than those removed earlier in patients without diabetes or IGT, such non-acute plaques remained highly unstable in patients with IGT or diabetes (difference in trend for overall instability: $\mathrm{p}=0.005$ ). Cap rupture was strongly associated with plaque macrophage infiltration in all groups but was only associated with plaque lymphocytes in diabetics (OR 3.26, 1.01-10.48, $\mathrm{P}=0.04$ )

Conclusion: Unstable carotid atherosclerotic plaque appears to be particularly persistent in patients with IGT and diabetes, which might partly explain their increased risk of recurrent stroke.
3 Stroke and diabetes

COMORBIDITY OF ATRIAL FIBRILLATION AND DIABETES MELLITUS IN PATIENTS WITH ISCHEMIC STROKE IN A POPULATION-BASED REGISTRY

F. Pistoia, T. Russo, L. Di Dionisio, C. Marini, A. Carolei

University of L'Aquila, L'Aquila, Italy

Background: Mortality after a first-ever stroke is high in patients with comorbidity of atrial fibrillation and diabetes mellitus. However, data on clinical characteristics and on short- and long-term follow-up are scarce. Aim of our study was to identify in a population-based registry the contribution of the comorbidity of atrial fibrillation with diabetes mellitus in patients with ischemic stroke.

Patients and Methods: All incident first-ever stroke cases in a 5-year period (1994-1998) were included in the prospective, population-based L'Aquila registry. For the purpose of the present study we considered only patients with ischemic stroke. Patients were followed up to December 31, 2005.

Results: Out of 3594 patients with a first-ever ischemic stroke $(47.2 \%$ men and $52.8 \%$ women), with a mean $[ \pm \mathrm{SD}]$ age of $75.3 \pm 10.7$ years, $223(6.3 \%)$ had comorbidity of atrial fibrillation and diabetes mellitus. Its prevalence was higher in women than in men $(8.5 \%$ vs $3.7 \%$; $\mathrm{P}<0.0001)$. Patients with comorbidity had more frequently high levels of hematocrit $(\mathrm{p}=0.003)$, coronary heart disease $(\mathrm{P}=0.007)$, and TACI $(43.9 \%$ vs $27.6 \%)$ rather than PACI $(38.6 .8 \%$ vs $43.7 \%)$ or LACI $(4.5 \%$ vs $17 \%)$ with respect to patients without $(\mathrm{P}<0.0001)$. By December 312005,2230 patients $(63.2 \%)$ had died; among them, $724(20.5 \%)$ died within 30 days and a total of $1166(33.0 \%)$ patients within 1 year. Besides, patients with the comorbidity had a higher 30 -day $(44.8 \%$ vs $18.8 \% ; \mathrm{P}<0.0001)$ and 1 -year case-fatality rate $(62.8 \%$ vs $31.0 \% ; \mathrm{P}<0.0001)$ with respect to patients without. Discussion: Comorbidity of atrial fibrillation and diabetes mellitus is more frequent in women. Short- and long-term prognosis was worse in patients with atrial fibrillation and diabetes mellitus than in those without, possibly because of a greater severity of the event and of late complications. The adequate management of comorbidities may help to improve survival and prognosis in patients with ischemic stroke.

\section{Stroke and diabetes}

TISSUE PLAQUE REMODELLING IS LINKED TO PROTHROMBOTIC STATE IN DIABETIC PATIENTS WITH ADVANCED CAROTID ARTERY DISEASE

A. Carvajal Collado, M. Miguel Turu, M.A. Font Padros, F. Rubio, L. Badimon, J. Krupinski

University Hospital of Bellvitge (HUB), Barcelona, L'Hospitalet de Llobregat (Barcelona), Spain

Background: Advanced atherogenesis is signaled by markers of enhanced prothrombotic capacity, attenuated fibrinolysis and by clinical conditions associated to defective coagulation. There is increasing clinical data suggesting that diabetes is associated with severe atherosclerosis and enhanced lesion instability leading towards atherosclerotic plaque rupture.

Methods: Plaques obtained from 291 diabetic and nondiabetic patients undergoing carotid endarterectomy were included in the study. Plaques were subjected to analysis of tissue factor, metalloproteinases- $2,-8,-9$, fibrin/fibrionogen related antigens by immunohistochemistry and western blot; in situ zymography was used to detect MMP activity, whereas plasma samples were quantified for TF procoagulant activity, CRP, fibrinogen and D-dimer.

Results: Diabetes in patients with carotid disease was associated with the presence of other vascular risk factors i.e. hypertension, hypercholesterolemia and coexisted with coronary artery disease $(\mathrm{p}<0.05)$. Diabetic and symptomatic patients with unstable, complicated plaques had increased plasma TF activity, D-dimer and MMP-8 levels than those with stable plaque $(\mathrm{p}<0,05)$. Further, there was correlation between intramural TF levels and intramural D-dimer in diabetic patients with symptomatic carotid disesae $(\mathrm{p}<0,005, \mathrm{r}=0,4)$.

Discussion: These data suggest that some of the currently available circulating markers of atherothrombosis are elevetaed in diabetic and symptomatic patients. Increased procoagulant activity in diabetic patients is linked to increased mural remodelling. 


\section{Stroke and diabetes}

HYPERGLYCAEMIA IN THE ACUTE POST-STROKE PHASE NEGATIVELY INFLUENCES MOOD OUTCOMES IN ISCHAEMIC STROKE

E.C. Lovett, C.R. Levi, J. Attia, M.W. Parsons, J. Lowe

John Hunter Hospital, Hunter Region Mail Centre, Australia

Background: Hyperglycaemia is associated with increased mortality and morbidity in acute ischaemic stroke in diabetic and non-diabetic patients. Similarly, poststroke depression, cognitive impairment, and health related quality of life and are associated with worse long term outcomes. Research investigating the relationship between hyperglycaemia and cognitive and behavioural outcomes in stroke has yielded mixed results, due in part to methodological difficulties. We hypothesize that hyperglycaemia in the acute post-stroke phase will negatively affect cognitive and behavioural outcomes at three months.

Methods: This study is an observational prospective case-control study. Patients presenting consecutively to the Emergency Departments of two tertiary referral hospitals in Newcastle (Australia), with first ever acute ischaemic stroke have been recruited. Clinical, cognitive and behavioural outcomes in hyperglycaemic and normoglycaemic stroke patients were compared to clinical, cognitive and behavioural assessments of age-matched non-stroke diabetic controls. Recruitment is ongoing.

Results: 133 participants ( 84 stroke patients and 49 controls) have been recruited. Increased acute blood sugar levels were positively correlated with higher Beck Depression Inventory (BDI-II) scores $(\mathrm{p}=0.018)$ in stroke patients. Conversely, there was no relationship found between hyperglycaemia and depressed mood in the control group. Paired sample t-tests indicate that stroke patients performed worse than diabetic non-stroke patients on cognitive assessment tasks. However, regression analysis failed to identify any predictive relationships between cognitive performance and hyperglycaemia.

Conclusion: Hyperglycaemia in the acute post-stroke phase indicates a significantly increased risk of depression, reflected by elevated BDI-II scores, at 3-month follow-up. Ongoing recruitment is expected to further clarify the relationship between hyperglycaemia and cognitive outcomes.

\section{Stroke and diabetes}

\section{LONG-TERM FOLLOW-UP OF PATIENTS WITH ISCHEMIC STROKE AND} DIABETES MELLITUS

T. Russo, L. Di Dionisio, D. Cerone, C. Marini, A. Carolei

University of L'Aquila, L'Aquila, Italy

Background: Recurrences and mortality after stroke are increased in patients with diabetes mellitus. However, data on short- and long-term follow-up are scarce. We evaluated stroke recurrences and causes of death in patients with ischemic stroke and diabetes mellitus in a population-based registry.

Methods: All incident cases of first-ever ischemic strokes were included in the population-based registry of patients living in the L'Aquila district over a 5-year period. Patients were followed up to December 31, 2005 with planned visits or phone interview either in person or with a close relative or the general practitioner. Causes of death were reviewed on death certificates and hospital records.

Results: Out of 3594 patients with a first-ever ischemic stroke $(47.2 \%$ men and $52.8 \%$ women), $923(26.2 \%)$ had diabetes mellitus. Prevalence was higher in women than in men $(30.2 \%$ vs $21.7 \%$; $\mathrm{P}<0.0001)$. Patients with diabetes mellitus were older than those without (mean age 76.0 vs 74.9 years; $P=0.009$ ).

By December 31, 2005, 2230 patients $(63.2 \%)$ had died: $721(20.4 \%)$ within 30 days and a total of $1162(33.0 \%)$ within 1 year. Patients with diabetes mellitus had a higher 30 -day $(28.4 \%$ vs $17.5 \%$; $\mathrm{P}<0.0001)$ and 1 -year case-fatality rate $(41.6 \%$ vs $29.7 \% ; \mathrm{P}<0.0001)$ that was confirmed at the long-term follow-up $(64.9 \%$ vs $51.2 \% ; \mathrm{P}<0.0001)$ with respect to those without the risk factor. In the long-term, patients with diabetes mellitus were more likely to die of cerebral (32.4\% vs $23.7 \%$; $\mathrm{P}=0.006)$ and of other vascular causes $(14.8 \%$ vs $18.0 \% \mathrm{p}=0.02)$. Recurrences tended to be more frequently fatal in diabetic patients than in the nondiabetic ones $(4.7 \%$ vs $4.1 \%$; $\mathrm{P}=0.30)$

Discussion: Diabetes mellitus is an unfavorable prognostic factor of ischemic stroke, especially in women and in older patients causing more frequently fatal events. Careful control of diabetes mellitus is relevant to improve survival and prognosis in patients with ischemic stroke.
7 Stroke and diabetes

DIABETES IS ASSOCIATED WITH IMPROVED OUTCOME FOLLOWING STROKE

R.E O'Brien, J.E. O'Connell, A.J. Hildreth, C.S. Gray

Newcastle University Sunderland Royal Hospital, Sunderland, United Kingdom

Background: Diabetes Mellitus (DM) is associated with increased stroke risk and poor outcome. Advances in management of DM and other risk factors may influence outcome following stroke. We examined the presentation and outcome from stroke in patients with DM.

Methods: Prospective study of consecutive acute stroke admissions to Sunderland Royal Hospital during a 7 year period. Demographics, stroke sub-type, glucose, HbA1c, CT findings, risk factors (including previous DM) were collected. Outcome (survival) was collected at 12-weeks and 12-months. Analysis was with SPSS.

Results: 2273 patients were included (1997 to 2004): 1155 (50.8\%) female; 337 (14.9\%) known to have DM; median age 75 yrs. Patients with DM had significantly more ischaemic heart disease (MI $17.5 \%$ vs $13.1 \% \mathrm{p}=0.05$; angina $24.6 \%$ vs $16.7 \% \mathrm{p}=0.001)$ and were more likely to be on antiplatelets $(48.8 \%$ vs $40.2 \%$ $\mathrm{p}=0.003)$ and lipid-lowering therapy $(17.0 \%$ vs $10.4 \% \mathrm{p}=0.002)$. Stroke sub-types were similar in each group, as were CT findings. Glucose \& HbA1c were higher in those with DM $(9.2$ vs $6.3 \mathrm{mmol} / 1 \mathrm{p}<0.001 ; 7.5 \%$ vs $5.9 \% \mathrm{p}<0.001)$. Overall survival was improved in patients with $\mathrm{DM}$ at 12 -weeks $(78.0 \%$ vs $72.3 \% \mathrm{p}=0.07)$ but not at 12 -months $(68.0 \%$ vs $66.1 \% \mathrm{p}=0.5)$. Those with poorly controlled DM had improved survival at 12 -weeks $(84.1 \%$ vs $65.9 \% \mathrm{p}<0.001)$ and 12 -months (73.9\% vs $59.9 \%$ p=0.003) compared to those without DM but with high glucose \& HbA1c. When compared with all DM patients, those patients without a history of DM who had raised glucose \& HbAlc had significantly reduced survival at 12 -weeks $(82.2 \%$ vs $65.9 \% \mathrm{p}<0.001)$ and 12 -months $(71.6 \%$ vs $59.9 \% \mathrm{p}=0.01)$.

Discussion: Previously recognised DM was associated with improved survival following acute stroke. The association between DM and poor outcome may no longer be valid due to improved management of vascular risk factors.

\section{Vascular surgery and PTCA}

\section{Vascular surgery and PTCA}

ECHOLUCENCY OF CAROTID ARTERY PLAQUES IS ASSOCIATED WITH HIGH RATE OF SOLID CEREBRAL MICROEMBOLISM DURING CAROTID ARTERY STENTING

M. Rosenkranz, J. Fiehler, G. Thomalla, A. Krützelmann, B. Eckert, W. Niesen, O. Wittkugel, T. Kucinski, U. Sliwka, H. Zeumer

University Medical Center Hamburg-Eppendorf, Hamburg, Germany

Background: Carotid artery stenting (CAS) may be associated with clinically silent cerebral embolic events. We prospectively evaluated the association of the sonographic echogenicity of carotid artery plaques with solid cerebral microembolism during CAS

Methods: Twenty-seven patients with symptomatic high-grade carotid artery stenoses scheduled to undergo CAS were included in the study. A computerassisted measure of echogenicity, the grey scale median (GSM), was used to quantify the echogenicity of carotid artery plaques on normalized sonographic B-mode images. Dual-frequency transcranial Doppler sonography was used to detect solid cerebral microemboli during CAS. No embolus protection devices were used in any of the cases.

Results: Solid cerebral microemboli were detected in 17 of the 27 patients. In patients with solid microemboli, GSM was lower (median, 37; 95\% CI, 27.2 to 48.7) than in patients without solid microemboli (median, 66; 95\% CI, 39.8 to 76.6) $(\mathrm{p}<0.05)$. ROC analysis identified a GSM cutoff value of 50 as threshold for the identification of patients at high risk of solid microembolism during CAS. In patients with echolucent plaques $(\mathrm{GSM}<50)$, the rate of solid microembolism was higher $(85 \%)$ than in patients with echogenic plaques $(\mathrm{GSM} \geq 50)(43 \%)(\mathrm{p}<0.05)$. Conclusion: CAS of echolucent carotid artery plaques is associated with a higher rate of solid cerebral microembolism than CAS of echogenic carotid artery plaques. Thus, quantitative evaluation of carotid artery plaque echogenicity may help to identify patients at high risk of procedure related embolic events. 


\section{Vascular surgery and PTCA}

ELEVATED HIGH-DENSITY LIPOPROTEIN CHOLESTEROL LEVELS ARE PROTECTIVE AGAINST RESTENOSIS AFTER CAROTID ARTERY STENTING

R. Topakian, M. Sonnberger, H.-P. Haring, K. Nussbaumer, J. Trenkler, F.T. Aichner

Wagner-Jauregg Hospital, Linz, Austria

Background: The long-term durability of carotid artery stenting (CAS) is affected by the incidence of neointimal proliferation and in-stent restenosis (ISR). Identification of predictors of restenosis would allow specific prophylactic measures.

Methods: The study included 102 consecutive patients who were prospectively followed up at our Department of Neurology after successful CAS for $\geq 60 \%$ symptomatic $(n=50)$, or $\geq 70 \%$ asymptomatic $(n=52)$ atherosclerotic internal carotid artery (ICA) stenosis. Color-coded duplex sonography, neurological evaluation, and routine laboratory tests were carried out at follow-up after 1 month and then every 6 to 12 months. A peak systolic velocity (PSV) $\geq 180 \mathrm{~cm} / \mathrm{s}$ defined ISR of $\geq 50 \%$ local diameter reduction.

Results: 10 patients $(10 \%)$ had ISR $\geq 50 \%$ after 1 year. On univariate analysis, only continued smoking, low 1-month high density lipoprotein cholesterol (HDL-C) levels, and high 1-month triglycerides levels were significantly associated with ISR $(\mathrm{p}<0.05)$. Other traditional cardiovascular risk factors, medication, pre-CAS stenosis, and procedure-related variables did not differ significantly in patients with and patients without ISR. In the best predictability-targeted multivariate logistic regression model 1-month HDL-C levels $>45 \mathrm{mg} / \mathrm{dl}$ were inversely associated with ISR ( $\mathrm{p}=0.05$, adjusted OR $0.07,95 \%$ CI $0.005-1.03)$, while all other variables clearly did not reach statistical significance.

Conclusion: Our findings indicate that high HDL-C levels may be protective against ISR. Whether elevation of HDL-C levels by lifestyle changes or specific medication can reduce the occurrence of ISR needs further elucidation.

\section{Vascular surgery and PTCA}

TCD MONITORING OF TRANSCERVICAL CAROTID STENTING WITH REVERSAL FLOW PROTECTION: A NOVEL CAROTID REVASCULARIZATION TECHNIQUE

M. Ribo, B. Alvarez, M. Rubiera, C. Molina, J. Alvarez-Sabín, M. Matas

Unitat Neurovascular Vall d'Hebron, Barcelona, Spain

Transfemoral carotid stenting despite becoming very frequent has some limitations such as difficult groin access in few patients, lack of distal protection during filter placement or embolization despite protection. Transcervical stenting (TCS) is a novel technique during which a common carotid to jugular vein shunt is placed creating a protective reversal flow in the internal carotid (ICA) after proximal common carotid (CCA) clamping. We aim to study with TCD cerebral flow changes and microemboli detection during transcervical stenting

From a total of 129 patients elegible for carotid revascularization, 39 were considered high risk (sapphire criteria) and underwent TCS. A neurological examination was performed before and after the procedure by a neurologist

After CCA clamping flow inversion is observed in anterior cerebral artery (ACA), supplying blood to middle cerebral artery (MCA) and ICA (reversal). TCD showed that the initial CCA occlusion technique (rubber band compression, $n=19$ ) was insufficient in some cases $(20 \%)$, however after adopting the CCA clamping method TCD did not detect any air/solid emboli during stent liberation or angioplasty $(\mathrm{n}=20 ; 0 \%)$ confirming the reversal flow protection hypothesis. Mean reversal flow time was 14 minutes, in all cases substantial MCA flow was present during CCA clamping (initial mean velocity $30 \mathrm{~cm} / \mathrm{s}$ ) and a slow gradual increase was observed traducing collateral flow recruitment (mean velocity after 5 minutes $38 \mathrm{~cm} / \mathrm{s}$ ). The only in-procedure complication was one TIA. After CCA unclamp normal antegrade flow was restored in ACA, mean final MCA velocity increased $16 \%$ according to pre-procedure flow

TCS with protective ICA flow reversal can eliminate cerebral microembolization during the procedure making it a promising carotid revascularization technique in high risk patients with carotid stenosis.
4 Vascular surgery and PTCA

SOLID/GAS DIFFERENTIATION OF MICROEMBOLI AND ENHANCED EMBOLI DETECTION: A NEW SYSTEM FOR THE OFF-LINE ANALYSIS OF TRANSCRANIAL DOPPLER DATA

C. Lucchesi, K. Zaheg, L. Studer, A. Herrmann, C. Gonin, R. Schuler, J.-M. Vesin, P. Ruchat, J. Bogousslavsky, G. Devuyst

ABMI SA, Lausanne, Switzerland

Background: Stroke and cardiovascular surgical patients would benefit from improved detection of microemboli and solid-gas differentiation systems. We have developed automated software for off-line, automated analysis of TCD data, which provides a useful inter-studies data analysis standard and gives good classification rates for separating HITS from bloodflow, artefacts from MES, and solid from gaseous emboli.

Methods: On a large collection of 700 solid (carotid stenosis) and 1400 gaseous (saline injections on PFO patients) microembolic signals (MES) recorded in-vivo, we computed numerous signal-processing features and applied machine-learning strategies. We compared various classifying strategies, including decision trees and neural networks, for separating MES from artefacts, and differentiating solid from gaseous emboli, we selected those which showed better classification performance and algorithmic robustness.

Results: The resulting system analyzes Doppler data files and produces separate counts for artefacts, solid and gaseous emboli. The classified signals can be reviewed with an interface providing details of each event. The classification rates depend on detection sensitivity, which can be adjusted, and influences the emboli detection rate. For a standard detection level at $12 \mathrm{~dB}$, the emboli-artefacts classification rate is $96 \%$, the solid-gas differentiation rate is above $86 \%$.

Conclusions: We describe a user-friendly system for automated, off-line analysis of brain embolization data. Such a system is useful for the analysis of long-term patient embolization monitoring, for re-analysis - with focus on embolization - of existing clinical data, and for prospective or retrospective clinical studies aimed at devising therapeutic strategies for neuroprotection in cardiovascular surgery and endovascular procedures.

\section{Vascular surgery and PTCA}

\section{IMPROVEMENT OF COGNITIVE FUNCTIONS AFTER CAROTID ENDARTERECTOMY}

G. Devuyst, C. Greber, I. Reverte, T. Karapanayiotides, V. Kémény, B. Piechowski-Jozwiak, P. Ruchat, J. Bogousslavsky

Centre Hospitalier Universitaire Vaudois, Lausanne, Switzerland

Background and Objective: Previous studies involving neuropsychological assessment of patients undergoing carotid endarterectomy (CEA) have shown conflicting results. We intended to deeply investigate the neuropsychological changes after CEA by assessing three cognitive domains: memory, executive functions, and attention.

Methods: 45 patients with asymptomatic $(n=19)$ and symptomatic $(n=26)$ internal carotid artery (ICA) stenosis $>70 \%$ were submitted to a battery of neuropsychogical tests evaluating verbal memory (three parallel versions of the Rey Auditory Verbal Learning Test), executive functions (Stroop, TMT part B, Verbal Fluency, Non Verbal Fluency) and attention (Digit Span-forwards and backwards from WAIS-R, TMT part A, ZVT, d2) before CEA, with follow-up after 5 days and 3 months. Results: There were no significant differences between baseline and both followsup in working $(\mathrm{p}=0.1)$ and short-term memory tests $(\mathrm{p}=0.25)$ as well as for the recognition sub-test of the Rey Auditory Verbal Learning Test $(\mathrm{p}=0.3)$. On the contrary, when the two specified follows-up are compared to baseline values patients had a clear improvement in most tests of executive function [fluency letter $(\mathrm{p}=0.04)$, fluency figural $(\mathrm{p}=0.02)$, Stroop $(\mathrm{p}=0.05)$, TMT B $(\mathrm{p}=0.05)$ ] and attention [TMTA $(\mathrm{p}<0.001)$, ZVT $(\mathrm{p}<0.001), \mathrm{d} 2$ production $(\mathrm{p}=0.001)$ and $\mathrm{d} 2$ errors $(\mathrm{p}<0.01)]$. Furthermore, tests involving verbal memory $(\mathrm{p}<0.001)$ and sustained attention $\mathrm{d} 2$ test $(\mathrm{p}<0.001)$ were significantly better between follow-up at 5 days and 3 months. Conclusions: Patients undergoing CEA seem to be associated with short and long-term cognitive improvement in the fields of executive functions and verbal learning, and these benefits are independent from test training. 


\section{Vascular surgery and PTCA}

COGNITIVE CHANGES AFTER CAROTID ENDARTERECTOMY ARE NOT RELATED TO NATURE OF MICROEMBOLIC SIGNALS, SOLID OR GASEOUS

G. Devuyst, C. Greber, I. Reverte, T. Karapanayiotides, V. Kémény, B. Piechowski-Jozwiak, P. Ruchat, J. Bogousslavsky

Centre Hospitalier Universitaire Vaudois, Lausanne, Switzerland

Background and Objective: Previous studies on neuropsychological assessment and mechanisms of patients undergoing carotid endarterectomy (CEA) are still debated. In this study, we investigated if cognitive changes after CEA were influenced by the nature -solid or gaseous- of microembolic signals (MES) detected by transcranial ultrasound (TCD).

Methods: 34 patients with an internal carotid artery stenosis $>70 \%$ were submitted to eight neuropsychogical tests evaluating verbal memory (Rey Auditory Verbal Learning Tests), executive functions (Stroop, TMT part B, Verbal Fluency, Non Verbal Fluency) and attention (Digit Span-forwards and backwards from WAIS-R, TMT part A, ZVT, d2) before CEA and had a follow-up at 3-5 days and at 3 months. These tests were correlated with the number and the nature, solid or gaseous, of MES before, during and after CEA. For this task, we used a new algorithm, based on 22 features, allowing the separation of solid from gaseous MES.

Results: There were no significant differences between baseline and both followsups in working and short-term memory tests as well as for the recognition sub-test of the Rey Auditory Verbal Learning Test. But when the two specified follows-ups are compared to baseline, patients had a clear improvement in tests $(\mathrm{p}<0.05)$ of executive function [fluency letter, fluency figural, Stroop] and attention [TMTA, ZVT, $\mathrm{d} 2$ production and $\mathrm{d} 2$ errors]. The total load of MES or solid MES or gaseous MES were statistically significant not related to cognitive changes at 3-5 days either at 3 months.

Conclusions: Patients undergoing CEA seem to show no correlation between cognitive changes and the total load and nature -solid or gaseous- of MES detected peroperatively by TCD.

\section{Vascular surgery and PTCA}

THE EFFICACY OF SURGICAL AND CONSERVATIVE METHODS IN SECONDARY PREVENTION OF CAROTID ISCHEMIC STROKE

V.I. Skvortsova, V.L. Lemenev, V.V. Akhmetov, G.S. Alexeeva,

L.V. Stakhovskaya, A.G. Gutsaluk, O.I. Guseva

Russian State Medical University, Skliphosophsky's Scientific Research

Institute of Emergency Care, Russian Federation

Background: The aim was to compare the efficacy of conservative and surgical methods for the secondary prevention of Ischemic Stroke (IS) or Transient Ischemic Attack (TIA) in patients with embologenic atherosclerotic plaque (heterogeneous morphology, surface ulceration, floatation signs) and a carotid stenosis of less than $70 \%$.

Methods: We conducted a clinical and ultrasonic (duplex) investigation of 1600 patients after IS or TIA and enrolled 209 patients (mean age 67.2 \pm 2.6 years). Patients with atrial fibrillation, heart failure, myocardial infarction, diabetes, intermittent claudiation were excluded. Carotid endarterectomy (CE) was offered to all enrolled patients. 101 patients consented to CE within 3 weeks after IS or TIA, followed with antiplatelet therapy; 108 patients rejected CE and received the same therapy. The end-points were secondary IS or TIA, other ischemic events during 3-year-follow-up. Platelet aggregation was controlled quarterly.

Results: Secondary IS or TIA developed in $3.9 \%$ of the operated patients, and in $52.8 \%$ of conservatively treated patients $(\mathrm{p}=0.0001)$. All secondary IS or TIA in the CE group occurred in the opposite ICA territory (3.9\% vs. $21.1 \%$ in the non-operated group, $\mathrm{p}=0.0001$ ). The relative risk of the end-points after $\mathrm{CE}$ was 0.07, after CE and followed permanent 3-year-lasting antiplatelet therapy -0.038 , without CE $-0.77(\mathrm{p}=0.0001)$. Absolute risks were $4 \%, 1.9 \%, 57 \%$ respectively $(\mathrm{p}=0.0001)$. Other vascular events were not observed.

Discussion: The results demonstrate CE may be recommended for the secondary IS and TIA prevention in patients with embologenic plaque with a stenosis of less than $70 \%$. Moreover, 3-year antiplatelet therapy compliance was significantly higher in operated patients $(97 \%$ vs. $75 \%$, $\mathrm{p}=0.0001)$.
8 Vascular surgery and PTCA

ESTIMATION OF THE SIZES OF SOLID EMBOLI THROUGH TRANSCRANIAL DOPPLER ULTRASOUND

C. Lucchesi, K. Zaheg, L. Studer, A. Herrmann, M. Inglin, J.-M. Vesin,

P. Ruchat, J. Bogousslavsky, G. Devuyst

ABMI SA, Lausanne, Switzerland

Background: Counting solid brain emboli in real time during embolizing surgical procedures would represent a definite progress in neuroprotection. However, the real challenge is in further estimating the sizes of solid emboli hitting the brain. Our objective is therefore to develop systems and algorithmics for estimating the total brain embolic load (that is, the total embolized volume) e.g. during cardiovascular surgery or endovascular procedures, based on real-time analysis of cerebral blood flows via transcranial Doppler (TCD) ultrasonography.

Methods: We have measured, via TCD in an MCA phantom, the microembolic signals (MES) for 604 artificial solid emboli and for 122 ateroma fragments. Signal processing features have been computed for training expert systems to classify the emboli according to their volume.

Results: For the 604 artificial emboli, our classification tree classifies $68 \%$ of these correctly into 5 classes (mean diameters $150,275,462,662$, and $800 \mathrm{u}$ ), a result significantly better than the performance of pure chance (which, for 5 classes, is $1 / 5=20 \%$ ). Allowing for error by one adjacent class raises the artificial emboli classification rate to $89 \%$. The 122 plaque fragments are correctly classified with a $52 \%$ success rate into 6 classes (mean diameters 150, 275, 462, 662, 1000 and $2000 \mathrm{u})$, a result which is again much better than chance $(1 / 6=17 \%)$. Allowing for error by one adjacent class raises the plaque fragments classification rate to $79 \%$.

Conclusions: Our preliminary results show that emboli size estimation based on expert systems and signal processing features do give interesting results. More efforts are needed to fine-tune the method and to come up with clinically validated emboli sizing algorithms.

Oral Session 


\section{New vascular imaging}

1 New vascular imaging

REAL TIME ULTRASONOGRAPHIC MICROVASCULAR IMAGING

S. Meairs

University Hospital Mannheim, Mannheim, Germany

Invited update.

\section{New vascular imaging}

IN VIVO HUMAN MIDDLE CEREBRAL ARTERY WALL IMAGING USING HIGH RESOLUTION MAGNETIC RESONANCE IMAGING

I.F. Klein, P.C. Lavallée, PJ. Touboul, E. Schouman-Claeys, P. Amarenco Bichat Stroke Centre, Paris, France

Background: Patients with symptomatic middle cerebral artery (MCA) stenosis are at high risk of recurrent stroke. Only indirect imaging of intracranial atherosclerosis is currently available.

We aimed at studying high-resolution MRI (HR-MRI) as direct and non-invasive imaging of MCA plaques.

Methods and Results: HR-MRI was performed in six patients, with MCA atherosclerotic stenosis as documented by angiographic indirect methods. For direct imaging of the vessel lumen and wall, we followed a black blood and multicontrast approach using high spatial resolution $(0,41 \mathrm{~mm}$ of in plane resolution and $2.5 \mathrm{~mm}$ slice thickness). HR-MR, short-axis, images were acquired at the level of MCA stenosis and of normal MCA segments. Data imaging and analysis showed a significant, focal, arterial wall thickening underlying each MCA stenosis. The mean wall (plaque) area was $6.99 \mathrm{~mm}^{2}$ (range $4.42 \mathrm{~mm}^{2}$ to $12.06 \mathrm{~mm}^{2}$ ). No significant wall thickening was measured in normal segments of the MCA.

Conclusion: HR-MRI provides a non-invasive method to study, in vivo, the burden and pathogenesis of intracranial atherosclerosis. Further studies on larger population of patients are required to confirm these data

\section{New vascular imaging}

MICROEMBOLIZATION FROM THE CAROTID PLAQUE IS ASSOCIATED WITH INTRAPLAQUE HAEMORRHAGE SEEN ON MAGNETIC RESONANCE IMAGING

N. Altaf, L. Daniels, A. Beech, A.R. Moody, J.R. Gladman, P.S. Morgan, S.T. MacSweeney, D.P. Auer

University of Nottingham, Nottingham, United Kingdom

Background: Magnetic Resonance Direct Thrombus Imaging (MRDTI) has been shown to identify intraplaque haemorrhage and thrombus within the complicated carotid plaque. However the true thromboembolic activity of the plaque may not always be reflected by the histological assessment of the carotid plaque.

The purpose of this study was to ascertain whether MRDTI hyperintense carotid plaques (MRDTI +ve) are associated with increased microembolization as detected by transcranial Doppler (TCD).

Methods: 61 patients with symptomatic high grade carotid artery disease were prospectively recruited between November 2003 and November 2005. The patients underwent MRDTI scanning of the carotid arteries and TCD recordings were made for 1 hour from the middle cerebral artery ipsilateral to the carotid stenosis. The MRDTI signals and embolic signals were analysed blinded to the clinical data.

Results: Of the 61 patients recruited, 7 did not have an adequate window for TCD monitoring and 4 did not tolerate the MRI. Of the remaining 50 patients, 36 were classed as MRDTI +ve and 14 as MRDTI -ve. There were no significant differences between the major demographic features or vascular risk factors between the MRDTI +ve and MRDTI -ve groups.

TCD monitoring detected spontaneous particulate embolization in 18 patients. Of the 18 patients with emboli, 17 had MRDTI +ve plaques and 1 had a MRDTI -ve plaque (chi square $=7.03, \mathrm{p}<0.01$ ).

Discussion and Conclusions: This study suggests that intraplaque haemorrhage or thrombus as shown by MRI is associated with spontaneous particulate embolization. This provides an important validation of the usefulness of MRDTI as surrogate marker for the risk of future stroke. In particular, lack of intraplaque haemorrhage/thrombus may be a powerful predictor of low risk of embolic stroke. Further studies are underway to investigate possible radiological differences between MRDTI +ve plaques with and without particulate emboli.

\section{New vascular imaging}

CAROTID PLAQUES: CORRELATION BETWEEN ULTRASOUND AND MAGNETIC RESONANCE CHARACTERISTICS AND HISTOPATHOLOGIC FINDINGS

F. Corea, A. Nicoletta, C, Foglieni, M. Sessa, M. Cadioli, A. Giazzon, E. Geppina, G. Scotti, G. Comi

DIBIT, INSPE, IRCCS San Raffaele, Milano, Italy

Background: Atherosclerosis is a systemic inflammatory disorder involving the vascular system leading to plaque vulnerability. Plaque components influence the risk of rupture and the severity of stenosis is no longer sufficient to predict ipsilateral stroke risk. The purpose of the study was to find correlations between plaque morphology and Ultrasound (US) and high spatial resolution 1.5 T Magnetic Resonance Imaging (MRI) characteristics.

Materials/Methods: 10 patients with asymptomatic $(\mathrm{A}=7)$ and symptomatic $(\mathrm{S}$ = 3) carotid stenosis admitted to the Stroke Unit or Vascular Surgery Department were studied. Patients underwent US study with an Acuson X5 machine and MRI on a Philips Integre $1.5 \mathrm{~T}$ scanner using time-of-flight-, T1-, proton density-, and T2-weighted images. Subsequent to endarterectomy the plaques were sectioned transversely, stained with Hematoxylin-Eosin and MOVAT, examined according to Virmani criteria, and defined as stable, vulnerable and unstable. Concordance analsyis (K-value) was performed between US/MRI characteristics and plaque morphology.

Results: Six patients had unstable plaques at the US assessment (at least 3 of the following parameters: stenosis $>70 \%$, echogenicity, irregular surface, and/or ulceration) with full concordance with MRI findings ( $\mathrm{p}<0.01$ ).

The agreement between MRI and MOVAT findings was larger in the definition of stable plaque ( $\mathrm{k}$ value $1 ; \mathrm{p}$-value 0,03 ). When single plaque characteristics were compared, the agreement between MRI and MOVAT findings was larger in the identification of calcification, fibrosis, lipid content, neovascularization, and thin cap ( $\mathrm{k}$ value 1 ; $\mathrm{p}$-value 0,03 ).

Conclusions: The multimodal approach to the assessment of plaque vulnerability may help in the stratification of the atherothrombotic risk.

\section{New vascular imaging}

\section{ACCURACY OF CT-PERFUSION IN PREDECTING MALIGNANT BRAIN} INFARCTION

R. Dittrich, S.P. Kloska, T. Fischer, E. Nam, E.B. Ringelstein, D.G. Nabavi University of Muenster, Muenster, Germany

Background and Purpose: With the advent of decompressive surgery for treatment of space occupying brain infarction, it is important to identify patients at risk. We performed a prospective study on patients with ischemic stroke to evaluate the accuracy of CT-based perfusion imaging (CTP) to predict the development of malignat brain infarction

Methods: 106 consecutive patients (women 37\%) with a mean age of 65 years underwent multislice CTP after a median of $2 \mathrm{~h}$ after stroke onset. We assessed the mean area of tissue ischemia according to cerebral blood flow (CBF), cerebral blood volume (CBV), time-to-peak (TTP) maps. Sensitivity, Specificity, negative (NPV) and positive predictive value (PPV) were calculated for the end-points "decompressive surgery" and/or midline shift $>5 \mathrm{~mm}$ on follow up imaging. By 
means of receiver operating characteristics, the size of ischemia with optimum prediction was calculated.

Results: All perfusion maps revealed a very high NPV (>95\%), a high sensitivity $(>85 \%)$ and specificity $(>70 \%)$ and moderate values for PPV $(44-47 \%)$. Best prediction was found for CBF maps with an ischemia of $>27.9 \%$ of the hemisphere: Sensitivity $85.0 \%$, Specificity $77.9 \%$, PPV $47.3 \%$, NPV $95.7 \%$.

Conclusion: CT-based perfusion mapping enable to discriminate between patients who are at high or low risk for the development of malignant brain infarction. However, the PPV is lower as compared with MRI techniques.

\section{New vascular imaging}

THREE-DIMENSIONAL DYNAMIC MAGNETIC RESONANCE ANGIOGRAPHY FOR THE EVALUATION OF RADIOSURGICALLY TREATED CEREBRAL ARTERIOVENOUS MALFORMATIONS

J.Y. Gauvrit, C. Oppenheim, F. Nataf, X. Leclerc, J.F. Meder

Hospital R Salengro, Lille; CH St Anne, Paris, France

Purpose: We assessed the value of three-dimensional (3D) dynamic magnetic resonance angiography (MRA) for the follow-up of patients with radiosurgically treated cerebral arteriovenous malformations (AVMs).

Methods: Fifty-four patients with cerebral AVMs treated by radiosurgery (RS) were monitored using conventional catheter angiography (CCA) and 3D dynamic MRA with sensitivity encoding based on the parallel imaging. Cerebral AVM was qualitatively classified by two radiologists into one of five categories in terms of residual nidus size and persistence of early draining vein (I, $>6 \mathrm{~cm}$; II, 3-6 cm; III, $<3 \mathrm{~cm}$; IV, isolated early draining vein; V, complete obliteration).

Results: 3D MRA findings showed a good agreement with CCA in 40 cases (kappa=0.62). Of 23 nidus detected on CCA, 3D dynamic MRA showed 14 residual nidus. Of 28 occluded nidus on 3D dynamic MRA, 22 nidus were occluded on CCA. The sensitivity and specificity of 3D dynamic MRA for the detection of residual AVM were $81 \%$ and $100 \%$.

Conclusion: 3D dynamic MRA after RS may therefore be useful in association with MRI and can be repeated as long as opacification of the nidus or early venous drainage persists, one CCA remaining indispensable to affirm the complete occlusion at the end of follow-up.

\section{New vascular imaging}

\section{DIFFERENCES IN ISCHEMIC LESION LOCALIZATION DUE TO} CARDIOEMBOLIC OR LARGE VESSEL SOURCES: A VOXELWISE APPROACH

S. Hermans, P. Dupont, V. Thijs

University Hospitals Leuven, Leuven, Belgium

Background: Etiologic mechanisms are often inferred when analyzing lesion patterns on brain imaging of patients with ischemic stroke. Previous studies have not used modern statistical imaging methods to describe these patterns. The goal of our study was to assess whether differences exist in brain regions affected by stroke due to cardioembolic sources or due to large vessel disease.

Methods: We analyzed the magnetic resonance imaging (MRI) scans of patients with atrial fibrillation or a significant carotid stenosis or occlusion with ischemic lesions on diffusion weighted imaging (DWI) within a single middle cerebral artery territory (MCA). DWI and fluid attenuated inversion recovery sequences (FLAIR) were performed two to 7 days after symptom onset. Ischemic lesions were manually outlined on FLAIR. Brain scans and regions of interest (ROI) were normalized to a standard brain template using SPM2. A chi-squared test was used to determine whether different voxels were affected in both groups.

Results: We enrolled 33 patients with isolated MCA infarction due to atrial fibrillation $(n=14)$ or high grade carotid stenosis $(n=9)$ or occlusion $(n=10)$. Although there was an important overlap between regions affected by cardioembolic sources and large vessel disease, twenty clusters of voxels were significantly more frequently affected with a cardioembolic source than with large vessel disease with p-values ranging between 0.03 to 0.001 . These clusters were mainly localized in superior temporal gyrus, insula and precentral gyrus.

Conclusions: These findings suggest that cardiac emboli may specifically cause ischemia damage to superior temporal gyrus, the insular region and precentral gyrus compared to ischemia due to large vessel disease. This approach may permit identification of lesion patterns on brain imaging that are typical of specific etiologic mechanisms.
8 New vascular imaging

STRATIFIED GRAY-SCALE MEDIAN ANALYSIS PREDICTS ACTIVE, COMPLICATED CAROTID PLAQUES ON ANATOMOPATHOLOGY WITH INCREASED FIBRIN/FIBRINOGEN TURNOVER

A. Palasí, P. Cardona, R. Vila, M. Miguel, F. Rubio, J. Krupinski

University Hospital of Bellvitge (HUB), Barcelona, L'Hospitalet de Llobregat (Barcelona), Spain

Background: Stratified gray-scale median (GSM) analysis of the carotid plaques has been demonstrated to correlate with anatomopathological state of carotid plaque. However, little is known about relation between GSM values and individual markers of altered haemostasis.

Methods: We included 49 patients, 31 symptomatic and 18 asymptomatic scheduled for carotid endarterectomy. The video signal from the ultrasound device was converted to a digital image and GSM measurements were performed by 2 independent investigators. The normalized gray scale was 0 for blood and 190 for adventitia. The GSM levels 30 and below were considered as high risk based on stratified analysis of our population. Fibrin/fibrinogen related products were evaluated by ELISA, Western blotting, and plaques were classified as ulcerated-complicated (UC), ulcerated non complicated (UNC) and fibrous by anatomopathology.

Results: In our study, elderly patients had lower GSM levels $(\mathrm{p}<0,05)$. Active, UC and UNC plaques with high cellular content had low GSM values as compared to stable fibrous plaques $(\mathrm{p}<0,05)$. Further, markers of intraplaque fibrin/fibrinogen turnover and circulating fibrionogen were increased in patients with plaques that demonstrated low GSM values $(\mathrm{p}<0,05)$.

Discussion: In this study, we confirm that GSM analysis could not only predict plaque anatomopathology but also correlated with markers of fibrin/fibrinogen turnover and subsequent risk of symptomatic atherothrombosis

\section{New vascular imaging}

\section{DETECTION OF UNSTABLE CAROTID ATHEROSCLEROTIC STENOSIS} USING NON-INVASIVE MAGNETIC RESONANCE IMAGING

L. Esposito, D. Sander, M. Sievers, P. Heider, O. Wolf, O. Greil, K. Sander, H. Poppert

Technical University of Munich, Munich, Germany

Background: Carotid artery stenosis has previously been classified by MRI as lesion type I-VIII according to a modified histological scheme based on American Heart Association (AHA) guidelines. Lesion types IV-V and VI, which are containing a large necrotic core or intraplaque hemorrhage, have been suggested to represent high risk atherosclerotic plaques. We aimed to evaluate the clinical relevance of this classification for identifying rupture prone plaque.

Methods: 35 Patients ( 26 male) with carotid stenosis $>70 \%$ (diagnosed by Doppler examination) were imaged with a $1.5 \mathrm{~T}$ scanner with bilateral phased array carotid coils. T1-, T2, time-of-flight (TOF) and proton-density (PD)-weighted studies were obtained. Field of view was $160 \times 100 \mathrm{~mm}$; slice thickness was $1 \mathrm{~mm}$ for the TOF images and $2 \mathrm{~mm}$ for the T1, T2 and PD weighted images. The images were evaluated by two reviewers who were blinded to the clinical history. The carotid plaque were classified as lesion type III-VIII according to the MRI-modified AHA criteria.

Results: 13 patients presented with a recently symptomatic stenosis, 22 patients were asymptomatic. Two patients (one symptomatic, one asymptomatic) were excluded because of insufficient image quality. There were significantly more symptomatic patients with lesion types IV-V and VI as compared to asymptomatic patients $(66.7 \%$ and $16.7 \%$ vs. $28.6 \%$ and $4.8 \% ; \mathrm{P}<0.05)$. These results indicate that lesion types IV-V and VI represent unstable plaques which are likely to cause embolic events.

Discussion: The distribution of lesion types differs significantly in symptomatic and asymptomatic carotid stenosis. Our data suggest MRI-plaque-classification according to the modified AHA-criteria to be a suitable tool for detection of unstable carotid lesions. 


\section{Dementia/cognition and gait}

\section{Dementia/cognition and gait}

SUBCORTICAL VASCULAR LESIONS ARE ASSOCIATED WITH COGNITIVE DECLINE IN MCI PATIENTS

S. Debette, S. Bombois, X. Delbeuck, C. Delmaire, A. Bruandet, F. Pasquier

Memory Centre, Lille University Hospital, Lille, France

Background: Whether there is an association between subcortical lesions on cerebral MRI and cognitive decline in patients with mild cognitive impairment (MCI) is not well known. Our aim was to test this hypothesis in consecutive MCI patients attending at Lille memory clinic.

Method: We included 148 patients diagnosed with MCI at first visit. Neuropsychological examinations were performed at baseline and at $\leq 12$ month follow-up. Subcortical hyperintensities $(\mathrm{SH})$ included hyperintensities of the periventricular white matter (PVH), lobar white matter (WMH), basal ganglia (BG) and infratentorial foci of hyperintensity (ITF) and were assessed on FLAIR, proton density and T2-weighted MRI scans using the Scheltens (1993) semi-quantitative rating scale (range: 0-84).

Results: The mean duration of follow-up was $3.6 \pm 1.5$ years. Over these follow-up period the MMSE score declined in $45.9 \%$ of the patients, remained unchanged in $24.3 \%$ and improved in $29.7 \%$. The median $\mathrm{SH}$ score was $8[1-29]$ in the patients who declined, $7[0-31]$ in those who remained stable and 3[0-28] in those who improved $(\mathrm{p}=0.004)$. The mean annual MMSE score decline was $0.3 \pm 1.2$ points per year. In a random effects model taking into account the MMSE scores at all follow-up visits cognitive decline was more important with increasing $\mathrm{PVH}$, independently of age, sex, vascular risk factors, educational level, temporal lobe atrophy and MMSE score at baseline $(\mathrm{p}=0.02)$. Additional adjustment for $\mathrm{MCI}$ subtype and executive dysfunction did not modify these results.

Conclusion: $\mathrm{SH}$ and in particular PVH were associated with increased cognitive decline in MCI patients, independently of vascular risk factors, temporal lobe atrophy and cognitive pattern.

\section{Dementia/cognition and gait}

\section{PREVALENCE AND SEVERITY OF MICROBLEEDS IN A MEMORY CLINIC} SETTING

C. Cordonnier, W.M. van der Flier, J.D. Sluimer, D. Leys, F. Barkhof,

P. Scheltens

Alzheimer Center, VU University Medical Center, Amsterdam, The Netherlands

Objective: There is mounting evidence for the involvement of vascular pathology in neurodegenerative disorders. We aimed to determine prevalence and severity of microbleeds (MBs) in a large cohort of patients attending a memory clinic.

Methods: We consecutively included patients attending our memory clinic, between January 2002 and April 2005. We analyzed prevalence and number of MBs according to demographic, diagnostic and MRI data.

Results: We included 772 patients (53\% male, age 66士11). One hundred and twenty-seven patients $(17 \%)$ exhibited at least $1 \mathrm{MB}$. The prevalence differed according to diagnostic groups $(\mathrm{p}<0.0001)$ : $65 \%$ of patients with vascular dementia exhibited MBs compared to $18 \%$ of Alzheimer's (AD) patients, $20 \%$ of mild cognitive impairment (MCI), and $10 \%$ in patients with subjective complaints. The presence of MBs was associated with age, white matter hyperintensities, lacunar infarcts and infarcts.

Conclusion: The prevalence of MBs in a large cohort of patients attending a memory clinic is higher than previously described in community samples and lower than reported in stroke patients. Our finding of a relatively high proportion of MBs in $\mathrm{AD}$ and $\mathrm{MCI}$ provides further evidence for the involvement of vascular factors in neurodegenerative diseases such as AD.

\section{Dementia/cognition and gait}

\section{NATURAL HISTORY OF POST-STROKE DEMENTIA (PSD): A 6-YEAR FOLLOW UP \\ M. Altieri, V. Di Piero, I. Maestrini, E. Sbardella, M. Gasparini, G.L. Lenzi}

University of Roma la Sapienza, Rome, Italy

Introduction: Previous studies indicate that stroke may disclose mixed dementia in the early phase of stroke followed by a vascular one later on. We performed a 6-year follow up in order to better define different patterns of PSD along the time. Methods: Non-demented patients after 6 months from stroke onset were followedup for 6 years. They were examined according to a standardized neurological and instrumental procedure, and underwent a complete neuropsychological (NP) assessment; dementia was diagnosed according to the NINCDS-ADRA and NINDS-AIREN criteria. The same evaluation, including neuroimaging and NP tests, was annually repeated at each control.

Results: 272 cerebrovascular patients (age 65 years $\pm 9,8 ; \mathrm{m} / \mathrm{f} 188 / 82$ ) were enrolled. At the end of follow-up $60(22.1 \%)$ developed dementia. At logistic regression analysis PSD was associated with with age $(\mathrm{p}=0.008)$, severe cortical atrophy $(\mathrm{p}=0.01)$ and leukoaraiosis $(\mathrm{p}=0.05)$ at admission, and progression of cortical and subcortical atrophy during follow up $(\mathrm{p}<0.001$ and $\mathrm{p}=0.01$ respectively). The proportion of patients with dementia was 5/272 at 6 months; $8 / 272$ at $1 \mathrm{y} ; 5 / 272$ at $2 \mathrm{y} ; 13 / 272$ at $3 \mathrm{y}, 10 / 272$ at $4 \mathrm{y}, 5 / 272$ at $5 \mathrm{y}$, and $14 / 272$ at $6 y$. Out of 60 patients, $39(65 \%)$ met the criteria for probable $\mathrm{VaD}$ and $21(35 \%)$ for mixed AD. The Kaplan-Meyer analysis showed a predominantly mixed AD in the first 2 years, and a VaD type in the following 2.5 years. Afterward, no difference in the incidence of PSD subtypes was observed (log-rank test; $\mathrm{p}=0.5$ ).

Conclusion: Our data indicate that stroke might act in the early phase as a disclosing factor for a subtle pre-existing cognitive decline. Later, a pure vascular mechanism seems more likely. Nevertheless, at the end of follow no prevalence of any type of dementia was observed. $\mathrm{VaD}$ and $\mathrm{AD}$ may be considered supplementary over the cognitive decline landscape. However, their borders show an extensive and variable representation.

\section{Dementia/cognition and gait}

\section{COGNITIVE DYSFUNCTION IN CHRONIC CHAGAS DISEASE} CARDIOMYOPATHY

J.S. Dias, A.M. Lacerda, R.M. Vieira-de-Melo, L.C. Viana, P.AP. Jesus, F.JFB. Reis, R. Nitrini, H. Charchat-Fichman, A.A. Lopes, J. Oliveira-Filho Federal University of Bahia, Salvador, Brazil

Background: Chagas disease (CD) remains a major cause of cardiomyopathy and stroke in developing countries. Cognitive repercussion of $\mathrm{CD}$ has not been well studied. We aimed to compare the frequency and pattern of cognitive dysfunction in patients with $\mathrm{CD}$ cardiomyopathy (CDC) and other cardiomyopathies (OC). Methods: We studied 37 patients with CDC and 42 patients with OC with similar age, educational level and cardiac systolic function. The following tests were applied to both groups by a single examiner blinded to CD status: Mini Mental State Exam (MMSE), Brief Cognitive Screening Battery, digit and visual span (forward and backward), Rey's Auditory Verbal Learning Test, Rey-Osterreith Complex Figure Test (ROCFT) and Hospital Anxiety and Depression Scale. Logistic regression multivariable models were constructed to detect predictors of cognitive dysfunction for each test.

Results: Cognitive dysfunction was detected in 9 (24\%) CDC patients and 6 (14\%) OC patients by MMSE corrected for educational level. Independent predictors of abnormal MMSE $(\mathrm{p}<0.05)$ included stroke history $(\mathrm{OR}=5.51 ; 95 \%$ $\mathrm{CI}=1.27-24.01)$ and digoxin use $(\mathrm{OR}=0.23,95 \% \mathrm{CI}=0.06-0.89)$, while $\mathrm{CD}$ showed a trend $(\mathrm{OR}=4.63 ; 95 \% \mathrm{CI}=0.87-24.73, \mathrm{p}=0.07)$. Delayed recall of $\mathrm{ROCFT}$ was significantly worse in CD patients (ROCFT score $4.8 \pm 3.7$ vs. $7.7 \pm 5.4, \mathrm{p}=0.02$ ). After correction for potential confounders, $\mathrm{CD}$ remained a significant predictor of abnormal delayed recall of ROCFT $(\mathrm{OR}=4.67 ; 95 \% \mathrm{CI}=1.23-17.68)$. The remaining cognitive tests did not differ between both groups.

Discussion: Chagas disease was associated with subtle cognitive abnormalities independently of age, educational level or cerebrovascular risk factors.

\section{Dementia/cognition and gait}

TREATING VASCULAR RISK FACTORS MAY SLOW COGNITIVE DECLINE IN ALZHEIMER DISEASE AND VASCULAR DEMENTIA Y. Deschaintre, C. Capele, F. Richard, F. Pasquier

EA 2691, Centre Mémoire de Ressource et de Recherche, Clinique Neurologique, CHRU de Lille, Lille, France

Background: There is growing evidence that vascular risk factors (VRF) contribute to cognitive decline in Alzheimer Disease (AD), AD with cerebro-vascular disease $(\mathrm{AD}+\mathrm{CVD})$ and vascular dementia $(\mathrm{VaD})$. We wanted to evaluate if VRF treatment slows cognitive decline in these dementias.

Methods: We analysed the charts of patients attending our memory clinic for the first time in 1997, with at least two mini-mental status examination (MMSE) more than 6 months apart and with a final diagnosis of AD, AD+CVD or VaD. Mention of high blood pressure, diabetes, dyslipidemia or atherosclerotic vascular disease was sought at their first complete evaluation. We considered these VRF treated if they received, respectively, an antihypertensive, an oral antihyperglycemic or insulin, a statin or a fibrate, an antiplatelet or an anticoagulant.

Results: Within the 142 cases included, 125 (88.0\%) had at least one VRF; 59 
$(47.2 \%)$ were treated and $66(52.8 \%)$ not or only partially treated. Among the four VRF studied, dyslipidemia was the least likely to be treated (25\% treated). The treated and untreated groups were similar for age (72.3 years old), sex $(55.2 \%$ women), education level (72.0\% low level), time since first symptoms (4.5 years), diagnosis $(54.4 \% \mathrm{AD}, 20.8 \% \mathrm{AD}+\mathrm{CVD}$ and $24.8 \% \mathrm{VaD})$, acetylcholinesterase inhibitor exposition $(61.6 \%)$ and follow-up (4.1 years). MMSE mean annual decline ( \pm standard deviation) was respectively $1.47 \pm 2.59$ and $2.80 \pm 4.03$ points $(\mathrm{p}=$ 0.029 , bilateral Student t test).

Discussion: In $\mathrm{AD}, \mathrm{AD}+\mathrm{CVD}$ and $\mathrm{VaD}$ taken as a whole, VRF treatment is associated with a slower decline at MMSE.

\section{Dementia/cognition and gait}

\section{THE PROGNOSTIC VALUE OF WORKING MEMORY IN SUBACUTE} STROKE: DOES IT WORK?

A. Jaillard, S. Trabucco-Miguel, B. Naegele, K. Garambois, O. Detante, M. Hommel

UnCentre Hospitalier Universitaire de Grenoble, Grenoble, France

Background: Cognitive impairment (CI), outside dementia, is an important problem after stroke which might be a key endpoint in prognosis after stroke. CI, and especially working memory (WM) impairment, may limit the participation in social and professional activities. in patients with apparent good recovery. The aim of our study was to evaluate the prognostic value of CI in recovery at 6 months.

Methodology: Patients with first-ever infarction had prospectively neuropsychological evaluation within 1 month after ischemic stroke documented by MRI if Mini Mental state Evaluation (MMSE) was $\geq 23 / 30$. Evaluation included NIHSS and at day $1 \& 15$, Rankin score at 6 months. Neuropsychological tests investigated depression, instrumental functions, memory, executive functions and explored the central executive of working memory (WM) using the PASAT and OWEN tests. The Rankin scored " $0-1$ " (versus $\geq 2$ ) 6 months after stroke was considered as the indicator of complete recovery. Statistical analysis were performed using logistic regression to evaluate the prognostic value of the Rankin score.

Results: Among the 177 stroke patients (mean age 50.6 years) evaluated 14.5 $( \pm 8.4)$ days after stroke, the Rankin score $\leq 1$ at 6 months was predicted by depression $(\mathrm{OR}=0.89$ per point of Beck depression index; $\mathrm{p}=0.001)$, NIHSS score at day $15(\mathrm{OR}=0.75$ per point; $\mathrm{p}=0.04)$, categorical fluency $(\mathrm{OR}=1.1$ per point; $\mathrm{p}=0.001)$, and Owen spatial WM $(\mathrm{OR}=0.98$ per between error; $\mathrm{p}=0.03)$.

Discussion: Impairment of the central executive of WM is a key feature in the evaluation of the prognosis of clinical recovery. These results should lead us to include WM assessment in the pronostic evaluation of stroke patients without serious neurological after-effects.

\section{Dementia/cognition and gait}

PREVALENCE OF SUBCORTICAL VASCULAR LESIONS IN MCI SUBTYPES S. Bombois, S. Debette, X. Delbeuck, C. Delmaire, A. Bruandet, F. Pasquier Memory Centre, Lille University Hospital, Lille, France

Background: While the association of subcortical lesions with Alzheimer's disease has been widely studied, no systematic evaluation of such lesions has been performed in patients with mild cognitive impairment (MCI). Our aim was to describe the amount and the distribution of subcortical lesions on cerebral MRI in consecutive MCI patients referred to a memory clinic.

Method: A standardized neuropsychological examination and a cerebral MRI with FLAIR, proton density and T2-weighted scans were performed at baseline. Subcortical hyperintensities ( $\mathrm{SH}$ ) included hyperintensities of the periventricular white matter (PVH), lobar white matter (WMH), basal ganglia (BG) and infratentorial foci of hyperintensity (ITF) were assessed with the Scheltens (1993)semi-quantitative rating scale (range:0-84).

Results: We included 148 consecutive patients diagnosed with MCI at first visit (mean age: $66.2 \pm 9.9$ years, women: $51.6 \%$, educational level $<8$ years: $57 \%$ ). The mean MMSE score was $28.1 \pm 1.2$ at baseline. The MCI subtype was amnestic in $27.5 \%$, multiple domain in $55.7 \%$ and single non-memory domain in $16.8 \%$ of patients.

SH were observed in 137 patients (92.6\% [95\%CI: 88.3-96.8\%]) and predominated in the white matter $(80.4 \%$ of the patients had PVH and $84.5 \%$ had WMH) compared to the BG $(23.7 \%)$ and the ITF $(23.0 \%)$. In a multivariable logistic regression a $\mathrm{SH}$ score $>6$ was more frequent with increasing age $(\mathrm{OR}=1.1$ [95\% CI: $1.0-1.2], \mathrm{p}=0.0004)$ and temporal lobe atrophy $(\mathrm{OR}=2.0$ [95\% CI: 1.1-3.8], $\mathrm{p}=0.02)$ and was not associated with MCI subtype. A PVH score $>1$ was more common in patients with executive dysfunction (OR=3.4 [95\% CI:1.3-8.9], $\mathrm{p}=0.01)$.

Conclusion: $\mathrm{SH}$ were highly prevalent in MCI patients, whatever the clinical subtype. Patients with executive dysfunction had a higher amount of PVH.
8 Dementia/cognition and gait

FRONTAL AND PERIVENTRICULAR BUT NOT BASAL GANGLIA AND PONTINE AGE-RELATED WHITE MATTER CHANGES CORRELATE WITH FALLS - NEWS FROM THE LADIS STUDY

C. Blahak, H. Baezner, A.A. Gouw, P. Scheltens, L. Pantoni, D. Inzitari, M.G. Hennerici, on behalf of the LADIS Study Group Universitaetsklinikum Mannheim, University of Heidelberg, Mannheim, Germany

Background: Results of the LADIS (Leukoariosis And DISability) study have demonstrated a positive correlation between general age-related white matter changes (ARWMC) and falls. Balance disturbances due to interruptions of corticosubcortical sensorimotor circuits reflect the most probable reason for falls in ARWMC, but studies correlating motor performance with the anatomic distribution of ARWMC are lacking to date.

Methods: 639 non-disabled individuals (mean age $74.1 \pm 5.0$ ) were followed-up for 3 years with clinical and functional tests including specific scores for motor testing. Centralized rating of baseline MRI scans was performed, using different scales like the Scheltens scale to quantify severity and anatomic distribution of AWRMC.

Results: Frontal $(p<0.001)$, periventricular $(p<0.05)$ and mesencephal $(p<0.05)$ ARWMC correlated significantly with the history of falls in the year prior to study inclusion, in contrast to basal ganglia, occipital and pontine ARWMC. Single leg stance time as a test for balance ability is significantly shorter in subjects with severe frontal ARWMC (12.1s $\pm 11.0 \mathrm{~s})$ than in those without lesions $(21.7 \mathrm{~s} \pm 10.6 \mathrm{~s}$; $\mathrm{p}<0.01)$.

Discussion: These data reveal a significant correlation of particularly frontal ARWMC with the history of falls in cross-sectional analysis of the LADIS study. This supports the hypothesis, that the most probable reason for falls in ARWMC are interruptions of frontal subcortical motor circuits causing balance disturbances, whereas lesions of descending motor fibers might be secondary.

\section{Dementia/cognition and gait}

DISTURBANCES OF GAIT AND BALANCE IN AGE-RELATED WHITE MATTER CHANGES - ONE YEAR FOLLOW-UP RESULTS OF THE LADIS STUDY

H. Bäzner, C. Blahak, L. Pantoni, D. Inzitari, M.G. Hennerici, on behalf of the LADIS study group

Universitätsklinikum Mannheim, University of Heidelberg, Mannheim, Germany

Background: In the LADIS (Leukoaraiosis And DISability) study eleven European centres collaborate with the aim to evaluate age-related white matter changes (ARWMC) as an independent predictor of the transition to disability in the elderly (65-84 years). Severe ARWMC are regarded as clinically relevant and potentially disabling due to cognitive and motor compromise, while the clinical significance of mild ARWMC is often doubted. Hence, we aimed at prospectively determining the association between different clinical scales and measures of gait and balance with varying degrees of ARWMC severity on cranial magnetic resonance imaging (MRI)

Methods: 639 non-disabled individuals were enrolled and are being followed-up for 3 years. At baseline and during follow-up, motor tests including the short physical performance battery (SPPB) are performed. The SPPB includes an assessment of standing balance, a timed walk at a normal pace, and a timed test of five repetitions of rising from a chair and sitting down. SPPB total scores range from 0-12, with scores below 10 indicating motor compromise. According to central MRI ratings, patients are stratified into three groups of mild, moderate, and severe ARWMC.

Results: 1-year follow-up data of 584 individuals demonstrated a progressive decline in motor performance in all three ARWMC severity groups (baseline data in brackets): $9.9 \pm 2.1(10.2 \pm 2.0, \mathrm{p}=0.005)$ in the mild, $9.4 \pm 2.6(9.9 \pm 1.9, \mathrm{p}=0.003)$ in the moderate, and significantly worse with a score of $8.5 \pm 2.8(9.2 \pm 2.3, \mathrm{p}=0.001)$ in the severe group.

Discussion: A progressive worsening of motor ability already in 1-year follow-up can be demonstrated independent of the severity of ARWMC on MRI.

Oral Session

Dementia/cognition and gait 


\section{Acute stroke: clinical patterns and practise}

1 Acute stroke: clinical patterns and practise

\section{RELIABILITY OF CLINICAL DIAGNOSIS OF THE SYMPTOMATIC VASCULAR TERRITORY IN PATIENTS WITH RECENT TIA OR MINOR STROKE}

E. Flossmann, J.N. Redgrave, U.G. Schulz, D. Briley, P.M. Rothwell University Radcliffe Infirmary, Oxford, United Kingdom

Background: Knowledge of the vascular territory of a recent TIA or minor stroke determines appropriate investigation and the need for territory-specific interventions, such as endarterectomy and stenting. However, there are few published data on the accuracy of clinical assessment of vascular territory.

Methods: We therefore studied the agreement of a clinical diagnosis of vascular territory in patients with TIA or minor stroke with the near "gold standard" of lesion location on DWI-MRI in consecutive patients attending a TIA clinic who had an acute ischaemic lesion(s) in a single vascular territory (determined by an independent neuroradiologist). Three independent neurologists diagnosed the most likely vascular territory (left or right carotid, or vertebrobasilar) of the presenting event for each patient blind to the results of brain imaging. One of the neurologists had seen and examined the patients and the others had a clinical summary.

Results: 133 (28.0\%) of 476 patients had a high signal lesion on DWI, of whom $115(86.5 \%)$ had a minor stroke and $18(13.5 \%)$ a TIA. Inter-observer agreement (kappa statistic) on territory ranged from 0.46 to 0.60 . The agreement with DWI was only moderate (observer 1: kappa $=0.5495 \%$ CI $0.36-0.72$, observer $2: 0.48$ $0.31-0.64$, observer $3: 0.48,0.28-0.67)$. Only the presence of visual symptoms improved the accuracy of vascular territory diagnosis (range of kappa: 0.63-0.77) but not the presence of motor, speech or sensory symptoms.

Discussion: The reliability of clinical diagnosis of vascular territory is only moderate, highlighting the importance of sensitive brain imaging after TIA or minor stroke. Further imaging-based research is required to determine the optimal clinical diagnostic criteria for classification of vascular territory.

\section{Acute stroke: clinical patterns and practise}

\section{THE EFFECT OF PRIOR ANTITHROMBOTICS USE ON EARLY}

FUNCTIONALOUTCOME IN PATIENTS WITH ISCHEMIC STROKE

M. Paciaroni, M. Acciarresi, K. Nardi, V. Caso, M. Venti, A. Alberti, P. Milia,

F. Palmerini, S. Biagini, G. Agnelli

University of Perugia, Silvestrini Hospital, Perugia, Italy

Background: Experimental reports have shown that antithrombotic agents could have a neuroprotective effect while clinical studies on this issue in patients with ischemic stroke have produced contradictory results. The aim of this study was to analyse the role of pre-treatment with antithrombotic agents (antiplatelets or anticoagulants) on functional outcome in ischemic stroke patients.

Subjects and Methods: Consecutive patients from the Perugia Stroke Registry were studied between January 2000 to December 2003. Outcome was evaluated at discharge using the modified Rankin Scale ( $\mathrm{mRS} \geq 3$ disabling stroke).

Results: 1,921 patients were studied (mean age $76.3 \pm 12.5$ years; $53 \%$ males); $662(34.5 \%)$ of these were on antithrombotic treatment (581 antiplatelets, 71 anticoagulants and 10 antiplatelets associated with anticoagulants). During the hospital stay 122 patients $(6.4 \%)$ died and $834(43.4 \%)$ died or were disabled: $54(44.3 \%)$ of the deceased patients and $324(38.8 \%)$ of deceased or disabled patients were on antithrombotic treatment while $608(33.8 \%)$ who survived and $338(31.1 \%)$ non-disabled patients were taking antithrombotics $(\mathrm{p}=0.023$ and $\mathrm{p}<0.0001$, respectively). From multivariate analysis age and stroke severity were associated with a worse outcome. Males, dyslipidemia, stroke due to small vessel disease and no history of previous stroke were associated with a better outcome while no correlation was found between pre-treatment with antithrombotics and outcome (mortality $\mathrm{OR}=1.32,95 \% \mathrm{CI} 0.85-2.04 ; \mathrm{p}=0.20$ and mortality or disability $\mathrm{OR}=0.95,95 \%$ CI $0.72-1.25 ; \mathrm{p}=0.80)$.

Conclusion: Pre-treatment with antithrombotics does not improve the functional outcome of stroke at discharge.
3 Acute stroke: clinical patterns and practise

DELAY IN SEEKING MEDICAL ATTENTION AFTER A TIA IS ASSOCIATED WITH PREDICTED STROKE RISK

M. Giles, E. Flossmann, P. Rothwell

University of Oxford, Oxford, United Kingdom

Background: Little research has been done on patients' behaviour following TIA Recent data on the high early risk of stroke after TIA mean that emergency action following TIA is essential for effective secondary prevention. We therefore studied patients' behaviour immediately after TIA according to their perceptions, clinical characteristics and predicted stroke risk.

Methods: Consecutive patients with TIA participating in a population based incidence study or attending dedicated hospital clinics in Oxfordshire, UK, were interviewed. Predicted stroke risk was calculated using two validated scores.

Results: Of 241 patients, $107(44.4 \%)$ sought medical attention within hours of the event, although only $24(10 \%)$ of these attended the Emergency Department. $107(44.4 \%)$ delayed seeking medical attention for one or more days. Correct recognition of symptoms ( $42.2 \%$ of patients) was not associated with less delay. However, patients with motor symptoms or duration of symptoms $\geq 1$ hour were more likely to seek emergency attention $(\mathrm{OR}=3.3,95 \% \mathrm{CI}=1.8-6.1, \mathrm{p}=0.00005)$, as were those at higher predicted stroke risk $(\mathrm{p}=0.002)$. The other main correlate with delay was the day of the week on which the TIA occurred $(\mathrm{p}<0.001)$, with greater delays at the weekend. Delay was unrelated to age, sex, or other vascular risk factors.

Discussion: Most patients delay seeking medical attention after a TIA irrespective of correct recognition of symptoms, although patients at higher predicted risk of stroke do act more quickly. Public education about both the urgency and nature of TIA is required.

\section{Acute stroke: clinical patterns and practise}

\section{HIPPOCAMPAL STROKE - A CLINICAL AND DIFFUSION-WEIGHTED MRI} ENTITY?

A. Förster, K. Szabo, S. Bukow, M. Griebe, R. Kern, M.G. Hennerici, A. Gass Universitätsklinikum Mannheim, University of Heidelberg, Mannheim, Germany

Background: The hippocampus, as part of the limbic system is a particularly interesting anatomical structure affected by various pathologies in acute and chronic neurological syndromes. In TGA and complex focal status epilepticus (CPSE) lesions of the hippocampus on diffusion-weighted MRI (DW MRI) have been identified. In the light of these findings, we analyzed clinical and MRI data of patients with acute stroke in the posterior circulation affecting the hippocampus. Methods: From a prospectively collected stroke and MRI data bank (2000-2005) we analyzed 32 patients with acute stroke in the A. cerebri posterior (PCA) territory involving the hippocampus with respect to clinical symptoms and lesion topography.

Results: In 29 cases hippocampal ischemic involvement was unilateral ( R 9, L 20), while $3 / 32$ cases showed bilateral mesiotemporal lesions. All patients had additional acute ischemic lesions on DW MRI in other parts of the PCA territory.

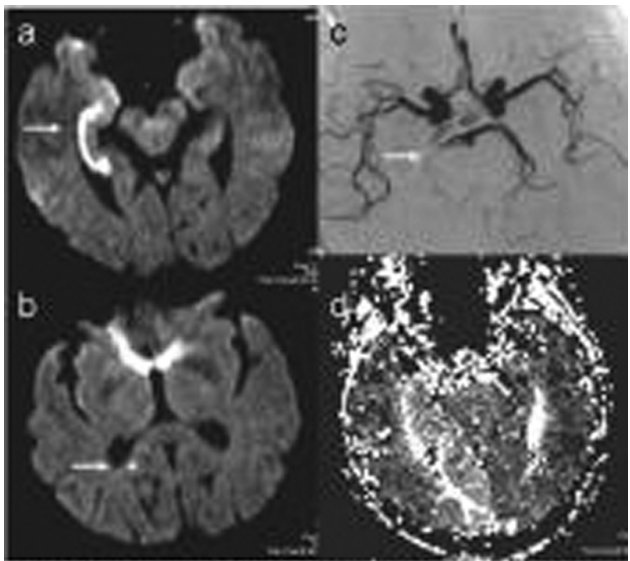

72-year old woman with transient sensory symptoms of the left leg, without neuropsychological symptoms. DWI shows acute ischemic lesion in the lateral portion of the right hippocampus and a punctuate lesion $(\mathrm{a}+\mathrm{b})$. Stenosis of the PCA and MRA (c) causes hypoperfusion of the right PCA territory (d). 


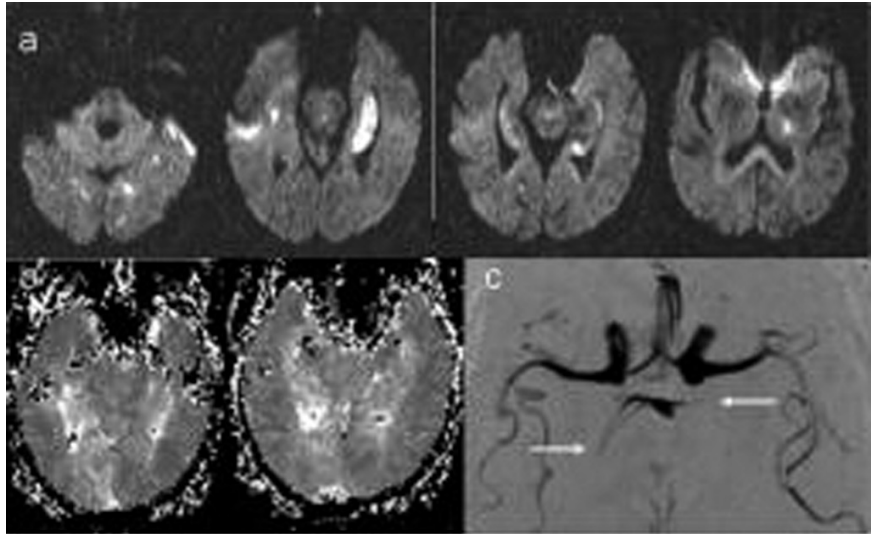

64-year old man with acute amnestic syndromes. DWI (a) shows bihemispheric multiple acute ischemic lesions in both PCA territories, including the hippocampus bilaterally. Bilateral PCA stenosis (c) leads to late contrast bolus arrival in both PCA territories on TTP perfusion images.

On MRA, ipsilateral PCA showed stenosis or occlusion in 28/30 (87.5\%). While all patients presented with typical symptoms of vertebro-basilar stroke, only $9 / 32$ $(28 \%)$ showed additional neuropsychological syndromes, and only one patient presented with initial seizures. Typical features of TGA or CPSE were not present in any patient of this series.

Discussion: 1. Acute stroke of the hippocampus is unlikely to occur as an isolated lesion, but rather in addition to further lesion within the PCA territory.

2. Clinical features in hippocampal stroke are mainly vertebro-basilar stroke syndromes - amnestic symptoms or seizures are not common.

3. DW MRI involvement of the hippocampus is present in hippocampal stroke,

TGA and after CPSE, each presenting as a distinct clinical entity.

\section{Acute stroke: clinical patterns and practise}

THE AUSTIN HOSPITAL ACUTE STROKE TEAM (AST): AN EFFECTIVE APPROACH TO HASTEN ASSESSMENT AND MANAGEMENT OF STROKE IN THE EMERGENCY DEPARTMENT

F. Nazir, I. Petre, H. Dewey

Austin Health, Melbourne, Heidelberg, VIC, Australia

Background: Intravenous recombinant tissue plasminogen activator (rtPA), alteplase, given within 3 hours of stroke onset, remains the only acute treatment proven to reduce death and disability after ischaemic stroke. Despite this, few patients receive alteplase, with delays in in-hospital evaluation and treatment being a key barrier to therapy. The Austin Hospital Acute Stroke Team (AST) was introduced on 17 January 2005 with the aim of improving the speed of assessment and management of acute stroke patients presenting to the emergency department. We sought to assess the effect of the AST on our already active stroke service. Methods: We analysed prospectively acquired data for all AST calls received between 17th January and 31st December 2005. Data recorded included: demographics; time of ED arrival, AST call, symptom onset, brain imaging and alteplase treatment; and diagnosis made by the AST.

Results: A total of 224 AST calls occurred. 54\% were female, median age was 75 years. $53 \%$ of AST calls occurred within working hours. 152 patients $(68 \%)$ had a final diagnosis of stroke, for which the median (Q1,Q3) NIH score was $6(3,13)$. 27 patients received treatment with alteplase (12\% of calls). Over the same time period in 2004, 11 patients received alteplase treatment. The most common reason for not treating was mild or rapidly resolving deficit. The median onset-needle time was $140(122.5,149.5)$ mins, AST call-CT time $28(16,33)$ mins, door-needle time $71(54.5,91)$ mins. Seizures and loss of consciousness without focal neurological deficit were the most common stroke mimics presenting within 3 hours.

Discussion: The introduction of the AST has increased the number of eligible patients receiving rtPA. Rapid door-CT and door-needle times are achievable with this approach
6 Acute stroke: clinical patterns and practise

DIAGNOSTIC SENSITIVITY OF RECOGNITION OF STROKE IN THE EMERGENCY ROOM (ROSIER) SCALE COMPARED TO OTHER STROKE RECOGNITION INSTRUMENTS IN AN EMERGENCY ROOM SETTING G.A. Ford, A. Mohd Nor

Newcastle University, Newcastle upon Tyne, United Kingdom

Background: Early stroke recognition in the Emergency Room (ER) is important We developed the ROSIER to aid early recognition of stroke in the ER. We compared the performance of ROSIER to existing paramedic stroke scales; MASS (Melbourne Ambulance Stroke Screen), FAST (Face Arm Speech Test), CPSS (Cincinnati Pre-Hospital Stroke Scale), LAPSS (Los Angeles Pre-Hospital Stroke Screen).

Methods: Data were prospectively collected on all patients with suspected stroke or TIA with symptoms seen in the ER over a 9 month period. ROSIER was completed by ER physicians prior to brain CT/MRI. Patients with suspected stroke, irrespective of the ROSIER score were then transferred to the Acute Stroke Unit All patients were examined by a research neurologist $(95 \%)$ or senior stroke unit physician. Diagnostic sensitivity, specificity, positive predictive value (PPV) and negative predictive value (NPV) were calculated for each scale; ROSIER (loss of consciousness, seizure, face/arm/leg weakness, speech disturbance, visual field defect, glucose $>3.5 \mathrm{mmol} / \mathrm{l}$ ); CPSS (face/arm weakness, speech disturbance); (FAST (face/arm weakness, speech disturbance, Glasgow Coma Score $>6$ ); LAPSS (face/arm/grip weakness, glucose 2.8- $22.2 \mathrm{mmol} / \mathrm{l}$, age $>45$ years, no seizure activity, symptoms $<24 \mathrm{hr}$, patient not wheelchair bound or bedridden); MASS (combined CPSS and LAPSS items).

Results: 160 suspected stroke patients were seen; 88 stroke ( $91 \%$ ischaemic), 59 non-stroke, 13 TIA with symptoms, age (mean, SD) stroke $(71 \pm 14)$, non-stroke $(72 \pm 16)$ years. Commonest stroke mimics were syncope $(24 \%)$, seizure $(14 \%)$, sepsis $(14 \%)$ and somatisation disorders $(12 \%)$. Diagnostic profiles were (sensitivity, specificity, PPV, NPV); ROSIER 93\%, 83\%, 90\%, 88\%; FAST (82\%, 83\%, $89 \%, 73 \%)$; CPSS $(85 \%, 79 \%, 88 \%, 75 \%)$; LAPSS $(59 \%, 85 \%, 87 \%, 55 \%)$; MASS $(78 \%, 85 \%, 90 \%, 69 \%)$.

Conclusions: The ROSIER scale demonstrates greater sensitivity than existing pre-hospital stroke recognition instruments in the ER setting.

\section{Acute stroke: clinical patterns and practise}

CIRCADIAN VARIATION OF ISCHAEMIC STROKE ACCORDING TO ONSET AND SYNDROME - AN 8 YEARS SURVEY FROM THE BASEL STROKE REGISTRY

P. Lyrer, M. Gostynski, F. Fluri, L. Bonati, S. Papa, S. Engelter University Hospital Basel, Basel, Switzerland

Background: The Basel Stroke registry was established in 1995 to systematically assess emergency admissions of acute stroke patients in the Basel Stroke Unit. Patients and Methods: From March 1995 to February 2003 data from 2603 patients with ischaemic stroke were prospectively registered. In 1714 (65.8\%) patients, symptom onset was known exactly. These comprised our study population. Stroke syndromes were classified according to the Oxfordshire Community Stroke Project and correlated to time and day of emergency admission. Chi square statistics were applied.

Results: 785 patients entered the hospital within 3 hours of symptom onset (45.8\%) Within this group, $65.6 \%$ patients had total anterior circulation infarcts $(p<0.001)$. On the opposite, among patients with lacunar infarcts, only $38.6 \%$ were hospitalised within 3 hours. Stroke onset occurred in $29.8 \%$ between 7 and 11 am and was not equally distributed over the weekdays. More strokes than expected occurred on a Tuesday $(+11.5 \%, \mathrm{p}=0.031)$. Stroke syndromes were equally distributed over the weekdays.

Conclusions: Among stroke patients with known symptom onset, almost half arrive at the emergency room within 3 hours. Patients with large cerebral infarcts were hospitalised earlier, while those with lacunar infarcts had the longest delay. More strokes occurred on Tuesdays and in the months from March to May. In one third of patients with confirmed ischemic stroke, symptom onset was unknown.

Oral Session

Acute stroke: clinical patterns and practise 
8 Acute stroke: clinical patterns and practise

SUDDEN-ONSET LIMBKINETIC DYSPRAXIA: AN ACUTE STROKE SYNDROME WITH A LESION AFFECTING THE CONTRALATERAL PRIMARY MOTOR CORTEX

C. Stapf, F. Buffon, A. Viswanathan, H. Chabriat, M.G. Bousser

Hôpital Lariboisière, Paris, France

Background: Limbkinetic dyspraxia (LD) constitutes a clinical syndrome characterized by loss of kinetic fluidity and impaired dexterity of distal limb movements. However, its significance as an acute stroke syndrome (regarding lesion location, stroke mechanism, and prognosis) has thus far not been investigated.

Methods: We prospectively studied patients presenting with isolated, acute LD using MR brain imaging (T1, T2, FLAIR, DWI) and standard diagnostic work-up (Doppler, TTE/TOE). LD was defined by isolated impairment and slowness of complex finger movements using the "penny pincher" test (the subject was asked to bring a coin from the palm of the hand to a position between the thumb and index finger). Patients showing additional focal deficits (i.e., aphasia, dysarthria, neglect, ataxia, and sensory or motor deficits interfering with distal limb movements) were excluded.

Results: Overall, 12 consecutive patients (all right-handed, mean age 56 years) with either left- $(\mathrm{n}=8)$ or right-sided LD were evaluated. In all cases, MRI demonstrated an acute vascular lesion affecting the contralateral primary motor cortex, including 11 infarcts ( 7 due to large artery disease, 2 cardioembolic, 2 cryptogenic) and one hemorrhage (AVM rupture). Clinical recovery at 3 months was generally good, but remained incomplete in 6 patients with preexisting subcortical white-matter lesions on MRI.

Conclusions: Sudden-onset LD represents an acute stroke syndrome suggesting a contralateral lesion in the primary motor cortex If ischemic, the lesion is likely due to large-vessel or cardiac disease. Our data argue against the commonly assumed involvement of the premotor area in the etiology of isolated LD.

\section{Acute stroke: clinical patterns and practise}

SPINAL CORD ISCHEMIA: CLINICAL AND IMAGING PATTERNS, PATHOPHYSIOLOGY, AND OUTCOME IN 27 PATIENTS

J. Novy, A. Carruzzo, P. Maeder, J. Bogousslavsky

CHUV/Lausanne, Lausanne, Switzerland

Background: Natural history and pathophysiology of ischemic spinal cord infarction remain largely unknown because most clinical studies included mostly patients with ischemic lesions consecutive to aortic surgery or prolonged hypotension.

Methods: We analyzed the clinical, laboratory, imaging and outcome data for $27 \mathrm{pa}-$ tients with acute spinal cord infarction admitted in our department between 1990 and 2003. There were 11 men and 16 women (age range $19-80$ years, mean age 56 years). Results: Ten $(37 \%)$ had anterior spinal artery patterns, 4 anterior $(15 \%)$ and 4 posterior $(15 \%)$ unilateral patterns, $3(11 \%)$ central syndromes, $2(7 \%)$ posterior spinal artery patterns, and 2 (7\%) transverse patterns, while two $(7 \%)$ had an unclassifiable clinical picture. Twenty patients $(74 \%)$ had no identifiable etiology. Patients with a central or transverse infarct showed a high frequency (40\%) of peripheral vascular disease, and all transverse infarcts occurred following prolonged arterial hypotension. The onset of all other infarcts was associated with mechanical triggering factors $(\mathrm{p}=0.02)$, and these patients frequently had diseases of the spine $(\mathrm{p}=0.003)$ at the level of the spinal lesion, the clinical data suggesting root involvement at the level of the spinal cord lesion and pointing to mechanical injury of a radicular artery. Outcome was favorable, with only 13 patients (48\%) showing significant gait impairment on leaving hospital.

Conclusion: There are two main types of spinal cord ischemia, i.e. radicular artery territory infarct (bilateral anterior or posterior spinal artery infarcts and unilateral infarcts) and extensive spinal cord hypoperfusion (central and transverse infarcts), each of which has characteristic clinical, imaging, pathophysiological and prognostic features.

\section{Acute stroke: clinical patterns and practise}

THE ABILITY OF SERIAL NIH STROKE SCALE SCORES TO IDENTIFY COMPLETE RECANALIZATION IN ACUTE ISCHEMIC STROKE

R. Mikulik, M. Ribo, M.D. Hill, J.C. Grotta, M. Malkoff, C. Molina,

M. Rubiera, R. Delgado-Mederos, AV. Alexandrov

University of Texas Health Science Center at Houston, Houston, TX, USA

Background: Early recovery after intravenous thrombolysis can be observed; however, the utility of measuring clinical improvement to diagnose recanalization has not been established. We sought to determine the potential of serial NIH Stroke
Scale (NIHSS) scores to detect complete recanalization of middle cerebral artery (MCA).

Methods: Data from the CLOTBUST trial was used to determine the most sensitive and specific NIHSS-derived parameter to identify complete recanalization. Then, reproducibility was tested against a separate patient population (Barcelona-dataset). NIHSS-scores were determined before t-PA bolus and at 60 and 120 minutes in both datasets. Receiver operating characteristic curves were used to compare test performance. The accuracy of individual cutoffs was demonstrated by sensitivity, specificity, positive and negative predictive values (PPV and NPV).

Results: A total of 122 patients in CLOTBUST and 98 in Barcelona-datasets received $0.9 \mathrm{mg} / \mathrm{kg}$ intravenous $\mathrm{t}-\mathrm{PA}$ : mean age $69 \pm 12$ vs $72 \pm 12$, men $57 \%$ vs $51 \%$, median NIHSS 16 vs 17 points, mean time from onset to treatment $140 \pm 32$ vs $177 \pm 59 \mathrm{~min}$, and complete MCA recanalization in $19 \%$ vs $17 \%$. For identifying recanalization the NIHSS-score reduction of $\leq 40 \%$ offered the best tradeoff with Sensitivity, Specificity, PPV, NPV of $65 \%, 85 \%, 50 \%, 91 \%$ at $60 \mathrm{~min}$ and $74 \%$, $80 \%, 58 \%, 89 \%$ at 120 minutes. Test performance was equal in Barcelona-dataset. Conclusions: Changes in serial NIHSS-scores can serve as a clinical indicator of early recanalization. Accuracy parameters are affected by "nutritional" incomplete recanalization or clinically "silent" complete recanalization.

\section{Behavior and mood}

\section{Behavior and mood}

PREDICTORS OF ABNORMAL MOOD FOLLOWING STROKE: RESULTS FROM THE AUCKLAND REGIONAL COMMUNITY STROKE (ARCOS) STUDY M.L. Hackett, C.S. Anderson, for the ARCOS Group

The George Institue for International Health, Sydney, NSW, Australia

Background: Clinically significant abnormal mood occurs in approximately one third of stroke survivors. Early identification of those at greatest risk is important for provision of early intervention and subsequent reduction of burden.

Methods: ARCOS was a prospective population-based stroke incidence study conducted in Auckland, New Zealand over a 12-month period (2002-2003). All stroke survivors were interviewed six-months after onset and mood was assessed using the 28-item General Health Questionnaire (GHQ-28). Multivariate stepwise logistic and linear regression were used to develop a predictive model for 'caseness' (score $\leq 5$ on the GHQ-28 using standard 0, 0, 1, 1 scoring) and severity of mood symptoms on the GHQ-28 at six months.

Results: 1172 patients were available for follow-up at six-months. Complete GHQ-28 data were available in $739(60 \%)$ patients and $27 \%$ were defined as cases. Pre-stroke patient and clinical variables measured during the acute phase did not predict a significant amount of the variation in the presence or severity of abnormal mood (C statistic 0.587). However, important baseline predictors were stroke severity and a history of depression before stroke (after adjustment for sex, age and co-morbidity), with pre-stroke 'social capital' providing some protection against the development and severity of abnormal mood.

Conclusion: These findings emphasise that multiple factors are likely to contribute to the development of abnormal mood after stroke and that a simple predictive model for clinical use during the acute phase of stroke is likely to be difficult to develop. In order to optimise stroke services further research to develop reliable methods for identifying those patients at greatest risk of abnormal mood is still required.

\section{Behavior and mood}

ADAPTIVE STRATEGIES IN THE ACUTE PHASE FOLLOWING A STROKE C. Donnellan, D. Hevey, A. Hickey, D. O'Neill

Trinity College Dublin, Dublin, Ireland

Psychological adjustment after stroke may be influenced by stroke severity and functional ability. This study aimed to investigate Baltes Profile of adaptive strategies Selection, Optimisation and Compensation (SOC) in the immediate aftermath of stroke and their relation to stroke severity, physical function as well as associations with psychological well-being.

Method: 80 stroke patients ( $49 \%$ male) mean age 69 years \pm 14.6 were interviewed within 6 weeks of admission. Stroke severity was assessed using the Orpington Prognostic Scale (OPS) and basic activities of daily living (BADL) and instrumental activities of daily living (IADL) were measured using the Barthel Index (BI) and the Nottingham Extended Activities of Daily Living (NEADL). Strategies of adaptation were measured using the 18 item Selection (Elective and Loss-based), Optimisation and Compensation questionnaire (SOC-18). The Hospital Anxiety and Depression Scale (HADS) was used to assess psychological well-being. 
Results: $57 \%$ of strokes were classified as mild to moderate, $39 \%$ as moderate to severe and $4 \%$ as severe to very severe. Loss-based Selection was the most commonly used SOC strategy by all three stroke severity groups $(\mathrm{p}<0.05)$. Significant positive relationships were seen between stroke severity and BADL $(r=-0.68, p<0.001)$ and IADL $(\mathrm{r}=-0.62, \mathrm{p}<0.001)$ and Loss-based Selection $(\mathrm{r}=-0.23, \mathrm{p}<0.05)$. Gender correlated with Elective Selection $(r=0.24, p<0.05)$ and anxiety $(r=0.28, p<0.05)$. No significant age differences were reported in the use of SOC strategies although a significant relationship was seen between age and IADL $(r=0.30, p<0.05)$.

Discussion: Stroke severity was associated with Loss-based Selection which tends to be the most frequently used strategy during periods of limited resources. Findings suggest that SOC strategies may be as effective for stroke adjustment as they are for their current use in successful ageing.

\section{Behavior and mood}

\section{APHASIA AFTER RIGHT HEMISPHERE STROKES: CHARACTERISTICS} AND ANATOMO-CLINICAL CORRELATIONS

G. Maillard, A. Carota, P. Maeder, J.M. Annoni, J. Bogousslavsky

University of Lausanne, Lausanne, Switzerland

Most of the studies on aphasia after right hemisphere (RH) stroke have been conducted within the frame of crossed aphasia. We studied the clinical characteristics of 26 patients with aphasia after $\mathrm{RH}$ stroke in a non selected population and compared it to 36 patients with aphasia after left hemispheric (LH) stroke. Aphasia (Boston Diagnostic aphasia (BDA), Montreal Toulouse protocol (MTP), Lausanne Acute Aphasia Battery (LAAB) and imaging data (CT and MRI using the Talairach Tournoux atlas) were correlated. On admission no significant difference was found for comprehension, repetition, naming, and spontaneous verbal fluency, but after 3 days impairment of comprehension (score LAAB RHSA $=1.46$ and LHSA $=$ 1.86) and repetition (1.54 and 2) was less severe after RHSA than LHSA $(p<0.05)$. Aphasia was less severe after RHSA (6.64 vs 7.87 for LHSA with LAAB score; $\mathrm{p}<.05)$. People with RHSA recovered better and quicker after RHSA (3.11vs 2.69 for LHSA) $(\mathrm{p}<0.05)$. Fluent speech was $38 \%$ in RHSA vs $56 \%$ in LHSA $(p<05)$ Dysgraphia was less common after RHSA than LHSA (38\% vs $64 \%$; $<<.05$ ). RH non-fluent aphasia was associated with lesion of the inferior frontal gyrus, insula, longitudinal superior fasciculus and basal ganglia. RH fluent aphasia was associated with lesion of superior, middle and inferior temporal gyri. Our findings highlight specific features of aphasia after RH stroke.

\section{Behavior and mood}

\section{THE PATTERN OF CHANGE IN EMOTION AFTER ACUTE STROKE}

A.K. Pasco, A. Bhalla

St Helier Stroke Service, Epsom and St Helier University Hospitals, Surrey, United Kingdom

Background: Emotional disorders, such as anxiety and depression, are common after stroke and influence stroke outcome. This study evaluates the pattern of change in emotion early after stroke and how this impacts on function and quality of life.

Method: 58 consecutive acute stroke patients admitted to the rehabilitation unit at St Helier Hospital were recruited between April and December 2005. Interviews took place at weeks 1, 6 and 12. Sociodemographic and clinical factors, details on mood (Hospital Anxiety and Depression scale (HADS), Montgomery-Asberg Depression scale), quality of life (Short-Form 12, Stroke Aphasia Quality of Life scale (SAQOL)) and function were recorded.

Results: Of the 58 eligible patients the mean age was 75.2 .35 patients were male. $29(50 \%)$ had a lacunar stroke. Subjects excluded include those with severe dysphasia or medical illness. $21(36 \%)$ patients were found to be depressed using HADS at week 1. Of these, $12(57 \%)$ had reduced function (Barthel less than 12) and all had reduced quality of life (SAQOL score less than 4). Depressive symptoms improved with time - $8(13 \%)$ cases at week 6 and $5(8 \%)$ at week 12 . $23(40 \%)$ patients were found to have anxiety at week 1 . Of these, $13(56 \%)$ had associated depression, $3(13 \%)$ had reduced function and $20(87 \%)$ had reduced quality of life. At week 6, $10(17 \%)$ patients had anxiety and at week 12 there were $5(8 \%)$. In addition, of those patients with normal mood (HADS), 21 (91\%) reported reduced quality of life during the three months following their stroke.

Discussion: Patients with symptoms of anxiety and depression early after stroke appear to improve with time and this may accompany a physical recovery. Not all patients with abnormal mood had reduced function, suggesting that either other factors contribute to mood or that patients attribute more meaning to their function than can be appreciated from the scales. Low quality of life appears to relate to low mood
5 Behavior and mood

CARE GIVERS HEALTH RELATED QUALITY OF LIFE IN STROKE: STRESS AND COPING INFLUENCE

A. Sgoifo, M.G. Celani, E. Righetti, A. Tufi, S. Ricci

UOSD Neurologia e Ictus, Città della Pieve, Italy

Background: Psychological (psy) components of stress are related to stroke patient recovery and health related quality of life $(\mathrm{hr}-\mathrm{QoL})$ : these conditions are dependent on positive caregiving. Caregivers (cg) must meet patient's needs and, in the mean time, carry on their daily routine. So $\mathrm{cg}$ health have to be promoted to save them and because of its link with patient well being.

It is important to know the relationship between $\mathrm{cg}$ hr-QoL and their personal coping strategies to find the best way to support stress.

Methods: We sought to investigate: the link between psy components of stress and coping strategies; the influence of psy stress on hr-QoL; the relevance of patien disability and psy stress on cg hr-QoL; the relevance of all variables on cg hr-QoL. We administered VRS, COPE, SF-36 to cg of stroke patients and BI to the patients; Spearman correlation and logistic regression were used for statistical analysis.

Results: We studied 52 stroke cg (80,8\% female, mean age 56).

Coping strategies, i.e. positive feelings for the future $(p, 002)$ and humour $(p, 04)$ have a negative correlation with depression (D) and religion has a negative correlation with D (p,001), lack of social support $(p, 002)$ and aggression $(p, 002)$. All the hr-QoL scales are correlated with stress components and predict these differently. On the contrary, patient's disability is not linked with cg stress, and discharge BI is a predictive factor only for general heath. $\mathrm{Cg}$ anxiety enters as predictor of hr-QoL mental component $(\mathrm{p}, 02)$ and mental disengagement of the physical one $(\mathrm{p}, 001)$.

Conclusions: Coping strategies are related to psy stress reaction and to hr-QoL of stroke cg. These strategies can improve the management of psy stress. Patient disability doesn't seem to have a relation with stress and hr-QoL of stroke cg. A model to evaluate hr-QoL in stroke cg should include psy reaction to stress (anxiety) and coping strategies (mental disengagement) rather then patient disability.

\section{Etiology of stroke}

\section{Etiology of stroke}

\section{ETIOLOGIC DIAGNOSIS OF STROKE SUBTYPES USING PLASMA} BIOMARKERS

J. Montaner, P. Delgado, M. Ribó, M. Rubiera, M.E. Palacios, C.A. Molina, I. Fernández-Cadenas, A. Rosell, P. Chacón, J. Alvarez-Sabín Vall d'Hebron Hospital, Barcelona, Spain

Background: Since there is no biological marker offering precise information about stroke etiology, many patients receive a diagnosis of undetermined stroke even after all available diagnostic tests are done.

Methods: To examine the value of a panel of blood-borne biochemical markers to differentiate stroke etiologies, consecutive patients with acute ischemic stroke $<24$ hours were evaluated. CT, ultrasonography, cardiac evaluations and other tests were done to make etiologic diagnosis following TOAST classification. Blood samples were drawn at emergency department arrival. Tested biomarkers were CRP, Ddimer, RAGE, MMP-9, s-100b, Brain Natriuretic Peptide (BNP), Neurotrophin-3, Caspase-3, Chimerin and Secretagogin (assayed by ELISA).

Results: The study protocol was performed in 776 consecutive ischemic stroke patients. Regarding etiology $35.4 \%$ were cardioembolic, $21.8 \%$ aterothrombotic $18.5 \%$ lacunars and $24.4 \%$ had an undetermined origin. The only identified biomarkers that were different regarding etiologies were high level of BNP $(\mathrm{p}<0.0001)$ and $\mathrm{D}$-dimer $(\mathrm{p}<0.0001)$ among patients with cardioembolic strokes Independent predictors of a cardioembolic stroke were: Atrial fibrillation OR 15.2 (8.4-27.7) $\mathrm{p}<0.001$; other embolic cardiopathies OR 14.7 (4.7-46) $\mathrm{p}<0.001$ TACI OR 3.9 (2.3-6.8) $\mathrm{p}<0.001$; BNP $>76 \mathrm{pg} / \mathrm{mL}$ OR $2.3(1.4-3.7) \mathrm{p}=0.001$ and D-dimer $>0.96 \mu \mathrm{g} / \mathrm{mL}$ OR $2.2(1.4-3.6) \mathrm{p}=0.001$. Even among those with transient symptoms $(\mathrm{n}=153)$, high BNP level identified cardioembolic TIA [OR 15 (3.6-60) $\mathrm{p}<0.001]$. The combination of D-dimer $<0.96$ and BNP $<76$ was present only in $10 \%$ of non-cardioembolic strokes and the opposite combination was present in $60 \%$ of cardioembolic strokes.

Discussion: Using a combination of biomarkers (including D-dimer and BNP) seem a promising strategy for making an etiologic diagnosis in the acute phase of stroke. These approaches will allow to rapidly guiding other diagnostic tests accelerating the onset of an optimal secondary prevention. 


\section{Etiology of stroke}

VARIATIONS IN SINGLE NUCLEOTIDE POLYMORPHISMS OF THE PDE4D GENE BETWEEN ISCHEMIC STROKE PATIENTS AND CONTROL SUBJECTS AMONG RESIDENTS IN THE SOUTH OF SWEDEN

H. Lövkvist, H. Luthman, P. Höglund, B. Norrving, U. Kristoffersson, A-C. Jönsson, B. Hallström, B. Petersen, A. Lindgren

Lund University, Lund, Sweden

Background: The previously reported increased prevalence of stroke or TIA among first-degree relatives of stroke patients may be explained by genetic liability. However, the impact of genetic factors may differ between populations because of allelic heterogeneity. We examined associations between genetic variants in the chromosome region for the phosphodiesterase 4D gene (PDE4D), a candidate gene for ischemic stroke suggested on genetic and functional grounds, in a case control material of patients with cerebral infarction and matched controls from a southern Swedish population.

Methods: A consecutive group of 932 ischemic stroke patients from the population based Lund Stroke Register and 396 randomly selected age and gender matched control subjects from the population register of the same geographical area were genotyped. Single nucleotide polymorphisms (SNP) in PDE4D on chromosome 5 were analysed on an ABI 7900 scanner. Haplotype association and analysis of single variants with correction of confounding phenotypes such as smoking, hypertension, and cardiovascular disease was performed. All SNPs were in Hardy-Weinberg equilibrium.

Results: Allelic associations analysis for two SNPs in PDE4D revealed association with ischemic stroke for one SNP in the 5'-end of the gene $(\mathrm{p}=0.005)$, but not for another SNP of the PDE4D gene $(\mathrm{p}=0.66)$. Haplotypes constructed with the two SNPs could not detect any haplotype specific association with stroke.

Discussion: Our study shows that genetic variation in the chromosomal region of PDE4D is involved in the expression of ischemic stroke in a southern Swedish population. This observation and similar observations in other populations confirm that this genomic region encodes important liability to ischemic cerebrovascular disease.

\section{Etiology of stroke}

HIGH RATE OF REVERSIBLE HIPPOCAMPAL PUNCTATE LESIONS WITHOUT PERFUSION ABNORMALITIES IN TRANSIENT GLOBAL AMNESIA

M. Toledo, F. Pujadas, E. Grivé, A. Rovira, M. Rubiera, C. Molina, N. Lara,

R. Rovira, J. Alvarez-Sabín

Vall dHebron Hospital, Barcelona, Spain

Background: Transient global amnesia (TGA) has been related to brain ischemia, epilepsy or migraine. However, evidence supporting these theories are scarce. Although hippocampal punctate (HP) lesions on diffusion weighted imaging (DWI) have been described in patients with TGA, combined DWI and perfusion weighted imaging (PWI) studies in the acute phase of TGA are lacking and also reversibility of HP lesions has been non demonstrate. We aim to determine the frequency, reversibility and extent of DWI lesion and PWI abnormalities in patients with TGA and their evolution during the first month.

Patients and Methods: We studied consecutive patients with TGA evaluated within the first 24 hours of symptom onset. All patients underwent multiparametric MRI examinations including DWI, PWI, T1, T2 and MRA at baseline, 48-96 hours and 4 weeks of onset. EEG, carotid and transcranial ultrasound doppler exams including right-to-left shunt (RLS) detection were also performed.

Results: A total of 24 patients were included (54\% female, age $63 \pm 9$ years). Mean duration of TGA was $6.8 \pm 4.6$ hours. Acute HP lesions on DWI were detected in 16 patients ( 1 lesion: 6 right and 3 in left hippocampus; 2 lesions: 2 right, 1 left and 4 bilateral). No relevant PWI abnormalities were observed. In $5(20 \%)$ patients a RLS was detected, and $4(80 \%)$ of them showed HP lesions on DWI. Neither significant extra o intracranial stenosis nor EEG epileptiform abnormalities were observed. HP lesions were apparent on MRI performed after 10 hours of onset of TGA symptoms, and T2 did not identified residual lesions at 4 weeks.

Conclusions: We confirm the high prevalence of DWI HP lesions in TGA, which were delayed and reversible. DWI HP lesions were not associated to hippocampal hypoperfusion during or short after TGA. The prevalence of RLS was low.
4 Etiology of stroke

IS THERE A LINK BETWEEN TRAUMATIC CERVICAL DISSECTION AND FIBROMUSCULAR DYSPLASIA?

J.M. de Bray, G. Marc, V. Pautot, A. Pasco, F. Dubas

University Hospital, Angers, France

Background: the associated role of Fibromuscular Dysplasia (FMD) in traumatic cervical artery dissections (CAD) has not yet been studied. Our aim was to determine the prevalence of FMD in dissections after trauma or chiropractic manoeuvres and the outcome of these patients.

Methods: a prospective study on symptomatic CAD recurrences after a first event of CAD was carried out from 1994 to 2004: 103 patients admitted for CAD assessed by an intramural hematoma visualised by cervical MRI or by suggestive aspects of CAD identified by 2 vascular investigations, were consecutively included after an informed consent and followed 4 years ( 3 months to 10 years). A digital subtraction angiography (DSA) was always performed. A cohort was selected from this study by 2 criteria: 1) blunt head or neck trauma or chiropractic manoeuvre $<15$ days before the onset of CAD, 2) presence of FMD assessed if DSA showed an image of 'string of beads 'located in a non dissected artery after a concordant evaluation by 2 radiologists.

Results: 14 males and 13 females were included for a CAD after a trauma (n of patients: 22) or after chiropractic manoeuvres (n: 5): 19 had a stroke, 5 an isolated TIA, 2 a Horner's syndrome and 1 a transient amnesic syndrome. 18 patients presented at least an ICA occlusion or stenosis, 9 a vertebral artery occlusion or stenosis. 9 patients had a dissecting aneurysm, 9 multiple dissections and $2(7 \%)$ an extensive FMD. 22 patients were treated by heparin without bad effects. Final Rankin scale was $>1$ in 11 patients.

Conclusion: This study shows that traumatic dissections often give single or multiple CAD without FMD identification. A traumatic dissection can exceptionally occurred in patients with FMD. Outcome was marked by a neurological deficit in $41 \%$ of the patients.

\section{Etiology of stroke}

DIRECT COMPARISON OF MICROVASCULAR ENDOTHELIAL FUNCTION AND CAROTID INTIMA-MEDIA THICKNESS IN PATIENTS WITH LARGE VESSEL AND SMALL VESSEL ISCHAEMIC STROKE

A. Fulton, A. Stanton, J. Moroney

Beaumont Hospital, United Kingdom

Objectives: To determine the role of endothelial dysfunction in small vessel stroke (SVS) versus large vessel stroke (LVS) using the novel paradigm of digital retinal imaging (DRI) in patients hospitalised with acute ischaemic stroke (AIS).

Background: Current vascular investigation focuses on the neck vessels and microvascular pathology has not been well studied. Our aim was to clarify if an association between functional changes in the retinal microvasculature and AIS existed and also if differences existed between our two stroke subtypes.

Design/Methods: Our sample consisted of one hundred and three patients consecutively hospitalised with AIS categorised into SVS or LVS using TOAST criteria. Endothelium-dependent retinal microvascular responses and endotheliumindependent retinal vasodilatation in response to sub-lingual glyceryl trinitrate were assessed with inhalation of three gaseous mixtures $\left(100 \% \mathrm{O}_{2}, 10 \% \mathrm{O}_{2}+\right.$ $90 \% \mathrm{~N}_{2}, 10 \% \mathrm{O}_{2}+10 \% \mathrm{CO}_{2}+80 \% \mathrm{~N}_{2}$ ). Carotid intima-media-thickness (IMT) measurements were obtained using a standard technique.

Results: Retinal endothelium-dependent responses to hyperoxia and hypoxia were significantly impaired in SVS versus LVS subtype (Student's unpaired t-test, $\mathrm{p}<$ 0.01 and $\mathrm{p}<0.05$ ), while no significant between group differences were found for endothelium-independent response to GTN. Additionally carotid IMT measurements were significantly higher in LVS versus SVS (Student's unpaired t-test, $\mathrm{p}<0.05$ ).

\section{Etiology of stroke}

\section{ASYMMETRIC DIMETHYLARGININE IN CEREBRAL SMALL VESSEI} DISEASE

U. Khan, A. Hassan, P. Vallance, H. Markus

St. George's University of London, London, United Kingdom

Background: Endothelial dysfunction has been proposed as a causal mechanism in cerebral small vessel disease (SVD), especially leukoaraiosis. Asymmetric dimethylarginine (ADMA) is a circulating endogenous inhibitor of nitric oxide. We determined if ADMA was elevated in SVD and correlated with disease severity. It may act via interaction with homocysteine, a known risk factor for SVD, and therefore we also determined serum homocysteine. 
Methods: Plasma ADMA and serum homocysteine levels were determined in 47 consecutive SVD cases and 38 community controls. Markers of endothelial dysfunction (intercellular adhesion molecule 1 (ICAM1) and thrombomodulin (TM)) were also measured in a subgroup. SVD was graded on the basis of increasing leukoaraiosis severity and number of lacunes ( $>0.5 \mathrm{~mm}$ diameter).

Results: Mean (SD) ADMA was higher in SVD cases $(0.814 \mu \mathrm{mol} / \mathrm{l}(0.145))$ compared to controls $(0.747 \mu \mathrm{mol} / \mathrm{l}(0.184), \mathrm{P}=0.014$ after controlling for age, gender, vascular risk factors and creatinine clearance. Mean (SD) homocysteine was higher in SVD cases (15.28 mmol/l (5.73)) compared to controls (12.49 mmol/l (4.15), $\mathrm{P}=0.035$. There was a positive correlation between overall leukoaraiosis grade and both $\mathrm{ADMA}(\mathrm{P}=0.050)$ and homocysteine $(\mathrm{P}=0.002)$. Homocysteine, but not ADMA ( $\mathrm{P}=0.888)$ correlated with the number of lacunes $(\mathrm{P}=0.015)$. Controlling for ICAM1, TM and homocysteine resulted in the association between ADMA and SVD no longer being significant.

Discussion: Elevated ADMA is an independent risk factor for SVD. It is a risk factor for leukoaraiosis severity but not the number of larger lacunar infarcts. Its effect may be mediated through endothelial dysfunction and via interaction with homocysteine. ADMA-lowering therapy may offer a novel treatment option in SVD with leukoaraiosis.

\section{Etiology of stroke}

\section{PROGNOSIS OF PATIENTS WITH CANCER AND STROKE DEPENDS ON} STROKE RATHER THAN ON CANCER

F. Taccone, P. Redondo, S. Jeangette, M. Pandolfo, S.A. Blecic

Erasme Hospital, Brussels, Belgium

Background: Stroke is frequent in patients (pts) with systemic cancer (SC). Etiologies are related to SC and are premature Large Vessel Disease (LVD), Non Bacterial Thrombotic endocarditis (NBTE), Coagulation Disorders (CD) and Tumor embolism (TE)

Objective: To find a difference between SC pts based upon the moment of stroke onset in the SC course.

Design/Methods: 5048 pts with first stroke were admitted between1991 and 2005 . Mean age was $66.4 \pm 8.1$ years. All risk factors were recorded and a complete stroke work-up was performed in all. In case of death all pts with SC had autopsy.

Results: $777 / 5048$ pts (15.4\%) were found to have SC. 752 pts had history of SC and in $25(0.5 \%)$ SC was eventually discovered during hospitalization. 522/752 pts $(69.5 \%)$ had SC on remission or presumably cured. Average age of these pts was $66.9 \pm 5$ years ( $\mathrm{p}=\mathrm{NS}$ vs general population (GP)). Stroke occurred on an average time of 6 months (mo) [2-18] after the last SC treatment. 445 pts (85.2\%) had LVD, $43(8.2 \%)$ had SVD, $20(3.8 \%)$ had atrial fibrillation (AF) and multiple causes were found in $20(3.8 \%)(\mathrm{p}<0.001 \mathrm{vs}$ GP for all etiologies). NBTE and CD were never found. On a 2 years follow-up $4 \%$ had vascular recurrence or died. In none death was the consequence of SC and none recurred a SC. 230/752 (30.5\%) pts had stroke while SC was active. LVD was found to be the cause in 168 pts (73\%), 24 pts NBTE, AF in 17 pts $(7.4 \%), 10 \%$ had SVD. The medial survival of was $7.5 \mathrm{mo}$. Analyzed by SC types, no difference was found between the outcome of pts with SC and stroke and pts with SC alone. In 25 pts a stroke was the initial presentation of SC. Average age of these pts was 52,1土4.1 years (24-80). LVD was found in $12 \mathrm{pts}$ (48\%), CD in 3 pts, NBTE in 7. Within the first month 19 pts had a second stroke. The mortality rate was $88 \%(22 / 25)$ with a medial survival of 59.4 days (3-180) after index stroke.

Conclusions: Stroke is a common event in pts with SC. History of SC could favor stroke by accelerating the development of LVD. Specific stroke etiologies are found in pts with active SC. Outcome is not modified by stroke in cancer pts, and is related to the SC specificity, except when stroke is the initial presentation of SC where the prognosis becomes particularly pejorative.

\section{Etiology of stroke}

\section{LACUNAR INFARCTION IS AN INDICATOR OF BENIGNITY ONLY IN} PATIENTS WITH CRYPTOGENIC STROKES

A. Dewindt, G.A. Remiche, P. Finet, S. Jeangette, M. Pandolfo, S.A. Blecic

Erasme Hospital, Brussels, Belgium

Background: Cryptogenic strokes (CS) represent in different series from 4 to $29 \%$ of patients (pts). Up to now, only few information is found about the recurrence predictors.

Aim and Objective: To determine if the outcome in terms of any vascular recurrence or death of pts with CS depends on the infarction' size.

Patients and Methods: From 1991 to 2005, 5048 pts pts (2602 males) were admitted to the Stroke Unit. They were distributed into 4 groups (G); G1: 1784 pts (35.3\%) with large stroke (LS) in the carotid artery territory (CAT), G2: 1630 pts (32.3\%)with lacunar infarction (LI) in the CAT, G3: 661 pts (13.1\%) with LS in the vertebral artery territory (VAT) and G4: 969 pts $(19.2 \%)$ with LI in the VAT. Mean age was $66.4 \pm 8.1$ years. 411 patients were found to have CS. Mean age of the CS pts was $51 \pm 4.1$ years $(\mathrm{p}<0.001$ compared to the general population (GP)). They were also distributed into the same 4 groups, G1: 118 pts $(28.7 \%)$ with LS in the CAT, G2: 149 pts (36.3\%)with LI in the CAT, G3: 54 pts (13.1\%) with LS in VAT and G4: 90 pts (21.8\%) with LI in the VAT, a statistical difference of $p<0.05$ being observed for all groups of CS compared to GP. All risk factors were recorded and a complete stroke work-up was performed in all. Trans-oesophageal echography, catheter angiography, extensive coagulation study, skin biopsy and lumbar puncture were performed in selected cases. All CS pts were followed twice a year for a maximum of 5 years (average 3 years).

Results: Within the follow-up period a new vascular event was found in 1408/5048 pts of GP (27. 9\%) 631 having a new stroke (12.8\%). No statistical difference being observed between the different groups in the GP population. In the CS population, only 21 pts $(5.1 \%)$ had a vascular recurrence ( $p<0.001$ CS vs GP). 17 pts $(81 \%)$ belonged to the LS groups and 3 to the LI group ( $p<0.01$ LS vs LI). 12 pts were found in the G1, 2 in the G2, 5 in the G3 and 1 in the G4. No difference was observed between the anterior and the posterior circulation territories. 9 pts had a new stroke, all being also new large strokes and 8 had a cardiac recurrence $(\mathrm{p}<0.001$ pts with LS versus pts with LI). No death was observed in all groups with CS.

Conclusions: This study demonstrated that pts with CS and GP differ widely in the follow-up, the former population being at a particular low risk to have any vascular recurrence. Despite over represented in the CS population the lacunar presentation constituted an entity with a very low rate of vascular recurrence that widely differs from the GP where lacunar stroke presentations did not differ in term of prognosis from the Large stroke presentations.

\section{Etiology of stroke}

POTENTIAL FACTORS ASSOCIATED WITH HAEMORRHAGE GROWTH AN EXPLORATORY ANALYSIS FROM THE STUDY OF RFVIIA IN ICH J.P. Broderick, T. Steiner, S.A. Mayer, N.C. Brun, M.N. Diringer, B.E. Skolnick, S. Davis, for the rFVIIa ICH Trial Investigators University of Cincinnati Medical Center, Cincinnati, OH, USA

Background: A recent report of the role of recombinant Factor VIIa (rFVIIa) in haemorrhage reduction suggested that $>70 \%$ of patients had some expansion in intracerebral haemorrhage (ICH). We report an exploratory analysis from the randomized study of rFVIIa in ICH patients that examines potential factors associated with haemorrhage growth.

Methods: Relationships between 5 different measures of change in haemorrhage volume (absolute and \% change in ICH volume, ICH growth - categorical [no growth if change $<33 \%$ and $<12.5 \mathrm{ml}$, and absolute and $\%$ change in ICH + intraventricular haemorrhage [IVH] volume) were assessed between baseline and 24-h CT scans and 31 demographic, clinical, imaging, historical and baseline laboratory variables.

Results: Treatment with rFVIIa was associated with decreased haemorrhage growth in all 5 models. Longer time-from-onset to baseline CT was related to limited observed haemorrhage growth in all 5 models. ICH volume at baseline CT was consistently associated with ICH growth. Other variables significantly related to growth of ICH or ICH+IVH in at least 1 of 5 models include: serum glucose (increased levels associated with increased growth), estimated body weight (heavier people have less growth), prior anti-platelet agent use (prior use associated with increased growth), serum cholesterol (higher levels associated with less growth) and serum creatinine (higher levels associated with more growth). Baseline blood pressure variables were not associated with ICH growth.

Conclusions: Treatment with rFVIIa reduces observed ICH growth. Most haematoma growth occurs early after ICH onset. Larger haematomas on baseline CT were associated with increased absolute growth of ICH. The relationship of other haemorrhage growth factors warrants further study.

\section{Epidemiology of stroke}

\section{Epidemiology of stroke}

STROKE AND VASCULAR MORTALITY TRENDS IN FRANCE: 1979 TO 2001 P.C. Lavallée, J. Labreuche, J-F. Spieler, E. Jougla, P. Amarenco Bichat Stroke Centre, Paris, France

Background: There have been improvements in the treatment of vascular risk factors in industrialized countries over the past several decades. We therefore studied 
vascular mortality trends in France between 1979 and 2001, and explored how these trends are related to stroke or non-stroke vascular mortality changes.

Materials and Methods: We included all deaths from vascular disease in the French population from 1979 to 2001 . Trends for total-, stroke- and nonstroke-vascular mortality are estimated using log-linear Poisson regression models. Gender-specific mortality rates were standardized directly by 5 -year groups to the 1990 France population.

Results: During 1979-2001 period, the mean annual percentage change in vascular death rates varied across age groups $(<35,35-54,55-64,65-74, \geq 75)$ between $-2.4 \%$ to $-3.3 \%$ in men and $-2.5 \%$ to $-4.1 \%$ in women. Respectively, $25.7 \%$ and $30.9 \%$ of total vascular death was attributed to cerebrovascular disease in men and women. Stroke mortality and non-stroke vascular mortality rates declined $60.9 \%$ and $34.6 \%$ in men, and $60.4 \%$ and $38.9 \%$ in women. Trend in rates of treated hypertension was negatively correlated with total vascular mortality, especially stroke mortality.

Conclusion: We observed a dramatic 60\% reduction of stroke death in France in the last 20 years. Stroke death decreased at about twice the rate as other causes of vascular death. Improved management of vascular risk factors could partly explain these results.

\section{Epidemiology of stroke}

HOSPITALIZATIONS, MORTALITY AND COSTS IN A SIX-YEAR SERIES OF STROKE AND TIA IN SPAIN AND ITS AUTONOMOUS COMMUNITIES

L.-C. Álvaro, P. López-Arbeloa, E. Gutiérrez, F. Sádaba

Basurto Hospital, Bilbao, Spain

Background: Stroke is a leading cause oh hospitalisation. Ageing, home management of minor forms, and a different specialisation among centres could explain a variability in hospitalisation and prognosis among different areas. We analyse: 1-The number of hospitalisations for stroke and TIA in Spain,1998-2003; 2-Differences among regional areas.

Methods: The Spanish Ministry of Health, through its MBDS (Minimum Basic Data Set) Office, provided data for stroke and TIA hospitalisation. Diagnoses were extracted according to ICD-9 (International Classification of Diseases) codes 14-17- and to GRD(Group Related Diagnoses) - codes 14-17, 532, 810.We included:a-Autonomous Community identification; b-Average stay; c- Age/sex, type of discharge (mortality); d- Number of diagnosis, weight/cost for GRD.

Results: Steady temporal incidence of hospitalisation for stroke(GRD14: 160/100000) and TIA(GRD15: 55/100000). Weight and costs increased between 2000 and 2003 and reached 3250 Euro(GRD14) and 2300 Euro(GRD15). Average stay ranged between 10-13,5 days (stroke) and 7,5-10,5 days (TIA), with no identifiable trend. Likewise, mortality was irregular, oscillating between $9,15 \%$ (2003) and $12,7 \%$ (2001) for stroke (GRD14). Average number of diagnoses was similar for stroke and TIA: both increased from 4 to 5 within 6 years.

There was a great variability in hospitalisations for stroke and TIA among the Autonomous Communities: from 250/100000 to less than 120/100000. The differences were similar in average stay and in mortality, with variations up to $40 \%$ among different areas.

Discussion: Hospitalisation rate for stroke and TIA is very high and sustained; in spite of an increasing complexity, average stay and mortality are stable, which points to a better management.Variability among regions is highlighted and should be analysed with epidemiological prospective studies.

\section{Epidemiology of stroke}

\section{INCIDENCE AND CASE-FATALITY OF FIRST-EVER STROKE IN COPENHAGEN, DENMARK}

T.S. Olsen, B.H. Rasmussen, L.P. Kammersgaard, U.J. Weber

Hvidovre University Hospital, Hvidovre, Denmark

Background: We studied rates of incidence and case-fatality of first-ever-stroke in Copenhagen 1999/2000 and compared these to the most recently recorded Danish rates in 1990.

Methods: We identified all strokes in residents of seven neighboring municipalities of Copenhagen County (population 273 095) September 1st, 1999 - April 30, 2000. Complete case ascertainment was ensured by registration of hospitalized and non-hospitalized patients (pts) and by scrutinizing all death certificates. WHO definition of stroke was used; TIA and subarachnoid hemorrhage were not included. CT-scan verification was available in $83 \%$ of pts. Death within five year after stroke onset was obtained from the Danish Central Registry of Persons. None were lost to follow-up.

Results: We identified 514 pts with stroke. Of these 394 pts had first-ever-stroke; 223 (57\%) women, $171(43 \%)$ men. Mean age (sd) 75 (13) years (women 77 (12) years, men 72 (12) years). Crude annual incidence was $2.19 / 1000 /$ year $(2.34 / 1000 /$ year in women and 1.97/1000/year in men). Adjusted to the Danish population year 2000 stroke incidence in Denmark was $1.80 / 1000$ /year (1.88/1000/year in women and 1.71/1000/year in men). Except for pts $<55$ and $>85$ years of age stroke incidence was higher in men; twice higher in the age group 65-74 years. One-month case-fatality was $19.5 \%$, one-year case fatality $32.3 \%$ and five-year case-fatality was $54.0 \%$. Stroke was unspecified in $17 \%$, hemorrhagic in $13 \%$ and ischemic in $70 \%$. Discussion: Compared to the most recent Danish stroke incident rates recorded in 1990 (all: 2.0, women: 1.9, men: 2.3/1000/year) stroke incidence has decreased slightly because of a marked decrease of stroke incidence in men. One-month case-fatality (30\% in Denmark 1990) has decreased by $1 / 3$ in ten years.

\section{Epidemiology of stroke}

TIME TRENDS IN INCIDENCE OF INTRACEREBRAL HAEMORRHAGE IN OXFORDSHIRE, UK, FROM 1981-2005

C.E. Lovelock, M.F. Giles, A.J. Molyneux, P.M. Rothwell

University of Oxford, Oxford, United Kingdom

Background: Death certification and autopsy data show a continued decline in mortality from primary intracerebral haemorrhage (PICH) over the last few decades in the UK, but it is not clear if this is due to decreased incidence or case fatality. Moreover, any trends in incidence of PICH might vary with age given the differing relative contributions of hypertension and amyloid angiopathy at different ages.

Methods: We determined the changes in incidence of PICH, risk factors and premorbid medication between 1981-86 (Oxford Community Stroke Project - OCSP) and 2002-2005 (Oxford Vascular Study -OXVASC).

Results: Among 417 incident strokes in OXVASC, there were 43 PICHs (7 associated with warfarin use). There was a trend towards a reduction in age and sex adjusted incidence from OCSP and OXVASC (ratio $=0.72,95 \%$ CI $0.48-1.08$, $\mathrm{p}=0.11$ ), but there was a significant interaction between the trend in incidence and age, with a fall in incidence in the population aged $<75$ years $(0.41,0.21-0.82$, $\mathrm{p}=0.01)$ and no change at older ages $(1.05,0.62-1.8, \mathrm{p}=0.85)$. Case fatality remained unchanged ( $54 \%$ vs $48 \%, \mathrm{p}=1.00$ ). In OXVASC, poorly controlled premorbid blood pressure $(\mathrm{SBP}>160 \mathrm{mmHg}$ ) was more common in patients with $\mathrm{PICH}<75$ years than in the older age group (67\% vs $20 \%$ ).

Conclusion: The incidence of PICH under age 75 years has fallen substantially over the last 20 years in the UK, but has remained unchanged in the older population. Poor control of hypertension does not appear to explain the continuing high rates of PICH in older patients, suggesting that other causes are more important in this group.

\section{Epidemiology of stroke}

MATERNAL TRANSMISSION OF STROKE IS STRONGER THAN PATERNAL: A NATION-WIDE STUDY OF 340,000 EVENTS

X. Li, K. Sundquist, S.E. Johansson, J. Sundquist

Karolinska Institute, Stockholm, Sweden

Background: The aim was to investigate the possible differential transmission of stroke risk from mothers, fathers, and siblings, as previous studies on this topic have been inconclusive.

Methods: The Multigeneration Register, where all women and men born in Sweden from 1932 onwards are registered together with their parents, was linked to hospital admission data. The registration of stroke cases started on January 1, 1987, and proceeded until death, emigration, or end of study on December 31, 2001. Using Poisson regression, standardized incidence ratios (SIRs) were calculated as the ratio of observed $(\mathrm{O})$ to expected $(\mathrm{E})$ number of cases for men and women with mothers or fathers affected by stroke, compared with men and women whose mothers or fathers were not affected by stroke.

Findings: In total, 341,716 cases of stroke were found. For both men and women the sibling transmission of stroke was stronger than the maternal. Furthermore, the maternal transmission to both sons and daughters was stronger than the paternal, especially pronounced in the youngest age groups. For example, among women aged $<30$, the SIR was 1.65 for those with a maternal history of stroke.

Interpretation: The finding that the maternal transmission was stronger than the paternal may indicate that stroke might be influenced by both genetic and intrauterine environmental factors. These findings are likely to modify prevention strategies for stroke and risk assessments by clinicians. 


\section{Epidemiology of stroke}

INCIDENCE AND 30-DAY CASE-FATALITY OF ISCHEMIC STROKE SUBTYPES IN TBILISI: RESULTS OF THE FIRST PROSPECTIVE POPULATION-BASED STUDY IN GEORGIA

A. Tsiskaridze, M. Djibuti, G. Lomidze, I. Burduladze, T. Vashadze, Z. Kharaishvili, I. Beridze, R. Shakarishvili

Sarajishvili Institute of Neurology and Neurosurgery, Georgia

Background: Although stroke is one of the major public health problems worldwide, information about epidemiological patterns of the disease in developing countries is scarce. The purpose of the present study was to determine incidence and short-term case-fatality of ischemic stroke (IS) subtypes in Tbilisi, capital of Georgia, a country with transition economy in the South Caucasian region.

Methods: In the framework of the joint Swiss-Georgian epidemiological project we identified all first-ever strokes from November 2000 to July 2003 in a defined population with 51,246 residents in Sanzona suburb of Tbilisi using overlapping sources of information and standard diagnostic criteria.

Results: The age-adjusted annual incidence rate for IS diagnosed by computed tomography, magnetic resonance imaging or autopsy and identified during the study period was 71 (95\% confidence interval (CI) 60 to 83) per 100,000 residents. Regarding IS subtypes (Lausanne Stroke Registry etiological classification), the annual incidence rates per 100,000 inhabitants were 31 (95\% CI 18 to 45$)$ for large-artery disease (LAD), 18 (95\% CI 6 to 30) for cardioembolism (CE), 11 (95\% CI 2 to 21 ) for small-artery disease (SAD) and 11 (95\% CI 2 to 21) for other (undetermined, mixed) etiology (OE). The case-fatality rates at one month were 17\% (95\% CI $12 \%$ to $23 \%$ ), $24 \%$ (95\% CI $16 \%$ to $31 \%$ ), $7 \%$ (95\% CI $1 \%$ to $24 \%$ ) and $20 \%(95 \% \mathrm{CI}$ $11 \%$ to $30 \%$ ) for $\mathrm{LAD}, \mathrm{CE}, \mathrm{SAD}$ and $\mathrm{OE}$, respectively.

Conclusion: While LAD is the most frequent cause of IS in Tbilisi, Georgia, $\mathrm{CE}$ (followed by OE) is characterized by the highest short-term mortality. These findings may have important implication for designing national stroke program aiming at reducing the burden of the disease throughout Georgia.

\section{Epidemiology of stroke}

\section{BASELINE CHARACTERISTICS AND ONE-YEAR OUTCOMES FOR} PATIENTS WITH CEREBROVASCULAR DISEASE IN THE REACH

\section{REGISTRY: US VS REST OF WORLD}

M.J. Alberts, J. Rother, J.L. Mas, M.D. Hill, P.G. Steg, D.L. Bhatt, on behalf of the REACH Registry Investigators

Northwestern University School of Medicine, Chicago, IL, USA

Background: Stroke is a global leading cause of death and disability. How worldwide differences in management affect the long-term outcomes of patients is a significant issue.

Methods: The REduction of Atherothrombosis for Continued Health (REACH) registry is an international, observational survey of subjects at risk of atherothrombotic events. Participants are $\geq 45$ years old with documented vascular disease or atherothrombotic risk factors. This abstract focuses on REACH patients enrolled with only a stroke or transient ischemic attack (TIA). Data on risk factors, treatments and outcomes after one year of follow-up were analyzed comparing the US population with the rest of the world (ROW).

Results: The REACH Registry enrolled 8,913 patients globally with only stroke or TIA. The US cohort had higher numbers of women than the ROW (57\% vs $42 \%$, $\mathrm{p}<0.001$ ), more Caucasians ( $77 \%$ vs $57 \%, \mathrm{p}<0.001$ ), more blacks ( $15 \%$ vs $0.5 \%$, $\mathrm{p}<0.001)$, and more retired persons $(69 \%$ vs $60 \%, \mathrm{p}<0.001)$. Hypertension was common in both groups ( $85 \%$ US, $80 \%$ ROW), as was diabetes (39\% and $32 \%$, respectively). Elevated cholesterol was more common in the US than ROW (65\% vs $49 \%, \mathrm{p}<0.001)$, but smoking was nearly identical in both regions ( $14 \%$ vs $13 \%)$. Aspirin was used in $63 \%$ of the US population vs $60 \%$ in the ROW. Statin use was $60 \%$ in US and $49 \%$ in ROW $(\mathrm{p}<0.001)$, diuretic use was $47 \%$ and $34 \%(\mathrm{p}<0.001)$, and anticoagulant use was $23 \%$ and $13 \%(\mathrm{p}<0.001)$ respectively. The one year rate of stroke/MI/vascular death was $5.9 \%$ overall, $6.6 \%$ in the US and 5.6\% in ROW $(\mathrm{p}=0.09)$, due largely to a higher rate of non-fatal MI in the US vs ROW $(1.2 \%$ vs $0.3 \%, \mathrm{p}<0.001$ ). The stroke rates at one year were similar at $4.1 \%$ for the US and $3.8 \%$ in ROW ( $=0.44)$.

Conclusion: This subgroup analysis found that stroke/TIA patients in the US compared to the ROW had higher rates of some vascular risk factors, of medication use and of vascular endpoints at one-year follow-up. Recurrent strokes were also common.
8 Epidemiology of stroke

TRENDS IN STROKE IN THE LAUSANNE STROKE REGISTRY, 1979 TO 2003 E. Carrera, A. Croquelois, M. Maeder-Ingvar, G. Devuyst, J. Bogousslavsky University Hospital, Lausanne-CHUV, Lausanne, Switzerland

Background: There are increasing information about evolution of stroke incidence during the last decades. However, in patients admitted for stroke, there is a lack of data regarding the trends in prevalence of cerebrovascular risk factors, distribution of stroke location and cause.

The goal of the study is to determine evolution of baseline characteristics, stroke location and causes over a 25 years period in patients admitted to a general hospital. Methods: We included all patients with first-ever stroke admitted to the Lausanne General Hospital, Lausanne Switzerland from 1979 to 2003 and prospectively included in the Lausanne Stroke Registry (LSR). Prevalence of cerebrovascular risk factors, presumed stroke locations and causes were compared between the 1996-2003 and 1979-1987 periods using the Pearson test with the Yates correction for continuity.

Results: From 1979 to 2003, 5759 patients were included in the LSR. In the 1979-1987 and 1996-2003 periods, there were respectively 1147 patients $(89.7 \%)$ and $2286(94.1 \%)$ patients with ischemic strokes. Comparison between 1996-2003 and 1979-1987 periods showed, for ischemic and hemorrhagic strokes, a significant increase in the proportion of older patients $\left(\mathrm{p}<0.0001 ; \chi^{2}=222.279\right)$, hypertension $\left(\mathrm{p}<0.0001 ; \chi^{2}=32.2241\right)$ and cholesterol blood level $\left(\mathrm{p}<0.0001 ; \chi^{2}=\right.$ $12.4396)$ and a reduction of smokers $\left(p<0.0001 ; \chi^{2}=78.57\right)$. In patients with ischemic strokes, there were additionally significant differences in the distributions of presumed stroke location and stroke causes with increase in posterior circulation strokes $\left(+13.1 \% ; \mathrm{p}<0.0001 ; \mathrm{df}=14 ; \chi^{2}=142.1537\right)$ and cardioembolic causes $\left(+10.8 \% ; \mathrm{p}<0.0001 ; \mathrm{df}=7 ; \chi^{2}=229.3236\right)$.

Conclusion: This study shows major trends in characteristics of stroke patients admitted in a general hospital over a 25 years period which result from the evolution of cerebrovascular risk factors and in-hospital management of stroke patients.

\section{Epidemiology of stroke}

OUTCOME AND SECONDARY PREVENTION OF PATIENTS WITH ISCHEMIC STROKE ASSOCIATED WITH ATRIAL FIBRILLATION IN ISRAEL:THE NATIONAL ACUTE STROKE ISRAELI SURVEY (NASIS) 2004 Y. Schwammenthal, N.M. Bornstein, U. Goldbourt, S. Koton, R. Schwarz, N. Koren-Morag, E. Gorossman, D. Tanne Sheba Medical Center, Tel Hashomer, Israel

Background and Purpose: Atrial fibrillation (AF), the most common sustained cardiac arrhythmia, is a major cause for stroke. Our aim was to assess the outcome of patients with ischemic stroke and $\mathrm{AF}$ and their secondary prevention in a comprehensive national survey in Israel.

Methods: Prospectively collected data from consecutive patients with acute ischemic stroke with and without AF, hospitalized in all 28 hospitals in Israel during February and March 2004 (the National Acute Stroke Israeli Survey), were analyzed with respect to patient characteristics, stroke severity, clinical course, outcome and secondary prevention

Results: Of 1558 patients with acute ischemic stroke, 282 patients (18\%) had a history of AF. Patients with AF were older ( $78 \pm 9$ vs. $69 \pm 13$ yrs.) and were more often female ( $60 \%$ vs. $42 \%$; $\mathrm{p}<0.0001)$ than counterparts without AF. Age-adjusted comparison to patients without $\mathrm{AF}$ revealed a higher prevalence of congestive heart failure, angina and renal failure and a lower prevalence of diabetes and smoking. Severe stroke (NIHS $>16$ ) on admission was significantly more frequent in patients with $\mathrm{AF}(24 \%$ vs. $9 \%, \mathrm{p}<0.0001)$, as was the incidence of total anterior circulation occlusion $(23 \%$ vs. $8 \%, \mathrm{p}<0.0001)$. Patients with $\mathrm{AF}$ had significantly more in-hospital complications. Adjusting for differences in age, sex and risk factors, ischemic stroke in patients with $\mathrm{AF}$ predicted poor outcome ( $\mathrm{mRS}>3$ or death) at discharge (OR 1.71; 95\% CI 1.26-2.31) and death after 3 month (OR $1.61 ; 95 \% \mathrm{Cl}$ 1.11-2.32). Secondary prevention by warfarin in patients with AF was provided to $44 \%$ of patients at hospital discharge and to $52 \%$ after excluding patients with predefined contraindications.

Discussion: Patients with ischemic stroke associated with AF have particularly severe strokes with poor outcome and a high case-fatality rate. Nevertheless, and in deviation from current recommendations, warfarin therapy for secondary prevention is underutilized. 
10

Epidemiology of stroke

SPONTANEOUS INTERNAL CAROTID ARTERY ATHEROMA EVOLUTION IN SYMPTOMATIC OR ASYMPTOMATIC SUBJECTS

G. Devuyst, M. Delgado, J. Bogousslavsky

CHUV, Lausanne, Switzerland

Background: Few recent studies are dedicated to analyzing the spontaneous evolution of the internal carotid artery (ICA) atheroma, symptomatic or asymptomatic, its maintenance or correction. The aim of our study is to evaluate the evolution of ICA atheroma by cervical ultrasounds during a long term follow-up.

Methods: We selected 54 patients producing 69 no stenotic ICA or ICA stenosis of lumen artery, including 30 symptomatic (TIA, stroke, amaurosis fugax) atheromatous ICA and 39 asymptomatic (carotid bruit or preoperative cardiac surgery) atheromatous ICA. Investigations of carotids were performed by ultrasounds, Bmode, color duplex flow imaging (CDFI) and power duplex imaging (PDI). The degree of ICA stenosis was established by hemodynamic criteria and by the reduction, in diameter and surface, of the artery lumen. Each ICA stenosis was followed once a year. To estimate the progression of ICA stenosis, we classified the degree of stenosis in 5 categories: $0:<50 \% ; 1: 50-59 \% ; 2$ : 60-69\%; 3: 70-79\%; 4: 80-89\% and $5:>90 \%$. All patients received antiplatelets.

Results: The mean follow-up was 5 years (ranging from 8 months to 17 years). We found out $31(45 \%)$ stable ICA stenoses, $3(4 \%)$ stenosis degree reductions and 35 $(51 \%)$ stenosis progressions. The distribution of the evolution of ICA stenosis was as follows: 0 to $5(17 \%), 1$ to $5(11 \%), 1$ to $2(11 \%), 0$ to $1(9 \%), 0$ to $4(9 \%), 4$ to $5(9 \%), 2$ to $4(9 \%), 3$ to $5(6 \%), 1$ to $4(6 \%), 3$ to $4(3 \%), 2$ to $3(3 \%), 0$ to $3(3 \%), 3-4-5(3 \%), 1-3-4(3 \%)$. The highest progression $(0-5$ and $1-5)$ represents $28.5 \%$ of the cases. Symptomatic ICA stenoses become severe (>90\%) statistically more frequently than asymptomatic ICA stenoses.

Conclusions: Our results confirm the dynamic evolution of the ICA stenoses. Only $45 \%$ of them were stable. Among the $51 \%$ ICA stenoses which worsened during the follow-up, $28.5 \%$ showed a rapid and important change in degree of stenosis. This information may be useful in the therapeutic approach.

\section{Epidemiology of stroke}

EPIDEMIOLOGY OF APHASIA DUE TO FIRST ISCHEMIC STROKE INCIDENCE, SEVERITY, FLUENCY, ETIOLOGY, AND THROMBOLYSIS S. Engelter, M. Gostynski, S. Papa, M. Frei, C. Born, V. Ajdacic-Gross, F. Gutzwiller, P. Lyrer

University Hospital Basel, Basel, Switzerland

Objective: In a geographically-defined population, we assessed incidence and determinants of aphasia due to first-ever ischemic-stroke (FEIS).

Methods: A one-year prospective, population-based study among the permanent residents of the canton Basle-City, Switzerland, was performed using multiple overlapping sources of information.

Results: Among 188'015 inhabitants, 269 patients had FEIS, of whom 80 (30\%; $95 \%$ confidence interval $(95 \% \mathrm{CI}) 24-36)$ had aphasia. The overall incidence rate of aphasia due to FEIS amounted to 43 per 100 '000 inhabitants (95\%CI 33-52). Aphasic stroke patients were older than non-aphasic. The risk of aphasia due to FEIS increased by $4 \%(95 \%$ CI $1-7 \%)$, and after controlling for atrial fibrillation by $3 \%$ (95\% CI 1-7\%) with each year of patients' age. Gender had no effect on incidence, severity, or fluency of aphasia. Cardioembolism was more frequent in aphasic stroke patients than in non-aphasic ones (OR 1.85; 95\%CI 1.07-3.20). Aphasic patients sought medical help earlier than non-aphasic stroke patients. Still, after controlling for stroke onset-assessment interval aphasic stroke patients were more likely to receive thrombolysis than non-aphasics (OR 3.5; 95\%CI 1.12-10.96).

Conclusion: Annually, 43 of 100'000 inhabitants had aphasia due to first ischemic stroke. Advancing age and cardioembolism were associated with an increased risk for aphasia. Severity and fluency of aphasia were not affected by demographic variables. The observation that the presence of aphasia may increase the odds for thrombolysis deserves further testing.

\section{Epidemiology of stroke}

STROKE PREVENTION PROGRAM RESULTS IN EXPERIMENTAL REGIONS OF RUSSIAN FEDERATION

V.I. Skvortsova, L.V. Stakhovskaya, N.Y. Airian, E.I. Gusev

Russian State Medical University, Moscow, Russian Federation

Background: National Stroke Association and Federal Stroke Institute established in 2002 a stroke secondary prevention Program based on individual approach to complex therapy depending on patient's risk factors and stroke subtype. The
Program was inculcated in two experimental regions of Russian Federation - Orel (Central part) and Krasnodar (Southern).

Methods: All cases of stroke were included in population-based registry in both regions during 48-month period of 2001 to 2004 that allow comparing the results before Program inculcation and after it.

Results: 4 years monitoring showed decreases of incidence from 3.2 to $1.2 / 1000$ /year and mortality from 1.9 to $0.4 / 1000 /$ year $(\mathrm{p}<0.01)$ between 2001 and 2004. Especially significant lowering of mortality rate (a 30\% from 2001 to 2004, p <0.01) was among able-bodied population (25-64 y.o.). Analysis of risk factors in stroke patients revealed cardiovascular diseases and atrial fibrillation reduction in both sites and arterial hypertension lessening in Orel from 2001 to $2004(\mathrm{p}<0.05)$.

Discussion: Present positive experience of prevention Program application in two experimental regions of Russian Federation evidences its efficacy and opportunity of successful replication of the Program in different regions. In 2005 an automated system for Prevention program was created to assist a doctor in decision-making and database maintenance.

\section{Acute stroke: complications and early outcome}

\section{Acute stroke: complications and early outcome}

HEMORRHAGIC TRANSFORMATION IS ASSOCIATED WITH RAPID RECOVERY FROM NEUROLOGICAL DEFICITS IN PATIENTS WITH ACUTE EMBOLIC STROKE

M. Kablau, J. Binder, K. Szabo, A. Gass, M.G. Hennerici Universitätsklinikum Mannheim, University of Heidelberg, Mannheim, Germany

Background: Magnetic resonance imaging (MRI) allows visualization of hemorrhagic transformation (HT) in ischemic stroke. We investigated potential predictive factors of HT and whether HT results in clinical deterioration.

Methods: 98 consecutive stroke patients were studied with MRI within $48 \mathrm{~h}$ after onset of symptoms. Occurrence of HT was correlated with age, previous aspirin medication, treatment with thrombolysis, etiology of stroke (TOAST criteria), presence of cerebral microbleeds as well as changes in clinical scores (NIH Stroke Scale (NIHSS), Barthel index (BI)).

Results: HT could be identified in 18 patients $(18,4 \%)$. Whereas age, chronic microbleeds or prior aspirin medication do not correlate with HT, patients with large vessel or cardioembolic stroke have a significantly higher incidence of HT than patients with small vessel disease $(26,8 \%$ versus $2,6 \%, \mathrm{p}<0.01)$. Treatment with thrombolysis is the major cause of HT $(11 / 19(57,9 \%)$ versus $7 / 79(8,9 \%))$. HT correlates with significant improvement in clinical scores (NIHSS: $-4,5$ [HT] vs $-0,2$ [non HT], p < 0.01; BI: 30 [HT] vs. 20 [non HT], p <0.01).

Discussion: Early HT in acute ischemia occurs frequently in patients with large vessel arterio-arterial and cardiogenic stroke. Previous medication with aspirin does not abet HT but treatment with thrombolysis does. Surprisingly, in stroke patients HT correlates significantly with rapid clinical improvement, especially in thrombolysis. Thus rather than predicting poor outcome HT characterizes the natural or treatment-triggered course of embolic stroke with rapid clot resolution and subsequent reperfusion. It should therefore not be mixed up with parenchymal hemorrhage as it was often the case in studies not particularly aware of the different pathomechanisms and time courses.

2 Acute stroke: complications and early outcome

EXTENT OF HEMORRHAGIC TRANSFORMATION ON CT AFTER THROMBOLYSIS PREDICTS OUTCOME IN THE CANADIAN ALTEPLASE FOR STROKE EFFECTIVENESS STUDY (CASES)

I. Dzialowski, P.A. Barber, J.H. Pexman, A.M. Demchuk, A.M. Buchan, for the CASES investigators

University of Calgary, Calgary, Canada

Background: There is ongoing controversy about the significance of hemorrhagic transformation (HT) after thrombolysis for long-term functional outcome. We sought to study the relationship between the extent of HT on computed tomography (CT) and functional outcome in the Canadian Alteplase for Stroke Effectiveness Study (CASES).

Methods: CASES was established as a registry to prospectively collect data for acute stroke patients receiving intravenous Alteplase from 02/99-06/01 within 3 hrs from onset. Follow-up was completed at 90 days and good functional outcome defined as modified Rankin Scale (mRS) score 0-1. Copies of head CT scans obtained at 24-48 hrs after treatment initialization were read in consensus by a central reading 
panel consisting of 1 Neuroradiologist and 1 stroke Neurologist. According to the European Cooperative Acute Stroke Study criteria, HT was classified into no HT, hemorrhagic infarction (HI)-1, HI-2, parenchymal hematoma (PH)-1, and PH-2. We compared outcome across HT groups and performed a multivariable analysis including previously determined important predictors of good outcome in acute ischemic stroke.

Results: From 1135 patients enrolled at 60 centers across Canada 954 follow-CTscans were assessable. We observed HT in 259/954 (23\%) patients (110 HI-1, 57 HI-2, 48 PH-1, 44 PH-2). Proportions of patients with good outcome were $41 \%$ with no HT, $30 \%$ with HI-1, $17 \%$ with $\mathrm{HI}-2,15 \%$ with $\mathrm{PH}-1$, and $7 \%$ with $\mathrm{PH}-2$ $\left(\mathrm{p}<0.0001, \chi^{2}\right.$-test $)$. After adjustment for age, baseline serum glucose, baseline Alberta Stroke program Early CT score and baseline NIHSS score, HI-1 was not a predictor of outcome. However, $\mathrm{HI}-2(\mathrm{OR}=0.38, \mathrm{CI} 95=0.17-0.83), \mathrm{PH}-1(\mathrm{OR}=0.32$, $\mathrm{CI} 95=0.12-0.80), \mathrm{PH}-2(\mathrm{OR}=0.14, \mathrm{CI} 95=0.04-0.48)$ were all negative predictors of good outcome.

Conclusion: In this large cohort of patients with acute ischemic stroke, the extent of HT on CT after thrombolysis with Alteplase is a predictor of functional outcome. The likelihood of bad outcome after thrombolysis was proportional to the extent of HT on CT.

\section{Acute stroke: complications and early outcome}

LEUKOARAIOSIS AND LACUNES ARE ASSOCIATED WITH SYMPTOMATIC INTRACEREBRAL HEMORRHAGE IN PATIENTS WITH ACUTE ISCHEMIC STROKE RECEIVING SYSTEMIC THROMBOLYSIS: THE CASES STUDY V. Palumbo, J.M. Boulanger, A.M. Buchan, M.D. Hill, on behalf of the CASES Investigators

University of Calgary, Calgary, Canada

Background: The presence of leukoaraiosis (LA) on baseline computed tomography $(\mathrm{CT})$ is commonly considered related to hemorrhagic complications in patients with acute ischemic stroke treated with intravenous thrombolysis. We aim to evaluate whether the presence of LA, lacunes and old infarcts is associated to symptomatic intracerebral hemorrhage ( $\mathrm{ICH})$, by systematically reviewing the $\mathrm{CT}$ scans in the CASES study.

Methods: Out of 936 CT scans, 820 were available for reading. LA was evaluated with Van Swieten Score (VSS), applied separately to each hemisphere. A VSS $>4$ accounted for the presence of severe LA (based on the sum of the score in the 2 hemispheres), while the number of lacunes was dichotomized into 2 or more versus 1 or none. A reliability test was performed in 40 scans. The remaining 780 scans were evaluated independently by 2 readers, blind to clinical information and follow-up imaging. Logistic regression was used to determine if an interaction existed between presence and degree of LA and risk of sICH. Similarly, the predictive role of lacunes and old infarcts was explored.

Results: An overall sICH rate of $3.5 \%$ was observed. The rate of sICH increased in patients with severe LA $(6 / 71,8.4 \%)$ and in presence of 2 or more lacunes $(7 / 70$, $10 \%$ ). A significant association was observed between sICH risk and both severe LA ( $R R=2.7$ [95\% confidence interval 1.1-6.5], $\mathrm{p}=0.03)$ and multiple lacunes $(\mathrm{RR}=3.4$ [95\% confidence interval 1.5-7.6], $\mathrm{p}=0.008)$. No relation was found with presence of old infarcts. In the multivariable logistic regression there was a preserved effect and only elevated blood glucose was also a predictor of sICH

Conclusion: The presence of multiple lacunes and severe LA significantly increases the risk of sICH in the CASES population.

\section{Acute stroke: complications and early outcome}

\section{ADMISSION FACILITY PREDICTS OUTCOME IN PATIENTS WITH BASILAR ARTERY OCCLUSION}

R. Müller, T. Pfefferkorn, B. Vatankhah, T. Mayer, D. Sander, M. Dichgans,

H.J. Audebert

Klinikum Harlaching, Städtisches Klinikum München GmbH, München, Germany

Background and Purpose: Basilar artery occlusion (BAO) is a stroke subtype with generally poor prognosis but recanalizing therapies have been reported to be effective. This study was conducted to determine whether initial admission to general hospitals with stroke centre transfer is related to poorer outcome than direct admission to a stroke centre.

Methods: All BAO cases in four stroke centres in Munich and Regensburg between Mar. 12003 and Dec. 312004 were included. BAO was defined as radiologically confirmed occlusion and/or acute infarcts in the basilar artery territory in patients with sudden onset of disturbed consciousness. 27 patients had initial admission to general hospitals and secondary transfer to stroke centers (group A). 26 patients (group B), were directly admitted into stroke centres. Baseline parameters (age, comorbidities, level of consciousness), and therapeutic procedures were mainly retrospectively recorded. One-year follow up was conducted prospectively per telephone.

Results: There were no statistically significant differences in baseline parameters. Time from onset to treatment decision was significantly longer in patients of group A (median: $340 \mathrm{~min}$ versus $232 \mathrm{~min}, \mathrm{p}=0.01$ ), mainly due to transfer duration. Group B patients got more often interventional treatment $(77 \%$ vs. $56 \%, \mathrm{p}=0.1)$. In-hospital mortality $(23 \%$ versus $52 \%, \mathrm{p}=0.031)$ and one-year-mortality $(31 \%$ versus $58 \%$, $\mathrm{p}=0.051$ ) were lower for group B patients. $50 \%$ of group B patients and $35 \%$ of group A patients $(\mathrm{p}=0.172)$ were living at home after one year.

Conclusions: Time to treatment is a critical prognostic factor in BAO cases. Patients with typical symptoms should have direct access to stroke centres, or may need bridging therapies.

\section{Acute stroke: complications and early outcome}

THE SAFETY AND EFFICACY OF T-PA IN THE INDEPENDENT ELDERLY

J. Bray, J. Lye, K. Coughlan, C. Bladin

Eastern Melbourne Neurosciences, Box Hill Hospital, Monash University, Deakin University, Melbourne, Box Hill, Australia

Background: The effectiveness of tissue-Plasminogen Activator (t-PA) is well established in ischaemic stroke, however there is limited evidence of its efficacy and safety in the elderly (80+ years). The aim of this study is to compare the outcomes between elderly and younger patients receiving t-PA.

Methods: A review of prospectively collected data for all patients receiving t-PA at Box Hill Hospital (Melbourne, Australia). The administration of t-PA was as per NINDS criteria, with the added exclusion of patients with a premorbid modified Rankin score (mRS) of 3 or greater (i.e. previously dependent patients). A statistical comparison between elderly ( $80+$ years) and younger $(<80$ years $)$ patients was performed for standard outcomes post-thrombolysis

Results: Over the 33-month period, 72 patients received t-PA. Of these, $19(26 \%)$ were aged 80 years or older. Median pre-treatment National Institutes of Health Stroke Scale (NIHSS) scores were similar, 13 vs. $13,(\mathrm{p}=0.99)$. There was no statistically significant difference in outcomes between the older vs. younger groups: NIHSS score improvement $\geq 4$ points post t-PA infusion ( $32 \%$ vs. $49 \%, \mathrm{p}=0.19$ ); favourable outcome (3-month mRS of $0-1)(32 \%$ vs. $38 \%$, $\mathrm{p}=0.63)$; independent outcome (3-month mRS of $0-2)(42 \%$ vs. $47 \%, \mathrm{p}=0.70)$; inhospital mortality ( $5 \%$ vs. $9 \%, \mathrm{p}=0.57)$; three-month mortality ( $16 \%$ vs. $9 \%, \mathrm{p}=0.45)$; asymptomatic intracerebral haemorrhage (haemorrhagic infarction or parenchymal haemorrhage by ECASS classification) $(5 \%$ vs. $11 \%, \mathrm{p}=0.44)$. Only one, 59 -year-old patient experienced a non-fatal symptomatic intracerebral haemorrhage.

Conclusion: This small study provides no indication for withholding t-PA in elderly patients with 'independent' premorbid level of functioning. Due to the small sample size of existing studies, a systematic review may be required to establish the efficacy and safety of t-PA in elderly ischaemic stroke patients.

\section{Acute stroke: complications and early outcome}

HAEMORRHAGIC COMPLICATIONS OF ROUTINE THROMBOLYSIS WITH TISSUE PLASMINOGEN ACTIVATOR IN ACUTE ISCHEMIC STROKE IN PATIENTS AGED 80 YEARS AND OLDER

M. Uyttenboogaart, P.C. Vroomen, J. De Keyser, G.J. Luijckx

University Medical Center Groningen, Groningen, The Netherlands

Background: thrombolysis with tissue Plasminogen Activator (tPA) has been proven effective for ischaemic stroke within 3 hours after onset. Little is known regarding the safety of this intervention in patients aged $\geq 80 \mathrm{yrs}$, because this age group was underrepresented in clinical trials. Until new data from randomised trials are available, data from cohort studies in this age group may be useful for clinicians as a guideline to treat these patients.

Methods: in a prospective patient registry we collected patient-characteristics, admission NIHSS, onset to treatment time (OTT) and the occurrence of symptomatic intracerebral haemorrhages (SICH). These data were analysed separately for patients $\leq 80$ yrs and their younger counterparts and compared with the results of other cohorts from the literature.

Results: 131 consecutive patients were treated with tPA; 101 patients were aged $<80$ yrs and 30 patients were aged $\geq 80$ yrs. The mean initial NIHSS and OTT were: 13 vs $14(\mathrm{p}=0.612)$, and 145 vs $155 \mathrm{~min}(\mathrm{p}=0.057)$ in respectively the younger and $\geq 80$ yrs group. SICH occurred in $4 \%(4 / 101)$ of the younger and in $10 \%(3 / 30)$ of the $>80$ yrs group $(p=0.196)$. From the literature analysis we found that $\mathrm{SICH}$ occurred in $7.5 \%(51 / 684)$ of patients aged $<80$ years and in $8.8 \%(15 / 171)$ of the patients aged $\geq 80$ yrs $(\mathrm{p}=0.564)$. Pooling our data with the previous data showed that SICH occurs in 7\% (55/785) of patients aged $<80 \mathrm{yrs}$ and in $9 \%(18 / 201)$ of patients aged $\geq 80 \mathrm{yrs}(\mathrm{p}=0.346)$.

Acute stroke: complications and early

outcome 
Discussion: Currently there is no statistical evidence that stroke patients aged $\geq 80$ yrs are at a greater risk for developing SICH following tPA treatment than their younger counterparts. Although these results are based on a small number of patients it may be considered to treat patients $\geq$ yrs with tPA.

\section{Acute stroke: complications and early outcome}

\section{HYPERTHERMIA AND LESION DWI-GROWTH IN ACUTE ISCHEMIC STROKE \\ M. Rodríguez-Yañez, M. Castellanos, J. García, M. Millán, M. Blanco, F. Nombela, Y. Silva, S. Arias-Rivas, R. Leira}

Hospital Clínico Universitario de Santiago, Santiago de Compostela, Spain

Objective: To study the relationship between hyperthermia and lesion growth measured by MRI-DWI (DWI-growth) in acute ischemic stroke.

Methods: 252 with acute ischemic stroke $<12$ hours of evolution were studied. Previous history, demographic data, fibrinogen and leukocyte count was register at admission. Temperature, glucose and blood pressure (BP) was measured at admission and every 6 hours during the first 3 days. Hyperthermia was defined as maximum temperature in first 24 hours (Tmax $24 \mathrm{~h}) \geq 37,5^{\circ} \mathrm{C}$. MRI-DWI was performed at admission and at 72 hours, defining DWI-growth as absolute difference between these determinations. MRI was performed at 30 days to determine final volume. NIHSS was performed at admission, 24, 48 and 72 hours, day 7 and 3 months. Modified Rankin scale was performed at 3 months.

Results: In 12 patients the second MRI was not performed. 240 patients were useful for the study. 121 were male, with 70,7 $\pm 9,4$ years. 43 patients developed hyperthermia. Higher DWI-growth was found in patients with hyperthermia $(93,6 \pm 95,4$ vs $27,1 \pm 41,8 \mathrm{cc}, \mathrm{p}<0,0001)$. Variables related with DWI-growth were NIHSS, diastolic BP, leukocyte and fibrinogen at admission, Tmax $24 \mathrm{~h}$, average of diastolic $\mathrm{BP}$ in first 24 hours and non-lacunar stroke. In a logistic model, only Tmax $24 \mathrm{~h}$ (OR 2,59, CI95\% 1,06-4,24; $\mathrm{p}=0,036)$ and non-lacunar stroke (OR 0,008, CI95\% $1,66-29,64, \mathrm{p}=0,008)$ were independently related with DWI-growth

Conclusions: In non-lacunar stroke, temperature increase is independently associated with DWI-growth in first 72 hours.

\section{Acute stroke: complications and early outcome}

LEUKOARAIOSIS IS A RISK FACTOR FOR SYMPTOMATIC INTRACEREBRAL HEMORRHAGE AFTER THORMBOLYSIS FOR ACUTE STROKE

T. Neumann-Haefelin, S. Hölig, J. Fiehler, A. Gass, M. Humpich, A. Kastrup, D.S. Liebeskind, Y. Samson, J. Berkefeld, for the MR Stroke Collaborative Group

University Hospital, Frankfurt, Germany

Background and Purpose: The aim of the study was to evaluate whether leukoaraiosis (LA) is a risk factor for symptomatic intracerebral hemorrhage ( $\mathrm{sICH}$ ) in patients treated with thrombolysis for acute stroke.

Methods: In this retrospective multi-center analysis, the authors evaluated data from acute anterior circulation stroke patients $(n=449 ;<6$ hours following symptom onset) treated with thrombolysis. All patients had received standard MRI evaluation prior to thrombolysis, including a high quality T2-weighted sequence. For the analysis, LA in deep white matter was dichotomized into absent or mild vs. moderate or severe (corresponding to Fazekas scores of 0-1 vs.2-3).

Results: The rate of sICH was significantly more frequent in patients with moderate to severe LA of the deep white matter ( $\mathrm{n}=12$ out of $114 ; 10.5 \%)$ than in patients without relevant $\mathrm{LA}$ ( $\mathrm{n}=13$ out of $335 ; 3.8 \%$ ), corresponding to an odds ratio (OR) of $2.9(95 \%$ confidence interval, 1.29 to $6.59 ; \mathrm{p}=0.015)$. In a logistic regression analysis (including age, NIHSS score at presentation, type of thrombolytic treatment), LA remained a significant independent risk factor (OR: 2.9, $\mathrm{p}=0.03$ ).

Conclusion: Leukoaraiosis of the deep white matter is an independent risk factor for sICH following thrombolytic treatment of acute stroke.

\section{Acute stroke: complications and early outcome}

INSULAR INFARCTS AND ELECTROCARDIOGRAPHIC CHANGES AT ADMISSION. RESULTS OF THE PROGNOSTIC OF INSULAR CEREBRAL INFARCTS STUDY (PRINCESS)

M. Pasquini, C. Laurent, M. Kroumova, I. Masse, D. Deplanque, X. Leclerc, R. Bordet, D. Leys

Lille University Hospital, Lille, France

Background and Purpose: Previous studies showed that insular strokes are asso- ciated with electrocardiographic (ECG) changes. However, they did not take into account the 1st ECG recorded at admission, but continuous ECG recorded up to 72 hours after onset. Whether these changes are the consequence of the infarct, or are associated with a cardiac source of cerebral ischemia, remain unsettled. If ECG changes are the consequence of insular infarcts, they should not yet be present at admission.

The aim of this study was to test the hypothesis that ECG changes in patients with insular infarcts are not yet present at admission.

Methods: We recruited consecutive patients admitted within 48 hours (median 3 hours) after the onset of symptoms of acute hemispheric cerebral ischemia. We compared ECG variables between patients with and without insular infarcts, and with left and right insular infarcts.

Results: The study population consisted of 208 patients ( 94 men; median age: 69 years). Seventy patients had a recent insular infarct (right in 33). ECG variables did not significantly differ between patients with and without insular infarcts, and with left and right insular infarcts. These results were not explained by a lack of statistical power (1-beta $>=0.90)$.

Conclusion: The lack of statistical link between insular infarcts and ECG changes at admission, suggests that ECG changes are not associated with the cause of insular infarcts, but are their consequence.

10 Acute stroke: complications and early outcome

ACUTE ISCHEMIC STROKE CARE AND OUTCOMES IN POLAND PRELIMINARY DATA FROM POLKARD (NATIONAL CARDIOVASCULAR DISEASE PREVENTION AND TREATMENT PROGRAM FOR 2003-2005) STROKE REGISTRY

M.P. Niewada, M. Skowrońska, I. Sarzyńska-Długosz, B. Kamiński,

D. Ryglewicz, A. Czlonkowska

Institute of Psychiatry and Neurology, Warsaw, Poland

Background: To estimate clinical presentation, the acute care and outcomes in stroke centers participating in POLKARD (National Cardiovascular Disease Prevention and Treatment Program for 2003-2005) STROKE Registry.

Methods: WHO Step Stroke and Swedish Stroke Registry web-based questionnaire was used to collect data on ischemic stroke patients admitted to participating centers between June the 1st 2004 and May 31st 2005. To ensure the quality only centers reported at least 100 patients were analyzed. Patients characteristic, in-hospital care and early outcomes were analyzed for ischemic stroke (I63 coding in International Classification of Diseases, 10th revision).

Results: There were 73 centers that met inclusion criteria and 20542 patients $(51.4 \%$ women, mean age: $70.6 \mathrm{yrs}$ ) were included in the final analyses. Mean time from onset to admission: 812 minutes. 14391 (70.1\%), 3138 (15.3\%), $1088(5.3 \%), 617$ $(3.0 \%)$ patients were alert, drowsy, stupor and in coma at admission, respectively. Risk factors distribution: history of stroke $-4577(22.3 \%)$, history of TIA - 1230 $(6.0 \%)$, hypertension $-14830(72.2 \%)$, atrial fibrillation $-4989(23.8 \%)$, history of myocardial infarction $-2270(11.1 \%)$, diabetes $-4911(23.9 \%)$, smoking -2976 (14.5\%), hiperlipidemia -5099 (24.8\%), alcohol abuse - 1058 (5.2\%). The ischemic stroke subtypes distribution of TOAST classification was as follows: large-artery atherosclerosis - $7396(36.0 \%)$, cardioembolism - $2794(13.6 \%)$, small-artery occlusion - $3887(18.9 \%)$, stroke of other determined cause - $1726(8.4 \%)$ and stroke of undetermined cause $-4723(23.0 \%)$. Thrombolytic therapy was introduced for 143 patients $(0.7 \%)$. Mean modified Rankin score following stroke was calculated on 3.02. 2383 patients (11.6\%) died and for $48,3 \%$ death was directly and $45.0 \%$ indirectly associated with stroke.

Conclusions: Ischemic stroke represents significant clinical matter and needs further monitoring in Poland.

\section{Acute stroke: complications and early outcome}

THE MOLECULAR TEMPORARY PROFILE OF SCD40L IN ACUTE ISCHEMIC STROKE: CLINICAL AND PROGNOSTIC SIGNIFICANCE M. Blanco, I. Cristobo, R. Leira, T. Sobrino, M. Rodríguez-Yañez, J. Vivancos, J. Serena, I. Lizasoain, A. Dávalos, J. Castillo

Hospital Clínico Universitario de Santiago, Santiago de Compostela, Spain

Objective: CD40 signalling in endothelial and smooth muscle cells, monocytes, and platelets promotes a wide array of proatherogenic and prothrombotic functions in vitro and in vivo. CD40L also occurs in plasma in soluble form. Elevated plasma concentrations of soluble CD40 ligand (sCD40L) were found in coronary syndrome and ischemic stroke, but its molecular temporary profile as well as its potential clinical and prognostic value is unknown.

Methods: We prospectively studied 67 patients (40 males, average $73.1 \pm 7.2$ y) within $12 \mathrm{~h}$ from stroke onset $(7.3 \pm 2.8 \mathrm{~h})$. Blood samples were obtained on 
admission, 24 and $72 \mathrm{~h}$ to determine $\mathrm{sCD} 40 \mathrm{~L}$. Early neurological deterioration (END) was defined as an increase $\geq 4$ in the NIHSS in first $24 \mathrm{~h}$. Lesion volume was measured by $\mathrm{CT}$ at $72 \mathrm{~h}$. We considered poor outcome a $\mathrm{mRS}$ score $\geq 3$ at 3 months.

Results: SCD40L basal levels were associated with early clinical, biochemical and neuroradiological factors of poor outcome (age, diabetes, hyperthermia, leukocytosis, hyperfibrinogenemia, hyperglycemia and early signs in $\mathrm{CT}$, all $\mathrm{p}<0.001$ ). sCD40L basal levels were significantly higher in patients with END (6.18 [5.1-7.8] vs $4.4[3.1-6.5] \mathrm{ng} / \mathrm{mL} ; \mathrm{p}=0.001)$; in patients that presented a bigger infarct volume $(\mathrm{r}=0.79, \mathrm{p}<0.0001)$ and worse outcome at 3 months $(6.18$ [4.96-8.57] vs 4.1 $[3.01-6.13] \mathrm{ng} / \mathrm{mL} ; \mathrm{p}=0.002)$. In the multivariate analyses, $\mathrm{sCD} 40 \mathrm{~L}$ basal levels were an independent factor for ultimate infarct volume (B:12.3; IC 95\%: 6.2-18.3; $\mathrm{p}<0.0001$ ), but not for END and outcome at 3 months. Plasma pick value was found on admission for $\mathrm{sCD} 40 \mathrm{~L}$ and its levels diminish progressively at 24 and $72 \mathrm{~h}$ (the decrease was more significant in patients who received antiplatelet drugs). However, sCD40L levels at 24 and $72 \mathrm{~h}$ don't offer more clinical and prognostic utility than basal levels.

Conclusion: sCD40L basal levels constitute a powerful marker of the ultimate infarct volume in patients with acute ischemic stroke.

\section{Acute stroke: complications and early outcome}

\section{ISCHEMIC STROKE IN PATIENTS UNDER 55: IS FEMALE GENDER A PROGNOSTIC FACTOR FOR POOR OUTCOME?}

P. Martinez, B. Fuentes, M.A. Ortega-Casarrubios, F.J. Rodriguez de Rivera,

S. Martin, R. Lobato, M. Valenti, E. Díez-Tejedor

Hospital Universitario La Paz, Universidad Autonoma de Madrid, Madrid, Spain

Background: Female gender has been related to a higher mortality and disability after ischemic stroke than male, however, few studies have examined these differences in young stroke patients.

Methods: Observational study with inclusion of consecutive stroke patients admitted to our Stroke Unit from January 1995 to February 2005. We selected patients under 55 years of age with acute cerebral infarction (CI), Vascular risk factors, subtype of stroke, neurological status at admission by the Canadian Stroke Scale (CSS), length of stay, in-hospital complications, mortality and functional outcome at discharge by the modified Rankin scale (mRS) were analysed to establish gender differences.

Results: 381 patients were included, 230 males and 151 females. Valvular heart disease, previous $\mathrm{CI}$, symptomatic migraine and thrombophilia were the risk factors more frequent in women than in men $(\mathrm{P}<0,001)$. Women had more cardioembolic infarcts, infarcts of unusual cause and infarcts of undetermined origin $(\mathrm{P}<0,05)$ than men. Although mortality was similar in both sexes, women had worse neurological status at admission and suffered more in-hospital complications. Women who survived remained more disabled (mRS $>2$ ) than men (Odds Ratio: 2,22; 95\% CI, 1.15 to 4.27). Multiple logistic regression analyses showed that female gender was an independent predictor for disability and for the combination of death and disability at discharge together with severe neurological deficit at admission $(\mathrm{CSS}<$ or $=3)$ and systemic complications.

Discussion: female gender is an independent factor for poor outcome, together with severe neurological deficit at admission and systemic complications, in ischemic stroke patients under 55 years of age. Female gender intrinsic factors could explain these results.

\section{Acute stroke: early management and stroke units}

\section{Acute stroke: early management and stroke units}

\section{CAROTID OCCLUSION AND ARTERIAL HYPOTENSION DURING THE} ACUTE PHASE OF ISCHEMIC STROKE

C. Arquizan, E. Collet-Sassere, E. Touzé, V. Domigo, J.L. Mas

Hôpital Sainte Anne, Paris, Montpellier, France

Background: Empiric experience suggests a relationship between carotid occlusion and arterial hypotension in acute ischemic stroke patients. Low blood pressure (BP) is associated with poor outcome in patients with ischemic stroke. The aim of our study was to determine whether carotid occlusion is associated with arterial hypotension in the acute phase of carotid ischemic stroke.

Methods: In this case-control study, we compared systolic (SBP) and diastolic (DBP) BP during a period of 72 hours (9 readings) after admission in a stroke unit in consecutive patients with carotid ischemic stroke, with $(n=26)$ or without $(n=56)$ carotid occlusion, after adjustment on age, sex and stroke severity (evaluated by the NIH Stroke Scale)

Results: The mean SBP during the first three days was $136 \pm 23 \mathrm{~mm} \mathrm{Hg}$ in cases and $149 \pm 24$ in controls $(\mathrm{p}=0.04)$. The mean DBP was respectively $77 \pm 15$ and $83 \pm 14$ $(\mathrm{p}=0.08)$. The mean BP $((\mathrm{SBP}+2 \mathrm{DBP}) / 3)$ was respectively $96.9 \pm 17$ and $105 \pm 16$ $(\mathrm{p}=0.04)$. Logistic regression analyses showed that carotid occlusion remained significantly associated with low mean BP $\leq 90 \mathrm{~mm} \mathrm{Hg}$ (odds ratio, 3.5; 95\% CI, 1.1 to 11.7 )

Discussion: This is the first study to show that carotid occlusion is significantly associated with arterial hypotension, in patients with acute ischemic stroke. In multivariate analysis, this effect is independent of stroke severity, and risk factors of autonomic failure (diabetes, age). If carotid sinus baroreflex impairment is a possible mechanism, others hypotheses should be investigated. In the acute phase of ischemic stroke in the carotid territory, the diagnosis of carotid occlusion justifies verifying arterial hypotension and patients with low BP might benefit from specific management.

\section{Acute stroke: early management and stroke units}

IMPLEMENTATION OF STROKE UNITS REDUCES STROKE MORTALITY BY 18\% IN DENMARK.EXPERIENCES FROM 24.791 STROKE ADMISSIONS K.K. Andersen, L.P. Kammersgaard, T.S. Olsen Informatics and Mathematical Modeling, Section for Statistics, Technical University of Denmark, Denmark

Background: The effect of stroke units (SU) is documented in randomized trials. Since 2001 survival after stroke in Denmark (DK) is followed in a nation-wide register, currently containing data on more than 30000 stroke admissions. We studied effect of implementation of SU in DK comparing survival of patients (pts) managed in SU and general medical wards (GMW).

Method: The Danish National Indicator Project introduced 2001 is expected to register all stroke admissions in DK and currently covers $75 \%$ of all stroke admissions. We analysed data on the first 30000 admissions. All pts underwent a pre-defined program: admission stroke severity using the Scandinavian Stroke Scale (SSS, 0-58), CT-scan and a cardiovascular risk factor evaluation. Survival was recorded through the Danish Death Registry. Mean follow-up was 1.2 years. We excluded readmissions and pts $<40$ years.

Results: We included 24791 pts. Males $51.6 \%$; mean age 72.3 (sd, 12.9) years; mean admission SSS-score 40.9 (sd, 17.2). Except for 1.108 pts $(4.4 \%)$ managed in GMW pts were managed in SU. In a logistic regression analysis pts in GMW and SU did not differ significantly in respect to age, sex, stroke severity and cardiovascular risk factors. In a univariate comparison odds ratio of dying within follow-up for pts in GMW was 1.67 compared to pts in SU $(\mathrm{p}<0.001)$.

A Cox proportional hazard model, with age modelled as delayed entry, was build to study the effect of SU/GMW on survival while controlling for relevant confounders (SSS, age, sex, cardiovascular risk factors). Pts in SU had a significantly higher chance of survival, Hazard Ratio 1.221 (CI 1.021 - 1.460), yielding reduced mortality by $18 \%$.

Discussion: A nation-wide implementation of SU in DK has resulted in a reduction of stroke mortality by $18 \%$.

\section{Acute stroke: early management and stroke units}

INTRACEREBRAL HEMORRHAGE MANAGEMENT:RESULTS OF THE AUSTRIAN ACUTE STROKE UNIT REGISTRY 2003-2005 - COMPARISON OF 758 CASES WITH CONCURRENT 5.926 CASES OF ISCHEMIC STROKE R. Eckhardt, M. Novotny, P. Bosak, C. Tatschl, K. Matz, G. Funk, M. Brainin Landesklinikum Donauregion Gugging, Maria Gugging, Austria

Background and Objectives: There is evidence that stroke units have a beneficial effect on the outcome of stroke but few data exist on the outcome of intracranial hemorrhages (ICH).

Methods: Descriptive analysis of the ÖBIG/ÖGSF-Austrian stroke registry which included 6.684 consecutively admitted stroke patients to 16 participating SUs from January 2003 to July 2005. A comparison of ICH ( $n=758)$ with ischemic stroke (IS, $\mathrm{n}=5.926$ ) was performed.

Results: Patients with ICH were more severely impaired on admission (NIHSS median ICH 10 vs. IS 5) and upon discharge (NIHSS median ICH 6 vs. IS 3). The in-hospital mortality was $14.8 \%$ vs. $3.7 \%$ (ICH/IS) and the 3 month mortality was $31.5 \%$ vs. $15.3 \%$. The need for invasive measures was significantly higher in ICH as seen in need for decompressive surgery (2.6\% vs.0.5\%), continuous iv medication $(33.5 \%$ vs. $14.4 \%)$, monitoring of intraarterial blood pressure $(9.4 \%$ vs. $1.6 \%)$ and central venous pressure $(3.5 \%$ vs. $0.8 \%)$, intubation $(4.8 \%$ vs. $1,0 \%)$ and artificial ventilation $(2.6 \%$ vs. $1.0 \%)$, urinary tract catheter $(63.3 \%$ vs. $39.9 \%)$ and nasogastric

Acute stroke: early management and stroke units 
tube feeding (26.0\% vs.13.3\%). ICH had more complications due to mass effect ( $20.6 \%$ vs. $14.4 \%)$, hydrocephalus ( $1.9 \%$ vs. $0.3 \%)$, pneumonia (12.3\% vs. $8.5 \%)$, seizures $(4.2 \%$ vs. $2,0 \%)$ and heart failure $(6.9 \%$ vs. $4.4 \%)$. The mean length of stay on the $\mathrm{SU}$ was $5.8 \mathrm{~d}$ vs. $4.6 \mathrm{~d}$ and more patients had to be transferred to neurosurgical departments (4.6\% vs. $0.5 \%)$ and ICUs (7.5\% vs. $1.9 \%)$.

Conclusions: Patients with ICH are in need of more intensive care and monitoring as they are in a clinically more severe condition and show higher rates of complications when compared to patients with IS. The system of semi-intensive SUs set up in Austria copes with most of the workload.

\section{Acute stroke: early management and stroke units}

\section{ADMISSION GLUCOSE LEVEL, C-REACTIVE PROTEIN, AND BLOOD PRESSURE IN STROKE PATIENTS TREATED WITH TPA: INFLUENCE ON CLINICAL OUTCOME AND INTRACRANIAL HEMORRHAGE \\ B. Dimitrijeski, A. Villringer, A. Hartmann \\ Charite - Campus Benjamin Franklin, Berlin, Germany}

Background: Hyperglycemia, elevated C-reactive protein (CRP) and hypertension during acute ischemic stroke may augment brain injury and worsen clinical outcome. Information about the impact of these parameters on clinical outcome in stroke patients treated intravenously with rt-PA is scarce.

Methods: Consecutive acute stroke patients treated with intravenous r-tPA in our department using the NINDS study protocol and additional CT exclusion criteria from ECASS were followed prospectively. Neurological status was assessed at admission, discharge, and after 3 months using the Rankin scale. The association of admission glucose level, CRP-level, leukocyte count, and blood pressure with unfavorable outcome (Rankin $>2$ ) at 3 months and symptomatic intracranial hemorrhage (ICH) was investigated using multiple logistic regression analysis.

Results: Of the 154 patients (mean age 69 years, $47 \%$ female, mean admissionNIHSS score 14,6), 54\% had a Rankin score $>2$ after 3 months. Symptomatic intracranial hemorrhage occurred in 4,5\%. Elevated admission glucose levels were significantly associated with an increased risk of unfavorable outcome (OR 1.01, CI $1.01-1.02$ per mg/dl glucose increase, $\mathrm{p}=0.006$ ) and for ICH (OR 1.01, CI 1.01 -1.03 per $\mathrm{mg} / \mathrm{dl}$ glucose increase, $\mathrm{p}=0.013$ ). No associations were found for CRP, leukocyte count and high blood pressure on admission.

Conclusion: In acute stroke patients treated with rt-PA, elevated admission glucose levels are associated with unfavorable outcome and a higher risk for symptomatic intracranial hemorrhage. Careful blood glucose monitoring and treatment in these patients is emphasized.

\section{Acute stroke: early management and stroke units}

\section{EVALUATING AN ORGANIZED APPROACH TO PALLIATIVE CARE}

\section{FOLLOWING DEVASTATING STROKE}

D. Blacquiere, G. Gubitz, S. Phillips, D. Macleod, D. Dupere

Queen Elizabeth II Health Sciences Centre, Halifax, Nova Scotia, Canada

Introduction: About $20 \%$ of patients will die following acute stroke. Stroke care guidelines rarely mention palliation. When mentioned, guidelines vary; none examine the efficacy of palliation. The role of the patient's family in decision making has not been evaluated. We sought to formally evaluate the use of palliative care guidelines on our acute stroke unit (ASU).

Hypothesis: An organized approach to management of patients with devastating stroke leads to better end of life care.

Methods: We retrospectively examined records of 104 patients who died on our ASU over two years to determine if existing palliative guidelines were reflected in clinical practice, and to identify conflicts. We collected medical and nursing data, palliative decisions, medications, and conflicts, and compared these to palliative care guidelines. Descriptive and qualitative analyses were used to examine the palliation process.

Results: Median age of the 104 patients was 80 years; 63\% were female. Most had large strokes (33\%) or hemorrhages (26\%). All received routine nursing and comfort care prior to death. $10 \%$ were receiving active treatment at the time of death; $90 \%$ of patients who died were palliated. Median time from admission to palliation was 3.6 days, and to death was 8.5 days. Most (99\%) had vital signs, investigations and diagnostic imaging stopped, $95 \%$ had non-palliative medications stopped, and $97 \%$ had feeding tubes and IV lines withdrawn. Most families (62\%) had no conflicts with staff. Conflicts arose from hydration and feeding (46\%), doubts about palliation (28\%) and comfort (18\%).

Conclusions: Death from acute stroke is common; patients are older, have larger strokes and live for a short time. A formal approach to palliation results in timely decisions regarding end of life care with relatively few conflicts. Further work to address the concerns of families is needed.
6 Acute stroke: early management and stroke units

THE PREVENTION OF COMPLICATIONS MAY EXPLAIN THE SURVIVAL BENEFIT OF ORGANISED (STROKE UNIT) CARE: A SECONDARY ANALYSIS OF A SYSTEMATIC REVIEW

L. Govan, P. Langhorne, C. Weir, Stroke Unit Trialists Collaboration University of Glasgow, Glasgow, United Kingdom

Background: Systematic reviews have shown that organised inpatient (stroke unit) care reduces the risk of death after stroke but it is uncertain how this is achieved. We tested whether stroke unit care could reduce deaths by preventing complications.

Methods: We updated the Stroke Unit Trialists Collaboration systematic review that currently contains 31 controlled clinical trials (6936 participants) to include the reported interventions and complications during early hospital care plus the certified cause of death during follow up. Complications were grouped as; a) neurological, b) cardiovascular, c) complications of immobility and d) others.

Results: Organised (stroke unit) care reduced case fatality during scheduled follow up (odds ratio $0.82 ; 95 \%$ confidence interval $0.73-0.92 ; \mathrm{p}=0.0009$ ).

Reported interventions: Data were available for 7 studies (1652 participants). Stroke unit care was associated with statistically significant $(\mathrm{P}<0.05)$ increases in the reported use of oxygen, antibiotics and paracetamol plus a reduced use of urinary catheterisation

Reported complications: Data were available for 8 studies (1824 participants). Stroke unit care was associated with statistically significant $(\mathrm{P}<0.05)$ reductions in complications of immobility (chest infections, other infections and pressure sores) but not any other group.

Certified cause of death: Data were available for 17 studies (3327 participants): Stroke unit care resulted in statistically significant $(\mathrm{P}<0.05)$ reductions in deaths certified as due to complications of immobility or cardiovascular but not for neurological or other causes.

Discussion: Organised inpatient (stroke unit) care appears to reduce the risk of death after stroke through the prevention and treatment of complications, particularly infections.

\section{Acute stroke: early management and stroke units}

\section{PROTOCOL ADHERENCE AND SAFETY OF INTRAVENOUS} THROMBOLYSIS AFTER TELEPHONE CONSULTATION WITH STROKE

\section{CENTER}

K. Uchino, L. Massaro, M. Hammer

University of Pittsburgh, UPMC Stroke Institute, Pittsburgh, PA, USA

Background: Intravenous tissue plasminogen activator (TPA) for acute ischemic stroke must be provided in appropriate setting. The best way to provide thrombolysis in small community hospitals remains uncertain.

Methods: From the registry of TPA treatments at a stroke center, randomly selected charts were reviewed retrospectively to determine the patient and treatment characteristics, protocol violation, and occurrence of symptomatic intracranial hemorrhage (sICH). The stroke center provides phone consultation for acute stroke to smaller hospitals and instructs thrombolysis before transferring patients.

Results: From January 2002 to October 2005, 124 patients were treated with TPA at referring hospitals before transfer to the university hospital (Treat and Transfer) and 157 patients were treated at the university hospital (University Treatment). sICH occurred in 3.5\% and rates were similar in the two groups. A subset of patient records was reviewed, 44 in Treat and Transfer group and 14 in University Treatment group. There were no significant differences in age or presenting NIH Stroke Scale, blood pressure, time from onset to CT, or time from onset to TPA treatment. But 25 protocol deviations occurred in 22 patients $(50 \%)$ among Treat and Transfer group and $7 \%$ among University Treatment group $(\mathrm{p}<0.01)$. No deviation was associated with sICH. The deviations were related to time to treatment $(\mathrm{n}=12)$, nonstandard TPA dosing $(n=9)$, medication coadminstration $(n=2)$ and 1 each related to seizure at onset, hypertension, and coagulation. Final diagnoses were cerebral ischemia in 91\% among Treat and Transfer and 93\% among University Treatment groups.

Discussion: For stroke thrombolysis among patients presenting in small community hospitals, protocol adherence can be improved. 
8 Acute stroke: early management and stroke units

TENECI: A FRANCO-SWISS PROJECT FOR REMOTE DIAGNOSIS OF STROKE AND EMERGENCY NEUROLOGY

G. Devuyst, J.P. Thiran, E. Garcia, A. Carruzzo, J.C. Lapayre, V. Bonnans,

B. Piechowski-Jozwiak, H. Guyennet, J. Bogousslavsky, T. Moulin

CHUV Lausanne, Lausanne, Switzerland

Background: The majority of stroke patients is still managed by neurologists in regional hospitals or by other departments. Telemedicine allows rapid access to the expertise of specialists through electronic, visual and audio communications. Still, clinicians need access to user-friendly high performance tools for diagnosis and treatment. $\mathrm{TeNeCi}$ (Collaborative Tele-Neurology) is a telemedicine platform for emergency neurology care, esp. in stroke.

Methods: TENECI is a multimedia cooperative application with networks, distributed systems, data consistency and Human-Machine Interfaces (HMI). It is programmed in JAVA and runs on $2.5 \mathrm{GHz}$ work stations with 2 output video cards. It uses a $8 \mathrm{Mb} / \mathrm{s}$ high speed connection with optimum performance. The server contains: user profile, connection and mail servers and observational/coordination functions. It is compatible with DICOM (standard communication method between different imaging modalities) including RIS and PACS systems managing electronic medical images. Databanks and exchanges have developed expertise in acute neurological patient care, including stroke patients, using standard tests for diagnosis/treatment (NIHSS, ASPECTS etc).

Results: The asynchronous mode allows the creation of an archive (package) containing downloaded images, patient data, modifications made to text, image or audio files. It can be saved, sent to another user's personal TeNeCi mailbox or used for a databank. The synchronous mode involves real-time interactions between $\mathrm{TeNeCi}$ users. Groupware functionalities are: tele-pointer, annotations and comments to medical images and tele-settings (zoom, image rotation, ...). Finally, a time-saving feature "the observer" records the observations of all participants in the collective consultation and produces a summary.

Discussion: After previous tests between Switzerland and France, the project has been developed since the beginning of 2006 with the first patients included. Rapid development of high-speed networks across both countries will ensure the project's application on a broader scale.

\section{Acute stroke: early management and stroke units}

PATIENTS ARE SATISFIED WITH THE STROKE CARE BUT LESS SATISFIED WITH THE GIVEN INFORMATION ABOUT STROKE. RESULTS FROM THE SWEDISH NATIONAL QUALITY REGISTER FOR STROKE CARE

A.B. Stegmayr, for the Riks-Stroke Steering Committee

Department of Public Health and Clinical Medicine/Medicine, Sweden

Background: Little is known how patients receive information given by personnel about stroke and how good are we to inform in an understandable way?

Methods: Hospitalized stroke patients have been included in "Riks-Stroke", the Swedish national quality register for stroke care. Riks-Stroke has been ongoing for more than 10 years and with the opportunity to evaluate routine stroke care. Riks-Stroke is established in all hospitals in Sweden caring for stroke patients $(\mathrm{n}=80)$ and almost all stroke patients are treated in hospitals in Sweden. Annually around 24,000 stroke events are included into the data bas. This report is based on data collection from 2004 and includes 24,017 events. A 3-month follow-up was performed in 88 per cent of all patients

Results: The mean age in men was 73.4 years and in women 77.9 years. Seventyeight percent of all stroke patients were treated at a stroke unit. At the 3-month follow-up, almost all patients $(93 \%)$ declared they were satisfied or very satisfied with the stroke care as well as with the staff in general. The ranges between the hospitals were small. A much larger difference was seen between the hospitals in how well the patients were informed about the stroke disease, ranging from $100 \%$ satisfied patients to $70 \%$. In patients treated in stroke units vs. general ward significantly more patients were very satisfied with care in stroke units (58\% vs. 48), the personnel (65\% vs, 56), rehabilitation (39\% vs. 26) and the given information about the stroke disease (30\% vs. $20 \%$ ).

Conclusions: Almost all patients are very satisfied with stroke care in general. The result shows that, from the patients' point of view, experience of the care is very high and that stroke units seem to be more superior than care in general wards.
10 Acute stroke: early management and stroke units

THE INFLUENCE OF AGE AND SEX ON THE QUALITY OF STROKE CARE IN ENGLAND, WALES AND NORTHERN IRELAND. DATA FROM THE NATIONAL STROKE AUDIT

A.G. Rudd, A. Hoffman, C. Down, M. Pearson, D. Lowe

Royal College of Physicians, London, United Kingdom

There is little data available to indicate whether age or sex influence access to stroke care.

Methods: The 2004 audit covered the stroke pathway, from admission to transfer of care to the community (www.rcplondon.ac.uk). 45 standards were analysed, 3 related to accessing stroke unit care and 42 covering other aspects of care. A composite patient score from 0 to 100 was formed from these standards, a ratio of the number of standards that were met, to the number of standards applicable. The association of age and gender with composite score was assessed with linear regression analyses adapted to account for clustering of patient data within sites. The association with each categorical standard was also assessed using adapted binary regression methods.

Results: All hospitals submitted data, 246 sites, 8718 cases, median 40 per site. $46 \%$ (4018) were treated in a stroke unit during their stay with $40 \%$ (3508) spending most of their stay there. Stroke unit treatment was associated with age group but not with gender. The risk ratio comparing the oldest (85+ years) with the youngest patients ( $<65$ years) was $0.82(95 \% \mathrm{CI} 0.75-0.90)$ with $8.5 \%$ fewer patients $(95 \% \mathrm{CI}$ 4.7-12.3\%) being treated. Provision of early care assessments (within 48 hours) was worse for older patients, especially brain scanning. Overall $71 \%(809 / 3348)$ of patients under 65 were scanned compared to 51\% (557/1087) of those over 85 . Older patients had better provision for screening of swallowing disorders within 24 hours, and assessment by a physiotherapist within 72 hours but worse secondary prevention by discharge. They were less likely to have lipids measured and to have received dietary, smoking and alcohol advice.

Discussion: The data show that increasing age results in lower probability of high quality acute stroke care, including access to stroke units and urgent brain imaging. The reasons for this are likely to include ageism and lack of an evidence base for treating older patients.

\section{Acute stroke: early management and stroke units}

A PILOT STUDY OF ENHANCED EXTERNAL COUNTERPULSATION (ECP) FOR ISCHEMIC STROKE PATIENTS WITH LARGE ARTERY OCCLUSIVE DISEASE

J.H. Han, T. Leung, L. Wynnie, S. Yannie, W. Ka Sing

Chinese University of Hong Kong, Department of Medicine \& Therapeutics, Prince of Wales Hospital, Hong Kong, China

Background: ECP is a noninvasive treatment that increases perfusion to internal organs such as the heart. It is an established treatment for ischemic heart disease but the experience for cerebral ischemia is unknown. We aimed to explore the effect of ECP on cerebral blood flow and neurological recovery after stroke.

Methods: We did a randomized, crossover, outcome assessment-blinded clinical trial in patients with recent ischemic stroke and relevant large artery occlusive disease. Patients were randomized to either Early (ECP week 1-7 and no ECP week 8-14) or Late Group (no ECP week 1-7 and ECP week 8-14). ECP treatment consisted of 35 daily one-hour sessions ( 5 days a week). NIHSS and cerebral blood flow estimate using ulstrasound color imaging quantification (CVIQ) were done at baseline, end of week 7 and week 14 .

Results: 50 patients ( 32 men; mean age 68; mean time after symptoms 19.6 days: range 1-95) were recruited. At baseline, the mean NIHSS for Early and Late groups were 5.5 vs 4.3 and for CVIQ 675 vs 653 respectively. After 7 weeks, there were significant changes in NIHSS between the 2 groups (Early $3.4 \pm 3.3$ vs Late $1.9 \pm 2.3$ $\mathrm{p}=0.042$ ) and a trend toward better CVIQ perfusion (Early $48.6 \pm 146$ vs Late $13.9 \pm 110 \mathrm{~mL} / \mathrm{min} ; \mathrm{p}=0.36$ ). There were no difference for both outcomes between week 7 and 14. Effect for a 7-week course ECP adjusted for treatment sequence showed a change of -2 on NIHSS vs 1.2 for no ECP ( $\mathrm{p}=0.09$ ); and for CVIQ 27.5 $\mathrm{mL} / \mathrm{min}$ vs 21.2 for no ECP ( $\mathrm{p}=0.07$ ). No major side effects was detected. Recurrent stroke developed in $1(4 \%)$ in Early group and in $4(16 \%)$ in Late group $(p=0.35)$. Conclusions: Early ECP, but not late ECP, appears to enhance neurological recovery and tends to increase cerebral blood flow in stroke patients with large artery disease.

Acute stroke: early management and stroke

units 
12 Acute stroke: early management and stroke units

MANAGEMENT AND STROKE RISK AFTER TIA IN PATIENTS ADMITTED IN A STROKE UNIT

D. Calvet, C. Lamy, C. Oppenheim, E. Touzé, J.-F. Meder, J.L. Mas

Hôpital Sainte-Anne, France

Recent studies have reported 7-day risks of stroke of up to $10 \%$ after transient ischemic attack (TIA). The purpose of this study was to assess management and stroke risk after TIA in patients admitted in a stroke unit.

Methods: Consecutive patients with a recent ( $<48 \mathrm{~h})$ TIA (NINDS criteria), admitted in our stroke unit from January 2003 to November 2005, were included. The occurrence of ischemic stroke and recurrence of TIA were assessed at 7 days and 3 months.

Results: Two hundred and three patients (mean age $=61.2$ years $\pm 15.7 ; 61 \%$ male) were included. The median delay between onset of symptom and admission was 12.2 hours. The median duration of TIA was $30 \mathrm{~min}$. On admission, all patients had standard blood tests, ECG and MRI (DWI abnormalities in 32\%). Arterial explorations were performed within 48 hours in $82 \%$ of patients. All patients had echocardiography. The initial treament was strated within 24 hours in $88 \%$ of patients and consisted of antiplatelet drugs in $86 \%$ of patients and anticoagulation in $14 \%$. This treament was modified in $31 \%$ of patients, according to the results of etiological work-up, including revascularization in $4 \%$, anticoagulation in $15 \%$, antiplatelet therapy modification in $10 \%$, other in $2 \%$. The incidence of stroke was $2.5 \%(0.34-4.66)$ at 7 days and $3.5 \%(0.97-6.11)$ at 3 months. In univariate analysis, DWI abnormalities, a prior stroke or TIA, a definite cause and TIA lasting more than 30 min were significantly associated with an increased risk of stroke. ABCD score was predicitve of stroke at 7 days and 3 months.

Early management in a stroke unit care leads to specific medical and interventional treaments in about $40 \%$ of cases. Compared to recent population based-studies, TIA patients admitted in stroke unit seem to have a lower risk of stroke.

\section{Brain imaging}

\section{Brain imaging}

IMPACT OF THE SPEED OF TPA-INDUCED CLOT DISSOLUTION ON DWI LESION EVOLUTION AND CLINICAL OUTCOME

O. Maisterra, R. Delgado-Mederos, M. Ribo, M. Rubiera, A. Rovira, E. Grivé, J. Alvarez-Sabín, C.A. Molina

Hospital Vall d'Hebron, Barcelona, Spain

To evaluate the impact of the speed of clot lysis during continuous TCD monitoring on the evolution of DWI lesion and outcome in stroke patients treated with tPA We studied 113 stroke patients due to MCA occlusion treated with iv tPA. All patients underwent MRI studies including DWI, PWI and MRA before and at 36-48h of tPA bolus. Patients were continuously monitored with TCD during the first $2 \mathrm{~h}$ after tPA bolus. Patterns of recanalization (RE) on TCD were defined according to the time to the maximum completeness of RE and categorized in sudden $(<1 \mathrm{~min})$ stepwise (1-29min) and slow (>30 min) RE. DWI lesion change ( $\triangle$ DWI: final-initial DWI), final DWI lesion, 24h-clinical improvement and 3-moth outcome were assessed in relation to the speed of tPA-induced clot lysis Mean initial DWI and PWI were 42.9 cc and $188 \mathrm{cc}$. RE on TCD started at $21 \pm 16 \mathrm{~min}$ and ended at $52 \pm 33 \mathrm{~min}$ of tPA bolus. $13(15 \%)$ patients recanalized suddenly, 32(30\%) in a stepwise and $18(17 \%)$ in a slow manner. Baseline NIHSS score occlusion site, time-to-treatment, extent of initial DWI and PWI defects were similar among RE subgroups. At 36-48h, $\Delta$ DWI was $37.2 \pm 48 \mathrm{cc}$. $\Delta$ DWI and final DWI volumes were lower $(\mathrm{p}<0.001)$ after sudden $(3.23 \pm 10.1$ and $26.4 \pm 15 \mathrm{cc})$ compared to stepwise $(24 \pm 37$ and $44.6 \pm 47 \mathrm{cc})$, slow $(44.3 \pm 68$ and $66 \pm 73 \mathrm{cc})$ and no $(51.7 \pm 34$ and $89.4 \pm 56 \mathrm{cc})$ RE. A correlation $(\mathrm{r}=0.52, \mathrm{p}<0.001)$ was found between time stroke onset-complete RE and degree of DWI lesion growth. Among patients who recanalized, slow pattern was associated with greater DWI growth $(\mathrm{p}=0.003)$, lesser degree of clinical improvement $(\mathrm{p}=0.021)$, worse 3 -month outcome $(\mathrm{p}=0.032)$ and higher mortality $(\mathrm{p}=0.003) .92 \%$, $59 \%$ and $12 \%$ of patients who showed sudden, stepwise and slow RE became independent at 3 months The speed of tPA-induced clot lysis predicts DWI lesion evolution and outcome. Unlike sudden and stepwise patterns, slow RE is associated with greater DWI lesion growth and poorer short and long-term outcome.

\section{Brain imaging}

DO CLINICAL RISK STRATIFICATION TOOLS IDENTIFY TIA PATIENTS WITH ISCHEMIC LESIONS ON DIFFUSION WEIGHTED IMAGING?

V. Thijs

University Hospitals Leuven, Leuven, Belgium

Background: Clinical risk stratification tools have been proposed to identify patients at higher risk of stroke after TIA. TIA patients with ischemic lesions on DWI appear to have a higher risk of early recurrent stroke.

We evaluated whether clinical statistical tools identified patients with ischemic lesions on diffusion weighted imaging (DWI).

Methods: Consecutive patients with TIA underwent DWI within 7 days after symptom onset. The California and Oxfordshire ABCD clinical risk scores were measured. The California model is a 5 point risk score that identifies patients with a higher 3 month stroke risk. The Oxfordshire ABCD model is a 6 point risk score that is highly predictive of stroke within 7 days.

Results: DWI was performed within a median of 3 days after TIA (interquartile range [IQR] 2-5)

Ischemic lesions were identified on DWI in 38 of 120 patients $(31.7 \%)$. There was a high correlation between the California and the ABCD risk score (correlation coefficient $0.72, \mathrm{p}<0.001$ ). The risk of finding a DWI hyperintensity was slightly higher with increasing scores on the ABCD model (test for trend, $\mathrm{p}=0.057$ ) and the California model (test for trend, $\mathrm{p}=0.031$ ). Still, a large proportion of patients with elevated risk scores did not have ischemic lesions on DWI. For instance, 17 of 29 patients (59\%) with the highest ABCD risk scores and 38 of 66 patients (58\%) with the highest California risk scores did not have ischemic lesions on DWI.

Conclusion: Clinical TIA stratification tools do not accurately identify patients with ischemic lesions on DWI. Further studies are needed whether the predictive ability of clinical risk scores can be improved by adding information derived from brain imaging.

\section{Brain imaging}

GLUCOSE AND TEMPERATURE: MAJOR DETERMINANTS OF DWI/PWI MISMATCH EXTENSION IN HIPERACUTE STROKE

M. Ribo, C. Molina, A. Rovira, M. Rubiera, E. Grivé, M. Rubiera, J. Munuera, R. Delgado-Mederos, J. Alvarez-Sabín

Unitat Neurovascular Vall d'Hebron, Barcelona, Spain

Background: In the following hours after stroke onset ischemic lesion progressively grows over the hypoperfused brain, however the recruitment speed of penumbra into infarct is very variable. We aim to determine the impact of clinical variables on the DWI growth velocity and the extend of DWI/PWI missmatch.

Method: A total of 234 acute stroke patients underwent emergent multiparametric MRI and MRI $<6 \mathrm{~h}$. Diffusion (DWI) and perfusion (PWI) volumes, and percentage of DWI/PWI mismatch ((PW-DW)/PW) were calculated. Time from onset to MRI (TFO) and admission clinical variables were prospectively recorded.

Results: 173 patients (mean age 71 years) presented an MCA occlusion on MRA and were included in the study $(50 \% \mathrm{M} 1,22 \% \mathrm{M} 2,28 \% \mathrm{MCA}+\mathrm{ACA}$ occlusion). Mean TFO was 163 minutes. MRI parameters were: DWI: $45 \pm 62 \mathrm{cc}$, PWI $195 \pm 86$ and mismatch $78 \pm 27 \%$. Twenty-two patients $(12,7 \%)$ had a mismatch $<50 \%$. Mean admission glycemia was $143 \mathrm{mg} / \mathrm{dl}$. The correlation between TFO and mismatch did not reach statistic significance $(r=-0,15 ; \mathrm{p}=0,06)$, the same happened for the clinical variables temperature $(\mathrm{r}=-0,18 ; \mathrm{p}=0,6)$ and glucose $(\mathrm{r}=-0,12 ; \mathrm{p}=0,1)$. However, the combination TFO $\mathrm{x}$ Glucose $\mathrm{x}$ Temperature (TGT) showed a good negative correlation with the percentage of mismatch $(r=-0,24 ; \mathrm{p}=0,005)$. Linear regression analysis demonstrated that TGT independently predicted mismatch (O.R. 88, 95\% CI 80-94; $\mathrm{p}=0.005$ )

Conclusions: Recruitment of penumbra into infarct depends on time but also on glycemia and temperature. Measures to lower these clinical variables may slow infarct growth.

\section{Brain imaging}

\section{CORRELATING EARLY ISCHEMIC CHANGE ON NON-CONTRAST CT WITH ACUTE PERFUSION CT: IS EARLY ISCHEMIC CHANGE ALWAYS IRREVERSIBLE?}

M.W. Parsons, E.M. Pepper, G.A. Bateman, C.R. Levi

John Hunter Hospital, University of Newcastle, Newcastle, NSW, Australia

Background: Early ischemic change (EIC) on non-contrast CT (NCCT) consists of parenchymal hypoattenuation (PH) and focal swelling. It is unclear whether isolated FS (IFS) has the same prognostic significance as $\mathrm{PH}$. 
Methods: Forty sub-6 hour stroke patients were studied with acute NCCT/perfusion CT (CTP), and day 3 MRI. On NCCT, the presence and type of EIC was recorded for Alberta Stroke Program Early CT Score (ASPECTS) cortical regions (M1-M6), Cerebral blood volume (CBV), and cerebral blood flow (CBF) were calculated for the same cortical ASPECTS regions, and from contralateral cortical regions, from co-registered acute CTP maps. Presence of infarction in cortical ASPECTS regions was recorded from follow-up MRI.

Results: There were 123 normal regions, 59 with PH, and 21 with IFS. A further 37 regions were excluded as they were not hypoperfused on acute CTP. For normal ASPECTS regions, acute $\mathrm{CBV}$ was not significantly different to contralateral $\mathrm{CBV}$, whereas $\mathrm{PH}$ regions had lower $\mathrm{CBV}$, and IFS regions had higher CBV than contralateral regions. However, acute CBF was similarly reduced (compared to contralateral regions) in both normal and IFS regions, with an even greater reduction in CBF seen in $\mathrm{PH}$ regions.

Virtually all PH regions progressed to infarction (98\%), compared to 53\% of normal regions, and $29 \%$ of IFS regions. When restricted to the 23 patients with major reperfusion by day 3 , progression to infarction occurred in $100 \%$ of $\mathrm{PH}$ regions, $44 \%$ of normal regions, and only $7 \%$ of IFS regions.

Discussion: Despite significant hypoperfusion on CTP, the majority of regions appeared normal on NCCT. Regions with PH had lower CBV and lower CBF than hypoperfused, normal NCCT regions, whilst those with IFS had elevated CBV. IFS identifies penumbral tissue, and has a different prognostic significance to $\mathrm{PH}$.

\section{Brain imaging}

\section{PERFUSION CT PROVIDES HINTS AGAINST PERIHEMORRHAGIC ISCHEMIA IN ACUTE CEREBRAL HEMORRHAGE}

C. Herweh, E. Jüttler, P.D. Schellinger, K. Sartor, W. Hacke, P. Schramm

Heidelberg Medical School, Heidelberg, Germany

Background: We sought to prove whether there is secondary tissue damage due to ischemia surrounding acute intracerebral hemorrhage (ICH). This question has been addressed by several groups applying different techniques in the past, but the results remain inconsistent so far.

Materials \& Methods: 20 patients suffering from acute ICH who were amenable to CT scanning within 3 hours from symptom onset were investigated with a perfusion CT (PCT) protocol. We employed a single section PCT protocol with the image level located at the largest extent of the hematoma. 40 images were acquired at that level continuously during contrast agent was administered. Parameter maps of cerebral blood flow (CBF), cerebral blood volume (CBV) and time-to-peak (TTP were calculated using a commercial software (Siemens). Regions of interest (ROI) were drawn manually on the CBF maps to encompass areas of reduced CBF but exclude the hematoma and were automatically transferred to the CBV and TTP maps. Perfusion parameters were also assessed in the contralateral hemisphere and relative values were calculated for the perihemorrhagic tissue. These values were compared with the literature.

Results: Relative values for CBF were $0,5 \pm 0,13$, for CBV $0,6 \pm 0,12$ and for TTP $1,6 \pm 1,51$. None of these values were within the range reported for acute ischemia. Discussion: Compared to perfusion weighted MRI or positron emission tomography, PCT has a higher spatial resolution and is free of image distortions. PCT is thus suitable to study perfusion changes occurring within a small rim surrounding ICH As we found significantly higher values for $\mathrm{rCBF}$ and $\mathrm{rCBV}$ and smaller values for rTTP than those reported for ischemic tissue, we conclude that our results suggest absence of ischemic tissue damage around ICH.

\section{Brain imaging}

ASSESSING SELECTIVE NEURONAL LOSS (SNL) IN THE REPERFUSED PENUMBRA USING 11C-FLUMAZENIL (FMZ): A CLINICAL PET STUDY J.V. Guadagno, P.S. Jones, F.I. Aigbirhio, D. Wang, T.D. Fryer, O. Golovko, D.J. Day, E.A. Warburton, J.C. Baron

University of Cambridge, Cambridge, United Kingdom

Background: SNL in the salvaged penumbra could account for suboptimal clinical recovery despite effective reperfusion. Previous studies were limited by use of SPECT, visually-defined ROIs and lack of partial volume effect correction (PVEc).

Methods: We prospectively recruited 6 patients $(3 \mathrm{M}, 43-75 \mathrm{yr} ; 4$ had T-Pa) with evidence of acute $(<6 \mathrm{~h})$ first ever extensive MCA stroke documented by CT Perfusion and NIHSS (8-23). All had good early improvement (NIHSS change $>6$ ) and good outcome (final NIHSS reduct. 6 - 20). 5/6 had small subcortical final infarcts. MR and FMZ PET were obtained 20-182 days post-stroke. 10 age-matched controls (7F, 48-71yr) were also studied. FMZ BP maps were produced. SPM was used to detect regions of sig. BP reduction in patients. Following spatial normalisation, a gray matter mask was applied, global normalization to the unaffected hemisphere was carried out, and individual infarcts masked out. Small volume correction was applied using a custom made MCA ROI. Based on permutation analysis on the controls, a $\mathrm{p}<0.001$ uncorrected cut-off was selected for the individual analysis (patient vs all controls). For the group analysis, the $\mathrm{p}<0.05$ FDR-corrected cut off was considered significant. Voxel-based PVEc was also applied to address potential confound of post-infarct atrophy.

Results: Individually, $6 / 6$ pts showed sig. clusters of reduced BP in the affected MCA ROI, extensive in 4/6. The group analysis revealed two large clusters of sig. reduced BP in the affected MCA ROI (peri-insular and prefrontal cortices), but none on the unaffected side. PVEc did not alter the results.

Conclusion: This is the first study to document loss of FMZ binding in the acutely ischaemic but ultimately non-infarcted cortical MCA territory, supporting the notion that reperfused penumbra can be affected by SNL. That our patients made good clinical recovery suggests effective plasticity compensated for SNL. Whether SNL affects outcome needs to be addressed.

\section{Brain imaging}

PREDICTION OF PENUMBRAL FLOW BY TIME TO PEAK MAPS WITH RESPECT TO VESSEL PATHOLOGY AND TIME POINT OF IMAGING: A PET/MR COMPARISON OF 41 CASES

J. Sobesky, O. Zaro Weber, M. Frackowiak, C. Dohmen, F.G. Lehnhardt,

M. Neveling, W. Moeller Hartmann, A.H. Jacobs, W.D. Heiss

Max-Planck-Institute for Neurologic Research, Cologne, Germany

Background: Perfusion weighted magnet resonance imaging (PW-MRI) is the clinical method of choice in acute ischemic stroke. In a large number of patients we describe the clinical utility of time-to-peak (TTP) maps in comparison with cerebral blood flow (CBF) measurements by positron emission tomography (PET) in acute and subacute stroke patients.

Methods: In 29 patients, 41 scans with paralleled DW/PW-MRI and PET imaging were performed (median 50 minutes between MRI and PET). Using 15O-wate PET, penumbral hypoperfusion was defined by CBF values below $20 \mathrm{ml} / 100 \mathrm{~g} / \mathrm{min}$. For PW imaging, the TTP maps were thresholded by a TTP prolongation of $>4$ sec compared to the unaffected hemisphere. The volumes obtained by PET and PW images were compared and a difference of $>10 \%$ was considered significant. Vessel pathology and time to imaging were included in the analysis.

Results: In the 41 measurements, the volume of penumbral flow $(<20 \mathrm{ml} / 100 \mathrm{~g} / \mathrm{min})$ was well represented in 39\%, overestimated in $41 \%$ and underestimated in $20 \%$ of the scans by TTP $>4 \mathrm{sec}$. Regarding the individual patients, overestimation of penumbral flow ranged from 10 to $90 \%$ (median 50\%), underestimation ranged from 15 to $70 \%$ (median 20\%). The highest TTP prolongation always pointed at the highest degree of ischemia. Vascular pathology ( $\mathrm{n}=15$ ipsilateral stenosis or occlusion of the internal carotid, $n=20$ of the medial cerebral artery), or time from stroke to imaging ( $0-6$ hours: $n=20,6-24$ hours: $n=12,1-14$ days: $n=7$ ) were not predictive for the degree of congruence.

Discussion: Time-to-peak (TTP) maps are easy to generate but are a very weak surrogate of the cerebral blood flow (CBF). However, in this large patient sample, the TTP threshold of $>4 \mathrm{sec}$ provided a clear localisation the ischemic core and a good estimate of the volume of penumbra. These findings were independent from vessel pathology or time point of the imaging.

\section{Brain imaging}

PERSISTING HYPERDENSE ARTERY SIGN, ASPECTS SCORE AND RISK OF DEVELOPING MASS EFFECT: AN ANALYSIS BASED ON THE FIRST 389 PATIENTS FROM THE IST-3 TRIAL

M. Skowrońska, J. Bembenek, P. Sandercock, I. Kane, A. Kobayashi,

A. Czlonkowska, S. Lewis, J. Wardlaw

Medical University of Warsaw, Warsaw, Poland

The hyperdense artery sign (HAS) is associated with acute major thrombotic arterial occlusion in acute ischaemic stroke. It is also a predictor of poor outcome. The HAS can disappear in case of reperfusion, or may persist if the artery remains occluded. The International Stroke Trial (IST-3) is an international, multicentre, randomised trial assessing the efficacy of i.v. alteplase in ischaemic stroke administered within 6 hours of onset. One of the principles of the trial is to assess the influence of early ischaemic changes on CT on the efficacy of thrombolysis. In this analysis we have included the first 389 randomised patients. CT scanning is the main imaging modality but MR scanning is permitted. The present analyses were restricted to patients with pre-randomisation CT. A follow up CT was performed 24-48 hours after randomisation. The $\mathrm{CT}$ scans were read centrally by a neuroradiologist blinded to all patient details according to a structured, validated coding system. We have 
assessed the frequency of HAS on initial and follow up CT in association with other ischaemic changes.

$152 / 389(39.1 \%)$ of patients had HAS on initial CT. In 88/152 (57.9\%) it was still visible on follow-up CT. There were no significant difference between patients with and without persisting HAS in the presence of ischaemic lesions, such as loss of grey/white matter cortex definition or hypodensity on follow-up scan. However, patients with persisting HAS more frequently had ventricular effacement and a significantly lower ASPECTS score on follow-up scan compared to patients in whom it has disappeared, mean 3.4 and 5.0 respectively $(\mathrm{p}<0.05)$.

Patients in IST-3 with a HAS which persisted on follow-up scan were more likely to develop mass effect and a lower ASPECTS score, than those in whom the HAS disappeared.

\section{Brain imaging}

\section{INTER-OBSERVER VARIABILITY IN THE DIFFERENTIATION BETWEEN}

\section{PICH AND HAEMORRHAGIC TRANSFORMATION OF INFARCTION (HTI)}

C.E. Lovelock, A.J. Coull, P. Anslow, J.V. Byrne, W. Kuker, A.J. Molyneux,

P.M. Pretorius, P.M. Rothwell

Stroke Prevention Research Unit, University of Oxford, Oxford, United

Kingdom

Background: The clinical management of primary intracerebral haemorrhage (PICH) and ischaemic stroke differ significantly. CT remains the gold standard for diagnosis of acute PICH, but inter-observer variability in distinguishing HTI from PICH has not been studied.

Method: We studied all CT scans showing any acute intra-parenchymal haemorrhage in the Oxford Vascular Study (prospective population-based study of all acute cerebrovascular events) from April 2002 to March 2005. Five neuroradiologists independently reviewed the scans after hearing the clinical history, and diagnosed either PICH or HTI

Results: Among 586 strokes, neuroimaging or post-mortem data were available in $557(95 \%)$. Acute intra-parenchymal haemorrhage was identified on 58 scans. The overall agreement between the five neuroradiologists for PICH vs HTI was only moderate (kappa $=0.59,0.4-0.79$ ) and the proportion of haemorrhages diagnosed as being due to HTI varied from $14 \%$ to $40 \%$. In 8 of the 18 cases in which there was some disagreement over the radiological diagnosis, the decision was relevant to clinical management.

Conclusion: Differentiation between HTI and PICH on CT scan in acute stroke has only moderate reproducibility. The variation in proportions of haemorrhages diagnosed as being due to HTI has implications for comparison of reported incidences of PICH in CT based epidemiological studies.

\section{Brain imaging}

DIAGNOSIS OF ACUTE ISCHEMIC VS. HEMORRHAGIC STROKE WITH TRANSCRANIAL ULTRASOUND IMAGING - A PROSPECTIVE MULTI-CENTER STUDY

R. Kern, C. Krogias, K. Meyer-Wiethe, G. Renault, M. Kablau, F. Sallustio, J. Eyding, S. Meves, G. Seidel, S. Meairs

Universitätsklinikum Mannheim, University of Heidelberg, Mannheim,

Germany

Objective: A prospective multi-center study was performed to determine sensitivity, specificity, positive (PPV) and negative predictive value (NPV) of transcranial ultrasound (US) for the diagnosis of acute ischemic vs. hemorrhagic stroke within 24 hours from onset.

Methods: 33 acute stroke patients ( 10 w., 23 m., mean age 68 y.) were included in three German stroke centers after abrupt onset (mean $13 \mathrm{~h}$.) of a focal neurological deficit suggestive of supratentorial stroke. US imaging studies were performed by an expert investigator unaware of neuroimaging findings, and according to a standard protocol: 1) transcranial color-coded duplex US (TCCD), 2) unenhanced US, 3) brain perfusion imaging using the echocontrast agent SonoVue, if no hemorrhage was detected on unenhanced US, 4) parametric post-processing of brain perfusion data sets including the FAMIS analysis (Factor Analysis of Medical Image Sequences). All patients underwent a baseline CT. In $78 \%$ of patients with ischemic stroke, MRI including diffusion-weighted imaging, MR perfusion imaging and MR angiography was obtained within 3 hours from US investigation.

Results: Stroke (mean NIHSS $=11$ ) was classified as ischemic in 23 patients $(60 \%)$, 10 had hemorrhagic stroke. Ischemic stroke was classified correctly in 22/23 patients (sensitivity $95.7 \%$, specificity $90 \%$, PPV $95.7 \%$, NPV 90\%). Hemorrhagic stroke was diagnosed with a sensitivity of $80 \%$ (specificity $100 \%$, PPV $100 \%$, NPV $92 \%$ ). US perfusion imaging detected hemodynamic impairment in patients showing a perfusion deficit on MRI with a sensitivity $80 \%$, a specificity $100 \%$ and a PPV $100 \%$, whereas NPV was low (50\%).

Conclusions: This prospective study is the first to provide information on the diagnostic validity of transcranial US including brain perfusion imaging performed by a blinded investigator in acute ischemic vs. hemorrhagic stroke. The high sensitivity and specificity of this method indicates that it may help in selecting patients for thrombolysis.

\section{Brain imaging}

\section{MEASUREMENT OF THE ACUTE MR PERFUSION LESION BY VARIOUS} METHODS AND CORRELATIONS WITH CLINICAL SCORES AND RADIOLOGICAL OUTCOME

I. Kane, T. Carpenter, C.S. Rivers, J. Wardlaw

University of Edinburgh, Edinburgh, United Kingdom

Background: Several methods are available to measure the acute MR perfusion (PWI) lesion with no consensus over which PWI measurement is best.

Methods: In 32 prospectively included acute stroke patients, with no prior history of stroke, imaged at median $7.63 \mathrm{hrs}$, we calculated the acute PWI lesions using 10 different methods (with AIF, with and without deconvolution, i.e. some relative, some quantitative, some assessing CBF, MTT and CBV). We compared PWI lesions to baseline NIHSS, outcome mRS ( 3 months) and outcome T2 ( $\geq 1$ month), and PWI/DWI mismatch (for all 10 PWI methods) with infarct expansion (DWI to $>$ 1month T2) using Spearman's rho.

Results: 2 patients had unusable data (movement artefact) and 3 had no PWI lesion identifiable by any method. In the remaining 27 patients, peak time fitted (PTF), full width half maximum (FWHM) and first moment (rMTT) correlated with baseline NIHSS and 3 month mRS (Spearmans, $\mathrm{p}<0.01$ ). Relative cerebral blood flow, quantitative CBF, cerebral blood volume and arrival time fitted (ATF) correlated with final T2 lesion (Spearmans, $\mathrm{p}<0.05$ ). PWI/DWI mismatch was not associated with infarct expansion (Fishers exact test). The proportion with mismatch ranged from $11 \%$ to $85 \%$ depending on which of the 10 PWI methods were used.

Conclusions: The PWI measurements which correlate with baseline and outcome clinical scores differ from those that correlate with the final infarct. PTF, FWHM and rMTT all measure mean transit time and do not require deconvolution, making them more practical for use in the acute situation. An association with clinical outcome may be more useful than one with an imaging surrogate

\section{Brain imaging}

PRESENCE OF ACUTE ISCHAEMIC LESIONS ON DIFFUSION-WEIGHTED IMAGING (DWI) IS ASSOCIATED WITH SIMPLE CLINICAL PREDICTORS OF EARLY STROKE RISK AFTER TIA

J. Redgrave, U. Schulz, D. Briley, T. Meagher, P. Rothwell

University of Oxford, Oxford, United Kingdom

Background: High early risk of stroke after a TIA can be reliably predicted with risk scores based on clinical features of the patient and event and also by the presence of an acute ischaemic lesion on DWI. However, it is unclear to what extent the presence of a lesion on DWI is associated with clinical features and risk score.

Methods: We related the presence of recent lesions on DWI to the clinical features associated with high stroke risk and to two validated risk scores (ABCD \& California score) in 200 consecutive patients with TIA attending a specialist clinic and in a meta-analysis of data from similar previous studies.

Results: 31 (16\%) patients had acute ischaemic lesions on DWI. Both risk scores were positively correlated with positive DWI (Ptrend $=0.017$ for both), independent of time interval between last symptoms and scan. In a meta-analysis with 14 smaller studies, the characteristics of the TIA, including symptom duration ${ }^{3} 60$ mins (10 studies, $\mathrm{OR}=1.78,1.32-2.42, \mathrm{P}<0.001)$, dysphasia ( 8 studies, $\mathrm{OR}=2.30,1.58$ $3.34, \mathrm{P}<0.001$ ) and motor weakness ( 8 studies, $\mathrm{OR}=2.17,1.51-3.10, \mathrm{P}<0.001$ ), were associated with lesions on DWI, but patient characteristics including age, sex, hypertension and diabetes were not. From an aetiological perspective, atrial fibrillation was associated with positive DWI ( 5 studies, $\mathrm{OR}=4.56,2.55-8.16, \mathrm{P}<0.001)$ and there was a trend for ipsilateral carotid stenosis ( 5 studies, $\mathrm{OR}=1.58,0.94-2.66, \mathrm{P}=0.13$ ). Conclusion: Presence of recent ischaemic lesions on DWI is associated with the clinical characteristics of TIAs that are known to predict early recurrent stroke. Any independent predictive value of DWI, and hence the need for inclusion of DWI findings in clinical risk scores, remains to be determined. 
STUDIED ON CONDUCTION APHASIA WITH TRACKING NEURONAL FIBERS TECHNOLOGY

Y. Yu-mei Zhang, Yong-jun Wang, Xi-ping Gong, Xing-quan Zhao

Tiantan hospital, affiliated with Capital University of Medical Sciences, China

Background: Anisotropic water diffusion in neural fibres such as nerve, white matter in spinal cord, or white matter in brain forms the basis for the utilization of diffusion tensor imaging to track fibre pathways. The fact that water diffusion is sensitive to the underlying tissue microstructure provides a unique method of assessing the orientation and integrity of these neural fibres, which may be useful in assessing a number of neurological disorders.

Objectives: We used tracking neuronal fibers technology to study conduction aphasia cases in order to explain the clinical characteristics of language disorders of conduction aphasia.

Methods: We used Aphasia Battery of China to evaluate five conduction aphasia cases oral fluency and used tracking neuronal fibers technology to analysis the fibers of Broca's area, Wernicke's area and arcuate fasciculus.

Results: Of five conduction aphasia cases, there were one non-fluent case and the rest cases were fluent oral spoken. The tracking neuronal fibers technology showed that arcuate fasciculus of all cases were damaged at different level, and the lesion of the patient who had non-fluent oral language were located near to Broca's area while the lesion of the patients who had fluent oral language were located near to Wernicke's area and the disturb of understanding were serious. On the other hand, Broca's and Wernicke's areas of some cases were not damaged.

Conclusions: Tacking neuronal fibers technology gives us an explanation for the clinical observation that conduction aphasias fall into three distinct groups: the Broca-like syndrome in which the deficit in repetition is accompanied by a relative impairment in fluency, the Wernicke-like syndrome in which the deficit in repetition is accompanied by a relative impairment in comprehension and the classical conduction aphasia.

\section{Brain imaging}

EVIDENCE OF ACUTE ISCHEMIA OF VARYING AGES PREDICTS HIGHER RISK OF SUBSEQUENT ISCHEMIC EVENTS IN STROKE/TIA PATIENTS P.N. Sylaja, S. Subramaniam, S.B. Coutts, M. Eliasziw, A.M. Demchuk Calgary Stroke Program, Clinical Neurosciences, Calgary, Alberta, Canada

Background and Purpose: Studies have shown that MR parameters can be useful in discriminating the age of cerebral infarcts. MRI findings at index event have demonstrated utility in predicting risk of future clinical and subclinical events. Specifically, multiple DWI lesions have been shown to predict risk of new events However the importance of lesion age has not been factored into this risk. Our goal was to evaluate whether the presence of ischemic lesions of varying ages in the baseline MRI predict a higher risk and to correlate with the stroke etiology.

Methods: Patients with acute stroke and TIA presenting within 12 hours of symptom onset who had a baseline MRI and one month follow-up MRI were enrolled in the study. Acute lesion was defined as DWI positive, ADC negative and subacute lesion defined as DWI positive, ADC normalized. The baseline MRI and the presence of new lesions in the followup MRI and the stroke etiology were analyzed.

Results: A total of 360 patients were prospectively enrolled and all had appropriate imaging. 145 were excluded since there were no DWI lesion and 81 excluded because they received rt-PA. 117 patients had DWI lesions of one age (acute or subacute) and 20 had evidence of lesions of varying ages (subacute and acute) on the baseline MRI. The new lesion rate on 30 day MRI was $18 \%$ when single acute; $0 \%$ when single subacute; $8 \%$ when multiple acute only, and $50 \%$ when multiple acute and subacute $(\mathrm{p}<0.001)$.Lesions of varying ages did not predict the pathogenetic stroke mechanism in these patients.

Conclusion: Attention to the age of DWI lesions on baseline MRI imaging can provide incite into the degree of risk for new events. Presence of both subacute and acute DWI lesions suggest very active disease over time and a portends a higher risk of future ischemic events.
CORRELATION OF STROKE SUBTYPE IN OCSP CLASSIFICATION AND EARLY ISCHAEMIC CHANGES ON CT: AN ANALYSIS BASED ON THE FIRST 389 PATIENTS FROM THE IST-3 TRIAL

A. Kobayashi, J. Bembenek, P. Sandercock, I. Kane, M. Skowrońska, A. Czlonkowska, S. Lewis, J. Wardlaw, the IST-3 Collaborative Group Medical University of Warsaw, Warsaw, Poland

The Oxfordshire Community Stroke Project (OCSP) classification is a simple method of assessing stroke subtype and lesion location, which in established stroke, has good interobserver reliability and is associated with changes in appropriate brain areas on CT. The clinical classification may be more difficult to apply in hyperacute stroke. The aim of this study is to assess the association of OCSP classification and early ischaemic changes on CT.

The International Stroke Trial (IST-3) is an international, multicenter, randomized trial assessing the efficacy of i.v. alteplase in ischaemic stroke administered within 6 hours of onset. In this analysis we have included the pre-randomization CT scans from the first 389 randomized patients. The patients were assessed according to OCSP classification and CT scans were assessed centrally according to a validated, structured classification of arterial territory by a blinded neuroradiologist, looking for early ischaemic changes (loss of grey/white matter cortex definition, loss of basal ganglia outline, mass effect, hyperdense artery sign)

The proportion of patients with each clinical subtype was: TACI $(50 \%)$, PACI $(35 \%)$, LACI $(11 \%)$, POCI $(4 \%)$. The clinical syndrome of TACI was associated with the highest frequency of early ischaemic change, mass effect, hyperdense artery sign and lowest mean ASPECTS score (TACI 5.0, non TACI 6.5). The correlation between lesion size and location according to a structured and validated coding scheme and the OCSP classification was statistically significant.

The results show that an OCSP clinical classification of TACI is associated with the likelihood of early ischaemic changes on CT, even within the first 6 hours.

\section{6}

\section{Brain imaging}

\section{LACTATE VALUES IN THE ACUTE STROKE DIFFUSION LESION VARY} WITH PERFUSION LEVELS

V. Cvoro, J.M. Wardlaw, K. Wartolowska, A.J. Farrall, I. Marshall, P.A. Armitage, C.S. Rivers, M.E. Bastin, T.K. Carpenter, M.S. Dennis University of Edinburgh, Edinburgh, United Kingdom

Background: Normal cerebral metabolites (N-Acetyl aspartate, NAA) are reduced and abnormal metabolites (lactate) increased in acute ischaemic stroke in the diffusion lesion. The relationship between metabolite concentration and different levels of perfusion, the presence of DWI/PWI mismatch or subsequent lesion growth is unknown. We examined these parameters and metabolite concentrations.

Methods: Patients with acute ischaemic stroke underwent diffusion tensor imaging (DTI), dynamic susceptibility contrast PWI, T2W and MR spectroscopic imaging (SI) within max. 24 hours of stroke, at days 5 and 14, and 1 and 3 months. Regions-of-interest were outlined on DTI images and metabolites extracted from the DTI region. A blinded assessor coded images for relative cerebral blood flow (rCBF, normal or reduced), mean transit time (rMTT, normal or prolonged), PWI/DWI mismatch (present or absent) and lesion growth (DTI to final T2W image, growth or no growth).

Results: 42 patients, median age 76 yrs (range 37-95) and NIHSS 9 (range 0-29) were scanned with a median of 7.8 hours (range 1.36-24) after stroke. Patients with more severe strokes (higher NIHSS) had reduced $\mathrm{rCBF}(\mathrm{p}=0.01)$, prolonged $\mathrm{rMTT}$ $(\mathrm{p}=0.01)$, and PWI/DWI mismatch $(\mathrm{p}=0.0002)$. Lactate was present in all diffusion lesions, but was higher in the lesions with reduced $\operatorname{rCBF}(\mathrm{p}=0.074)$. NAA did not differ with rCBF. Neither lactate nor NAA levels were associated with MTT levels, DTI/PWI mismatch or lesion growth.

Conclusion: Higher lactate values (implying worse ischaemia) may be associated with more abnormal CBF in acute stroke, but metabolites are not associated with DTI/PWI mismatch or infarct growth. Metabolites are not associated with MTT levels, possibly because MTT includes oligaemic as well as ischaemic tissue. 
17 Brain imaging

VOXEL-BASED SUB-REGIONAL ANALYSIS OF DIFFUSION AND PERFUSION IMAGES IN ACUTE ISCHAEMIC STROKE -PERFUSION CHANGES THAT ALTER TISSUE OUTCOME OCCUR SURPRISINGLY LATE J.M. Wardlaw, C.S. Rivers, P.A. Armitage, M.S. Dennis, V. Cvoro, P.J. Hand, M.E. Bastin

University of Edinburgh, Edinburgh, United Kingdom

Background: MR DWI/PWI mismatch may predict infarct expansion yet the fate of mismatch tissue varies and no ADC or PWI threshold reliably identifies "tissue at risk". We performed a voxel-based analysis of MR DWI/PWI mismatch to identify correlates of infarct growth.

Methods: Patients with acute stroke underwent DWI (tensor), contrast PWI, and T2WI on admission, at 5+14 days, $1+3$ months after stroke. DWI and PWI images were co-registered to baseline DWI. From a $0.5 \mathrm{~cm}$ diam. voxel grid superimposed on the baseline DWI, each voxel was classified as 'abnormal', or 'normal' on DWI appearance. Infarcts were also classified into those that "grew", stayed the same size, or shrank between baseline DTI and $>1$ month T2WI. Relative values (abnormal/contralateral normal values) of DTI, ADC, CBF, MTT for lesions that grew, stayed the same, or shrank, were compared.

Results: Of 46 patients, mean age 72 years (range 37-94), NIHSS 8 (range 1-25), $40 \%$ were first imaged $<6 \mathrm{hrs}$, $24 \%$ from $6-12 \mathrm{hrs}$ and $36 \%$ from $12-24 \mathrm{hrs}$. At baseline, NIHSS, time to scan, $\mathrm{ADCr}, \mathrm{CBFr}$ and MTTr in the DWI-abnormal tissue did not differ between patients whose infarcts grew, were the same or shrank. In patients whose infarcts grew, $\mathrm{CBFr}$ improved more rapidly, peaked earlier (5 vs 14 days) and at a higher value than in those whose infarcts grew ( $\mathrm{p}=0.03)$. $\mathrm{ADCr}$ values recovered more rapidly in those whose infarcts shrank than in those staying the same or growing $(p<0.02)$. MTTr values did not differ between the groups.

Conclusion: Changes in CBF occurring over several days (not just the first few hours) after stroke were associated with tissue outcome, more than DWI or PWI features at baseline. Factors influencing tissue recovery do not just occur in the first few hours after stroke.

\section{Acute stroke: treatment concepts}

\section{Acute stroke: treatment concepts}

\section{THROMBOLYSIS FOR ACUTE ISCHEMIC STROKE IN PATIENTS OLDER} THAN 80 YEARS: RESULTS FROM SITS (SAFE IMPLEMENTATION OF THROMBOLYSIS IN STROKE) REGISTER

G. Ford, V. Larrue, N. Ahmed, N.G. Wahlgren, SITS Collaborators

SITS International and Karolinska University Hospital, Stockholm, Sweden

Background and Purpose: SITS-ISTR (International Stroke Thrombolysis Register) is an internet-based register open to all countries. In Europe, i.v thrombolysis (rt-PA) for acute ischaemic stroke is provisionally licensed within $3 \mathrm{~h}$ of onset for patients up to 80 years under the condition of registration in SITS-MOST (MOnitoring STudy). The risks and benefits of rt-PA in patients over 80 years are currently unknown. We examined outcomes and rates of symptomatic intracerebral haemorrhage (SICH) in $\leq 80 \mathrm{yr}$ and $>80 \mathrm{yr}$ patients registered in SITS.

Methods: 7392 patients entered SITS-ISTR (4961 within SITS-MOST) from 349 centres in 27 countries up to 31 December 2005 of which $643(9.5 \%)$ were $>80$ years old. Data collected: baseline, demography, time delays, NIHSS at baseline, $2 \mathrm{~h}, 24 \mathrm{~h}$ and 7 days, imaging at baseline and within 22-36h after treatment, global outcome at 24 hours and 7 days, modified Rankin Score (mRS) at 3 months and adverse drug reactions. Definitions: SICH: primary ICH type 2 plus NIHSS worsening $\geq 4$ points within $24 \mathrm{~h}$ after treatment. Deaths and independence (mRS 0-2) were determined at 3 months.

Results: Data are presented $>80$ vs. $\leq 80$ years. The median (Inter quartile range) age 83 (82-86) vs. 68 (59-75) years; females $63 \%$ vs. $40 \%$, Pre-Stroke independency $91 \%$ vs. $95 \%$, median NIHSS 15 (10-19) vs. 13 (8-18), mean stroke onset to treatment time 147 vs. 151 minutes. SICH rates: $2.0 \%(1.1-3.5)$ vs. $1.5(1.2-1.8)$, mortality within 3 months: 31 (27-36) vs. 15 (14-16), independence at 3 months: 30 (26-34) vs. 52 (51- 53).

Conclusions: Selected patients over 80 yr olds with severe acute ischaemic stroke treated with i.v rt-PA have almost similar rate of SICH but, as expected, a higher mortality and poorer functional outcome than younger patients. These observations suggest patients just over 80 years can be considered for thrombolysis but further trials are needed to define risks and benefits in the very elderly.
2 Acute stroke: treatment concepts

MICROBUBBLE POTENTIATED TRANSCRANIAL COLOR-CODED DUPLEX ULTRASOUND ENHANCES SYSTEMIC THROMBOLYSIS IN ACUTE MIDDLE CEREBRAL ARTERY STROKE

F. Perren, J. Loulidi, D. Poglia, T. Landis, R. Sztajzel

HUG, University Hospital and Medical School of Geneva, Geneva, Switzerland

Background and Objective: Transcranial Doppler ultrasound is reported to accelerate thrombolysis, and microbubbles, contained in an echocontrast agent (ECA) have been shown in vitro to potentiate this effect. Transcranial color-coded duplex (TCCD) allows to visualize basal cerebral arteries and thus localizes precisely blood clot site. We studied whether TCCD, combined with ECA enhances intravenous (IV) rtPA-thrombolysis in the acute phase of middle cerebral artery (MCA) stroke. Methods: Non-randomized stroke patients treated with $0.9 \mathrm{mg} / \mathrm{kg}$ TPA ( $10 \%$ bolus) within 3 hours of onset (group 1) were compared in terms of MCA recanalization and/or clinical improvement to those in whom additional TCCD monitoring (60 minutes) alone (group 2) or associated with continuous ECA injection (group 3) was performed. Recanalization of the MCA was recorded pre- and postthrombolysis using the Thrombolysis in brain ischemia (TIBI) grading system. Clinical outcome was assessed at admission and 24 hours after treatment using the NIHSS and improvement was considered when the score improved of at least $\geq 4$ points.

Results: Analysis of the 53 patients revealed significant improvement of the NIHSS (Kruskal-Wallis $\mathrm{H}=8.0 ; \mathrm{p}<0.005)$. Pairwise comparisons showed group $3(\mathrm{~N}=14)$ to improve the NIHSS significantly more than both group $2(\mathrm{~N}=16)$ (Mann-Whitney $\mathrm{U}=60 ; \mathrm{p}<0.03)$ and group $1(\mathrm{~N}=23)(\mathrm{U}=72 ; \mathrm{p}<0.002)$. Moreover, the flow signal improved more in group 3 than in group 2 (Mann-Whitney $\mathrm{U}=65 ; \mathrm{p}<0.03$ ).

Conclusion: The results of this pilot study show that the use of ECA in addition to TCCD monitoring lead to a greater clinical improvement than both iv-thrombolyses+TCCD monitoring and iv-thrombolysis alone. Moreover, the flow signal improves more when ECA is added to iv-thrombolysis+TCCD monitoring. This result encourages the evaluation of the thrombolytic effect of ECA on a larger clinical scale.

3 Acute stroke: treatment concepts

\section{SAFETY AND FEASIBILITY OF INTRA-ARTERIAL AUTOLOGOUS BONE} MARROW MONONUCLEAR CELLS TRANSPLANTATION IN ACUTE ISCHEMIC STROKE

D.C. Bezerra, M.L. Mendonca, G.R. de Freitas, S.A. Silva, R. Mendez-Otero, C. Andre, C.S. Gonzalez, CH. Falcao, A.F. Haddad, F.A. Tuche Pro Cardiaco Hospital, Federal University of Rio de Janeiro; Biophysics and Histology, Federal University of Rio de Janeiro; Neurology, Pontificia Universidade Catolica do Rio Grande do Sul, Brazil

Objective: To assess if intra-arterial autologous bone marrow mononuclear cells (BMMC) transplantation is safe and feasible in acute ischemic stroke.

Background: In animal models, stem cells enhanced functional recovery after stroke. Moreover, recent studies have demonstrated that the injection of autologous BMMC in coronary arteries is safe in humans.

Methods: This phase I open label multicenter trial involved ischemic stroke patients in the territory of the middle cerebral artery (MCA) with a NIHSS score between 4 and 20 and with spontaneous recanalization confirmed by transcranial Doppler (TCD) and/or magnetic resonance angiography. Between the 3rd and the 7th day after stroke onset, bone marrow cells were aspirated from the posterior iliac crest. On the same day, 30 million BMMC were injected in the MCA via catheter angiography. The procedure was monitored by TCD and electroencephalography. MRI was performed at baseline, 7 days, 3 and 6 months after months after BMMC injection. In one of the centers, brain perfusion with $99 \mathrm{mTc}-\mathrm{ECD}$ SPECT and PET-FDG were also performed. Clinical examination was quantified by the NIHSS, Barthel Index and the modified Rankin Scale (mRS). 25 patients are planned to be enrolled in the trial.

Results: We report the results of the first 13 patients that completed the 3 month evaluation. Mean age was $61.3 \pm 8$ years and 8 were male. In 10 patients the infarct size on baseline DWI was greater than $1 / 3$ of the MCA territory. Median mRS at 3 months was 2. One patient died of myocardial infarction after discharge from the hospital and one patient had an ischemic stroke during arterial catheterization and BMMC were not injected. Serial MRI performed at 3 months did not show any structural changes (including tumor formation) within the brain.

Discussion: These initial data suggests that intra-arterial injection of autologous $\mathrm{BMMC}$ in the MCA is feasible and safe in acute stroke. 


\section{Acute stroke: treatment concepts}

RECANALIZATION AFTER INTRAVENOUS THROMBOLYSIS IN STROKE PATIENTS - RATES, PREDICTORS AND PROGNOSTIC SIGNIFICANCE M. Furtner, S. Kiechl, A. Zangerle, M. Spiegel, M. Knoflach, T. Gotwald, S. Felber, J. Willeit

Innsbruck Medical University, Innsbruck, Austria

Background and Purpose: Although intravenous thrombolysis is a mainstay of acute stroke therapy, the rate of vessel recanalization is not firmly established. In this study, we aimed at estimating rates, predictors and prognostic significance of recanalization in an unselected series of stroke patients treated with intravenous (IV) thrombolysis.

Methods: A total of 64 patients with middle cerebral artery (MCA) strokes had a computed tomography angiography or transcranial doppler examination prior and 24 hours after IV thrombolysis. Recanalization was defined by a rating of 3 on the TIMI (thrombolysis in myocardial infarction) and $4 / 5$ on the TIBI (thrombolysis in brain infarction) scales, respectively. Data on risk factors, clinical features and outcome were prospectively collected by standardized procedures.

Results: In 36 of the 64 patients $(56.3 \%)$, vessel recanalization was achieved. There was a non-significant trend of recanalization rates to decline with a more proximal site of occlusion: $68.4 \%$ (M2 segment of MCA), 53.1\% (M1 segment) and 46.2\% (carotid ' $\mathrm{T}$ ') ( $\mathrm{P}$ for trend $=0.28$ ). Frequencies of vessel re-opening were markedly reduced in subjects with diabetes $(9.1 \%$ vs. $66.0 \%$ in non-diabetics, $\mathrm{P}<0.001)$ and less so in subjects with additional extracranial carotid artery occlusion $(\mathrm{P}=0.03)$. Finally, successful recanalization significantly predicted a favorable stroke outcome at the day 90 (modified Rankin $\leq 2$ ) on top of the information provided by age, National Institutes of Health Stroke Scale score and onset-to-needle time.

Discussion: This study documents a high rate of successful vessel recanalization following standard intravenous thrombolysis and indicates that information on recanalization represents a powerful and easily obtainable predictor for clinical outcome. Importantly, thrombolysis did not work well in the large segments of patients with diabetes and those with co-existent extracranial carotid occlusions.

\section{Acute stroke: treatment concepts}

DELETERIOUS ROLE OF APOPTOSIS IN HUMAN STROKE: CASPASE-3 IS RELATED TO POOR NEUROLOGICAL OUTCOME AMONG T-PA TREATED PATIENTS AND ACTIVATED IN THE ISCHEMIC BRAIN

A. Rosell, J. Alvarez-Sabín, A. Ortega-Aznar, P. Delgado, R. Delgado-Mederos, I. Fernández-Cadenas, E. Cuadrado, M. Ribó, C.A. Molina, J. Montaner Laboratorio de Investigación Neurovascular, Hospital Vall d'Hebron, Barcelona, Spain

Background: Stroke-related apoptosis is a highly regulated mechanism that requires a complex activation cascade of caspases. Caspase- 3 plays an important role related to DNA fragmentation and cell death. Our aim was to investigate caspase- 3 blood level, its correlation with neurological outcome and to explore caspase- 3 activity in the ischemic brain

Methods: Caspase- 3 level was determined by an ELISA in plasma samples from 116 patients that received t-PA (pre-treatment). Moreover, in a subgroup of 41 patients serial determinations were done at 1 and 2 hours after initial bolus and at 12 and 24 hours after stroke onset. NIHSS scores were serially obtained until discharge and functional outcome evaluated with the modified Rankin Scale (mRS) at three months. To achieve tissue lesion, MRI studies were done (pre-treatment and at follow-up). In addition, caspase- 3 content was studied at different brain areas (infarct, periinfarct and contralateral) of 4 deceased stroke patients.

Results: Caspase- 3 level at 24 hours was associated to a worse neurological outcome as shown by NIHSS correlations $(\mathrm{r}=0.334, \mathrm{p}=0.035$ at $24 \mathrm{~h} ; \mathrm{r}=0.376, \mathrm{p}=0.018$ at $48 \mathrm{~h} ; \mathrm{r}=0.412, \mathrm{p}=0.011$ at discharge) and positively correlated with lesion growth assessed by DWI images $(\mathrm{r}=0.582, \mathrm{p}=0.018)$. This deleterious association was still evident at three months as shown by the positive correlation between mRS and caspase- 3 level at 24 hours $(r=0.378, p=0.030)$. Regarding brain tissue, active caspase- 3 was identified in periinfarct but not in infarct core.

Discussion: Higher plasmatic level of caspase- 3 one day after stroke is related to a worse neurological outcome and caspase- 3 is activated in the ischemic brain Altogether our data suggests that caspase- 3 may be involved in the recruitment of ischemic brain tissue.
6 Acute stroke: treatment concepts

\section{DIFFUSION WEIGHTED IMAGING EVALUATION FOR UNDERSTANDING} STROKE EVOLUTION (DEFUSE) STUDY

V. Thijs, M. Lansberg, R. Bammer, W. Kakuda, S. Kemp, E. Skalabrin, G. Schlaug, L. Wechsler, M. Moseley, G.W. Albers, DEFUSE Investigators Stanford Stroke Center, Palo Alto, CA, USA

Background: MRI patterns might identify patient subgroups that derive benefit from early reperfusion therapy or may be harmed.

Methods: In this prospective, open-label, 7 center, NIH-funded study, an MRI was obtained in 74 consecutive acute ischemic stroke patients (NIHSS $\geq 6$ ) immediately prior to, and 3-6 hrs (median $4.25 \mathrm{hr}$ ) after, treatment with IV tPA in the 3-6 h window. Early reperfusion required $\mathrm{a} \geq 30 \%$ and $\leq 10 \mathrm{cc}$ reduction in PWI. The Target mismatch profile was defined as PWI $>10 \mathrm{cc}$ and $>120 \%$ of DWI. The Malignant profile was a DWI $\geq 100 \mathrm{cc}$ or severe PWI lesion $(\geq 8$ seconds delay on Tmax) of $\geq 100 \mathrm{cc}$. The Benign profile was DWI and PWI volume both $\geq 10 \mathrm{cc}$. The primary endpoint was the "clinical response" defined as an NIHSS $0-1 / \geq 8 \mathrm{pt}$ improvement at 30 days.

Results: Recanalization based on MRA occurred in 44\%. More complete recanalization was associated with more important decreases in PWI volumes $(p<0.001)$. The clinical response associated with early reperfusion differed depending on the baseline MRI profile. For Target mismatch patients ( $41 \%$ of all patients) early reperfusion was associated with a favorable clinical response (64\% vs. $19 \%$ withou reperfusion, $\mathrm{p}<0.011)$. The Benign profile $(26 \%)$ was associated with a favorable clinical response regardless of reperfusion status. The Malignant pattern ( $8 \%$ of patients) was associated with a high rate of poor outcomes with or without reperfusion. The SICH rate was $9.7 \%$ (minor $2.8 \%$, major $6.9 \%$ ). DWI lesion volume was the only independent predictor of SICH ( $\mathrm{p}=0.002$; Odds Ratio 1.41 ; $95 \%$ CI 1.07-1.87; AUC 0.89).

Conclusions: The DEFUSE results support the concept that MRI patterns can differentiate patient subgroups that benefit from early reperfusion from those who do not benefit, or may be harmed. Baseline DWI lesion volume may be a more potent predictor of SICH than traditional clinical variables in patients treated with tPA in the three to six hour time-window.

\section{Acute stroke: treatment concepts}

PRE-TREATMENT WITH ANTIPLATELET AND RISK OF SYMPTOMATIC HAEMORRHAGE AFTER THROMBOLYSIS: A MULTICENTRIC STUDY Y. Bravo, J. Martí-Fàbregas, D. Cocho, M. Rodríguez-Yañez, M. Castellanos, N. Pérez de la Ossa, J. Roquer, V, Obach, J. Maestre, J.L. Martí-Vilalta Hospital de la Santa Creu i Sant Pau, Spain

Background: The influence of antiplatelet agents (AP) in the development of a symptomatic parenchymal haemorrhage after intravenous rt-PA is poorly know. We assessed the hypothesis that pre-treatment with AP may increase that risk.

Methods: We studied consecutive patients with ischemic stroke treated with intravenous rt-PA within the first 3 hours after symptom onset. The patients were treated by experienced neurologists at seven Spanish hospitals. We recorded whether the patients were currently receiving any AP. A control CT was obtained routinely 24-36 hours after the infusion of rt-PA or before in case of neurological worsening. Intracranial bleeding was categorized according to the ECASS II criteria in hemorrhagic infarction type 1 and 2, and parenchymal haemorrhage type 1 and 2 (PH1, PH2). Symptomatic haemorrhage was diagnosed if it was of the PH1 or PH2 type, occurred within the first 36 hours, and was associated with a neurological deterioration (increase $>3$ points in the NIHSS score).

Results: A total of 422 patients were studied. Their mean age was $67.7 \pm 10.9$ years and $57.1 \%$ of them were men. The mean time to treatment was $150.9 \pm 40.9$ minutes and the median NIHSS score was 15 . Seventy-nine patients $(18.7 \%)$ were pretreated with AP, most of them $(n=61)$ with aspirin. $\mathrm{PH}$ was observed in 36 patients $(8.7 \%)$ and was symptomatic in only $17(4 \%)$. There were no differences between AP and non-AP groups in the frequency of PH-1 and $\mathrm{PH} 2(6.3 \%$ vs $5 \%$ and $6.3 \%$ vs $2,6 \%$ $\mathrm{p}=0.17$ ). There was a non-significant increase in the frequency of symptomatic haemorrhage in the AP group compared with the non-AP group (7.6\% vs $3.2 \%$ $\mathrm{p}=0.10$ ).

Discussion: Pre-treatment with AP increases non-significantly the risk of symptomatic haemorrhage. 
8 Acute stroke: treatment concepts

MAGNESIUM (MG) FOR TREATMENT OF ACUTE LACUNAR STROKE SYNDROMES: FURTHER ANALYSIS OF THE INTRAVENOUS MAGNESIUM EFFICACY IN STROKE (IMAGES) TRIAL

S. Aslanyan, C.J. Weir, K.W. Muir, K.R. Lees, for the IMAGES Study Group Glasgow University, Glasgow, United Kingdom

Background: A prespecified interaction analysis of the neutral IMAGES trial revealed significant benefit from $\mathrm{Mg}$ in patients with non-cortical stroke. Post hoc analysis indicated that this effect was seen in lacunar syndromes (LS), interaction $\mathrm{p}=0.005$. We have now examined whether this interaction could be explained by confounding baseline factors.

Methods: LS was defined by Oxfordshire clinical classification and did not include imaging findings. We investigated the interaction between baseline variables and $\mathrm{Mg}$ treatment on global outcome. We used logistic regression models to test if the $\mathrm{Mg} / \mathrm{LS}$ interaction remained significant after adjusting for stratification variables (age group, side of symptoms and time from onset), gender, a novel IMAGES stroke severity score and baseline variables that had an interaction with treatment $(\mathrm{p}<0.1)$. Results: The $\mathrm{Mg}(\mathrm{n}=383$; including 31 with primary haemorrhage) and placebo $(\mathrm{n}=382 ; 37$ with haemorrhage) groups of LS patients were well matched on baseline factors. In addition to LS we found an interaction between beneficial $\mathrm{Mg}$ treatment effect and younger age $(\mathrm{p}=0.003)$, higher baseline diastolic $\mathrm{BP}(\mathrm{p}=0.02)$, higher mean BP $(\mathrm{p}=0.02)$ and not having ischaemic heart disease $(\mathrm{p}=0.07)$. Even so, the adjusted $\mathrm{Mg} / \mathrm{LS}$ interaction remained significant (OR 0.57; 95\% CI, 0.39-0.83, $\mathrm{p}=0.003)$. In the LS subgroup, Mg improved Barthel Index $(\mathrm{BI})<95(0.73 ; 0.55-$ $0.98)$, modified Rankin Scale $>1(0.67 ; 0.50-0.91)$ and global outcome $(0.70$; $0.53-0.92$ ), but not $\mathrm{BI}<60$ or mortality endpoints.

Discussion: We cannot ascribe the positive $\mathrm{Mg} / \mathrm{LS}$ interaction to general issues of severity, time to treatment, BP or other baseline factors. Our analysis cannot distinguish a chance finding from a real independent effect of $\mathrm{Mg}$. A large trial of $\mathrm{Mg}$ treatment in LS appears essential.

\section{Acute stroke: treatment concepts}

DOES BUBBLE CHARACTERISTICS AFFECT RECANALIZATION IN STROKE PATIENTS WITH MICROBUBBLE-ENHANCED SONOTHROMBOLYSIS?

M. Rubiera, M. Ribo, R. Delgado-Mederos, E. Santamarina, O. Maisterra,

J. Alvarez-Sabín, C.A. Molina

Hospital Vall d'Hebron, Barcelona, Spain

Administration of microbubbles (MB) may augment the effect of ultrasoundaccelerated systemic thrombolysis in acute stroke. Bubble structural characteristics may influence the effect of $\mathrm{MB}$ on sonothrombolysis. We aimed to compare the effects of galactose-based air-filled MB (Levovist) and sulphur hexafluoride-filled MB (Sonovue) on recanalization and clinical outcome.

One hundred and one iv tPA-treated patients with middle cerebral artery (MCA) occlusion were studied. Presence and location of arterial occlusion and recanalization (RE) was assessed using the TIBI flow grading system. Patients underwent 2-hours continuous TCD monitoring and received 3 bolus of MB after 2, 20 and 40 minutes of tPA bolus. Fifty-nine patients received Levovist (LV) and 42 received Sonovue (SV). NIHSS scores were obtained at baseline and after 24h. Modified Rankin Scale (mRS) score was used to assess outcome at 3 months.

Median admission NIHSS was 16 . On TCD, $73(72.3 \%)$ patients had a proximal and $28(27.7 \%)$ a distal MCA occlusion. Age, baseline NIHSS, clot location, stroke subtypes and time to treatment were similar between LV and SV groups. Recanalization rates after $1(42.4 \% / 37.5 \%), 2(55.2 \% / 51.3 \%)$ and 6 hours $(63 \% / 52 \%)$ were similar in LV/SV-groups $(\mathrm{p}>0.4)$. Clinical improvement (NIHSS decrease $>4$ points) at 24 hours was similar in both groups $(59.3 \% / 54.8 \%, \mathrm{p}=0.687)$, as well as symptomatic intracranial haemorrhage $(1.7 \% / 2.4 \%, \mathrm{p}=0.654)$ and in-hospital mortality $(7 \% / 8.3 \%, \mathrm{p}=0.638)$. Similarly, the type of MB administered did not affect long-term outcome after sonothrombolysis. Fifty percent of patients in the LV group and $43.8 \%$ in the SV group achieved functional independence $(\mathrm{mRS}<2)$ at 3 months $(\mathrm{p}=0.626)$.

MB administration during sonothrombolysis associates with a high RE rate However, RE rates, clinical course and long-term outcome are comparable when administering galactose-based air-filled MB (Levovist) or sulphur hexafluoride-filled MB (Sonovue)
10 Acute stroke: treatment concepts

TRENDS OF ANTITHROMBOTIC USE IN PATIENTS WITH NON-VALULAR ATRIAL FIBRILLATION IN THE COMMUNITY AND IN A TEACHNG HOSPITAL

V. Peppes, K. Takis, J. Revela, K. Xynos, M. Saliaris, C. Zafeiriou, M. Synetou, K. Vemmos

Alexandra Hospital, University of Athens, Athens, Greece

Background: Limited data are available concerning trends in use of oral anticogeulants $(\mathrm{OAC})$ or aspirin over time in patients with atrial fibrillation $(\mathrm{AF})$. The objective of this study was to evaluate physicians' attitudes in current clinical practice of $\mathrm{OAC}$ or aspirin use among patients with $\mathrm{AF}$.

Methods: Medical records of hospitalized patients for the years 1991,

1996 and 2001 were searched to identify cases with AF diagnosis in a University Hospital. Stroke risk stratification was based on Atrial Fibrillation Investigators guidelines in high risk and low risk group.

Antithrombotics were recorded a) for the last week prior to admission (attitude of primary community physicians) and b) recommendation on hospital discharge (attitude of academic physicians). Statistical analysis by $\chi^{2}$ test for trend was used. Results: Based on our inclusion criteria we recruited 192, 181, and

191 cases with AF for the years 1991, 1996, and 2001 respectively. In the high risk group OAC use by community physicians increased from $15.7 \%$ in 1991 to $37.6 \%$ in 2001 ( $\chi^{2}$ for trend $15.9, \mathrm{p}<0.001$ ), while the corresponding percentages for academic physicians were $15.1 \%$ in 1991 to $60.7 \%$ in 2001 ( $\chi^{2}$ for trend 70.1 , $\mathrm{p}<0.001)$. A substantial proportion of high risk patients in 2001 were on aspirin $(29 \%)$ or no therapy $(33.5 \%)$ in the community setting, while the corresponding values for patients discharged from an academic hospital were $22.5 \%$ and $16.8 \%$. In the low risk group conversely the majority of AF patients were on $\mathrm{OAC}(61 \%)$ in the community setting and in academic practice $(83 \%)$.

Discussion: Despite the increased use of OAC over time for primary prevention in $\mathrm{AF}$ patients, many patients were treated inappropriately based on risk stratification criteria, especially those under primary care physicians.

\section{Acute stroke: treatment concepts}

\section{EFFECT OF THROMBOLYSIS ON RELATIONSHIP BETWEEN BLOOD} PRESSURE AND STROKE OUTCOME

M. Yong, H.C. Diener, M. Kaste, J. Mau

Institute of Statistics in Medicine, Heinrich Heine University Hospital, Duesseldorf, Germany

Background and Purpose: In the blood pressure (BP) management of acute stroke thrombolytic therapy has to be given special consideration. Additive or interactive effects of thrombolysis on the relationship between BP and stroke outcome are unknown. We investigated the presence of such effects with respect to baseline BP and 90-day outcome.

Methods: The study is based on 615 patients from the first European Cooperative Acute Stroke Study (ECASS 1) who had been randomized to either $1.1 \mathrm{mg} / \mathrm{kg} \mathrm{IV}$ rt-PA or placebo and had received trial medication within 6 hours after symptom onset. Endpoints are assessments of modified Rankin 0 or 1 on day 90, as good outcome, and 90-day mortality. Each endpoint was represented as a function of sliding-average BP values. Suggested cut-off points were confirmed by classification and regression tree (CART) methodology

Results: S-shape relationships between systolic BP (SBP) and probability of good outcome were found in rt-PA and in placebo patients. For patients with baseline SBP between 140 and $160 \mathrm{mmHg}$, there was a persistent benefit of $10 \%$ per $1 \mathrm{mmHg}$ for the rt-PA treated patients in comparison with placebo. An excess mortality attributable to rt-PA is lowest for SBP between 150 and $160 \mathrm{mmHg}$. Patients with diastolic BP (DBP) between 80 and $90 \mathrm{mmHg}$ benefit from rt-PA, while there is an excess mortality attributable to rt-PA up to $60 \%$ for DBP larger than $90 \mathrm{mmHg}$

Conclusions: We found an additive effect of rt-PA on the relationship between SBP and 90-day good outcome. Subgroups of patients with baseline SBP between 140 and $160 \mathrm{mmHg}$ or DBP between 80 and $90 \mathrm{mmHg}$ had highest benefit and lowest risk of death attributable to rt-PA. 
EFFECTS OF MICROBUBBLES-ENHANCED SONOTHROMBOLYSIS AMONG STROKE SUBTYPES

M. Rubiera, M. Ribo, R. Delgado-Mederos, E. Santamarina, O. Maisterra,

J. Montaner, P. Delgado, J. Alvarez-Sabín, C.A. Molina

Hospital Vall d'Hebron, Barcelona, Spain

We aimed to evaluate the impact of MB administration during sonothrombolysis on the success of recanalization among different stroke subtypes.

We studied 155 consecutive stroke patients due to MCA occlusion treated with iv tPA. Patients were allocated to receive tPA plus continuous 2-h TCD monitoring ( $\mathrm{n}=37$; US group), or tPA plus placebo monitoring ( $\mathrm{n}=36$; tPA group), or tPA/US plus 3 doses of MB given at $2 \mathrm{~min}, 20 \mathrm{~min}$ and $40 \mathrm{~min}$ after tPA bolus $(\mathrm{n}=67$; MB group). Recanalization on TCD during at $2 \mathrm{~h}$ of tPA bolus was recorded. Stroke subtypes were assessed by means of the TOAST criteria.

Median admission NIHSS was 16 . On TCD, 118 (76\%) patients had a proximal and $37(24 \%)$ a distal MCA occlusion. Stroke was categorized as cardioembolic (CE) in $76(49 \%)$ aterothrombotic (AT) in $37(24 \%)$ and undeterminated (UD) in 35 (23\%) patients, others $4 \%$. Baseline NIHSS, clot location, and time to treatment were similar among stroke subtypes Two-hour complete recanalization rate was significantly $(\mathrm{p}=0.039)$ higher in MB group $(52 \%)$ as compared to tPA/US $(40.2 \%)$ and tPA (24\%) groups. MB administration significantly $(\mathrm{p}=0.021)$ increased de rate of 2-h recanalization in patients with AT stroke. Among patients with AT stroke (96\% tandem ICA/MCA occlusions), MB increased in 1.5- and 2-fold, the rate of 2-h recanalization compared to tPA+US (39\% vs 26\%) and tPA (39\% vs $21 \%$ ). Treatment allocation did not affect recanalization rates in CE and UD stroke patients. At 3 months, AT stroke was significantly associated with a poor outcome in US $(\mathrm{p}=0.043)$ and $\mathrm{TPA}(\mathrm{p}=0.021)$ groups, but not in the MB group. In the MB group, $36 \%$ of AT, $48 \%$ of CE, and $47 \%$ of UD strokes became independent (mRS $\leq 2)$.

Efficacy of MB-enhanced sonothrombolysis varies among stroke subtypes. MB administration during sonothrombolysis increases recanalization rates and improves outcome in patients with tandem ICA/MCA occlusion.

\section{Venous diseases/Heart \& brain}

\section{Venous diseases/Heart \& brain}

MRI OF CLOT IN CEREBRAL VENOUS THROMBOSIS: HIGH DIAGNOSTIC VALUE OF SUSCEPTIBILITY-WEIGHTED IMAGES

I. Crassard, A. Idbaih, M. Boukobza, R. Porcher, M.G. Bousser, H. Chabriat Lariboisiere Hospital, Paris, France

Background: In cerebral venous thrombosis (CVT), the sensitivity of conventional MRI sequences to detect the clot in sinuses or veins is incomplete and largely depends on the time elapsed since the thrombus formation. Little is known concerning the diagnostic value of fluid-attenuated inversion recovery (FLAIR), echo-planar $\mathrm{T} 2 * \mathrm{SW}$-weighted imaging $(\mathrm{T} 2 * \mathrm{SW})$ or diffusion-weighted images (DWI) for this purpose.

Material and Methods: a retrospective analysis was performed for 114 MRI examinations from 39 patients with CVT using a structured assessment. The sites of venous occlusion were systematically assessed, as the aspect of signal at each site of thombosis on T1SE, T2SE, DWI, FLAIR and T2*SW images. The time course of sensitivity in the detection of clot ( $\mathrm{n}=166$ clots) was analyzed for different MR sequences using a multilevel logistic model. The sensitivity of different MR sequences for diagnosis of cortical venous thrombosis was tested separately $(\mathrm{n}=38$ clots)

Results: The sensitivity of T2*SW and T1SE sequences to detect the clot in sinuses or veins was estimated at $90 \%$ and $71 \%$ between day 1 and day 3 and was found much higher than that of T2SE, FLAIR or DWI during the first week of clinical onset. Only the sensitivity of $\mathrm{T} 2 * \mathrm{SW}$ was relatively stable during this period. Furthermore, after day 7 , the sensitivity of T2*SW decreased less than that of T1SE in the presence of a venous clot. Thrombosed cortical veins, even in the absence of visible occlusion on MRV, were detected more frequently with $\mathrm{T} 2 * \mathrm{SW}(97 \%)$ and T1SE $(78 \%)$ than with FLAIR or DWI $(<40 \%)$.

Discussion: $\mathrm{T} 2 * \mathrm{SW}$ imaging appears of additional diagnostic value in CVT usually investigated with the combination of VRM, T1SE and T2SE. The T2*SW sequence may be particularly useful during the acute phase of CVT when the sensitivity of the other sequences is incomplete and for the diagnosis of isolated cortical venous thrombosis.

\section{Venous diseases/Heart \& brain}

RISK OF NEW EARLY SEIZURES AND ANTIEPILEPTIC DRUG (AED) PROPHYLAXIS IN ACUTE CEREBRAL VEIN AND DURAL SINUS THRMBOSIS (CVT). A STRATIFIED CASE CONTROL STUDY J.M. Ferro, P. Canhão, J. Stam, M.G. Bousser, F. Barinagarrementeria, ISCVT Investigators

Hospital de Santa Maria, Lisboa, Portugal

The efficacy of AED prophylaxis in acute CVT, has never been investigated in randomized or in case-control studies.

Methods: we evaluated the risk of seizures occurring within 2 week of admission (new early seizures) in 624 CVT patients included in the International Study on Cerebral Vein and dural sinus Thrombosis (ISCVT). Dates of start/stop of AEDs were registered but the prescription of AED was left to the initiative of the local investigators. We compared the risk (OR and 95\% CI) of new early seizures in patients who were/were not prescribed AEDs, stratified for supratentorial lesions on admission CT/MR seizures before or at presentation (presenting seizures) Results: $43(6.9 \%)$ patients experienced new early seizures. Stratum "no supratentorial lesions no presenting seizures,"197 patients were not prescribed AEDs: 5 (2.5\%) had new early seizures; 11 received AEDs, none had new early seizures. Stratum "no supratentorial lesions, presenting seizure": 14 patients were not prescribed AEDs, seizures recurred in 1 (7.1\%); 35 received AEDs, none had recurrent seizures. Stratum "supratentorial lesions, no presenting seizure": 134 were not prescribed AEDs: $11(8.2 \%)$ had new early seizures; 35 received AEDs and only $1(2.9 \%)$ had had new early seizures $(\mathrm{OR}=0.3 ; 95 \% \mathrm{CI}=0.04-2.6)$. Stratum "supratentorial lesion, presenting seizure,": 47 were not prescribed AEDs, seizures recurred in $24(51 \%) ; 148$ received AEDs, only $1(0.7 \%)$ had recurrent seizures $(\mathrm{OR}=0.006$; $95 \% \mathrm{CI}=0.001-0.05$ ).

Conclusion: the risk of new early seizures in CVT patients without supratentorial lesions or presenting seizures was very low, whether or not they are prescribed AEDs. In patients with supratentorial lesions, in particular in those with presenting seizures. The risk was ower in those who received AEDs. These results support (case-control study - evidence class III) the prescription of AEDs in acute CVD patients with supratentorial lesions who present with seizures.

\section{Venous diseases/Heart \& brain}

EARLY SEIZURES IN ACUTE CEREBRAL VEIN AND DURAL SINUS THROMBOSIS (CVT). PREVALENCE, RISK OF EARLY RECURRENCE AND ASSOCIATED CONDITIONS

J.M. Ferro, P. Canhão, M.G. Bousser, J. Stam, F. Barinagarrementeria, ISCVT Investigators

Hospital de Santa Maria, Lisboa, Portugal

Background and Purpose: Seizures are a frequent initial manifestation of acute CVT. After admission, seizures can recur or appear "de novo". The risk of early recurrence and associated conditions have not been investigated in large CVT cohorts.

Methods: In a multicentre prospective observational study (ISCVT) including 624 CVT patients, we analysed the risk factors for seizures occurring before confirmation of the diagnosis of CVT (presenting seizures) or within the following 15 days (new or recurrent).

Results: 245 patients (39.3\%) experienced presenting seizures: 122 focal and 187 with generalization. $43(6.9 \%)$ patients suffered 17 new and 26 recurrent seizures. Predictors of presenting seizures: supratentorial parenchymal lesion on CT/MR $(\mathrm{OR}=4.05)$, thrombosis of cortical vein $(\mathrm{OR}=2.31)$, of saggital sinus $(\mathrm{OR}=2.18)$ and puerperal CVT $(\mathrm{OR}=2.06)$; predictors of new+recurrent seizures: supratentorial parenchymal lesion on admission CT/MR ( $\mathrm{OR}=3.09)$, presenting seizure $(\mathrm{OR}=1.74)$ Variables associated with recurrent seizures: supratentorial lesion $(\mathrm{OR}=7.01)$, motor deficit (3.49); variables associated with new seizures after admission: supratentorial lesion $(\mathrm{OR}=3.10)$ and aphasia $(\mathrm{OR}=4.86)$.

Conclusion: CVT patients with supratentorial parenchymal lesions have a higher frequency of both seizures at the onset of CVT and of seizures early after admission. Patients with presenting seizures have a high risk of recurrence. 


\section{Venous diseases/Heart \& brain}

\section{CEREBRAL VENOUS THROMBOSIS: A STUDY OF MEDICAL} MANAGEMENT IN FRANCE

F. Vuillier, T. Moulin, F. Ricolfi, F. Cattin, F. Woimant

University of Franche-Comté, Federation of Neurosciences, Jean Minjoz

University Hospital, Besançon, France

Background: Although anticoagulant randomised trials have been carried out, medical management of cerebral venous thrombosis (CVT) varies to a large extent. The implementation of guidelines and routine management have not been evaluated. Method: We developed a series of 31 open questions about the medical management of acute CVT and in the first few months of follow-up. This questionnaire was sent to 51 Neurology Units in French hospitals, with 37 responses.

Results: In 27/37 centres initial emergency treatment was begun based on clinical findings in 15, and on radiological evidence of CVT in the remaining 12 centres. All centres administered initial anti-thrombotic treatment to achieve effective anti-coagulation.

In 30/37 centres $(80 \%)$ intravenous heparin was most often used. The main contraindication for anticoagulants is the risk of bleeding. Intracranial hypertension was most often treated by osmotic diuretics and based on clinical and imaging arguments. The main deciding factor for prescribing anti-epileptics was a seizure in the acute phase in $35 / 37$ centres $(94 \%)$ and intravenous clonazepam was used in $38 \%$.

Oral anticoagulants were prescribed in all centres, peaking on day 8 in 13/37 centres $(35 \%)$ depending on the clinical course and aspect of cerebral lesions. The first follow-up visit was routinely organised at month 3, any decision to discontinue anti-coagulants depended on CVT etiological origin and on the aspect of the dural sinuses.

Discussion: Diagnostic methods and emergency management were not yet standardised enough while anticoagulants were systematically used. Data is more variable concerning the investigation of etiology, follow up and treatment duration.

\section{Venous diseases/Heart \& brain}

NOVEL NEUROPROTECTIVE APPROACHES IN EXPERIMENTAL MODELS OF CEREBRAL VENOUS INFARCT

H. Nakase, T. Nishioka, R. Kimura, R. Tamaki, K. Tamura, T. Sakaki,

A. Heimann, O. Kempsk

Nara Medical University, Nara, Japan

Background and Purpose: Cerebral venous disorders are potentially good outcome if diagnosed and treated promptly. Therapeutic window in time and space is considered to be much wider in cerebral venous infarct than arterial stroke. Therefore, neuroprotective candidates with the success obtained with animal models are more promising in human trial in CVI. In the paper, novel neuroprotective approaches in experimental model of cerebral venous infarct (CVI) are described.

Methods and Results: We used the rat two cortical veins occlusion (2-VO) model. $<$ Experiment-1 $>$ Male Wistar rats were divided into two groups: the control and the treated group with Vascular endothelial growth factor (VEGF) antagonist. They were evaluated by magnetic resonance imaging (MRI) 24 hours after the operation. VEGF expression was observed exclusively in the area of vasogenic edema which was shown as a high intensity area in apparent diffusion coefficient of water (ADCw) map. The both volumes of the infarction and the vasogenic edema in MRI were significantly smaller in the treated group than the control $(\mathrm{P}<0.05) .<$ Experiment- $2>$ Caspase-inhibitor z-VAD.fmk was applied intraventricularly 3, 24, 48, or 96 hours after 2-VO. Vehicle was injected $3(n=6)$ or 24 hours $(n=7)$ after 2-VO. Inhibition of caspases by z-VAD.fmk given 3 and even 24 hours after 2-VO significantly reduced infarct volume $(\mathrm{P}<0.05)$.

Conclusion: On the basis of these data, the inhibition of VEGF and apoptosis might be new therapies against CVI.
6 Venous diseases/Heart \& brain

OMISSION OF ANTICOAGULATION TREATMENT IN PATIENTS WITH STROKE AND ATRIAL FIBRILLATION (AF) DOUBLES THE RISK OF PREMATURE DEATH. A DANISH NATION-WIDE STUDY OF STROKE WITH AF

K.K. Andersen, T.S. Olsen

Informatics and Mathematical Modeling, Section for Statistics, Technical

University of Denmark, Denmark

Background: Anticoagulation (AC) with warfarin protects against recurrent stroke in stroke patients (pts) with AF but due to fear of bleeding complications treatment is often omitted. Survival after stroke in Denmark is now followed in a nation-wide register. We studied survival of stroke pts with $\mathrm{AF}$ who were/were not treated with $\mathrm{AC}$.

Method: The Danish National Indicator Project established 2001 is expected to register all stroke admissions in Denmark. Coverage is currently $75 \%$ of all admissions. We present data from the first 30000 admissions. All pts underwent a pre-defined evaluation: Admission stroke severity using the Scandinavian Stroke Scale (SSS, 0-58), a cardiovascular risk factor evaluation and electrocardiography. AC treatment was registered. Survival was recorded through the Danish Death Registry. Mean follow-up was 1.2 years. We excluded readmissions and pts $<40$ years.

Results: We included 24791 pts of which 4185 (16.9\%) had AF. Males 45.1\%; mean age (sd) 79.5 (9.2) years. Of these 1517 pts $(36.2 \%)$ had AC treatment; in $2668(63.8 \%)$ AC treatment was omitted. Of pts who had AC 19\% died during follow-up vs. $43 \%$ of pts who had not AC. Pts in AC treatment were younger (76.7 (9.4) years vs. $81.1(9.1)$ years, $\mathrm{p}<0.0001)$ and had less severe strokes (SSS (sd): 42.5 (14.9) vs 32.0 (18.6), $\mathrm{p}<0.0001)$.

A Cox proportional hazard $(\mathrm{CPH})$ model with age modelled as delayed entry was build to study effect of AC treatment on survival while controlling for relevant confounders (SSS, age, sex, cardiovascular risk factors). Pts who did not have AC were at greater risk of dying having a hazard ratio of 2.09 (95\% CI: $1.76-2.47$ ) compared to pts who had the treatment.

Discussion: Omission of AC protection in stroke pts with AF doubles the risk of premature death.

\section{Venous diseases/Heart \& brain}

\section{PROGNOSTIC RELEVANCE OF CAROTID DISEASE IN} CARDIOPULMONARY BYPASS SURGERY AND THE IMPORTANCE OF AN EXHAUSTED CEREBROVASCULAR RESERVE CAPACITY

J. Schoof, W. Lubahn, C.W. Wallesch, C. Huth, M. Goertler

University of Magdeburg, Magdeburg, Germany

Background: Carotid stenosis in cardiopulmonary bypass surgery (CPBS) is linked to an increased stroke risk. We aimed to assess this risk in relation to the degree of stenosis and whether stenosis related haemodynamic compromise predicts perioperative stroke.

Methods: 2809 of 7120 (39.5\%) consecutive patients (pts) who underwent CPBS had carotid duplex before surgery $(73.3 \%$ coronary artery bypass graft, $14.1 \%$ valve repair, $12.6 \%$ both). Transcranial Doppler carbon dioxide testing for cerebrovascular reserve capacity was performed in all pts with high-grade stenosis $(>80 \%$ local diameter reduction) and occlusion. 12 pts underwent synchronous carotid endarterectomy and were excluded from further analyses.

Results: $2.2 \%$ of pts had (bilateral) occlusion, $4 \%$ high-grade $(0.6 \%$ bilaterally/with contralateral occlusion), $12.6 \%$ medium-grade ( $0.9 \%$ with contralateral occlusion), $81.1 \%$ low-grade/no stenosis. Reserve capacity was exhausted in $11(0.4 \%)$ pts distal to 9 occlusions and 2 high-grade stenoses. $2.4 \%$ of pts suffered ischemic stroke. Stroke occurred distal to an occluded, high-grade, medium-grade and low-grade/not stenosed artery in 5.7, $0.8,1.3$, and $0.9 \%$, respectively. When arteries with exhausted reserve capacity were considered as a separate category, corresponding percentages were 27.3, 1.3 (occlusion), 0 (high), 1,3 (medium), and 0.9 (low-grade/no stenosis). Exhausted reserve capacity was the strongest of all selected predictors for ischemic stroke (adjusted hazard ratio 20.6, 95\% CI 4.7-90.8; $\mathrm{p}<0.001$; adjusted for sex, age, prior cardiac surgery, prior (ipsilateral) stroke, peripheral arterial disease, atrial fibrillation).

Conclusion: Doppler carbon dioxide testing can be used to identify pts, who have an increased stroke risk at CPBS from carotid stenosis/occlusion. 


\section{Venous diseases/Heart \& brain}

DIFFUSION WEIGHTED IMAGING IN STROKE DUE TO PATENT FORAMEN OVALE - THE SIGNIFICANCE OF CONCOMITANT ATRIAL SEPTUM ANEURYSM

L. Bonati, A. Kessel-Schaefer, A.Z. Linka, P. Buser, S.G. Wetzel, E.W. Radue, P. Lyrer, S.T. Engelter

University Hospital Basel, Basel, Switzerland

Background: Patent foramen ovale (PFO) is an established cause of stroke in young patients without other determined etiologies (i.e. cryptogenic stroke). Additional presence of atrial septum aneurysm (ASA) possibly increases stroke risk, but it remains undetermined which factors best predict thromboembolism in patients with PFO. Diffusion weighted imaging (DWI) may help to distinguish characteristics of cerebral embolism associated with different features of the interatrial septum in PFO stroke.

Methods: In a stroke-databank based cohort study, DWI and transesophageal echocardiography findings were assessed in 48 consecutive patients with cryptogenic ischemic stroke associated with PFO. Number, size and distribution of acute ischemic lesions on DWI were correlated with PFO size, degree of interatrial right-to-left shunt (RLS) and presence of ASA

Results: Patients with PFO plus ASA combined more often had multiple acute DWI lesions $(16 / 30,53 \%)$ than those with PFO alone $(3 / 18,17 \%$; $=0.01)$. This association remained significant after correction for PFO size, degree of RLS and vascular risk factors in a logistic regression analysis $(\mathrm{p}=0.04)$. No significant associations between DWI lesion characteristics and PFO size or degree of RLS were found.

Conclusion: The presence of concomitant ASA is independently associated with multiple cerebral ischemic lesions in PFO stroke, which may indicate an increased embolic risk.

\section{Venous diseases/Heart \& brain}

RELIABILITY OF TRANSTHORACIC ECHOCARDIOGRAPHY PERFORMED BY NEUROLOGISTS IN STROKE PATIENTS: A PILOT STUDY

D. Cocho, Y. Bravo, R. Leta, J. Martí-Fàbregas, F. Carreras, G. Pons,

J.L. Martí-Vilalta

Hospital de la Santa Creu i Sant Pau, Spain

Background: Transthoracic echocardiography (TTE) is the most frequently used method in screening for cardioembolic sources in stroke patients. The aim of this study was to compare the reliability of TTE when performed by neurologists and cardiologists screening in ischemic stroke patients.

Methods: We prospectively examined consecutive patients with stroke. All patients were evaluated with TTE by a cardiologist and also by a neurologist who had been trained in TTE for one month. TTE were performed from all standard echocardiographic windows, and the examiners were blinded to the results.

Cardioembolic sources were classified according to the TOAST criteria. The agreement between the two examinations for the diagnosis of a cardioembolic source was analyzed with the Cohen's Kappa Test (K). A K > 0.6 was considered good. The cardiologist's results were considered the gold standard.

Results: We studied 27 stroke patients: Their mean age was $68.7 \pm 10.2$ years and $70 \%$ were men. Cardioembolic sources were detected in nine patients by the cardiologist and in eight of these by the neurologist (one patient with mitral annulus calcification was not detected, one false negative). In two patients with a deficient echocardiographic window, the neurologist under-estimated the ventricular function (two false positive). Comparison of the two examinations showed an agreement of $88.9 \%(\mathrm{~K}=0.77, \mathrm{p}=0.001)$

Discussion: Neurologists performed the TTE with good reliability in comparison with cardiologists.
ORAL ANTICOAGULATION IN ELDERLY PATIENTS AS SECONDARY PREVENTION OF CARDIOEMBOLIC STROKE

L. Benavente, S. Calleja, J. García-García, S. Rodríguez, J. Villafani, C.H. Lahoz

Hospital Universitario Central de Asturias, Oviedo, Spain

Background: Stroke incidence increases with age. Atrial fibrillation (AF) is a potent risk factor for ischemic stroke and its incidence also increases with age However, the elderly population is thought to have a greater risk of hemorrhagic complications, and oral anticoagulant therapy (OAT) tends to be underused.

Methods: Elderly patients ( $\geq 80$ years) with an ischemic or hemorrhagic stroke admitted in our department from 1/7/2003 to $31 / 6 / 2005$ were prospectively evaluated. Baseline characteristics, risk factors, treatment and aetiology according to TOAST criteria were recorded. Patients treated with OAT were followed in order to asses side effects and recurrence of stroke. Mean follow-up was 19.5 months (7-45) from discharge.

Results: 52 out of 150 elderly patients $(34.7 \%)$ were classified as cardioembolic, mean age was 84.5 years (80-97) and $64.6 \%$ were women. AF was previously known in $60 \%(16.9 \%$ were on OAT and $40.6 \%$ on antiplatelet therapy). At discharge 32 patients $(49.2 \%)$ were on OAT. In the follow-up 4 patients $(12.5 \%)$ suffered from a systemic haemorrhage ( 3 urinary, 1 gastrointestinal bleeding), with no change in functional status. Mean INR in this group was 5.9 (3-11) and, in 3 of them, OAT was stopped. No brain haemorrhages were recorded. Ischemic stroke recurred in 4 patients (INR $<1.8$ in 3 of them, the forth INR 2.35). Three patients had died at the end of the follow-up, one of them as a consequence of ischemic stroke recurrence. Discussion: $28.8 \%$ of stroke patients admitted in the period of study were $>80$ years. The high proportion of cardiembolic strokes in this age segment contrast with the general underuse of OAT as antithrombotic prophylaxis. Our study suggest that OAT is a safe strategy when it is carefully indicated, even in elderly people.

\section{Venous diseases/Heart \& brain}

SILENT CORONARY ARTERY DISEASE AND SUBCLINICAL PERIPHERAL VASCULAR DISEASE IN PATIENTS WITH LACUNAR AND LARGE VESSEL NON-CARDIOEMBOLIC STROKE

J. Calleja, L. Hoyos, E. Vallejo, L. Quintero, A. Leyva, A. Arauz

Instituto Nacional de Neurologia, Mexico City, Mexico

Background: Stroke, coronary artery disease (CAD) and peripheral vascular disease (PVD) share common risk factors and pathophysiologic mechanisms. The relationship between lacunar stroke and occult CAD and PVD has not been evaluated sufficiently. The purpose of the study was to investigate the prevalence of silent CAD and subclinical PVD in patients with large-vessel atherosclerotic and lacunar stroke and to identify factors associated with it presence.

Methods: During a 1 year period, 102 patients with large vessel or lacunar stroke who had no symptoms of CAD or PVD were included. Exclusion criteria were a potential cardioembolic source or a modified Rankin scale $>4$. Demographic data and relevant medical history were collected. Presence of CAD was detected with myocardial perfusion SPECT imaging. PVD was detected using the ankle-brachial index (ABI). Patients were divided in groups according to their subtype of stroke (lacunar vs large vessel).

Results: We included patients with 69 lacunar infarcts and 33 large artery strokes Hypertension was significantly more prevalent in patients with lacunar stroke $78 \%$ vs $45 \%$, p $=0.001)$. Silent CAD was detected in 35 patients (34\%) including $30 \%$ of patients with lacunar stroke and $42 \%$ of patients with large artery stroke $(p=.23)$ The difference between the groups was not significant. Among the different risk factors, only diabetes was independently associated with CAD $(\mathrm{p}=0.32) .6$ patients $(5 \%)$ had severe perfusion deficits and had to undergo further study with invasive procedures. $26 \%$ of patients had an abnormal ABI. Differences between groups were also not significant.

Conclusions: There is a high prevalence of silent CAD and subclinical PVD in patients with non-cardioembolic stroke. Only diabetes was independently associated with CAD. These results warrant further investigation in patients even in the absence of suggestive clinical findings. Lacunar and large vessel subtypes behaved similarly in this respect.
Oral Session

Venous diseases/Heart \& brain 


\section{Meta-analysis and review papers}

\section{Meta-analysis and review papers}

SOURCES OF BIAS IN ANIMAL MODELS OF STROKE

M. Macleod, E. Sena, P. Sandercock, for the CAMARADES collaboration

University of Edinburgh, Clinical Neurosciences, Edinburgh, United Kingdom

Background: A plausible explanation for the failure of potential neuroprotective drugs to show efficacy in clinical trials is that the animal studies may have overestimated efficacy. This might be due to biases introduced by aspects of study design or by limited study quality. Here we report the impact of these potential biases in data drawn from systematic reviews of animal experiments evaluating 13 neuroprotective drugs.

Methods: (1) stratified weighted mean difference random effects meta-analysis; (2) weighted multivariate linear regression analysis.

Results: Data from 516 experiments involving 7,956 animals were included. Study quality was modest (mean score 4/10 items, IQR 3-5), and in stratified meta-analysis low study quality was associated higher estimates of efficacy. A regression model using only 11 factors (including 3 of 10 study quality items - use of diabetic or hypertensive animals, blinded assessment of outcome, and avoidance of ketamine anaesthesia) explained one third of the variation in efficacy ( $r 2=0.303, \mathrm{~F}=19.9$, $\mathrm{p}<10-11$ ). We used this model to estimate the impact these methodological factors might have on the results of an experiment which appeared to show that treatment reduced the volume of infarction by $30 \%$. The table shows the efficacies predicted by the model after adjustment for the impact of each of the study quality items.

Predicted efficacy after adjustment

\begin{tabular}{lcc}
\hline Factor & Observed effect $(\%)$ & Adjusted effect $(\%)$ \\
\hline Comorbidity & $-30 \%$ & $-8.5 \%$ \\
Blinding & $-30 \%$ & $-23.3 \%$
\end{tabular}

Discussion: These observations suggest that study quality and design issues impact significantly on estimates of efficacy. These effects are so large that they might lead some observers erroneously to conclude that a drug had significant neuroprotective actions when in fact it had no protective effect at all.

\section{Meta-analysis and review papers}

AN INDIVIDUAL DATA META-ANALYSIS OF NON-INVASIVE IMAGING OF CAROTID STENOSIS COMPARED WITH INTRA-ARTERIAL ANGIOGRAPHY IN 1982 PATIENTS - THE LITERATURE MAY OVERESTIMATE ACCURACY

J.M. Wardlaw, F. Chappell, J. Gillard, G. Young, M.M. Brown, P.M. Rothwell, G. Roditi, M. Randall, G. Venables, UK NHS Health Technology Assessment Panel Carotid Stenosis Imaging Project

University of Edinburgh, Cambridge, Oxford, UCL, Sheffield, and Glasgow and South Tees NHS Trusts,United Kingdom

Background: Carotid stenosis is a preventable cause of stroke. Accurate diagnosis of carotid stenosis is essential for the correct patients to receive carotid endarterectomy. The literature estimates of accuracy may be over-optimistic.

Methods: We performed an individual patient data (IPD) meta-analysis to determine the accuracy of non-invasive tests for carotid stenosis: Doppler ultrasound (US), CT angiography (CTA), MR angiography (MRA), and contrast enhanced MRA (CEMRA) compared with intra-arterial angiography (IAA). We performed sensitivity analyses on symptomatic status of the artery, patient age and gender, routine audit or research setting, and compared IPD with literature estimates of accuracy.

Results: Amongst 12 datasets (1982 patients), CEMRA was most accurate (sensitivity $81 \%$, specificity $87 \%$ ) for $70-99 \%$ stenosis (NASCET method). Data were inadequate to assess accuracy at moderate (50-69\%) stenoses. Sensitivity/specificity were lower for IPD compared with literature values and in symptomatic than asymptomatic arteries. Age and gender did not affect sensitivity and specificity but there were few older patients.

Conclusion: The literature overestimates non-invasive carotid imaging accuracy. More data are needed to inform routine clinical practice, especially for 50-69\% stenoses. Imaging tests appeared to perform less accurately in symptomatic than asymptomatic arteries. Primary studies should analyse per patient (not per artery), carefully describe the symptomatic status of the patients and the setting in which the tests are performed
3 Meta-analysis and review papers

SHOULD DATA MONITORING COMMITTEES ASSESS EFFICACY WHEN CONSIDERING SAFETY IN TRIALS IN ACUTE STROKE?

P.M.W. Bath, L.J. Gray

University of Nottingham, Nottingham, United Kingdom

Introduction: The primary role of a trial's Data Monitoring Committee (DMC) is to ensure the safety of enrolled patients. Conventionally, safety is monitored by comparing death and stroke specific events such as deterioration and recurrence between treatment groups. Although the DMC may have the remit for monitoring efficacy (functional outcome), trialists may choose for this to be excluded from assessment. We hypothesised that functional outcome is a powerful measure of safety and tested this in a semi-quantitative research synthesis.

Methods: Acute stroke trials with a negative outcome (active treatment worse than control) or which were stopped prematurely on the grounds of safety were sought from searches of electronic libraries. Information on early and late death, impairment, functional outcome, and the presence of a DMC were recorded for each trial. The results of significance tests for each outcome were ranked within each trial to determine which was most efficient statistically in detecting hazard.

Results: 11 negative trials were included (AASI, ASK, ASSIST, EAST, INWEST, MAST-E, MAST-I, DCLHb, sipatrigine, STIPAS, TESS II); insufficient data were present for 2 other trials. The most efficient outcomes were: late death or disability, 5 trials; early death, 4 trials; late death, 1 trial; late death or impairment, 1. Early death was insensitive to hazard in all 5 trials where late death or dependency was most sensitive. Death was the most sensitive measure of hazard in the three trials of thrombolysis. Two trials (both phase II) did not report the presence of a DMC.

Conclusions: All multicentre trials should have an independent DMC. End of trial functional outcome should be included in all assessments of safety, whether or not efficacy itself is being assessed.

\section{Meta-analysis and review papers}

IV THROMBOLYSIS IN STROKE PATIENTS AGED 80 YEARS AND MORE VERSUS $<80$ YEARS OF AGE - A SYSTEMATIC REVIEW ACROSS COHORT STUDIES

S. Engelter, L. Bonati, P. Lyrer

University Hospital Basel, Basel, Switzerland

Objective: Elderly stroke patients were excluded or underrepresented in the randomized controlled trials of iv thrombolysis with recombinant tissue plasminogen activator (rtPA) applied within 3 hours. Cohort studies comparing iv rtPA in stroke patients aged 80 years and more versus $<80$ years of age were limited by small sample sizes. Thus, we peformed a systematic review across all such studies.

Methods: A systematic literature search (PubMed; Sience Citation Index) was performed to retrieve all eligible studies. Two reviewer independently extracted data on "death", "favorable 3-month-outcome (modified Rankin Scale 0-1)" and "symptomatic intracranial hemorrhage (sICH)". Across studies, weighted odds ratios (OR) with $95 \%$ confidence intervals $(95 \% \mathrm{CI})$ were calculated

Results: Five cohort studies ( $\mathrm{n}=1021$ patients) were included. Lacking information about the distribution of missing data was the most important methodological flaw. Compared to younger patients, older patients had a 3.96 times (95\% CI 2.44-6.42) higher mortality. No significant differences were shown for "favorable outcome" (OR 0.69; 95\%CI 0.45-1.07), "favorable outcome among survivors" (OR 0.95; 95\%CI 0.59-1.53); and for "sICH" (OR 1.25; 95\%CI 0.42-2.42).

Conclusion: Present evidence suggests that there is scope for benefit from iv rtPA for stroke patients aged 80 years and over. Hence, in order to obtain reliable evidence on the balance of risk and benefit, it is reasonable to include such patients in randomized controlled trials.

\section{Meta-analysis and review papers}

THE ASSOCIATION BETWEEN CHLAMYDIA PNEUMONIAE AND STROKE IS BIASED BY POLYMERASE CHAIN REACTION METHODOLOGY

P. Apfalter, E. Diabl

Medical University Vienna, Vienna, Austria

Background: Polymerase chain reaction (PCR) for detection of Chlamydia (C.) pneumoniae is not standardized, but has been widely used to study a possible link between the bacterium and atherosclerosis. As of 2005, 28 out of 38 studies addressing the question of whether or not C. pneumoniae is present in cerebrovascular atherosclerosis were PCR-based. The aim of this study was to review the literature with special focus on methodological details in order to find explanations for the highly variable positivity ratios reported. 
Material and Methods: Studies were identified by searching Medline.

Results: Data on 971 atherosclerotic carotid arteries and 731 blood specimens from 1485 patients analyzed by 35 in-house PCR protocols revealed positivity ratios between 0 and $100 \%$, even apparently when the same methods were used. Steps where problems could have arised include pre-analytical procedures, DNA extraction, assay design, format, and set-up, interpretation, confirmation of results and quality control issues. The results of this analysis reveal major inconsistencies with PCR methodology. Nearly all the investigators used nested PCR which is highly prone to produce false positive results and did not confirm their positive findings.

Conclusions: The variations in results from study to study are probably due to technical issues rather than differences in clinical settings or geographical regions. It seems as if detection of C. pneumoniae-DNA failed when the specificity of the reaction was optimized. Unless a standardized PCR is available, the true prevalence of C. pneumoniae-DNA in tissue from patients with cerebrovascular atherosclerosis cannot be accurately determined.

\section{Meta-analysis and review papers}

SYSTEMATIC REVIEW AND META-ANALYSIS OF THE EFFICACY OF TISSUE PLASMINOGEN ACTIVATOR (TPA) IN EXPERIMENTAL STROKE E. Sena, C. Briscoe, P. Sandercock, M. Macleod

University of Edinburgh, Clinical Neurosciences, Edinburgh, United Kingdom

Background: tPA is a highly effective treatment for selected patients with acute ischaemic stroke. However, uncertainty remains regarding efficacy beyond 3 hours and the risk of haemorrhage. Animal experiments are held to "model" human stroke, and may therefore provide some insights on these issues. We report a systematic review and meta-analysis of the efficacy of tPA in animal models of stroke.

Methods: Systematic review of animal studies assessing the effects of tPA on infarct volume, neurobehavioural score or haemorrhage in models of focal cerebral ischaemia.

Results: An extensive search identified 2771 studies of which 131 were included. Study quality was modest (mean score 4/10, IQR 3-5). Funnel plotting and Egger regression suggested a substantial publication bias. tPA reduced infarct volume by $24.2 \%$ (95\% CI $20.3 \%-28.0 \%$ ), improved neurobehavioral score by $23.4 \%$ (95\% CI $17.3 \%-29.4 \%)$, and was associated with increased risk of haemorrhage (odds ratio $1.96,95 \%$ CI 1.63 to 2.35 ). Efficacy was time dependent, and the risk of haemorrhage increased with delay to treatment. Although most animal studies post-dated the pivotal clinical trials, many of these later animal studies reported the efficacy of tPA alone compared with efficacy in combination with potential neuroprotectants.

Discussion: While tPA appears effective in animal models of acute ischaemic stroke, this conclusion must be qualified because of the potential effects of study quality and publication biases. The animal data support a time dependence for efficacy, and suggest that the risk of haemorrhage following tPA in human stroke may increase substantially with delays to treatment beyond 4-5 hours. An analysis of animal data reporting outcome following combined treatment is ongoing.

\section{Genetic disorders}

\section{Genetic disorders}

\section{BLOOD PRESSURE AND HEMOGLOBINA1C ARE ASSOCIATED WITH} CEREBRAL MICROHEMORRHAGE IN CADASIL: A MULTICENTER COHORT STUDY

A. Viswanathan, J.-P. Guichard, A. Gschwendtner, F. Buffon, R. Cumurcuic,

C. Boutron, M. Holtmannspötter, M.G. Bousser, M. Dichgans, H. Chabriat Hôpital Lariboisière, Paris, France

Background and Purpose: Cerebral autosomal dominant arteriopathy with subcortical infarcts and leukoencephalopathy (CADASIL) is a hereditary small-vessel arteriopathy caused by mutations of the Notch3 gene. The incidence and risk factors for cerebral microhemorrhages (CM) in CADASIL and their relationship to other MRI lesions is not defined. Our purpose was to examine the frequency, number, and location of microhemorrhages in a multicenter cohort study, defining predisposing factors and associated MRI markers.

Methods: We reviewed data from 147 consecutive patients enrolled in an ongoing prospective cohort study. Clinical variables, cognitive status and degree of neurologic disability were recorded. MRI was performed in all patients. Volume and location of lacunar infarcts and white matter hyperintensity (WMH) were assessed. Number and location of CM were recorded.
Results: CM were present in $38 \%$ patients, most commonly occurring in the thalamus $(61.5 \%)$, brainstem and basal ganglia (38.5\%). Location of CM was different from areas of lacunar infarction and $\mathrm{WMH}$. There was a significant association between presence of CM and systolic blood pressure $(\mathrm{p}=0.014), \mathrm{HbA1c}(\mathrm{p}=0.004)$, and the volume of lacunar infarcts $(\mathrm{p}=0.010)$ and WMHs $(\mathrm{p}=0.046)$. The number of $\mathrm{CM}$ was independently associated with $\mathrm{WMH}$ volume $(\mathrm{p}=0.0159)$. There was no association between these risk factors and the load of WMH or lacunar infarcts. The presence of $\mathrm{CM}$ was associated with functional dependence but not with cognitive impairment.

Conclusions: CM are common in CADASIL and are associated with blood pressure, $\mathrm{HbA1c}$, lacunar infarct and WMH volume. Risk factors for $\mathrm{CM}$ are not associated with other MRI markers, suggesting that they may represent an independent pathologic process that has an impact on clinical outcome.

\section{Genetic disorders}

\section{GENETIC VARIANTS IN THE LEUKOTRIENE SYNTHESIS PATHWAY} CONFER AN INCREASED RISK OF ISCHAEMIC STROKE

S. Bevan, P. Patak, M. Sitzer, H. Markus

Saint George's, University of London, Tooting, London, United Kingdom

Background: Leukotrienes are short lived, potent pro-inflammatory modifiers produced by inflammatory cells in response to a number of stimuli. Modification of leukotriene action has been used effectively in the treatment of asthma and has recently been postulated as a treatment for cardiovascular disease. Identification of the gene encoding 5-lipoxygenase activating protein as conferring an increased risk of MI and stroke has now placed the leukotriene pathway at the centre of cardiovascular genetics research. We have sought to assess the effect of a series of genetic variants in members of the leukotriene pathway on ischaemic stroke risk using a case control methodology.

Methods: Stroke cases $(n=900)$ and controls ( $n=933)$ were recruited in the UK from a single centre with cases having standard stroke investigation including risk factor evaluation, brain CT or MRI. Subtyping was performed by a single physician. SNPs in LTA4H, 5-LO, LTB4R1/2, LTC4S and CYSLTR1/2 were typed by fluorescent allele calling with all typing performed blind to patient details. Statistical analysis was undertaken globally and by stroke subtype.

Results: A variant in LTC4S was associated with a 1.8 fold increase in risk for all ischaemic stroke $(\mathrm{p}=0.00695 \%$ CI $1.181-2.763)$. The same variant also imparted a significant risk in large vessel disease, lacunar stroke and stroke of unknown aetiology independently. Two variants in the leukotriene B4 receptor conferred a 2 fold increase in risk in cardioembolic stroke $(2.31(1.282-4.387) \mathrm{p}=0.006$ and 2.04 $(1.15-3.62) \mathrm{p}=0.015$ respectively) and remained significant on haplotype analysis. Discussion: Genetic variation within members of the leukotriene synthesis pathway confer a risk in both ischaemic stroke and specific ischaemic stroke subtypes in our population. A replication of these findings is underway in a second population, which if confirmatory may have implications for the use of leukotriene modifiers as therapeutic agents.

\section{Genetic disorders}

CLINICAL SPECTRUM IN 14 ITALIAN CADASIL PATIENTS: ADVANCED AGE OF PRESENTATION AND ATYPICAL SYMPTOMS

F. Pescini, L. Pantoni, C. Sarti, S. Bianchi, L. Bartolini, M.T. Dotti, A. Federico, D. Inzitari

University of Florence, Florence, Italy

Objective: CADASIL (Cerebral Autosomal Dominant Arteriopathy with Subcortical Infarcts and Leukoencephalopathy) is an inherited vascular disease clinically characterized by TIA/strokes, cognitive deficits, migraine, mood disturbances, and epilepsy. The phenotypic expression is however variable and new clinical features have been reported.

We describe clinical aspects of 14 consecutive CADASIL patients (5 males) belonging to 9 families.

Results: Time of first neurological evaluation was at advanced age (mean $51 \pm 15$ years), despite an earlier onset of the disease (mean age 29 years \pm 16 ; 64\% with migraine). Clinical features were: TIA/strokes (57\%), cognitive deficits (71\%), migraine $(86 \%)$, and mood disturbances $(79 \%)$. Patients presented with a high frequency of atypical symptoms such as somnolence (36\%), acute mental confusion $(29 \%)$, language and visual field deficits $(21 \%)$, consciousness alterations $(14 \%)$, psychosis $(7 \%)$. Neurological presentation was at advanced age in 3 patients $(67$, 72 , and 77 years) with 2 patients suffering from gait disturbances and 1 from epilepsy. All but 2 patients presented confluent or partly-confluent MRI white matter changes. Involvement of the temporal poles was variable in terms of severity. Family history was positive for at least one CADASIL clinical hallmark in all cases. Notch3

Genetic disorders 
mutations involved exon 11 (3 families), 19 (2 families), 3, 4, 6, and 10 in one family each.

Conclusions: CADASIL should be suspected also in patients of older age-groups, with minimal disturbances, or presenting atypical symptoms. Since genetic testing for CADASIL diagnosis is expensive and time consuming, selection criteria for Notch3 analysis likely based on clinical, historical, and MRI evaluation is badly needed.

\section{Genetic disorders}

\section{APOE GENE PROMOTER POLYMORPHISMS AS DETERMINANT OF} MIDDLE-AGE ISCHEMIC STROKE

S. Abboud, L. Viiri, S. Blecic, D. Luetjohann, A. Peteers, P. Laloux, P. Karhunen, T. Lehtimaki, R. Laaksonen, M. Pandolfo

Erasme Hospital, Brussel, Belgium

Background: Apolipoprotein E (APOE) E4 as a candidate gene for ischemic stroke (IS) has shown conflicting results in association studies, probably due to studies designs and the heterogeneity of IS

Objective: We investigated the possible association between 4 single nucleotide polymorphisms (SNPs) of APOE gene and IS in a selected Belgium stroke population. We used cerebral atherosclerosis (CA) as intermediate phenotype

Design: This multi-centre retrospective case control study included 200 patients (45-60 yrs) with lacunar and atherotrombotic stroke, and 180 gender and ethnicity matched controls ( $>60 \mathrm{yrs}$ ) with no history of stroke or MI. We measured the plasma concentration of $\mathrm{CH}$, lathosterol (a $\mathrm{CH}$ precursor, using its ratio to $\mathrm{CH}$ as a marker of endogenous $\mathrm{CH}$ synthesis) and plant sterols (using their ratio to $\mathrm{CH}$ as markers of intestinal sterol absorption). We genotyped the APOE E2E3E4 SNPs and two SNPs 219G/T and 113G/C located in the promoter region of the gene. Positive results were then tested in a Finnish population of 640 autopsy cases with an assessment of atherosclerosis score in the nine branches of the circle of Willis

Results: The GG homozygosity of the two promoters SNPs and the 219G/113G haplotype associate significantly with stroke cases $(\mathrm{p}=0.026 \mathrm{p}=0.022 \mathrm{p}=0.011)$ as compared to TT, CC homozygosity and the 219T/113C haplotype, after adjustment for known vascular risk factors. The 219G/T SNP did not associate with CA Conclusion: We demonstrate that the GG homozygote of the two APOE gene promoter SNPs as well as their haplotype carriers are at increased risk of developing IS. APOE - $219 \mathrm{G} / \mathrm{T}$ is a functional SNP producing variations in the transcriptional activity of the gene; our finding suggests that quantitative rather than qualitative variation of APOE is determinant in the development of IS. Moreover our results suggest that APOE is implicated in IS by another mechanism than changes in $\mathrm{CH}$ metabolism or development of CA

\section{Genetic disorders}

PARAOXONASE 1 Q192R AND M55L AND PARAOXONASE 2 C311S POLYMORPHISMS AND RISK FOR DIFFERENT ETIOLOGIES OF ISCHEMIC STROKE

A. Slowik, D. Partyka, W. Turaj, P. Wolkow, M. Malecki, T. Dziedzic, J. Pera, A. Szczudlik

Jagiellonian University, Krakow, Poland

Oxidative stress plays a role in atherothrombosis. The human paraoxonase (PON) gene family consists of PON1, PON2 and PON3, which are located on chromosome 7. PON gene products exert antioxidant properties. We studied the significance of Q192R and M55L polymorphisms of the PON1 gene and C311S polymorphisms of the PON2 gene in different etiologies of ischemic stroke.

Data were obtained in the setting of a single center, hospital-based study. 136 patients with large vessel disease (LVD) stroke, 140 with small vessel disease stroke and 218 with cardioembolic stroke and their age and sex matched controls were included. The distribution of PON1 polymorphisms was similar in each group of stroke patients as compared with controls. The distribution of PON2 C311S genotypes (chi2 $2 \mathrm{df}=5.88, \mathrm{P}=0.02$ ) and alleles (chi2 $1 \mathrm{df}=5.96, \mathrm{P}=0.01$ ) differed significantly only in patients with LVD stroke as compared with controls. Genotypes with the $\mathrm{C}$ allele were over-represented in LVD patients as compared with controls, both in univariate and multivariate $(1.59,95 \% \mathrm{CI}: 1.01-2.49, \mathrm{p}<0.05)$ analyses. A gene-environment interaction was found between PON2 C311S and hypertension. Adjusted OR of stroke for hypertensive subjects with the SS genotype when compared with normotensive subjects with the SS genotype was 6.16 (95\%CI: 2.85-13.29, $\mathrm{p}<0.05)$. Adjusted OR of stroke for normotensive subjects with the $\mathrm{C}$ allele as compared with normotensive subjects with the SS genotype was 1.35 (95\%CI: 1.21-1.50, $\mathrm{p}<0.05$ ). A further increased risk of stroke was found in hypertensive subjects who concomitantly carried the $\mathrm{C}$ allele as compared with normotensive subjects with the SS genotype [adjusted OR for stroke: 8.11 (95\% CI: 3.78-17.38, $\mathrm{p}<0.05$ ].
Genotypes with $\mathrm{C}$ allele of PON2 gene and hypertension display synergistic effects on the risk of LVD stroke. This result emphasizes the importance of gene-environmental interactions in stroke patients.

\section{Genetic disorders}

\section{ASSOCIATION BETWEEN APOLIPOPROTEIN E GENOTYPE (APOE) AND} CAROTID INTIMA-MEDIA THICKNESS (CIMT)

N.A Martinez-Gonzalez, L. Paternoster, S. Lewis, C. Sudlow

University of Edinburgh, Edinburgh, United Kingdom

Background: APOE influences cholesterol levels and ischaemic heart disease, but no convincing association with ischaemic stroke has been identified. CIMT may be an informative intermediate phenotype, particularly for large artery ischaemic stroke. Studies assessing the effect of APOE on CIMT have produced conflicting results. We aimed to evaluate the association more reliably with a systematic review and meta-analysis.

Methods: A comprehensive search strategy found 18 relevant published studies (12888 subjects) with appropriate data. We extracted data on the types of subjects studied (including high vs low risk, ie. those with vs without risk factors for or a history of vascular disease, and ethnicity), study methods, and mean (\& SD) CIMT for those with E4 (e4/e4 or e4/e3), E2 (e2/e2 or e2/e3) and E3(e3/e3) genotypes We compared these means for each study and overall using the weighted mean difference (WMD) random effects method in Cochrane Revman. We examined funnel plots, and did several pre-specified sensitivity subgroup analyses to assess whether effects varied with high vs low risk status, ethnicity, study size and method of CIMT measurement.

Results: As predicted from their known effects on cholesterol levels, E4 genotypes increased and E2 genotypes decreased mean CIMT, with the greatest contrast between E4 and E2 genotypes (WMD [in mm] E4 vs E2 = 70, 95\% CI 40 to 100). However, there was substantial heterogeneity between studies $(\mathrm{p}<0.00001)$ and funnel plots showed small study bias. Results from larger studies ( $>600$ subjects) were homogeneous and found a significantly smaller difference (WMD 20 (95\% CI 6 to 30$)$.

Discussion: APOE probably does influence CIMT but small study bias means that the published literature substantially overestimates the effect size.

\section{Brain imaging - new developments}

\section{Brain imaging - new developments}

BRAIN VOLUME CHANGES CORRELATE WITH CLINICAL PROGRESSION IN CADASIL: A SERIAL MRI STUDY IN PURE SUBCORTICAL ISCHEMIC VASCULAR DISEASE

N. Peters, M. Holtmannspötter, C. Opherk, A. Gschwendtner, J. Herzog, P. Sämann, M. Dichgans

Klinikum Grosshadern, Munich, Germany

Objective: CADASIL is monogenic microangiopathy leading to stroke and subcortical vascular dementia. Recent studies in sporadic subcortical ischemic vascular disease have found cerebral atrophy to be a clinically important marker of disease progression. However, little is known about the role of brain atrophy and its clinical correlates in CADASIL.

Method: Using MRI, we determined the normalized brain volume (NBV) and percent brain volume change (PBVC) over two years in 76 CADASIL subjects $(45.1+9.7$ years) using the SIENA (structural image evaluation using normalization of atrophy) software and its adaptation for cross-sectional measurement (SIENAX). Baseline assessments included systolic blood pressure (SBP), smoking, hypercholesterolemia, homocysteine levels, body mass index (BMI), and APOE genotyping. Additionally, T2-lesion volumes and clinical scales were assessed at both time points.

Results: The NBV significantly correlated with all clinical scores (Rankin, NIHSS, Barthel, SIDAM, MDRS) at both time points independently of age and gender. PBVC significantly correlated with changes of all clinical scores (all $\mathrm{p}<0.01)$ except for the MDRS. In a linear regression model, age $(\mathrm{p}<0.001)$, male sex $(\mathrm{p}<0.01)$, and $\operatorname{SBP}(p=0.08)$ were the main risk factors for a lower NBV at baseline. Age $(p<0.001)$ and SBP $(\mathrm{p}=0.01)$ were risk factors for brain volume loss during follow-up. No association was found with T2-lesion volume. Sample size calculation showed that the number of individuals needed to demonstrate a treatment effect in a treatment trial can be reduced when $\mathrm{PBVC}$ is used as an endpoint compared to clinical endpoints.

Conclusions: This study identifies brain atrophy as an important aspect of the 
disease process in CADASIL and establishes significant correlations with multiple clinical aspects including cognition. Age and SBP are risk factors for brain volume loss during follow-up. PBVC seems promising as additional outcome measure in future interventional trials.

\section{Brain imaging - new developments}

\section{NON-CONTRAST CT EARLY ISCHEMIC CHANGES UNDERESTIMATE ISCHEMIC CORE IN THE PRESENCE OF AN INTRACRANIAL ARTERIAL OCCLUSION}

I. Dzialowski, S. Subramaniam, A. Krol, J. Roy, M.D. Hill, P.A. Barber,

J.M. Boulanger, S.B. Coutts, T. Watson, A.M. Demchuk

University of Calgary, Calgary, Canada

Background: Recent work with multimodal computed tomography (CT) in acute ischemic stroke suggests that $\mathrm{CT}$ angiography-source images (CTA-SI) more accurately predict ischemic core than non-contrast CT (NCCT). We studied the hypothesis that a mismatch between NCCT and CTA-SI is associated with intracranial arterial occlusion.

Methods: We retrospectively studied patients with acute ischemic stroke from 04/2002-04/2005. Patients were included in the study if NCCT and CTA were performed from skull base to supraganglionic level, within $12 \mathrm{hrs}$ from symptom onset and within a $30 \mathrm{~min}$ interval. We independently applied the Alberta Stroke Program Early CT Score (ASPECTS) to the baseline NCCT and CTA-SI by $3-$ reader-consensus. An ASPECT score $=10$ indicates a normal scan, a score $=0$ a complete acute middle cerebral artery infarction. Clinical baseline variables and intracranial vessel status on CTA were recorded from the patient chart. According to the ASPECTS-difference between NCCT and CTA-SI, we classified patients into 3 groups: 1) NCCT $>$ CTA-SI by $=>2$ points, 2$)$ NCCT $=$ CTA-SI \pm 1 and 3) NCCT $<$ CTA-SI by $\leq 2$ points.

Results: We identified 175 patients meeting inclusion criteria with a median age of 71 years, onset-to-CTA-time of $140 \mathrm{~min}$, baseline NIHSS of 9, NCCT ASPECTS of 10 and CTA-SI ASPECTS of 9. Intracranial occlusion was present in 88/175 patients $(50 \%)$. Proportions of patients with intracranial occlusion were $81 \%(\mathrm{n}=36)$ for group 1, 43\% ( $\mathrm{n}=116)$ for group 2, and 39\% $(\mathrm{n}=23)$ for group $3(\mathrm{p}<0.001$, Chi-square-test).

Conclusion: Applying a semi-quantitative scoring system (ASPECTS) to NCCT and CTA-SI in acute ischemic stroke, the finding of a significant NCCT $>$ CTA-SImismatch is associated with high likelihood of intracranial arterial occlusion. In the presence of intracranial occlusion, CTA source images should be reviewed as it better demonstrates the full extent of irreversible ischemic injury and may factor to treatment decision making.

\section{Brain imaging - new developments}

FEASIBILITY OF MULTI-PLANAR ULTRASOUND PERFUSION IMAGING IN ACUTE STROKE PATIENTS

F. Sallustio, R. Kern, M. Kablau, M.G. Hennerici, S. Meairs

Universitätsklinikum Mannheim, University of Heidelberg, Mannheim, Germany

Background: Ultrasound perfusion imaging (UPI) analyzing bolus kinetics after echo-contrast agent injection was shown to be useful for the assessment of cerebral perfusion. However, one of the major shortcomings of this approach is that only a single plane can be evaluated. Microbubble destruction imaging (MDI) is a new and fast approach for UPI which can be repeated, and therefore seems to be suitable for evaluation of multiple planes. This is a first study on the feasibility of multi-planar MDI to assess the extension of perfusion deficits in patients with acute middle cerebral artery (MCA) stroke.

Methods: Twelve patients with acute MCA stroke and sufficient transtemporal bone windows were investigated with multi-planar MDI, diffusion weighted (DWI) - and perfusion MRI (PW-MRI). US images were adjusted from both sides to 3-5 standardized axial planes, depending on the quality and size of the temporal bone window. Local perfusion status was analyzed in selected regions of interest using destruction curves and acoustic intensity differences (AID) before and after microbubble destruction. The extension of the perfusion deficit on multi-planar MDI was graded semiquantitatively and compared to DWI and PW-MRI

Results: Mean AIDs from the ischemic MCA territory were significantly diminished compared with the normal hemisphere as a result of hypoperfusion. Multi-planar assessment of at least 3 planes of each hemisphere using MDI was possible in 9/12 patients. The extension of the perfusion deficit could be classified correctly using multi-planar MDI in these 9 patients. In 3 patients, however, only 2 planes for each hemisphere could be evaluated due to the small size of the temporal bone window.

Oral Session

Brain imaging - new developments
Discussion: The study confirms multi-planar MDI as suitable method to evaluate the extension of perfusion alteration in acute stroke patients. Provided that good insonation conditions are present, the assessment of at least three planes on each hemisphere is possible.

\section{Brain imaging - new developments}

DIFFERENCES IN LESION EVOLUTION ON DIFFUSION-WEIGHTED BRAIN IMAGING (DWI) IN RELATION TO CAROTID STENOSIS

U. Schulz, J.N. Redgrave, S. Mazzucco, E. Flossmann, P.M. Rothwell

Stroke Prevention Research Unit, University Department of Clinical Neurology, Oxford, United Kingdom

Background: Haemodynamically significant carotid stenosis changes the diffusion characteristics of brain tissue in the ipsilateral hemisphere. Carotid disease may therefore also influence the temporal evolution of ischaemic lesions on DWI. We studied the association between carotid disease and the presence and evolution of ischaemic lesions on DWI in a large cross-sectional cohort and in a follow-up study. Methods: 500 consecutive patients investigated late ( $>3$ days) after TIA or minor stroke underwent DWI and carotid imaging. In a further study, 40 patients presenting within 2 weeks of minor stroke or TIA underwent carotid imaging and serial DWI at baseline and at 1,2 and 3 months or until endarterectomy (if indicated). The signal evolution of any lesion over time was recorded and the occurrence of any new lesions during follow-up noted.

Results: In the cross-sectional study, presence of a recent ischaemic lesion on DWI was associated with $>50 \%$ ipsilateral carotid stenosis $(\mathrm{OR}=1.8,1.1-3.2, \mathrm{p}=0.04)$. In the longitudinal study, all 4 patients who had new lesions on follow-up had $\geq 50 \%$ carotid stenosis. However, in both studies, DW-signal intensity was higher $(\mathrm{p}=0.007)$ and the ADC tended to be lower $(\mathrm{p}=0.05$ ) in patients with no significant stenosis In the longitudinal study, the ADC also normalized more quickly in patients with carotid disease.

Conclusion: As well as influencing diffusion characteristics of normal brain, carotid stenosis also influences the temporal evolution of ischaemic lesions, with reduced intensity on DWI and more rapid normalisation of the ADC. The high prevalence of ischaemic lesions on DWI in the subacute phase in patients with carotid stenosis is likely to be due to recurrent asymptomatic ischaemia rather than to lesion persistence.

\section{Brain imaging - new developments}

TISSUE AT RISK IN PATIENT WITH SEVERE ICA OR MCA STENOSIS CAN BE EASILY ASSESSED BY FMRI WITH BREATH HOLDING C. Dannenberg, H. Hentschel, T. Goldhagen, T. Scholle, C. Disque, A. Werner, G. Gahn, R. von Kummer

University Hospital, Technische Universität Dresden, Dresden, Germany

Background/Purpose: Measurement of increase of cerebral blood flow velocity in patients with severe ICA- or MCA-stenosis by breath-holding transcranial Doppler ultrasound is used to evaluate the risk for ischemic events. It gives vessel flow, but no parenchyma information. It is impossible in about $15 \%$ of patients because transtemporal bone ultrasound window is missing. The aim of our study was to develop a simple, fast and reliable MRI method for cerebrovascular reactivity in healthy volunteers and test its sensitivity in patients with severe ICA- or MCA-stenosis.

Methods: We optimised the MRI measurement protocol in 4 healthy volunteers and included so far 8 patients with moderate to severe ICA or MCA stenosis. We used BOLD imaging for breath-holding fMRI: EPI sequences, 30 transversal slices, TR $3 \mathrm{sec}, 150$ runs, Block design, 1,5T scanner (Sonata, Siemens), data analysis with Brainvoyager (Brain Innovation B.V.): general linear model, FDR $<0,05$. We performed CE-MR angiography in all patients, perfusion MRI in 7 patients, additional breath-holding transcranial doppler ultrasound in 6 patients.

Results: Breath-holding fMRI was successfully performed in all cases. All volunteers and 4 patients had no regional deficits of BOLD response. In 4 patients, different parts of vascular territories had a reduced BOLD response, 1 of these patients developed ischemic events in the involved brain area. TTP shortening in perfusion MRI was not able to predict the area of diminished BOLD response. Results of transcranial Doppler ultrasound during breath holding were in accordance with fMRI results.

Conclusion: Breath-holding fMRI seems to be a useful and feasible technique in assessment of brain tissue at risk for infarction in patients with severe ICA- or MCA-stenosis. We are currently studying whether angioplasty can reduce the brain tissue volume with impaired cerebral vasoreactivity. 


\section{Cerebral haemorrhage and SAH}

\section{Cerebral haemorrhage and SAH}

ASSESSMENT OF FEASIBILITY OF ENDOVASCULAR TREATMENT OF RUPTURED INTRACRANIAL ANEURYSMS WITH 16-DETECTOR ROW COMPUTED TOMOGRAPHY ANGIOGRAPHY

M. van der Jagt, H. Flach, H.L.J. Tanghe, S.L.M. Bakker, M.G.M. Hunink, P.J. Koudstaal, A. van der Lugt

Erasmus MC, Rotterdam, Rotterdam, The Netherlands

Background: The value of computed tomography angiography (CTA) in the assessment of feasibility of endovascular treatment (EVT) of ruptured intracranial aneurysms has not been studied. We therefore investigated whether 16-detector row CTA was sufficient as pretreatment investigation to assess feasibility of EVT of ruptured intracranial aneurysms, and whether digital subtraction angiography (DSA) had additional value compared with CTA.

Methods: 80 consecutive patients with aneurysmal subarachnoid hemorrhage who were referred to our university center between 2002 and 2004 and who underwent both CTA and DSA were included in the study. Two experienced interventional neuroradiologists independently scored 3D-CTA and-immediately thereafter- DSA hard-copies with respect to feasibility to treat the ruptured aneurysm endovascularly. We determined whether CTA alone was sufficient for a definite judgment. We also assessed interobserver agreement with respect to feasibility judgments based on CTA alone or the combination of CTA and DSA.

Results: Reader 1 and 2 were able to make a definite judgment on feasibility of EVT with CTA alone in $58(73 \%)$ and $57(71 \%)$ of the patients, respectively. DSA results never changed definite judgments by CTA alone, except in one patient who underwent DSA later than CTA when vasospasm had subsided. DSA yielded additional or affirmative anatomical information in 19\% (reader 1) and 14\% (reader 2), whereas in $33 \%$ and $12 \%$ DSA was considered inferior to CTA. Interobserver agreement on feasibility of EVT both before and after viewing DSA was just fair (kappa is 0.35 and 0.33 , respectively).

Discussion: 16-Detector row CTA without DSA is a reliable pretreatment investigation to assess feasibility of EVT of ruptured intracranial aneurysms. However, interobserver disagreement on feasibility of EVT is considerable.

\section{Cerebral haemorrhage and $\mathrm{SAH}$}

AGE-DEPENDENT INCIDENCE OF AVM HEMORRHAGE: THE NEW YORK ISLANDS AVM STUDY

C. Stapf, C. Hofmeister, H. Mast, J.H. Choi, J.P. Mohr, for the New York Islands AVM Study Collaborators

Columbia University, New York, NY, USA

Background: Prospective data on the effect of age on incident rupture of brain arteriovenous malformations (AVMs) are scarce.

Subjects \& Methods: The New York Islands AVM Study is an ongoing prospective population-based survey since March 15, 2000. The catchment area (i.e., Manhattan Island, Staten Island, and Long Island) comprises 9,429,541 residents according to the 2000 census. All major NY islands hospitals prospectively report demographic data on consecutive patients with a diagnosis of brain AVM and whether or not the patient had suffered AVM hemorrhage. Referral patients living outside the ZIP code-defined study area were excluded from the study population.

Results: As of March 14, 2005, 638 prospective AVM patients (mean age 41 years, $\pm 18 \mathrm{SD}, 53 \%$ women) were encountered leading to a calculated AVM detection rate of 1.35/100,000 person-years (95\% CI: 1.25 to 1.46) with an estimated incidence of first-ever AVM hemorrhage ( $\mathrm{n}=246$, mean age 38 years $\pm 19 \mathrm{SD}, 52 \%$ women) of $0.52 / 100,000$ person-years (95\% CI: 0.46 to 0.59 ). The prevalence of AVM hemorrhage among detected cases $(\mathrm{n}=301)$ was $0.64 / 100,000(95 \%$ CI: 0.57 to 0.71$)$. The relative frequency of hemorrhagic presentation decreased with age $(\mathrm{p}<0.0001)$ and was significantly higher in patients aged $<20(58 \%)$ when compared to those $\geq 20$ years $(45 \% ; \mathrm{p}=0,02)$.
Conclusions: Our 5-year data suggest stable rates for AVM detection and incident AVM hemorrhage in this geographically defined population. Overall, approximately half of AVM patients may suffer intracranial hemorrhage. The relative proportion of incident AVM rupture among detected cases appears to be inversely associated with age.

\section{Cerebral haemorrhage and $\mathrm{SAH}$}

RECURRENT CORTICAL SPREADING DEPRESSIONS AS A MECHANISM OF DELAYED ISCHEMIC NEUROLOGICAL DEFICIT AFTER SUBARACHNOID HEMORRHAGE

J.P. Dreier, J. Woitzik, M. Fabricius, R. Bhatia, S. Major, C. Drenckhahn, T.-A. Lehmann, A. Sarrafzadeh, M. Lauritzen, A.J. Strong, for the COSBID study group

Charité University Medicine, Berlin, Germany

Background: Animal research has provided evidence that spreading depression (SD) is a key component of cortical infarcts induced by breakdown products of erythrocytes in the subarachnoid space. Cortical infarcts crossing the vascular boundaries represented the predominant lesion type associated with delayed ischemic neurological deficit (DIND) after subarachnoid hemorrhage (SAH) in human autopsy studies ( $80 \%$ of patients). Here, we studied whether SD occurs in patients with SAH.

Methods: Four centers of the Co-operative Study on Brain Injury Depolarizations (COSBID) consecutively recruited 18 patients with aneurysmal SAH undergoing open surgical treatment. A subdural strip electrode was placed on cortex to record SD. SD was defined as a propagating depression of electrocorticographic activity associated with a spreading, slow potential shift.

Results: Thirteen of 18 patients (72\%) showed 298 spreading depressions in 2110 hours of recording time. In seven cases, clusters of SD were observed time-locked to the onset of DIND. In those subdural electrodes with close spatial relation to a newly developing infarct on CT, the electrophysiological evolution of stroke was characterized by a pattern change of SD towards progressively prolonged electrocorticographic depression periods in all three patients. This pattern change is typical of SD under energy compromise in animals as in the ischemic penumbra or during spreading ischemia induced by subarachnoid breakdown products of erythrocytes. Discussion: Recurrent spreading depressions represent the electrocorticographic correlate of DIND after SAH. This is the first study which demonstrates SD in the evolution of infarcts in the human brain.

\section{Cerebral haemorrhage and $\mathrm{SAH}$}

PRE-TREATMENT WITH ANTIPLATELETS DOES NOT CONTRIBUTE TO MORTALITY AND UNFAVORABLE OUTCOME IN INTRACEREBRAL HEMORRHAGE

C. Foerch, B. Misselwitz, M. Sitzer, T. Neumann-Haefelin, Arbeitsgruppe Schlaganfall Hessen (ASH)

Johann Wolfgang Goethe-University, Frankfurt am Main Germany

Background: Oral anticoagulation increases the mortality of intracerebral hemorrhage $(\mathrm{ICH})$. Recent studies indicated that pre-treatment with antiplatelet agents could also be associated with a bad prognosis. This study investigated the effect of antiplatelet therapy on outcome, as well as the effects of potential confounders.

Methods: Our analysis is based on data from a large stroke registry in Germany. All parameters relevant to this analysis including age, sex and ICD-based diagnosis as well as pre-hospital status (modified Rankin Scale, mRS) and pre-treatment with antiplatelets or oral anticoagulation were recorded prospectively.

Results: Over a two year period, 1691 patients with ICH were documented $(48 \%$ male, mean age $72 \pm 12$ years). At symptom onset, $26 \%$ were on antiplatelets, and $12 \%$ received oral anticoagulation. Compared to patients without pre-treatment, those on antiplatelet therapy or anticoagulation were older and had a worse prehospital status (all $\mathrm{p} \geq 0.001$ ). Using univariate logistic regression, pre-treatment with antiplatelets or anticoagulation were found to be predictors of in-hospital 
mortality (OR 1.42, $\mathrm{p}=0.008$; OR 1.53, $\mathrm{p}<0.001$ ) and of unfavorable functional outcome (mRS $>2$ and death; OR 1.34, $\mathrm{p}=0.032$; OR 1.51, $\mathrm{p}<0.001$ ). However, after adjustment for age and pre-hospital status, pre-treatment with antiplatelets was no longer an independent predictor of in-hospital death $(\mathrm{p}=0.257)$ or unfavorable functional outcome $(\mathrm{p}=0.886)$, whereas the influence of pre-treatment with oral anticoagulants remained significant (OR 1.46, $\mathrm{p}<0.001$; OR 1.40, $\mathrm{p}=0.011$ ).

Discussion: Patients on antiplatelet therapy are significantly older and their prehospital status is worse compared to patients without antithrombotic medication. After adjusting for this confounders, the overall effect on mortality and functional outcome attributable to pre-treatment with antiplatelets is small, if present at all.

\section{Cerebral haemorrhage and $\mathrm{SAH}$}

SURGERY FOR INTRACEREBRAL HEMORRHAGE (ICH): RE-BLEEDING OCCURS EARLY AND PREDICTS IN-HOSPITAL MORTALITY

A. Hug, P. Hand, A. Kaye, P. Teddy, L. McGregor, J. Brown, B. Tress, S. Davis Royal Melbourne Hospital, University of Melbourne, Parkville, VIC, Australia

Background and Aims: All randomised clinical trials comparing surgery versus conservative treatment in supratentorial ICH have been generally negative. One explanation for this lack of benefit from hematoma evacuation might be recurrent bleeding. Little is known about the frequency of recurrent hemorrhage after hematoma evacuation. Furthermore, the effect of re-bleeding on early mortality is unknown. If re-bleeding after evacuation predicts poor outcome, there may be a role for a combined approach with a hemostatic agent.

Methods: Retrospective single centre analysis of 75 patients with spontaneous or warfarin-related infra- and supratentorial ICH treated by craniotomy and hematoma evacuation between 2000 and 2005. CT-scans before and after hematoma evacuation were analysed applying the $(\mathrm{a} \times \mathrm{b} \times \mathrm{c}) / 2$ method, blinded to the clinical data. We dichotomized the patients into re-bleeders and non re-bleeders, using the median of the relative residual volume, compared to the initial scan. Predictors of in-hospital mortality were assessed using multivariate logistic regression. The Chi-square test was used to analyse the time course of re-bleeding, by dichotomization of the onset-surgery time ( $<$ or $>12$ hours).

Results: The patients included 60/75 with supratentorial, 15/75 with infratentorial and 9/75 with warfarin-related ICH, aged 23-86 years. Initial hematoma volumes ranged between 1-147 ml, median $55 \mathrm{ml}$. Post-evacuation volumes ranged between $0-119 \mathrm{ml}$, median $4 \mathrm{ml}$. Re-bleeding was more likely in ICH-patients treated surgically within 12 hours after symptom onset, compared to later hematoma evacuation (OR 2.94; CI 1.13-7.64; $\mathrm{p}=0.027$ ). Furthermore, re-bleeding was an independent predictor of in-hospital mortality (OR 5.75; CI 1.77-18.72; $p=0.004$ ). Age, initial hematoma volume, prior anticoagulant therapy, admission GCS, history of diabetes and initial blood pressure were not significant.

Conclusions: Early craniotomy and hematoma evacuation within 12 hours after symptom onset was associated with re-bleeding and greater in-hospital mortality. A trial of surgical evacuation combined with rFVIIa would be worthwhile.

\section{Cerebral haemorrhage and SAH}

COST-EFFECTIVENESS OF RECOMBINANT ACTIVATED FACTOR VII IN THE TREATMENT OF ACUTE INTRACEREBRAL HAEMORRHAGE IN THE UNITED KINGDOM

K. Lees, M.C. Christensen, A. McGuire

University Department of Medicine \& Therapeutics, Gardiner Institute,

Bagsvaerd, Denmark

Background: Recombinant activated factor VII (rFVIIa) was recently demonstrated to reduce haematoma expansion and improve survival following intracerebral haemorrhage (ICH). We have assessed the potential cost-effectiveness of rFVIIa in the treatment of acute ICH in the United Kingdom (UK).

Methods: We applied UK estimates of drug and hospital bed-day costs to the outcomes and resource use observed in the published rFVIIa ICH trial (ICH confirmed by $\mathrm{CT}$ scan within $3 \mathrm{~h}$ of onset; randomisation to receive placebo or 40 $\mathrm{mcg}, 80 \mathrm{mcg}$ or $160 \mathrm{mcg}$ per kilogram of rFVIIa [NEJM 2005; 352:777-785] to estimate incremental short-term (days 1-90) healthcare costs per day-90 survivor. We used retrospective long-term outcome and healthcare data from a cohort of ICH patients originally treated in Scotland in 1993 and UK national statistics to estimate incremental lifetime healthcare costs per life-year gained (LYG). Analyses were limited to hospital costs and used a discount rate of $3.5 \%$.

Results: Over the first 90 days, rFVIIa increased costs by $£ 3,991, £ 610$, and $£ 5,479$ in the 40,80 and $160 \mathrm{mcg} / \mathrm{kg}$ dose groups respectively, and survival by $0.11,0.11$ and 0.10 lives saved per treated patient. Thus, rFVIIa costs $£ 36,282, £ 5,545$ and $£ 54,790$ per life saved in the $40 \mathrm{mcg} / \mathrm{kg}, 80 \mathrm{mcg} / \mathrm{kg}$ and $160 \mathrm{mcg} / \mathrm{kg}$ dose groups. Projected to lifetime, rFVIIa increased costs by $£ 9,171, £ 3,581$ and $£ 6,869$ in the three dose groups and survival by $2.17,2.13$ and 2.05 LYGs per treated patient. Thus, rFVIIa costs $£ 4,226, £ 1,681$ and $£ 3,351$ per LYG in the $40 \mathrm{mcg} / \mathrm{kg}, 80 \mathrm{mcg} / \mathrm{kg}$ and $160 \mathrm{mcg} / \mathrm{kg}$ dose groups. Estimates were robust to changes in discount rate and long-term survival gains.

Conclusion: In the UK, rFVIIa could be a highly cost-effective therapy for control of bleeding in acute ICH compared with standard care.

\section{Cerebral haemorrhage and $\mathrm{SAH}$}

\section{INTRACRANIAL DISSECTIONS WITH SUBARACHNOID HEMORRHAGE} (SAH): CLINICAL CHARACTERISTICS AND LONG TERM FOLLOW-UP J. Sedat, Y. Chau, M.H. Mahagne, A. Dunac, V. Bourg, N. Boissier Hopital St Roch, France

Background: Intracranial dissection is a rare and serious cause of SAH. We have reviewed initial diagnostic features, treatment, and long-term outcome in 15 patients. Methods: At our hospital, 15 patients (6 males, 9 females) presented SAH related to intracranial dissection between 1999 and 2003. The patient's ages ranged from 40 to 71 years. 6 patients were admitted in Hunt and Hess grade II, 1 in grade III, 6 in grade IV, and 2 in grade V. In all cases SAH was confirmed by a computed tomographic scan. In the Fisher classification, 1 patient was grade II, 1 was grade III, and 13 were grade IV. Hydrocephaly required ventricular drainage in 4 patients Diagnosis of dissection was established with cerebral angiography in all patients. Dissection was located in 8 cases on the intracranial vertebral artery, in 4 cases on the Postero-inferior cerebellar artery, in 2 cases on the intracranial carotid artery and in 1 case on the middle cerebral artery.

Results: Endovascular treatment was performed in 12 patients. No procedurerelated complication and no rebleeding were observed. Therapeutic abstention was indicated in 3 patients. Every patient underwent angiographic follow-up at one year. Clinical outcome was assessed at one and 2 years. No angiographic recurrence was observed. Each patient's outcome was graded according to the Glasgow Outcome Score (GOS). 6 patients died in the acute stage; all of them presented a high clinical grade on admission. 7 patients were GOS 1 after 2 years, 1 was GOS 2, and 1 was GOS 3 .

Conclusions: Mortality of intracranial dissections with SAH is high. Clinical outcome depends mainly on the clinical status on admission. Endovascular treatment is effective to prevent rebleeding.

\section{Cerebral haemorrhage and $\mathrm{SAH}$}

UNRUPTURED BRAIN ARTERIOVENOUS MALFORMATIONS PRESENTING WITH FOCAL NEUROLOGICAL DEFICITS

C. Stapf, J.H. Choi, R.R. Sciacca, H. Mast, J.P. Mohr

Columbia University, New York, NY, USA

Background: Isolated focal neurological deficits constitute an infrequent clinical presentation type in patients diagnosed with an unruptured brain arteriovenous malformation (AVM)

Subjects \& Methods: A total of 735 consecutive patients from the prospective Columbia AVM Database were analyzed using univariate and multivariate logistic regression models testing the effect of age, sex, AVM size, anatomic location (lobar, deep, infratentorial), venous drainage pattern (superficial, deep, or both), and venous ectasia on focal neurological deficits at initial presentation in patients with unruptured AVMs.

Results: Fifty-four (7.3\%) patients were initially diagnosed with a focal neurological deficit unrelated to AVM hemorrhage. The clinical course was chronic progressive in $15(2.5 \%)$, chronic and persistent in $12(1.6 \%)$, and fluctuating in $27(4 \%)$. Univariate testing showed significant associations with age $(\mathrm{p}=0.005)$ and female $\operatorname{sex}(\mathrm{p}=0.02)$. The multivariate model confirmed the independent effect of age (OR $1.027,95 \%$ CI 1.01 to 1.04 ) and female sex (OR 2.09, 95\% CI 1.18 to 3.68), while no was associations were found for AVM size, location, venous drainage pattern, and presence of venous ectasia.

Conclusion: Increasing age and female gender appear to be independently associated with the occurrence of focal neurological deficits in a setting of unruptured brain AVMs. The findings suggest that women and older AVM patients may have increased neuronal vulnerability in the surrounding brain tissue. Further studies are needed to further determine the underlying mechanism. 


\section{Cerebral haemorrhage and SAH}

SAFETY PROFILE OF RECOMBINANT FACTOR VIIA IN PATIENTS WITH INTRACEREBRAL HAEMORRHAGE

M.N. Diringer, S.A. Mayer, N.C. Brun, J.P. Broderick, S. Davis, B.E. Skolnick,

T. Steiner, on behalf of the rFVIIa ICH Trial Investigators

Washington University School of Medicine, St. Louis, Missouri, USA

Background: Safety data of recombinant Factor VIIa (rFVIIa) use in patients (pts) with spontaneous intracerebral haemorrhage $(\mathrm{ICH})$ were analysed to better define the risk of thromboembolic (TE) complications.

Methods: Data were pooled from 3 randomized, placebo (pbo)-controlled studies of rFVIIa in ICH pts (mean age 65) diagnosed within $3 \mathrm{~h}$ of onset who received a single dose of rFVIIa $(5-160 \mathrm{mcg} / \mathrm{kg} ; \mathrm{n}=371)$ or pbo $(\mathrm{n}=115)$. Safety parameters included adverse events (AEs) until discharge/day 15, serious AEs and mortality to day 90. TE event monitoring (including clinical/laboratory evaluations, lower extremity Doppler, oedema/haematoma ratio on $72 \mathrm{~h} \mathrm{CT} \mathrm{scan)} \mathrm{was} \mathrm{performed.}$

Results: AEs were comparable across rFVIIa and pbo groups; $50 \%$ were of mild severity. Mortality was significantly lower in rFVIIa pts (combined) vs pbo ( $\mathrm{p}=$ 0.02). Regression analysis indicated no significant treatment effect on overall risk of TE events including DVT and PE. However, increased risk for arterial TE events was observed for the $160 \mathrm{mcg} / \mathrm{kg}$ dose vs pbo). For the entire study group arterial events occurring within 90 days of drug administration included myocardial ischaemia ( $\mathrm{n}=11,2$ with sequelae, 1 fatal, with 5 of these being non-Q-wave myocardial ischaemia with troponin elevation) and ischaemic stroke ( $n=13,4$ with sequelae, 1 fatal).

Conclusions: No significant differences in AEs were found between rFVIIa-dosed pts vs pbo. TE events also did not differ between groups, including risk of DVT or PE. However, frequency of arterial events was significantly greater in the 160 $\mathrm{mcg} / \mathrm{kg}$ group than pbo suggesting that the $160 \mathrm{mcg} / \mathrm{kg}$ dose, in this principally older pts with atherosclerosis risk factors, although reducing overall mortality, may increase the frequency of arterial TE events.

\section{Cerebral haemorrhage and $\mathrm{SAH}$}

\section{BASELINE MARKERS OF HEMOSTASIA AND HEMATOMA} ENLARGEMENT IN ACUTE INTRACEREBRAL HEMORRHAGE

J. Martí-Fàbregas, D. Cocho, Y. Bravo, L. Molina-Porcel, J. Díaz-Manera,

M. Borrell, D. Alcolea, E. Martínez, J. Fontcuberta, J.L. Martí-Vilalta

Hospital de la Santa Creu i Sant Pau, Spain

Background: Recent studies suggest that treatment with procoagulant drugs in the acute stage of intracerebral hemorrhage $(\mathrm{ICH})$ may limit the risk of hematoma enlargement (HE). We tested the hypothesis that the measurement of markers of hemostasia and fibrinolysis at baseline could predict HE in untreated patients.

Methods: We studied prospectively consecutive patients with spontaneous supratentorial ICH, within the first 6 hours after symptom onset. HE was defined as an increase $>33 \%$ in the volume of hematoma measured on the Computed Tomography (CT), obtained 24-48 hours after the onset of symptoms, in comparison with the admission CT. The volume was calculated using the formula AxBxC/2. Immediately after CT diagnosis, we collected blood samples by venipuncture in sodium citrate. Plasma was frozen at $-40{ }^{\circ} \mathrm{C}$ until used. We measured fibrinogen $(\mathrm{g} / \mathrm{l})$, Thrombin-antithrombin complex $(\mu \mathrm{g} / \mathrm{l})$, Thrombomodulin $(\mathrm{ng} / \mathrm{ml})$, t-PA $(\mathrm{ng} / \mathrm{ml})$, Plasminogen Activator Inhibitor (UI/ml), D-dimer $(\mu \mathrm{g} / \mathrm{ml})$, Thrombin Activatable Fibrinolysis Inhibitor (\%), alfa2-antiplasmin (\%), plasminogen (\%), and factor XIII (\%). Statistics: t-test.

Results: We included 32 patients, with a mean age $71.5 \pm 10.3$ years, and $56 \%$ were men. HE was observed in 16 patients $(50 \%)$. The baseline CT was obtained $155 \pm 74$ minutes after symptom onset, while the control CT was performed $31.9 \pm 11.4$ hours after the onset. There were no statistically significant differences between HE and non-HE groups in the values of hemostatic markers.

Discussion: These preliminary results suggest that there is no association between any of the measured baseline hemostatic markers and the risk of hematoma enlargement in patients with acute intracerebral hemorrhage.

\section{Cerebral haemorrhage and $\mathrm{SAH}$}

COST-EFFECTIVENESS OF LONG-TERM FOLLOW-UP SCREENING FOR NEW ANEURYSMS IN PATIENTS WITH CLIPPED ANEURYSMS AFTER SUBARACHNOID HEMORRHAGE

M.J.H. Wermer, H. Koffijberg, I.C. van der Schaaf, for the ASTRA study group University Medical Centre Utrecht and the Academic Medical Centre Amsterdam, The Netherlands

Background: Patients who survive after subarachnoid hemorrhage (SAH) are at risk for a recurrence despite successful treatment of the ruptured aneurysm and may therefore benefit from screening for new aneurysms.

Methods: We screened 610 SAH patients with CT-angiography 2-18 years after operative clipping of the aneurysm(s). Results of screening were used as input for a Markov decision model. We compared the expected number of recurrent hemorrhages, life-expectancy and quality-adjusted life-years (Qaly's) of the strategies "screening every 5 years" and "no screening" and performed a cost-effectiveness analysis.

Results: According to the model, screening SAH patients in general prevented almost half of the recurrences, slightly increased life-expectancy (from 21.06 to 21.08 years), but reduced Qaly's (from 12.18 to 12.04 ) and increased costs (from $\$ 2750$ to $\$ 4165$ per patient). Screening was cost-saving in patients with a twofold risk above baseline of both aneurysm formation and rupture and was cost-effective if both risks were at least 4.5 times higher. In patients with reduced quality of life because of fear for a recurrence, screening increased Qaly's at a maximum cost of $\$ 17422$ per Qaly.

Conclusion: In general, screening patients with a history of SAH cannot be recommended. Screening can be cost-saving and cost-effective in patients with a relatively high risk of both aneurysm formation and rupture. In patients who fear a recurrence, screening increases Qaly's at acceptable costs. More data on risk factors for aneurysm formation and rupture in patients with a history of SAH are needed to identify patients who can benefit from screening.

\section{Cerebral haemorrhage and $\mathrm{SAH}$}

\section{PREDICTORS OF RECURRENT PRIMARY INTRACEREBRAL HEMORRHAGE:A STUDY USING T2*-WEIGHTED GRADIENT-ECHO IMAGING}

J.S. Kim, S.B. Jeon, D.W. Kang, S.W. Kwon, A.H. Cho, C.G. Choi Asan Medical Center, Asan, South Korea

Background: Intracerebral microbleeds (MBs) are frequently observed in intracerebral hemorrhage ( $\mathrm{ICH})$ patients. Although $\mathrm{MBs}$ have been shown to be pathogenetically related with ICH, it is unknown whether MBs are predictors of recurrent ICHs. We sought to assess predictors for recurrent primary ICHs in patients with ICH with special reference to the role of MBs.

Methods: Among 220 acute symptomatic primary ICH patients, 112 patients who underwent gradient-echo T2*-weighted MR images (GRE) within 10 days after symptom onset were considered for this study. Among them, the final 63 patients who consented to follow-up clinical, laboratory and GRE studies were included. The presence and number of radiological ICHs (mean diameter $>5 \mathrm{~mm}$ ) and MBs on baseline and follow-up GRE were evaluated. The relationship of recurrent ICHs with initial and follow-up clinical and laboratory data as well as the MBs was assessed. Results: Among 63 patients, $43(68.3 \%)$ had MBs on baseline GRE. Seven $(11.1 \%)$ patients developed recurrent ICHs, and $19(30.2 \%)$ had new MBs during median 23.3 months (range, 8.3 to 33.0 months) of follow-up. The numbers of initial MBs $(\mathrm{p}<0.0001)$ and ICHs $(\mathrm{p}=0.010)$ on baseline GRE were significantly associated with development of recurrent ICHs whereas other clinical and laboratory data were not.

Conclusions: Recurrent ICHs and MBs are common during long-term follow-up of primary ICH. The number of MBs on baseline GRE predicts the recurrence of the $\mathrm{ICH}$

13 Cerebral haemorrhage and $\mathrm{SAH}$

\section{ROLE OF CHOLESTEROL IN PAZIENTS WITH INTRACEREBRAL} HEMORRHAGE

L. Olivieri, S. Sacco, D. Cerone, C. Marini, A. Carolei

University of L'Aquila, L'Aquila, Italy

Background: Low values of cholesterol were reported to be associated with an increased risk of intracerebral hemorrhage. We evaluated, the prevalence and the prognostic role of cholesterol serum levels in patients with a first-ever intracerebral hemorrhage. 
Methods: For the purpose of the present study we considered only patient with intracerebral hemorrhage included, in a 5-year period, in the prospective populationbased registry of patients living in the L'Aquila district.

Results: Data on cholesterol values were available in $434(79.1 \%)$ out of 549 patients with intracerebral hemorrhage. Three hundred thirty eight patients had levels of total cholesterol $\leq 220 \mathrm{mg} / \mathrm{dl}$ and 96 had levels $>220 \mathrm{mg} / \mathrm{dl}$. Patients with cholesterol levels $\leq 220 \mathrm{mg} / \mathrm{dl}$ were more frequently women $(50.9 \%$ vs $65.6 \%$ $\mathrm{p}=0.0105)$ and had a lower proportion of peripheral arterial disease $(8.1 \% \mathrm{vs}$ $17.0 \% ; \mathrm{p}=0.0106$ ) with respect to those with higher levels, while proportions of arterial hypertension, coronary heart disease, and cigarette smoking were similar in both groups. Thirty-day $(43.8 \%$ vs $47.0 \% ; \mathrm{P}=0.5682)$ and 1 -year case-fatality rates $(52.1 \%$ vs $56.8 \%$; $\mathrm{P}=0.4111)$ were also similar in both groups of patients as well as the long-term survival $(74.0 \%$ vs $73.4 \%$; $\mathrm{P}=0.9087)$. According to the Cox regression analysis which included age, sex, arterial hypertension, diabetes mellitus, coronary heart disease, cigarette smoking, and peripheral arterial disease as covariates, hypercholesterolemia was not an independent predictor of 30-day and 1-year mortality.

Discussion: Despite a reported increased incidence of intracerebral hemorrhage in patients with high cholesterol levels, our results do not support an unfavorable prognostic role of this risk factor in patients with intracerebral hemorrhage.

\section{Cerebral haemorrhage and $\mathrm{SAH}$}

\section{SUPERFICIAL CORTICAL AND SUBARACHNOID HEMOSIDEROSIS IN}

\section{CEREBRAL AMYLOID ANGIOPATHY}

J. Linn, M. Wiesmann, G. Fesl, J. Herm, H. Brückmann

University Hospital Munich, Grosshadern, Munich, Germany

Background: Intracerebral hemorrhage (ICH) is the most important clinical presentation of cerebral amyloid angiopathy (CAA). Identifying CAA as cause of an ICH and differentiating it from hypertensive hemorrhage is challenging. In this study we propose superficial cortical and subarachnoid hemosiderosis as new magnetic resonance imaging (MRI) criteria for CAA.

Methods: 27 patients with an acute ICH were included in this study $(16 \mathrm{~m} / 11 \mathrm{f}$, mean age $65,6 \pm 9,5 \mathrm{y}$ ). In these patients the presumed cause of the hemorrhage was either chronic hypertension or CAA. T2*-weighted gradient-echo MR images of these patients were analysed by three readers. They noted the localisation of blood residues (intraparenchymal, intraventricular, subarachnoid, or superficial cortical) and the occurrence and number of macro- and microbleeds. In a consensus reading the most likely reason for the acute $\mathrm{ICH}$ was defined and patients were grouped accordingly (HTN group, CAA group). In a last step, differences in the two groups concerning findings in the $\mathrm{T} 2 *$-weighted gradient-echo MR images were analysed. Results: In the CAA group, microbleeds were detected in $94 \%$ of the cases, in $75 \%$ of the cases superficial cortical and/or subarachnoidal blood residues occurred. In $60 \%$ of the patients in the HTN group, microbleeds were observed, whereas in none of these patients subarachnoidal or superficial cortical hemosiderosis was found.

Discussion: The presence of subarachnoid hemosiderosis in CAA patients suggest that they had suffered from clinically occult SAH and that CAA should be considered as a possible cause of primary SAH in elderly patients.

We detected superficial cortical hemosiderosis in 13 of 17 patients with CAA but in none of the HTN patients. CAA preferentially affects leptomeningeal as well as small and medium sized cortical vessels. Thus, we suspect that these vessels cause recurrent, small bleedings leading to superficial cortical hemosiderosis.

In conclusion, we propose superficial cortical and subarachnoid hemosiderosis as new MRI criteria for discriminating between hypertensive ICH and CAA.

\section{Cerebral haemorrhage and SAH}

\section{IS ADMISSION BLOOD PRESSURE RELATED TO HEMORRHAGIC} TRANSFORMATION IN PATIENTS WITH ACUTE ISCHEMIC STROKE?

K. Takis, J. Zafeiriou, A.A. Liodou, M.S. Trianti, S. Scandalidi, T. Pappa,

M. Skoufi, K. Vemmos

Alexandra Hospital, University of Athens, Athens, Greece

Background: Hemorrhagic transformation (HT) is a natural event in the evolution of a cerebral infract. It is unknown if the common elevated blood pressure (BP) during the acute phase of stroke leads to HT of the infract tissue. The aim of this study was to evaluate if the presence of HT is related to admission BP in patients with acute ischemic stroke.

Methods: From a prospective case series of 1717 patients with first-ever acute ischemic stroke we selected 1008 cases with territorial infarct based on the following criteria: a first brain CT scan $<24$ h from symptoms onset and at least a second CT scan within the first week in order to assess the occurrence of the HT and no thrombolytic agents were administered. BP was measured by the conventional method at the time on admission. Logistic regression analysis was used for identify the independent predictors of HT after adjustment for all possible confounding factors such as age, risk factors, severity of stroke, CT findings and treatment.

Results: HT was observed in $313(31.1 \%)$ patients: $285(28.3 \%)$ had hemorrhagic infarction and $28(2.8 \%)$ had parechymal hemorrhage. After adjustment for baseline factors HT was positive associated with brain edema on the 2nd CT scan, presence of atrial fibrillation, absence of previous antihypertensive medication use and an increase of systolic Blood Pressure (BP) on admission (OR 1.11, CI=1.04-1.19, $\mathrm{p}=0.002$ )

Discussion: Elevated systolic BP on admission is associated with subsequent HT in patients with a territorial acute ischemic infarct.

Treatment strategies should be considered in such patients with a marked elevated admission BP.

\section{Experimental studies}

\section{Experimental studies}

\section{THE EFFECT OF SB-737004/S-0139 ON INFARCT SIZE AND CORTICAL PERFUSION FOLLOWING PERMANENT OCCLUSION OF THE MIDDLE CEREBRAL ARTERY IN THE CAT}

A. Strong, H. Watts, P. Anderson, A. Lloyd, T. Nagafuji, M. Ninomiya, D. Virley

King's College London, London, United Kingdom

Backgound: The highly specific endothelin-A receptor antagonist SB-737004/S0139 reduces infarct size in rats subjected to middle cerebral artery occlusion (MCAO). We (1) examined whether this treatment effect can be replicated in a larger animal stroke model (gyrencephalic brain) and (2) explored the mechanism of any treatment effect, imaging cortical perfusion quantitatively (laser-speckle).

Methods: Observer-blind study in cats undergoing permanent MCAO (pMCAO). From $15 \mathrm{~min}$ after pMCAO until termination at 4 hours, four groups received continuous infusion of either: (i) vehicle (bicarbonate-buffered saline at $\mathrm{pH} 7.4,4$ $\mathrm{ml} / \mathrm{kg} / \mathrm{hr}$ ); SB-737004/S-0139 (ii) $0.1 \mathrm{mg} / \mathrm{kg} / \mathrm{hr}$ (iii) $0.3 \mathrm{mg} / \mathrm{kg} / \mathrm{hr}$ (iv) $0.9 \mathrm{mg} / \mathrm{kg} / \mathrm{hr}$ in same vehicle at same volume rate.

Results: Quantitative histology showed that treatment with $0.1 \mathrm{mg} / \mathrm{kg} / \mathrm{hr} \mathrm{SB}$ $737004 / \mathrm{S}-0139$ had no effect on infarct size. Treatment with $0.3 \mathrm{mg} / \mathrm{kg} / \mathrm{hr}$ produced a moderate reduction in infarct volume, although this was not statistically significant. Treatment with the highest dose of SB-737004/S-0139 $(0.9 \mathrm{mg} / \mathrm{kg} / \mathrm{hr})$ dramatically reduced the volume of ischaemic damage in the cortex by $50.7 \%$ (from $1119 \pm 176$ $\mathrm{mm}^{3}$ in the vehicle-treated group $(\mathrm{n}=11)$ to $552 \pm 178 \mathrm{~mm}^{3}$ in the $0.9 \mathrm{mg} / \mathrm{kg} / \mathrm{hr}$ treated group $(\mathrm{n}=10) ; \mathrm{P}<0.05)$ and in the caudate by $67.4 \%$ (from $30.5 \pm 5.1 \mathrm{~mm}^{3}$ to $11.5 \pm 5.4 \mathrm{~mm}^{3} ; \mathrm{P}<0.05$ ), compared with the vehicle control group.

Discussion: Number of peri-infarct depolarisations (PIDs) influences infarct size, and PIDs vasoconstrict. Here, no significant reduction in number of PIDs during pMCAO was observed with any dose, relative to vehicle treatment. Instead, a significant increase in perfusion was seen in the core with $0.9 \mathrm{mg} / \mathrm{kg} / \mathrm{hr}(\mathrm{p}<0.05 \mathrm{vs}$ vehicle). Protection may therefore relate to reduced impact of individual PIDs on perfusion.

\section{Experimental studies}

POWERFUL NEUROPROTECTION IN A MOUSE STROKE MODEL WITH I.V. ADMINISTRATION OF XG102

K. Wiegler, J. Bogousslavsky, C. Bonny, L. Hirt

CHUV, Lausanne, Switzerland

Background: XG102 (formerly D-JNKI1), a TAT-coupled dextrogyre peptide which inhibits the c-jun N-terminal kinase (JNK), is a powerful neuroprotectant in mouse middle cerebral artery occlusion (MCAo) models (Nat Med 2003; 9:1180-6; Stroke $2004 ; 35: 1738-43$ ) when administered by intra-cerebro-ventricular injection (i.c.v.). The degree of protection is very high even with delayed treatment and this compound is a promising new candidate drug for ischemic stroke. As i.c.v. administration is not convenient in patients, the aim of the present study is to determine whether neuroprotection can be achieved with intravenous (i.v.). administration

Methods: Young adult male mice ICR-CD1 were subjected to $30 \mathrm{~min}$ transient suture MCAo, with laser Doppler monitoring of regional cerebral blood flow. Different doses of XG102 were administered i.v. 6 hours after ischemia onset to determine the dose range and the efficacy; then a single dose was chosen for a blinded experiment. Lesion size was determined after 48 hours by HE staining. Neurological outcome was evaluated by neurological scores and rotarod test Results: XG102 administered i.v. 6 hours after ischemia onset strongly reduces 
the infarct volume at 48 hours. The lowest dose with maximal neuroprotection is $0,3 \mu \mathrm{g} / \mathrm{kg}$, which reduces the infarct volume from $61 \pm 14 \mathrm{~mm}^{3}(\mathrm{n}=16)$ for the vehicle-treated group to $18 \pm 9 \mathrm{~mm}^{3}(\mathrm{n}=5, \mathrm{P}<0.001)$. A dose of $1 \mathrm{mg} / \mathrm{kg}$ tested blindly also reduces the lesion volume, from $52 \pm 22 \mathrm{~mm}^{3}(\mathrm{n}=8)$ to $13 \pm 2 \mathrm{~mm}^{3}(\mathrm{n}=9$, $\mathrm{P}<0.0001$ ). The behavioural outcome is also improved

Discussion: XG102 is a powerful neuroprotectant in our mouse stroke model, also with i.v. administration. The feasibility of systemic administration is an important step towards the development of XG102 as drug candidate in human ischemic stroke. This compound is a powerful agent and remains very promising, with good hopes for future clinical trials. We are currently investigating its mechanisms of action in neuroprotection after stroke.

\section{Experimental studies}

MICRODIALYSIS ANALYSIS UNDER ULTRASOUND AND MICROBBUBBLES TREATMENT IN EXPERIMENTAL STROKE

M. Fatar, M. Stroick, M. Griebe, S. Kreisel, A. Alonso, R. Kern,

M.G. Hennerici, S. Meairs

Klinikum Mannheim, University of Heidelberg, Mannheim, Germany

Background: As little is known on interactions between ultrasound + microbubbles (US+MB) and acute cerebral infarction, we performed combined ultrasound and microbubbles in a middle cerebral artery (MCA) occlusion model in rats to evaluate possible effects upon infarct volume and influence on tissue damage metabolites obtained by microdialysis

Methods: MCA occlusion was induced in two groups of Wistar rats $(n=16)$. Rats were insonated with ultrasound for $30 \mathrm{~min}(2 \mathrm{MHz}$ sector transducer on a Philips HDI-5000 platform, mechanical index 1.1, focal depth $3.0 \mathrm{~cm}$ ) during a continuous infusion of microbubbles (Sonovue ${ }^{\mathrm{TM}} 0.2 \mathrm{ml} / \mathrm{min}$ ) and compared to controls. Animals were sacrificed 24 hours after MCA infarction. Brains were removed and silver-stained for quantification of infarct volume. 5 animals of each group received an intracerebral microdialysis probe (AP-0.3, Lat -3.5, caudal -4.5 $\mathrm{mm}$ ) before stroke induction and glutamate, glycerole, lactate and pyruvate were measured by HPLC during and after ultrasound and microbubbles treatment for $6 \mathrm{~h}$.

Results: Infarct volume in the microbubble + ultrasound group was $186.9 \pm 137.2$ $\mathrm{mm}^{3}$ compared to control $352.8 \pm 92.2(\mathrm{p}<0.05)$. Glutamate levels in controls were significantly higher in the first 3 hours of infarction $(\mathrm{p}=0.028)$, whereas lactate, glycerole and pyruvate did not differ in both groups.

Discussion: This is the first study demonstrating biomarker changes during ultrasound/microbubbles treatment in vivo and suggesting an inhibition of the glutamate expression during acute stroke.

\section{Experimental studies}

\section{INTRATHECAL STEROIDS - NEUROPROTECTIVE EFFECT IN FOCAL} CEREBRAL ISCHEMIA IN RATS

S.L. Goericke, A. Doerfler, T. Engelhorn, W.P. Becker, U. Speck, M. Forsting Institute of Diagnostic and Interventional Radiology and Neuroradiology, Essen, Germany

Purpose: The aim of our study was to evaluate the neuroprotective efficacy of intrathecally administered triamcinolonacetonide (TCA) on infarction volume in acute focal cerebral ischemia in rats.

Materials \& Methods: Focal cerebral ischemia was induced in 102 Wistar rats using an endovascular occlusion technique of the middle cerebral artery (MCAO). In a first dose-finding study, different doses of TCA $(0.3,0.03,0.012,0.006$, or 0.003 $\mathrm{mg} / \mathrm{kg}$ bodyweight) were administered into the cisterna magna of 12 rats each 30 minutes after MCAO. Twelve animals received equivolumetric saline intrathecally. In a second MR-controlled confirmation study, the neuroprotective efficacy of the most effective dose was compared to controls in 15 rats each. Infarction volume was calculated 24 hours after MCAO by TTC-staining in all animals.

Results: Experiment 1: Compared to controls (18.2 $\pm 5.0 \%)$, infarction volume was significantly reduced using TCA at a dose of $0.012 \mathrm{mg} / \mathrm{kg}(13.4 \pm 5.3 \%, \mathrm{p}=0.04)$ TCA $0.03 \mathrm{mg} / \mathrm{kg}(17.7 \pm 6.9 \%, \mathrm{p}=0.84), 0.006 \mathrm{mg} / \mathrm{kg}(15.9 \pm 4.2 \%, \mathrm{p}=0.24)$, and $0.003 \mathrm{mg} / \mathrm{kg}(14.5 \pm 5.2 \%, \mathrm{p}=0.11)$ did not reduce infarction size significantly, whereas TCA $0.3 \mathrm{mg} / \mathrm{kg}$ resulted in bilateral infarction with increased infarction volume $(19.8 \pm 5.0 \%, p=0.49)$. Experiment 2 : MR imaging (diffusion-weighted images, T1-weighted images) confirmed successful MCAO and intrathecal administration in all animals. Compared to controls $(20.0 \pm 8.0 \%)$ infarction volume was reduced significantly in animals treated with TCA $0.012 \mathrm{mg} / \mathrm{kg}(13.4 \pm 6.5 \%, \mathrm{p}=$ $0.02)$.

Conclusion: Intrathecally administered steroid triamcinolonacetonide may reduce infarction volume significantly in permanent focal cerebral ischemia in rats. Further studies, focusing on long-term effects and clinical outcome, are necessary to assess the therapeutic value in stroke.

\section{Experimental studies}

\section{ANGIOGENESIS IS REACTIVATED IN HIGH-RISK ADVANCED HUMAN} CAROTID ARTERY PLAQUES

M.A Font, M. Miguel, M. Slevin, J. Martinez, A. Carvajal, F. Rubio, J. Krupinski

University Hospital of Bellvitge (HUB), Barcelona, L'Hospitalet de Llobregat (Barcelona), Spain

Background: Hemorrhagic transformation of advanced carotid plaque is a frequen finding and is probably responsible for atherothrombotic complications. The molecular mechanisms involved in new blood formation in patients with carotid disease are poorly studied. We suggest that during plaque development many pro-angiogenic pathways are reactived and lead to formation of immature blood vessels prone to rupture.

Methods: We included ulcerated non-complicated (UNC, n=10) and fibrose $(\mathrm{F}$, $\mathrm{n}=10$ ) carotid specimens taken during endarterectomy. Additionally, post-mortem carotid specimens $(n=10)$ with type III/IV atherosclerotic lesions were studied. Differential gene expression was analysed by Taq Man Low Density Array technology. The panel of antibodies to identify endothelial cells (flk-1, CD105, CD31, trk and Eph receptor families) was used for immunohistochemistry.

Results: UNC, highly cellular plaques and PM-III/IV specimens but not F plaques had abundant expression of mRNAs typical for endothelial progenitor cells $(p<0,001)$. Interestingly, UNC plaques contained high levels of mRNA for pathways usually reactivated in adult neoangiogenesis. Further, these mRNA levels correlated with immunohistochemical findings. In UNC plaques endothelial cells were strongly positive for VEGF-R2 and TGF-beta1.

Discussion: This study demonstrates that both in high-risk human carotid plaques as well as in specimens with developing atherosclerosis angiogenesis is reactivated. Angiogenesis can become an interesting new target for antiangiogenic therapies in patients with progressing carotid atherosclerosis.

\section{Experimental studies}

\section{EXPERIMENTAL SONOTHROMBOLYSIS OF HUMAN CLOTS WITH} ABCIXIMAB IMMUNOBUBBLES

A. Alonso, M. Fatar, C.-E. Dempfle, A. Della Martina, M. Stroick, E. Allemann, M.G. Hennerici, S. Meairs

Klinikum Mannheim, University of Heidelberg, Mannheim, Germany

Background: There is increasing evidence that ultrasound in combination with microbubbles can be used for clot lysis. We hypothesized that abciximab (ReoPro ${ }^{\mathrm{TM}}$ ), a glycoprotein $\mathrm{IIb} / \mathrm{III}$ a receptor inhibitor licensed for prevention of platelet aggregation before percutaneous coronary intervention and after acute coronary syndrome could be tagged to microbubbles (immunobubbles) to enable enhanced sonothrombolysis Methods: A partial thrombotic occlusion of the right common carotid artery of 16 rats was produced by insertion of human clot material via an external carotid artery catheter. Five rats received abciximab immunobubbles, in each case six rats had nonspecific immunobubbles or saline, respectively. The solutions were administered intravenously over 30 minutes in combination with pulsed $2 \mathrm{MHZ}$ ultrasound. Blood samples were taken at baseline and 5,10,20,30 and 60 minutes after beginning of treatment. Human d-dimer levels for quantification of thrombolysis were analysed by ELISA.

Results: Plasma d-dimer levels increased significantly in the abciximab immunobubblestreated group ( $\mathrm{p}=0.013$ at the end of treatment). Rats treated with a combination of abciximab immunobubbles and ultrasound showed significantly higher d-dimer levels after treatment compared to rats in the non-specific immunobubble group $(\mathrm{p}=0.027)$ and to rats treated with ultrasound alone $(\mathrm{p}=0.017)$. Ultrasound in combination with non-specific immunobubbles tended to result in non-significantly increasing d-dimer levels with treating time while ultrasound alone did not effect an increment in d-dimer levels.

Conclusions: $2 \mathrm{MHz}$ ultrasound in combination with abciximab tagged immunobubbles can induce thrombolysis of human clots. 


\section{Experimental studies}

THE EFFECTS OF ARTERIAL WALL, VENOUS WALL, ARTERIAL BLOOD, VENOUS BLOOD AND THEIR DIFFERENT COMBINATIONS TO THE VASOSPASM AFTER SUBARACHNOID HEMORRHAGE

M.T. Cansever, A. Sencer, A. Canbolat, T. Kiris, A. Karasu

Istanbul School of Medicine, Istanbul, Turkey

The risk of rebleeding after subarachnoid hemorrhage (SAH), seen after ruptured intracranial aneurysms, has decreased by early diagnosis and immediate interventions. However the vasospasm seen after $\mathrm{SAH}$, with ischemic neurological deficits, is still the most important cause of morbidity and mortality.

Many agents, most importantly oxyhemoglobin, are known to cause vasospasm which is a dynamic process. The experimental SAH models and the clinical observations on patients with SAH have shown variability in the severity of the vasospasm. The metabolites released from damaged arterial wall by the hemorrhage should have an important role in the process.

To test the hypothesis, 46 male Wistar rats were used in our experimental model. Cisterna Magna injections were performed on the rats with arterial and venous blood, arterial and venous wall homogenate, arterial blood and wall homogenate, venous blood and wall homogenate which were categorized in 7 groups. To make morphometric measurement and morphological examination on the basilar artery, brainstem was excised, protecting basilar artery.

The morphometric examinations has revealed that there was significant difference between the degree of vasospasm created by the arterial blood and by the venous blood $(\mathrm{p}<0,0001)$. On the measurements of the luminal area of the basilar artery, the groups in which, arterial blood, arterial wall homogenate and their mixture were used $(45-50 \%)$ revealed significant constriction $(p<0,0001)$ relative to the groups in which, venous blood, venous wall homogenate and their mixture were used (25-30\%).

The study has shown that the metabolite(s) released from vessel wall may also be as responsible as blood and its degradation products for vasospasm. We believe that discovering the vasospastic metabolite(s) released from the vessel wall with the use of advanced techniques, may help finding the cause and treatment of vasospasm.

\section{Experimental studies}

PENUMBRA AND HYPOXIA IMAGING IN MCA OCCLUDED RATS USING18F-FLUOROMISONIDAZOLE (FMISO) AND PET; AN EARLY RESULT

M. Takasawa, J.S. Beech, T.D. Fryer, J.L. Hughes, K. Igase, P.S. Jones, R. Smith, F.I. Aigbirhio, J.C. Clark, J.C. Baron

University of Cambridge, Cambridge, United Kingdom

Background: In acute stroke, the target of therapy is the severely hypoxic but potentially salvageable tissue. Previous work using FMISO-PET has shown foci of high late-phase tracer retention indicative of tissue hypoxia, which had cleared at repeat scan $48 \mathrm{~h}$ later. However, the only validation study of FMISO used ex vivo autoradiography in rats, and unexpectedly showed increased uptake in the infarct (assessed with TTC) as late as $22 \mathrm{hrs}$ after thread retraction, raising questions about validity of FMISO as a hypoxia tracer. Here we implemented longitudinal MicroPET in spontaneously hypertensive rats with permanent or temporary MCA occlusion (pMCAo and tMCAo, respectively) using the distal clip method to control reperfusion, and obtained full histopathology.

Methods: We studied 7 rats: 3 normals, 1 with pMCAo (two sessions: 30min and $48 \mathrm{~h}$ after clip), and 3 with tMCAo (45min, $\mathrm{n}=1 ; 120 \mathrm{~min} \mathrm{n}=2$; scanning started $30 \mathrm{~min}$ after clip removal). PET acquisition lasted $3 \mathrm{hrs}$. Experiments were terminated by perfusion-fixation. We obtained the affected/contralateral MCA territory FMISO uptake ratio $(\mathrm{A} / \mathrm{C}) 3 \mathrm{~h}$ after tracer injection.

Results: In normal rats the $\mathrm{A} / \mathrm{C}$ ratio was $0.97,1.01$ and 1.05 . In the pMCAo rat, the $\mathrm{A} / \mathrm{C}$ was high at 2.49 at session 1 , but normal at 0.99 at session 2 ; histopathology showed pan-necrosis. The A/C was 1.01 in the 45-min, and 1.04 and 0.94 in the 120-min tMCAo rats, respectively; histopathology showed early and severe ischemic changes, respectively.

Conclusions: These early results document abnormal uptake of FMISO in the stroke area only in the early phase of MCA occlusion, but not when tissue necrosis has developed nor if reperfusion has been implemented. These findings strongly support the validity of FMISO as a marker of brain hypoxia/penumbra after stroke.
9 Experimental studies

ROLE OF CANDESARTAN ON NEUROPROTECTION AGAINST CEREBRAL ISCHEMIA IN NORMOTENSIVE RATS

H. Liu, K.T. Kitazato, M. Uno, A. Suzue, S. Nagahiro

The University of Tokushima Graduate School, Tokushima, Japan

Background: Oxidative stress plays a key role in various pathological processes in brain ischemia. Angiotensin II (Ang-II) stimulates the production of reactive oxygen species including superoxide anion through Ang II AT1-receptor (AT1-R) activation. AT1-R blocker (ARB) has been reported to reduce ischemic cerebral damages. However, the detail neuroprotective mechanism of ARB remains unclear The goal of this study is to clarify the role of ARB on the protection from brain oxidative damage. We assessed the candesartan can protect normotensive rats against oxidative brain damage to clarify the role of ARB in brain ischemia.

Subjects and Methods: After 2-week pretreatment with candesartan, 5 weeks normotensive Wistar rats were subjected to 2-h middle cerebral artery occlusionreperfusion (MCAO-R) and $24 \mathrm{~h}$ later, the lumen diameter of the middle cerebral artery, the infarct volume, and the level of 8-hydroxy-2'-deoxyguanosine (8-OHdG), iNOS, and eNOS mRNA in the internal carotid artery was recorded and compared. Immunohischemically, $8-\mathrm{OHdG}$ and hypoxic inducible factor (HIF) - 1 alpha were evaluated.

Results: Restoration of the MCA diameter via an increase in eNOS mRNA, and the reduced expression of HIF-1 alpha and $8-\mathrm{OHdG}$ were observed after candesartan pretreatment, resulting in a decreased cortical infarct volume $(0.5 \mathrm{mg} / \mathrm{kg}$ candesartan, $46.8 \pm 13.2 \mathrm{~mm}^{3} ; 1.0 \mathrm{mg} / \mathrm{kg}$ candesartan, $19.3 \pm 15.3 \mathrm{~mm}^{3}$ vs. control, $111.7 \pm 14.3 \mathrm{~mm}^{3} ; \mathrm{p}<0.05, \mathrm{p}<0.01$, respectively). The decreased infarct volume and $8-\mathrm{OHdG}$ positive cells in cortical region were associated with the plasma level of 8 -OHdG. Candesartan treatment tends to reduce the blood pressure and neurological deficits.

Conclusions: In normotensive rats exposed to MCAO-R, candesartan protected against brain ischemia and oxidative damage though inhibition of endothelial dysfunction. This effect may be represent a significant therapeutic advantage and may induce end-organ protection even at normal blood pressure.

\section{Experimental studies}

HYPERBARIC OXYGEN MORE POTENTLY PROTECTS AGAINST MURINE PERMANENT FOCAL ISCHEMIA THAN NORMOBARIC HYPEROXIA

A.R. Veltkamp, L. Sun, O. Herrmann, G. Wolferts, H.H. Marti, M. Schwaninger University Heidelberg, Heidelberg, Germany

Background and Purpose: Recent experimental studies suggested a protective potential of both normobaric (NBO) and hyperbaric (HBO) oxygen therapy in transient cerebral ischemia. We compared the effectiveness of NBO and $\mathrm{HBO}$ in permanent focal ischemia, evaluated the combination of both modalities, and studied critical variables such as ischemia model and time window.

Methods: In mice $(n=140)$, permanent occlusion of the middle cerebral artery (MCAO) was induced either by coagulation or by filament. Animals received air, $\mathrm{NBO}$, a single or repeated $\mathrm{HBO}$ (3 ata) treatments. Infarct sizes and TUNEL-staining were quantified at $7 \mathrm{~d}$ and $24 \mathrm{~h}$, respectively

Results: Infarct volume was $12.9 \pm 1.6 \mathrm{~mm}^{3}$ in mice breathing air after MCA coagulation. When started $45 \mathrm{~min}$ after MCAO, NBO (10.8 \pm 2.2$)$ and significantly more potently $\mathrm{HBO}(7.8 \pm 0.9)$ reduced infarct size. $\mathrm{HBO}$ significantly decreased the number of TUNEL staining cells $24 \mathrm{~h}$ after MCAO compared to NBO. Repetative $\mathrm{HBO}$ treatments had no additional effect. HBO was also significantly more effective than NBO when initiated $2 \mathrm{~h}$ after MCAO. Combination of early NBO plus delayed $\mathrm{HBO}$ resulted in significantly smaller cortical infarcts than $2 \mathrm{~h}$ NBO treatment alone. In contrast, in filament-induced permanent MCAO, infarct volumes did not differ among mice receiving air $\left(50 \pm 24 \mathrm{~mm}^{3}\right)$, NBO $(46 \pm 21)$ or $\mathrm{HBO}(48 \pm 16)$. After filament-induced $2 \mathrm{~h}$ transient $\mathrm{MCAO}$, however, $\mathrm{HBO}$ treatment reduced infarct volume $\left(24 \pm 11 \mathrm{~mm}^{3}\right)$ compared to NBO $(41 \pm 19)$.

Conclusion: NBO and more effectively HBO protect the brain against permanen cortical ischemia. In more extensive focal ischemia, however, oxygen therapy is only effective in case of early recanalization.

Experimental studies 


\section{Experimental studies}

EFFECT OF S 18886, A THROMBOXANE A2 RECEPTOR ANTAGONIST, ON THE OCCURRENCE OF SPONTANEOUS BRAIN DAMAGE IN STROKE-PRONE RATS

E. Nobili, A. Gianella, P. Gelosa, U. Guerrini, V. Blanc-Guillemaud, L. Lerond, C. Banfi, M. Brioschi, E. Tremoli, L. Sironi

Department of Pharmacological Sciences, University of Milan, Milan, Italy

Background: Stroke-prone spontaneously hypertensive rats (SHR-SP) constitute the only animal model that spontaneously develop cerebrovascular events. In SHRSP, stroke is preceded by development of hypertension, proteinuria and inflammation. The aim of the present investigation was to assess the efficacy of S 18886 , an orally active antagonist of TP-receptors (the receptors for thromboxane A2), in preventing the pathological events in SHR-SP. S 18886 is being developed as an anti-atherothrombotic agent which possesses anti-inflammatory properties.

Methods and Results: Male SHR-SP ( $n=7$ per group), fed with a high-salt diet, received by gavage vehicle or $\mathrm{S} 1888630 \mathrm{mg} / \mathrm{kg} / \mathrm{day}$. The vehicle-treated animals showed a progressive increase of the protein loss per day (24-hour proteinuria) that sharply raised after four weeks. In these animals, brain lesions, detected by magnetic resonance imaging, developed spontaneously after $38 \pm 4$ days of diet and the animals died after $42 \pm 2$ days. S 18886 significantly delayed the increase of proteinuria $(\mathrm{p}<0.005)$, the appearance of brain lesion $(69 \pm 10$ days; $\mathrm{p}<0.001$ vs vehicle) and increased survival $(70 \pm 10$ days; $\mathrm{p}<0.001$ vs vehicle). S 18886 also exerted protective effects when it was administered after the first occurrence of proteinuria ( $>40 \mathrm{mg} /$ day $)(\mathrm{p}<0.05)$. Moreover, $\mathrm{S} 18886$ prevents the accumulation of acute-phase proteins and thiobarbituric acid-reactive substances observed in the body fluids of control animals.

Conclusion: These data indicate that, in SHRSP, S 18886 treatment, dosedependently prevents the occurrence of spontaneous brain damage and consequently increases the survival rate likely due to a reduction in inflammation and lipid peroxidation. These findings support the clinical use of S 18886 in prevention of cerebrovascular events.

\section{Experimental studies}

\section{ALTERED BEHAVIOUR OF NEURAL STEM CELLS IN INTACT AND LESIONED BRAIN AREAS}

V.A. Ágoston, A. Zádori, K. Demeter, E. Madarász, Z. Nagy

National Institute of Psychiatry and Neurology, National Stroke Centre,

Budapest, Hungary

Introduction: Our investigations were to compare survival and differentiation of neural stem cells implanted into intact brain, and lesioned cortical areas. NE-4C neuroectodermal stem cells harvested from E9, p53-/- mouse embryos are able to differentiate into neurons and astrocytes if induced by all-trans retinoic acid. These cells are pheno- and genotypically identical therefore serve as a unique model system for implantation studies on brain repair

Methodolgy: We implanted PLAP-, and GFP-expressing NE-4C neuroectodermal stem cells into the intact and lesioned cortices of rodent one week after the lesion. GFP-4C cells were shown to integrate into the embryonic brain. But in the intact adult rodent brain the cells did not migrate and could not survived longer than 4-6 weeks. On the other hand, GFP-4C cells showed long (>2 months) survival and sustained proliferation in the lesioned cortical areas. GFP-4C cells repopulated both the infarct core and the peri-lesion area, but showed a very low rate of differentiation. On the 62nd post-operative day, implanted cells were re-cultured from the lesioned brain region. Re-cultured GFP-4C cells showed no changes in morphology, chromosome-number, immunocytochemic properties, viability and in differentiation potential in comparison to the mother-cultures.

Conclusions: Results indicates that the altered behavior of stem cells was consequence of modified environment. The host environment strictly determines the fate of grafted stem cells. No malignant transformation of implanted stem cells was detected. The environmental niche provided by the injured/regenerating cortical tissue offers similar developmental opportunities for implanted cells, as it offered by the neonatal brain tissue.
13 Experimental studies

PROFOUND BUT TRANSIENT DEFICITS IN LEARNING AND MEMORY AFTER GLOBAL ISCHEMIA USING A NOVEL WATER MAZE TEST A. von Euler, O. Bendel, J. Sandin, T. Bueters, G. von Euler Department of Clinical Neuroscience, Karolinska Institutet, Karolinska University Hospital, Stockholm, Sweden

In search for neuroprotective treatment after ischemic brain injury it is essential with reproducible experimental animal models with reliable methods of evaluation of outcome, not only post-mortem, but also functionally. The pyramidal CA1 neurons of the hippocampus are critically involved in spatial learning and memory. These neurons are especially vulnerable to cerebral ischemia, but in spite of this, it has been consistently difficult to show any learning and memory deficits in 2-vessel occlusion models of global ischemia. In the present study transient global ischemia was induced in adult male rats under general anaesthesia administered by artificial respiration to prevent respiratory arrest. Systemic blood pressure was reduced to below $50 \mathrm{~mm} \mathrm{Hg}$ by instant adjustments of the halothane concentration, before and during 10 minutes of bilateral occlusion of the carotid arteries. Cerebral blood flow was monitored by laser-Doppler flowmetry. Learning and memory was assessed in a novel water T-maze with three successive left-right choices and also in a conventional Morris water maze. We found severe impairments in learning at 13 days after ischemia (DAI) and in memory, as tested $24 \mathrm{~h}$ afterwards. At 90 DAI the deficiency had disappeared and remained similar to controls up to 250 DAI. The impairment at 14 DAI was associated with a selective and profound cell death in CA1 as detected by TUNEL staining. Only about $3 \%$ of the CA1 neurons, as visualized with NeuN staining, remained at this time. The recovery of learning and memory performance at 90-125 DAI was associated with a reappearance of CA1 neurons suggesting that these newly formed neurons contributed to the functional improvement.

\section{Experimental studies}

MICROPLASMIN MAY IMPROVE RECOVERY IN A RAT EMBOLIC STROKE MODEL

R.S. Rasmussen, K. Overgaard, S. Pakola, G. Boysen

University Hospital of Copenhagen, Copenhagen East, Denmark

Introduction: The purpose of the present study was to examine effects of microplasmin on behavioural performance and infarct volume after middle cerebral artery occlusion in rats. Some experiments support that microplasmin may have neuroprotective properties in addition to its thrombolytic properties.

Methods: 80 rats underwent surgery and were embolized in the right carotid territory and randomly assigned to: 1) $5 \mathrm{mg} / \mathrm{kg}$ microplasmin, 2) $10 \mathrm{mg} / \mathrm{kg}$ microplasmin or 3 saline (control). Groups treated with microplasmin received $50 \%$ bolus injection 10 minutes after embolization and 50\% continuous infusion during the following hour. Animals from all groups were trained to obtain high baseline scores in Montoya's Staircase Test before embolization and were retested during days 7 to 14 after surgery.

Results: Including prematurely dead animals in group 1 achieved improved behavioural recovery compared to group 2 and 3 and had lesser infarct volume than group 2. Excluding prematurely dead animals no differences were observed among groups regarding infarct volumes. Mortality was significantly lower in group 1 than $2(\mathrm{P}<0.05)$ and when evaluating performances on days 7 to 14 after surgery, group 1 was better than group 2 concerning fine motor performance $(\mathrm{P}<0.05)$ and also achieved more normal bodyweight $(\mathrm{P}<0.05)$.

Conclusions: Including prematurely dead animals $5 \mathrm{mg} / \mathrm{kg}$ microplasmin treatment was associated with improved recovery compared to saline and $10 \mathrm{mg} / \mathrm{kg}$ microplasmin. Among surviving animals $5 \mathrm{mg} / \mathrm{kg}$ microplasmin treatment had no effect compared to saline treated control animals. $5 \mathrm{mg} / \mathrm{kg}$ microplasmin reduced mortality and improved both behavioural rehabilitation and bodyweight compared to $10 \mathrm{mg} / \mathrm{kg}$ microplasmin treatment, while saline treated animals did not differ from animals treated with $10 \mathrm{mg} / \mathrm{kg}$ microplasmin. Overall these results point toward a beneficial effect of $5 \mathrm{mg} / \mathrm{kg}$ microplasmin treatment, while $10 \mathrm{mg} / \mathrm{kg}$ was associated with a worsening of outcome.

\section{Experimental studies}

THROMBIN MEDIATES ISCHEMIC PRECONDITIONING IN VITRO

M. Price, J. Thevenet, J. Bogousslavsky, J. Badaut, L. Hirt

CHUV, Lausanne, Switzerland

Background: Prothrombin, the precursor of the coagulation factor thrombin is expressed in the brain. Low concentrations of thrombin can induce tolerance and 
protect the brain while high concentrations induce neuronal death. Using an in vitro ischemia model of hippocampal slice cultures subjected to oxygen and glucose deprivation, we have shown that thrombin is activated in response to ischemia and that it mediates ischemic neuronal death (de Castro et al, in press). Mild ischemia prior to a severe ischemia induces (ischemic preconditioning, IPC) protection. Interestingly, IPC appears to involve thrombin in vivo. We have now studied the role of thrombin in IPC in vitro, with the aim of comparing the role of thrombin in cell death or survival.

Material and Methods: Rat organotypic hippocampal slice cultures from P10-12 rats were subjected to an initial mild oxygen and glucose deprivation (OGD, 10\% O2, $1 \mathrm{mM}$ glucose for 15 minutes), followed 24 hours later by a more severe OGD (5\% O2, $1 \mathrm{mM}$ glucose for 30 minutes). Cell death was evaluated in the hippocampal CA1 region by propidium iodide uptake after 48 hours. Data were compared using the Kruskall-Wallis non-parametric ANOVA followed by the Dunn multiple comparison test.

Results: Mild OGD 24 hours before severe OGD reduces neuronal death in the CA1 region from $36 \pm 16 \%$ to $8 \pm 5 \%(\mathrm{p}<0.01)$. Treatment with the selective thrombin inhibitor after mild ischemia prevented the induction of ischemic tolerance (neuronal death $28 \pm 14 \%, \mathrm{p}<0.05$ compared to ischemic preconditioning).

Discussion: Thrombin is present in the central nervous system. It is activated in response to ischemia and plays a complex role. We and others have shown previously that it mediates ischemic neuronal death. We now show that it mediates also ischemic preconditioning in vitro. A better understanding of the role of coagulation factors in the CNS is critical for an optimal management of stroke patients.

\section{Long-term outcome of stroke}

\section{Long-term outcome of stroke}

\section{RISK OF CORONARY VASCULAR DEATH IN RELATION TO THE PRESENCE AND EXTENT OF ATHEROSCLEROTIC DISEASE AT THE CAROTID BIFURCATION \\ E. Touze, C. Warlow, P. Rothwell \\ University Department of Clinical Neurology, Oxford, United Kingdom}

Background: It has been proposed that unilateral carotid stenosis is due mainly to local haemodynamic factors whereas more diffuse disease reflects a systemic as well as a local predisposition and should therefore be associated with higher rates of arterial disease in other territories.

Methods: We studied arterial angiograms from 2741 patients with a recently symptomatic carotid stenosis from the European Carotid Surgery Trial and categorised them as showing purely unilateral disease (no plaque in the contralateral carotid artery) or bilateral disease.

Results: The degree of symptomatic carotid stenosis was unrelated to either the prevalence of symptomatic arterial disease in other territories at baseline or to risk of non-stroke vascular death during follow-up, whereas there were strong associations with the presence of bilateral carotid disease. After adjustment for traditional vascular risk factors, bilateral carotid disease $(n=2076)$ was associated with previous myocardial infarction $(\mathrm{OR}=1.7 ; 95 \% \mathrm{CI}: 1.2-2.4)$ or peripheral vascular disease $(\mathrm{OR}=1.5 ; 1.2-2.0)$ and with subsequent non-stroke vascular death $(\mathrm{HR}=2.0 ; 1.5-2.6)$. The 5-year risk of non-stroke vascular death ranged from $2.7 \%$ (1.5-4.6) in patients with no history of coronary or peripheral arterial disease and purely unilateral carotid stenosis to $21.4 \%$ (17.6-26.1) in those with bilateral carotid stenosis and a history of either coronary or peripheral arterial disease $(\mathrm{p}<0.0001)$.

Conclusion: Purely unilateral carotid stenosis is associated with low rates of symptomatic arterial disease in other territories, suggesting a local hemodynamic aetiology, whereas bilateral carotid disease indicates a systemic predisposition to atherosclerosis and hence the need for more aggressive preventive treatment.

\section{Long-term outcome of stroke}

\section{INTRACRANIAL STENOSIS INCREASES THE RISK OF RECURRENT VASCULAR EVENTS 6 MONTHS AND 1 YEAR AFTER ACUTE ISCHEMIC STROKE \\ J.L. Pascual, M. Lee, H. Gan, L. Wang, X. Xie, C. Teo, H.M. Chang, M.C. Wong, C.P. Chen \\ Singapore General Hospital, Singapore}

Background: Significant stenosis of the intracranial vessels is commonly found in Asian stroke patients. Previous studies have shown that the long-term outcome of ischemic stroke patients with intracranial atherosclerosis is poorer in that they are at higher risk of suffering death or further vascular events. We sought to determine the 1 year prognosis of acute ischemic stroke patients with intracranial atherosclerosis.

Methods: All patients admitted the Stroke Programme with a recent ischaemic stroke or TIA between November 2003 to July 2004 were eligible. Patients were investigated according to the SGH stroke care pathway; which includes investigations for vascular risk factors as well as neurovascular imaging. Study follow-up was arranged at 1 year with data collected on vascular outcome events.

Results: Of the 889 eligible patients, 610 (69\%) had complete ultrasound \& transcranial Doppler examinations of their extra- and intra-cranial vessels. Of these, $196(22 \%)$ had absent temporal windows and were therefore excluded. 43 patients were lost to follow up at 1 year. A total of 351 patients were included of whom $8 \%$ had significant extra- and $56 \%$ intra-cranial disease. There were 34 recurrent vascular events and 6 vascular deaths at 1 year - the majority being strokes. Univariate analysis showed that only the presence of significant intra- or extra-cranial disease were associated with recurrent vascular events or vascular deaths $(\mathrm{OR}=1.98,95 \%$ CI: $1.0-3.7$, and $\mathrm{OR}=3.1,95 \% \mathrm{CI}: 1.3-7.3$ respectively) at one year.

Conclusions: Significant stenosis of the intracranial vessels is a prognostic factor at 1 year post-stroke in that such patients are at an increased risk for recurrent vascular events or vascular death. These findings provide data for planning future randomized clinical trials in this group of stroke patients.

\section{Long-term outcome of stroke}

FURTHER ARGUMENTS FOR TWO LACUNAR STROKE SUBTYPES: RESULTS FROM A 15-YEAR FOLLOW-UP STUDY ON MORTALITY IN 782 ISCHEMIC STROKE PATIENTS

J. Staals, L. van Raak, A. Hilton, J. Lodder

University Hospital Maastricht, Maastricht, The Netherlands

Background: Earlier we hypothesized that two lacunar stroke phenotypes can be distinguished during life and differences in short-term follow-up favored such distinction. Differences in long-term outcome would further strengthen this concept. There are few mortality studies in lacunar stroke in comparison to other stroke types with a long-term follow-up. There are no long-term follow-up studies distinguishing two lacunar subtypes.

Methods: 782 first-ever ischemic stroke patients (42.6\% atherothrombotic (AT), $23.5 \%$ cardioembolic (CE), 33.9\% lacunar (LACI) strokes) were prospectively included between 1987 and 1992 in a stroke database. Survival status was collected in 2005.

Results: End of follow-up mortality in LACI (76.6\%) was significantly lower than in CE $(90.2 \%$; OR 0.54 , 95\% CI 0.30-0.97), but not significantly lower than in AT (80.8\%; OR 0.78, 95\%CI 0.53-1.15). Kaplan-Meier curve showed best survival in LACI and worst in CE (Log Rank test, $\mathrm{P}=0.000$ ). Cox regression analysis showed age, male gender, diabetes mellitus and stroke type (CE vs. LACI and AT) to be independent predictors of mortality. There were $179(69 \%)$ LACI type 1 patients (without silent lacunar lesions on CTscan) and $80(31 \%)$ LACI type 2 patients (with silent lacunar lesions). End of follow-up mortality in LACI type 2 was significantly higher than in LACI type 1 (87.5\% vs. 70.9\%; OR 2.87, 95\%CI 1.37-5.99). KaplanMeier curve showed better survival in LACI type 1 (Log Rank test, $\mathrm{P}=0.001$ ). Cox regression analysis showed age, male gender, diabetes mellitus, ischemic heart disease and lacunar subtype to be independent predictors of mortality.

Discussion: On the long term LACI stroke has no better prognosis on mortality than AT stroke and should therefore not be considered a benign stroke type. Differences in long-term prognosis further validate the concept of two different subtypes of lacunar stroke. Such validated "phenotyping" may facilitate future research into underlying differences in genotype.

\section{Long-term outcome of stroke}

LONG-TERM SURVIVAL AFTER STROKE

H. Hassanpour, M. Grønbæk, G. Boysen, T. Truelsen

Rigshospitalet, Copenhagen, Denmark

Background: Data on long-term survival after stroke is available from only few studies. Knowledge about survival is importment for predicting the future burden of stroke. The present study presents up to 16 years of follow-up of patients after a first-time-ever stroke.

Methods: 19,698 participants in the Copenhagen City Heart Study were followed from 1976 to 1997. First-time-ever stroke events were validated using the World Health Organization's definition of stroke. Linkage to the Danish National Register of Death enabled complete identification of all patients who died and provided data on time of death and cause of death. Cox regression analyses adjusting for age and sex were used to estimate survival $4,8,12$, and 16 years after stroke onset. To demonstrate changes beyond the acute phase the analyses were restricted to patients

Long-term outcome of stroke 
who survived the initial seven days after stroke. Causes of death were obtained from death registration forms.

Results: A total of 1659 patients with first-ever stroke were registered. Of these 927 $(56 \%)$ were fatal during follow-up. Compared with the reference period 1978-81 four-years survival improved significantly during the period of surveillance and Relative Risk (RR) of death was 0.60 (95\% CI: 0.34-0.93) in 1994-1997 compared with 1978-1981. 8, 12, and 16 years survival also improved: RR 8 years $=0.84$ $(95 \%$ CI $0.60-1.30) ;$ RR 12 years $=0.95(95 \%$ CI $0.64-1.40)$; and RR 16 years $=$ 0.81 (95\% CI 0.60-1.20). Causes of death in stroke patients were cerebrovasculare diseases in $27 \%$, cardiovascular diseases in $37 \%$, cancer in $11 \%$, and $25 \%$ died from other causes.

Conclusions: long-term survival has improved since the periode 1978-1981. Two-thirds of stroke patients died from cardiovascular diseases, including cerebrovasculare disease.

\section{Long-term outcome of stroke}

\section{LONG-TERM EFFECT OF INTRA-ARTERIAL THROMBOLYTIC THERAPY \\ IN ACUTE STROKE}

K. Nedeltchev, U. Fischer, M. Arnold, L. Kappeler, T. Haefeli, C. Brekenfeld,

L. Remonda, G. Schroth, H.P. Mattle

Inselspital, University of Bern, Bern, Switzerland

Background: Thrombolysis has been shown to improve the 3-months outcome of patients with acute ischemic stroke. Knowledge regarding the long-term effects of thrombolysis in stroke is limited.

Patients and Methods: Monocenter, retrospective study comparing the long-term outcome of patients who were treated with intra-arterial urokinase (IAT) with that of patients receiving aspirin or heparin only (non-IAT). The long-term outcome was assessed using the modified Rankin scale (mRS). 173 IAT and 261 non-IAT patients from the Bernese Stroke Registry were eligible for the study. A matching algorithm that took into account patients' age and initial stroke severity (as measured by the National Institute of Health Stroke Scale, NIHSS) was used to assemble a IAT and a non-IAT group. Matching was blinded for clinical outcome.

Results: 144 IAT patients (mean age 63 years, 50\% women) and 147 non-IAT patients (mean age 62 years, $45 \%$ women) were included in the comparative analysis. The median NIHSS was 14 in each group. At 2 years, $57 \%$ of the IAT patients and $45 \%$ of the non-IAT had a favourable outcome when defined as mRS 0 to 2 ( $\mathrm{p}=$ 0.037 ), or $41 \%$ and $26 \%$, when favourable outcome was defined as mRS 0 to 1 ( $\mathrm{p}=$ 0.008 ). Mortality was $23 \%$ in the IAT and $24 \%$ in the non-IAT group.

Conclusion: The present study provides evidence for a sustained effect of IAT when assessed 2 years after the stroke. The results support the more widespread use of IAT for treatment of acute stroke.

\section{Long-term outcome of stroke}

LONG-TERM EFFECTS OF SUBARACHNOID HEMORRHAGE ON EMPLOYMENT, RELATIONSHIPS, PERSONALITY AND MOOD

M.J.H. Wermer, H. Kool, K.W. Albrecht, G.J.E. Rinkel

University Medical Center Utrecht, Utrecht, The Netherlands

Background: Only one third of patients regains functional independence after aneurysmal subarachnoid hemorrhage (SAH). Despite this recovery, many of such patients experience psychosocial problems. We assessed the long-term effects of SAH on employment, relationships, personality and mood.

Methods: We included patients who had been treated by clipping after SAH between 1985 and 2001 and resumed independent living. Patients underwent structured interviews regarding employment, relationships and personality before and after the SAH. Anxiety and depression were assessed by the Hospital Anxiety and Depression Scale (HADS) and scores were compared between the study group and a reference population.

Results: 610 patients were interviewed (mean follow-up after SAH 8.9 years). Of the employed patients $26 \%$ stopped working and $24 \%$ worked shorter hours or had a position with less responsibility. On average, patients returned to work 9.4 months after discharge (range 0-96). Seven percent of patients got divorced because of SAH related problems. Fifty-nine percent of the patients reported changes in personality, most commonly increased irritability (37\%) or emotionality $(29 \%)$. SAH patients had a statistically significant higher mean depression score than the reference population. Approximately $10 \%$ of the patients had a HADS score in the range of a probable depression or anxious state. Only $25 \%$ reported a complete recovery without psychosocial or neurological problems.

Conclusions: The long-term psychosocial effects of SAH are considerable even in patients who regain functional independence. Treating physicians should be aware of these long-term effects of SAH when discussing prognosis and reintegration to work after initial recovery with patients and family

\section{Long-term outcome of stroke}

\section{ACETYLCHOLINESTERASE AND LONG-TERM OUTCOME AFTER ACUTE} ISCHEMIC STROKE

S. Shenhar-Tsarfaty, I. Bova, K. Ofek, N.M. Bornstein, L. Shopin, S. Berliner, I. Shapira, H. Soreq, E. Ben-Assayag

Tel-Aviv Sourasky Medical Center, The Hebrew University, Tel-Aviv, Israel

Background: Evidence from recent years pointed at the stress response as key components in the clinical deterioration following acute ischemic stroke. The acetylcholinesterase enzyme (AChE) regulates acetylcholine levels in both the CNS and periphery. The role of AChE has been previously established in muscle malfunctioning, prolonging the recovery from brain injury, Parkinson disease, Alzheimer and predisposition to anxiety.

Therefore, we aimed to associate between impaired cholinergic regulation, as reflected by variable AChE activity, who shown to reflect anxiety/stress parameters, and neurological outcome in acute ischemic stroke.

Methods: We evaluated AChE activity in 120 acute ischemic stroke patients within $24 \mathrm{~h}$ from hospital admission and 6 months following the event. Neurological deficit was evaluated using the NIH Stroke Scale (NIHss).

Results: Serum AChE activity on time 1 (first day of acute stroke) correlated significantly with the neurological score NIHss 6 months following the event (time 2) $(\mathrm{r}=0.221 \mathrm{p}=0.015)$. A predictive model was derived by stepwise linear regression analysis with the neurological score NIHss on time 2 as a dependent variable and age, gender, BMI, diabetes mellitus, hyperlipidemia, hypertension, past and present smoking, NIHss on time 1 and AChE activity on time 1 as the independent ones. The results demonstrate that AChE activity and NIHss score on time 1 $(\mathrm{p}=0.002, \mathrm{p}=0.0001$, respectively, Model $\mathrm{R} 2=0.419)$ are valuable predictors for the neurological outcome of acute stroke patients 6 months following the acute event. Conclusions: We found strong association between AChE activity on first day of acute stroke and long-term outcome. These findings demonstrate that cholinergic imbalance may have clinical manifestations in acute stroke.

Understanding the contribution of the stress response to the neurological state in acute stroke may shed light on new approaches for the treatment of stroke patients.

\section{Long-term outcome of stroke}

\section{ISCHEMIC STROKE IN MEXICAN YOUNG ADULTS: PREDICTORS OF} OUTCOME AND RECURRENCE

A. Leyva, M. López, F. Barinagarrementeria, C. Cantú, A. Arauz

National Institute of Neurology and Neurosurgery, Mexico City, Mexico

Background and Objective: Ischemic stroke in young people has a high frequency in our institution and there is limited information about predictors of outcome and recurrence; the objective was to define the frequency of stroke etiology in Mexican young adults and to determine the predictive value of risk factors for outcome and recurrence.

Methods: Clinical data for 792 patients of the Stroke Clinic data base aged 15 to 45 years were reviewed; risk factors, etiology (TOAST), severity (NIHSS) and subtype (Bamford criteria) were evaluated; the outcome was evaluated using modified Rankin scale (mRS) at 3 months as favorable (0-2) and unfavorable (3-6); recurrence was assessed in months after 1 st clinical event.

Results: Stroke in the young comprised $33.7 \%$ of 2345 consecutive patients with ischemic stroke (men 48.7\%); stroke was caused by atherosclerotic large artery disease in $4.4 \%$, cardiac embolism in $23.2 \%$ (patent foramen ovale [PFO] $13.8 \%$ ), small vessel disease in $3.2 \%$, another determined etiology in $39.4 \%$ (arterial dissection $15.2 \%$ ) and undetermined etiology $29.7 \%$. Clinical outcome at three months was favorable in $58 \%$, unfavorable in $38.6 \%$ and death $3.4 \%$. The median follow up was 25 months; recurrence was $7.1 \%$. Alcohol use $(\mathrm{p}=0.005)$ and large artery disease $(\mathrm{p}=0.02)$ were independent predictors of unfavorable outcome or death; previous stroke and prothrombotic state predicted recurrence.

Conclusions: Arterial dissection and PFO were the most frequently stroke etiology; alcohol use and large artery disease were predictors for unfavorable outcome and previous stroke and prothrombotic state predicted recurrence. 
9 Long-term outcome of stroke

IMPACT OF LEVEL OF DEPENDENCY AT 6 MONTHS ON LONG-TERM SURVIVAL AFTER ISCHAEMIC STROKE IN 5651 PATIENTS FROM OCSP AND IST-1

K. Bruins Slot, E. Berge, P. Sandercock, S. Lewis, on behalf of the OCSP and IST-1 UK Collaborative Groups

Ullevål University Hospital, Oslo, Norway

Background: To inform clinical practice and health economic analyses, we sought to examine the effect of the degree of dependency at 6 months on long-term survival in 2 large prospective cohorts of patients with ischaemic stroke.

Methods: The two cohorts were patients from the Oxford Community Stroke Project (OCSP) registered between 1981 and 1986 and patients enrolled in the First International Stroke Trial (IST-1) in the UK between 1991 and 1996, who were alive and whose degree of dependency (modified Rankin scale (mRs)) was known at six months after their index stroke. We divided patients into two groups based on their $\mathrm{mRs}$ at 6 months (mRs 0-2 and mRs 3-5). We recorded all deaths occurring in both cohorts up to the end of 2000. Survival was estimated using Kaplan-Meier methods. Results: In the OCSP, $513(81 \%)$ patients had a definite ischaemic stroke; $97(15 \%)$ patients died $<6$ months. Of survivors at 6 months, $281(55 \%)$ patients were mRs $0-2$ and $153(30 \%) \mathrm{mRs} 3-5$. Median survival in the mRs 0-2 group was 9.5 years (95\% CI 8.5-10.5) vs. 4.2 years (95\% CI 3.2-5.2) in the mRs 3-5 group. There was a significant difference in survival rates between these two groups (log rank test $\mathrm{p}<0.001)$. Of UK patients in IST-1, $5138 \mathrm{had}$ an ischaemic stroke and $1298(25 \%)$ died $<6$ months. Of survivors at 6 months, $1101(21 \%)$ were mRs $0-2$ and 2739 $(53 \%) \mathrm{mRs} 3-5$. Five years after stroke onset $75 \%$ of patients in the mRs $0-2$ group were still alive vs. $57 \%$ in the mRs 3-5 group. There was a significant difference in survival rates in the 2 outcome groups $(\log$ rank test $\mathrm{p}<0.001)$.

Conclusions: Dependency at 6 months post stroke has a significant effect on long-term survival after ischaemic stroke. Treatments, which have even modest effects on dependency at six months, could therefore have a significant impact both on long-term clinical outcome and cost-effectiveness.

\section{Long-term outcome of stroke}

RISK OF VASCULAR DEATH AND MYOCARDIAL INFARCTION IN PATIENTS WITH STROKE OR TIA: RESULTS FROM THE REDUCTION OF ATHEROTHROMBOSIS FOR CONTINUED HEALTH (REACH) REGISTRY

J.L. Mas, E. Touze, J. Rother, M.J. Alberts, S. Goto, A.T. Hirsch, E.M. Ohman, P.W.F. Wilson, D.L. Bhatt, P.G. Steg, on behalf of the REACH Registry Investigators

Hôpital Saint-Anne, Paris, France

Background: Patients with history of stroke or TIA are at risk of subsequent cardiac events. However, the absolute risk and predictors of such events are not well known. Methods: The REACH Registry enrolled $>68,000$ outpatients with documented atherothrombotic disease or with $\geq 3$ atherothrombotic risk factors, from 44 countries all over the world, including 18,450 patients with previous stroke and/or TIA.

Results: Preliminary analysis of follow-up data on 15,481 patients with previous stroke and/or TIA, followed for a mean duration of 11.3 months, showed there were $410(2.65 \%)$ non-stroke vascular deaths and 178 (1.15\%) non-fatal myocardial infarctions (MI). Patients with previous history of symptomatic coronary artery disease had a higher risk of non-stroke vascular death $(2.6 \%$ vs. $1.1 \%, \mathrm{p}<0.0001)$ or non-fatal MI $(2.0 \%$ vs. $0.7 \%, \mathrm{p}<0.0001)$ than those without such history. In the 9,575 patients without history of symptomatic coronary artery disease at baseline, age $(\mathrm{HR}=1.5 ; 95 \% \mathrm{CI}: 1.2-1.9)$, diabetes $(\mathrm{HR}=1.6 ; 1.1-2.3)$ and peripheral arterial disease $(\mathrm{HR}=2.0 ; 1.2-3.4)$ were independently associated with a higher risk of nonstroke vascular death or non-fatal MI. However, history of hypertension, abdominal obesity, smoking (current, former), male gender and elevated total cholesterol at baseline did not predict future cardiac events.

Conclusion: Patients with stroke or TIA without past history of symptomatic coronary artery disease have a fairly high risk of subsequent non-stroke vascular death or non-fatal MI. Traditional risk factors do not fully predict the risk of those events, emphasizing the need for identifying other determinants.
11 Long-term outcome of stroke

WOULD FIRST-DAY BODY TEMPERATURE DYNAMICS CONTRIBUTE INDEPENDENTLY TO LONG-TERM HANDICAP AFTER ACUTE ISCHEMIC STROKE?

J. Mau, S. Jayavel, M. Yong

Heinrich Heine University Hospital, Insitute of Statistics in Medicine, Duesseldorf, Germany

Background and Aims: Studies of associations between body temperature (BTp) in acute ischemic stroke and long-term outcome have not led to satisfactory conclusions. The dynamics of BTp during the first day were expected to be more clearly linked to functional outcome than individual measurements of BTp at baseline or later. This was to be studied in a data base from a trial of thrombolytic treatment.

Methods: In the first European Cooperative Acute Stroke Study (ECASS-I) patients had been randomized to either $1.1 \mathrm{mg} / \mathrm{kg}$ IV rt-PA or placebo within 6 hours after symptom onset; 615 patients received trial medication. BTp was measured at baseline, and again after 2 and after 24 hours. Long-term handicap was graded on the modified Rankin scale around day 90 . Good outcome was considered as no or negligible persisting handicap (grades 0 or 1), and poor outcome as severe or complete dependency (grades 4 or 5). Profiles of individual BTp readings were grouped as constant, either only early or only late rising, persistently rising, dropping in any way, concave, or convex. Stepwise multifactorial logistic regression included all anamnestic and baseline clinical, neurological, and neuroradiological assessments.

Results: Constant and only late rising BTp profiles suggested reduced risks of poor outcome at levels of nominal significance, already. No impact on mortality was found after adjusting for thrombolytic treatment and known contributing factors. Instead, constant profiles still somewhat reduced the risk of poor outcome among 90-day survivors, $\mathrm{OR}=0.268$ (95\% CI $0.078-0.925 ; \mathrm{P}=0.037)$, while profiles rising only late significantly reduced the risk of complete dependency or death among all first-day survivors, OR=0.427 (95\%CI 0.232-0.767; P=0.0047), independently of all other known significant predictors and thrombolytic treatment.

Conclusion: The dynamics of body temperature within the first 24-30 hours add to the known risk profiles of thrombolysis independently.

\section{Long-term outcome of stroke}

THE OXFORDSHIRE COMMUNITY STROKE PROJECT STROKE SUBTYPE PREDICTS FOR OUTCOME AND DISABILITY AT 1 YEAR AFTER ACUTE ISCHEMIC STROKE

J.L. Pascual, C. Teo, H.M. Chang, M.C. Wong, C.P. Chen

Singapore General Hospital, Singapore

Background: The Oxfordshire Community Stroke Project (OCSP)classification for acute stroke has been shown to be an independent predictor for survival in a number of studies limited to Western populations. We aim to investigate the

prognostic value of OCSP subtypes in Singaporean acute ischemic stroke patients who are of Chinese, Malay and Indian ethnicity.

Method: All acute ischemic stroke and transient ischemic attack (TIA) admitted from October 2002 to July 2004 were classified as total anterior circulation infarcts (TACI), partial anterior circulation infarcts (PACI), posterior circulation infarcts (POCI), and lacunar infarcts (LACI) using the OCSP criteria. They were followed up after 1 year and assessed for disability using the modified Rankin score (mRS). Results: Of 1299 eligible patients, 129 were lost to follow-up. 1170 patients were included in this study. $55 \%$ of patients had LACI. TIA patients were significantly younger by 4 years than the other subtypes (mean age $=62 \pm 12$ years). On univariate analysis, OCSP subtype,ischemic heart disease, atrial fibrillation (AF), diabetes, peripheral artery disease (PAD),hyperlipidemia, and smoking were associated with higher mortality. Among OCSP subtypes TACI $(\mathrm{p}<0.0001)$ and PACI $(\mathrm{p}<0.0001)$ had significantly higher mortality. PACI/TACI patients also had significantly higher MRS scores $(\mathrm{p}<0.0001)$. On multiple logistic regression, TACI /PACI remained independent predictors for mortality or disability at 1 year.

Conclusions: The OCSP classification is useful for identifying Asian patients who may be at high risk for death and significant disability. 


\section{Management and economics}

\section{Management and economics}

\section{SETTING PRIORITIES IN STROKE CARE: THE 2006 COMPREHENSIVE SWEDISH STROKE GUIDELINES}

B. Norrving, the Stroke Guidelines Working Group, National Board of Health and Welfare, Sweden

Lund Univerity Hospital, Lund, Sweden

Background: Traditional clinical guidelines usually focus on levels of evidence and strength of recommendations. For disorders causing a heavy societal burden the Swedish National Board of Health and Welfare now produces a series of more comprehensive guidelines which extend conventional ones by including open (transparent) priority-setting between various interventions for prevention, diagnostics, treatment and rehabilitation, and taking basic ethical principles (human dignity, need and solidarity, and cost-efficiency) approved by the Parliament into account. The guidelines on stroke will be issued in early 2006

Methods: For a number of well-defined management issues in stroke care, possible interventions were identified. Each 'medical condition - intervention' pair was assessed according to needs, baseline risks, effects, quality of evidence, and costeffectiveness. A modified Delphi approach was used to set priorities, ranging from 1 (top priority) to 10 (no priority). Procedures judged to be outdated, too hazardous, too cost-ineffective or lacking scientific support were assigned to a do-not-do list. Results: 102 'medical condition - intervention' pairs were listed; 49 were assigned a priority grade of 1-3,30 a grade of 4-7, and 23 a grade of 8-10. Twenty interventions (7 diagnostic and 13 therapies) that should not be applied in routine clinical practise were separately listed. One diagnostic procedure and 15 treatments were assigned to a list of interventions awaiting more solid scientific evidence.

Conclusions: This process used shows that open/translucent priority-setting through consensus across different interests (professionals, decision-makers and patients) is feasible. Examples of priorities for different management issues will be presented.

\section{Management and economics}

\section{LIFETIME COST OF ISCHEMIC STROKE IN GERMANY - RESULTS AND NATIONAL PROJECTIONS FROM A POPULATION-BASED STROKE REGISTRY: THE ERLANGEN STROKE PROJECT}

P. Kolominsky-Rabas, P.U. Heuschmann, D. Marschall, O. Schöffski, K. Krobot Unit for Stroke Research \& Public Health Medicine, Interdisciplinary Center for Public Health Studi, Erlangen, Germany

Background: The number of stroke patients and the healthcare costs of strokes are expected to rise. The objective of this study was to determine the direct costs of first ischemic stroke and to estimate the expected increase in costs in Germany.

Methods: An incidence-based, bottom-up, direct-cost-of-ischemic-stroke study from the third-party payer's perspective was performed, incorporating 10-year survival data and 5-year resource use data from the Erlangen Stroke Registry (ESPro). The ESPro is a prospective population-based stroke registry based in Bavaria, Germany covering a total study population of 102,000 residents (census 2003) in the Community of Erlangen. The unique feature of the ESPro is its continuing case ascertainment and long-term follow-up, irrespective of age, without any stop since 1994.

Results: A total of 1637 and 821 patients from the ESPro were available for the survival analyses and the healthcare resource utilization analyses, respectively. First-year cost accounted for about $35 \%$ of the present value of the lifetime cost of first ischemic stroke. Mean undiscounted lifetime cost was higher in men (54,552 EUR) than in women $(47,596$ EUR). Overall, the lifetime cost per ischemic stroke was 50,507 EUR undiscounted and 43,129 EUR discounted. National projections for the period 2006-2025 showed 1.5 million and 1.9 million new cases of ischemic stroke in men and women, respectively, at a present value of 51.5 and 57.1 billion EUR

Discussion: The number of stroke patients and the healthcare costs of strokes in Germany will rise continuously until the year 2025. Therefore, stroke prevention and reduction of stroke-related disability should be made priorities in health planning policies.
3 Management and economics

EFFECTIVENESS OF STROKE CENTERS IN FINLAND - IMPACT ON CASE FATALITY AND RISK OF INSTITUTIONAL CARE IN ISCHEMIC STROKE PATIENTS

A. Meretoja, S. Roine, U. Hakkinen, M. Linna, A. Liski, A. Puumalainen, M. Kaste, S.O. Roine

Helsinki University Central Hospital, Helsinki, Finland

Background: Stroke centers have been shown effective in reducing death and risk of institutional care. It is not known which components of care contribute to this effect. Our study compares patient outcome in differently equipped stroke centers. Methods: All non-institutionalized first-ever ischemic stroke patients over 18 years of age treated in Finnish hospitals in 2001-2003 were included in the registry-based analysis. Patients were selected by joining the causes of death registry and the national hospital discharge registry, including all acute and long term institutional care in Finland. Case fatality and institutional care were assessed up to one year, and Odds ratios adjusted for age and sex were calculated. The treating hospitals were classified as primary stroke centers (PSC) and comprehensive stroke centers (CSC), according to previously published criteria (JAMA; 283:3102, Stroke; 36:1597) Hospitals not meeting these criteria were classified as general hospitals $(\mathrm{GH})$ Classification was based on an on-site audit of stroke resources in each hospital.

Results: The 4 hospitals identified as CSCs treated 6083 patients (mean age 69.0 years, $52 \%$ male). The 8 PSCs treated 6355 patients $(70.8,51 \%)$ and the $30 \mathrm{GHs}$ $10395(76.0,43 \%)$

The patients treated in stroke centers were more likely to survive and to return home than patients treated in GHs, even when adjusted for age and sex. Similarly, CSCs were superior to PSCs (Table).

Case Fatality and Risk of Institutional Care

\begin{tabular}{lccccc}
\hline & CSC & PSC & GH & OR CSC vs PSC & OR PSC vs GH \\
\hline Case Fatality (7 d) & $4.5 \%$ & $5.7 \%$ & $5.7 \%$ & $1.24(1.05-1.45)$ & $1.03(0.88-1.20)$ \\
Case Fatality (28 d) & $8.4 \%$ & $10.2 \%$ & $14.0 \%$ & $1.15(1.02-1.30)$ & $1.22(1.09-1.36)$ \\
Case Fatality (365 d) & $16.6 \%$ & $19.4 \%$ & $30.3 \%$ & $1.13(1.03-1.24)$ & $1.46(1.34-1.59)$ \\
Institutional Care (28 d) & $28.8 \%$ & $31.0 \%$ & $40.2 \%$ & $1.07(0.99-1.16)$ & $1.41(1.32-1.52)$
\end{tabular}

Adjusted Odds ratios (with $95 \% \mathrm{CI}$ ) for age and sex

Discussion: In Finland, patients with acute stroke are only treated in public hospitals. Differences in resource allocation to stroke care are large between different parts of the country. This study suggests that investment in stroke care saves lives and leads to meaningful recovery, as reflected by lower institutionalization rates.

\section{Management and economics}

ECONOMIC BURDEN OF STROKE IN THE UK

Ö. Saka, A. McGuire, C. Wolfe

Kings College, London, United Kingdom

Background: Although stroke is a major public health issue there are few studies analysing the resource implications of treatment in this area. This study presents the cost of stroke in the UK from a societal perspective.

Methods: The study uses a bottom up approach to calculate the costs accrued as a result of inpatient and outpatient services and informal care. Productivity losses attributable to stroke from morbidity and mortality are calculated separately. Incidence and prevalence rates were based on previously published studies supplemented by data obtained from the South London Stroke Register. These were combined with various estimates of treatment costs drawn from published and NHS sources. The resulting total cost of illness was estimated for the UK for the year 2003 .

Results: Stroke treatment and productivity losses results in total costs of $£ 8$ billion a year, with treatment costs accounting for 5-6\% of total NHS costs per annum. Annual direct care accounts for approximately $40 \%$ of this total; informal care for $35 \%$; and the indirect costs for approximately $25 \%$. Sensitivity analyses was conducted on this baseline figure by increasing and decreasing the prevalence and incidence rates, health care unit costs and the volume of their use. Using different prevalence rates had the largest impact on the costs. Variations in the cost of care had a small effect, although the impact of variation in specialist time spent on patients was significant. Total annual direct care costs varied between $£ 2.5$ - $£ 3.3$ bn when the resource use items were alternated by $20 \%$.

Discussion: Stroke is a very costly disease, mainly because of the long-term nature of the. The calculations showed a high sensitivity to prevalence rates, not so much to the incidence rates; due to that nature. Given the current unprecedented increases in the expenditure on health care in the UK the importance of stroke, as a major disease area would support the use of increased resources in this area. 


\section{Management and economics}

COMPARING PATIENT PERCEPTIONS OF THE QUALITY OF STROKE CARE WITH AUDIT OF HOSPITAL RECORDS IN ENGLAND A.G. Rudd, D. Lowe, A. Hoffman, C. Down, R. Reeves, E. Howells

Royal College of Physicians, London, United Kingdom

Health services are increasingly using patient surveys to assess the quality of care provision. There has been no previous attempt to correlate how their views correlate with evidence based measures of quality.

Methods: The national audit of stroke care involved detailed analysis of case records of consecutive admissions to all hospitals in England. Concurrently with the 2004 audit the Picker Institute conducted a patient survey in a randomly selected third (51) of these hospitals. Further details of the audits can be found on www.rcplondon.ac.uk. and http://www.nhssurveys.org/docs/Stroke_Development_ Report.pdf. Patient data for patients discharged alive were matched between the national audit and patient survey on the basis of trust, year of birth, date of admission and date of discharge.

Results: There were 1042 discharged patients in both the national stroke audit and the patient survey. There was evidence that in better organised sites a higher percentage of patients rated their care as very good or excellent (Spearman 0.29, $\mathrm{P}=0.02$ ) Eleven process indicators were analysed which were those that were identified as best representing the total clinical process for each hospital. There was generally an absence of correlation between patient satisfaction and compliance with evidence based standards. Using Fisher test $\mathrm{P}<0.01$ as a sieve then only the assessment of weight and performance of a home visit were associated with requiring help from others for everyday activities, assessment of mood with the feeling of not having made a complete recovery and being assessed by an occupational therapist with higher ratings of care.

Discussion: There is some evidence to show that patients appreciated better organised stroke care but little correlation was found between patient perception and better performance on the audit of process of care in hospital. These data suggest that patient surveys cannot be used alone as a measure of quality of care.

\section{Management and economics}

\section{DEVELOPMENT AND VALIDATION OF A SIMPLE DEFICIT SCORE FOR} MEASURING ISCHEMIC STROKE SEVERITY

C. Prugger, P.U. Heuschmann, A. Ray, J. Schenkel, H.J. Audebert

Institute of Epidemiology and Social Medicine, University of Muenster, Muenster, Germany

Background: Initial stroke severity is important in predicting stroke outcome National Institute of Health Stroke Scale (NIHSS) is gold standard for measuring severity but requires specific training of raters. Use of NIHSS might be hampered in settings where no regular training could be secured. We developed and internally validated a Simple Deficit Score (SDS) for estimating stroke severity based on routinely collected data.

Methods: Data were collected within the Telemedic Pilot Project for Integrative Stroke Care (TEMPiS). Neurological deficits and NIHSS were assessed in consecutively admitted stroke patients within $24 \mathrm{~h}$ of admission by trained physicians or interviewers. SDS was calculated as cumulative number of following deficits: motor deficit, aphasia, dysarthria, disturbed consciousness. For comparing SDS with NIHSS the dataset was split randomly into two equal subsets; first served as exploratory, second as validation set. Psychometric properties and prognostic accuracy of SDS in terms of seven day mortality were assessed in exploratory and reproduced in validation set.

Results: Overall, 2052 patients with ischemic stroke were included. SDS showed good agreement with NIHSS $(r=0.7)$. SDS and NIHSS demonstrated similar discrimination abilities for seven day mortality in multivariate logistic regression mode (c-statistic 0.90 and 0.94, respectively). Sensitivity (SDS 88.6; NIHSS 93.2) and Positive Predictive Value (SDS 20.5; NIHSS 20.3) for seven day mortality were comparable between both measures. Good performance of SDS could be replicated in validation set.

Conclusion: SDS is a reliable and valid tool for measuring stroke severity. Its predictive properties are comparable to those of NIHSS. SDS should be used in settings where NIHSS cannot be documented reliably.
7 Management and economics

OBSTACLES TO THE USE OF INTRAVENOUS TISSUE PLASMINOGEN ACTIVATOR FOR ACUTE ISCHEMIC STROKE IN BELGIUM. IS TIME THE ONLY BARRIER?

P. Laloux, V. Thijs, A. Peeters, P. Desfontaines

UCL University Mont-Godinne, University Hospitals Leuven, UCL University Brussels, Centre Hospitalier Chrétien Liège, Yvoir, Belgium

Background and Aims: the short time window ( $<3$ hours) is frequently cited as the main reason for exclusion of iv thrombolysis in acute stroke. Identifying an circumventing barriers to thrombolysis other than time could increase the frequency of treatment. The main goal of this study was to identify whether the rate of treatment with iv tPA would increase if time window was not an obstacle to treatment.

Methods: in four hospitals we prospectively collected (1) the rate of iv tPA use in consecutive patients admitted with acute ischemic cerebrovascular disease and (2) in those admitted within 3 hours, the reasons why thrombolysis was not given, and (3) the potential gain in the rate of tPA use if all patients were admitted within 3 hours by taking into account all exclusion criteria other than time.

Results: we recruited 335 patients (mean age, $70 \pm 14 ; 183$ men, 152 women), of whom $100(29.8 \%)$ were admitted within 3 hours. The time of stroke onset was unknown in $16(4.8 \%)$. The rate of tPA use was $9.5 \%$ in the study population. The main exclusion criteria for treatment were delayed admission (51.9\%), mild stroke $(33.7 \%)$, age $>80(21.2 \%)$, unknown time of onset $(19.4 \%)$, TIA $(16.4 \%)$, and rapidly recovering stroke $(5.3 \%)$. Treatment was not given in $7.8 \%$ despite the lack of any exclusion criteria. Of the 100 patients admitted within 3 hours, the rate of tPA use was $32 \%$. TIA $(21.0 \%)$, age $(20.0 \%)$, mild $(19.0 \%)$ or recovering stroke $(14.0 \%)$ were the main reasons for exclusion in this subgroup. If all patients had hypothetically been admitted within 3 hours, the rate of tPA use would have increased from $9.5 \%$ up to $21.4 \%$ and up to $33.71 \%$ if aged patients and those with no documented exclusions had been treated.

Conclusions: a significant minority of stroke patients are not treated despite having no contraindications. Delayed presentation to hospital is the main factor limiting the use of thrombolysis in acute stroke. If there were no delay in presentation to the hospital, additional increases would have been observed especially if aged patients and those without contraindications had been treated.

\section{Management and economics}

\section{WHY INVEST IN A NATIONAL HEALTH PROGRAM FOR STROKE? AN EXAMPLE FROM AUSTRALIA}

D.A. Cadilhac, R.C. Carter, A.G. Thrift, H.M. Dewey

National Stroke Research Institute, Heidelberg Heights, VIC, Australia

Background: Given the expected rise in stroke numbers due to population ageing and evidence that access to best-practice management is variable, the National Stroke Foundation (Australia) launched a 10-year public health program (strokesafe ${ }^{\mathrm{TM}}$ ) in 2004 , at an expected annual cost of AUD $\$ 12$ million. However, program success will require upfront commitment by several partners. We undertook an exploratory economic analysis to: 1) predict potentially achievable health-gains and cost-offsets; and 2) determine the economically warranted size of budget-spend as an investment argument to policy-makers.

Methods: We estimated the likely impact of strokesafe ${ }^{\mathrm{TM}}$ in a representative year (2015) assuming maximum achievable effectiveness using local epidemiological and cost data. Costs and outcomes (Disability Adjusted Life Years [DALYs]) were calculated for the additional cases that would benefit if current practice were improved to reflect 'best-practice'. Interventions: blood pressure lowering; warfarin for atrial fibrillation; access to stroke units; increased use of intravenous thrombolysis and aspirin; and carotid endarterectomy. Value-for-money threshold: AUD\$30,000 per DALY recovered. Only first-ever cases were considered.

Results: Given age-sex adjusted stroke incidence predictions, about 27,000 strokes could be prevented from better management in that one-year (2015). For these cases, about 85,000 DALYs could be recovered. The present-value (2004) of lifetime cost-offsets for these cases was about AUD\$1.06 billion. The net level of warranted investment was AUD\$3.63 billion per annum, a substantial increase over current spending

Conclusion: Improving access to effective prevention and treatment interventions will produce important health gains and warrants substantial investment. 


\section{Management and economics}

GENDER ASPECTS IN THE TREATMENT OF ACUTE STROKE: DATA FROM THE AUSTRIAN STROKE UNIT DATABASE

S. Horner, for the Austrian Stroke Unit Database Working Group

Medical University Graz, Graz, Austria

Background: Admission to a Stroke Unit (SU) may be subject to some bias because of specific selection criteria. We therefore searched for gender related differences in the management and outcome of patients with acute cerebral stroke admitted to SUs in Austria.

Methods: Our database contains prospective information from 17 centres and provided 10356 documented cases (5349 men, 5007 women) for this comparative analysis.

Results: The proportion of stroke subtypes or other disorders admitted to the SUs were distributed similarly between both genders but women had a significantly higher mean age than men $(72.1 \pm 15.8$ vs. $67.4 \pm 14.4 ; \mathrm{p}<0.001)$. Women with stroke also had a significantly more severe neurologic deficit at admission (NIHSS: 8.3 vs. 6.5 ; BI: 50.5 vs. $59.7 ; \mathrm{p}<0.001$ ). The delay between stroke onset and hospital admission as well as the frequency of performed examinations and of specific treatments were comparable between both groups, e.g. intravenous thrombolysis was performed in $5.9 \%$ of female and $5.7 \%$ of male patients. The gender distribution for patients in whom a follow-up documentation after three months had been performed was similar to that at admission. In this subcohort the proportion of more severly handicapped patients (Rankin score $\geq 2$ ) was higher in women $(51.9 \%)$ than in men $(40.6 \%)$.

Discussion: This analysis of a large patient population did not show any gender differences in the care for stroke patients treated in Austrian SUs. The worse outcome of female patients appears related primarily to their higher mean age including a more sever deficit at admission.

\section{Management and economics}

DATA ON THE INCIDENCE OF TIA SUBSTANTIALLY UNDER-ESTIMATE THE NEED FOR CLINICAL SERVICE PROVISION

M. Giles, P. Rothwell

University of Oxford, Oxford, United Kingdom

Background: Rapid assessment and treatment is required after TIA in order to prevent stroke. Accurate data on the numbers of patients needing assessment are required to plan services, predict future demand, and determine the economic viability of investment in the development of new treatments. Such health-economic estimates are usually based on data from incidence studies of definite, first-ever-ina-lifetime TIA, with a "rule of thumb" doubling of numbers to account for recurrent events and patients with non-TIA diagnoses.

Methods: To assess the validity of this approach, we determined rates in a population-based $(n=91,000)$ study (Oxford Vascular Study) of: 1) Definite, firstever-in-a-lifetime TIA; 2) Any definite (incident or recurrent) TIA; 3) Any probable or definite TIA requiring investigation and secondary vascular prevention; 4) All referrals of suspected TIA to clinical services. Patients with stroke were excluded.

Results: During the three years from April 2002, 615 episodes of suspected TIA were assessed by clinical services in 568 patients. Average age was 69.4 years. Crude annual incidence per 1000 population (95\% confidence interval) for definite, first-ever-in-a-lifetime TIA was 0.51 (0.43-0.6). However, the rate of any first or recurrent probable or definite TIA requiring investigation and vascular prevention was double this rate $(1.08(0.96-1.21))$ and the overall rate of presentation of any suspected TIA to clinical services was over four times higher (2.25 (2.08-2.43)). Rates in all categories rose sharply with age and substantial increases are projected in future decades.

Discussion: Estimates of demand for TIA services should be based on a rate of presentation four times higher than estimates of incidence of definite first-ever-in-alifetime TIA.

\section{Management and economics}

MISADMISSIONS TO A STROKE UNIT. A POPULATION-BASED STUDY OF 5250 ADMISSIONS

T.K. Sørensen, T.S. Olsen

Hvidovre University Hospital, Hvidovre, Denmark

Background: Stroke units (SU) dedicated to manage patients with stroke/TIA often receive patients having another diagnosis than stroke. We studied frequency and type of non-stroke admissions to a SU.
Methods: In a community of 133.000 inhabitants all citizens with acute stroke 1998-2005 were admitted to the same 35-bed SU. The evaluation program included neurological examination, CT-scan, electrocardiogram, Doppler-sonography, and relevant blood samples. We registered final diagnosis of patients with another diagnosis than stroke.

Results: Totally 5250 patients were admitted to the SU. The stroke diagnosis was confirmed in $3117(59 \%), 430(7 \%)$ had TIA while $202(4 \%)$ had sequelae from previous stroke in. In 449 patients $(9 \%)$ another specified neurological disease was final diagnosis (epilepsy in $191(4 \%)$, migraine in $104(2 \%)$, Bell's paralysis in 47 $(1 \%)$ ). Malignancy was diagnosed in 86 patients $(2 \%)$. In 287 patients $(5 \%)$ the diagnosis was internal medical disease (syncope in $75(1 \%)$, infection in $51(1 \%)$, vertigo in $68(1 \%)$ ). CNS trauma was the diagnosis in 88 patients $(2 \%)$ of which 38 $(1 \%)$ had subdural hematoma. In 13 patients $(<1 \%)$ the diagnosis was diseases of eyes/ears and in $517(10 \%)$ it was "non-specified neurological disease".

Discussion: One third of patients admitted to an acute SU had not stroke. Epilepsy, migraine, Bell's paralysis, subdural hematoma, syncope, vertigo and infection are relevant differential diagnoses.

\section{Management and economics}

\section{ACCURATE, PRACTICAL AND COST-EFFECTIVE ASSESSMENT OF CAROTID STENOSIS WITH NON-INVASIVE IMAGING FOR STROKE PREVENTION}

J.M. Wardlaw, F. Chappell, J. Gillard, S. Thomas, G. Roditi, J,J. Best, E. De Nigris, E. Berry, P.M. Rothwell, G. Young

University of Edinburgh, Edinburgh, United Kingdom

Background: Stroke prevention with carotid endarterectomy depends on accurate carotid imaging. We aimed to determine if non-invasive imaging tests (Doppler ultrasound, MRA, CTA, contrast-enhanced MRA), alone or combined, were costeffective compared with intra-arterial angiography (IAA) for stroke prevention.

Methods: We obtained data on the accuracy of non-invasive carotid imaging from a systematic review and individual patient data meta-analysis; calculated costs of non-invasive imaging, stroke care and endarterectomy; and developed a mathematical model of the process of care following a TIA/minor stroke. We evaluated the effect of 22 different combinations of carotid imaging modalities on short and long term outcomes, quality adjusted life years (QALYs), and Net Benefit.

Results: Strategies allowing patients with $70-99 \%$ stenosis to reach endarterectomy most quickly, and where (if assessed early after TIA) patients with 50-69\% stenosis would be offered surgery, prevented most strokes, produced most QALYs and greatest Net Benefit. This included most strategies with ultrasound as first or repeat test (despite $85-90 \%$ sensitivity/specificity), but not those with IAA (too slow). However, the model was sensitive to the accuracy of non-invasive tests, cost and timing of endarterectomy. In patients seen late (ie $>1$ month) after TIA, test accuracy was crucial and \% stenosis should be confirmed with contrast-enhanced MRA or possibly IAA prior to surgery.

Conclusions: Non-invasive tests can replace IAA if used carefully by specialists. Patient assessment for stroke prevention should be speeded up at all stages. Delays in assessment increase the need for contrast-enhanced MRA or IAA.

\section{Management and economics}

RANDOMISED CLINICAL TRIAL ON AN EMERGENCY SYSTEM CLINICAL PATHWAY FOR THE MANAGEMENT OF CRITICAL STROKE PATIENTS IN THE LAZIO REGION (ITALY)

A. De Luca, D. Toni, M. Barbolini, M. Ferri, C. Francia, E. Puca, M. Prencipe, M.L. Sacchetti, G. Guasticchi

Public Health Agency, Roma, Italy

Background: In the Lazio Region (Italy) management of stroke in the emergency system needs to be improved, both in terms of knowledge and of organizational set up. To this purpose, an Emergency Clinical Pathway (ECP) protocol for the management of critical stroke patients was elaborated, focusing on early detection and treatment in the most appropriate ward.

Objective: To evaluate the efficacy and safety of introducing the ECP.

Methods: A cluster-randomized controlled trial (ISRCTN41456865) was planned comparing the practice of a test group of health professionals (HP) trained to use the ECP with that of a control group. The training program included residential education of selected HP who, in turn, performed on-site training of their coworkers. Discussion of the cases in terms of medical practice and appropriateness of the organizational choices was performed and a feed back on the patient's final health status was given to HPs.

Results: The educational program involved 324 emergency HPs pertaining to Emergengy Medical Services (118) and to Emergency Departments of peripheral 
hospitals. Preliminary data from the test area showed that, out of 395 suspected stroke patients, $33(8.5 \%)$ were appropriately referred to the Emergency Department Stroke Unit, of whom $5(15 \%)$ had successful thrombolysis.

Discussion: Preliminary results suggest that the educational diffusion of standardized treatment protocols and organizational procedure, may improve stroke patient management in the Lazio Region. Following these encouraging results, the regional health authorities decided to extend the ECP and the educational program to other areas of the Region.

\section{Management and economics}

CAN PEOPLE WITH STROKE BE INVOLVED IN DEVELOPING STROKE SERVICES?

N. Fudge, C.D.A. Wolfe, C. McKevitt

King's College London, London, United Kingdom

Background: A programme of stroke service improvement has been undertaken in southeast London. This programme prioritises the involvement of people with stroke and their families in developing the work of the programme. We evaluated the involvement of people with stroke in the programme to identify the barriers to and facilitators of involvement.

Methods: We used an ethnographic approach including: participant observation at events and meetings to document the processes of involving people with stroke in the programme and in depth interviews $(n=20)$ with participants and those who declined to be involved.

Results: People with stroke and their families were recruited to the programme via a community stroke register, clinical services and community organisations. 80 people responded to invitations to participate. At 9 months 41 people were active in the programme.

People with stroke and their families have been involved in: overseeing the programme as members of the management team; improving stroke information provision; training stroke professionals; peer support.

Factors that facilitated involvement were: encouragement from trusted contacts; introductory events; feeling listened to; sociable aspect of being involved. Barriers to involvement were transport; inflexibility of health service organisation; illness; caring commitments and communication.

Conclusion: We have demonstrated how people with stroke can begin to actively contribute to the development of stroke services. However if the political imperative is for greater involvement of people with stroke in developing services, several issues will need to be addressed: the resource implications of involvement; the impact involvement has on services and ensuring the culture of the health service is open to involvement.

15 Management and economics

ATTITUDES AND CURRENT PRACTICE OF GENERAL PRACTITIONERS IN ACUTE STROKE MANAGEMENT

S. Roebers, M. Wagner, M. Ritter, B. Mohn, F. Dornbach, K. Wahle,

P.U. Heuschmann

Institute of Epidemiology and Social Medicine, University of Muenster,

Muenster, Germany

Background: Stroke is a medical emergency requiring immediate diagnosis and treatment. Little is known about management and referral patterns of acute stroke patients by general practitioners (GP). Therefore, we studied GP attitudes and current practice in management of acute stroke patients.

Design and Methods: A cross-sectional survey was conducted among 744 GP providing acute primary care in four different regions of North Rhine-Westphalia, Germany, using self-administered mailed questionnaires. To evaluate GP attitudes and practice, case vignettes and standardised questions were used. Multivariate logistic regression was performed to investigate impact of GP characteristics on attitudes and practice.

Results: Between September and December 2005350 GP participated in the study. Mean age of GP was $48 y, 25.9 \%$ were female. Regarding the referral patterns for acute stroke patients, $29.0 \%$ of GP stated to admit patients always to nearest available hospitals, $43.2 \%$ always to hospitals specialised in acute stroke care. Location of GP practice in metropolitan areas increased (OR 2.2; 95\% CI 1.2-4.1) and distance to next specialised hospital $>20 \mathrm{~min}$ decreased (OR 0.3; $95 \% \mathrm{CI}$ $0.2-0.6)$ probability of admitting stroke patients to a hospital specialised in stroke care. Most GP agreed that stroke (95.9\%) and TIA (88.9\%) are urgent medical emergencies, but $38.2 \%$ of GP stated that TIA patients could also be diagnosed and treated outside hospitals. Location of GP practice in rural areas increased (OR 2.5; 95\% CI 1.4-4.5) probability of diagnosing and treating TIA outside hospital.

Oral Session

New clinical trials II
Conclusion: Although a high proportion of GP rated TIA as urgent medical emergency, there is a substantial proportion of GP who would diagnose and treat these patients outside the hospital.

\section{New clinical trials II}

1 New clinical trials II

ABCIXIMAB IN EMERGENT STROKE TREATMENT TRIAL - II (ABESTT-II): RESULTS OF A RANDOMIZED, DOUBLE-BLIND PLACEBO-CONTROL PHASE 3 STUDY

The AbESTT-II Investigators

Background: The Abciximab in Emergent Stroke Treatment Trial-II (AbESTT-II) was a phase 3 , multinational, randomized, double-blind placebo-controlled study testing the usefulness of abciximab for treatment of patients with acute ischemic stroke. The primary population (planned $\mathrm{n}=1200$ ) randomized patients within 4.5 hours with planned treatment within 5 hours of stroke onset. The companion population of patients (planned $n=600$ ) were randomized either 4.5-5.5 hours after stroke onset with planned treatment within 6 hour or who awaken with stroke symptoms and can be randomized within 2.5 hours of awakening. Patients received a bolus of $0.25 \mathrm{mg} / \mathrm{kg}$ abciximab or placebo followed by continuous infusion for 12 hours. Design: The primary efficacy endpoint at 3 months was the proportion of responders using modified Rankin Scale mRS score as defined as 0 if baseline National Institutes of Health Stroke Scale (NIHSS) 4-7, $\leq 1$ if baseline NIHSS 8-14 or $\leq 2$ if baseline NIHSS 15-22. The primary safety endpoint was the incidence of fatal or non-fatal symptomatic intracranial parenchymal hemorrhage (ICH) and other symptomatic ICH though the earlier of discharge or day 5 . Both the efficacy and safety analyses were based on the outcomes in the primary population.

Results: The study was halted prematurely due to unfavorable risk/benefit profile as assessed by the trial's independent Safety and Efficacy Monitoring Committee. A total of 808 patients were enrolled at the time of study termination, 441 in primary, 367 in companion population ( $45=$ wake-up stroke patients). Because finalization of the database including follow-up data is not complete, an unblinded review of the data by the investigators has not yet occurred.

Discussion: The final efficacy and safety results of AbESTT-II will be presented.

\section{New clinical trials II}

\section{RESULTS OF THE SPACE STUDY(STENT-PROTECTED PERCUTANEOUS} ANGIOPLASTY OF THE CAROTID VS. ENDARTERECTOMY)

The SPACE-Study Group (Germany)

The SPACE-trial is is a multicenter, prospective, randomized trial in Germany, Austria and Switzerland. The Space study was designed to test the hypothethis that carotid artery stenting (CAS) and carotid endarterectomy (CEA) are equivalent in treating patients with symptomatic carotid artery stenosis. Symptomatic patients who suffered from amaurosis fugax, TIA oder non-disabling stroke with high-grade carotid stenosis $(>70 \%$ ECST or $>50 \%$ NASCET based on ultrasound examination) within 180 days before randomization could be included. Primary endpoint was ipsilateral stroke or death of any cause from randomization until 30 days after intervention. Secondary endpoints were ipsilateral stroke or vascular death within 24 months after randomization; ipsilateral stroke with permanent handicap (modified Rankin Score $\geq 3$ ) within 30 days after intervention; stroke of any localisation within 30 days and 24 months after intervention; residual stenosis and restenosis $\geq 70 \%$ in ultrasound and procedural failure.

Up to January 20061200 patients were randomized in 35 centers. The primary endpoint of these patients are currently analyzed. The 30day primary and secondary endpoint-result will be presented.

\section{New clinical trials II}

DESTINY - DECOMPRESSIVE SURGERY FOR THE TREATMENT OF MALIGNANT INFARCTION OF THE MIDDLE CEREBRAL ARTERY PRELIMINARY RESULTS

The DESTINY Study Group (Germany)

Background: Patients with a hemispheric infarct and massive space-occupying brain oedema have a poor prognosis. Despite maximal conservative treatment, the case fatality rate may be as high as $80 \%$ and most survivors are left severely disabled. Non-randomised studies suggest that decompressive surgery reduces 
mortality substantially, and may improve functional outcome of survivors. Yet, there are no published randomised, controlled, prospective studies that have tested whether decompressive surgery reduces mortality and improves functional outcome as compared to maximal conservative treatment.

Methods: DESTINY is a multi-centre, randomised, controlled, sequential clinical trial, including patients with malignant middle cerebral artery (MCA) infarction aged between 18 and 60 years. Patients were randomised to either conservative treatment plus decompressive surgery or conservative treatment alone. To include only as many patients as absolutely necessary to demonstrate the superiority of decompressive surgery concerning the reduction of mortality a sequential design was chosen. The primary outcome measure is functional outcome using the modified Rankin Scale (mRS) at 6 months. Mortality at 30 days is the secondary outcome measure.

Results: 32 patients have been included between February 2004 and October 2005 According to the sequential design recruitment of patients was stopped as soon as one treatment arm proofed to be superior to the other concerning mortality at 30 days: 15 of 17 patients (88\%) who were treated by decompressive surgery survived, whereas 7 of 15 patients $(47 \%)$ who received conservative treatment alone survived. Discussion: DESTINY demonstrates the benefit of decompressive surgery in malignant MCA infarction to reduce mortality in a randomised, controlled, prospective study design. There are no data yet concerning functional outcome. 6-month follow-up (mRS) will be concluded in April 2006.

\section{New clinical trials II}

\section{SAFETY OF TIROFIBAN IN ACUTE ISCHEMIC STROKE (SATIS) The SaTIS Investigators}

The highly selective platelet glycoprotein IIb/IIIa-receptor-antagonists have been shown to improve the treatment of the acute coronary syndrome and the lesion development in experimental cerebral ischemia. We investigated tirofiban, a non peptide substance with fast acting and deactivating properties in a multicenter, prospective, randomized and placebo-controlled trial, targeting the frequency of cerebral hemorrhages as primary endpoint and clinical outcome as secondary endpoint.

Study design: Patients with ischemic stroke, symptom onset $<22$ hours, NIH Stroke Scale between 4-18 and who were not eligible for thrombolysis received either placebo or tirofiban continuously for 48 hours according to the dosage described in the PRISM-Plus study. Patients' cCT-scans at the time of admission and 2-4 days after symptom onset were subjected to central, blinded evaluation. Secondary endpoint were the neurological outcome (NIHSS at second CT scan) and clinical disability scales (Barthel Index, modified Rankin Scale) within 3-5 months after enrolment

Results: After enrolment of 250 patients ( 127 verum/123 placebo, median age 67 years, median inclusion NIHSS 6 points) in 4 communal and 7 university hospitals - all with a certified stroke unit - the recruitment was finished in December 2005. The average time between symptom onset and start of infusion was 12.2 hours $( \pm 1.2) .12$ severe adverse events were reported. The total hemorrhage rate including hemorrhagic transformation was $28 \%$. Parenchymal bleeding was $5 \%$ none of them symptomatic. The final data are under evaluation and will be presented on the meeting.

Conclusions: Tirofiban, a strong platelet inhibitor, seems to be safe. The results of the study encourage for further clinical trials to prove the efficacy in acute stroke.

\section{New clinical trials II}

\section{ESPRIT: RESULTS OF A RANDOMISED COMPARISON OF THE EFFECTS OF ASPIRIN + DIPYRIDAMOLE VERSUS ASPIRIN ALONE IN PATIENTS AFTER CEREBRAL ISCHAEMIA OF ARTERIAL ORIGIN \\ The ESPRIT Study Group}

Background: Aspirin (ASA) $\geq 30 \mathrm{mg} /$ day prevents only $13 \%$ of subsequent vascular events after minor cerebral ischaemia of arterial origin. The 2nd European Stroke Prevention Trial reported a $22 \%$ (95\% CI 9 to 33\%) relative risk reduction (RRR) of the combination of ASA and dipyridamole (DIP) above that of ASA only. Pooled results of four smaller trials, however, had shown an RRR of only 3\% (95\% CI -22 to $+22 \%$ ). Hence, uncertainty exists on the efficacy of ASA+DIP.

Methods: ESPRIT, the European/Australasian Stroke Prevention in Reversible Ischaemia Trial, is a multicentre, randomised, open, parallel group, controlled trial. Patients with cerebral ischaemia of arterial origin (TIA or stroke; modified Rankin $<4)$ were randomised between ASA (30-325 mg/day) + DIP (2x200 mg/day) and ASA only (30-325 mg/day) within six months of the event. Primary outcome was the composite of vascular death, nonfatal stroke, nonfatal myocardial infarction or major bleeding. Outcome assessment was blinded.
Results: We randomised 1390 patients to ASA+DIP and 1407 to ASA only. Mean follow-up was 3.5 years. Baseline characteristics were similar in the two treatment groups. Mean age was 63 years, $65 \%$ was male. Thirty-four percent had a TIA (5\% transient monocular blindness) and $66 \%$ a minor ischaemic stroke; $32 \%$ was randomised within a month of the event. The overall annual rate for the primary outcome event (first events only) was 4.4\%. Outcome events will be reported according to allocated trial medication; an updated meta-analysis will also be presented.

\section{New clinical trials II}

\section{ENDARTERECTOMY VERSUS ANGIOPLASTY IN PATIENTS WITH} SYMPTOMATIC SEVERE CAROTID STENOSIS: A RANDOMIZED TRIAL

The EVAS-3S Investigators (France)

Background: Carotid stenting is less invasive than endarterectomy but it is unclear whether it is as safe as endarterectomy and provides a degree of long-term protection against ipsilateral stroke similar to that of surgery. Methods. We established EVA-3S - a multicenter, randomized, non-inferiority trial, with national research organization funding - to evaluate whether stenting is not inferior to endarterectomy concerning (a) the risk of stroke or death within 30 days of procedure and (b) the long-term risk of ipsilateral stroke, in patients with recently symptomatic, severe carotid stenosis. The study is conducted in 20 academic and 10 non-academic centers in France. To join the trial, each center was required to assemble a team of physicians comprising at least one neurologist, one vascular surgeon and one interventionalist. The vascular surgeon had to document at least 25 endarterectomies in the year preceding enrolment. The interventionalist had to document at least 12 cases of carotid stenting or at least 35 stenting procedures in the supra-aortic trunks, of which at least 5 should be in the carotid artery. Patients were eligible if they had experienced a hemispheric or retinal transient ischemic attack or a nondisabling stroke within the 120 days before entry and had a atherosclerotic stenosis of 60 to 99 percent in the symptomatic carotid artery (NASCET method). The primary end point was a composite of any stroke or death occurring within 30 days of treatment. Secondary outcomes included: myocardial infarction; transient ischemic attack; cranial nerve injury, major local complications and systemic complications. Results. In September 2005, the Safety Committee recommended to stop inclusions into the trial because of safety concern. The results on the incidence of stroke or death within 30 days of procedure will be presented. Long-term follow-up is ongoing. 


\section{Stroke and diabetes}

\section{Stroke and diabetes}

GLYCOSYLATED HEMOGLOBIN A1 (HBA1C) IS A PROGNOSTIC FACTOR FOR THROMBOLYSIS ASSOCIATED HEMORRHAGE IN STROKE E. Jüttler, M. Köhrmann, P.D. Schellinger, H.B. Huttner, C. Schwark, P.A. Ringleb, W. Hacke University of Heidelberg, Germany

Background: Only $3 \%$ to $4 \%$ of all stroke patients are currently treated with rtPA. Many patients are withheld from treatment because of safety concerns, most of all the fear to cause intracerebral haemorrhage (ICH), which leads to reluctance especially on the part of non-specialists. One risk factor for ICH after thrombolysis is thought to be severe microangiopathy due to hypertension or diabetes. In some trials baseline hyperglycemia has been demonstrated to predict ICH after thrombolysis. Glycosylated haemoglobin A (HbAlc), however, is a better marker for diabetic micoangiopathy. In this study we assessed the value of HbAlc and baseline blood glucose levels as predictors for ICH and outcome after thrombolysis in acute middle cerebral artery (MCA) infarction.

Methods: We evaluated prospectively collected clinical, biochemical, and neuroimaging data of 400 consecutive patients with MCA infarction treated with rtPA, including age, gender, time to treatment, NIHSS score at baseline, TOAST classification, blood glucose at baseline, $\mathrm{HbAlc}$ at day $1, \mathrm{ICH}$ on $\mathrm{CT}$ at day 1 or 2, and outcome using the modified Rankin Scale (mRS) at day 90 (mRS 0-1 = favourable, mRS 0-2 = independent).

Results: ICH occurred in $20,3 \%$ of patients, $6,3 \%$ suffered symptomatic ICH (sICH). 38,0\% had favourable, 50,8\% had independent outcome. Using multivarate analysis NIHSS score at baseline and $\mathrm{HbA} 1 \mathrm{c}$ remained as independent predictors for ICH. No variable independently predicted sICH. Age and NIHSS score were independent predictors for favourable. Age, NIHSS score, and blood glucose predicted independent outcome.

Discussion: $\mathrm{HbAlc}$ as a marker for diabetes associated microangiopathy is a predictor for thrombolysis associated ICH, but not for outcome, whereas increased blood glucose level is a predictor for poor outcome, but not for ICH, which implies that hyperglycemia exerts its harmful effects through other pathomechanisms than an increased bleeding risk.

\section{Stroke and diabetes}

PREVALENCE OF IMPAIRED GLUCOSE TOLERANCE IN PATIENTS WITH TIA OR MINOR ISCHEMIC STROKEPREVALENCE OF IMPAIRED GLUCOSE TOLERANCE IN TIA PATIENTS

S.E. Vermeer, P.J. Koudstaal, D.W.J. Dippel

Erasmus MC, Rotterdam, Rotterdam, The Netherlands

Background: Impaired glucose tolerance, an intermediate metabolic state between normal glucose tolerance and diabetes, nearly doubles the risk of stroke in patients with TIA or minor stroke, but its prevalence is largely unknown. In a very recent North-American study, $28 \%$ of patients with TIA or non-disabling stroke had impaired glucose tolerance. We investigated the prevalence of impaired glucose tolerance in Dutch patients with a recent TIA or minor ischemic stroke.

Methods: Since March 2005, patients visiting our university outpatient clinic with a TIA or minor ischemic stroke undergo an oral glucose tolerance test as part of the standard risk factor screening, unless they are known to have diabetes. We assessed the prevalence of impaired glucose tolerance (2-hr post-load glucose levels of 7.8-11.0 mmol/L), impaired fasting glucose (fasting glucose levels of 5.6-6.9 $\mathrm{mmol} / \mathrm{L}$ ), and diabetes mellitus in the first 52 consecutive patients.

Results: Twelve (23\%, 95\% confidence interval 12 to $37 \%$ ) of the 52 patients had impaired glucose tolerance, 1 (2\%) had impaired fasting glucose, and none newlydiagnosed diabetes (figure). Five (10\%) patients were already known diabetics. As this study is an ongoing survey, we will be able to report on more than 150 patients by May 2006.

Poster Session First Visit

Stroke and diabetes

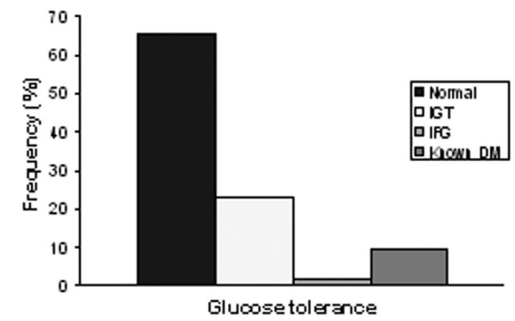

Conclusion: Impaired glucose tolerance is very frequent among patients with TIA or minor ischemic stroke, with a prevalence of $23 \%$. Without an oral glucose tolerance test, only the $2 \%$ of the patients with impaired fasting glucose would be identified. New secondary prevention trials should determine whether intensive glucose control reduces stroke incidence in these patients.

\section{Stroke and diabetes}

RECURRENT VASCULAR EVENTS IN DIABETIC STROKE PATIENTS

A.K. Ghosh, I.M. Shah, S.K. Ghosh, A. Collier

The Ayr Hospital, Ayr, United Kingdom

Introduction: Diabetes and the Metabolic Syndrome (MetS) are both associated with increased vascular risk. We analysed the incidence of recurrent stroke and vascular events in a group of type 2 diabetic (T2D) stroke patients. We further investigated for any differences in the incidence of recurrent events among patients diagnosed with the MetS

Methods: Information was obtained from our Diabetes Centre database and retrospective case record analysis. We analysed 243 T2D stroke patients for recurrent vascular events over a 2-year period after their initial stroke. The diagnostic values used for the MetS were low HDL $<1 \mathrm{mM}$, elevated triglycerides $>1.7 \mathrm{mM}$, obesity BMI $>30 \mathrm{~kg} / \mathrm{m}^{2}$ and $\mathrm{BP}>130 / 85 \mathrm{mmHg}$.

Results: Stroke was diagnosed in 149 male (61.3\%) and 94 female (36.7\%) patients, with a mean age of $72.9 \pm 9.7$ years. Recurrent vascular events recorded in these patients were stroke $(n=46)$, ischaemic heart disease $(n=35)$ and peripheral vascular disease $(n=12)$. MetS was diagnosed in 151 patients ( 86 male: 65 female; age: $71.7 \pm 9.7)$. Comparing the MetS and non-MetS groups, recurrent vascular events were stroke $16.6 \%(\mathrm{n}=25)$ v $22.8 \%(\mathrm{n}=21)$, ischaemic heart disease $13.2 \%(\mathrm{n}=20)$ v $16.2 \%(n=15)$ and peripheral vascular disease $5.3 \%(n=8)$ v $4.3 \%(n=4)$. Risk of recurrent vascular event, adjusted for age, sex and use of secondary prevention treatment showed no significant difference between the two groups (OR: 0.8; $95 \%$ CI: $0.46-1.44)$.

Conclusion: Stroke was the most common recurrent vascular event in our T2D stroke patients. There was no significant difference in the incidence of recurrent vascular events among patients diagnosed with the MetS.

\section{Stroke and diabetes}

\section{STROKE RISK FACTORS IN A SCOTTISH DIABETIC POPULATION}

I.M. Shah, A.K. Ghosh, S.K. Ghosh, A. Collier

The Ayr Hospital, Ayr, Scotland, United Kingdom

Introduction: Diabetes is an important risk factor for cerebrovascular disease and its incidence is rising with the obesity epidemic. We analysed the risk factor profile of type 2 diabetic (T2D) patients diagnosed with stroke, from a West of Scotland population

Methods: Information was obtained from our Diabetes Centre database. The data was collected between Sept 1996 - Feb 2004. We analysed 2,762 T2D patients with complete available data. Multivariate logistic regression was used to analyse the risk factor profile of patients diagnosed with stroke.

Results: Stroke was diagnosed in 243 patients (8.8\%). There were 149 males 
(61.3\%) and 94 females (36.7\%), with a mean age of $72.9 \pm 9.7$ years. The different stroke sub-types were TACS $(\mathrm{n}=8)$, PACS $(\mathrm{n}=15)$, POCS $(\mathrm{n}=11)$, lacunar stroke $(n=106)$, TIA $(n=94)$ and intracerebral haemorrhage $(n=9)$. Multivariate analysis, adjusted for age and sex, showed hypertension (OR: 1.6; 95\% CI: $1.2-2.3$ ) as the only significant risk factor for stroke. There was a trend towards increased risk associated with smoking (OR: $1.23 ; 95 \%$ CI: $0.9-1.7)$. There was no significant risk associated with HbA1c (OR: 1; 95\% CI: $0.9-1.11$ ), BMI (OR: 0.9; 95\% CI: $0.9-1.02$ ), HDL (OR: 1; $95 \%$ CI: $0.9-1.01$ ) or triglyceride levels (OR: $0.9 ; 95 \%$ CI: $0.9-1.02)$

Conclusion: These results emphasise the importance of hypertension as a risk factor for stroke in T2D patients. Aggressive treatment of high blood pressure should be mandatory in all diabetic patients.

\section{Stroke and diabetes}

\section{BRAIN NATRIURETIC PEPTIDE AND RISK OF EARLY STROKE IN DIABETIC PATIENTS}

C.M. Brisc, P. Mihancea, C. Brisc, L. Matcau, A.R. Popa, L.A. Vatca, S.A. Iuhasz

University of Oradea, Faculty of Medicine and Pharmacy, Oradea, Romania

Aim: There are some recent studies which provides the fact that the elevated level of serum brain natriuretic peptide is a reliable cardiovascular risk factor in diabetics, especially for cardiac failure.

The aim of our study was to note if there is a correlation between serum BNP level and clinical and biological picture in diabetic patients with ischemic stroke.

Material and Method: We perform a comparative statistic study of 2 groups of 15 diabetics with stroke - lot A and without stroke-lot B, admitted in the Clinical County Hospital Oradea and in Neurology and Psychiatry Hospital Oradea (total of 30 diabetics). All the patients were without cardiac dysfunction.

Results: The statistical study provided the following outcomes: Feminine gender was most affected in both groups-lot A 53,3\%, lot B- $60 \%$, the average age of the first lot being $65,7 \pm 8,1$ and of the second lot $57,2 \pm 7$. The lot A contented $46,7 \%$ overweight patients and lot B $40 \%$ obese. Lot A of patients had many modified clinical and biochemical parameters than lot B, with statistic significant differences involving glucose, fibrinogen and pro-BNP serum level. The average value for pro-BNP was superior in diabetics with stroke, with a sensibility of 0,93 and an accuracy index of 6,1 .

Conclusion: Diabetic patients with high BP, without cardiac failure, overweight, with bad metabolic balance, multiple location of macrovascular complications and serum levels of pro-BNP higher than $500 \mathrm{pg} / \mathrm{ml}$ have an over $80 \%$ risk of developing a stroke.

\section{Stroke and diabetes}

PREVALENCE OF IMPAIRED GLUCOSE METABOLISM AND METABOLIC SYNDROME IN NON-DIABETIC PATIENTS WITH ACUTE POST STROKE HYPERGLYCAEMIA

M.T. McCormick, K.W. Muir

Institute of Neurological Sciences, University of Glasgow, Southern General Hospital, Glasgow, United Kingdom

Background: Acute post stroke hyperglycaemia (PSH) affects 40-60\% of patients and is associated with poor outcome. It is not known whether acute PSH signifies an underlying abnormality of glucose metabolism in non-diabetic subjects, rather than a stress response. We sought to establish the prevalence of impaired glucose metabolism and metabolic syndrome in stroke patients.

Methods: Patients with PSH (capillary blood glucose $>7.0 \mathrm{mmol} / \mathrm{l}$ ) within 48hours of ischaemic stroke were identified from a database. Known diabetic patients were excluded. Consenting patients underwent an oral glucose tolerance test (OGTT) at least 3 months from ictus. Impaired glucose metabolism was defined as diabetes, impaired glucose tolerance (IGT), or impaired fasting glucose (IFG). Fasting lipids, blood pressure and waist circumference were measured to allow diagnosis of metabolic syndrome (International Diabetes Federation criteria).

Results: 39 patients (22 females) median age 66 years (IQR 59,74) underwent OGTT. Two patients were excluded from analysis due to (I) hyperglycaemia occurring $>48$ hours from ictus and (II) non-stroke diagnosis. Three patients were unable to complete the OGTT. Of the 34 patients with completed OGTT, 13/34 (38\%) had abnormal glucose metabolism: diabetes in $7 / 34(21 \%)$ (3 had normal fasting glucose), IGT in 4/34 (12\%) and IFG in 2/34 (6\%). Criteria for metabolic syndrome were fulfilled in 16/37 (43\%). Four patients with abnormal glucose metabolism did not fulfil criteria.

Discussion: Almost $40 \%$ of non-diabetic patients with hyperglycaemia within the first 48 hours of ischaemic stroke had abnormal glucose metabolism at late follow- up, 43\% had metabolic syndrome, and 54\% had either. Convalescent screening of patients with acute PSH may be important for secondary prevention.

\section{Stroke and diabetes}

\section{ALGORITHM FOR DIAGNOSIS EARLY ISCHEMIC STROKE IN PATIENTS} WITH DIABETES MELLITUS

C.M. Brisc, P. Mihancea, C. Brisc, L. Matcau, L.A. Vatca, S.A. Iuhasz, E. Drambarean

University of Oradea, Faculty of Medicine and Pharmacy Oradea; University of Medicine Timisoara, Oradea, Romania

Aim: the aim is to emphasize the most important parameters in diabetic patients, in order to develop an algorithm for early detection of stroke(before onset of the motor deficit).

Method: we compared 3 batches of 60 patients: diabetics with and without ischemic stroke and non diabetics with ischemic stroke, in order to discover the most important parameters involved in early brain damage. Thus, we performed a statistic study concerning age, gender,family and personal history,type of diabetes mellitus and complications, toxic intake, body mass index, symptoms, serum fibrinogen, $\mathrm{C}$ reactive protein-CRP, glucose, $\mathrm{HbAlc}$, cholesterol, tryglicerides and pro-Brain Natriuretic Peptide(pro-BNP). Also we assessed blood pressure values, EEG, Doppler cervical ultrasound, measuring media+intima thickness-IMT, CT scan and spinal fluid biological assessment, treatment. For statistic analyses we used test $t$, significance test $\chi^{2}$, Bravais-Pearson coefficient, positive predictive value, relative risk.

Results: Parameters included into an algorithm for detecting risk of early stroke in diabetics are: symptoms, age, type of diabetes, at least 2 vascular complications, toxic intake, elevated serum levels of cholesterol, HbAlc, fibrinogen, CRP, proBNP, IMT over $1,2 \mathrm{~mm}$ and high levels in spinal fluid of lactic acid, pyruvic acid and LDH. We developed an algorithm for diagnosing early stroke in diabetics.

Conclusions: overweight diabetic males, over 50, with high blood pressure and uncontrolled $\mathrm{HbAlc}$, with 2 macrovascular complications or a IMT over $1,2 \mathrm{~mm}$ and high serum levels of fibrinogen, CRP, pro-BNP, with mycrocalcifications on previous CT, presenting sudden headache, half body numbness, gait troubles, dizziness, nausea or speaking problem, most likely are developing an ischemic stroke.

\section{Etiology of stroke}

\section{Etiology of stroke}

EARLY RISK OF RECURRENCE BY ETIOLOGICAL SUBTYPE OF TRANSIENT ISCHEMIC ATTACK

F. Purroy, J. Montaner, C.A. Molina, P. Delgado, E. Santamarina, M. Rubiera, M. Ribo, M. Quintana, J. Alvarez-Sabín

Hospital Universitari Arnau de Vilanova de Lleida; Hospital Universitari Vall d'Hebron de Barcelona, Spain

The risk of recurrent stroke is highest in the first few weeks after a TIA and it is likely to be related to the underlying pathology.

Objective: To study the early risk of recurrent stroke by etiologic subtype.

Methods: We prospectively studied 388 TIA patients. The cause of TIA was classified according to the TOAST criteria: large-artery atherosclerosis (LAA=96), cardioembolism $(\mathrm{CE}=85)$, small vessel $(\mathrm{SV}=68)$, undetermined $(\mathrm{UND}=123)$ and other determined cause $(\mathrm{OC}=16)$. Patients were follow-up at 3 months. Risk factors and clinical symptoms for each subtype were recorded.

Results: Duration of symptoms and clinical symptoms varied significantly among the different subtypes. LAA was associated with recurrent short episodes of weakness while speech impairment and cortical symptoms were associated with $\mathrm{CE}$ $(\mathrm{p}<0.05)$. The association of risk vascular factors was highest in LAA $(\mathrm{p}<0.05)$. Recurrent stroke risk varied between subtypes $(\mathrm{p}<0.001)$ : LAA $29.2 \%$; CE $15.3 \%$; UND $8.9 \%$; SV $5.9 \%$; OC $0 \%$. Cox proportional hazards multivariate analyses identified congestive heart disease (HR 6.02; 1.41-25.70, $\mathrm{p}<0.015)$ as independent predictors of further cerebral ischemic events in LAA. History of previous stroke (HR 5.09; 1.66-15.59, $\mathrm{p}<0.01$ ) and multiple TIAs (HR 4.23; 1.10-16.33, $\mathrm{p}=0.03$ ) predicted stroke recurrence in UND. No independent predictors were identified for CE, SV and OC.

Conclusions: The risk of early recurrent stroke is highest in patients with LAA This supports the need for urgent carotid and transcranial imaging for identifying those patients at highest risk. Some risk factors and clinical symptoms are related with some etiologic subtypes but we need stronger predictors of stroke recurrence in order to identify those patients with highest risk in each TIA subtype. 


\section{Etiology of stroke}

VIRCHOV-ROBIN SPACES RELATE TO SILENT LACUNES AND WHITE MATTER LESIONS; FURTHER EVIDENCE FOR DISTINCTION OF TWO TYPES OF CEREBRAL SMALL VESSEL DISEASE

R. Rouhl, R. van Oostenbrugge, J. Lodder

University Hospital Maastricht, Maastricht, The Netherlands

Background: Virchov-Robin spaces (VRS) are perivascular spaces surrounding the deep perforating brain arteries and the small medullary branches in the cerebral white matter. The presence of (dilated) VRS has been associated with hypertension related arteriolosclerosis. We hypothesize that, if VRS would differentiate the two lacunar stroke types we distinguished earlier, this would further strengthen this concept of two lacunar stroke types caused by either small vessel atherosclerosis or arteriolosclerosis.

Methods: 84 new lacunar stroke patients from the prospective Maastricht Stroke Registry were included, and various baseline characteristics, including findings on Magnetic Resonance Imaging (MRI), were noted. We distinguished those with only the symptomatic lacunar lesion ( $\mathrm{n}=18$, type 1$)$ from those with at least one silent lacunar lesion plus white matter lesions on MRI $(n=28$, type 2$)$. VRS were counted at the level of the basal ganglia (BG), and the supraventricular white matter (WM), and divided into 3 categories on both levels: $<20$ (cat1), 20-50 (cat2), $>50$ (cat3).

Results: Results are shown in the table. Multivariate regression analysis with clinical and radiological characteristics in the model showed VRS at BG level independently associated with type $2(\mathrm{p}<0.001)$, but not VRS at the WM level with type $2(\mathrm{p}=0.82)$. VRS at BG level were also (statistically significantly) related to hypertension (chi-square: $8.994 ; \mathrm{p}=0.01$ ).

VRS on BG and WM level in type 1 and 2

\begin{tabular}{lcc}
\hline & $\begin{array}{c}\text { BG level } \\
\text { cat1 }- \text { cat2 }- \text { cat3 }\end{array}$ & $\begin{array}{c}\text { WM level } \\
\text { cat1 }- \text { cat2 }- \text { cat3 }\end{array}$ \\
\hline Type 1 $(\mathrm{n}=18)$ & $5-11-2$ & $6-7-5$ \\
\hline
\end{tabular}

Discussion: The formation of VRS relates to the clinically identifiable lacunar stroke type which is caused by small vessel arteriolosclerosis, and is strongly associated with hypertension.

\section{Etiology of stroke}

\section{PRO12ALA (P12A) POLYMORPHISM OF THE PPAR-GAMMA NUCLEAR} RECEPTOR AND ISCHEMIC STROKE SUBTYPE

M. Millan, J. Mallolas, T. Sobrino, J.F. Arenillas, Y. Silva, M. Rodríguez-Yañez, J. Vivancos, J. Serena, M.A Moro, A. Dávalos

Hospital Universitari Germans Trias i Pujol, Badalona, Spain

Background: PPAR-gamma nuclear receptors modulate the expression of genes implicated in the atherogenesis. The P12A polymorphism of PPAR-gamma has been associated with a lower risk of diabetes mellitus and myocardial infarction. We sought to investigate whether the P12A polymorphism is associated with a lower risk of atherothrombotic stroke

Methods: Retrospective study of 361 patients with an acute ischemic stroke admitted in two university hospitals.Stroke subtypes were classified according to TOAST criteria in atherothrombotic $(n=189)$ and non-atherothrombotic $(n=172)$ (cardioembolic $(n=84)$, undetermined $(n=59)$ and lacunar $(n=29)$ ).Patients initially underwent Transcranial Doppler and Echo-Doppler, and large artery stenoses were confirmed by MR- or conventional angiography. Transthoracic or transesophagic echocardiography were performed to rule out a cardioembolic source or in patients with undetermined etiology.P12A polymorphism was analyzed by SNapShot technology in blood samples obtained on admission

Results: The P12A polymorphism was detected in $10.5 \%$ of patients with atherothrombotic stroke and in $22.9 \%$ of those with non-atherothrombotic stroke $(\mathrm{p}=0.001)$. In the non-atherothrombotic group, P12A distribution was $26.2 \%$ cardioembolic, $22 \%$ undetermined and $13.8 \%$ lacunar. There were no significant differences in the P12A genotype regarding hypertension, diabetes, hyperlipidemia, ischemic heart disease and history of prior stroke. However, P12A polymorphism was significantly associated with a lower frequency of history of peripheral artery disease $(2.5 \%$ vs $18.3 \%, \mathrm{p}=0.012)$. $\mathrm{P} 12 \mathrm{~A}$ polymorphism showed an OR for atherothrombotic stroke of $0.41(95 \% \mathrm{CI}, 0.22-0.77)(\mathrm{p}=0.006)$ after adjustment for vascular risk factors

Conclusions: P12A polymorphism was independently associated with a lower frequency of atherothrombotic stroke. These findings suggest that the P12A PPARgamma polymorphism may modulate the expression of different vascular diseases underlying stroke subtypes.

Poster Session First Visit

Etiology of stroke
4 Etiology of stroke

PRESENTING CHARACTERISTICS IN PATIENTS WITH AND WITHOUT CERVICAL PSEUDOANEURYSM DUE TO CAROTID DISSECTION. A CASE-CONTROL STUDY

D. Benninger, J. Gandjour, M. Arnold, D. Georgiadis, R.W. Baumgartner University Hospital of Zürich, Zürich, Switzerland

Background: The treatment of cervical pseudoaneurysm due to spontaneous dissection of the internal carotid artery (ICAD) is controversial. We performed case-control study to compare the risk of ischemic events, local symptoms and signs such as Horner syndrome and cranial nerve palsy and other characteristics at presentation in SICAD patients with and without associated cervical pseudoaneurysm. Methods: Consecutive patients with sICAD were included $(n=222,132$ men; mean age $46 \pm 11$ years) presenting 248 sICADs and 15 spontaneous vertebral artery dissections (sVAD). Thirty-four patients ( 22 men; mean age $46 \pm 11$ years; sICAD, $\mathrm{n}=41 ;$ sVAD, $\mathrm{n}=8$ ) had $41 \mathrm{ICA}$ - and 5 VA-aneurysms. All patients underwent clinical, laboratory, color duplex ultrasound (CDS) of the cerebral arteries, MR imaging (MRI) of the neck, cerebral artery MR (MRA) and/or catheter angiography, and cerebral MRI in case of brain ischemia. Unpaired t-tests were used to compare parametric data between groups, and Chi-square tests or Fisher exact test for nominal data

Results: Patients with cervical pseudoaneurysms presented more often multiple CAD $(p=0.001)$ and normal cerebral artery ultrasound findings $(p<0.001)$, and showed a trend to cause less often carotid territory ischemia and local symptoms and signs that was significant for ischemic events $(\mathrm{p}=0.001)$. There were no differences in demographics and prevalence of vascular risk factors between both groups.

Discussion: Our data indicate that sICAD with cervical aneurysms cause less carotid ischemia and in particular not more local symptoms and signs than their non-aneurysmatic counterparts supporting the view that conservative, non-invasive management is appropriate in most cases.

\section{Etiology of stroke}

THE ASSOCIATION OF CEREBRAL WHITE MATTER HYPERINTENSE LESIONS WITH CAROTID PLAQUE INSTABILITY

N. Altaf, L. Daniels, P.S. Morgan, A.R. Moody, J.R. Gladman,

S.T. MacSweeney, D.P. Auer

University of Nottingham, Nottingham, United Kingdom

Background: White matter hyperintense lesions (WMHL) on fluid attenuated inversion recovery (FLAIR) brain scans represent a risk factor for stroke, and are assumed to reflect small vessel disease. However, we recently found an association between the extent of WMHL and unstable carotid plaques defined by histology. To further determine this interrelation in vivo, we studied a larger cohort of patients exploiting the high prediction of plaque instability afforded by MR direct thrombus imaging (MRDTI)

Methods: 178 patients with unilateral symptomatic carotid artery disease underwent MRDTI and FLAIR scans. Analysis was carried out by a blinded, trained researcher. The volumes of periventricular (PV), subcortical (SC) and total WMHL were determined per hemisphere and compared in a repeated measures multivariate design with symptom as within subject factor, and ipsi- and contra-lateral MRDTI status as between subject factors controlling for age, sex and hypertension.

Results: Age, but not hypertension or gender covaried significantly. There was a strong interaction between symptom and MRDTI status for both the ipsilateral and contralateral hemisphere $(\mathrm{F}=9.95 \& 9.90, \mathrm{p}<0.05$ respectively), but no significant association of the individual factors. MRDTI + plaques on the symptomatic hemisphere were associated with significantly larger total WMHL volumes compared to the asymptomatic hemispheres ( 114.1 vs. $68.8 \mathrm{ml}, \mathrm{p}<0.05)$. The MRDTI status of the asymptomatic hemisphere also modulated the WMHL volumes, being larger for MRDTI+ compared to MRDTI - plaques (total 87.0 vs. $66.9 \mathrm{ml}, \mathrm{p}<0.05$ ). Similar interactions were seen for PV, but not for SC volumes.

Conclusion: The extent of total and PV, but not SC WMHL in the symptomatic and asymptomatic hemispheres is associated with the unstable carotid plaque as defined by MRDTI. This suggests that microembolic ischeamic events contribute to the development of WMHL in patients with symptomatic carotid artery disease. 


\section{Etiology of stroke}

\section{ADIPOPHILIN EXPRESSION IN CAROTID ATHEROSCLEROSIS}

P.M. Isoviita, K. Nuotio, J. Saksi, P. Ijäs, J. Pitkäniemi, P.T. Kovanen, M. Kaste, P.J. Lindsberg

Neuroscience Program, Biomedicum Helsinki, Helsinki, Finland

Background: Adipophilin is an adipose differentiation-related protein, expressed in lipid-containing cells. It is involved in the foam cell differentiation, and the lipid metabolism of the atherosclerotic plaque. Based on DNA microarrays, we have found that adipophilin is overexpressed in symptomatic carotid plaques (CP). Here we examined the localization of adipophilin protein and mRNA in advanced CPs, and compared its relation to microhemorrhages and free cholesterol in symptomatic and asymptomatic CPs.

Methods: Paraffin sections from 87 high-grade CPs (48 symptomatic, 39 asymptomatic) were obtained in carotid endarterectomy and immunostained against adipophilin. The relative expression of adipophilin mRNA was measured by quantitative real-time RT-PCR in a subgroup of 22 CPs ( 13 symptomatic, 9 asymptomatic). Results: Adipophilin immunostaining was confined to macrophages and colocalized with extravasated red blood cells $(\mathrm{P}<0.001)$, free cholesterol $(\mathrm{P}<0.001)$, and necrotic area $(\mathrm{P}<0.001)$ within the $\mathrm{CP}$. The relative expression of adipophilin mRNA was increased in the symptomatic compared to asymptomatic CPs $(1.85 \pm 0.31 \mathrm{SE}$ vs. $0.89 \pm 0.19$ respectively, $\mathrm{P}=0.012$ ).

Conclusions: Our results suggest that microhemorrhages and release of cholesterol from erythrocyte membranes may participate in the differentiation of macrophages to adipophilin expressing foam cells, which may lead to symptomatic plaque phenotype.

\section{Etiology of stroke}

\section{GENE EXPRESSION IN UNSTABLE ATHEROSCLEROTIC CAROTID} ARTERY PLAQUES

J. Saksi, P. Ijäs, K. Nuotio, M. Mäyränpää, P.T. Kovanen, M. Kaste, P.J. Lindsberg

Helsinki University Central Hospital (HUCH), Neuroscience Research Program, Biomedicum Helsinki, Helsinki, Finland

Background and Purpose: The symptomatic progression of atherosclerotic plaques leading to ischemic insult is still poorly understood. In this study we adapted largescale DNA microarray analysis to compare gene expression profiles of high-grade symptomatic versus asymptomatic carotid artery plaques to identify genes potentially involved in symptom generation.

Methods: The Helsinki Carotid Endarterectomy Study (HeCES) included 92 consecutive patients operated due to severe $(>70 \%)$ symptomatic (stroke/TIA) or asymptomatic carotid artery stenosis. A subcohort of completely asymptomatic patients with normal brain CT/MRI $(n=9)$ and patients with confirmed ipsilateral ischemic stroke $(n=13)$ were analyzed using Affymetrix HG-U133 arrays.

Results: Statistically significant $(\mathrm{p}<0.05)$ differential gene expression was observed in 78 genes when symptomatic plaques were compared to asymptomatic ones. Increased gene expression was observed in 71 genes including hemioxygenase 1 (HMOX1, 2.8-fold), vascular endothelial growth factor (VEGF, 2.3-fold), and adipophilin 1.9 -fold. Downregulation was observed in 7 genes including regulator of G-protein signalling 5 (RGS5, -1.9-fold), myosin heavy polypeptide 10 (MYH10, -1.9-fold), and calponin 1 (CNN1, -1.8-fold) all related to smooth muscle cell function.

Discussion: Here we describe changes in gene regulation, which associated with such biological processes as cellular macromolecule catabolism, immune response, angiogenesis, cellular lipid metabolism, and smooth muscle cell function. We identified genes with potential link to the symptomatic progression of atherosclerotic carotid artery disease, validating the global genomic approach.

\section{Etiology of stroke}

\section{SERUM LIPOPROTEIN (A) IS ASSOCIATED WITH SYSTEMIC} INFLAMMATION IN ACUTE ISCHEMIC STROKE

S. Chou, K. Furie, N. Hagan, B. Decisso, M. Lederer, D. Stein, R. Betensky, F. Buonanno

Massachusetts General Hospital, Boston, MA, USA

Objective: To determine relationship between lipoprotein (a) $[\mathrm{Lp}(\mathrm{a})]$ and serum inflammatory markers in acute stroke.

Background: Though Lp(a) is implicated cardiovascular diseases, its role in stroke is controversial. Elevations in inflammatory markers have been associated with increased risk and poorer outcome after stroke. While $\mathrm{Lp}(\mathrm{a})$ level is known to increase with inflammation in rheumatological diseases and infection, data from prior studies have not resolved the controversy surrounding the effect of the acute phase response on serum $\mathrm{Lp}(\mathrm{a})$ level in acute stroke.

Method: We measured serum Lp(a), ESR, LDL, and homocysteine in 167 acute stroke patients patients within 48 hours of admission to an academic medical center over one year. We collected data on stroke severity, mechanism, age, gender, ethnicity, co-morbidities, and medications. Mean log-transformed Lp(a) levels across stroke subtypes were compared using ANOVA. Determinants of $L p(a)$ were analyzed using stepwise linear regression.

Results: Stroke subtypes included 21\% atherothrombotic, 22\% cardioembolic, $7 \%$ lacunar, and 38\% cryptogenic, and $11 \%$ other. Mean Lp(a) level was $29.6 \mathrm{mg} / \mathrm{dl}$. Using multivariate regression, ESR was shown predict $\mathrm{Lp}(\mathrm{a})$ level $(\mathrm{P}=0.0002)$. $\mathrm{Lp}(\mathrm{a})$ did not correlate with of stroke severity or age. Although $\mathrm{Lp}$ (a) levels were higher in patients with an atherothrombotic mechanism of stroke, this association did not reach statistical significance.

Conclusion: Serum Lp(a) level is strongly associated with ESR, a marker of the acute phase response after ischemic stroke. The role of inflammation and elevated $\mathrm{Lp}(\mathrm{a})$ in the pathogenesis of strokes remains to be elucidated. Further studies are needed to determine if chronic low-grade inflammation is associated with elevated Lp(a) levels.

\section{Etiology of stroke}

THE ANALYSIS OF THE LESION PATTERN AND MECHANISM OF ACUTE STRIATOCAPSULAR INFARCTIONS

K.B. Lee, H. Roh, M.Y. Ahn

Soonchunhyang University Hospital, Seoul, South Korea

Background \& Objectives: There is no clear description about the patterns of each mechanism of SC infarcts. The aim of our study was to investigate the differential points between embolic SC infarcts and those originated from MCA disease.

Methods: We have prospectively enrolled the patients with acute infarcts which located in the lenticulostriate artery territory and were larger than $20 \mathrm{~mm}$ in axial DWI. Brain coronal DWI and MRA were done for the evaluation of distribution of infarcts and MCA stenosis in all patients. The infarct patterns were divided into 3 categories; dominant in proximal territory (DP), dominant in distal territory (DD), and distributed equally (DE). The upper limit of proximal territory, or the lower limit of distal territory, was the point which was thought to be the exact half of the total length of perforator in each section, based on the template map of lenticulostriate artery territories. We executed tests for embolic sources (TTE, TEE, Holter monitoring and MRA including aortic arch) in almost all patients. Stroke mechanism was determined by TOAST classification and Korean Stroke Registry classification

Results: This study has recruited 21 patients ( 9 men and 12 women; mean age, 57 years) until now. The patients with DP or DE infarct have significantly more proximal embolic sources than those with DD infarct. Most of the proximal embolic sources were of cardiac origin. On the contrary, the patients with DD infarct had more MCA stenoses which were related with the SC infarction.

Conclusion: These results suggest that the dominant area of SC infarction on coronal DWI can be an important clue of stroke etiology. It could be helpful to the determination of mechanism and treatment in many undetermined etiology patients of TOAST classification

\section{Etiology of stroke}

\section{SILENT INFARCTS ARE FREQUENT IN PATIENTS WITH ASYMPTOMATIC CAROTID ARTERY STENOSIS, BUT ARE THEY REALLY DIRECTLY} RELATED?

S. Fernández, A. Carvajal, M. Miguel, P. Cardona, F. Rubio, J. Krupinski UH of Bellvitge. CRC, IIBB/CSIC-HSCSP-UAB, Barcelona, L'Hospitalet de Llobregat, Barcelona, Spain

Background: Little is known about the underlying mechanisms and risk factors for silent infarcts (SI) in the setting of asymptomatic carotid artery disease (ACD). Currently, presence of SI is considered as an additional indication for carotid endarterectomy (CEA) in patients with high-grade ACD

Methods: We reviewed the magnetic resonance images (MRI) of 162 consecutive patients undergoing CEA at our centre, 53 had high-grade (>70\%) ACD. Subsequently, 24 patients without contralateral carotid stenosis were included. Risk factors profile, plaque anatomopathology and circulating atherothrombotic markers were measured. MRIs were examined for presence and distribution of infarcts.

Results: Mean age was 70 years, and 22 were men. Fifty percent of patients had diabetes, $71 \%$ hypertension, $46 \%$ were active smokers and $42 \%$ had ischaemic cardiopathy. In $75 \%$ patients plaques were hyperechogenic on ultrasound. Serum 
levels of MMP-8 (mean:10.6ng/ml), MMP-9 (mean:112.8ng/ml) and circulating tissue factor activity (mean:14.8pM) were elevated as compared to age-matched healthy controls $(\mathrm{p}<0,05)$. There were SI in 17 patients $(71 \%)$ on MRI. In 8 patients(33\%), MRIs presented with bilateral SI, 6 patients (25\%) had ipsilateral SI and $3(12.5 \%)$ patients had contralateral SI. All lesions were distributed in the white matter, corona radiata or centrum semiovale, except two patients with lacunar infarcts.

Discussion: SI on MRI is a frequent finding in patients with ACD. These patients present with higher proinflammatory and procoagulant state as compared to healthy controls. The high proportion of patients with bilateral lesions suggests that other factors than unilateral ACD contribute to its etiology.

\section{Etiology of stroke}

\section{HIGHER SEVERITY OF FRONTAL, OCCIPITAL, AND BASAL GANGLIA} WHITE MATTER HYPERINTENSITIES IN FIRST-EVER LACUNAR STROKE WITH MULTIPLE SILENT LACUNES

M. Grau-Olivares, A. Arboix, D. Bartrés-Faz, V. Cendrós, C. Garro, M. Besa, C. Junqué

Hospital del Sagrat Cor, University of Barcelona, Barcelona, Spain

Lacunar infarcts (LI) appears to be more closely associated with white matter hyperintensities (WMH) than in cortical ischemic stroke. However little is known about the differences in the frequency, severety and topography of WMH between patients with first-ever LI and single lacune versus patients with multiple silent LI.

Methods: Fourty two consecutive patients with a first-ever acute LI presenting a lacunar syndrome according to Miller Fisher's classification were recruited. Structural magnetic resonance images (MRI) were acquired from all cases using T1 weighted, T2 weighted, FLAIR and Difussion sequences to distinguish between acute and chronic LI. Patients were classified into two groups according whether the LI was isolated or it coexisted with multiple silent LI. WMH were assessed by visual inspection from T2-weighted MRI sequences with the Scheltens Scale.

Results: 19 patients $(45.2 \%)$ presented an isolated LI and 23 patients $(54.2 \%)$ multiple silent lacunes. There were not significant differences between groups in gender, age, education, clinical syndrome and MMSE. Multiple LI patients showed more hypertension $(82.6 \%$ vs $52.6 \% ; \mathrm{p}=0.04)$ and higher presence of WMH $(100 \%$ vs $73.7 \% ; \mathrm{p}=0.01$ ). The total Scheltens Scale score was higher in patients with multiple LI vs. single LI (19.3 \pm 8.1 vs. $10.3 \pm 10.4 ; \mathrm{p}=0.003)$. Multiple LI group showed higher severity of periventricular WMH in frontal lobe $(\mathrm{p}=0.001)$, occipital lobe $(\mathrm{p}=0.02)$, caudate nucleus $(\mathrm{p}=0.02)$ and thalamus $(\mathrm{p}=0.01)$

Conclusions: Multiple silent LI are closely associated with higher severity of cortical and basal ganglia white matter hyperintensities. This affectation might be related with a worst prognosis and frontosubcortical cognitive decline.

\section{Etiology of stroke}

\section{DIFFUSION WEIGHTED MRI LESIONS OF INTERNAL BORDERZONE INFARCT HAVE LINEAR CORRELATION WITH THE DEGREE OF} CEREBRAL PERFUSION ABNORMALITY

S.H. Lee, M.-K. Han, J.-U. Chun, H.J. Bae, S.-H. Park, S.I. Sohn

Seoul National University Bundang Hospital, Seoul, South Korea

Background and Objective: Internal borderzone (IB) infarct is associated with higher frequency of severe carotid stenosis and occlusion, diabetes mellitus, heart disease. The purpose of the present study is to examine diffusion-weighted imaging (DWI) and single photon emission computed tomography (SPECT) patterns of IB infarct and the relationship between the IB infarct and the degree of large artery diseases.

Methods: We selected borderzone infarcts on DWI using templates for the identification of subcortical vascular territories. IB infarcts were identified as hyperintense areas in the vascular IB area. All patients had DWI, MRA and SPECT with carbonic anhydrase inhibitors, the majority of them within the first 48 hours of symptom onset. The association between DWI lesions and the degree of cerebral perfusion in SPECT was evaluated by visual inspection and statistical parametric mapping (SPM)

Results: Of 33 borderzone infarcts, twelve patients (36\%) had IB infarcts. All patients with IB infarct had MCA (8) diseases or ICA (4) disease. DWI patterns of IB infarcts were divided into 2 groups: scattered DWI lesions $(<2 \mathrm{~mm})$ less than 3 (4 patients), confluent DWI lesions (8 patients) in IB vascular territories. All patients with scattered pattern had mild MCA stenosis and mild hypoperfusion of basal ganglia or cortex. The confluent pattern was associated with severe stenosis or occlusion of large arteries (4 ICA and 4 MCA) and extensive hypoperfusion of basal ganglia and cortices in SPECT with impaired vasoreactivity.

Conclusion: We postulate that DWI lesions of IB infarcts have linear relation with the degree of large artery stenosis and the cerebral perfusion abnormality in symptomatic patients.

\section{Etiology of stroke}

\section{LARGE ANTERIOR CHOROIDAL ARTERY (ACHA) INFARCTS. ITS} ETIOLOGY AND PROGNOSIS

P. Cardona, J. Krupinski, S. Fernández, A. Palasí, F. Rubio

Bellvitge Hospital, Barcelona, Spain

Background: The underlying cause of large AChA infarcts is not well known. A typical clinical triad is similar as for non-AchA superficial infarcts.

Methods: We reviewed retrospectively our database of 2256 patients who suffered from ischaemic stroke between 1998 and 2005. We compared distribution of vascular risk factor and most likely etiology in AChA and non-AchA superficial infarcts by multivariant regression analysis.

Results: Large AChA infarcts were present in 43 patients $(1,7 \%)$. The classical triad of clinical symptoms was observed in $32(80 \%)$ of these patients. Hypertension was found in 8 patients $(18 \%)$, diabetes in $6(16 \%)$, and hypecholesterolaemia in $3(8 \%)$. The presumed cause of stroke was cardioembolism in 19 patients, large vesse disease (severe carotid stenosis or occlussion) in 9, small-vessel disease in 6 and cryptogenic infarcts in 8 . On multivariant regression analysis cardioembolic source and carotid stenosis was more frequent in large AChA infarcts than in non-AchA superficial infarcts $(\mathrm{p}<0.005)$. Mortality and functional independence (Rankin $0-1)$ were more favorable for patients with AChA $(\mathrm{p}<0.0006)$. Five patients received intravenous rt-PA. Three patients had complete recovery at day 7 , and all 5 patients had final good outcome at day 90 (Rankin 0-1).

Conclusions: Large AChA infarcts are very rare $(1,7 \%)$. Cardiac or carotid embolism is a common cause of large AChA infarct. The good prognosis and possible better response to rtPA support the idea that it is a different stroke subtype as compared to other small or large vessel infarcts.

\section{Etiology of stroke}

ISCHEMIC STROKES IN THE ANTERIOR CHOROIDAL ARTERY: MULTIFACTORIAL ANALYSIS ACCORDING TO THE INFARCT SIZE E. Cuadrado, A. Ois, A. Rodríguez, E. Munteis, M. Gomis, J. Jiménez, J. Martínez, J. Roquer

Hospital del Mar, Barcelona, Spain

Background: The pathophysiological mechanisms of anterior choroidal artery (AchA) strokes are poorly understood. Some studies reported that these strokes are similar to those found in small vessel disease (SVD) or lacunar infarcts. The aim of our study was to compare clinical, ultrasonographic and radiological data in patients with AchA and lacunar strokes.

Methods: Patients with acute ischemic stroke were studied in our centre in the past 3 years. We selected 47 patients with established ischemic stroke in the AchA and 190 with lacunar infarcts. We determined the size of the lesion based on radiological studies (176 with DWI-MRI and 61 with CT scan) within the first week after the stroke onset. The size of the AchA infarcts was dichotomised in large $(>15 \mathrm{~mm})$ or small $(<15 \mathrm{~mm})$. We analysed the following data: presence of vascular risk factors, initial clinical severity, evolution and outcome at three months after stroke onset, previous SVD in radiological study and a embolic source according to the TOAST criteria.

Results: When analysing AchA infarcts according to the size, large AchA infarcts were more associated with an embolic source $(\mathrm{p}=0.019$. OR=4.28 IC 95\% 1.23 14.83 ), a higher rate of early progression ( $\mathrm{p}=0.009$ OR $5.7595 \% \mathrm{CI} 1.45-22.78)$ and had a worse functional prognosis at three months ( $\mathrm{p}=0.38$ OR $4.1895 \%$ CI 1.03 16.93) than small ACh infarts. Comparing large AchA infarcts with lacunar infarcts, we found a higher frequency of embolic source and a less presence of previous small vessel disease in AchA infarcts. We did not found significant differences between small AchA infarcts and lacunar infarcts in other territories.

Conclusions: Large infarcts in the AchA territory may have a different pathophysiology than lacunar strokes and should be considered as a different etiological group. Further accurate neurovascular studies on AchA infarcts are needed. 
RISK FACTORS, CLINICAL FEATURES OR NEUROIMAGING FINDINGS. HOW DO WE DIAGNOSE LACUNAR INFARCTIONS?

M.A. Ortega-Casarrubios, B. Fuentes, I. Ybot, B. San José, R. Madero, E. Díez-Tejedor

Hospital Universitario La Paz. Universidad Autónoma de Madrid, Madrid, Spain

Background: Current diagnostic criteria for lacunar infarctions (LI) are based on the combination of risk factors (hypertension and/or diabetes), clinical features (lacunar syndromes) and neuroimaging findings,together with no other possible etiologies.Our goal is to analyse the impact of those criteria in LI diagnosis and in outcome.

Methods: Observational study from the data bank of the Stroke Unit, with inclusion of consecutive ischemic stroke (IS) patients (in the period 2000-2004). Parameters analysed:Risk factors, clinical syndrome, neuroimaging findings, stroke subtype, stroke severity and functional state at discharge.

Results: 1522 IS patients,460 LI.They had higher frequency of hypertension $(67.8$ vs $62.4 \%$, p=0.04) than Non-LI, without differences in diabetes. Lacunar syndrome was present in $88.9 \%$ of LI patients (sensorimotor $38 \%$; pure motor $35.9 \%$; pure sensory $9.6 \%$; dysarthria-clumsy hand $4.1 \%$ and ataxic hemiparesis $1.3 \%$ ) Brain CT showed LI in $63.7 \%$ Final diagnosis of LI was based on the combination of risk factors and classical lacunar syndrome in $41.7 \%$; risk factors, classical lacunar syndrome and neuroimaging data in $24.3 \%$; classical lacunar syndrome alone in $14.5 \%$, classical lacunar syndrome and neuroimaging in $8.2 \%$; only risk factors in $5.8 \%$; risk factors and neuroimaging in $2.8 \%$,only neuroimaging findings in $0.8 \%$. Diagnosis of LI based on the combination of two or more of those criteria was associated to a higher stroke severity on admission and poor functional state at discharge $(\mathrm{p}<0.05)$

Discussion: The most frequently used diagnostic criteria for LI is the presence of a classical lacunar syndrome, followed by the risk factors profile (hypertension and/or diabetes). The combination of two or more diagnostic criteria is associated to worse evolution.

\section{Etiology of stroke}

\section{ETIOLOGY OF ISCHEMIC STROKE IN YOUNG ADULTS}

I. Divjak, M. Jovicevic, A. Jovanovic

Institute of Neurology, Clinical Centre Novi Sad, Medical Faculty Novi Sad,

Serbia and Montenegro, Novi Sad, Yugoslavia

Background: The aim of the study was to analyse the etiology of ischemic stroke (IS) in 100 successive stroke patients aged 15-45 years treated at our Institute in the period 2002-2005.

Methods: The patients were divided into three age groups: 15-25, 26-35, and 36-45 years. Diagnostic procedures applied in all patients included brain CT and/or MRI, carotid arteries Doppler, transcranial Doppler, coagulation tests and routine laboratory tests. Contrast and/or transesophageal echocardiography, immunological blood assays, MRA and/or DSA, C, protein S, antitrombin III, antiphospholipid antibodies, toxicological examination and pregnancy test were performed in selected patients.

Results: The results showed men to be at higher risk of IS in the overall study population as well as in the age groups $26-35$ and 36-45. The most frequent risk factor was smoking, recorded in $55.65 \%$ of all patients and it was similarly represented in all groups. A significant difference was found among the age groups with regard to the average number of risk factors. The group $36-45$ was associated with significant increase in the number of risk factors $(\mathrm{p}=0.004)$. Large vessel disease was the most frequent cause of IS in the group 36-45, found in $29.2 \%$. Carotid dissection was the most frequent cause $(16.7 \%)$ in the group $15-25$. Fibromuscular dysplasia and systemic vasculitis were diagnosed in $12.6 \%$ of patients in the group 26-35. Moya-Moya, Takayasu's disease, infectious and isolated CNS angiitis were not found in the study sample. Cardiac embolism was significantly more frequent $(\mathrm{p}=0,006)$ in the group 26-35. Paradoxical embolism was found in $6 \%$ of all study patients and was more frequent in the age group 15-25. Small vessel disease was significantly more frequent in the age group $36-45(\mathrm{p}=0.032)$. None of the study patients was diagnosed with migraine infarction, rare coagulopathy or drug abuse. Discussion: Our results are similar to other series of young patients with IS in Europe.

\section{Interesting cases}

\section{Interesting cases}

SUPERFICIAL SIDEROSIS OF THE CENTRAL NERVOUS SYSTEM- AN UNCOMMON CHRONIC CEREBROVASCULAR DISEASE K. Spengos, G. Tsivgoulis, S. Sameli, G. Koutsis, G. Karahalios, N. Zakopoulos, D. Vassilopoulos University of Athens, Athens, Greece

Background: Superficial siderosis (SS) is an unusual affection of the central nervous system caused by chronic subarachnoid hemorrhage of different, mostly undetermined sources leading to hemosiderin deposition in the leptomeninges of the brain and the spinal cord. Progressive cerebellar ataxia, sensoneuronal hearing loss and pyramidal tract signs are the cardinal clinical features.

Case presentations: We present the cases of 3 males, (A: $72 y$; B: $63 y$, C: $63 y$ ) admitted with suspected adult onset spinocerebellar atrophy. Patients A and B reported a since 10 years progressing gait disorder. On examination both patients presented severe bilateral cerebellar and pyramidal features and were both deaf. Patient $\mathrm{C}$ reported a since 2 years persisting gait instability. A prominent bilateral pyramidal syndrome was accompanied by slight dysmetria and dysdiadochokinesia. Hearing loss affecting high frequencies was also documented. In all 3 cases SS diagnosis was set based on the typical MRI findings on GRE sequences that revealed the characteristic hemosiderin rim on the hemisphere's, brainstem's and cord's surface. A bleeding source, namely a lumbar ependymoma, could get identified in only one case (C). Treatment with steroids led to clinical improvement only in case C.

Conclusion: SS is an uncommon and probably underdiagnosed chronic cerebrovascular disease that in its progression may imitate different degenerative diseases including spinocerebellar ataxia. Since in some cases causative treatment is possible and effective, early diagnosis is of crucial importance. Gradient echo weighted MRI reliably sets the diagnosis and should therefore be included in the imaging evaluation protocol of patients presenting with slowly progressive pyramidal and cerebellar features.

\section{Interesting cases}

\section{SEVERE PROLONGED AURA IN A SPORADIC MIGRAINE ASSOCIATED WITH CACNA1A MUTATION: EVIDENCES FOR A NON ISCHEMIC} MECHANISM

S. Debiais, I. Bonnaud, C. Hommet, B. Lucas, G. Rival, E. Tournier Lasserve, A. Autret

University Hospital, Tours, Tours, France

Background: Hemiplegic migraine is characterised by motor weakness during aura. In familial hemiplegic migraine HM (FHM), mutations of two genes have been identified: CACNA1A and ATPA12. In sporadic hemiplegic migraine (SHM), mutations (involving CACNA1A) have been reported in only four cases. The pathophysiology of prolonged aura remains uncertain, with two mains hypothesis: an ischemic mechanism, or a spreading cortical depression (SCD) mechanism.

Objective: To report evidences of a non-ischemic mechanism of prolonged aura in a case of SHM with a CACNA1A mutation

Case report: A 25 y.o. woman in the 19th week of her pregnancy, was admitted for a diffuse headache, associated with photophobia and vomiting, followed 24 $\mathrm{h}$ later by a sudden left hemiparesis, impaired consciousness and hyperthermia. Blood and CSF examinations were normal. The EEG mapping showed a prolonged right hemispheric delta activity, while hemiparesis persisted. Cerebral MRI, with diffusion-weighted (DW) sequences and angio MRI, performed at 24 and 48 hours after the onset, was strictly normal, as well as cardiac and vascular investigations. Fever lasted for 3 days, motor weakness and headache resolved after 5 days. During her adolescence, she had experienced 5 similar, but less severe, attacks (visual disorders and arm paresis lasting less than one hour followed by headache). There was no familial history of migraine. Genetic screening demonstrated a S218L mutation in CACNA1A gene.

Conclusion: Diagnosis of SHM is difficult, particularly when hemiparesis is prolonged. Diagnoses of stroke or cerebral venous thrombosis have to be ruled out before evoking HM. This is to our knowledge the first report of S218L mutation causing SHM. In our case, normality of repeated DW MRI associated with prolonged right hemispheric dysfunction (persisting left hemiplegia and EEG focal abnormalities) excludes an ischemic mechanism, and argues for the hypothesis of SCD phenomena. 


\section{Interesting cases}

CAROTICO-CAVERNOUS FISTULA ASSOCIATED WITH FIBROMUSCULAR DYSPLASIA

D. Manawadu, O. Malik

Hillingdon Hospital, Hillingdon, United Kingdom

Case Presentation: A 29-year-old Afro-Caribbean woman presented 5 weeks post-partum with sudden onset right sided weakness. She had no significant past medical or family history and was not on any medications. On examination she had expressive dysphasia, left sided Horner's syndrome and mild right sided weakness. A CT brain scan revealed a left middle cerebral artery infarct. Doppler ultrasound revealed a dissection of the left internal carotid artery. A vasculitic screen and echocardiogram were normal. She was anticoagulated with warfarin.

One week later she presented with a swollen painless eye. On examination her left eye was swollen with pulsatile proptosis, ptosis and chemosis. There was a third cranial nerve palsy with a dilated pupil. Four-vessel angiography revealed a carotico-cavernous fistula with a hypoplastic left middle cerebral artery and retrograde filling from the vertebro-basilar circulation. Both the carotid and vertebral arteries had a 'string of beads' appearance strongly suggestive of fibromuscular dysplasia. Dissection of the left internal carotid artery was demonstrated. Her fistula was occluded with 16 coils via an endovascular approach with a good result.

Discussion: There are only a few case reports of carotico-cavernous fistula in the presence of fibromuscular dysplasia. This is a non-inflammatory disease of the arterial wall of unknown aetiology more commonly found in females. The internal carotid artery is most commonly affected after the renal vessels. It is associated with dissection and can lead to carotico-cavernous fistula formation. Diagnosis of this is by carotid angiography. Untreated fistula may lead to visual loss. Endovascular techniques are used increasingly over surgical approaches to obliterate the fistula.

\section{Interesting cases}

\section{ISOLATED BILATERAL OCULOMOTOR NUCLEAR PALSY DUE TO A} MIDBRAIN INFARCTION

J.H. Park, S.W. Han, J.Y. Kim, J.S. Paik

Sanggye Paik Hospital, Inje University College of Medicine, South Korea

Background: While the bilateral oculomotor nuclear palsy with more widespread rostral brainstem lesion has been reported, isolated bilateral oculomotor nuclear palsy due to a midbrain infarction had not been reported. We report a patient who had an isolated bilateral oculomotor nuclear palsy with pupil sparing and no ptosis. Case report: A 52-year-old man with a history of hypertension presented with a sudden onset of double vision. On neurological examination, he did not have motor weakness, sensory changes, tremor or dysdiadochokinesia. On ocular examination, there was no ptosis. The pupillary size was equal and the light reaction was normal on both eyes. On forward gaze, the both eyes were deviated outward and slightly downward. Supraduction, infraduction and adduction of the both eyes were significantly limited but abduction was normal. The limitations were also observed during the test of Bell's phenomenon and Doll's-head maneuver. Brain MRI revealed an acute infarction in the midbrain, adjacent to the midline and ventral to the aqueduct of sylvius, at the level of the mid portions of oculomotor nucleus. Discussion: Our patient had an isolated bilateral oculomotor nuclear palsy and brain MRI revealed a small midbrain infarction, adjacent to the midline. In this patient, the occlusion of the single artery, which originates from an unpaired trunk, may be the most plausible explanation for this rare type of infarction. The organization of the oculomotor nuclear complex consists of the Edinger-Westphal nucleus supplying fibers to the pupils, the motor nucleus supplying the extra ocular and levator palpebrae muscles in the rostro-caudal order. The findings for this particular patient showed that the occlusion of the single artery might result in bilateral oculomotor nuclear infarctions. It also showed that the oculomotor nucleus supplying the extra ocular muscles was located at the level of the mid portions of oculomotor nucleus.

\section{Interesting cases}

\section{ENTIRE CORPUS CALLOSUM INFARCTION - AN UNUSUAL VARIANT OF} WATERSHED ISCHEMIA

K. Spengos, G. Tsivgoulis, A. Papapostolou, E. Manios, M. Papadopoulou,

N. Zakopoulos, V. Zis

University of Athens, Athens, Greece

Background: Infarction of the corpus callosum (CC) is uncommon due to its rich blood supply and resistance to small vessel ischemic changes. Lesions involving the entire CC are extremely rare and have been related to bilateral internal carotid artery (ICA) occlusion, underlining the importance of hemodynamic insufficiency in such cases. The pericallosal branch of the anterior cerebral artery is the dominant vascular supply of the body of the CC whereas perforating branches supply rostrum and genu. The splenium is covered by the posterior pericallosal artery; a posterior cerebral artery branch. The watershed area between anterior and posterior pericallosal artery is quiet variable explaining the unpredictable size and location of CC infarcts.

Case report: We present the uncommon case of a hypertensive man who developed hemihypoesthesia and confusion shortly after receiving $20 \mathrm{mg}$ Nifedipine sublingually, due to a hypertensive episode $(195 / 110 \mathrm{mmHg}$ before; $150 / 85 \mathrm{mmHg}$ after medication). Detailed examination revealed also an impressive disconnection syndrome with left hemialexia, visual and tactile anomia and bilateral apraxia. MRI revealed an entire CC infarction and a typical posterior borderzone infarct. Conventional angiography showed a subtotal stenosis of the intracranial portion of the left ICA, in combination with contralateral intracranial moderate ICA stenosis and ipsilateral A1 hypoplasia.

Conclusion: Therefore, we assume hypoperfusion due to distal hemodynamic insufficiency after a drug induced abrupt blood pressure fall as cause of stroke and suggest that this case of extensive $\mathrm{CC}$ infarction might be a highly uncommon variant of watershed stroke. The acute onset of all symptoms and the co-existence of a typical ipsilateral posterior borderzone infarct with identical findings on DWI and ADC suggest the concomitant onset of both ischemic lesions, implies a common underlying pathogenetic mechanism and supports our hypothesis of terminal circulation failure.

\section{Interesting cases}

CEREBRAL VASOREACTIVITY ASSESSMENT IN UNILATERAL COMMON CAROTID ARTERY OCCLUSION WITH PATENCY OF EXTERNAL AND INTERNAL CAROTID ARTERIES. AN FMRI STUDY OF THE MOTOR CORTEX

R. Herzig, P. Hlustik, J. Mares, S. Burval, M. Herman, P. Kanovsky Stroke Center University Hospital, Olomouc, Olomouc, Czech Republic

Background: Patients with cerebrovascular occlusive disease may develop compensatory changes with a resultant vascular reactivity loss. These alterations can affect the blood oxygen level-dependent (BOLD) signal that is the basis for functional magnetic resonance imaging (fMRI). Occlusion of the common carotid artery (CCA) with reversed blood flow in the patent external carotid artery (ECA) and with proper blood flow direction in the patent internal carotid artery (ICA) is quite uncommon. The aim was to examine whether these patients suffer from a decreased vasodilatory capacity in the corresponding ICA territory.

Methods: fMRI examination of the motor cortex was performed in 2 females, 63 and 79-year old, suffering from left CCA occlusion with patent ECA and ICA. The patients performed a bimanual hand motor task alternated with rest within an event-related paradigm for assessment of hemodynamic response.

Results: Significantly active areas included both motor cortices. There were no significant inter-hemispheric differences in active M1 volume or the size, delay and integral of the regional hemodynamic response.

Discussion: Unlike previous observations in occluded ICA, our patients did not show impaired cortical hemodynamic response ipsilateral to the occluded CCA thanks to sufficient blood supply through patent ECA and ICA. These cases show that (1) fMRI of the motor cortex appears to have clinical merit in the assessment of cerebrovascular conditions and (2) reversed blood flow in ECA may serve as a "natural extracranial-intracranial bypass" in patients with occluded CCA.

Acknowledgement: Supported by the IGA MH CR grants number NR/7830-3/2004 and NR/8367-3/2005

\section{Interesting cases}

\section{SEVERE AUTO-HETERO-TOPAGNOSIA AND IDEOMOTOR APRAXIA} WITHOUT APHASIA AFTER STROKE. REPORT OF TWO CASES A. Carota, S. Dieguez, P. Michel, J. Bogousslavsky CHUV, Lausanne, Switzerland

Background: Auto-hetero-topagnosia (e.g., the inability of localizing body parts respectively on oneself and the examiner) and ideomotor apraxia are both related to left parietal damage. The rarity of presentation of these syndromes in pure forms is due to the concomitant presence of aphasia in most cases.

Method: We assessed two young patients during the first week after stroke, a $32 \mathrm{y}$ old left handed man and a 44 y old ambidextrous man. Stroke aetiology was right internal carotid occlusion and hyperhomcystinemia (MTHR mutation) for the first, and left internal carotid subocclusion of undetermined cause for the second patient. Results: Both patients showed severe (more than $90 \%$ error rate, flagrant difficulties) auto-hetero-topagnosia and ideomotor apraxia notably without aphasia. We considered the two syndromes (topagnosia and apraxia) to be independent because 
difficulties of pointing were essentially limited to body parts. The patients did not show any difficulty ( $0 \%$ error rate) in recognizing either body parts or gestures and they recovered almost completely during the first week after stroke onset. The MRI showed for both the involvement on the respective hemispheres of the supramarginal gyrus of the parietal lobe and the dorsolateral frontal cortex.

Discussion: In patients with unusual handedness, auto-hetero-topagnosia and ideomotor apraxia might present in a severe and pure form. We think that, in these rare cases, despite remarkable errors in pantomimes or designation of body parts, the unimpaired knowledge of body parts and gestures is probably due to the effective dominance of the intact hemisphere for related semantic aspects.

Auto-hetero-topagnosia and ideomotor apraxia should be accurately and systematically assessed during the earliest phases of stroke especially in patients with variant handedness.

\section{Interesting cases}

\section{POSTERIOR SUBTYPE ALIEN LIMB SYNDROME IN BILATERAL} HEMISPHERIC STROKE

J. Wöhrle, H. Bäzner, C. Blahak, M.G. Hennerici

Universitätsklinikum Mannheim, University of Heidelberg, and Department of

Neurology Katholisches Klinikum, Mannheim, Germany

Background: Alien limb syndrome is a rare manifestation of stroke. The existence of a posterior subtype of alien limb syndrome is a matter of debate.

Case report: Within 3 days prior to admission, a right-handed 78-year-old woman with arterial hypertension and hyperlipidemia experienced two episodes of 15 minutes with numbness of the left arm accompanied by a strange feeling that the arm no longer belonged to her. On the morning of admission, a third episode recurred, then accompanied by purposeless movements of her left arm that seemed to be under external, extravolitional control. She told her hand off "you don't have to help me", when using her right hand, but she did not notice any weakness. The symptoms completely remitted after 5 hours, and on presentation to the emergency unit, neurological examination was normal, apart from mild action tremor of the right hand and elevated blood pressure of 170/90 $\mathrm{mmHg}$. After 24 hours, cranial magnetic resonance (MR) diffusion weighted imaging showed a small acute hyperintense lesion in the hand area of the right postcentral gyrus and additional small hyperintense lesions in the left parietooccipitotemporal cortex and subcortical white matter in the area of the posterior borderzone. MR angiography revealed a reduced flow signal in the left internal carotid artery (ICA) and MR perfusion weighted imaging showed a delayed contrast bolus arrival in the left middle cerebral artery territory. Ultrasound studies demonstrated a $90 \%$ ICA occlusion and collateral flow via the anterior cerebral artery from the right to left side. There was no cardiac source of embolism; blood laboratory values including coagulation studies were normal.

Discussion: The alien hand syndrome was described as a consequence of callosal or medial frontal lobe damage and distinct clinical syndromes of callosal type and frontal type were characterized. As in our patient, modern imaging techniques help to diagnose the recently described posterior type of alien hand syndrome that is attributed to damage to the lateral thalamus and parietal and temporal cortex.

\section{Interesting cases}

VIAGRA (SILDENAFIL) TEMPORARILY IMPROVES HEMINANOPIA AFTER POSTERIOR INFARCTION

M. Eicke, P. Schlindwein, P. Stoeter, M. Dieterich

Klinikum Idar-Oberstein, University of Mainz, Idar-Oberstein, Germany

Background: Animal trials in rats with acute stroke showed improved neurological function with sildenafil (Zhang 2005). In healthy human subjects no effects of sildenafil on visual fields and intraocular pressure (Ermis 2004) were found while the pulsatile ocular blood flow increased (Paris 2001). To date no data of patients with hemianopia due to stroke is available.

Case Report: A 65 year old male had suffered from a bilateral ischemic stroke of the posterior cerebral arteries (PCA) probably due to a cardiac embolisation resulting also in the occlusion of the proximal left vertebral artery in 2002. After partial recovery, he had persisting hemianopia to the right and less to the left with some day-to-day fluctuations. In 2004 he experienced improvement of the heminaopia, every time he took $25 \mathrm{mg}$ Sildenafil. This effect started approximately 10 minutes after oral administration, had its maximum effect after 60-90 minutes and lasted for at least 4-7 days. Since then he took 50-100 mg sildenafil regularly every 4-7 days. The improvement of scotomas and brightness of the visual field could be verified in the visual field examinations (Humphrey and Goldmann).

Functional MRI during a visual stimulation by horizontal optokinetic nystagmus showed significant differences in activations of the visual cortex (BA 17, 18 and 19) bilaterally between t-contrast images before and after the administration of $100 \mathrm{mg}$ sildenafil. Overlaying the t-contrasts of the fMRI experiment on a high resolution T1-image of the patient, we located these new activations after medication mainly along the margins of the old bilateral posterior infarctions (lingual and fusiform gyri, cuneus bilaterally).

Discussion: This is the first report on objectively and reliably tested effects of sildenafil in a patient with bilateral hemianopia.

\section{0}

Interesting cases

FMRI IN ISCHEMIC STROKE: A CASE OF CROSSED APHASIA

L. Caputi, M.R. Carriero, P. Vitali, M.G. Bruzzone, L. D'Incerti, E. Colombo,

A.R. Giovagnoli, E.A. Parati

National Neurological Institute C. Besta, Italy

Only a few cases of crossed aphasia (CA) with functional neuroimaging data are described in literature demonstrating the involvement of the right hemisphere. Func tional magnetic resonance imaging (fMRI) in aphasic subjects is complex because the language tasks activate mostly the residual areas close to the lesion. We report a case of CA after ischemic stroke studied with fMRI.

A 75-year-old woman, five years schooling, right-handed housewife, with no history of familial sinistrality, affected by hypertensive heart disease, chronic atrial fibrillation and mitral valve disease, suffered sudden onset of speech difficulties and neglect in May 2004, with partial recovery. CT scan showed right temporo-parietal ischemic stroke. After the first event, she presented 3 episodes of transient left hemiparesis and speech disorders. We re-evaluated her 12 months later.

Methods: a complete neuropsychological evaluation was carried out in May 2005. fMRI was obtained using a modified version of the semantic discrimination correctly performed by the patient: during auditory presentation of word sequence, she had to recognize the word "lion" indicating it with a slight movement of the tips.

Results: Severe aphasia involving all language components was recognized. Writing, comprehension and the semantic competences were mostly impaired. Oro-facial, ideomotor and constructive praxis were also impaired

MRI twelve months later showed a non-acute ischemic stroke in the right temporoparietal region. At fMRI only a little cluster in medium temporal gyrus was found without activation in left inferior frontal gyrus. Many activation clusters were found within right frontal operculum, residual part of medial temporal gyrus and inferior temporal gyrus.

Discussion: the heterogeneous impairments of oral and written language abilities suggest greater involvement of the posterior regions. Clinical presentation and conventional neuroimaging suggested right hemispheric language dominance, but only fMRI demonstrated the hypothesis. fMRI could, therefore, help at confirming diagnosis in $\mathrm{CA}$

\section{Interesting cases}

ISCHEMIC NEURO-OPHTALMIC COMPLICATIONS OF COCAINE ABUSE: REPORT OF TWO CASES

M.E. Erro, B. Zandio, M. Herrera, D. Aliseda, R. Muñoz, J. Gállego

Servicio de Neurología. Hospital de Navarra, Pamplona, Spain

Background: Cocaine abuse is a well known risk factor for ischemic and haemorrhagic stroke. Neuro-ophtalmic ischemic complications related to cocaine abuse have been seldom described.

Methods: Our purpose has been to describe two patients with acute visual loss secondary to retinal artery occlusion and ischemic optic neuropathy respectively occurring immediately after the nasal inhalation of cocaine. The pathogenic mechanisms are discussed.

Results: Case 1, A 34 years old woman was admitted with right sudden visual loss, without eye pain occurring four hours after the nasal consumption of cocaine. Ophthalmoscopy showed a whitish platelet-fibrin emboli in the inferior retinal artery that produced whitening of the retina and a macular cherry red spot. Carotid artery disease was ruled out with Doppler exam. Cranial RM was normal. Right visual evoked potentials revealed high amplitud and latency response on right eye and electroretinogram showed rod involvement. The diagnosis of cocaine associated retinal artery occlusion was considered.

Case 2, A 21 years old man consulted for acute and painless right visual loss. Optic disc on funduscopy was normal. Inferior altitudinal visual field loss was found. Urine toxic screening was positive for cocaine. Empiric corticosteroid treatment was initiated without any response. Possible inflamamatory systemic conditions such as lupus erytematosus, sarcoidosis and Lyme disease were ruled out. Cerebrospinal fluid was normal without oligoclonal bands. Cranial RM was normal. Visual evoked potentials were not obtained in the right eye. The diagnosis of ischemia of the retrobulbar optic nerve was established. 
Discussion: Cocaine related vasospasm, vasculitis or hypercoagulable state leading to platelet-fibrin emboli are the mechanisms that may have contributed to retinal artery occlusion and optic nerve infarction in these two cases.

\section{Interesting cases}

\section{T2*-WEIGHTED GRADIENT-ECHO MRI FOR DIAGNOSIS OF A CASE OF} ISOLATED CORTICAL VEIN THROMBOSIS

S. Rodríguez, S. Calleja, L. Benavente, J. Villafani, J.M. Fernández, C.H. Lahoz Hospital Universitario Central de Asturias, Oviedo, Spain

Background: Isolated cortical vein thrombosis (ICVT), without concomitant dura sinus thrombosis, is only rarely diagnosed, although it may commonly be overlooked. Clinical symptoms are non-specific and radiological studies are crucial in establishing the diagnosis.

Case report: A 72-year-old man with hypertension, hypercholesterolemia and myocardial infarction was admitted after suffering recurrent episodes of right arm paresthesias and dysphasia. Neurological and electroencephalographic examination, vascular and cardiologic studies were normal. Thrombophilia workup and CSF exam were also normal. Brain CT showed no abnormalities but on the MRI a narrowing of the sulci of the left central region was observed on T1-weighted images, with enhancement of the superficial vein after gadolinium injection. FLAIR images did not show abnormal hyperintensities and there were no abnormalities on the DWI sequence either. T2*-weighted gradient-echo (GRE) sequence showed the ICVT with areas of reduced intravasal signal in both hemispheres. Treatment with high doses of low-molecular-weight heparins, gabapentin and levetiracetam was started in order to stop seizures. The patient improved and continued to take oral anticoagulants for six months. Follow-up MRI was obtained four months later showing no remarkable abnormalities.

Discussion: T2*-weighted GRE sequence is very helpful for the detection of ICVT and might be positive sooner than other techniques such as FLAIR or DWI sequences. Therefore it may be of great diagnostic value to integrate this sequence into the MR protocol for visualization of ICVT. Previous extracerebral venous thrombosis, presence of hereditary thrombophilia or evidence of previous microbleeds in the T2*-weighted GRE could help the therapeutic decision

\section{Interesting cases}

\section{PROLONGED LOW-DOSE INTRAVENOUS THROMBOLYSIS IN A STROKE}

\section{PATIENT WITH DISTAL BASILAR THROMBUS}

R. Veltkamp, C. Jacobi, J. Regula, P.A. Ringleb, B. Kress, W. Hacke

UniversityHospital Heidelberg, Heidelberg, Germany

Patients with high grade basilar artery stenosis secondary to thromboembolism are at high risk of developing subsequent vessel occlusion. Optimal medical management of this condition is unclear. We present a patient with a small subacute brainstem infarction and filiform distal basilar residual lumen due to arterioarterial or cardiogenic embolism. Beginning 3 days after symptom-onset, low-dose intravenous thrombolysis with $0.125 \mathrm{mg} / \mathrm{kg} \mathrm{rtPA}$ was continuously infused for 48 hours Follow-up MR angiography revealed complete resolution of the embolus. No further cerebral ischemic episodes occurred during 3 months follow-up and the basilar artery remained patent. Our observation suggests a potential for prolonged low-dose intravenous thrombolysis in basilar artery embolism but further data are needed to judge the effectiveness and risk of this intervention.

\section{Interesting cases}

\section{A PATIENT WITH DISSEMINATED INTRAVASCULAR COAGULOPATHY} WHO WAS RECEIVED ARGATROBAN TREATMENT

K.B. Lee, J.Y. Park, S.Y. Hwang, R. Hakjae, M.Y. Ahn

Soonchunhyang University Hospital, Seoul, South Korea

Background \& Significance: Both ischemic stroke and ICH may occur in DIC. When ischemic thromboembolic events are the prevailing process, anticoagulation with heparin or low molecular weight heparin is generally advised to prevent additional events, although this approach is controversial. Argatroban, direct thrombin inhibitor, recently received FDA approval for HIT patients who are at high risk for developing for thrombosis.

Case: A 67-year-old women was referred for GI bleeding. She had been in good health previously until showed confusion and decreased response 1 month ago, and was received antiplatelet therapy for acute multiple cerebral infarction. Three days later, sudden weakness in the left limb made her under heparin treatment, but the platelet count reduced to $67 \times 10^{3} / \mu 1$. It was slightly restored after heparin withdrawal and steroid therapy $\left(>100 \times 10^{3} / \mu 1\right)$. Her initial laboratory findings in our hospital revealed anemia $(9.0 \mathrm{~g} / \mathrm{dl})$, leukocytosis $\left(14,400 \times 10^{3} / \mu \mathrm{l}\right)$, and thrombocytopenia $\left(38 \times 10^{3} / \mu \mathrm{l}\right)$. PT (INR) was 1.4 , and aPTT was $28.7 \mathrm{sec}$. FDP $(>20 \mu \mathrm{g} / \mathrm{ml})$ and D-dimer $(>10 \mu \mathrm{g} / \mathrm{ml})$ were positive, but lupus anticoagulant was negative. In hospital day (HD) 3 , however, melena and severe epistaxis developed in spite of warfarin withdrawal. In HD 5, there were aggravating necroses in both leg and pulmonary embolism with decreased platelet count $\left(11 \times 10^{3} / \mu 1\right)$. In HD 7 , we started argatroban $(2 \mu \mathrm{g} / \mathrm{kg} / \mathrm{min})$, and detected increased platelet count $\left(22 \times 10^{3} / \mu \mathrm{l}\right)$ the next day. The gangrene of both legs also stopped worsening and no bleeding occurred thereafter.

Conclusions or Comments: This patient is considered to have DIC and suspected HIT-type II. In this situation which was at high risk of thrombosis with severe thrombocytopenia, argatroban could be a possible treatment option.

\section{Interesting cases}

\section{INCIDENT OF DYSARTHRIA AND DIFFICULTY IN READING AND WRITING IN A YOUNG FEMALE OF 22 YEARS OLD, DUE TO IDIOPATHIC} HYPEREOSINOPHILIC SYNDROME (HES)

A. Andrikopoulou, P. Agelidakis, K. Kioulahidis, D. Kentroti, N. Matikas

Evagelismos Hospital, Athens, Greece

Introduction: The Idiopathic Hypereosiniphilic Syndrome (IHES) is a rare group of myeloproliferative disorders of unknown etiology, marked by a sustained overproduction of eosinophils and an end organ damage, most commonly involving the heart, with related pathologies.

Case report: A young female of 22 years old, right handed, with a history of eosinophilia and valvular disease for 3 years under follow up, was admitted to our Department for an incident of dysarthria and difficulty in reading and writing that happened 4 days before. The neurological physical examination was normal. The perfomed cerebral MRI-MRA showed a pathological cortical area in the posterior border zone of the left parietal lobe -infarct in subacute phase. The performed paraclinical tests showed: leukocytosis (up to $23.94 \mathrm{~K} / \mathrm{ul}$ ) with eosinophilia (up to $76.6 \%$ ) and anemia (Hct $34.8 \%$ ), the transesophageal echocardiography showed rheumatic heart disease with incompetence of the aorta, the mitral and tricuspid valve, without endomyocardial fibrosis and the abdominal imaging with $\mathrm{u} / \mathrm{s}$ and CT showed hepatosplenomegaly. The remaining laboratory workup was without any pathological findings. The patient is treated with coumadin, corticosteroid and hydroxyurea.

Discussion: The HES is a rare entity of unknown etiology with sustained overproduction of eosinophils $>1500 / \mathrm{mm}^{3}$ in the peripheral blood for at least 6 month and definite end organ damage. Fifty four percent of the patients have neurological manifestations of the CNS, focal or difuse, as well as of the PNS. Embolic strokes, permanent or transient -as with our patient, are mainly from thrombi of endocardial origin and may relapse even under efficient anticoagulant therapy.

\section{Interesting cases}

\section{FIRST CASE OF AN INTERNAL CAROTID ARTERY (ICA) DISSECTION IN} AN ADULT AFTER AN ADENOTONSILLECTOMY

I. Bonnaud, S. Debiais, K. Mondon, D. Saudeau, B. De Toffol, A. Autret

Hopital Bretonneau, Tours, France

Background: Internal carotid artery (ICA) dissections account for $10 \%$ of strokes in young patients. It may be caused by sudden or prolonged movements of cervical rotation and hyperextension. We report the first case of ICA dissection in a female adult occurring a few hours after an adenotonsillectomy.

Case report: A 50 y.o. woman was admitted for a bilateral adenotonsillectomy following recurrent episodes of tonsillar abcesses. The surgical procedure was performed under general anesthesia, without complications. Twenty four hours after waking up, the patient presented with a global aphasia and a right hemiplegia. The NIH Stroke Scale was 25. At 72 hours, the cerebral angio MRI demonstrated a massive cerebral ischemia, a left ICA dissection, a mural hematoma within the subpetrous segment of the ICA, and an occlusion of the left middle cerebral artery. Search for ultrastructural abnormalities of connective tissue and underlying pathologies (Ehlers Danlos syndrome, fibromuscular dysplasia) was negative. Six months later, the NIHSS was 4, and the angio MRI showed a complete recanalization of all arteries

Discussion: A few cases of ICA dissection, attributed to ventilation procedures during anesthesia, were reported in the 3 to 24 hours after surgery (Gould et al, Stroke, 1994). There is only one case reported of ICA dissection after an adenotonsillectomy in a child (Wraige and al, Dev Med Child Neurol 2003). Presumed mechanisms are the fragilization of vessels by a chronic infectious process, prolonged opening of the mouth and stretching of cervical soft tissue during surgery and anesthesia. 
Conclusion: Diagnosis of ICA dissection should be evoked in case of stroke in hours following adenotonsillectomy, a procedure frequently performed in young adults.

\section{Interesting cases}

\section{REDUCTION OF SALIVATION AND PREVENTION OF ASPIRATION WITH} TRANSDERMAL SCOPOLAMINE

O.M. Rønning, M. Dahl

The Acute Stroke Unit Akershus University Hospital, Nordbyhagen, Norway

Background: Incidence of dysphagia is high in stroke. In the first days the proportion with swallowing difficulties is reported to be $50 \%$. One third of stroke patients with dysphagia develop pneumonia and mortality is high. Nasogastric feeding has limited effect on reducing aspiration pneumonia. Aspiration occurs due to difficulties in swallowing normally secreted amounts of saliva. Transdermal application of scopolamine is used to prevent nausea and vomiting in motion sickness but reduced salivation is a common side effect.

Methods: We report three patients with sever dysphagia that accumulated saliva and were successfully treated with transdermal scopolamine (TDS) in the form of a $1 \mathrm{mg} / 2.5 \mathrm{~cm}^{2}$ patch applied every third day.

Results: Patient 1 was a 43 year old male with dysphagia after a unilateral right-sided lateral medullary infarction. He accumulated saliva and needed tracheal suctioning to maintain normoxemia. After administration of TDS there was no need to continue suctioning. Patient 2 was a man 73 year old, with a unilateral left sided upper lateral medullary infarction. He was in need of endotracheal intubation, mechanical ventilation and frequent tracheal suctioning. He was extubated after treatment with TDS. Patient 3 was a 67 year old male with a large right-sided internal capsular stroke. He had previously suffered an internal capsular stroke on the left side. The patient had a severe pseudobulbar palsy with anarthri, dysphagia and bilateral facial palsy. He was not able to swallow saliva and he was treated with TDS from the first day. After 15 days he was discharged to a nursing home and scopolamine was discontinued. He was readmitted due to respiratory failure two days later.

Conclusion: The cases presented give support for the effect of TDS to reduce salivary secretion in patients accumulating saliva due to dysphagia after stroke. Benefits and risks of TDS in stroke should be evaluated in randomised controlled trials.

\section{Interesting cases}

AWARE OF AN ABSENT LIMB, UNAWARE OF A PLEGIC ONE. ANOSOGNOSIA AFTER STROKE IN AN AMPUTATED PATIENT

S. Dieguez, F. Staub, A. Carota, J. Bogousslavsky

CHUV Lausanne, Lausanne, Switzerland

Background: Anosognosia refers to the loss of insight into an existing deficit.

Methods: We investigated AD, a 66-year-old man who suffered a right hemispheric stroke with severe hemiplegia following a cardio-embolic occlusion of the right carotid artery. 4 years earlier, AD underwent an amputation of the left leg from the knee down, from which he kept a vivid and painful complete phantom.

Results: MRI showed a large perisylvian involvement, including underlying structures. While AD was aware of having suffered a stroke and never denied his hemiplegia, he underestimated the severity of his impairment and denied that his hemiplegia was a cause of disability. Importantly, he would also underestimate his past disabilities due to amputation. His painful phantom leg was still present after the stroke and he did not deny the amputation. His wife confirmed that his claims about his prestroke abilities were frankly exaggerated, e.g. he said that he could easily drive a car.

Discussion: This case confirms that anosognosia can be restricted to the functional consequences of an impairment, even as the latter is fully recognized. What is new is that this specific form of unawareness can extend retrospectively to a previous and unrelated impairment, adding weight to approaches of anosognosia in stroke as a domain-general higher-order deficit. Further, the subjective experience of a contralesional phantom limb can survive an extensive stroke leading to anosognosia.

\section{Interesting cases}

ACUTE WEIGHT LOSS FOLLOWING ACUTE STROKE

A.L. Dalabie, S. Jeangette, A. Dewindt, S. Dethy, S.A. Blecic Stroke Unit. CHU de Tivoli, La Louvière, Belgium

Background: Gustatory and olfactory disorders are common after stroke and could induce appetite disorders. They can be due to several different conditions. However acute drop in appetite for food has been rarely described.

Patients: We report here 5 consecutive patients (pts) who were admitted into our two stroke units. Within the month after first acute stroke all experienced a severe and rapid weight loss of an average of $10 \mathrm{~kg}$ [8-13].

All described a decrease in the hedonic character of olfactory and gustatory perception and an altered perception of taste with modified food preference. All had also a severe drop in appetite.

4 pts were found to have ischemic stroke involving the posterior right parietal cortical area and one had a right parietal hemorrhage. In this last pt 18FDG-PETscan disclosed a right parietal cortical hypo metabolism and also hypo metabolism in the right polar thalamic area.

During hospitalization, one pt had a status epilepticus, which was treated with a loading dose of valproate (VP). During the treatment, appetite dramatically improved without a dramatic change in taste disorders. Because of a diagnosis of focal seizure, VP was then changed to carbamazepine. A recurrence in appetite disturbance was immediately observed. VP was the re-introduced with at a dramatic appetite improvement. At the light of this single-case observation VP was then tried in the two last consecutive pts with drop in appetite. A complete recovery was observed in one and a partial recovery of appetite was observed in the second Conclusions: Despite gustatory and olfactory disorders are commonly described after stroke and could theoretically induce food intake modification, we found in 5 consecutive patients that appetite drop is probably the mechanism inducing more frequently severe weight loss. The mechanism of appetite involvement could be due to thalamic or hypothalamic de-afferentation observed after parietal stroke. In these pts following a single case positive observation VP could be useful to restore appetite but has little effect on gustatory and olfactory disorders.

\section{Cerebral haemorrhage and SAH}

\section{Cerebral haemorrhage and SAH}

POLYMORPHISMS IN THE COAGULATION FACTOR VII GENE AND RISK OF INTRACRANIAL HEMORRHAGE

S. Greisenegger, M. Funk, G. Endler, W. Lang, C. Mannhalter, W. Lalouschek Medical University Vienna, Vienna, Austria

Little is known about genetic risk factors influencing the occurrence intracranial hemorrhage. The impact of polymorphisms of the factor VII gene on development of ischemic or hemorrhagic stroke is still a matter of discussion. One recent study found a significantly higher prevalence of the FVII (-402)G $>$ A mutation among ischemic stroke patients than in controls. The same study showed that the $(-323)$ insertion/deletion, the $353 \mathrm{R}>\mathrm{Q}$ and the $(-401) \mathrm{G}>\mathrm{T}$ polymorphism of the factor VII gene were in strong linkage disequilibrium. Previously, it has been indicated that carriers of the -323 Ins allele of factor VII carry a higher risk for intracranial hemorrhage compared to matched control subjects. We analyzed therefore wether the $(-401) \mathrm{G}>\mathrm{T}$ and the $(-402) \mathrm{G}>\mathrm{A}$ polymorphisms of the factor VII gene are associated with an increased risk for intracranial hemorrhage.

We performed a case control study in 85 with intracranial hemorrhage and in 85 healthy control subjects individually matched for age, gender and hypertension to each patient. Patients with hemorrhagic transformation of a previous infarct, arteriovenous malformations (AVM) or a traumatic cause of hemorrhage were excluded. Furthermore, we excluded patients on previous oral anticoagulant therapy and patients with subarachnoid hemorrhage. We did not detect significant differences in allele frequency between control subjects and patients neither for the A Allele of the FVII (-402) $\mathrm{G}>\mathrm{A}$ polymorphism ( $42 \%$ vs. $46 \%$; $\mathrm{p}=0.760$, ns.) nor for the $\mathrm{T}$ Allele of the FVII(-401)G $>\mathrm{T}$ polymorphism ( $18 \%$ vs. $21 \%$; $\mathrm{p}=0.678$, ns.). A further analysis of haplotype distributions revealed no signifinant differences.

Our results do not support the hypothesis that polymorphisms of the FVII gene are associated with the risk for intracranial hemorrhage.

\section{Cerebral haemorrhage and SAH}

\section{VASCULAR ANOMALIES AND MALFORMATIONS ASSOCIATED WITH INTRACRANIAL ANEURYSMS}

A. Borratynska, A. Slowik, A. Klimkowicz-Mrowiec, D. Partyka, A. Szczudlik Medical College, Jagiellonian University, Poland

Patients harbouring congenital anomalies and malformations of cerebral arteries are considered to be predisposed to intracerebral aneurysms. The purpose of the present study was to determine the frequency of vascular anomalies and malformations coexisting with intracranial aneurysms in a Polish population. 
A total of 241 patients (139 women and 102 men, mean age 50.4 \pm 12.4 ) with aneurysmal subarachnoid hemorrhage were prospectively assessed for the location, volume, and number of aneurysms and coexisting vascular variations and malformations.

Three hundred and thirteen aneurysms were identified. Fifty six (23.2\%) patients had multiple aneurysms. The most frequent locations of the aneurysms were middle cerebral artery $(112-35.8 \%)$ and anterior communicating artery $(86-27.5 \%)$. Fifty one $(21.1 \%)$ patients had vascular anomalies or malformations coexisting with aneurysms. Hypoplasia or aplasia of A1 segment of the anterior cerebral artery (ACA) was found in 22 cases, persistent fetal origin of the posterior cerebral artery (PCA) in 17, coexistence of hypoplasia of A1 segment of ACA and persistent fetal origin of PCA in 7, bilateral aplasia of internal carotid artery (ICA) in 1, hypoplasia of vertebral artery (VA) in 3, and arteriovenous malformation in 1 . There was no statistical difference neither in demographics and clinical presentation nor in the number, volume and location of aneurysms between patients with and without vascular anomalies. There was also no difference in the frequency of vascular anomalies and malformations compared to that reported from normal populations. The coexistence of intracerebral aneurysms and vascular malformations may result from impaired hemodynamics and increased shear stress or may be the manifestation of "congenital vascular malformation syndrome". However, the results of the present study indicate that additional vascular anomalies and malformations do not influence the natural history of intracranial aneurysms.

\section{Cerebral haemorrhage and $\mathrm{SAH}$}

THE SOCIETAL COSTS OF INTRACEREBRAL HAEMORRHAGE IN SPAIN

P. Navarrete, M.C. Christensen, W.M. Hart, J. Lopez, F.J. Carod-Artal

Hospital Virgen de las Nieves, Bagsvaerd, Denmark

Background: Stroke imposes a substantial economic burden on individuals and society. This study estimates the direct and indirect lifetime costs associated with intracerebral haemorrhage $(\mathrm{ICH})$ in Spain

Method: We developed a model of the lifetime costs of incident ICH in Spain. An epidemiological model of ICH incidence, survival and morbidity was developed using retrospective data from hospital records in three regions (Andalusia, Madrid, and Canary Islands), patient interviews and a review of the literature. Data on health outcomes and hospital care were obtained from 33 regional hospitals and one hospital in Madrid, whereas cost of outpatient care, cost of informal care and lost productivity associated with premature morbidity and mortality were obtained from patient interviews.

Results: The lifetime cost of a first ICH in Spain is estimated at $€ 49,199$ per patient. Direct medical costs accounted for $29.4 \%$ of total costs, while $70.6 \%$ were related to indirect costs. Indirect costs were dominated by costs of informal care $(58.5 \%)$, followed by cost related to lost productivity $(12.1 \%)$. Close to half $(42.5 \%)$ of direct medical costs over the first year were attributable to follow-up care, including rehabilitation. Furthermore, more than half $(63.1 \%)$ of the direct medical costs were incurred after the first 3 years following a first ICH. In total, two-thirds $(65 \%)$ of total lifetime costs occurred after the first 3 years of ICH onset. The aggregated lifetime cost for the estimated 12,534 Spanish patients with a first-ever ICH in 2004 was $€ 616.7$ million.

Conclusion: ICH entails substantial ongoing costs beyond the first 3 years after onset. The need for effective intervention is critical to reduce disability and thereby the long-term economic burden of ICH.

\section{Cerebral haemorrhage and $\mathrm{SAH}$}

\section{VARIANTS OF THE BASAL VEIN OF ROSENTHAL AND PERIMESENCEPHALIC NONANEURYSMAL HAEMORRHAGE \\ T. Daenekindt, G. Wilms, V. Thijs, F. Van Calenbergh \\ University Hospital Leuven, Leuven, Belgium}

Background and Purpose: The cause of a perimesencephalic nonaneurysmal subarachnoid hemorrhage (PMH) is not known. Earlier studies reported a possible contribution of a primitive variant of the basal vein of Rosenthal (BVR) in the pathogenesis of PMH. We compared the variants of BVR between patients with PMH and aneurysmal subarachnoid hemorrhage (SAH) by studying the venous phase of the digital substraction angiography (DSA).

Methods: Two observers reviewed the DSAs of 59 patients with PMH and 59 patients with SAH. The variants of BVR were classified into: (1) normal continuous: BVR is continuous with the deep middle cerebral vein and drains mainly to the vein of Galen (VG); (2) normal discontinuous: drainage anterior to uncal veins and posterior to VG; (3) primitive variant: drainage to other veins than VG.

Results: 118 patients were enrolled with a mean age of $49 \pm 12$ years. There were 31 males and 28 females in both groups. Patients with PMH were older than patients with SAH ( 52 versus $46, \mathrm{p}=0.01$ ). Primitive variants were found in $21 \%$ on the left side, and $29 \%$ on the right side $(\mathrm{p}=0.27)$. There was no association between PMH and the presence of a primitive variant on the left $(25 \%$ in PMH versus $19 \%$ in SAH, $\mathrm{p}=0.65$ ) or on the right side ( $31 \%$ in $\mathrm{PMH}$ versus $30 \%$ in $\mathrm{SAH}, \mathrm{p}=0.92)$ in univariate analysis. After correction for age and sex, variants on neither side were associated with PMH (OR 1.4, $\mathrm{p}=0.53$ for left variants, OR 1.2, $\mathrm{p}=0.67$ for right variants). Conclusions: In this large controlled study we were unable to confirm a contribution of a primitive variant of the basal vein of Rosenthal in the pathogenesis of PMH.

\section{Cerebral haemorrhage and SAH}

\section{HEMATOMA, EDEMA VOLUME AND HEMODYNAMIC CHANGES IN THE} ACUTE AND SUBACUTE PHASE OF SPONTANEOUS INTRACRANIAL

\section{HEMORRHAGE}

L. Csiba, B. Fulesdi, J. Settakis, I. Fekete, D. Bereczki

Debrecen University, Debrecen, Hungary

ICH (intracranial hemorrhage) is a common disorder, with an estimated frequency of 37 000-52 000 each year in the USA. The two most important new concepts are that haemorrhages continue to grow and expand over several hours after onset of symptoms-a process known as early haematoma growth-and that most of the brain injury and swelling that happens in the days after ICH is the result of inflammation caused by thrombin and other coagulation end-products. Ca. 38\% of patients had an increase in haematoma volume within $3 \mathrm{~h}$ of onset. The authors review the mechanisms that lead to early haematoma growth during the acute stage of ICH and summarize their observation on ICH pts.

Methods: 20 patients with supratentorial ICH were examined. Brain, hematoma and edema volumes were calculated from CT scans performed at admission and two weeks later and were compared with the values obtained from bilateral TCD recordings of the middle cerebral arteries.

Results: TCD blood flow velocities (FV) did not change, cerebral perfusion pressure $(\mathrm{CPP})$ and resistance area product (RAP) decreased $(\mathrm{P}=0.007$ and $\mathrm{P}=0.003$ respectively) while cerebral blood flow index (CBFI) remained constant $(\mathrm{P}=0.91)$ in the affected side, indicating preserved autoregulation. Hemorrhage volume did not correlate with RAP in the acute phase $(\mathrm{r}=0.07 \mathrm{P}=0,76)$, but in the subacute phase a significant positive correlation could be detected $(r=0.44, \mathrm{P}=0.04)$.

Conclusions: The calculation of CPP, RAP and CBFI contributed more to the understanding of the hemodynamic changes developing after a spontaneous ICH than the absolute TCD velocity values. The sudden appearance of a new mass temporarily impairs cerebral circulation but cerebral autoregulation remains intact in small to middle size brain hemorrhages

\section{Cerebral haemorrhage and SAH}

POSTERO-INFERIOR CEREBELLAR ARTERY (PICA) DISSECTION WITH SUB-ARACHNOID HAEMORRHAGE: CLINICAL CHARACTERISTICS AND LONG-TERM FOLLOW-UP IN 4 CASES

J. Sedat, Y. Chau, A. Dunac, M.H. Mahagne, M. Chatel

Hopital St Roch, St Roch, France

Background: Most of the arterial dissections which provide subarachnoid haemorrhage are located on the vertebral artery; spontaneous isolated PICA dissection is rare. We report here 4 cases treated endovascularly.

Methods: from 1999 to 2003, four patients were hospitalized for sub-arachnoid haemorrhage due to PICA dissection. Apart from one patient who presented symptoms after cervical manipulation, no predisposing factor or traumatic cause were found. 2 patients were classified grade 2 in the Hunt and Hess classification, 1 grade 3 , and 1 grade 4 . On the CT scanner, 3 patients were grade 4 in the Fisher classification, and one was grade 2 . The diagnosis of dissection was carried out on the cerebral angiography in every patient. 3 patients presented acute hydrocephalus and were treated with surgical derivation before endovascular therapy. All the patients were treated endovascularly by occlusion of the PICA. No occlusion testing was carried out before sacrifice. The clinical follow-up was up to 2 years. We assessed long term results with the Glasgow Outcome Score, and the Karnovsky classification.

Results: Angiography before treatment showed focal stenosis with aneurysmal dilatation on the PICA. Occlusion of the PICA was performed because of evidence of good haemodynamic balance with the others cerebellar arteries, and because of the high risk of rebleeding. No ischemic complication was observed after sacrifice of the PICA. No re-bleeding occurred after treatment. After 2 years, a good recovery was achieved for 3 patients (GOS 1 and Karnovsky 100); however, the patient Hunt and Hess grade 4 on admission was GOS 3 and Karnovsky 50, three years later. Conclusion: Isolated PICA dissecting aneurysm is much less frequent than vertebral pseudoaneurysm. Endovascular occlusion may be performed safely after prethera- 
peutic anatomic analysis. Long term clinical results depend mainly on the clinical status on admission.

\section{Cerebral haemorrhage and $\mathrm{SAH}$}

\section{NOT ONLY HAEMATOMA GROWTH BUT ALSO HAEMATOCEPHALUS,} HYDROCEPHALUS AND MIDLINE SHIFT PLAY A ROLE FOR ICH OUTCOME

R. Eckhardt, M. Novotny, P. Bosak, C. Tatschl, K. Matz, G. Funk, M. Brainin Landesklinikum Donauregion Gugging, Maria Gugging, Austria

Background: Several characteristics of intracerebral haematomas (ICH) - large volume, haematoma expansion, internal haematocephalus and hydrocephalus - have been reported to be related to outcome of ICH. The role of each of these factors was studied.

Methods: Prospective study of 79 consecutive patients admitted to our stroke unit within 24 hours, the mean admission time was $5.4 \mathrm{hrs}$

Results: The mean volume on admission was $31.5(1-182) \mathrm{ml} .45 \mathrm{pts}$ had a second CT scan of which 13 pts (29\%) showed a haematoma growth. 34 patients had no follow-up CT scan due to early death $(\mathrm{n}=17 ; 21.5 \%)$, transfer to neurosurgery ( $=2 ; 2.5 \%)$, ICUs $(n=3 ; 3.8 \%)$ ) or to other medical wards $(n=3 ; 3.8 \%)$ or had MR-imaging for follow up and were therefore not included. The mean time between first and second CT-scan was 5.7 (1-22) days

We found no significant correlation between volume increase within the first days and age, gender, history of diabetes or hypertension and depression of consciousness on admission. Regarding the site of haematoma and volume increase there was a significant correlation only for thalamic location $(\mathrm{p}=0.042)$, but not for lobar, putaminal, cerebellar, intraventricular or brain stem location.

Regarding the CT characteristics of ICH we found a significant association for a haematoma volume larger than $15 \mathrm{ml}$ on the first CT-scan $(\mathrm{p}=0.003)$, intraventricular haematoma extension $(\mathrm{p}=0.000)$, midline shift $(\mathrm{p}=0.000)$ and hydrocephalus $(\mathrm{p}=0.006)$ and bad outcome, defined as NIHSS $>14$ or death on discharge from the stroke unit.

Conclusion: Not only haematoma growth but also rupture into ventricles, hydrocephalus and visible midline shift play a role for ICH outcome. These factors are interrelated and should be considered in addition to the parenchymal expansion of the haematoma.

\section{Cerebral haemorrhage and SAH}

\section{EFFECT OF HYPOTHERMIA ON INFLAMMATORY RESPONSE AFTER POOR GRADE ANEURYSMAL SUBARACHNOID HEMORRHAGE: PRELIMINARY RESULTS \\ C. Muroi, K. Frei, Y. Yonekawa, E. Keller \\ University Hospital of Zurich, Zurich, Switzerland}

Background: Interleukin-6 (IL-6) and tumor necrosis factor-alpha (TNF-alpha) are reported to reflect the inflammatory response. Cytokine production in the central nervous system (CNS) following aneurysmal subarachnoid hemorrhage (SAH) and its relation to clinical outcome and occurrence of vasospasm has been reported. Therapeutic hypothermia may attenuate inflammatory response but carries the risk of severe side effects. The aim was to assess the influence of hypothermia on IL-6 and TNF-alpha-levels systemically and in the cerebrospinal fluid (CSF) in patients with SAH.

Methods: Daily CSF and plasma samples were collected. IL-6 and TNF-alpha was measured by enzyme-linked immunoabsorbent assay. In case of intractable intracranial hypertension and/or refractive vasospasm, hypothermia $\left(33-34^{\circ} \mathrm{C}\right)$ was induced by systemic endovascular cooling.

Results: In 4 of 9 patients, hypothermia was applied. IL-6 levels in the CSF were lower in the hypothermia group: $5.56( \pm 0.84) \mathrm{ng} / \mathrm{ml}$ vs. $28.24( \pm 0.49) \mathrm{ng} / \mathrm{ml}$, $\mathrm{p}=0.007$ (Mann-Whitney Test). TNF-alpha levels in CSF were higher in the hypothermia group: $2.85( \pm 0.48)$ vs. $1.46( \pm 0.27) \mathrm{pg} / \mathrm{ml}, \mathrm{p}<0.001$. Systemic levels of IL-6 and TNF-alpha were lower in the patients with normothermia compared to hypothermia: $7.92( \pm 2.64) \mathrm{pg} / \mathrm{ml}$ vs. $38.45( \pm 7.30) \mathrm{pg} / \mathrm{ml}$, and $1.08( \pm 0.12)$ vs. $2.36( \pm 0.45) \mathrm{pg} / \mathrm{ml}$, both $\mathrm{p}<0.001$. C-reactive protein-levels tended to be lower in patients with normothermia while leukocyte-counts were significantly higher. Time and course of systemic IL-6 correlated with CRP-level, while IL-6 in the CSF correlated more with the occurrence of vasospam.

Discussion: The results support the beneficial effects of hypothermia regarding suppression of IL-6 in the CNS. High systemic levels of IL-6 in patients with hypothermia reflects its side effect. The impact on TNF-alpha remains unclear.

\section{Venous diseases/Heart \& brain}

\section{Venous diseases/Heart \& brain}

CEREBRAL VENOUS THROMBOSIS AND SEVERE IRON DEFICIENCY

\section{ANEMIA}

I. Crassard, A. Ameri, V. Biousse, V. Wolff, M.G. Bousser

Lariboisiere Hospital, Paris, France

Background: Though considered as a hypercoagulable state, iron deficiency anemia has rarely been reported in association with CVT.

Material and Methods: Database of 320 patients with CVT (1975-2005).Diagnosis of CVT based on MRI+MRA and/or CT venography and/or intra-arterial angiography.

Results: 13 patients (all women) with iron deficiency anemia were identified Six had isolated hypertension, 6 focal deficits and 1 focal seizures. Parenchymal abnormalities on neuroimaging were found in 6 patients. The mean level of $\mathrm{Hb}$ was $7.5 \mathrm{~g} / \mathrm{dl}$ (range 5.6-9). Four patients had transient thrombocytosis. Anemia was secondary to gynecological bleeding in 8 and to gastrointestinal bleedings in 2 patients. No source of bleeding was found in 3 patients. Eight patients had other risk factors for thrombosis: OC (7), Crohn's disease (1) and tranexamic acid intaking(1). Despite the potential risk of further bleeding, CVT was treated with heparin in all patients. They received iron therapy and were treated for a local cause of bleeding if present (at the acute phase of CVT for 2 patients). Blood transfusions were required in 3 patients. Eleven patients recovered completely, 2 had mild sequelae.

Discussion: Iron deficiency anemia was found in $6 \%$ of women with CVT. Their clinical and neuroimaging presentation was not different from that of other etiologic varieties. All patients were treated with heparin and had a good prognosis. Blood transfusion and/or local hemostatic treatment at acute phase of CVT were required in $30 \%$ of patients. The mechanism of hypercoagulation is suggested to be secondary to thrombocytosis often developing in case of iron deficiency. The absence of thrombocytosis in the majority of our patients suggests that other factors are involved in the increased risk of CVT.

\section{Venous diseases/Heart \& brain}

\section{INTERLEUKIN 6 G-174C GENE POLYMORPHISM AND RISK OF} CEREBRAL VENOUS THROMBOSIS

C. Lichy, J. Genius, M. Kloss, K. Reuner

University of Heidelberg, Heidelberg, Germany

Background: Proinflammatory mediators as interleukin 6 (IL-6) have been shown to be risk indicators for peripheral venous thrombotic disease. The CC genotype of the common G-174C polymorphism of the IL-6 gene is strongly associated with increased plasma levels of IL-6.

Methods: We performed a case-control-study including 74 consecutive patients with non-septic cerebral venous thrombosis and 203 population-based controls free from thrombotic disease. In all subjects, the IL-6 G-174C genotype was determined using PCR technique.

Results: A history of recent non-septic systemic infection was more common in patients (OR 2.94, 95\%CI 1.0-8.69). In patients, the IL-6 CC genotype was not more prevalent $(14 / 74)$ as compared to controls $(28 / 203$; OR 1.46 ; $95 \%$ CI 0.72 2.95). By contrast, the prothrombotic FVLeiden and the G20210A prothrombin polymorphisms both were more prevalent in patients.

Discussion: Our study could not confirm the hypothesis of a genetic proinflammatory diathesis in patients with non-septic cerebral venous thrombosis. As compared to established prothrombotic genetic variants, the role of the IL-6 G-174C polymorphism seems to be of minor importance in cerebral venous disease.

\section{Venous diseases/Heart \& brain}

PREDICTORS OF VISUAL LOSS IN CEREBRAL VENOUS THROMBOSIS G.S. Silva, D.L. Gomes, A. Pieri, J. Fiorot, M.M. Alves, M. Gadelha, A.R. Massaro

Federal University of São Paulo - UNIFESP, São Paulo, Brazil

Background: CVT has increasingly been recognized as a cause of cerebrovascular disease. Visual loss is one of the most threatening complications and may lead to permanent disability in patients with CVT. The purpose of this study was to investigate predictors of visual loss in patients with CVT.

Methods: Records of all patients with the diagnosis of CVT followed in the Stroke Service of our University Hospital were reviewed.

Demographic characteristics, predisposing factors for CVT and baseline clinical 
features including visual impairment were recorded. Neuroimaging and CSF exams were reviewed. Treatment received, visual status and outcome (MRS) during the follow-up were recorded at 6 months.

Results: Forty-two patients (26 women) with a mean age (SD) of 35.5 (13.6) years were identified from 1998 through 2005. Headache was the most common symptom (93\%). Visual impairment was present in $22 \%$ of the patients at the day of the diagnosis. At a 6-month follow-up, $33.5 \%$ of the patients had visual impairment and $10 \%$ of the patients were blind. Visual impairment was associated with the presence of visual symptoms at diagnosis $(\mathrm{p}=0.01)$, higher values of cerebral spinal fluid pressure $(\mathrm{p}=0.06)$ and altered fundoscopic examination $(\mathrm{p}=0.08)$ at onset. The history of hypertension and diabetes $(\mathrm{p}=0.03$ and $\mathrm{p}=0.02$, respectively) and lack of heparin use in the acute phase $(\mathrm{p}=0.02)$ were also related with visual impairment. Older patients and those with a chronic presentation were at increased risk for blindness ( $\mathrm{p}=0.04$ and 0.008 respectively).

Discussion: Visual loss is a common complication of CVT. Older, hypertensive and diabetic patients, with visual impairment at diagnosis, altered fundoscopic examination, increased CSF pressure or chronic presentation seem to be associated with increased risk for visual impairment after six months. Early identification of such patients and acute treatment with anticoagulation may prevent this severe and permanent complication.

\section{Venous diseases/Heart \& brain}

CEREBRAL VENOUS THROMBOSIS IN ROMANIA - REVIEW OF 39 CASES I. Geana, C. Popa, A. Stuparu, A. Popescu, Al. Sasaran

"Prof. Dr. V. Voiculescu" Institute of Cerebrovascular Diseases, Bucharest, Romania

Background: Cerebral venous thrombosis (CVT) is a rare and underdiagnosed cause of stroke. The clinical spectrum of the disease is wide, and treatment is a challenge for the medical team. Early diagnose is important because of its unpredictable evolution.

Objectives: To collect clinical data, identify causes and risk factors for cerebral venous thrombosis and to highlight the characteristics in Romania; to evaluate the applied treatment, the complications and evolution (prospective study).

Material and Method: 39 patients included, admitted between 01.01.1998 31.12.2005, diagnosed by MRI, CT + angiography or by necropsy.

Results: Sex ratio women/males: 1.3/1; average age 37.9 years, median age 37 years; $68 \%$ of women were fertile. Average hospitalization time 21 days, average time from debut to admission 10 days, average time from admission to diagnosis 3 days. Risk factors: trombophillic states (26\%), postpartum18\%, infections $15 \%$, lumbar punction $8 \%$, pregnancy $5 \%$, oral contraceptives $5 \%$, other causes $10 \%$; no risk factors identified $31 \% .54 \%$ debuted as an acute illness, while in $41 \%$ cases the debut was subacute; $5 \%$ had cronic onset. Localization: superior sagital sinus $51 \%$; multiple sinuses $46 \%$, cortical veins $21 \%$. Heparin was administrated in all but 3 cases ( 2 died before diagnose, 1 received ASA). $15 \%$ of the patients died, $85 \%$ were discharged showing mild or no disability.

Conclusions: The percentage of women is lower than recently reported for other sites. Oral contraceptives are not an important risk factor in Romania (socioeconomical motivation). More than $60 \%$ of women were of fertile age. The CVT is probably underdiagnosed in Romania (low percentage of admissions for benign intracranial hypertension). There is a local consensus concerning the use of heparin.

\section{Venous diseases/Heart \& brain}

\section{THE PREVALENCE OF CORONARY ATHEROSCLEROSIS IN PATIENTS WITH STROKE}

P. Amarenco, F. Gongora-Rivera, J. Labreuche, A. Jaramillo, P.G. Steg, J.J. Hauw

Bichat Stroke Centre, Paris, France

Background: Cardiac death is the leading cause of long-term mortality in stroke patients, but the prevalence of coronary atherosclerosis in these patients is unknown. Methods: Using an autopsy data bank, we studied the prevalence of coronary plaques and coronary stenoses greater than 50 percent in 811 consecutive autopsies of neurological patients.

Results: Coronary plaques and stenoses were present in 72 and 37 percent of the 347 patients with cerebrovascular disease and 27 and 10 percent of the 464 patients with other neurological diseases $(\mathrm{P}<0.001)$. Two thirds of myocardial infarction were unexpected autopsy findings. After adjusting for age, gender and heart weight, the odds ratio (95 percent confidence interval) of presence of coronary plaques, coronary stenosis and myocardial infarction were 3.65 (2.54 to 5.24 ), 2.60 (1.71 to 3.94 ) and 2.30 (1.55 to 3.42 ) respectively. Frequency of coronary atherosclerosis and myocardial infarction was similar between stroke subtypes. The prevalence of coronary plaques, coronary stenosis and myocardial infarction was 79, 46 and 46 percent in the presence of plaques in any segment of extra- and intracranial brain arteries, and 51, 17 and 23 percent in the absence of plaques, respectively (adjusted p-values $<0.01$ ). Coronary atherosclerosis was also related to the severity of atherosclerosis in any segment of cerebral arteries (adjusted p-values for linear trend $<0.003)$. Similar results were observed in relation to internal-carotid-artery atherosclerosis.

Conclusions: Coronary atherosclerosis is highly prevalent in stroke patients regardless of the etiology, and more prevalent when atherosclerosis is present in carotid and cerebral arteries.

\section{Venous diseases/Heart \& brain}

PREVALENCE OF PATENT FORAMEN OVALE AND ATRIAL SEPTAL ANEYRYSM IN YOUNG ADULTS WITH CRYPTOGENIC STROKE -STUDY IN 129 PATIENTS IN GREECE

E. Karavasili, A. Tavernarakis, V. Antrian, I. Xydakis, A. Andrikopoulou, N. Matikas

Evagelismos Hospital, Athens, Greece

Background: Prevalence of crypyogenic stroke (CS) in young adults is high and in consecutive series of patients is approximately $40 \%$. A significant proportion of CS is of cardiac aetiology. The aim of this study was to evaluate the frequency of patent foramen ovale (PFO) and atrial septal aneurysm (ASA) in this subgroup.

Methods: 129 patients with ischemic stroke (age less than 45 years), admitted to our Neurology Department between 2001 and 2005, were evaluated with a complete aetiological workup. A thorough risk profile was obtained. The type of stroke (permanent or transient) and infarct location was determined.

Results: In129 patients (pts), based on TOAST subtyping, 36 pts (27.9\%) were found to have a CS, 26 pts (20.2\%) dissections and 19 pts (14.7\%) cardio embolic stroke.Evidently CS was the most frequent cause in this subgroup.All the pts with CS underwent trans- oesophageal

echography (TEE) and 7 pts were found to have PFO, 2 pts PFO and ASA and 3 pts ASA (25\% PFO and 13,9\%ASA).These 12 pts fulfil the criteria to have suffered a cardio embolic stroke.Three of these pts underwent successfully endovascular closure and the other 9 pts were treated with antiplatelet medecation (aspirin or warfarin). During the follow-up period (3-48 months) no one relapsed.

Conclusion: The results of this study are consistent with the current literature. The prevalence of CS in young pts is increased and a significant proportion of them are due to cardio embolism,especially in pts with PFO and ASA. A systemic investigation of this pathology of TEE in young pts with CS is necessary. The absence of relapse coincides with other studies results that the former population is at a particular low risk to have any vascular recurrence, independently of the treatment.

\section{Venous diseases/Heart \& brain}

\section{INAPPROPRIATE CLOSURE OF PFO}

P. Michel, E. Eeckhout, P. Ruchat, J. Bogousslavsky

Centre Hospitalier Universitaire Vaudois, Lausanne, Switzerland

Background: Neurological symptoms in young patients are often attributed to a patent foramen ovale (PFO) found during work-up. Effective prevention of PFO-related strokes depends on the correct initial diagnosis.

Methods: Medical records and follow-up data were reviewed of all patients who underwent PFO-closure at a single institution. Only patients with presumed cerebrovascular symptoms and a presumed high risk of recurrence (associated atrial septum aneurysm, large shunt, recurrent events before closure) were eligible for closure. If neurological symptoms reoccurred after closure, a new thorough work-up was performed.

Results: From 228 patients with surgical $(n=107)$ or percutaneous $(n=121)$ PFO-closure, follow-up data were available for 190 for a mean of 6.3 years. During follow-up, 4 patients $(2.1 \%)$ with inappropriate PFO closure were identified: a 35 year old man with a mild hemiparesis was found to have multiple sclerosis; a 43 year old woman with vertigo and gait ataxia was diagnosed with right peripheral vestibulopathy; a 41 year old man with lacunar strokes was found to have CADASIL; and a 30 year old man with a carotid territory stroke had severe genetical hyperhomocysteinemia. Retrospectively, three of the four patients had neuroimaging findings before closure that were not compatible with embolic stroke.

Conclusions: We identified inappropriate closure of PFO in $2.1 \%$ of patients. A thorough initial work-up may avoid unnecessary interventions. Symptom recurrences after closure should prompt a search of rare causes of stroke and of stroke mimics. 


\section{Venous diseases/Heart \& brain}

ARE SEGMENTAL LEFT VENTRICULAR WALL MOTION ABNORMALITIES A POTENTIAL CAUSE FOR ISCHEMIC STROKE

D. Deleu, S. Kamran, A.A. Hamad

Hamad Medical Corporation, Doha, Qatar

Objective: The aim of this study is to determine whether segmental left ventricular wall motion abnormalities (SLVWMA) are a potential cause for ischemic stroke (IS).

Methods: Population. Data from three groups of patients were reviewed over a 6 month period: patients with IS with and without SLVWMA, and patients with SLVWMA but without IS or TIA (cardiac patients). Demographics, cardiovascula risk factors and echocardiographic parameters of patients with IS (with and without SLVWMA) were collected and compared with those of patients who had SLVWMA but without history of IS. Primary outcome measures were cardiovascular risk factors, echocardiographic parameters and subtype of stroke.

The Student s t-test or non-parametric testing $(\mathrm{p}<0.05)$ was used where appropriate Results: 298 patients with IS were identified: 187 patients without and 72 with SLVWMA, and 79 cardiac patients. The number of risk factors in IS patients without SLVWMA was significantly lower compared with IS patients with SLVWMA (2.7 vs. 3.7). There was no difference in age or gender between stroke patients with SLVWMA and the cardiac patients. However, the number of risk factors was significantly higher in the cardiac patients compared with stroke patients with SLVWMA (4.4 vs. 3.7). The ejection fraction was normal in both groups of stroke patients but significantly lower in the cardiac patients (37\%). Significantly more lacunar IS were observed in IS patients with SLVWMA than in those without SLVWMA (76\% vs. $68 \%$ ).

Conclusion: Our data indicate that in IS patients with multiple cardiovascular risk factors and SLVWMA a higher frequency of lacunar strokes can be found. The latter could be a marker of small-vessel disease and/or be a potential contributing factor, perhaps through a mechanism of cardiac microembolism, in the development of lacunar IS. The mechanisms of the association between SLVWMA and lacunar IS remain however unclear.

\section{Venous diseases/Heart \& brain}

ISCHEMIC STROKE AND TIA IN ATRIAL FIBRILLATION PATIENTS TAKING ORAL ANTICOAGULANT THERAPY: A CASE-CONTROL STUDY A.M. Basile, S. Tonello, A. Corfini, L. Costa, L. Busson, V. Argentiero, M. Armani, V. Pengo, B. Tavolato

University of Padua, Padova, Italy

Background: Ischemic stroke in atrial fibrillation (AF) is not fully suppressed by oral anticoagulant therapy (OAT) with target international normalized ratio (INR) of 2-3. Aims were to verify if TIA/ischemic stroke onset in AF patients on OAT is correlated with INR and to identify any associated risk factors and coagulation disorders

Methods: Thirty-one AF cases (mean age:78 $\pm 7.3 \mathrm{y} ; \mathrm{F} / \mathrm{M}: 15 / 16$ ) with acute TIA/ischemic stroke during OAT, were assessed wholly, including coagulation tests and transesophageal echocardiogram. Cases were divided into 2 groups on entry INR: INR $>2$ and INR $<2$. Previous 3 months' INR values were collected. Cases were compared with a control group of AF patients on OAT without ischemic events: for each case, 3 age- and sex-matched controls were randomly taken from the Padova Thrombosis Centre database.

Results: Of the 31 cases, 15 had entry INR $>2$ and 16 INR $<2$. Risk factors and comorbidities did not differ significantly between the 2 groups. Compared with controls, cases presented significantly more often with hypertension $(87 \%$ vs $67 \%$; $\mathrm{P}=0.030$ ), diabetes ( $45 \%$ vs $16 \% ; \mathrm{P}=0.009)$, previous stroke ( $58 \%$ vs $8 \% ; \mathrm{P}<0.001)$, and $\mathrm{G} 20210 \mathrm{~A}$ factor II mutation $(16.0 \%$ vs $3.4 \%$; $\mathrm{P}=0.022)$. On logistic regression analysis, diabetes (OR: 4.6; 95\%CI: 1.1-18.8), previous stroke (OR: 25.9; 95\%CI: 6.1-109.8), and G20210A mutation (OR: 11.5; 95\%CI: 1.4-90.3) predicted cerebral ischemic events in AF during OAT, independently of all other factors. Cases had decreasing INR values with wide fluctuations in all measurements preceding stroke, while controls showed more stable anticoagulation intensity.

Discussion: risk factors, comorbidities, and coagulation disorders contribute to ischemic stroke/TIA in AF during OAT. Anticoagulation intensity variability may affect the efficacy of such therapy.

\section{Cerebrovascular autoregulation}

\section{Cerebrovascular autoregulation}

SHORT-TERM HIGH-DOSE ATORVASTATIN IMPROVES CEREBRAL VASOREACTIVITY IN PATIENTS WITH HIGH-GRADE CAROTID STENOSIS T. Karapanayiotides, I. Zaloni, G. Aposporos, A. Margherita, K. Lianos, V. Kotsi, A. Mamantas

"Pammakaristos" Hospital, Athens, Greece

Objectives: Data suggest that statins improve cerebral vasoreactivity (CVR) in patients with small vessel disease by propagating NO-mediated vasodilation. Hitherto, it is unknown whether this benefit occurs: a) independently from the lipid lowering properties of statins and $b$ ) in patients with high-grade carotid stenosis. We investigated the influence of short-term high-dose atorvastatin treatment on CVR in patients with high-grade carotid stenosis.

Methods: We evaluated CVR in 21 patients with $>70 \%$ carotid stenosis (S) (women $=12$, mean age $=61 \pm 8.2$ ) by recording with trancranial Doppler the MCA ipsilateral to the stenosis before and after the iv. administration of $1 \mathrm{~g}$ acetazolamide and by calculating the relative increase (RI\%) of the MCA mean velocity. The test was performed at baseline and after 3 days of administration of $80 \mathrm{mg}$ atorvastatin/day. Results were compared with a control group (C) of 15 healthy age- and sex-matched individuals that received the same regimen.

Results: Baseline CVR was lower in S compared to C (median [quartiles]: 21.3 [12.7, 35.1] vs 41.4 [18.8, 61.3]; $\mathrm{p}<0.001)$. Baseline cholesterol values were higher in $\mathrm{S}$ (median [quartiles] mg/dl: 171.3 [122.5, 245.6] vs 149.1 [109.9, 181.6]; $\mathrm{p}<0.005)$ and were not influenced by atorvastatin treatment $(\mathrm{p}=0.88)$. Atorvastatin improved VSR in $\mathrm{S}(29.3[13.5,44.2] ; \mathrm{p}<0.008)$ but only tended to improve VSR in $\mathrm{C}(49.3[18.5,74.2] ; \mathrm{p}=0.057)$. The degree of vasoreactivity improvement in $\mathrm{S}$ was higher compared to $\mathrm{C}$ (RI\%: $38.5[13.8,53.2]$ vs $17.3[6.9,28.7] ; \mathrm{p}<0.002)$.

Conclusions: Our results suggest that high doses of atorvastatin administered for very short periods can improve the impaired cerebral vasomotor reactivity in patients with high-grade carotid stenosis through other mechanisms than lowering blood lipids.

\section{Cerebrovascular autoregulation}

\section{CEREBRAL AUTOREGULATION IS ALTERED IN OBSTRUCTIVE SLEEP} APNEA SYNDROME

N. Nasr, A. Pavy-Le Traon, M. Tiberge, M. Czosnyka, E. Schmidt, V. Larrue Hôpital Rangueil, Toulouse, France

Backgound: Evidence is gathering to point out obstructive sleep apnea syndrome (OSAS) as an independent risk factor for ischemic stroke. Several mechanisms may underlie the predisposition to ischemic stroke in OSAS patients, with failure of cerebrovascular reactivity playing a potential role. Dynamic cerebral autoregulation (DCA) has not been investigated so far in OSAS patients. The aim of this study was to determine whether cerebral autoregulation is altered in OSAS patients compared to controls.

Methods: Patients and controls were explored after an overnight complete polysomnography. Main exclusion critera were an history of cerebrovascula disease, intracranial arterial stenosis, and carotid stenosis as detected by ultrasound Mean cerebral blood flow velocity (CBFV) in the middle cerebral artery and mean arterial blood pressure (ABP) were continuously recorded using transcranial doppler and Finapres. Correlations between $\mathrm{ABP}$ and $\mathrm{CBFV}$ spontaneous fluctuations were averaged to form the coefficient correlation indice $\mathrm{Mx}$.

Results: All examinations were performed in the morning, within 2 to 3 hours after awakening. Eleven OSAS patients (mean age \pm sd; 52.6 \pm 7.9 ) and 9 controls (mean age \pm sd; 49.1 \pm 5.3 ) were enrolled. The mean apnea-hypopnea index (AHI) in the OSAS group was of 22.7. No significant difference was found between the two groups as for age, body mass index, mean $\mathrm{ABP}$ and end-tidal $\mathrm{CO} 2$ pressure. $\mathrm{Mx}$ was significantly higher in OSAS patients compared to controls $(0.414$ vs 0.233 ; $\mathrm{p}=0.009)$. Furthermore, in OSAS patients, Mx was correlated to the severity of OSAS as defined by the AHI ( $\mathrm{p}=0.003)$.

Conclusion: DCA is altered in OSAS patients. This finding may contribute to the undestanding of the increased risk of stroke in OSAS. 


\section{Cerebrovascular autoregulation}

\section{POSTPROCEDURAL HEMODYNAMIC INSTABILITY AFTER CAROTID} ARTERY STENT PLACEMENT

J. Staszewski, J. Swistak, K. Brzozowski, J. Kotowicz, A. Stepien

Military Medical Institute, Poland

Background: Acute hemodynamic instability (HI) during carotid endarterectomy (CEA) is a well-recognized phenomenon. The frequency, risk factors and consequences of long term fluctuations in blood pressure after carotid artery stenting (CAS) are not well known.

Methods: Prospective analysis of 25 patients with 6-month follow-up undergoing CAS (self-expanding stent with an emboli-protection device) due to symptomatic or asymptomatic stenosis who were ineligible for CEA. A validated 24-hour ABPM was taken 24 hours before and after CAS. Neurological assessment was performed at baseline, 24hours after CAS and at 6 month follow-up visit.

Results: Median age was 68 years, $73 \%$ were male, $86 \%$ of patients had symptomatic carotid stenosis. $80 \%$ of patients experienced intraprocedural HI (bradycardia and hypotension $40 \%$, bradycardia $13 \%$, hypotension $27 \%$ ). The cumulated postprocedural mean of SBP and DBP decreased from baseline $128 / 67 \mathrm{mmHg}$ to $108 / 54$ $\mathrm{mmHg}(\mathrm{p}<0.01)$. Postprocedural hypotension was found in $67 \%$, bradycardia in $87 \%$ of cases. In the multivariate analysis the predictors of postprocedural $\mathrm{HI}$ were: male sex (OR 1.5, p=0.04), ulcerated plaque (OR 1.2, $\mathrm{p}=0.03)$, contralateral carotid stenosis $>50 \%$ (OR 2.1, p=0.05), episode of intraprocedural hypotension (OR 4.2, $\mathrm{p}=0.02$ ). Chronic $\mathrm{Ca}$-antagonists treatment and smoking reduced the risk of $\mathrm{HI}$ (OR $0.5, \mathrm{p}=0.01$; OR $0.8, \mathrm{p}=0.008$, respectively). We found no association of $\mathrm{HI}$ with age, ischemic heart disease, bifurcation involvement, baloon size, inflation pressure, longer lesion length. During 6 month follow-up only 1 case of neurological deterioration was observed.

Conclusions: Postprocedural HI is a common phenomenon after CAS. Patients a risk can be possibly identified through clinical and angiographic variables. HI was not linked with neurological complications in the studied group.

\section{Cerebrovascular autoregulation}

THE EFFECT OF CAROTID STENT INSERTION UPON INTRACRANIAL HAEMODYNAMICS AND CEREBROVASCULAR REACTIVITY IN PATIENTS WITH SEVERE CAROTID STENOSIS

C.J. Harrow, K. Oldroyd, L. Malcolm, M. Walters

University of Glasgow, Glasgow, United Kingdom

Background: Impaired cerebrovascular reactivity (CVR) predicts stroke in patients with carotid disease. CVR is improved by carotid endarterectomy (CEA) however few studies have examined the effect of carotid artery stenting (CAS) on CVR. We compared the breath hold index (BHI, a measure of CVR) in patients with asymptomatic carotid disease pre and post CAS.

Methods: In our institution, patients awaiting coronary artery surgery are screened for severe $(>70 \%)$ carotid stenosis and such patients undergo carotid revascularisation using CEA or CAS as part of routine clinical care. Patients selected for CAS underwent assessment of $\mathrm{BHI}$ prior to CAS and 3 weeks post CAS. The middle cerebral artery was insonated ipsilateral to the diseased ICA and MFV measured before and after 30 second breath-hold. BHI was calculated as [MFV (at end of breath-holding) - MFV (rest)/[MFV (rest) $\times 100 /$ duration of breath-hold (secs)]. Both assessments of BHI occurred prior to surgery.

Results: 7 patients were recruited $(5(71 \%)$ males, mean age $68 \mathrm{y}( \pm 10.6))$. Patient were similar with regard to risk factors, degree of carotid disease and medication. All underwent uncomplicated CAS with distal protection using nitinol self expanding carotid stents. There was a significant increase in mean BHI post CAS insertion. Mean (SD) BHI prior to stent insertion was $0.84( \pm 0.53)$ compared with $1.41( \pm 0.36)$ three weeks post stent insertion $(\mathrm{p}=0.024$, paired $\mathrm{t}$-test). There was no significant difference in blood pressure either pre- or post CAS or before or after breath-hold.

Discussion: This is the first study to evaluate the effect of CAS on CVR in patient with severe asymptomatic carotid arterial disease. Reactivity of the cerebral vessels to a hypercapnic stimulus improved significantly following CAS. Larger trials will be required to determine the clinical significance of this observation and to inform future decisions about the role of CAS in patients with asymptomatic carotid stenosis.
5 Cerebrovascular autoregulation

POSTERIOR REVERSIBLE ENCEPHALOPATHY SYNDROME (PRES): A CASE SERIES WITH VARIABLE CAUSES

C. Odier, G. Matte, C. Chayer, D.K. Nguyen, S. Lanthier

Centre hospitalier de Montréal (CHUM), Montréal, Canada

Background: Posterior reversible encephalopathy syndrome (PRES) frequently mimics cerebral venous or basilar artery thrombosis. Typically, PRES presents with headaches, altered consciousness, seizures, or visual deficits, and posterior cerebral oedema on brain imaging. PRES results from endothelial dysfunction or overwhelmed autoregulation, due classically to immunosuppression therapy, severe hypertension, or eclampsia.

Objective: To identify other potential causes of PRES.

Methods: We analyzed conditions associated with typical PRES from two hospitalbased populations in 2005 .

Results: Six individuals presented with blood pressure of 130-180/70-101 $\mathrm{mmHg}$, headaches $(n=5)$, seizures $(n=4)$, altered consciousness $(n=4)$, cortical blindness $(n=3)$, Balint syndrome $(n=2)$, visual hallucinations $(n=2)$, aphasia $(n=2)$, and hemiparesis $(\mathrm{n}=1)$. Brain CT showed posterior abnormalities consisting of bilateral subcortical hypodensities $(n=4)$ or hyperdense gyri reflecting cortical hyperperfusion or subarachnoid bleeding $(n=2)$, or normal finding $(n=1)$. Initial diagnoses included cerebral venous or basilar artery thrombosis, which were excluded by selective cerebral or MRI angiography. Brain MRI revealed vasogenic predominant posterior cerebral oedema without diffusion restriction, consistent with PRES $(n=6)$. PRES resulted from immusuppression therapy $(n=2)$, eclampsia $(n=1)$, or none of these classical causes $(n=3)$. In the latter group, associated conditions were moderate hypertension $(177-180 / 96-101 \mathrm{mmHg} ; \mathrm{n}=2)$, low-dose methotrexate $(\mathrm{n}=1)$, remote cisplatin therapy $(n=1)$, blood transfusion $(n=1)$, and hypomagnesaemia $(n=1)$ Symptoms resolved within 1-22 days with treatment of underlying causes and associated conditions.

Discussion: PRES may mimic cerebral venous or basilar artery thrombosis. Severe hypertension, immunosuppression therapy, and eclampsia are frequently absent in typical PRES. Potential causes include moderate hypertension, lowdose methotrexate, remote cisplatin therapy, blood transfusion, and hypomagnesaemia.

\section{Cerebrovascular autoregulation}

\section{DECREASED VASOMOTOR REACTIVITY OF THE BASILAR ARTERY IN PATIENTS WITH OCCLUSIVE VASCULAR DISEASES IN THE ANTERIOR CIRCULATION}

P. Tai Hwan, P. Soo-Hyun, Y. Young-Chul, K. Oh-Sang

Chungang University Hospital, Seoul, South Korea

Background: Decreased cerebral vasomotor reactivity (VMR) indicates the pres ence of preexisting vasodilatation in response to cerebral hypoperfusion. Vertebrobasilar circulation can be an important intracranial collateral to compensate hypoperfused anterior circulation. The aim of our study was to determine whether VMR of the basilar artery (BA) would be affected in patients with occlusive vascular diseases in the anterior circulation (OAC).

Methods: Sixty five patients who performed MRI including MRA for various minor ischemic symptoms or headache were divided into two groups according to the presence of OAC. The OAC included $>70 \%$ stenosis or occlusion of either internal carotid artery or M1 segment of middle cerebral artery. VMR to apnea was calculated with transcranial Doppler ultrasonography (TCD) by means of the breath-holding index (BHI). TCD parameters including VMR of the BA and clinical factors were compared between groups.

Results: Patients with OAC ( $\mathrm{n}=21$; mean age, 60 years) showed significantly lower basilar BHI $(0.68 \pm 0.41$ versus $1.08 \pm 0.39, \mathrm{P}<0.01)$ and higher mean flow velocity $(\mathrm{mFV})(\mathrm{P}<0.01)$ compared to those of patients without OAC $(\mathrm{n}=45$; mean age, 61 years). Increased $\mathrm{mFV}$ was observed at whole length of the BA. Baseline pulsatility index as well as rates of diabetes, hypertension, and ischemic lesions in posterior circulation were not different between groups. In patients with OAC, the mean basilar BHI was significantly lower in patients with ischemic symptoms than that of patients without $(\mathrm{P}<0.01)$.

Discussion: Assessing VMR of BA by using BHI can be an easily applicable and noninvasive method to evaluate collateral flow via posterior circulation in patient with $\mathrm{OAC}$, which will be more useful for patients with poor echogenic transtemporal windows. 


\section{Cerebrovascular autoregulation}

STROKE SECONDARY TO SLE IS ASSOCIATED WITH ALTERED CEREBROVASCULAR REACTIVITY

R.J. Davey, P. Emery, J. Bamford

Leeds General Infirmary, Leeds, United Kingdom

Background: SLE is an autoimmune disease with frequent neuropsychiatric (NP) complications, including stroke. Brachial artery flow studies indicate that systemic endothelial dysfunction (ED), a precursor of atherosclerosis, is common in SLE. The pathogenesis of NP SLE is uncertain, but cerebrovascular ED is a candidate mechanism. Changes in cerebral blood flow induced by hypercapnia (cerebrovascular reactivity, CVR) reflect endothelial function in cerebral resistance vessels. We investigated the role of CVR in SLE.

Methods: Patients with SLE were assessed to identify NP syndromes according to American College of Rheumatology definitions. Both middle cerebral arteries were assessed by transcranial doppler ultrasound to ascertain mean CVR to hypercapnia (\% increase in flow velocity $/ \mathrm{mmHg}$ rise in end-tidal $\mathrm{CO} 2$ ). Findings were compared to those amongst healthy control subjects matched for age and gender.

Results: 57 subjects ( 54 female, 3 male) with SLE of median age 46 years were assessed, along with 67 control subjects. There was no significant difference in CVR between cases and controls (3.06 vs 3.06, $\mathrm{p}=1.00$ ). However, SLE patients with a history of stroke or TIA $(n=19)$ had a significantly higher CVR than those without (3.79 vs $2.70, p=0.01$ ). Other NP syndromes (mood disorders, headache, seizures, cognitive dysfunction, psychosis, chorea) were not associated with altered CVR CVR was independent of SLE disease activity and treatment, antiphospholipid antibodies, smoking and hypertension.

Conclusions:

- Although abnormal CVR is not linked to many NP SLE manifestations, increased CVR is associated with stroke or TIA.

- In non-SLE studies, stroke has been associated with low CVR. Our results suggest a different stroke mechanism exists in SLE.

- Increased CVR may reflect ED. Cerebral ischaemia may result from vasospasm or increased vascular permeability. Although ED in SLE is likely to be immunemediated, its precise cause merits further study.

\section{Cerebrovascular autoregulation}

CEREBRAL VASOREACTIVITY IN PATIENTS WITH ASYMPTOMATIC AND SYMPTOMATIC HIGH-GRADE ICA STENOSIS FOLLOWING STENT-PTA

I. Brcic, S. Horner, D. Thaler, G.E. Klein, K. Niederkorn

Medical University Graz, Graz, Austria

Background and Purpose: Some studies have suggested that carotid endarterectomy normalizes impaired cerebral vasoreactivity. The aim of this study was to assess the effects of percutaneous transluminal angioplasty with stenting on cerebral vasoreactivity and the possible changes after carotid artery stenting.

Methods: We studied the changes of blood flow velocity in the middle cerebral arteries after breath-holding in thirty-three patients (15 symptomatic and 18 asymptomatic) undergoing PTA with stenting for high-grade carotid artery stenosis. Transcranial Doppler evaluation of both middle cerebral arteries was performed before, one day and one month after stenting. Breath-holding index (BHI) was used to evaluate cerebral vasoreactivity.

Results: Before and one day after stenting, a significant difference $(\mathrm{p}<.01)$ in $\mathrm{BHI}$ values was detected when comparing the stenotic and the contralateral side of symptomatic $(0.59 \pm 0.30$ and $0.80 \pm 0.29)$ and asymptomatic $(0.71 \pm 0.29$ and $0.83 \pm 0.36)$ patients with that of controls $(1.24 \pm 0.25)$. One day after stenting, BHI significantly increased $(\mathrm{p}<.01$ for each) on the previously stenotic side of asymptomatic $(0.92 \pm 0.35)$ and symptomatic patients $(0.96 \pm 0.16)$ while remaining substantially stable on the contralateral side of both asymptomatic and symptomatic patients. On the other hand, one month after stenting, BHI was significantly higher on the previously stenotic side of asymptomatic $(1.15 \pm 0.25 ; \mathrm{p}<.01)$ and symptomatic $(1.10 \pm 0.32 ; \mathrm{p}<.01)$ patients and on the contralateral side of asymptomatic $(1.19 \pm 0.38 ; \mathrm{p}<.05)$ and symptomatic patients $(1.15 \pm 0.36 ; \mathrm{p}<.01)$

Conclusions: These findings suggest that PTA with stenting results in improvement close to normalization of impaired cerebral vasoreactivity in patients with symptomatic and asymptomatic high-grade carotid artery stenosis.
9 Cerebrovascular autoregulation

CORRELATION OF VASOMOTOR REACTIVITY BY TCD AND PRESENCE OF SILENT INFARCTS IN MRI IN MEN WITH ARTERIAL HYPERTENSION A.G. Kozera, C.M. Dubaniewicz, B.M. Mielczarek, B.A. Madej-Dmochowska, A.D. Gletterasecki, A.K. Chwojnicki, D.J. Wojczal, B.T. Zdrojewski, B.B. Wyrzykowski, A.W.M. Nyka

Medical University of Gdansk; Dept. Neurology, Lublin School of Med., Poland

Aim: We accessed a relationship between middle cerebral artery (MCA) vasomotor reactivity and presence of asymptomatic vascular lesions in MRI examination.

Materials: We examined 47 hypertensive men (14 with type II of diabetes) all 60 years old, without history of stroke, neurological deficits and pathological findings in carotid arteries. Mean risk of stroke calculated with PRECARD protocol was $6,35 \%$ (range 1-16).

Methods: Magnetic resonance imaging (MRI) was performed with Picker (1,5 T) device. Silent infarcts was defined as vascular lesions with diameter $\geq 3 \mathrm{~mm}$. Transcranial doppler examination (TCD) was performed with MULTIDOP 2 (DWL) device, $2 \mathrm{MHz}$ PW probe, with assistance of Datex capnograph. Vasomotor reactivity $(\mathrm{Vr})$ of MCA was assessed by monitoring of mean velocity in MCAs (Vmca) from hyperventilation (hypocapnia) to hypercapnia (obtained during Breath Holding Test) and it was expressed as a percentage change of Vmca. Statistical analysis was performed using U-Mann-Whitney and Spearmann rank correlation tests.

Results: There was insignificant trend to lower vasomotor reactivity in group of 22 $(47 \%)$ subjects with vascular lesions compared with $25(53 \%)$ men without brain infarction in MRI (median 54\%; quartile range $43-65 \%$ vs. $61 \%$; quartile range $42-80 \%) \mathrm{p}=0.082$. Otherwise calculated risk of stroke was greater in patients MR abnormalities ( $7 \%$ vs. $6 \% \mathrm{p}<0,05$ ). There was also a good correlation between vasomotor reactivity of MCA and calculated risk of stroke, $r=0.28, p<0.05$.

Conclusions: Our results support hypothesis that that patients with impaired $\mathrm{Vr}$ of $\mathrm{MCA}$ are in bigger risk of brain infarction (also asymptomatic). Calculated risk of stroke correlates well with $\mathrm{Vr}$ of MCA and have an impact on presence of silent infarcts in MRI examination.

\section{Cerebrovascular autoregulation}

\section{EFFECTS OF SHORT TERM ATORVASTATIN TREATMENT ONCEREBRAL} HEMODYNAMICS IN CADASIL

T. Freilinger, N. Peters, C. Opherk, A. Gschwendtner, T. Pfefferkorn, M. Dichgans

Klinikum Grosshadern, Munich, Germany

Background: HMG-CoA-reductase-inhibitors (statins) exhibit pleiotropic beneficial effects on the vascular system including induction of endothelial nitric oxide synthase (eNOS) expression which is critical for vasodilation. Recent studies suggest a beneficial effect of statins on cerebral vasoreactivity in patients with cerebral smal vessel disease (SVD). CADASIL is a monogenic form of SVD caused by mutations in the NOTCH3 gene. Treatment options are limited and little is known about the therapeutic role of statins in CADASIL.

Methods: Twenty-four CADASIL subjects were treated with atorvastatin for eight weeks. Treatment was started with $40 \mathrm{mg}$, followed by a dosage increase to $80 \mathrm{mg}$ after four weeks. Transcranial doppler sonography measuring mean flow velocity (MFV) in the middle cerebral artery was performed at baseline and the end of the treatment period. Vasoreactivity was assessed by hypercapnia and intravenous application of L-Arginine, which is the substrate for eNOS.

Results: There was no significant treatment effect on MFV ( $\mathrm{p}=0.5)$ and cerebral vasoreactivity as assessed by hypercapnia $(\mathrm{p}=0.5)$ and intravenous L-Arginine $(\mathrm{p}=0.4)$ in the overall cohort. However, an inverse correlation was found between vasoreactivity at baseline and changes of both $\mathrm{CO} 2$ and L-Arginine-induced vasomotor response (both $\mathrm{p}<0.05$ ).

Conclusions: Short term treatment with atorvastatin resulted in no significant improvement of hemodynamic parameters in the overall cohort of CADASIL subjects. However, a beneficial effect in subjects with pronounced impairment of cerebral vasoreactivity seems possible. 


\section{Genetic disorders}

\section{Genetic disorders}

PREMATURE BORDERZONE INFARCTIONS IN HEREDITARY PROTEIN S DEFICIENCY ASSOCIATED WITH A NOVEL PROTEIN S GENE (PROS1) MUTATION

T.W. Leung, S.F. Yip, C.W. Lam, Y.H. Fan, P.H. Chan, L.C. Chan, K.S. Wong Prince of Wales Hospital, The Chinese University of Hong Kong, Hong Kong

Background: Protein S deficiency (PSD) is an independent risk factor for venous thromboembolism. However, controversy surrounds the role of PSD in arterial ischemic stroke as the association is rarely supported by genotypic and infarct topographic correlation.

Methods: In a pedigree of young strokes with hereditary PSD identified as a tight co-segregating factor, we correlated the biochemical, genotypic and radiological findings.

Results: Three out of 11 family members had premature stroke at their early forties. Six members, including those 3 with premature strokes, were found to have PSD type III phenotype. Polymerase chain reaction and sequencing on all exons of protein S gene (PROS1) identified a novel mis-sense mutation in exon 10 resulting in Arginine 314-Cysteine (R314C). The mutation co-segregated fully with the PSD phenotype. Bilateral subcortical white matter infarctions in external and internal borderzones were consistently revealed by cranial magnetic resonance imaging (MRI) in all 5 PSD adult members (including 3 with strokes and 2 asymptomatic members). MRI was normal in all 5 non-PSD members, and in the youngest PSD member (aged 13).

Discussion: The novel PROS1 R314C mutation is most likely a causative mutation leading to PSD type III. The homogeneous infarct topography indicated a strong association between PSD and borderzone infarctions. The crescendo infarct volume from adolescence onwards suggested that the emergence of stroke in the fifth decade might have been preceded by a silent evolution of borderzone infarctions from as early as late teenage. PSD may cause cerebral infarctions through disturbing the hemostasis in borderzone. Awareness for screening and stroke prevention in PSD patients may be warranted.

\section{Genetic disorders}

\section{ACTIVATED PROTEIN C RESISTANCE AND FACTOR V GENE} MUTATIONS/POLYMORPHISMS IN CHILDREN WITH ISCHAEMIC STROKE

A. Komitopoulou, H. Platokouki, Z. Kapsimali, N. Komitopoulos,

H. Pergantou, S. Aronis

Aghia Sophia Children's Hospital, Athens, Greece

The most common inherited thrombophilic defect is the resistance to activated protein C (APCR) caused in $>90 \%$ of cases by the factor V Leiden (FVL) mutation. HR2 polymorphism of the FV gene (His1299Arg-FVR2) has also been suggested as a mild risk factor for thrombosis. The aim of this study was to investigate the frequency of the FVL mutation and FVR2 polymorphism and to correlate them with APCR in children with ischaemic stroke. PCR technique (Vienna Lab Kit) and a coagulation test (Coatest APCR, Chromogenix) were used for the detection of mutation/polymorphism and APCR, respectively, in 90 children (62.2\% males) with CNS thrombosis mean age 5.6 years (31 cases intrauterine), The patients were compared with 103 age-matched controls.

Results: CNS thrombosis was more frequent in boys $(\mathrm{p}<0.01)$. FV Leiden genotype distribution was significantly higher in patients compared to controls $(\mathrm{p}=0.006$ ), whereas no difference was found in FVR2. The risk of stroke was higher in the presence of FVL (OR 4.2, 95\% CI 1.5-12.1). FVL carriership was found to increase the probability for stroke in utero (OR 6.82,95\% CI 2.0-22.8). The joint distribution of FVL and FVR2 was mutually exclusive. Additionally, the risk for stroke in FVL carriers was furthermore increased (OR 5.76, 95\% CI 1.6-6.4) when FVR2 was absent. APCR ratio $<2$ had a true causal association with FV Leiden. The presence of FVR2 had no impact on APCR.

Conclusion: Since FVL mutation seems to contribute in the pathogenesis of ischaemic stroke in children, the role of FVR2 polymorphism is still not quite clear. A hint for a protective rather than a risk factor for thrombosis is presumed for FVR2. However, FVR2 was found to have no impact on the activated protein $\mathrm{C}$ resistance and as a consequence on thrombus formation.
3 Genetic disorders

MORPHOLOGY OF CORPUS CALLOSUM AND NEUROPSYCHOLOGICAL PERFORMANCES IN CADASIL PATIENTS WITH MILD COGNITIVE DECLINE

C. Sarti, F. Pescini, E. Salvadori, M. Moretti, G.P. Giordano, S. Bianchi, M.T. Dotti, A. Federico, D. Inzitari, L. Pantoni

University of Florence, Florence, Italy

Background: CADASIL is an inherited vascular disease mainly characterized by recurrent strokes and cognitive deficits (mostly frontal-executive functions impairment). The MRI hallmarks of the disease are subcortical, bilateral T2-hyperintense areas frequently associated with multiple lacunar infarcts. Corpus callosum (CC) has not been extensively studied in CADASIL. We studied CC morphology in CADASIL patients with mild cognitive decline and the association with neuropsychological performances.

Methods: T1-weighted sagittal MRI scans from 8 genetically diagnosed CADASIL patients (mean age: 57.4 yrs $\pm 10.9,4$ males) with mild cognitive decline were analyzed. The following CC measures were considered: 1) CC thickness at the genu, splenium, and 2 intermediate and equidistant points (body 1 and body 2) (single measure and total sum); 2) CC area using a computed assisted method; 3) presence and location of hypointense lesions. The association between neuropsychological tests (Mini-Mental State Examination, VADAS scale, Trial Making Test, Color Word Stroop Test) and CC measures was tested using non-parametric correlations (Kendall's tau).

Results: Mean CC area was $6.88 \pm 0.9 \mathrm{~cm}^{2}$, and mean total thickness $2.87 \pm 0.6 \mathrm{~cm}$ Thirteen hypointense lesions were detected in 3 patients. The following correlations were found: $\mathrm{CC}$ area, splenium and body 2 thickness with short term verbal memory; $\mathrm{CC}$ area with attention; $\mathrm{CC}$ hypointense lesions with frontal executive functions but not with memory.

Conclusions: Although limited by the low number of patients, this study suggests that CC measures could be of interest in the evaluation of cognitive profile of CADASIL patients. Larger studies with possibility to explore the independence of the found correlations are needed.

\section{Genetic disorders}

PCSK9 GENE AS DETERMINANT FOR STEROL ABSORPTION AND ATHEROTROMBOTIC STROKE

D. Abboud, S. Blecic, D. Luetjohann, S. Jeangette, M.D. Gazagnes, P. Desfontaines, P. Karhunen, T. Lehtimaki, R. Laaksonen, M. Pandolfo Erasme Hospital, Brussel, Belgium

Background: Recently mutations in PCSK9 gene have been identified in autosomal dominant hypercholesterolemia. The E640G single nucleotide polymorphism (SNP) of PCSK9 gene has been then implicated in coronary atherosclerosis severity and LDL cholesterol $(\mathrm{CH})$ levels. Dyslipidemia is a clear risk factor for ischemic heart disease but its implication in ischemic stroke (IS) is controversial.

One way to increase the chance of identifying genes in IS is to study stroke subtypes, in combination with intermediate phenotypes.

In this study we investigated the possible association between E640G gene, IS, sterol metabolism and severity of cerebral atherosclerosis

Method: E640G SNP of the PCSK9 gene was analysed in two different populations. The multi-centre Belgium retrospective case-control study including 200 patients (45-60 yrs), with lacunar and atherotrombotic stroke (large vessel disease LVD), and 180 gender and ethnicity matched controls ( $>60 \mathrm{yrs}$ ) with no history of stroke or MI. We measured the plasma concentration of $\mathrm{CH}$, lathosterol (a $\mathrm{CH}$ precursor, using its ratio to $\mathrm{CH}$ as a marker of endogenous $\mathrm{CH}$ synthesis) and plant sterols (using their ratio to $\mathrm{CH}$ as markers of intestinal sterol absorption).

The Finnish population constituted of 640 autopsy cases with an assessment of atherosclerosis score in each of the nine branches of the circle of Willis

Results: After adjustment for all known risk factors, the minor allele $(\mathrm{G})$ carriers of the PCSK9 gene associated significantly with LVD $(\mathrm{p}=0.012)$, with campesterol a plant sterol and campesterol to $\mathrm{CH}$ ratio $(\mathrm{p}=0.011, \mathrm{p}=0.05)$. This SNP did not associate with $\mathrm{LS}, \mathrm{CH}$ level and $\mathrm{CH}$ synthesis rate. The $\mathrm{G}$ allele carriers tended to associate with the severity of cerebral artery atherosclerosis ( $\mathrm{p}=\mathrm{ns}=0.1$ ).

Conclusion: PCSKA E640G SNP is associated with markers of $\mathrm{CH}$ absorption. PCSKA 640G SNP is an independent predictor of IS considering LVD subtype, and has a small influence in cerebral artery atherosclerosis. 


\section{Genetic disorders}

MMP-9 SINGLE NUCLEOTIDE POLYMORPHISMS (SNP) ARE NOT ASSOCIATED WITH TERRITORIAL STROKE IN ISCHEMIC STROKE PATIENTS

M. Fatar, M. Stroick, M. Steffens, S. Bukow, A. Alonso, M. Griebe, P. Bugert, T. Wienker, M.G. Hennerici

Klinikum Mannheim, University of Heidelberg, Mannheim, Germany

Background: In recent years different SNP polymorphisms were associated with cerebrovascular diseases and their potential role was postulated increasingly. Elevated MMP-9 protein levels were found in experimental animal models as well as in human stroke. We therefore examined whether SNP polymorphisms of the MMP-9 gene are associated with territorial stroke

Patients: Patients with territorial stroke ( $\mathrm{n}=108)$, diagnosed both clinically and with brain imaging, were recruited from our stroke unit. Patients with transient ischemic attacks, lacunar infarcts and primary brain haemorrhages were excluded. Ischemic strokes were classified according to the TOAST criteria for large vessel disease $(\mathrm{n}=35)$, cardioembolic sources $(\mathrm{n}=54)$ and undiagnosed after full vascular work-up $(n=19)$. Control subjects were recruited from voluntary donors of the local blood bank $(n=57)$ and the out-patient unit of the department of ophthalmology $(n=85)$.

Methods: DNA samples were analyzed by MALDI-TOF (matrix assisted lase desorption/ionization-time of flight) analysis and all participants had given informed consent for genetic analysis. 15 SNPs of MMP-9 gene were examined. Differences between samples with respect to genotype distribution and allele frequencies were tested in a pair wise fashion by a Chi-Square test and Armitage's trend test. Phenotypes including risk factors, drugs and all available clinical data were documented as designed in the study protocol.

Results and Discussion: All samples were at the Hardy-Weinberg Equilibrium and mean call rate was $98.7 \%$. Despite current data demonstrating the role of MMP-9 protein levels in stroke, no genetic risk according to MMP-9 SNPs could be identified. All examined 15 SNPs were not associated with territorial stroke.

\section{Genetic disorders}

\section{ASSOCIATION BETWEEN SILENT CEREBRAL INFARCTION AND POLYMORPHISMS IN THE ENDOTHELIAL NITRIC OXIDE SYNTHASE GENE}

O. Ok Joon Kim, K. Kim Kyoung Keun, Y. Young Ho Ku, N. Nam Keun Kim, I. Il Hyung Lee, H. Hyun Sook Kim, W. Won-Chan Kim

Pochon CHA University, Pundang CHA Hospital, Sungnam, Kyonggi-do, South Korea

Background: Endothelium-derived nitric oxide is formed from L-arginine by endothelial nitric oxide synthase (eNOS) encoded by the NOS 3 gene on chromosome 7. eNOS plays a key role in vascular regulation and atherosclerosis. It means that the impairment of nitric oxide production could facilitate the atherosclerotic process or cerebral infarction. There are three clinically relevant eNOS polymorphisms; T786C in the promoter, 27-bp repeat in intron 4, and the G894T in exon 7. We investigated whether the common variants in the eNOS gene are associated with the risk of silent cerebral infarction.

Methods: We studied 272 patients with the silent cerebral infarction and 72 healthy controls. The presence of cerebral infarction was confirmed by brain MRI. 1 intronic (intron 4) polymorphism and 2 exonic (T786C, G894T) polymorphisms of the eNOS gene were explored by PCR.

Results: The distribution of exonic polymorphism in G894T was significantly different between cases and controls; the GT and TT genotypes were more frequent in patients $(42.9 \%)$ than in controls $(15.4 \%)(\mathrm{P}=0.01$; OR, $0.38 ; 95 \% \mathrm{CI}, 0.16$ to 0.89$)$. In addition, the distribution of genotypes in intron 4 polymorphism was significantly different; 4R4R and 4R5R repeats were more frequent in patients (19.9\%) than in controls $(9.3 \%)(\mathrm{P}=0.026 ; \mathrm{OR}, 0.40 ; 95 \% \mathrm{CI}, 0.20$ to 0.80$)$. TC and CC genotypes in T786C polymorphism were more frequent in cases $(18.0 \%)$ than in controls $(9.3 \%)$, but there was no significance.

Discussion: The genotypes containing T allele of G894T and 4R variant of intron 4 in the eNOS gene are risk factors for the silent cerebral infarction in this study population. These findings suggest that it may help us to understand the cause of the vascu! lopathy underlying silent brain infarction.

\section{Genetic disorders}

POLYMORPHISMS OF NITRIC OXIDE SYNTHASE INTERACTING PROTEIN (NOSIP) AND SMALL VESSEL DISEASE

K. Gormley, S. Bevan, H.S. Markus

St George's, University of London, London, United Kingdom

Background: Endothelial dysfuntion has been implicated in the pathogenesis of cerebral small vessel disease (SVD). One of the key mediators of endothelial dysfunction is nitric oxide (NO). Genetic variation in NOSIP, a novel protein which controls the localisation and therefore the activity of the enzyme responsible for NO production, could play a role in the pathogenesis of SVD through effects on NO and endothelial dysfunction. It has been suggested that there are two subtypes of SVD, isolated lacunar infarction (ILI), resulting from microatheroma, and multiple lacunar infarcts with leukoaraiosis (ILA), due to a diffuse arteriopathy.

Methods: 8 single nucleotide polymorphisms (SNPs) spanning the NOSIP gene were genotyped. Haplotypes were constructed using PHASE. Individual SNPs and haplotypes were examined for associations with SVD as a whole and the two subtypes. Linkage Disequilibrium (LD) structure was analysed with Haploview.

Results: None of the individual SNPs were associated either with SVD or its subtypes. SNPs 2 (rs3745474) and 7 (rs4594362) were in LD with each other (D'=0.79) but not any of the remaining SNPs. Strong LD (D' $\geq 0.86$ ) was observed spanning a $10 \mathrm{~kb}$ region covered by SNPs 3-6. Haplotypes with a frequency of over $5 \%$ in the control population were analysed. There were no significant associations between haplotypes and SVD or its subtypes.

Discussion: There were no significant associations between either individual SNPs or haplotypes within NOSIP and SVD or its subtypes. This not support a role for the genetic variation in NOSIP in the pathogenesis of cerebral SVD.

\section{Genetic disorders}

\section{A NOVEL MUTATION OF THE GENE NOTCH3 IN A PATIENT WITH CADASIL}

E. Azevedo, S. Ferreira, J. Reis, J. Guimarães, J.P. Oliveira, C. Garrett Hospital S. João - University of Porto, Porto, Portugal

Background: CADASIL is a hereditary vascular dementia characterized by midadulthood onset of cerebrovascular disease, with diffuse white matter lesions and subcortical infarcts. It is due to mutations of Notch3, a gene that maps to chromosome $19 \mathrm{p} 13$ and codifies a transmembrane receptor. The majority of these mutations have been described on exons 3 and 4 . We report a novel Notch3 mutation in a CADASIL patient.

Case Report: Mood and emotional disturbances, evolving with agressive behaviour and cognition decline, were first noted in a previous healthy female at age 46. At admission, the patient had little spontaneous speech and showed a pseudobulbar affect. Neuropsychological evaluation disclosed a global cognitive decline involving multiple domains, suggesting a predominantly subcortical dementia. There were no focal neurological signs. Known family history was irrelevant. Routine laboratory tests, including screening for prothrombotic states, serology for Lyme disease, syphilis and HIV were unremarkable. Cervical and transcranial ultrasound duplex, electrocardiogram and echocardiogram were normal. Electroencephalography showed a theta-delta slowing indicating diffuse cerebral dysfunction. Brain magnetic resonance imaging demonstrated multiple subcortical lacunar infarctions and diffuse white matter abnormalities, including anterior temporal pole and external capsule. Molecular genetic analysis of gene Notch3 detected a mutation W1028C on exon 19.

Discussion: Mutation W1028C has not been previously reported in CADASIL. However, the clinical evidence for the diagnosis, together with the lack of evidence for other aetiologies, plus the similarity of this mutation to other transitions to cysteine already described in CADASIL, strongly support its pathogenic role.

\section{Experimental studies}

\section{Experimental studies}

HOW CANDESARTAN PROTECTS AGAINST CEREBRAL ISCHEMIA: ON THE EXPRESSION OF ANGIOTENSIN II TYPE I AND II RECEPTOR Y. Kanematsu, K.T Kitazato, H. Liu, M. Uno, A. Suzue, T. Tamaki, S. Nagahiro The University of Tokushima, Tokushima, Japan

Background: Angiotensin II (AngII) and its receptors (AT1R and AT2R) are involved in the initiation and processing of cerebral ischemia. However, details 
regarding the role of $\mathrm{ARB}$ in regulating these receptors during ischemia remain to be elucidated. We examined the effect of ARB on the expression of AT1R and AT2R in vivo and in vitro.

Methods: Normotensive, 5-week-old Wistar rats received daily oral doses of 0.5 or $1.0 \mathrm{mg} / \mathrm{kg}$ candesartan for 14 days before middle cerebral artery occlusion followed by reperfusion (MCAO-R). At $24 \mathrm{hr}$ post-MCAO-R, AT1R and AT2R were examined by immunohistochemical and quantitative real-time PCR (qRT-PCR) analysis to determine the possible correlation between their expression levels and infarct size. Plasma Ang II levels were measured by radioimmunoassay. To study the protein and gene expression of AT1R and AT2R in human umbilical vein endothelial cells (HUVEC) treated with 10-7 M candesartan and/or 10-7 M Ang II, we performed Western blotting and qRT-PCR analysis.

Results: Candesartan pretreatment decreased the infarct volume in the cortical region but not the caudate putamen. There was a positive correlation between the reduction in infarct size in the cortical area and the protein- and mRNA expression of AT1R $(\mathrm{p}<0.01)$. There was no obvious effect on AT2R expression. Plasma Ang II levels were higher in ARB-treated- than ischemic control rats $(\mathrm{p}<0.05)$. In HUVEC exposed to Ang II, candesartan reduced the protein and mRNA expression of AT1R. Our findings suggest that the down-regulation of AT1R expression contributes to the inhibition of brain damage after ischemic insult.

Conclusions: We first demonstrated the expression of AT1R and AT2R in a rat MCAO-R model with and without ARB pretreatment. Our results indicate that in rats, AT1R was associated with the development of ischemic brain damage and that candesartan inhibited the activity of AT1R via down-regulation of its gene expression, thereby limiting the infarct size.

\section{Experimental studies}

\section{CELL TRACKING OF GADOLINIUM LABELLED STEM CELLS IN A} HEMORRHAGE STROKE MODEL

M. Stroick, F. Giesel, M. Griebe, M. Fatar, C. Kern, S. Zechmann, H. Tröster,

N. Radzwill, F. Kiessling, M.G. Hennerici

Klinikum Mannheim, University of Heidelberg, Mannheim, Germany

Cell transplantation is used to reduce behavioural deficits after focal cerebral damage in animals and first human trials have been initiated for safety controls. To evaluate migration of the transplanted cells MRI tracking by iron labelling is performed. In stroke hemorrhagic transformation after ischemia prevents the separation between hemorrhage and the iron labelled cells. Therefore a new labelling technique is needed. Here we evaluated the possibility of gadolinium chelates as agent for cell tracking experiments.

Mesenchymal stem cells were incubated with different gadolinium chelates between $1 \mathrm{~h}$ and $168 \mathrm{~h}$, parts of the experiments used lipofectamin as transfection reagent. Afterwards cells were examined histologically and by MRI. In exemplaric in vivo experiments hemorrhage was induced by stereotactic collagenase infusion. Gadolinium and BrdU labeld cells were implanted contralateral to the hemorrhage. MRI and histological examinations were performed one day afterwards using a brucker biospin 9.4T MRI-scanner.

In vitro experiments demonstrate a continuous linear uptake of the gadolinium chelates into the cells which is not mediated by the transfecting agent lipofectamin. Fluorescence microscopy reveals a nearly $100 \%$ labelling success after an incubation period of $48 \mathrm{~h}$. In vivo experiments show a hyperintense area in the T1w images in the region of the implanted cells while the hemorrhage is hypointense. The corresponding T2w images show succeptibility effects in both areas. BrdU staining afterwards marks positive cells only in the area of the implanted cells.

These results illustrate a successful labelling with complete cell tracking ready to discriminated between hemorrhage and labelled cells.

\section{Experimental studies}

GLIAL GAP-JUNCTIONS IN THE RAT BRAIN: ARE THEY ESSENTIAL FOR BRAIN FUNCTION DURING BRAIN ISCHEMIA?

R. Tamaki, K. Tamura, A. Heimann, B. Alessandri, O. Kempski

Institute of Neurosurgical Pathophysiology, Mainz, Germany

Background: Gap junctions are reported to be important to maintain the physiological function in brain, e.g. spatial buffering of potassium. And they form gap junction intercellular communications (GIJC). But, it is still unclear which role gap junctions play in pathophysiological processes. We investigated 1) effect of gap junction blockade under physiological conditions with the gap junction blocker Carbenoxolone (CBX). 2) effect of gap junction blockade under ischemic conditions with $\mathrm{CBX}$ using our rat 2 vein occlusion model, which has rather wide penumbra area compared to infarct core size.

Method: 1) Male Wistar rats were used. After anesthetizing with chloral hydrate, lt. temporal craniotomy was made. $\mathrm{CBX}$ was injected into the rt. ventricle before spreading depression induction $(25,50,250 \mu \mathrm{g}$, saline, $10 \mu \mathrm{g}$ each).Spreading depression was induced with $\mathrm{KCl}$ injection into the cortex $(150 \mathrm{mM}, 5 \mu \mathrm{l}$, every 10 minutes, 7 times). Impedance and cerebral blood flow (CBF) were monitored. 2) After craniotomy, 2 adjacent cortical veins were occluded photochemically. CBX was injected into the rt. ventricle $(250 \mu \mathrm{g}$ or saline, $10 \mu \mathrm{l})$. Spreading depressions were induced with $\mathrm{KCl}$ injection (150mM, $5 \mu 1$, every 7 minutes, 10 times). Histological analysis was done 7 days after the operation (HE stain).

Results: 1) After injection of CBX, CBF was elevated in the $250 \mu \mathrm{g}, 50 \mu \mathrm{g}$ treated group compared to $25 \mu \mathrm{g}$ or saline treated group. Velocity of spreading depression was accelerated with $250 \mu \mathrm{g}$ CBX injection compared to saline treated group.2) Ischemic damage was aggravated with $250 \mu \mathrm{g}$ CBX injection compared to saline treated group $(\mathrm{P}<0.05)$

Conclusion: Disturbed function of gap junctions can aggravate outcome from venous ischemia

\section{Experimental studies}

\section{MOLECULAR IMAGING OF HUMAN THROMBUS WITH ULTRASOUND} AND ABCIXIMAB IMMUNOBUBBLES

A. Alonso, A. Della Martina, M. Stroick, M. Fatar, M. Griebe, E. Allemann, M.G. Hennerici, S. Meairs

Klinikum Mannheim, University of Heidelberg, Mannheim, Germany

Background: Molecular imaging of therapeutic interventions through development of novel agents serving both as ultrasound contrast agents and as carriers of drugs or genes is appealing. In this study we investigate the ability of a novel microbubble carrier (immunobubble) for GP IIb/IIIa receptor inhibitor abciximab to allow ultrasonographic molecular imaging of human clots.

Methods: Human blood thrombi were incubated in vitro in immunobubbles conjugated with abciximab. Control clots were incubated with either immunobubbles conjugated with a non-specific antibody or saline. Immunobubble suspensions with variable concentrations of encapsulated gas $(0.02 \mu 1$ to $0.8 \mu 1)$ were analysed at variable MI. Mean acoustic intensity was assessed with pulse inversion harmonic $(n=60)$ and high resolution B-mode imaging $(n=18)$. In vivo molecular imaging was performed on human clots pre-incubated in immunobubbles in a rat model of carotid artery occlusion $(n=19)$. Ultrasonic detectability of thrombotic material was compared.

Results: Specific abciximab immunobubble contrast enhancement was significantly higher compared to binding of control immunobubbles with a maximum difference of intensity at a gas volume of $0.2 \mu 1(\mathrm{p}=0.0013$ for MI $0.05, \mathrm{p}=0.0001$ for MI $0.7)$. Ultrasonic detectability of carotid thrombi in vivo was significantly higher for abciximab immunobubbles-targeted clots $(\mathrm{p}=0.016)$. Quantification of in vivo contrast enhancement in mean acoustic intensity displayed a highly significant increment for abciximab immunobubble targeted clots compared to non-specific immunobubble targeted clots $(\mathrm{p}=0.0001)$ and to native clots $(\mathrm{p}=0.0001)$.

Conclusion: This study demonstrates the feasibility of using a therapeutic agent tagged to microbubbles for selective targeting in diagnostic imaging.

\section{Experimental studies}

INFLUENCE OF CLOPIDOGREL, ASPIRIN AND A ASPIRIN-CLOPIDOGREL COMBINATION THERAPY ON THE NEUROLOGICAL- AND MICROVASCULAR SYSTEM FOLLOWING TRANSIENT ISCHEMIA

A. Trinkl, M. Vosko, N. Wunderlich, M. Dichgans, G.F. Hamann

Klinikum Großhadern/Experimental Stroke Research, Munich, Germany

Acetylsalicylic acid (ASA) and clopidogrel (CLO) could be protective through various pharmacological action sides, but earlier animal studies showed that a single dose administration of either of these drugs didn't reduce the infarct size in a focal ischemia model. This study evaluated the effects of an ASA-, CLO- and ASA CLO combo pre-treatment on the general outcome and the microvascular system of rats following transient ischemia. Using the suture model, we subjected 24 rats to focal ischemia ( $3 \mathrm{~h})$ and reperfusion (24h). Rats received either ASA (5 $\mathrm{mg} / \mathrm{kg} / \mathrm{d})$, or CLO $(1 \mathrm{mg} / \mathrm{kg} / \mathrm{d})$, or ASA_CLO $(2.5 \mathrm{mg} / \mathrm{kg} / \mathrm{d} ; 0.5 \mathrm{mg} / \mathrm{kg} / \mathrm{d}$ resp. $)$, or saline (control group) for 4 weeks prior to the experiment. The general outcome was determined by a behavior test (best: 18 points) and the infarct size. Collagen type IV, a marker for an intact basal lamina, was measured by western blot analysis. Only the CLO pre-treated animals had a significantly reduced infarct size $(167 \% \pm 13$, $74 \% \pm 15$ resp.; $\mathrm{p}<0.01)$ and they improved significantly the behavioural outcome $(11.4 \pm 0.6,14.5 \pm 0.5$ resp.; $\mathrm{p}<0.05)$ compared to control animals.

The collagen western blot in the cortex and in the basal ganglia showed in controls a loss to $64 \% \pm 5$ and $43 \% \pm 4$ compared to the non-ischemic side. This loss was partly inhibited by the ASA pre-treatment (cortex: $86 \% \pm 3, \mathrm{p}<0.05$;

Experimental studies 
basal ganglia: $70 \% \pm 9, \mathrm{p}<0.05)$ and strongly inhibited by the CLO- and the ASA_CLO_combo pre-treatment (cortex: $98 \% \pm 4, \mathrm{p}<0.01 ; 96 \% \pm 6, \mathrm{p}<0.01$ resp.; basal ganglia: $95 \% \pm 4, \mathrm{p}<0.01 ; 92 \% \pm 5, \mathrm{p}<0.01)$. CLO pre-treatment, and not ASA or ASA_CLO_combo, resulted in a ameliorated general outcome of animals following transient ischemia. CLO also protects the microvascular basal lamina against degradation.

\section{Experimental studies}

UP-REGULATION OF GLUTATHIONE-S-TRANSFERASES (GSTS) AND ACTIVATION OF NRF2 TRANSCRIPTION FACTOR: RELEVANCE TO ATHEROSCLEROSIS AND STROKES

F.M. Yatsu, K. Ranganna, O.P. Mathew

University of Texas - Houston Texas Southern University, Houston, TX, USA

Background and Purpose: VSMC proliferation is a critical element in the pathogenesis of atherosclerosis, an important cause of strokes. Butyrate, a fermentation product of dietary fiber, inhibits VSMC proliferation, potentially by modulating cellular redox state via up-regulation of glutathione (GSH)/GST anti-oxidant system. We show up-regulation of GST-P1 is linked to activation of Nrf2, which is implicated in transcriptional activation of antioxidant response element-mediated detoxifying enzyme expression, such as GSTs.

Methods: Proliferating VSMC were treated with or without $5 \mathrm{mM}$ butyrate for $48 \mathrm{hr}$ and $96 \mathrm{hr}$. At the end of treatment period, GST expression was monitored by western blotting and immunocytofluorescence. Activation of Nrf2 was evaluated by its translocation from cytoplasm to nuclear compartment both by western blotting and immunocytofluorescence.

Results: Butyrate treated VSMC exhibit increased expression of GST compared to untreated VSMC as assessed by western blotting. Interestingly, immunocytofluorescence analysis discloses that butyrate-induced GST-P1 expression is localized in nucleus in contrast to untreated VSMC. Furthermore, the immunocytofluorescence demonstrates that the up-regulated GST-P1 and Nrf2 are co-localized in nucleus. In contrast, the basal levels of GST-P1 and Nrf2 in untreated VSMC are confined to the periphery of the nucleus.

Discussion: The present study indicates a relationship between butyrate-induced GST-P1 expression and activation of Nrf2. Furthermore, the cytosolic GST-P1 translocates to nucleus in response to butyrate and co-localizes with activated $\mathrm{Nrf} 2$. The nuclear co-association of GST-P1 and Nrf2 in butyrate treated VSMC implies that GST-P1 may have other regulatory role(s) in addition to detoxification and regulation of cellular redox state, such as in cell cycle control and cell proliferation, both critical to atherogenesis and therefore to strokes. (Support: G12RR0345 grant from NIH/NCRR)

\section{Experimental studies}

DECREASED BRAIN EDEMA AFTER EXPERIMENTAL INTRACEREBRAL HEMORRHAGE IN MICE LACKING THE INDUCIBLE NITRIC OXIDE SYNTHASE GENE

S.W Jeong, E.S. Choi, S.H. Kim, J.S. Lee, J.Y. Cho, Y.J. Cho, E.M. Park, G.T. Oh, E.Y. Kim, D.W. Kim

Ilsan Paik Hospital, Inje University College of Medicine, Goyang-City,

Gyeonggi-do, South Korea

Background and Purpose: Brain edema after intracerebral hemorrhage (ICH) is an important prognostic factor. Inducible nitric oxide synthase (iNOS) is not normally expressed in brain, but induced after ischemia, and known to be involved in secondary neuronal injury. However, its significance in intracerebral hemorrhage was unknown. We tested whether iNOS influenced hematoma size and brain edema after ICH.

Methods: C57BL/6 mice $(\mathrm{n}=32)$ and iNOS knockout mice $(\mathrm{n}=24)$ were used. Experimental ICH was induced by intrastriatal stereotaxic administration of bacterial collagenase type VII $(0.075 \mathrm{U})$. Brain tissue was obtained at $72 \mathrm{hrs}$ after ICH. Volume of hematoma was quantified by spectrophotometric assay and brain water content was measured. Expression of iNOS was examined by reverse-transcriptionpolymerase chain reaction (RT-PCR) and immunohistological staining 24 hours after ICH.

Results: There was no significant difference in hematoma size between two groups. Brain water content of the lesional hemisphere was $79.71 \pm 0.78 \%$ in C57BL/6 mice and $78.96 \pm 0.69 \%$ in iNOS knockout mice $(p=0.03)$. Brain water content in non-lesional hemisphere was not significantly different between the two groups $(78.49 \pm 0.67 \%$ vs $78.58 \pm 0.64 \%, p=0.745)$. RT-PCR showed no iNOS mRNA in iNOS knockout mice after ICH. Immunohistochemistry showed iNOS immunoreactivity in peri-hematomal areas of C57BL/6 mice but not in iNOS knockouts.
Conclusions: We have demonstrated that although there was no difference of hematoma volume, brain edema was reduced iNOS knockout mice compared with littermate. These findings suggest that iNOS modulation could be an anti-edematic therapy for ICH.

\section{Experimental studies}

\section{PHARMACOLOGICAL ATTENUATION OF APOPTOSIS OF HUMAN BRAIN CAPILLARY ENDOTHELIAL CELL BY (-)BAP IN A MODEL OF} HYPOXIA/REOXYGENATION

L. Dénes, A. Gál, G. Szilágyi, Z. Bori, I. Miklya, Z. Nagy

Hung. Natl. Inst. Psychiatry and Neurology, Dept. Vasc. Neurol., Semmelweis Univ., Budapest, Hungary

Introduction: (-)-BPAP [R-(-)-1-(benzofuran-2-yl-)-2-propylamino- pentane] the newly developed selective mesencephalic enhancer substance has neuroprotective feature even at low doses (fm-pm) induction of $\mathrm{Bcl}-2$ and and neurotrophic factors. The cytoprotective of this drug candidate was studied on human brain capillary endothelial cells (HBEC)

Methods: The effect of (-)-BPAP (1 pM-100 micro-M) was tested on HBEC either treated before $(1 \mathrm{~h})$ and/or straight after the oxygen deprivation. The activity of living cells was studied by the alamar Blue assay (0, 2, 4, and $20 \mathrm{~h})$. Distribution of apoptotic and necrotic cells were determined by confocal laser microscope with AnnexinV, propidium iodide (PI) double staining.

Results: Following the hypoxia/reoxygenation stress (-)-BPAP improved with bimodal, bell-shaped - dose-response curves (at $4 \mathrm{hrs}$ and $20 \mathrm{hrs)}$ the survival of vascular EC. Addition of the compound to cells prior to hypoxia or at start of reoxygenation significantly reduced the percentage of caspase 3 -active cells $(75.8 \%)$. The number of dead cells decreased significantly, particularly post ischemia treatments at low (pM) and high (micro M) doses of drugs. Two different protocols of (-)-BPAP administration, namely prior to hypoxia and at start of reoxygenation, yielded rather similar effects on cell death and survival $(-58.1 / 67.7 \%$ versus $-66.4 / 73.8 \%)$. One hour pre- and post treatment with (-)-BPAP resulted in significant cell protection in HBEC.

Discussion: Cytoprotection is lead to an increased number of living cells decreasing the ratio of apoptotic and necrotic cells pre and/or post stress treatment. The cytoprotective effect of (-)-BPAP on HBEC at hypoxia-reoxygenation makes this new compound a promising drug candidate in the field of cerebrovascular injury. Supported by the Hungarian National Science Foundation (OTKA 2001 T-037887) and Health Scientific Council (ETT 096/2003 and ETT 140/2003).

\section{Experimental studies}

\section{APOPTOSIS INVOLVEMENT AFTER HUMAN SPONTANEOUS INTRACEREBRAL HEMORRHAGE: SOLUBLE FAS AND CASPASE-3 IN} BLOOD AND BRAIN TISSUE

P. Delgado, J. Alvarez-Sabín, A. Rosell, E. Santamarina, S. Abilleira, C. Molina, I. Fernández-Cadenas, L. Ortega, M. Quintana, J. Montaner Vall d'Hebron Hospital, Barcelona, Spain

Background: Apoptosis has been involved as a prominent form of cell death in intracerebral hemorrhage (ICH) animal models. Fas system and caspases activation play a central role in apoptosis. We aimed to investigate the presence of soluble Fas (s-Fas) and caspase-3 in plasma and brain tissue after acute intracerebral hemorrhage $(\mathrm{ICH})$ and to determine its influence on clinical and radiological features.

Methods: Soluble Fas (s-Fas) and caspase- 3 were determined in plasma by means of ELISA on admission in 78 consecutive ICH patients and serially $(24 \mathrm{~h}, 48 \mathrm{~h}, 7 \mathrm{th}$ day and $3 \mathrm{rd}$ month) in a subgroup of them $(n=21)$ just at the time of neurological and radiological assessment. Caspase- 3 activity was investigated in post-mortem brain tissue (perihematoma and contralateral areas) in four patients who died as a consequence of ICH

Results: Baseline s-Fas level in ICH patients was significantly lower than in healthy controls ( $199.51 \mathrm{vs} 269.57 \mathrm{pg} / \mathrm{mL}, \mathrm{p}<0.001$ ), while no difference was found between both groups in caspase- 3 level ( 2.26 vs $2.83 \mathrm{ng} / \mathrm{mL}, \mathrm{p}=0.29)$. S-Fas and caspase-3 temporal profile will be discussed. On admission, neither s-Fas nor caspase-3 level were associated with any clinical variable at baseline or follow-up and among the radiological features, s-Fas was found inversely correlated to perihematomal edema growing at follow-up $(\mathrm{r}=-0.33, \mathrm{p}=0.041)$. In addition, caspase- 3 activity was found increased at perihematomal area from 3 patients who died beyond 12 hours from ICH onset. In contrast the patient who died before 12 hours did not show any caspase- 3 activity at perihematoma area.

Conclusions: Decreased plasma s-Fas level together with increased caspase- 3 activity at perihematomal brain tissue is found early after $\mathrm{ICH}$, both suggesting an apoptosis involvement in this disease. 
REDUCTION OF SOD ACTIVITY, WHICH CAN REACT MORE EASILY WITH NO TO PRODUCE PEROXINITRITE AND INCREASE OXLDL, CONTRIBUTES TO PLAQUE VULNERABILITY

M. Uno, K.T. Kitazato, A. Suzue, K. Nishi, S. Nagahiro

The University of Tokushima Graduate School, Tokushima, Japan

Background: SODs have the potential for regulating redox signals modulated by ROS and RNS. The local steady-state of O2- depends on both the rate of superoxide production and the activity of endogenous SODs. Cu/ZnSOD and MnSOD are inactivted by $\mathrm{H} 2 \mathrm{O} 2$ and peroxynitrite, respectively. As the $\mathrm{O} 2$ - scavenged by SOD competes with NO for O2-, it is assumed that reduction of SOD activity predominantly promote the interaction with $\mathrm{NO}$ for $\mathrm{O} 2-$, leading to the increase of peroxynitrite and oxidized low-density lipoprotein (OxLDL). Here we provide evidence for the overwhelmed pro-oxidant systems in patients requiring carotid endarterectomy.

Subjects and Methods: Carotid plaques from 79 patients who underwent carotid endarterectomy were classified as vulnerable or stable based on histopathological findings. OxLDL as a biomarker of oxidative status was measured by a sandwich ELISA using mAb against oxidized phosphatidylcholine (FOH1a/DLH3; DLH3) and apoB IgG antibody.

Results: OxLDL levels in vulnerable plaques was significantly higher $(\mathrm{p}<0.01)$ and the total SOD activity was significantly lower than those in stable plaques $(\mathrm{p}<0.05)$, although MnSOD level was increased in vulnerable plaque. The plaque and plasma OxLDL level were inversely correlated with plaque SOD activity $(\mathrm{p}<0.01)$. The uric acid which the plasma physiological level can preserve the activity of $\mathrm{Cu}-\mathrm{ZnSOD}$ from degradation by $\mathrm{H} 2 \mathrm{O} 2$ was reduced one-forth to one-eight of plasma levels in all plaques. Immunohistochemical findings manifested the increased peroxinitrite and OxLDL in vulnerable plaque.

Conclusions: Ours is the first study to demonstrate an overwhelmed oxidant system due to decreased SOD activity in vulnerable- compared to stable plaques. In patients with vulnerable carotid plaques, an imbalance between the oxidant and antioxidant systems may induce high levels of OxLDL, which may play an important role in plaque instability.

\section{Experimental studies}

A NOVEL THERAPEUTIC STRATEGY (EPO AND HCG) IMPROVES FUNCTIONAL RECOVERY AFTER FOCAL ISCHEMIA IN RATS

L. Beleyev, L. Khoutorova, K.L. Zhao, F.M. Salzman, A.F. Moore,

A.W. Davidoff, J.T. Tucker, S.C. Cramer

Stem Cell Therapeutics Corp., Calgary, Canada

Human chorionic gonadotropin (hCG) and erythropoetin (EPO) induce neurorepair through neurogenesis by signaling endogenous neural stem cells. This study tested the hypothesis that the combination of hCG + EPO, given 24 hours after stroke onset, and significantly improves behavioral outcome.

Methods: Long-Evans rats recieved $90 \mathrm{~min}$ MCAo stroke by retrograde insertion of an intraluminal suture coated with poly-L-lysine. At 24 hours after stroke, animals were randomized into 4 treatment groups in a double-blinded manner: hCG was given IM, 300IU/kg, days 1,3,5; EPO, IV, $1440 \mathrm{IU} / \mathrm{d}$, days 6,7 and 8. Primary endpoint was Composite Neurological Score (placing and reflex tests, $0-12$ points, $0=$ normal, $12=$ maximal deficit) measured $1 \mathrm{hr}, 1,2,7,14,21,28$ adn 42 days after stroke; primary analysis was repeated measures ANOVA examining the time $\mathrm{X}$ treatment group factor.

Results: Body and cranial temperature showed no significant differences between groups. The dosing scheduling regimen of hCG + EPO ( $\mathrm{p}>0.0001)$, as well as each drug alone $(\mathrm{p}>0.005)$, resulted in lower scores as compared to saline over the 6 weeks. Beginning at 3 weeks after stroke onset, a trend favored hCG + EPO over either drug alone. Among secondary endpoints, time $\mathrm{X}$ treatment group was also significant $(\mathrm{P}>0.009)$ for the cylinder test, but not for swimming or sticky tape tests. Conclusion: The current therapeutic strategy improves behavioral outcome after stroke with a time window of 24 hours after stroke onset. Extensive human experience exists for both hCG and EPO, suggesting high potential for translation into studies of acute human stroke.
12 Experimental studies

IMAGING CELLULAR CHANGES IN THE REPERFUSED PENUMBRA USING 11C-FLUMAZENIL (FMZ) \& 11C-PK11195 (PK) IN THE SPONTANEOUS HYPERTENSIVE RAT (SHR) USING MICROPET

J.L. Hughes, J.S. Beech, T.D. Fryer, M. Cleij, R. Smith, O. Golovko, F.I. Aigbirhio, D.K. Menon, J.C. Clark, J.C. Baron University of Cambridge Clinical School, Cambridge, Cambridge United Kingdom

Background: Although early reperfusion improves outcome after stroke, the salvaged penumbra may be affected by post-ischemic cellular changes such as selective neuronal loss (SNL) and inflammation, potentially hampering recovery. Previous PET studies suggest FMZ and PK may be useful for in vivo investigation of SNL and microglia activation (MA). However, histological validation is lacking, while the relationship between SNL and MA has not been studied. To address these issues, we used both FMZ and PK in correlation with histopathological assessment of SNL and MA in a rodent temporary MCAo model that generates extensive cortical SNL and MA with very limited pan-necrosis.

Methods: SHRs received 45min distal clip MCAo. 14 days later, high specific activity FMZ ( $n=6)$ and PK $(n=4)$ were successively administered and MicroPET data acquired. We implemented fully quantitative PET and generated FMZ distribution volume (DV) and PK binding potential (BP) maps. After the scan, brains were processed immunohistochemically for SNL and MA using NeuN and OX42, respectively. Using an MR template, 44 MCA territory ROIs were defined, and FMZ affected/unaffected ratios (A/U Rs) and PK BP were correlated with ROI histopathology scores using Kendall's tau_b.

Results: Across ROIs, there was a decrease in FMZ A/U R $(\mathrm{P}<0.001)$, particularly significant in the striatum, insular cortex and somatosensory cortex S1 and S2; this decrease was significantly but weakly correlated with NeuN pathology (tau= -0.237 ). In parallel, significant increase in PK BP was found in affected MCA cortex with a strong correlation with $\mathrm{OX} 42$ scores across all ROIs (tau $=0.563$ ). Across ROIs, NeuN and OX42 scores strongly correlated (tau= 0.664), but ipsilateral PK $\mathrm{BP}$ and FMZ A/U Rs only weakly correlated (tau $=-0.180$ ).

Conclusions: These results validate PK as an in vivo marker of MA in the reperfused penumbra, corroborating its usefulness as an in vivo tool to image inflammation in the penumbra. These results also suggest that factors other than SNL, such as receptor plasticity, may be contributing to the observed changes in FMZ

\section{Experimental studies}

THROMBOELASTOGRAPHY IN ASPIRIN RESISTANCE IN ACUTE STROKE D. Brenner, J. Payne, B. Coull, P. McDonagh University of Arizona, Tucson, AZ, USA

Background: Although aspirin (ASA) is a very effective anti-platelet agent, many patients suffer strokes despite taking therapeutic doses. These patients may be aspirin resistant (ASA-R), or have other mechanisms of platelet activation not affected by ASA. In an ongoing study, we are evaluating platelet function with a novel system: thromboelastography (TEG, Haemoscope Corp) with the platelet mapping assay. This system isolates platelet activation from arachidonic acid (AA) stimulation, the pathway usually blocked by ASA.

Methods: To date, we have recruited acute stroke or TIA patients $(n=9)$ who were taking ASA, and performed TEG-platelet mapping within 48 hours of symptom onset. Results are expressed as reduction of AA-induced platelet activation. We compared the TEG results to the platelet function analyzer (PFA)-100.

Results: One patient had no inhibition $(8 \%)$, two patients had moderate inhibition (45-55\%), and six patients had complete inhibition (88-100\%) of AA-induced platelet activation. Four patients were ASA-R by PFA-100 (closure time $<184 \mathrm{sec}$ ). The patient with no platelet inhibition by TEG was also ASA-R by PFA-100. There were 3 patients with complete inhibition of AA-induced platelet activation, but were ASA-R by PFA-100.

Discussion: The TEG results indicated that most of these patients had strong anti-platelet responses to ASA, therefore suggesting they were not resistant to ASA and had alternate pathways of platelet activation causing their strokes. There was poor correlation between the TEG and PFA-100, which could be due to differences in blood flow velocity and shear stress used by the two tests. More research is needed to better understand how platelets are activated in stroke. 
14

Experimental studies

IROXANADINE A HSP CO-INDUCER DRUG CANDIDATE DOWNREGULATES THE PROLIFERATION OF CELLS ISOLATED FROM MYOINTIMAL HYPERPLASIA AND UPREGULATE VASCULAR ENDOTHELIAL CELLS

L. Dénes, Z. Bori, E. Csonka, L. Erntz

Hung. Natl. Inst. Psychiatry Neurol., Dept. Vasc. Neurol., Semmelweis Univ.,

Budapest, Hungary

Introduction: Early carotid restenosis is characterized by permanent cell proliferation following mechanical intervention of carotid artery. This limits the efficacy of stroke prevention output. Iroxanadine ${ }^{\circledR}$ (Cytrex\&Biorex) can moderate the endothelial cell dysfunction by co-induction of heat shock proteins in several vascular syndromes. The inhibitory action of Iroxanadine ${ }^{\circledR}$ treatment $(1,2,4 \mathrm{H}$ oxidiazinetype derivative) on the myointimal cell proliferation has been tested in culture system in our laboratory.

Methods: Myointimal hyperplasia (MIH) cells were isolated from surgical material of carotid restenosis and cultured to test the effect of Iroxanadine ${ }^{\circledR}$ (1 micro M 10 nano M) on cell proliferation by BrdU incorporation. Drug effects on MIH were compared to the effects on control human vascular smooth muscle cells (VSMC) and HBEC. There were determined the amount of Hsp 70 by western blot analysis, the cyclin dependent kinase (Cdk1A) mRNA expression by PCR techniques and the cell cycle by FACS.

Drug effect was tested in normal condition and after hypoxia/reoxygenation.

Results: Without treatment in hypoxia/reoxygenation the proliferation of upregulated MIH cells were enhanced while the activity of HBEC downregulated $\mathrm{MIH}$ : $0.266 \pm 0.016$ versus $0.336 \pm 0.024, p<0.05$; HBEC: $1.249 \pm 0.10$ versus $0.878 \pm 0.11$, $\mathrm{p}<0.05$ ). Iroxanadine ${ }^{\circledR}$ treatment significantly reduced the cell-proliferation of MIH cells ( 1 micro $\mathrm{M}$ and 0.1 micro $\mathrm{M}=45.1 \%$ and $40.0 \%$ ), while the HBECs react contrary ( 1 micro $M=46.2 \%$ ). The proliferation of healthy VSMC did not change significantly in those conditions. In MIH cells after Iroxanadine ${ }^{\circledR}$ treatment the amount of HSP 70 and Cdk1A mRNA level increased significantly.

Conclusion: These data confirmed the notion, that Iroxanadine ${ }^{\circledR}$ (BRX-235) has a therapeutic potential in the prevention of early restenosis following vascular surgery or balloon angioplasty.

\section{Experimental studies}

\section{A CHRONIC TREATMENT WITH CDP-CHOLINE INCREASES NEURONAL PLASTICITY AND IMPROVES SENSORIMOTOR RECOVERY AFTER} STROKE

I. Lizasoain, A. Cardenas, O. Hurtado, J. Serena, J. Vivancos, T. Sobrino, J.J. Secades, A. Dávalos, J. Castillo, M.A. Moro

Fac Medicina. Universidad Complutense de Madrid, Madrid, Spain

Introduction: Stroke is the third cause of death and disability in most of the developed countries and, unfortunately, the majority of neuroprotective drugs have failed in the clinical practice. An explanation for this could be the early action of these drugs (6-24h.), enhancing the necessity of new drugs acting in later phases of the disease. Therefore, we decided to investigate if a chronic administration of Citicoline (CDP-choline), started 24 hours after stroke, could improve the functional recovery after stroke.

Methods: Citicoline ( $1 \mathrm{~g} / \mathrm{kg}$ day; i.p.) was administered 24 hours after the permanent medial cerebral arterial occlusion (pMCAO) and during 28 days to adult male Sprague-Dawley rats $(250-275 \mathrm{~g})$. To assess the functional recovery we have performed the staircase reaching test and the elevated body swing test (EBST), for studying sensorimotor integration and asymmetrical motor function respectively. In addition, to study potential neuronal substrates of the improved function, we examined the dendritic morphology of layer $\mathrm{V}$ pyramidal cells in the undamaged motor cortex using a Golgi-Cox procedure.

Results: The treatment with CDP-choline improved functional outcome in both the staircase test $(\mathrm{MCAO}+\mathrm{CDP}=87.0 \pm 6.6 \%$ pellets eaten vs. $\mathrm{MCAO}+\mathrm{SAL}=40.0 \pm 4.5$ $\mathrm{p}<0.05)$ and the EBST $(\mathrm{MCAO}+\mathrm{CDP}=70.0 \pm 6.8 \%$ vs. $\mathrm{MCAO}+\mathrm{SAL}=88.0 \pm$ 5.4 ; contralateral swing $\mathrm{p}<0.05$ ). The animals treated with $\mathrm{CDP}$-choline showed enhanced dendritic complexity and spine density compared with saline group.

Conclusion: Our results suggest that a chronic treatment with CDP-choline initiated 24 hours after the insult is able to increase the neuronal plasticity within noninjured and functionally connected brain regions as well as to promote functional recovery.
16 Experimental studies

(-)-BPAP BEFORE AND AFTER FOCAL PHOTOTHROMBOTIC ISCHEMIC CORTICAL INJURY

L. Dénes, I. Miklya, F. Erdö, G. Szilágyi, E. Wappler, Z. Bori, Z. Nagy Hung. Natl. Inst. Psychiatry Neurol., Dept. Vasc. Neurol., Semmelweis Univ., Budapest, Hungary

Introduction: The effect of (-)-BPAP, the newly developed selective mesencephalic enhancer substance, on histological and functional outcome after experimental stroke in rats was evaluated.

Method: Permanent focal ischemia was induced by cortical photothrombosis in Wistar rats with rose Bengal $(20 \mathrm{mg} / \mathrm{kg}$, i.v.), irradiated by cold light source (for 10 min.). Rats treated by (-)-BPAP $(0.2 \mathrm{mg} / \mathrm{kg}$, i.p./day for 3 days $)$. The infarction was visualized with $2 \%$ TTC. The lesion size has been evaluated by morphometry using image analysis software (AxioVison/Zeiss).

The effect of (-)-BPAP on the learning ability of rats was measured in the shuttle box. The acquisition of a two-way conditioned avoidance reflex (CAR) was analyzed in the box during 5 consecutive days. The rats were trained with 100 trials per day. At each learning session, the number of CARs, escape failures (EFs) and intersignal reactions (IRs) were automatically counted and evaluated by multi-way analysis of variance (ANOVA).

Results: The light-irradiation for $10 \mathrm{~min}$ after injection of rose Bengal caused welldemarcated tissue damage in the cerebral cortex and decreased the performance of the lesioned rats in the shuttle box.

(-)-BPAP treatment caused statistically significant reduction of the infarct size (from $1.190 \pm 0.096 \mathrm{~mm}^{2}$ to $0.256 \pm 0.057 \mathrm{~mm}^{2} ; \mathrm{n}=18$ ) and improved the performance of the rats $(45.75 \pm 15$ to $75.67 \pm 7.2)$.

Conclusion: The extension of focal ischemia and the behavioral data well correlated. The neuronal enhancer (-)-BPAP produced a remarkable protection in the cortex. The infarct size reduced and functional activity has been recovered.

Supported by the Hungarian National Science Foundation (OTKA 2001

T-037887) and Health Scientific Council (ETT 096/2003 and ETT 140/2003).

\section{Experimental studies}

QUANTIFICATION OF HEMIANOPIA AS A NEW MEANS OF OUTCOME ASSESSMENT IN EMBOLIC MCA OCCLUSION IN RATS

M. Nedelmann, T. Wilhelm-Schwenkmezger, B. Alessandri, A. Heimann, F. Schneider, B.M. Eicke, M. Dieterich, O. Kempski

Johannes Gutenberg University, Mainz, Germany

Background: Assessment of functional deficits improves the impact of recanalisation studies in the embolic MCA occlusion model in rats. This study evaluates quantification of hemianopia in an 8-arm maze as a new means of outcome assessment.

Methods: Male Wistar rats were submitted to MCA clot embolism $(n=14)$ or sham surgery $(n=7)$. In order to achieve a larger variety of lesion size, subgroups were subjected to differently sized emboli (30 and $40 \mathrm{~mm}$ in 7 animals, respectively). During a follow-up period of 6 days, the turning angle of the animals' exploration behavior in an 8-arm maze was determined. The turning angle was defined as the animal's deviation from straight movement through the centre of the maze. A turn to the neighbouring arm would thus signify a turn of $135^{\circ}$, omitting one arm a turn of $90^{\circ}$ and omitting two arms a turn of $45^{\circ}$. Turns to the right were counted as positive, turns to the left as negative angles. The angles of every turn during one session were added and divided by the total number of passages through the centre, so as to receive a mean angle per turn. Animals were perfusion-fixed for histology on day 7 (blinded examination).

Results: There was a strong tendency of the ischemic animals to explore the maze towards their right, whereas sham animals displayed a more random exploration behavior. Results were significant for both experimental groups as compared to sham on day 2 and additionally for the $40 \mathrm{~mm}$ group at the end of the observation period. Furthermore, the correlation between total infarct volume and the turning angle was statistically significant throughout the observation period (day $1: \mathrm{R}=0.68$; day 2 : $\mathrm{R}=0.87$; day $3: \mathrm{R}=0.74$; day $4: \mathrm{R}=0.84$; day $5: \mathrm{R}=0.83$; day $6: \mathrm{R}=0.66$ ).

Discussion: Hemianopia in rats submitted to cerebral clot embolism is frequent. Assessment in an 8-arm maze allows, to some extent, quantification of hemianopia. This testing of exploration behavior is a novel approach to quantification of functional impairment. It may be valuable in future studies, since it tests for deficits that are not detected by more conventional outcome scores based on motor functions. 
SECONDARY REMOTE LESIONS FOLLOWING FOCAL ISCHEMIC DAMAGE OF SENSORYMOTOR CORTEX IN ADULT RAT

P. Holmberg, S. Liljequist, A. Wagner

Karolinska institutet, Stockholm, Sweden

Background: We have recently developed a model of focal cerebral ischemia in rat, this model produce a reproducible and transient neurological deficit.It is induced by extradural compression and leads to an ischemic lesion. The lesion produces a neurological deficit presented as a left side hemiparesis. The functional disturbance is accompanied by moderate brain damage in the sensorimotor cortex.The aims of the present study are to develop the compression model further by describing the locations of secondary remote lesions and the development of these lesions over time. In addition we report data about physiological parameters important for the interpretation of our findings.

Methods: Rats were subjected to a beam-walking test before and after surgery to measure neurological deficits. After the brain area of interest had been identified the defined area of the skull was cut out, leaving the dura intact. A plexiglass piston was lowered $3 \mathrm{~mm}$ at the rate of $1 \mathrm{~mm} / \mathrm{min}$. The compression was kept for $30 \mathrm{~min}$. The temperature was kept constant. Blood gas analysis, blood glucose, electrolytes, Laser Doppler Flow (LDF) and intracranial pressure were measured. Following different time points the animals were anaesthetized the brain removed and cryoprotected. Serial sections were cut and degenerating neurons were detected with Fluoro-Jade and examined.

Results: Physiological measurements did not differ significantly between different time points.Secondary lesion areas were found both in Thalamus and Hippocampus.The cell lesions were most pronounced on day 5. No secondary lesion was presented in any animal on day 1 .

Discussion: The study of secondary remote lesions in experimental stroke has been limited, depending in part by the large size lesion produced by many stroke models. We think that this model might be a valuable tool for future studies concerning the mechanisms behind secondary remote lesions and how these lesions affect cognitive and behavioural parameters.

\section{Experimental studies}

\section{CEREBROLYSIN ENHANCES FUNCTIONAL RECOVERY IN A RAT MODEL} OF FOCAL CEREBRAL INFARCTION

J.M. Ren, D. Sietsma, S.M. Qiu, S.P. Finklestein, H. Moessler, E. Doppler EBEWE Pharma Ges.m.b.H. Nfg.KG, Unterach/Attersee, Austria

Background: Stroke recovery treatments are designed to augment recovery processes in the brain after stroke, including neural sprouting and progenitor cell proliferation, migration, and differentiation. Cerebrolysin, a preparation with neurotrophic and neuroprotective peptides, is an approved drug and well established for the treatment of neurological disorders like stroke. This current in vivo study seeks to exploit the property of Cerebrolysin, to promote recovery after stroke.

Methods: Rats were subjected to middle cerebral artery occlusion followed by a once daily intraperitoneal administration of Cerebrolysin $(1.0 / 2.5 / 5.0 \mathrm{ml} / \mathrm{kg}$ body weight) starting 24, 48 or 72 hours after stroke onset for 21 days. As this focal cerebral infarction involves cortical regions important in sensorimotor function, enhancement of sensorimotor recovery was assessed by forelimb and hindlimb placing and body swing tests.

Results: Comparing to vehicle-treated animals, Cerebrolysin treatment significantly enhanced recovery of sensorimotor function after stroke when treatment was started within 48 hours after stroke onset. A dose of $2.5 \mathrm{ml} / \mathrm{kg}$ turned out to be most effective.

Discussion: These data showed a discrete time window for initiation of Cerebrolysin treatment to obtain efficacy in enhancing sensorimotor recovery following stroke. However, extrapolating these findings to human stroke patients, the duration of this window may well be longer. Most importantly, and in contrast to naturally occurring neurotrophic factors, the described pharmacodynamic actions are observed after peripheral administration of the drug. These results support the usefulness of Cerebrolysin as a treatment to enhance neurological recovery after stroke.
20 Experimental studies

TALAMPANEL A NON-COMPETITIVE AMPA ANTAGONIST ATTENUATES CASPASE-3 DEPENDENT APOPTOSIS IN MOUSE BRAIN AFTER TRANSIENT FOCAL CEREBRAL ISCHEMIA

L. Dénes, G. Szilágyi, A. Gál, Z. Nagy

Hung. Natl. Inst. Psychiatry Neurol., Dept. Vasc. Neurol., Semmelweis Univ., Budapest, Hungary

Introduction: Talampanel (IVAX) is a non-competitive AMPA-antagonists and an allosteric modulator of AMPA receptors has a remarkable neuroprotectiv effect in different rodent stroke models. Modulation of AMPA-type receptors can regulate production of trophic factors, promote synaptic plasticity and functional activity after ischemic brain injury.Data from our laboratory can confirm and even extend the findings that were recently published. (Erdö et al., Brain Res Bull, 66 (2005) 43-49). In the present study we analyze the effect of talampanel, the non-competitive glutamate receptors antagonist, on apoptotic and necrotic cell death by double immunohistochemical staining.

Methods: The focal cerebral ischemia in mice (male, C57B16/J/Charles River) was induced by transient $(60 \mathrm{~min}$.) MCA occlusion and $24 \mathrm{~h}$ reperfusion and treated with talampanel $(6 \times 2 \mathrm{mg} / \mathrm{kg}$ i.p., started at $15 \mathrm{~min}$ after the occlusion and then in every $15 \mathrm{~min}$ ). The areas of both the ischemic damage and the whole hemispheres (stained by cresyl violet) were measured in $\mathrm{mm}^{2}$, using a morphometric program (AxioVision/Zeiss).

The apoptotic\& necrotic cells were counted by double immunohistochemical staining on confocal laser microscope.

Results: The infarct size is decreased significantly by talampanel treatment (from $57.1 \pm 7.2 \mathrm{~mm}^{2}$ to $\left.18.9 \pm 2.6 \mathrm{~mm}^{2} ; \mathrm{p}<0,001\right)$.

The number of TUNEL-positive cells is reduced significantly after talampanel treatment (from $962 \pm 13.0$ to $604 \pm 6.9$; $p<0.01$ ). Moreover, an eliminated caspasedependent necrosis was resulted by talampanel treatment.

Conclusion: A neuroprotective effect of talampanel has been demonstrated in MCA occlusion stroke model by morphometry and cellular level by confocal microscope. The reduced number of apoptotic cells and inhibition of caspase-3 activity reflect the mechanism of cytoprotection by talampanel.

Study was supported by the Hungarian National Science Foundation (OTKA 2001 T-037887) and Health Scientific Council (ETT 096/2003).

\section{Experimental studies}

\section{ADENOVIRUS CONTANING BCL-2 OR BCL-XL ANTIAPOPTOSIS GENES REDUCE CELL LOSS AFTER HYPOXIA IN PC12 CELL CULTURE}

A. Agal, G. Szilágyi, E. Wappler, L. Dénes, Z. Nagy

National Institute of Psichiatry and Neurology, National Stroke Center, Budapest, Hungary

Introduction: Hypoxia induces cell necrosis and/or apoptosis. Antiapoptotic gene therapy could be an option to prevent the cell death. In this paper we tested Bcl-2 and Bcl-XL gene therapy using an adenovirus construct for transfection in PC-12 cell culture system.

Materials and Methods: The cells have been treated by Argon gas ( 1 hour) for induction hypoxic cell injury followed by $4-48$ hours of restored oxygen. The cells were infected with different concentration (10-200 PFU/cell) of beta-galactosidase to test the rate of adenovirus transfection. Virus constructs contaning Bcl-2 or $\mathrm{Bcl}-\mathrm{XL}$ genes are utilized before or after hypoxia. We examined the expression of Bcl-2, Bcl-XL and Gap-43 protein levels with immuncytochemistry and western blotting. The apoptotic and necrotic cell death was visualized with annexin V propidium iodide double staining, and the mitochondrial membrane potential with TMRE staining.

Results: After LacZ transfection practically all the cells were take up the signal gene using the adenovirus vector. Gene transfers of $\mathrm{Bcl}-2$ and $\mathrm{Bcl}-\mathrm{XL}$ resulted in a significant increase of protein expression. Hypoxia and reoxygenization caused apoptotic $(39 \pm 2 \%)$ and necrotic $(6 \pm 1 \%)$ cell death in the culture while the genes delivery reduced the number of apoptotic cells (Bcl-2: $5 \pm 2 \%$ and Bcl-XL: $9 \pm 1 \%$ ). The mitochondrial membrane potential was decreased after hypoxia, while the $\mathrm{Bcl}-2$ and $\mathrm{Bcl}-\mathrm{XL}$ gene transfers were protectiv in mitochonrial membran. The Gap43 protein increased significantly after $\mathrm{Bcl}-2$ and $\mathrm{Bcl}-\mathrm{XL}$ transfections following hypoxic condition.

Conclusions: The PC12 cells could be transfected by adenovirus constructs. In our in vitro model the Bcl-2 and Bcl-xl antiapoptotic gene transfer decreased the hypoxic impairments by reducing apoptosis. These genes are increasing the plasticity protein as well GAP-43. These double action of antiapoptotic genes are promising to in the repair mechanisms following hypoxic and ischaemic injury in the brain. 
(-)-DEPRENYL EFFECT ON BCL-2, SOD1, GAP-43 GENES UPREGULATION IS RELATED TO THE NFKB PATHWAY

Z. Bori, P. Koska, E. Duda, K. Kolev, L. Dénes, Z. Nagy

Semmelweis University, Budapest, Hungary

Introduction: (-)-Deprenyl is a selective monoamine oxidase-B inhibitor that exerts protective effects in various experimental and pathological conditions. These effects could be related to the upregulation of genes involved in anti-apoptosis $(\mathrm{Bcl} 2)$ and elimination of free radicals (SOD1). Both genes are known to be regulated by NFkB activity. (-)-Deprenyl upregulates GAP-43 expression also that may be responsible to neuronal plasticity.

Methods: To study the mechanism by wich (-)-deprenyl exerts its action PC12 cells, and L-929 cell line transfected with an NFkB-lucirefase reporter construct were used. Cells were subjected to hypoxia or hyperoxia. The cell death were visualised with propidium-iodide staining, the level of proteins were measured by Western-blot. NFkB pathway activity was studied using a lucirefase assay.

Results: (-)-Deprenyl reduces significantly the hypoxia/H2O2-induced cell death Furthermore (-)-deprenyl induces the translocation of NFkB to the nucleus and the transcriptional activity of NFkB in a time- and dose-dependent manner. Paralel with the induction of NFkB activity a significant increase of the expression of all the selected proteins (Bcl2, SOD1, GAP43) were detected (treated vs control GAP43 $13.8 \pm 1.6$ vs $7.2 \pm 1.5$; BCL $5.9 \pm 0.6$ vs $3.7 \pm 0.4$; SOD1 $14.4 \pm 2.3$ vs $7.9 \pm 1.1 ; \mathrm{p}<$ $0.05 \mathrm{n}=3$ ).

Discussion: The cytoprotection effect of (-)-deprenyl has been confirmed in our model system. The augmented gene expression and increased protein levels are related to the NFkB/IkB pathway. Anti-apoptosis genes and plasticity gene GAP-43 could be connected by this pathway.

This work was supported by the Hungarian National Science Foundation (OTKA 2001 T-037887) and Health Scientific Council (ETT 96/2003).

\section{Experimental studies}

PLASMA AND CSF GLUCOSE-6-PHOSPHATE DEHYDROGENASE ACTIVITY IN ACUTE ISCHEMIC STROKE

V. Selakovic, R. Raicevic, L. Radenovic, M. Jovanovic, B. Mrsulja

MMA, Belgrade, Yugoslavia

Introduction: Glucose-6-phosphate dehydrogenate (G6PD; EC1.1.1.49) is the key regulatory enzyme of the pentose phosphate pathway. G6PD determines the amount of NADPH, the principal intracellular reductant that is necessary in regeneration of reduced glutathione. Considering the importance of G6PD for cellular antioxidant defenses and the documented role of oxidant stress in neuronal cell death during ischemia, we investigated the activity of G6PD in the plasma and cerebrospinal fluid (CSF) of patients with different kinds of acute ischemic stroke.

Methods: The study included 85 patients of both sexes, mean age $65 \pm 8$ years, divided in three groups with: brain infarction (BI), reversible ischemic attack (RIA), and transient ischemic attack (TIA). Control group consisted of 15 patients with radicular lesions of discal origin, subjected to diagnostic radiculography, without signs of interruption in the passage of CSF. Samples were collected within first seven days after the cerebrovascular insults. History, clinical examination and cerebral CT scan established the diagnosis. Activity of G6PD was determined by spectrophotometric method.

Results: During the first seven days of ischemia there was significant decrease in G6PD activity in the plasma and CSF, compared to controls. The decrease was the highest in BI $(p<0.001)$, somewhat lower in RIA $(p<0.01)$ and the lowest in TIA $(\mathrm{p}<0.05)$ patients. In the plasma and CSF of stroke patients, the G6PD activity was decreased progressively in relation to the severeness of the disease, indicating an acute inactivation or modification of enzymes, probably mediated by free radicals.

Conclusion: The G6PD activity in the plasma and CSF of patients with acute ischemic stroke could be an indicator of cellular antioxidant defenses dysfunction and neuronal cell damage.

\section{Experimental studies}

CHANGES IN HYALURONAN EXPRESSION AND METABOLISM FOLLOWING ISCHAEMIC STROKE IN MAN

M. Slevin, A. AlQteishat, J. Gaffney, F. Rubio, D. West, S. Kumar, P. Kumar, N. Mitsios, J. Krupinski

Manchester Metropolitan University, Manchester, United Kingdom

Background: The extent of recovery from stroke is associated with the survival of neurons, particularly in peri-infarcted regions. Angiogenesis is critical for the development of new microvessels and leads to re-formation of collateral circulation, reperfusion, and better recovery. Hyaluronic acid (HA) is a major component of the brain extra-cellular matrix and is also a regulator of cellular differentiation, migration, proliferation and angiogenesis

Methods: Here, we have studied the expression of total HA and low molecular weight oligosaccharides of HA (o-HA) in post-mortem tissue and in the serum of patients 1, 3, 7 and 14 days after stroke using enzyme studies, Western blotting, and immunohistochemistry (IHC).

Results: Both HA and o-HA were significantly increased in stroke-affected tissue and at all measured time points in the serum. IHC performed using a HA-specific biotinylated binding protein showed enhanced expression of HA in blood vessels and both the cytoplasm and nuclei of peri-infarcted neurones. Up-regulation of HA synthases 1 and 2 and hyaluronidases 1 and 2 occurred in inflammatory cells from stroke and peri-infarcted regions of the brain as well as in microvesssels and the cytoplasm and nucleus of neurones in peri-infarcted areas. RHAMM was increased in the cytoplasm of neurons, in the intima of larger blood vessels, and in the endothelial cells of microvessels in stroke-affected tissue. TSG-6 was mainly increased in infiltrating cells from inflammatory regions.

Discussion: HA breakdown is a feature of the acute stage of stroke injury. Increased o-HA expression after stroke might by detrimental through enhancement of the inflammatory response, whilst activation of $\mathrm{HA}$ and/or o-HA-induced cellular signalling pathways in neurones and microvessels might impact on the remodelling process by stimulating angiogenesis and revascularisation, as well as the survival of susceptible neurones.

\section{Epidemiology of stroke}

\section{Epidemiology of stroke}

OLDER PATIENTS WITH ACUTE STROKE IN DENMARK: TREATMENT, CARE AND MORTALITY. A NATIONWIDE FOLLOW-UP STUDY K.D. Palnum, P. Petersen, A. Ingemann, J. Mainz, P. Bartels, S.P. Johnsen Aarhus University Hospital, Aarhus, Denmark

Background: Age-related differences in treatment and care may exist among patients with stroke.

We examined quality of treatment and care according to age and the possible impact of any differences on mortality in a population-based nationwide follow-up study in Denmark.

Methods: Using data from the Danish National Indicator Project (DNIP), we identified a total of 29549 patients, who had been admitted with acute stroke between January 2003 to October 2005. Data on key processes of treatment and care and patient characteristics were obtained from DNIP. Data on 30- and 90-days mortality were obtained from The Danish Civil Registration System. We compared the proportion of patients who received adequate treatment and care across age groups ( $\leq 65,>65-80$ and $>80$ years). Further, we used Cox proportional regression to compare 30- and 90 days mortality rates across age groups adjusted for patient characteristics and quality of treatment and care.

Results: The proportion of eligible patients who received adequate treatment and care declined with age for all the examined processes. The relative risk (RR) of receiving specific processes of treatment and care ranged from 0.66 (95\% confidence interval (CI):0.60-0.73) to 0.97 (95\% CI:0.95-0.99) when comparing patients $>80$ years with patients $<65$ years. The mortality increased with age, however, adjusting for the age-related differences in treatment and care did not influence the age-related differences in mortality.

Discussion: We found indications that elderly stroke patients in Denmark may receive a poorer quality of treatment and care compared to younger stroke patients; however, the differences were not substantial for most of the examined processes of treatment and care and the differences did not appear to explain the higher mortality among older patients.

\section{Epidemiology of stroke}

ACUTE ISCHEMIC STROKE CARE IN HISPANIC MESTIZOS. A MEXICAN MULTICENTRIC ISCHEMIC STROKE REGISTRY

C. Cantu, J.L. Ruiz-Sandoval, R. Rangel, J. Villarreal, A. Arauz, L. Murillo, F. Barinagarrementeria, J.A. Fernandez, A. Roman, E. Garcia, for the PREMIER Collaborative Study Group

Mexican Stroke Association, Mexico City, Mexico

Introduction: Data on acute stroke care in Hispanic mestizos is negligible. This first Mexican ischemic stroke registry is being developed to improve our knowledge 
regarding risk factors profile, outcome, current diagnostic and treatment strategies, and long-term follow-up.

Methods: We analyzed data from patients with acute cerebral ischemia who are being enrolled in a large multicentric stroke data bank in Mexico. From November 2004 to December 2005, 905 patients were evaluated. Standardized data assessment was used by all centers.

Results: There were 806 cerebral infarctions (89\%) and 99 TIAs (11\%) in 453 $(50 \%)$ men (age 65.7) and $452(50 \%)$ women (age 67.6). Risk factors: hypertension $64 \%$, diabetes mellitus $34 \%$, current smoking $18 \%$, dislipidemia $31 \%$, previous ischemic stroke $16 \%$, previous AITs $17 \%$, ischemic heart disease $13 \%$, and atrial fibrillation $12 \%$, BMI $>25$ in $65 \%$. Stroke onset time and arrival to hospital was documented in $85 \%$ of patients: $14 \%$ arrived within 3 hours, $21 \%$ within 3 to 6 hours, $16 \%$ within 6 and 24 hours, and $49 \%$ after 24 hours. All patients received cerebral imaging, either CT scan $(90 \%)$ or MRI (24\%), patients were examined by duplex sonography in $28 \%$, TCD $3 \%$, by angiography in $13 \%$, and echocardiography in $20 \%$ of patients. Stroke subtype classification was: large-artery atherosclerosis $27 \%$, cardioembolism $22 \%$, small-vessel disease $20 \%$, other $5 \%$, concurrent etiology in $6 \%$, and undetermined in $21 \%$. Thrombolysis was carried out in $2.5 \%$ of patients; carotid endarterectomy or angioplasty was performed within 30 days of stroke in $1.3 \%$. Clinical outcome at day 30 in patients with infarctions was: mRS $0-2$ in $37 \%$, $\mathrm{mRS} 3$ in $13 \%$, mRS $4-5$ in $28 \%$, and death in $22 \%$.

Conclusions: More than $60 \%$ of patients with acute ischemic stroke had poor outcome in this Hispanic Mestizo population. A minority of patients were treated with thrombolysis. Quality improvement interventions are needed to improve acute stroke care in our population.

\section{Epidemiology of stroke}

\section{IMPACT OF FAMILY HISTORY ON THE KNOWLEDGE, ATTITUDE, BELIEF AND PRACTICE OF STROKE AND ITS RISK FACTORS AMONG SINGAPOREANS}

N. Venketasubramanian, A. Yin, B.H. Heng, M. Toh, L.Y. Wong, T.S. Cheah

Disease Management, National Healthcare Group, Singapore, Singapore

Background and Purpose: It is unclear if people with a family history of stroke(FHS) are more aware about stroke than those without. This study was performed compare the impact of FHS on knowledge, attitudes, beliefs and practices of stroke among Singaporeans.

Methods: Singaporeans aged 15 to $69 \mathrm{yr}$ were randomly selected to participate in this population-based survey from December 2004 to October 2005. Trained survey officers conducted face-to-face interviews to elicit information on demographics, family history of stroke, presence of risk factors, their knowledge of stroke, and lifestyle behaviour and practices. Findings of respondents with at least one family member with stroke were compared with that without, using SPSS v13.

Results: There were a total of 2,600 respondents, whose demographics were similar with that of the Singapore population. 266(10.2\%) of respondents reported FHS Respondents with FHS had a higher level of knowledge of causes of stroke $(83.1 \%$, $69.3 \%, \mathrm{p}<0.001)$, that stroke can $\operatorname{recur}(95.1 \%, 91.6 \%, \mathrm{p}=0.43)$, and that stroke can be prevented $(85.0 \%, 76.3 \%, \mathrm{p}=0.001)$. There was no difference in knowledge between the two groups on stroke morbidity i.e. it can get worse, and an afflicted person can die from it. A higher percentage of respondents with FHS believed that they can get stroke sometime in their life compared with those without FHS(80.1, $71.7 \%, \mathrm{p}=0.004)$. There were fewer $\operatorname{smokers}(9.4 \%, 14.5 \%, \mathrm{p}=0.025)$ and more regular exercise $(35.0 \%, 27.6 \%, \mathrm{p}=0.014)$ amongst respondents with FHS, but no difference in alcohol consumption. More respondents with FHS had been screened for high blood pressure $(88.3 \%, 83.6 \%, \mathrm{p}=0.045)$, high cholesterol( $66.2 \%, 54.2 \%$, $\mathrm{p}<0.001)$ and diabetes $(75.6 \%, 66.8 \%, \mathrm{p}=0.004)$.

Conclusion: There was better knowledge among people who had a family member with stroke. Having a family member afflicted by stroke had a favourable impact on their attitudes and belief, and generally translated into healthy lifestyle behaviour and practices.

\section{Epidemiology of stroke}

STEPS STROKE - RESULTS FROM THE FEASIBILITY STUDY

T. Truelsen, P.U. Heuschmann, R. Bonita

World Health Organization, Noncommunicable Diseases and Public Health,

Geneva, Switzerland

Background: The World Health Organization's (WHOs) Stepwise approach to Stroke Surveillance project (STEPS Stroke) was pilot tested in year 2005. The present study presents hospital data (step 1) from 7 participating centres.

Methods: All sites used the same definitions for stroke symptoms, subtype classification, and time to hospitalisation. Events were hospitalised stroke patients from

Poster Session Second Visit

Epidemiology of stroke study sites in India (3), Mozambique (1), Russia (1), Nigeria (1), and Iran (1). Continuous and complete registration of all events was done at all sites using the WHO definition of stroke. Duration of data registration ranged from 2-13 months (median 8 months). 6 study sites used STEPS Stroke manual version 1.4/2.0, whereas one centre incorporated the STEPS Stroke questionnaire into a local questionnaire. Analyses were done at the WHO collaborating centre, Muenster University.

Results: A total of 4,507 stroke patients were registered during follow-up. Mean age was 63.5 years (SD15.3), ranging from 2- 105 years. There were $2,520(55.9 \%)$ men, $1,977(43.9 \%)$ women, and missing values for $10(0.2 \%)$ patients. $2,010(45 \%)$ of patients were aged less than 65 years. There were 849 patients (18.9\%) who had had a previous stroke. Subtype of stroke was: 2,924 (64,9\%) ischemic; $963(21.4 \%)$ intracerebral hemorrhage; $74(1.6 \%)$ subarachnoid hemorrhage; while unspecified in $507(11.3 \%)$; missing data in $39(0.9 \%)$. Type of stroke was verified by diagnostic techniques in 4,214 (93.5\%) of patients. Nearly half, 2,015 (44.7\%), of stroke patients were admitted on the same day of stroke symptoms onset. Two-thirds were admitted within the first day after stroke, while 241 (5.4\%) were admitted later than one week after stroke symptoms onset.

Conclusions: The WHO STEPS Stroke questionnaire is a useful tool for collecting standard data on stroke in different countries. Expansion with more sites will improve the understanding of the stroke burden.

\section{Epidemiology of stroke}

\section{Epidemiology of stroke}

\section{SEX DIFFERENCES IN THE STEPS STROKE PROJECT - RESULTS FROM THE FEASIBILITY STUDY}

P.U. Heuschmann, T. Truelsen, R. Bonita, for the STEPS Stroke Project The STEPS Stroke International Stroke Surveillance Coordinating Unit, Muenster, Germany

Background: The World Health Organization's (WHOs) Stepwise approach to Stroke Surveillance project (STEPS Stroke) was pilot tested in different study sites in different countries in the year 2005. Data were analysed regarding sex differences in patient characteristics, stroke subtypes and hospital management.

Methods: The present study presents hospital data (step 1) from 7 study sites in India (3), Mozambique (1), Russia (1), Nigeria (1), and Iran (1). Duration of data registration in study sites ranged from 2-13 months (median 8 months). All sites used the same definitions for stroke symptoms, subtype classification, and time to hospitalisation. Analyses were carried out at the WHO collaborating centre, Muenster University.

Results: A total of 4,497 stroke patients with known sex were registered. Mean age of all patients was 63.5 years (y) (SD 15.3), ranging from 2-105y. Women were older 
compared to men (Mean age 65.0y (SD 15.5) versus 62.3y (SD 15.0), respectively; $\mathrm{p}<0.001$ ). This pattern was found in all study sites. $39.6 \%$ of women and $48.7 \%$ of men were aged less than 65 years. Previous stroke was more often diagnosed in women compared to men $(20.0 \%$ versus $17.9 \%$, respectively; $\mathrm{p}<0.001)$. Women tended to be hospitalized more often within the first day after stroke compared to men $(46.8 \%$ versus $43.2 \%$, respectively; $\mathrm{p}<0.001)$. No statistically significant differences between women and men were found for stroke subtypes and for use of diagnostic techniques to verify stroke subtype.

Conclusions: The STEPS Stroke project is a useful tool for investigating differences in acute stroke management among hospitalized stroke patients from different countries. More data are needed on potential sex differences with respect to clinical characteristics and risk factors of stroke patients.

\section{Epidemiology of stroke}

\section{IN-HOSPITAL ACUTE STROKE: FREQUENCY AND POSSIBLE}

\section{THROMBOLYTIC TREATMENT}

P. Nencini, M. Nesi, I. Romani, M. Martini, G. Pracucci, D. Inzitari, on behalf of the Careggi Hospital Stroke Team

Careggi University Hospital, Florence, Firenze, Italy

Background: Information on stroke occurring in patients already in hospital is limited. This group of patients could potentially be assessed more rapidly than others and could be candidate for intravenous or intra-arterial thrombolysis.

Methods: Careggi University Hospital is a large tertiary referral hospital where is available a Stroke Team for acute interventional therapies. A prospective series of in-hospital acute stroke between February 2004 and December 2005 was derived from the Careggi Hospital Acute Stroke Registry.

Results: 73 (6.5\%) out of 1115 consecutive acute stroke occurred during hospitalization (45 males, mean age 71 years, median NIHSS 9). Sixty-seven strokes were ischemic and six hemorrhagic. Twenty-seven ischemic stroke patients had surgery (13 general, 8 cardiac, 6 vascular), 13 interventional procedures (7 percutaneous coronary and 2 carotid angioplasty, 4 angiography), while 27 had no invasive procedure. Thirty-four (51\%) patients with ischemic stroke were evaluated within 3 hours from symptom onset. Among these patients, 9 were treated with intra-arterial thrombolysis ( 7 males, mean age 72 years, median NIHSS 17), and 1with rtPA intravenosus thrombolysis (male, 78 years, NIHSS 6). The 3-month modified Rankin Scale score was 0-2 in 5 patients and 3 in 1 patient; 3 patients died for myocardial infarction and 1 for symptomatic intracranial hemorrhage.

Conclusions: In-hospital stroke is not rare. Thrombolytic therapies seem feasible and reasonably safe in selected patients. The awareness by hospital physicians of such interventions may reduce delay in assessment of patients who have strokes while in hospital.

\section{Epidemiology of stroke}

POTENTIAL FOR ACUTE STROKE INTERVENTIONS AMONG IN-HOSPITAL STROKE PATIENTS. A SUBGROUP ANALYSIS OF THE NORTH EAST MELBOURNE STROKE INCIDENCE STUDY (NEMESIS) A.K. Gilligan, H.M. Dewey, J.W. Sturm, R.A. Macdonell, G.A. Donnan National Stroke Research Institute, Melbourne, Australia

Background: In-hospital stroke patients avoid the pre-hospital delays that preclude many stroke patients from acute therapies. A study was performed to assess the eligibility for acute stroke interventions among in-hospital stroke patients registered in a community-based stroke incidence study.

Methods: Eligibility for tissue plasminogen activator (tPA), aspirin, stroke unit management and a hypothetical neuroprotectant were assessed among all stroke cases occurring between May 1997- April 1998 within NEMESIS.

Results: Among 306,631 people, 721 strokes were recorded. 79 strokes (11\%) occurred in hospitalised patients. In-hospital stroke patients were older (79 years vs. 76 for presenters, patients who presented to hospital following stroke onset). Atrial fibrillation, recent myocardial infarction and peripheral vascular disease were more common among in-hospital stroke patients. Less investigations were performed among in-hospital stroke patients (Brain imaging: $80 \%$ vs. $96 \%, \mathrm{P}<0.001$ ) and anticoagulation following stroke was more common ( $66 \%$ vs. $25 \%, \mathrm{P}<0.001)$ compared to presenters. The proportions of in-hospital stroke patients that would have been eligible for treatment were: $11(14 \%)$ for intravenous tPA, $65(82 \%)$ for acute stroke unit care, $38(48 \%)$ cases for aspirin and $33(41 \%), 40(51 \%)$ and $48(61 \%)$ for a neuroprotectant within 3, 6 and 12 hours respectively. These proportions are similar to the proportions eligible for treatment among presenters (11\%, 94\% and $48 \%$ for tPA, SCU and aspirin respectively) but higher than presenters for neuroprotectant $(24 \%, 32 \%$ and $41 \%)$

Conclusions: In-hospital stroke patients represent a small but significant group who could benefit from acute stroke interventions and who currently may receive sub-optimal treatment.

\section{Epidemiology of stroke}

\section{Epidemiology of stroke}

EDUCATION AND COUNTRY OF ORIGIN AS DETERMINANTS OF FATAL STROKE OVER A LONG-TERM FOLLOW-UP IN A MULTICULTURAL SOCIETY

U. Goldbourt, D. Tanne

Tel Aviv University Sackler Medical Faculty, Tel Aviv, Israel

Background: There is limited research associating educational attainment and occupation with the incidence of stroke. In a migrant country, these associations may be further intertwined with geographic/ethnic origin.

Methods: We followed up 10,059 men of diverse countries of origin, civil servants and municipal employees, who participated in the Israeli Ischemic Heart Disease (IIHD) study in 1963, of whom $86 \%$ had migrated form Europe, North Africa and the Near-East area of Asia. Extensive demographic, biochemical, socioeconomic and clinical information was collected on these men in 1963, 1965 and 1968.

Results: Over a 23 -yr follow up, 3473 men died, among whom for 364 the recorded underlying cause of death was stroke. Reported formal education was grouped into 3 categories i.e. elementary, high school and any higher education. Fatal stroke was associated with education, the age-adjusted hazard ratio being $0.78(95 \% \mathrm{CI}=0.68$ 0.89) per "step". Adjustment for blood pressure, smoking and diabetes made little difference. Area of origin was closely related to incident fatal stroke, as the Africanborn migrant exhibited the highest rates. Relative to the latter, the HR of stroke mortality among migrants from Balkan countries and central Europe were $0.61 \mathrm{CI}$ $0.40-0.91$ ) and 0.63 (CI 0.40-0.97), respectively. Nevertheless, additional adjustment for area- of- origin modified the HR of decreasing education scale only somewhat to 0.82 (CI, 0.71-0.96). Substituting Socio-economic status (SES) index, combined from reported education and civil service occupational scales, for education, had no appreciable effect on these results ( $\mathrm{HR}=0.87, \mathrm{CI}$ 0.79-0.95 per "step" in a 5-step SES scale)

Discussion: It appears that both education, as reflected by years of schooling, and area-of-origin may be independently related to the risk of fatal stroke in a multi-cultural society such as Israel of the 1960-1980s. 


\section{Epidemiology of stroke}

KNOWLEDGE AND PREVALENCE OF TIA IN THE SWISS POPULATION ABOVE 55 YEARS

P. Michel, S. Engelter, P. Lyrer, R. Sztajzel, D. Georgiadis, B. Tettenborn,

J. Bogousslavsky, R.W. Baumgartner

Centre Hospitalier Universitaire Vaudois, Lausanne, Switzerland

Background: Knowledge about TIA may influence utilisation of highly effective secondary prevention. In a recent US survey, only $9 \%$ of the adult population knew the meaning of TIA, and $6.6 \%$ above age 55 were once given a diagnosis of TIA.

Methods: A telephone survey of non-institutionalised persons above 55 years of age living in Switzerland was performed. Demographic data as well as risk factors, knowledge of the meaning of TIA, of its symptoms, and its prevalence were collected. Descriptive and comparative statistics were used.

Results: Among 1002 persons with a mean age of $68.5( \pm 0.3)$ years, $51 \%$ knew the meaning of TIA, and two thirds knew at least one of its cardinal features. Knowledge was significantly better in the German speaking than in the French speaking regions; however, it was not better in patients with previous TIAs or strokes nor in patient with one or several risk factors. $6 \%$ of the population had symptoms compatible with TIA in the past and $4.4 \%$ had once been given a diagnosis of TIA. Of patiens with TIA, $64 \%$ saw a physician within 24 hours, and $14 \%$ later than 7 days.

Conclusion: Understanding of the term "TIA" is higher in Switzerland than in a recent US survey, but knowledge about symptoms is poor, especially in the French speaking regions. Patients with a diagnosis of TIA are not more knowledgeable despite their higher risk. The $4.4 \%$ prevalence of TIA is similar to that reported in other parts of the world.

Supported by the Swiss Stroke Foundation.

\section{Epidemiology of stroke}

\section{DIFFERENT PROGNOSIS IN PATIENTS WITH ISCHEMIC STROKE WITH}

\section{AND WITHOUT ARTERIAL HYPERTENSION}

S. Sacco, L. Olivieri, P. Sucapane, C. Marini, A. Carolei

University of L'Aquila, L'Aquila, Italy

Background: Arterial hypertension has been associated to a worse prognosis in ischemic stroke patients, even if in the early stroke phase it might improve cerebral perfusion. We evaluated differences in characteristics and prognosis of patients with ischemic stroke according to blood pressure status.

Materials and Methods: All incident first-ever ischemic strokes in a 5-year period (1994-1998) were included in the prospective, population-based L'Aquila registry. Patients were followed-up to 10 years.

Results: Data on hypertension were available in 3,569 out of 3,594 patients: 2,307 $(64.7 \%$ ) were hypertensive (mean age \pm SD $75.8 \pm 9.5$ ) and 1,262 were normotensive (mean age \pm SD $74.2 \pm 12.4)$. Proportions of women $(56,0 \%$ vs $46,6 \% ; \mathrm{P}<0.0001)$ and of diabetes mellitus ( $27.9 \%$ vs $22.9 \% ; \mathrm{P}=0.0012)$ were higher in the hypertensive than in the normotensive group while proportions of atrial fibrillation, cigarette smoking, hypercholesterolemia, coronary heart disease, and peripheral arterial disease were similar. The 30-day case fatality rate was $20.4 \%$ for hypertensive and $21.6 \%$ for normotensive patients $(\mathrm{P}=0.4238)$ and the 1 -year case-fatality rate was $32.1 \%$ for hypertensive and $35.8 \%$ for normotensive patients $(\mathrm{P}=0.0235)$. During the 10-year follow-up, the annual mortality rates were higher for hypertensive with respect to normotensive patients (2-year: $9.6 \%$ vs $9.0 \%$, 3-year: $7.8 \%$ vs $7.5 \%$; 4-year: $7.3 \%$ vs $6.6 \%$; 5 -year: $8.9 \%$ vs $6.4 \%$, 6-year: $6.7 \%$ vs $4.7 \% ; 7$-year: $8.0 \%$ vs $6.8 \%$; 8 -year: $7.6 \%$ vs $5.2 \%$; 9 -year: $10.6 \%$ vs $5.2 \%$, and 10 -year: $9.4 \%$ vs $5.2 \%$ ). Besides, the long-term survival was $31.0 \%$ for the hypertensive and $35.8 \%$ for the normotensive patients $(\mathrm{P}=0.8928)$ while the long-term stroke recurrence rate was similar (13.0\% vs $12.1 \%$; $\mathrm{P}=0.6142)$.

Discussion: Even if arterial hypertension might provide benefits early after the stroke possibly increasing cerebral perfusion, in the following years mortality of hypertensive overcomes that of normotensive patients.

\section{Epidemiology of stroke}

CEREBROVASCULAR ACCIDENT IN HIV/AIDS PATIENTS: THE TWO YEARS RETROSPECTIVE STUDY IN HIGHLY ACTIVE ANTI RETROVIRAL TREATMENT (HAART) ERA

K. Subsai, S. Kanoksri

Rajavithi Hospital, Bangkok, Thailand

Previous population base study, in pre-highly active anti retroviral treatment (HAART) era, showed AIDS to be closely associated with both ischemic stroke and hemorrhagic stroke. In HAART era, several studies continue reported the evidence of rare cardiovascular events such as myocardial infarction and ischemic stroke following treatment with protease inhibitor base regimens. Interestingly, incidence rate of cerebrovascular events in non-protease inhibitor base regimens, such as non nucleotide reverse transcriptase inhibitor (NNRTI) plus nucleotide reverse transcriptase inhibitor (NRTI) (GPO-vir: Stavudine-D4T + Lamivudine-3TC + Nevirapine-NVP) had not been reported. So we performed two years retrospective study to identify incidence rates of cerebrovascular events in HAART era by comparison with previous incidence in the pre-HAART era. Altogether 506 AIDS patients were treated with GPO-vir during the specified period. The overall incidence of ischemic stroke and hemorrhagic stroke were increased. Based on a comparison of the prevalence rates of IS and ICH detected here with those reported before the HAART era in different parts of the world, the prevalence rates of both ischemic and hemorrhagic strokes were more than twofold. The average prevalence of ischemic stroke before the HAART era was $1.17 \%$ (95\% CI $0.87-1.54 \%$ ), and the incidence of ischemic stroke in this study was $2.35 \%$ (95\% CI $0.64-6.02 \%)$. The average prevalence of ischemic stroke before the HAART era was $0.21 \%(95 \% \mathrm{CI}$ $0.09-4.01 \%)$, and the prevalence of ischemic stroke in this study was $1.17 \%(95 \%$ CI $0.14-4.24 \%)$.

In conclusion, the incidence rate and prevalence of cerebrovascular accident in GPO-vir HAART era had increased.

\section{Epidemiology of stroke}

\section{ELECTIVE AND ISOLATED CAROTID ENDARTERECTOMY IN} CALIFORNIA: HEALTH DISPARITIES IN UTILIZATION AND OUTCOMES, BUT NOT READMISSION

B.S. Kennedy, S.P. Fortmann, M.A. Winkleby, R.S. Stafford

Stanford Prevention Research Center, Stanford University School of Medicine, Stanford, CA, USA

Background: Few studies have examined the effects of race upon carotid endarterectomy (CEA) use and perioperative outcomes, and among these, there are conflicting results. Further, studies considering the effects of race upon CEA readmission risk are lacking.

Methods: For whites and nonwhites (blacks, Hispanics, Asians/Pacific Islanders), CEA rates were calculated using an inpatient database, and logistic regression was used to examine the effects of race upon in-hospital death and stroke, as well as CEA readmission.

Results: In California, a total of 8,080 whites, 228 blacks, 643 Hispanics, an 325 Asians/Pacific Islanders underwent at least one elective, isolated CEA between January 1 st and December 31st, 2000. Symptomatic disease was less common among whites than other groups. However, whites underwent CEA at rates about 3 -fold greater than that of blacks and Hispanics, and about 4-fold greater than that of Asians/Pacific Islanders. The odds of perioperative death and stroke were greater for nonwhites than for whites, but after adjustment for patient and hospital factors, these differences were only significant for stroke (OR=1.7, $\mathrm{P}=0.013)$. For both outcomes, the final models had good predictive accuracy. Overall, CEA readmission risk was about $7 \%$, and no significant racial differences were observed ( $\mathrm{P}=0.110)$.

Conclusions: These findings have several implications. First, CEA may be overused by whites and/or underused nonwhites. Second, if racial disparities exist in CEA outcomes, they are likely to be mediated, in part, by patient and hospital factors The models developed here may help identify patients at high-risk of complications. Further research with larger databases (or pooled data) is needed in this area. Finally, significant racial disparities in first-ever CEA use do not persist with subsequen CEA use. These results may imply that whites and nonwhites are similar in their progression of carotid artery disease after initial treatment.

\section{Epidemiology of stroke}

\section{EPIDEMIOLOGY OF HEMORRHAGIC STROKE IN THE COAST AND GORSKI KOTAR COUNTY - CROATIA DURING THE PERIOD 2001-2005: A HOSPITAL BASED STUDY}

L. Tuškan-Mohar, I. Antonc, S. Dunatov, I. Strenja-Linic, K. Bla?ina, M. Buc, A. Jurjevic, K. Willheim

University Hospital Center, Rijeka, Croatia

Background: The aim of the study was to examine the frequency of hemorrhagic stroke in the Coast and Gorski Kotar County - Western Croatian region.

Methods: Most of these patients had been treated at the Department of Neurology, Rijeka University Hospital Center. Using medical records and protocols the epidemiological charachteristics of hemorrhagic strokes (HS) were analyzed for five-year period (2001-2005). Average annual age and sex incidence rates per 100000 population were calculated. Brain hemorrhages were grouped according to

Epidemiology of stroke 
age, gender, location, volume of hematomas, Glasgow Comma Score (GCS), risk factors and treatment outcome were analysed.

Results: During the five-year period there were in total 3699 stroke patients out of which $590(15.9 \%)$ had had HS. Four hundred and forty five of them were residents of Coast and Gorski Kotar County (239 men and 206 women). The age-adjusted incidence rate was 28.22/100 000 (CI 95\% 22.54-34.91), for males 30.57 (CI 95\% 22.28-40.90), for females 26.02 (CI 95\% 18.58-35.44). The mean age for men was $68.2 \pm 11.2$, women $67 \pm 10.8$. The most common localisations of hemorrhages were basal ganglia and thalamus $200(44.9 \%)$. Seventhy patients $(15.7 \%)$ had had subarrachnoidal hemorrhages. Hypertension was the most common risk factor followed by hyperlipidemy and obesity. More than half of patients had more than one risk factor.

Discussion: In this study we have examined preliminary epidemiological and clinical data of brain hemorrhages, which, as a separate group, were not proper followed up in this region of Coast and Gorski Kotar County. Further prospective studies have been predicted.

\section{Epidemiology of stroke}

\section{ULTRASONIC CHARACTERISTICS OF ATHEROSCLEROTIC PLAQUES IN} SYMPTOMATIC AND ASYMPTOMATIC PATIENTS WITH SEVERE

UNILATERAL CAROTID STENOSIS

G. Telman, E. Kouperberg, D. Yarnitsky

Rambam Medical Center, Haifa, Israel

Background and Purpose: Duplex ultrasound (DU) is a useful tool for investigation of extracranial cervical vessels in patients with suspected disease of internal carotid artery (ICA). The aim of this study was to compare the ultrasonic characteristics of atherosclerotic plaques in symptomatic and asymptomatic patients with severe unilateral carotid stenosis.

Methods: Two hundred eighty one patients with severe unilateral carotid stenosis were included into the study. 183 patients were asymptomatic and 98 patients were symptomatic. According to the ultrasonic appearance all plaques found in stenotic ICA were classified as heterogenous calcified, heterogenous noncalcified, homogenous hypo-, iso- or hyperechoic.

Results: The homogenous hypoechoic plaqies were significantly more frequent in symptomatic then asymptomatic patients - $29.4 \%$ and $20 \%$ correspondently $(\mathrm{p}=0.05)$. There was no difference in frequency of other plaques in both groups. Conclusion: The presence of homogenous hypoechoic plaques by DU in patients with severe carotid stenosis can predict unfavorable course of the disease.

\section{Epidemiology of stroke}

\section{A PRIOR TRANSIENT ISCHEMIC ATTACK IS INDEPENDENTLY ASSOCIATED WITH DECREASED IN-HOSPITAL CASE FATALITY IN DIABETIC AND NON-DIABETIC ACUTE STROKE PATIENTS \\ J. Zsuga, R. Gesztelyi, I. Fekete, L. Mihálka, L. Csiba, D. Bereczki \\ University of Debrecen, Debrecen, Hungary}

Background: The phenomenon of ischemic preconditioning (IP) is well established concerning healthy human hearts; however limited information is available about its occurrence or its integrity in diabetes mellitus following transient ischemic attacks (TIA) of the brain. Therefore we set out to assess if a prior cerebral ischemic episode (stroke or TIA) is able to confer protection as reflected by in-hospital case fatality, in the presence and absence of diabetes mellitus.

Methods: We investigated 2874 acute stroke patients included in the prospective, hospital-based Debrecen Stroke Database; of whom 673 had previous stroke and 195 had prior TIA.

Results: Following adjustment for active confounders, TIA in the history was associated with decreased odds for in-hospital case fatality in the un-stratified model (OR, 0.53; CI, 0.29 to $0.98, \mathrm{p}=0.041$ ) and following stratification by the absence (OR, 0.53; $\mathrm{CI}, 0.27$ to $1.04, \mathrm{p}=0.068$ ) or the presence (OR, 0.36; $\mathrm{CI}, 0.075$ to 1.77 , $\mathrm{p}=0.211$ ) of diabetes for patients having prior TIA. Conversely, IP phenomenon could be detected when stroke severity characterized by the Mathew score was chosen as the outcome measure (coefficient being 3.47 ; $\mathrm{CI}, 1.05$ to $5.90 \mathrm{p}=0.002$ ). Discussion: These data suggest that TIA may have a cerebral ischemic preconditioning effect in the human brain. Whether the lack of statistical significance in diabetes is due to the effect of restricted power or the loss of IP is yet to be determined by future studies.
18 Epidemiology of stroke

\section{SPONTANEOUS CERVICAL ARTERY DISSECTION IN A MULTIETHNIC} POPULATION

A. Pieri, M.M. Alves, R.A. Valiente, G.S. Silva, M.S. Rocha, A.M. Massaro São Paulo Federal University - São Paulo Hospital, Santa Marcelina Hospital, São Paulo, Brazil

Background and Methods: Spontaneous cervical artery dissection (SCAD) is a frequent cause of ischemic stroke in young adults. There are no published studies to date analyzing ethnic differences in SCAD. The aim of our study was to compare the demographic and clinical features, predisposing factors and vascular territory among White $(\mathrm{W})$ and Afro-Brazilians $(\mathrm{AB})$ patients with SCAD evaluated at two Stroke Services - Hospital São Paulo and Santa Marcelina, São Paulo, Brazil.

Results: From May 2001 through May 2005, we evaluated 36 patients with SCAD using a comprehensive protocol. The majority of patients were white (32), 4 were $\mathrm{AB}$, and only one was Asian-Brazilian. The mean age was similar between the two groups ( $\mathrm{W}=40.4 \mathrm{yrs}$; range $20-57$ and $\mathrm{AB}=39.3 \mathrm{yrs}$; range $32-44$ ), whereas male gender was more frequent in $\mathrm{W}$ patients $(\mathrm{p}=0,02)$. A history of a minor predisposing event, such as head flexion or rotation during sports activities, had a tendency to be more common in W. Few patients had past history of modifiable stroke risk factors and hypercholesterolemia was more frequent in $\mathrm{AB}$ patients $(\mathrm{p}=0,09)$. History of migraine was common in both groups. The most frequent clinical manifestation at onset in $\mathrm{W}$ and $\mathrm{AB}$ patients was headache. Severe headache with an abrupt onset and vertebral location (VAD) was more frequent in $\mathrm{W}(\mathrm{p}=0.04)$. Horner's syndrome was only observed in $\mathrm{W}$ patients. There was no difference in the 3 months follow-up between $\mathrm{W}$ and $\mathrm{AB}$ patients, using modified Rankin scale.

Conclusion: Despite having similar access to our public hospitals, SCAD patients were more frequently $\mathrm{W}$ and this was particularly significant for male patients with VAD. These differences may be crucial in the etiology of SCAD. Differences in minor predisposing events may be a reflection of ethnic socio-economic activities. In addition, the relationship between heritable disorders of connective tissue and ethnicity in SCAD deserves further investigation.

\section{Epidemiology of stroke}

ISCHEMIC STROKE SUBTYPES IN KERALA, SOUTH INDIA B.V. Maramattom, G.V. Kuruttukulam Lourdes Hospital, Kochi, India

Introduction: There is limited data on stroke classification in developing countries such as India.

Aims: To determine ischemic stroke subtypes presenting to a large neurology department in an urban setting in south India.

Materials and methods: We planned to conduct a retrospective-prospective study from 1994 January to 2006 March. We analyzed the medical records of all ischemic stroke patients admitted or transferred to the neurology service during this period. From 1994 January till 2005 June, the charts were analyzed retrospectively. From 2005 June to 2006 March, patients were collected prospectively. For patients encountered in the last one year, hospital arrival times following the onset of stroke were also determined. Ischemic stroke subtypes were subclassified according to a modified Oxfordshire community stroke project classification.

Results: Approximately 2000 patients with ischemic stroke were encountered during the study period. The vast majority of ischemic stroke subtypes were lacunar strokes (LACS) or partial anterior circulation strokes (PACS). Total anterior circulation strokes, posterior circulation strokes, multi infarct states, diffuse white matter hypodensities, internal borderzone or external borderzone (watershed) infarctions and venous infarctions were encountered in descending frequency. Less than $5 \%$ of patients arrived within the 3 hour time window for thrombolysis in the prospective group.

Conclusions: Our study provides the data on ischemic stroke subtypes in an urban population in south India in a state with a high quality of health care. The vas majority of patients suffered a lacunar stroke (LACS) or partial anterior circulation strokes (PACS). Only a minority of patients arrived within the time window for IV thrombolysis with rtPA.

\section{Epidemiology of stroke}

\section{THE STROKE OBSERVATIONAL STUDY (SOS)}

S.A. Blecic, V. Thijs

Erasme Hospital, Brussels, Belgium

Background and Aim of the Study: The stroke Observational Study (SOS) is a survey performed in Belgium among the general practionners (GPs) under the 
supervision of the Belgian Stroke Council, during a 3 month-period and designed to assess:

1. The proportion of patients suffering a vascular disease (i.e. stroke, MI, PAD, ...) seen in a GP surgery.

2. How the GPs managed patients with stroke and more globally patients with known risk factor for stroke

3. If the fact to have a stroke or any vascular disease (i.e. stroke MI or PAD) changed the patient lifestyle

Patients and methods: 9273 consecutive pts from 334 GPs were included. The average age of the analyzed population was $61.1 \pm 18$ years range 21-101]. Khi square test and logistic regression were used for data comparisons

Results: 9273 consecutive pts from 334 GPs were included. The average age of the analyzed population was $61.1 \pm 18$ years range $21-101.31 \%$ of the sample was aged more than 75 , while $10.7 \%$ was under 25 years. Hypertension $(45 \%)$ and dyslipidemia $(37.5 \%)$ were the most frequent finding in the medical history, 28, 5\% suffered both conditions while $46 \%$ suffered a cardiac disesase $20.5 \%$ were current smokers $37 \%$ of the whole population was treated with an antiothrombotic agent among them $73.7 \%$ were under ASA, $10 \%$ under Clopidogrel and $12.7 \%$ under Warfarin. $1715 \mathrm{pts}(18.5) \%$ had a stroke and $2345 \mathrm{pts}$ (25.3\%) suffered a MI or PAD. The comparison of vascular profile disclosed several differences between the Stroke Population (SP) and the Other Vascular Population (OVP). Increase in age (78 vs 61 years, $\mathrm{p}<0.001)$, sedentary lifestyle $(78 \%$ vs $47 \%, \mathrm{p}<0.001)$, hypertension ( $73 \%$ vs $44 \%, \mathrm{p}<0.0001)$, diabetes $(24 \%$ vs $12 \% \mathrm{p}<0.001)$ favoured stroke, while dyslipidemia ( $17 \%$ vs $34 \% \mathrm{p}<0.001)$ and smoking ( $13 \%$ vs $21 \% \mathrm{p}<0.001)$ were less frequently encountered in the SP than in pts with OVP. Treatments vary widely since $56 \%$ of the SP was treated with diuretics, $42 \%$ with $\beta$-blockers and $40 \%$ received ACE inhibitors. In the OVP $44 \%$ were on diuretics, $49 \%$ on $\beta$-blockers and $32 \%$ on ACE inhibitors. $45 \%$ received statins in the SP for $25 \%$ in the OVP. In The SP 59\% were treated with AAS, $21 \%$ with Clopidogrel and $14 \%$ with Warfarin while in the OVP $78 \%$ were on ASA, $7 \%$ on Clopidogrel and $12 \%$ on Warfarin

$18 \%$ of the SP suffered atrial fibrillation (AF) while $5 \%$ of the general population had AF $(\mathrm{p}<0001) .50 \%$ of the SP with AF being treated with warfarin, $41 \%$ with AAS and $9 \%$ with clopidogrel while $6 \%$ of the GP was treated with Warfarin, 37\% with AAS and $10 \%$ with clopidogrel.

Logistic regression disclosed that combination of Hypertension, sedentary life style and AF favoured stroke while smoking and dyslipidemia favoured pts with OVP

Conclusions: Almost one fifth of the population followed by the Belgian GP had a stroke and one fourth had OVP. This SP is older than the general populationa nd also than the OVP and is more frequently strikken by hypertension and AF while dyslipidemia and smoking favoured OVP

We saw the treatment in both OVP and SP met the international criteria except for statins which were much more prescribed in the SP than in the OVP and AF where only $50 \%$ of the SP was on anticoagulation.

\section{Epidemiology of stroke}

\section{KNOWLEDGE OF RISK FACTORS AND STROKE SIGNS IN CROATIAN POPULATION}

V. Vukovic, M.J. Kesic, M. Roje Bedekovic, S. Morovic, I. Mikula, M. Budišc, A. Lovrenc Huzjan, V. Demarin

University Hospital "Sestre Milosrdnice", Croatia

Aims: The aim of this community-based survey was to determine baseline stroke knowledge in Croatian population.

Methods: A multiple choice questionnaire was designed and divided into three sections:1)demographic data,2)knowledge regarding stroke risk factors and warning signs for stroke, 3 ) action the patients would undertake if confronted with risk of stroke and information resources regarding health.The data were collected as "door to door" survey.

Results: There were 720 completed and analysed questionnaires (54.9\% women).The respondents were divided into groups according to demographic data (gender, age, level of education and living place).The respondents indicated stroke symptoms as following: speech disorder $82 \%$, paresthesiae in half of the body $71 \%$, weakness of arm or leg $55 \%$, unsteady gait $55 \%$, malaise $53 \%$, monocular loss of vision $44 \%$. The risk factors most frequently identified were hypertension $64 \%$, stress $61 \%$, smoking $59 \%$, elevated lipids $53 \%$, obesity $52 \%$, coagulation disorder $47 \%$, alcoholism $45 \%$, low physical activity $42 \%$, elderly age $39 \%$, cardiac diseases $38 \%$, weather changes $34 \%$, drugs $33 \%$ and diabetes $32 \%$.If confronted with stroke signs $37 \%$ of respondents would consult the general practitioner and $31 \%$ a neurologist.Among patients with a risk factor, only diabetics were aware that their risk factor might cause stroke $(\mathrm{p}<0,001)$. Multiple logistic regression analysis showed that risk factors more frequently listed by the oldest group were elderly age and diabetes $(\mathrm{p}<0.05)$; coagulation disorders by females $(\mathrm{p}<0.01)$; physical activity, stress and oral contraceptives by respondents living in city $(\mathrm{p}<0.05)$. Respondents with lowest education had the least knowledge regarding stroke signs $(\mathrm{p}<0.01)$;
Discussion: The results of this study indicate that respondents showed a fair knowledge about stroke signs and risk factors for stroke.The results of this study will help to create and plan programmes for improvement of public health.

\section{Epidemiology of stroke}

\section{PREVALENCE AND CONTROL OF ARTERIAL HYPERTENSION IN THE} POPULATION WITH A HISTORY OF A STROKE. THE POLISH 400 CITIES PROJECT RESULTS

K. Chwojnicki, B.T. Zdrojewski, Ł. Wierucki, P. Popowski,

M. Zarzeczna-Baran, A. Czlonkowska, A. Januszewicz, G. Kozera,

B. Wyrzykowski, A.W.M. Nyka

Medical University of Gdansk; Institute of Psychiary and Neurology, Institute of Cardiology, Warsaw, Poland

Aim: The aim of the study was to assess the prevalence and control of arterial hypertension $(\mathrm{AH})$, in subjects with history of stroke, living in small Polish towns Material and Methods: The Polish 400 Cities Project (P400CP) is a programme of cardiovascular diseases (CVD) prevention for the years 2004-2006. It concerns the citizens of 418 small towns with population under 8 thousand. In years 2004-2005, 81959 subjects, aged 18-98 years underwent medical examination and a series of tests, including: blood pressure measurements (BP), anthropometrical measurements and laboratory tests (total cholesterol and glycaemia strip tests). The prevalence of stroke and $\mathrm{AH}$ were analyzed on the basis of a questionnaire filled in by the subjects. The assessment of AH control was based on the mean of two BP measurements taken during one visit $(<140 / 90 \mathrm{mmHg})$.

Results: All together, 1076 subjects $(1,31 \%$ ), aged 26-91 years (Women, W $67,1 \pm 9,4$ vs. Men, M 64,3 $\pm 10,1 ; \mathrm{p}<0,001$ ) had a history of stroke (W $1,14 \%$ vs. M $1,66 \% ; \mathrm{p}<0,001)$. A history of AH or newly increased BP was detected during the first visit in $89,5 \%$ of the subjects after stroke (W $91 \%$ vs. M $87 \%$; $=0,02$ ). Only $14,1 \%$ of the subjects with history of $\mathrm{AH}$ had $\mathrm{BP}<140 / 90 \mathrm{mmHg}$ and in $34,9 \% \mathrm{BP}$ was $<160 / 95 \mathrm{mmHg}$.

Conclusions: It should be noted that there is a distinct prevalence of $\mathrm{AH}$ in the subjects with a history of stroke. AH in secondary prevention after stroke is not effectively controlled and calls for urgent preventive and therapeutical interventions in small towns in Poland.

\section{Epidemiology of stroke}

\section{ASSESSING THE IMPACT OF THE REQUIREMENT FOR CONSENT IN A} HOSPITAL-BASED STROKE STUDY

C. Jackson, L. Crossland, M. Dennis, J. Wardlaw, C. Sudlow

University of Edinburgh, Western General Hospital, Edinburgh, United

Kingdom

Background: We attempted to recruit all subjects attending our hospital with a stroke or transient ischaemic attack into a hospital-based cohort study. Ethical approval required patients or their relatives to give consent for: use of data for research; follow-up via the patient's general practitioner; follow-up by postal questionnaire; and storage of a research blood sample. We investigated how the need for consent may have biased our cohort.

Methods We compared our cohort with a stroke audit occurring in our hospital at the same time, recruiting the same target population, but with no requirement for consent. We obtained numbers of consenters, refusers, and those missed (consent not sought for logistical reasons). We compared several characteristics (age, sex, OCSP stroke subtype, socioeconomic status, admission to stroke unit) of consenters vs non-consenters (refusers + those missed) and consenters vs refusers.

Results: Of 1202 patients included in the audit over an 18 month period, 1049 $(87 \%)$ consented to inclusion in the cohort study. Only $11(0.9 \%)$ refused any participation, while $4 \%$ did not consent to a research blood sample or questionnaire follow-up. $12 \%$ were missed. Compared with non-consenters, consenters were more likely to have TIAs or mild strokes, be admitted to the stroke unit, and to be less socioeconomically deprived, but age and sex distributions were similar. Refusers were slightly older than consenters (mean 78 vs 71 years). We found no other significant differences, but the number of refusers was very small.

Conclusions: Very few patients refused inclusion in our cohort, but the need for consent introduced bias. An opt-out system for observational studies would minimise bias and reduce the considerable time and costs associated with the consent process.

Epidemiology of stroke 
24 Epidemiology of stroke

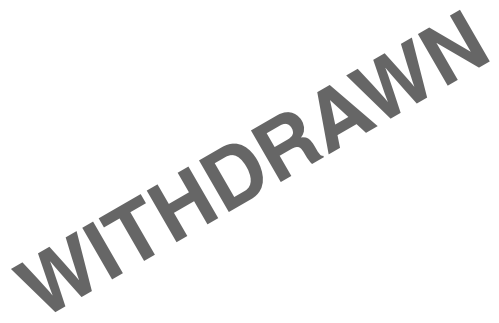

\section{Risk factors of stroke}

\section{Risk factors of stroke}

TISSUE-TYPE PLASMINOGEN ACTIVATOR -7,351C > T POLYMORPHISM DOES NOT INFLUENCE THE RISK OF STROKE. A PROSPECTIVE CASE-REFERENT STUDY

P.G. Wiklund, L. Johansson, P. Ladenvall, A. Tjärnlund Wolf, A.B. Stegmayr, C. Jern

Umeå University Hospital, Umeå, Sweden

Background: Prospective studies have shown an association between plasma levels of tPA and the risk of stroke. A functional polymorphism at position -7,351 in the tissue plasminogen activator (tPA) gene has been associated with impaired local net-release of tPA, but also with an increased risk of myocardial infarction and ischemic stroke. The present study prospectively examined the influence of the $-7,351 \mathrm{C}>\mathrm{T}$ polymorphism on the risk of first-ever stroke.

Methods: This study was a nested case-referent study within the Västerbotten Intervention Program (VIP) and the Northern Sweden MONICA cohorts. Up to the year 2000, 74,500 subjects had been screened and 386 individuals who subsequently suffered a first-ever stroke, defined according to the WHO MONICA stroke criteria, were identified and analyzed. 775 matched controls were selected from the same cohort.

Results: Cases had a significantly higher prevalence of diabetes, hypertension, smoking and higher levels of BMI and cholesterol as compared with controls. There were no differences in genotype distribution between cases and controls among all stroke cases nor when women and men were analysed separately. The conditional logistic regression analysis showed no association between genotype and first-ever stroke, ischemic or hemorrhagic stroke.

Discussion: The tPA $-7,351 \mathrm{C}>\mathrm{T}$ polymorphism did not influence the risk of a future first-ever stroke in this northern Swedish study population. The ischemic stroke subgroup of 320 cases in the present study retained $80 \%$ power to detect association with an OR $>1.7$ at the 5\% level. Although the possibility that the present study failed to detect an existing association cannot be excluded, a major effect of this polymorphism on the risk of ischemic stroke is less likely.
2 Risk factors of stroke

HSCRP IS INDEPENDENTLY ASSOCIATED WITH EARLY CAROTID ARTERY PROGRESSION IN WOMEN BUT NOT IN MEN. THE INVADE STUDY

K. Sander, C. Schulze-Horn, C. Briesenick, D. Sander

Technical University of Munich, Munich, Germany

Background and Purpose: High-sensitivity C-reactive protein (hsCRP) is known to be associated with atherosclerosis and cardiovascular events. Limited information exists regarding the importance of gender differences for the association between hsCRP and the progression of early stages of atherosclerosis. Therefore, we investigated the effect of hSCRP on early carotid atherosclerosis progression and major vascular risk factors in men and women.

Methods: We analyzed the data of INVADE (Intervention project on cerebrovascular diseases and dementia in the community of Ebersberg, Bavaria), a prospective, population-based study. In addition to common risk factors, measurements of carotid intima-media-thickness (IMT) and hsCRP were performed at baseline and after 2 years.

Results: Complete baseline data were available in 3387 patients including 2001 women. In this study population women were older, had higher systolic blood pressure, and cholesterol levels. The prevalence of smoking and ischemic heart disease was more frequent in men. The baseline carotid IMT was significantly higher in men as compared to women $(0.823 \mathrm{~mm}$ versus $0.771 \mathrm{~mm} ; \mathrm{p}<0.0001)$ The interaction hsCRP $x$ gender with baseline IMT was highly significant $(\mathrm{F}=6.54$; $\mathrm{p}=0.01$ ). The IMT progression was significantly associated with hsCRP only in women after risk factor adjustment $(\mathrm{p}=0.005)$ but not in men $(\mathrm{p}=0.39)$.

Conclusions: The association between hsCRP and progression of early carotid atherosclerosis shows gender differences. In further studies analysing the role of inflammation for cardiovascular diseases and atherosclerosis these differences should be taken into account.

3 Risk factors of stroke

POOR FUNCTIONAL STATUS BASED ON THE NYHA CLASSIFICATION EXPOSES THE CORONARY PATIENT TO ELEVATED RISK OF FIRST-EVER ISCHEMIC STROKE

D. Tanne, N. Koren-Morag, Y. Adler, U. Goldbourt

Sheba Medical Center and Tel Aviv University, Tel Hashomer, Israel

Background: Patients with coronary heart disease (CHD) are at increased risk of stroke. We examined the relationship between the New York Heart Association (NYHA) classification, a well established clinical index of functional status of patients with cardiac disease, with incident first-ever ischemic stroke in a large cohort of CHD patients.

Methods: We followed up 15,524 patients with documented CHD, screened for inclusion in a clinical trial, for 4.8 to 8.1 years. Functional status at baseline was categorized according to the NYHA classification. Among 14,703 patients free of stroke with recorded NYHA functional class, $1086(7.4 \%)$ developed an ischemic cerebrovascular event, of which $604(4.1 \%)$ had a confirmed ischemic stroke or TIA Results: the cumulative rate of ischemic stroke increased from $6.7 \%$ in patients with NYHA functional class 1 to $9.2 \%$ and $9.7 \%$ for patients with NYHA functional class 2 and 3 respectively $(\mathrm{P}<0.001)$. Adjustment was made in a Cox proportional-hazard model for age, gender, body mass index, past myocardial infarction, current smoking, diabetes, hypertension, peripheral vascular disease, percent of cholesterol in HDL, triglyceride level and antidiabetic, antiplatelet and antihypertensive therapy. The hazard ratio associated with NYHA functional class 2 and 3 were 1.29 (95\% C.I. 1.13-1.49) and 1.69 (95\% C.I. 1.35-2.14), as compared with patients with NYHA class 1. Similar results were obtained for confirmed ischemic stroke or TIA. Discussion: Our findings indicate that, in addition to the already established risk of mortality, poor functional status based on the NYHA classification exposes the coronary patient to elevated risk of first-ever ischemic stroke.

\section{Risk factors of stroke}

POTENTIAL APPLICABILITY OF THE ABCD SCORE IN IDENTIFYING INDIVIDUALS AT HIGH EARLY RISK OF STROKE FOLLOWING A TRANSIENT ISCHEMIC ATTACK: A HOSPITAL-BASED CASE SERIES STUDY

G. Tsivgoulis, K. Spengos, E. Manios, A. Papapostolou, M. Papadopoulou, E. Koroboki, N. Zakopoulos, D. Vassilopoulos

University of Athens, Athens, Greece

Background: A simple score derived in the Oxfordshire Community Stroke Project 
(ABCD score) and validated in a population- and a hospital-based cohort was recently reported as a practical tool able to identify individuals at high risk of early stroke after transient ischemic attack (TIA). Our aim was to further validate this score in a cohort of hospitalised TIA patients.

Methods: All hospitalised patients with definite TIA according to the WHO criteria during a 5-year period were identified. Medical charts and emergency room records were retrospectively reviewed. Patients with previous stroke and individuals who missed the planned follow-up evaluation at 1 month following hospitalisation were excluded. The 6-point ABCD score [Age $(<60 \mathrm{y}=0, \leq 60 \mathrm{y}=1)$; Blood pressure (systolic $\leq 140 \mathrm{mmHg}$, and diastolic $\leq 90 \mathrm{mmHg}=0$; systolic $>140 \mathrm{mmHg}$ and/or diastolic $>90 \mathrm{mmHg}=1$ ); Clinical features (unilateral weakness $=2$, speech disturbance without weakness $=1$, other symptom $=0$ ); Duration of symptoms in min $(<10=0,10-59=1, \geq 60=2)]$ was used to stratify the 30 -day risk of stroke in all cases. Statistical analysis was performed using the Kaplan-Meier product-limit method and stepwise Cox's proportional hazards model.

Results: The 30-day risk of stroke in our case series $(n=226)$ was $9.7 \%$ (95\%CI:5.8 $13.6 \% ; \mathrm{n}=22)$. The ABCD score was highly predictive $[\leq 2.1 \%$ (95\%CI:0.0$4.9 \%) ; 4: 7.6 \%$ (95\%CI:1.2-14.0\%); 5: $21.3 \%$ (95\%CI:10.4-33.0\%); 6: $31.3 \%$ $(95 \% \mathrm{CI}: 8.6-54.0 \%)$; log-rank test $=22.81 ; \mathrm{df}=3 ; \mathrm{p}<0.0001 ; \mathrm{p}$ for linear trend for factors $<0.00001]$. After adjustment for risk factors, vascular comorbidities, history and number of TIA and secondary prevention treatments a score of 5-6 was independently $(\mathrm{p}<0.001)$ associated with an 8-fold greater 30-day risk of stroke (HR:8.01, 95\% CI:3.21-19.98).

Discussion: The present results validate the predictive value of the ABCD score in identifying TIA patients with high risk of early stroke and provide further evidence for its potential applicability in clinical practice.

\section{Risk factors of stroke}

\section{FACTORS ASSOCIATED WITH NEUROIMAGING EVIDENCE OF} INFARCTION IN PATIENTS WITH TRANSIENT ISCHEMIC ATTACKS

G. Tsivgoulis, K. Spengos, E. Manios, M. Papadopoulou, A. Papapostolou, E. Koroboki, N. Zakopoulos, D. Vassilopoulos

University of Athens, Athens, Greece

Background: Recent data suggests that patients with transient ischemic attack (TIA) and evidence of new infarct on brain CT carry a higher short-term risk for stroke than TIA patients with no visualized ischemic lesions. Our aim was to evaluate which factors (demographics, risk factors, history and number of prior TIAs, duration and clinical features) are associated with the presence of a new infarction on CT in a cohort of hospitalised TIA patients.

Methods: All hospitalised patients with definite TIA according to the WHO criteria during a 5-year period were identified. Medical charts and emergency room records were retrospectively reviewed. Patients with previous stroke and those with no CT performed within the first $24 \mathrm{~h}$ were excluded. Brain CTs obtained after the onset of symptoms leading to the diagnosis of a new stroke or recurrent TIA were excluded. The presence of a new ischemic lesion located on the appropriate side to the TIA symptoms was investigated in a total of 204 cases. Statistical analysis was performed using logistic regression models

Results: The presence of new infarcts was identified in 14 cases (6.8\%, group A). TIAs lasted significantly ( $\mathrm{p}$ for Mann-Whitney U-test: 0.017 ) longer in this group (group A: median $=210 \mathrm{~min}$, interquartile range $=405 \mathrm{~min}$ ) than in subjects without new lesions on CT (group B: median time $=33 \mathrm{~min}$, interquartile range $=168 \mathrm{~min}$ ). Unilateral weakness was significantly $(\mathrm{p}=0.030)$ more prevalent in group A $(11.2 \%$ vs. $6.7 \%$ ). Multiple logistic regression models revealed only 2 factors independently associated with the presence of a new infarction: duration of symptoms $>60 \mathrm{~min}$ (OR:3.88, 95\%CI:1.15-13.08; $\mathrm{p}=0.029)$ and unilateral weakness as presenting TIA symptom (OR:3.96, 95\% CI:1.18-13.34; $\mathrm{p}=0.026)$.

Discussion: Longer TIA duration and unilateral weakness are features that help identifying patients with rapidly resolving symptoms and concomitant new ischemic CT lesions, who constitute a subgroup vulnerable to recurrent ischemic events.

\section{Risk factors of stroke}

\section{ASSOCIATION BETWEEN SELF-PERCEIVED PSYCHOLOGICAL STRESS AND ISCHEMIC STROKE}

K. Jood, P. Redfors, A. Rosengren, C. Blomstrand, C. Jern

Institute of Clinical Neuroscience, The Sahlgrenska Academy at Göteborg University, Göteborg, Sweden

Background and Purpose: A growing body of evidence suggests that psychological stress contributes to coronary artery disease. However, associations between stress and stroke are less clear. We examined the possible association between self-perceived psychological stress and ischemic stroke in a large case-control study.
Methods: In the Sahlgrenska Academy Study on Ischemic Stroke (SAHLSIS), 600 consecutive patients with acute ischemic stroke (18-69 years) and 600 age and sex matched controls were recruited. Ischemic stroke subtype was determined according TOAST criteria. Self-perceived psychological stress preceding the stroke was assessed retrospectively during the acute stage using a single-item questionnaire. Stroke outcome was assessed after 3 months using the modified Rankin Scale.

Results: Permanent self-perceived psychological stress during the last year or longer, but not periodic stress, was associated with overall ischemic stroke (multivariateadjusted odds ratio [OR] 2.85; 95\% CI 1.89-4.29). Analyses by stroke subtype showed that this association was present for large vessel disease (OR, 4.06; $95 \%$ CI 1.61-10.19), small vessel disease (OR, 3.01; 95\% CI 1.54-5.89), and cryptogenic stroke (OR, 3.72; 95\% CI 2.18-6.35), but not for cardioembolic stroke (OR, $1.38 ; 95 \%$ CI $0.60-3.18)$. No significant association between stress and functional outcome at 3 month follow-up was observed.

Conclusion: Results from this case-control study support that permanent psychological stress is an independent risk factor for ischemic stroke. While this association was observed for the ischemic stroke subtypes large vessel disease, small vessel disease and cryptogenic stroke, it was not observed for cardioembolic stroke, suggesting that stress may contribute to primary vascular mechanisms.

\section{Risk factors of stroke}

\section{BLOOD PRESSURE IMPACT ON THE INCIDENCE OF PRIMARY} INTRACEREBRAL HAEMORRHAGE SUBTYPE AND CEREBRAL INFARCTION.A PROSPECTIVE COHORT-STUDY

E. Zia, B. Hedblad, H. Pessah-Rasmussen, G. Engström

Inst of Clinical Science, Malmö; University of Lund; Malmö University Hospital, Sweden

Background: Data regarding the association of blood pressure (BP) levels and incidence of stroke subtype, especially primary intracerebral haemorrhage $(\mathrm{PICH})$ subtypes, in Western population, is scanty.

Methods: Baseline data were retrieved from Malmö Diet and Cancer Study, a population-based prospective cohort study $(\mathrm{n}=28$ 449), with examinations between March 1991 and October 1996. Mean BP values were grouped according to European guidelines from 2003. Incidence of first-ever stroke and death was monitored until Dec 31, 2001, by data linkage with local and national registers. PICH cases were classified as lobar or nonlobar after review of CT and MR-scans. During the mean observation time of 7.5 years, 566 cases of CI and 88 cases of PICH (38 lobar and 45 nonlobar, 5 not classified) were identified.

The risk of stroke was calculated by COX regression model. The incidence was expressed as events/1000 person year.

Results: At baseline, 11766 had normal BP (NBP) $(<140 / 90 \mathrm{mmHg}), 9675 \mathrm{had}$ hypertension (HT) grade 1 (140-159 and/or 91-99 $\mathrm{mmHg}), 5047$ had HT grade 2 (160-179 and/or 100-109 mmHg), and 1554 had HT grade 3 ( $\geq 180$ and/or 110 $\mathrm{mmHg}$ ).

In terms of absolute risk (AR), incidence of CI was 1.3,2.8,4,8 and 8.3, respectively, for subjects with NBP and HT grade 1-3. The corresponding incidence for PICH was $0.1,0.3,1.2$ and 2.1 .

Adjusted (age, sex, smoking, alcohol, use of lipidlowering and/or antidiabetic drugs, BMI) relative risk (RR) for stroke subtypes, related to NBP and HT grade 1-3, respectively:

CI: $1.00,1.6(1.3-2.1), 2.2(1.7-2.8)$ and $3.3(2.5-4.5)$. PICH: $1.00,2.6(1.2-5.7)$, 6.3(3.0-13.7) and 14.7(6.5-33.0). Non-lobar PICH: 1.00, 3.0(0.94-9.5), 7.8(2.524.2) and 26.5(8.4-84.3). Lobar PICH: 1.00, 3.1(0.94-9.4), 5.2(1.6-16.6) and $10.2(2.9-35.6)$

Conclusion: 1) In terms of AR, the association to BP elevation is greater for $\mathrm{CI}$ than for PICH. 2) In terms of RR, the association is stronger to $\mathrm{PICH}$, particularly nonlobar, than to $\mathrm{CI}$.

\section{Risk factors of stroke}

IS HYPERHOMOCYSTEINEMIA ASSOCIATED WITH A SPECIFIC ISCHEMIC STROKE SUBTYPE?

P. Laloux, N. Gospodaru, V. Cornil, M. Ossemann, J. Jamart

UCL Mont-Godinne University Hospital, Yvoir, Belgium

Hyperhomocysteinemia is considered to be a risk factor for ischemic stroke, but its relationship to some etiologic subtypes remains controversial. In this study, we addressed the issue of whether the homocysteine $(\mathrm{Hc})$ level is influenced by some risk factors and whether $\mathrm{Hc}$ is associated with a particular subtype such as large vessel disease (LVD), small vessel disease (SVD) or cardioembolic stroke (CES). Samples of plasma $\mathrm{Hc}$ were obtained from 244 stroke/TIA patients (142 men, 102 women; mean age, 72.3 years \pm 12.7 ), who were prospectively evaluated for 
the risk factors (age, sex, hypertension, diabetes, alcohol consumption, smoking, hypercholesterolemia, triglycerides, intermittent claudication, atrial fibrillation) and for 4 stroke subtypes (TOAST): LVD, 23; SVD, 27; CES, 67; other, 124. The mean $\mathrm{Hc}$ levels for each risk factor were compared using Wilcoxon test or Spearman correlation. Hypertension (15.19 vs. $17.87 \mu \mathrm{mol} / \mathrm{l} ; \mathrm{p}=0.033$ ) and a positive history of intermittent claudication ( $16.58 \mathrm{vs.} 18.38 \mu \mathrm{mol} / \mathrm{l} ; \mathrm{p}=0.037)$ significantly influenced the Hc level. Correlation analysis showed a significant association between age and the $\mathrm{Hc}$ level $(\mathrm{p}<0.001)$. Logistic regression analysis showed that the $\mathrm{Hc}$ level was independently associated with age (b-coefficient, $0.206 ; \mathrm{p}=0.002$ ) and hypertension (b-coefficient, $0.159 ; \mathrm{p}=0.021$ ), but when only these two independent factors were entered in an additional logistic regression analysis, only age was retained ( $\mathrm{b}$-coefficient, $0.167 ; \mathrm{p}=0.011$ ). There was no significant difference in the distribution of the mean $\mathrm{Hc}$ level among the 3 etiologic ischemic stroke subtypes (Wilcoxon test). In conclusion, age is the most important determinant of the Hc level which does not seem to be related to a specific etiologic stroke subtype.

\section{Risk factors of stroke}

PREVALENCE OF METABOLIC SYNDROME IN HISPANIC MESTIZO PATIENTS WITH ISCHEMIC STROKE. A MEXICAN MULTICENTRIC ISCHEMIC STROKE REGISTRY

C. Cantu, J.L. Ruiz-Sandoval, R. Rangel, J. Villarreal, A. Arauz, L. Murillo,

F. Barinagarrementeria, M. López, R. Leal, S. Reyes, for PREMIER

Collaborative Study Group

Mexican Stroke Association, Mexico City, Mexico

Background and Purpose: The combination of vascular risk factors known as metabolic syndrome (MS) is receiving increased clinical attention. Data on metabolic syndrome in Hispanic Mestizo patients with stroke is unknown. This first Mexican ischemic stroke registry is being developed to improve our knowledge regarding risk factors profile, outcome, and long-term follow-up in patients with cerebral ischemia. Methods: We analyzed data from patients with acute cerebral ischemia who are being enrolled in a large multicentric stroke data bank in Mexico. Standardized data assessment was used by all centers which included information on demographics and risk factors including lipid profile. From November 2004 to December 2005, 586 had available data for the component conditions of the metabolic syndrome.

Results: There were 518 cerebral infarctions (89\%) and 64 TIAs (11\%). Metabolic syndrome was documented in 284 patients $(49 \%)$ including $136(48 \%)$ men (age 66.6) and $148(52 \%)$ women (age 69.5). Frequency of individual components of the MS: hypertension 89\%; diabetes mellitus 64\%; abnormal lipid profile $94 \%$ including hypertriglyceridemia in 73\%, low HDL cholesterol in 76\%, and combined in $57 \%$; $\mathrm{BMI}>25$ in $85 \%$ (BMI $>30$ in $35 \%$ ). Previous ischemic stroke or AITs was more common in patients with MS (38\%) compared with those without MS $(25 \%)[\mathrm{P}=0.001]$. Stroke subtype classification in patients with MS was: large-artery atherosclerosis $30 \%$, small-vessel disease $21 \%$, cardioembolism $19 \%$, concurrent etiology $8 \%$, other $2 \%$, and undetermined $19 \%$. Clinical outcome at day 30 was worse in patients with metabolic syndrome: mRS $0-2$ in $41 \%$ and mRS 3-6 in 59\%, compared with mRS 0-1 in 51\% and mRS 3-6 in 49\% in patients without MS $(\mathrm{p}=0.017)$.

Conclusions: Metabolic syndrome was documented in half of the Hispanic Mestizo patients with ischemic stroke with a high frequency of atherogenic dyslipidemia. MS was associated with previous ischemic stroke events and a poor outcome.

\section{0}

\section{Risk factors of stroke}

DECREASED INSULIN SENSITIVITY AND DYSLIPIDEMIA ARE STRONGLY ASSOCIATED WITH ISCHEMIC STROKE AND ASYMPTOMATIC CAROTID ARTERY STENOSIS IN NON DIABETICS

N. Covickovic-Sternic, M. Mijajlovic, N. Lalic, A. Jotic, A. Pavlovic,

J. Zidverc-Trajkovic, Z. Jovanovic, V. Kostic

Institute of Neurology, Clinical Center of Serbia, Belgrade, Yugoslavia

Background and Aims: Decreased insulin sensitivity (IS) with compensatory hyperinsulinemia plays a crucial role in the pathogenesis of atherosclerosis, but their role in ischemic stroke (IST) has not yet been elucidated. This study was aimed to analyze IS as the indicator of atherosclerotic activity,as well as plasma insulin (PI) levels and dyslipidemia pattern in 50 patients with atherothrombotic IST confirmed on computerized tomography/magnetic resonance imaging- (group A), 50 patients with asymptomatic stenosis of one or both internal carotid arteries $>50 \%$ - (group B), and 40 healthy controls-(group C) Patients with diabetes mellitus and ischemic heart disease were excluded from the study.

Material and Methods: IS was determined by Homeostasis Assessment Model (HOMA-IR), PI levels were determined by Radioimunoassay.We measured total cholesterol, LDL and HDL cholesterol and trygliceride levels in all groups.
Results: IS was significantly lower in group A compared to group B and C ( $3.82 \pm 0.27$ vs. $2.69 \pm 0.22, \mathrm{p}<0.05 ; 3.82 \pm 0.27$ vs. $1.51 \pm 0.22, \mathrm{p}<0.01$ ). PI levels were significantly higher in group A in comparison to group B and C $(16.00 \pm 1.2$ vs. $11.95 \pm 0.88, \mathrm{p}<0.05 ; 16.00 \pm 1.2$ vs. $7.12 \pm 0.19, \mathrm{p}<0.01)$. Different patterns of dyslipidemia were observed in ACAS in comparison to IST.

Conclusion: Our results indicate that atherothrombotic IST as well as ACAS are strongly associated with decreased IS and increased PI levels, although all the patients were non diabetics.Also, different patterns of dyslipidemia between ACAS and IST were observed.

\section{Risk factors of stroke}

STATINS AND THEIR INFLUENCE ON THE MACROPHAGE INFILTRATION OF INTERNAL CAROTID ARTERY (ICA) STENOSIS

H. Kunte, N. Amberger, R.-I. Rückert, L. Harms

Charité-Universitätsmedizin, Berlin, Germany

Background: For statins a lipid independent effect on the composition of atherosclerotic plaques has generating considerable clinical interest. This could be partly responsible for the risk reduction of vascular events. The role of monocyte-derived macrophages has become critically important to the scientific understanding of plaque instability.The following study investigates the percentage of macrophages infiltration (PMI) found in internal carotid artery (ICA) stenosis plaques as an indicator of inflammation and assesses whether or not statins influence the PMI Methods and Results: In a prospective study 94 consecutive patients with highgrade ICA-stenosis and planned carotid endarteriectomy were included. Immunohistochemistry and quantitative image analysis techniques were used.

The PMI of asymptomatic $(\mathrm{n}=68)$ was lower than in symptomatic $(\mathrm{n}=26)$ ICAstenosis $(0.67 \% \pm 0.58 \%$ vs $3.10 \% \pm 1.91 \%, \mathrm{p}<0.0005)$. In patients with "previous" (symptomatic older than 120 days)-symptomatic ICA-stenosis $(\mathrm{n}=5)$ PMI was lower than in symptomatic ICA-stenosis $(n=26)(0.64 \pm 0.42$ vs $3.10 \%$ $\pm 1.91 \%, \mathrm{p}<0.0005)$. The use of statins in asymptomatic ICA stenosis showed significant effects (statin $\mathrm{n}=37$ vs. no statin $\mathrm{n}=31)(0.50 \% \pm 0.40 \%$ vs $0.87 \% \pm$ $0.70 \%, p=0.01$ ). Significant effects were also apparent when statins were given after an ischemic event $(\mathrm{n}=4)$ vs. when no statins were given $(\mathrm{n}=15)(1.40 \pm 0.47 \%$ vs $3.18 \% \pm 1.66 \%, p=0.01)$ and when patients received statins both before and after an ischemic event $(\mathrm{n}=8)(1.40 \pm 0.47 \%$ vs $3.79 \% \pm 2.25 \%, \mathrm{p}=0.03)$.

Conclusions: Our study showed that patients with asymptomatic and "previous"symptomatic ICA-stenosis had a lower PMI compared to patients with symptomatic ICA-stenosis and statin intake results in lower PMI. This findings could give evidence for plaque stabilizing properties by statins.

\section{Risk factors of stroke}

\section{YEARS ON: - DOES BEHAVIOUR MODIFICATION AFFECT POST STROKE} RISK FACTOR CONTROL?

J.A. McManus, A. Craig, G. Ellis, C. McAlpine, P. Langhorne

Glasgow Royal Infirmary, Glasgow, United Kingdom

Background: A previous randomised controlled trial evaluated the effects of nurseled health education and counselling on risk factor control, satisfaction, mood an perceived health status, in post stroke patients. In the initial study, blood pressure control and satisfaction were significantly improved in the intervention group. The aim of this follow-up study was to determine if these benefits were maintained 3 years after the initial intervention.

Methodology: The survivors of the original cohort were contacted and asked to attend for repeat interview. Details of risk factor control, medication compliance and recurrent events were documented. Questionnaires used in the initial study were repeated, including, Geriatric Depression score, Euroqol perceived health status and stroke services satisfaction questionnaire.

Results: Mean length of follow-up was 3.6years (SD 0.43). Of the 205 patients enrolled in the initial study, 25 were dead (11 experiment/14 control, $\mathrm{p}=0.39)$. 102 patients attended for repeat interview ( 49 experiment $/ 53$ control). There were no significant differences in the percentage of controlled risk factors between groups (Experiment $51.7 \%$ vs Control $55.9 \%, \mathrm{p}=0.528$ ). No overall difference was seen between groups in Euroqol percent score (Experiment 61.8\% vs Control $60.3 \% \mathrm{p}=0.725$ ), Depression score (Experiment 3.59 vs Control 4.29, $\mathrm{p}=0.355$ ), satisfaction scores or recurrent events.

Discussion: Initial benefits from a 3-month intervention of behaviour modification appear not to be maintained long-term. These results must be cautiously interpreted in light of the small study numbers. 
13 Risk factors of stroke

SYNERGISTIC EFFECT OF HYPERTENSION AND ESTROGEN DEFICIENCY CONTRIBUTE TO ENDOTHELIAL DYSFUNCTION AND INCREASE THE RISK FOR CEREBRAL ANEURYSM FORMATION IN RATS

T. Tamura, M.A. Jamous, K.T. Kitazato, M. Uno, K. Sato, S. Nagahiro

The University of Tokushima, Tokushima, Japan

Background: Cerebral saccular aneurysms (CSAs) are a major cause of catastrophic subarachnoid hemorrhage. Although their pathogenesis is multifactorial pathogenesis, the mechanisms underlying their formation, growth, and rupture remain unclear. We examined the role of endothelial cells in the pathogenesis of CSAs in a rat model.

To assess whether the protection from endothelial damage inhibits the formation of CSAs we treated female rats with estrogen and angiotensin II AT1 receptor blocker(ARB), which manifest endothelial protection and anti-hypertensive effects, respactively.

Materials and Methods: Female Sprague-Dawley rats aged 7 weeks underwent ligation of the right common carotid- and bilateral posterior renal arteries to induce hemodynamic stress and hypertension. One week later, their drinking water is changed to a $1 \% \mathrm{NaCl}$ and 1 month post-ligation they underwent bilateral oophorectomy. They were divided into 4 groups; 2 groups were treated with ARB, 0.5 or $1.0 \mathrm{mg} / \mathrm{kg}$, another received $17 \mathrm{beta}$-estradiol implanted hormone replacement therapy(HRT), and the 4th served as the control. The CSAs formation was detected with vascular corrosion casts under a scanning electron microscope. We used quantitative RT-PCR to assess the eNOS mRNA level in the vessel wall.

Results: The blood pressure in the HRT- and control groups exceeded $170 \mathrm{mmHg}$; it was less than $130 \mathrm{mmHg}$ in rats treated with ARB. CSA changes were observed in 8 of 15 control rats. In contrast, 1 of 8 rats treated with $0.5 \mathrm{mg} / \mathrm{kg}$ ARB, none of 8 rats treated with $1.0 \mathrm{mg} / \mathrm{kg}$, and 1 of 15 rats subjected to HRT developed CSAs. The eNOS mRNA levels in all 3experimental groups were higher than in the controls, suggesting that ARB treatment and HRT were associated with a decrease in the incidence of CSA formation.

Conclusions: We found a lower incidence of aneurysmal changes in rats treated with HRT or ARB. Our findings suggest that hypertension and hemodynamic stress play a role in the formation and progression of CSAs while estrogen exerts a protective effect and that improved endothelial function may contribute to prevention of CSAs.

\section{Risk factors of stroke}

\section{A STUDY OF PLASMA RESISTIN LEVELS AND ITS GENE POLYMORPHISMS IN STROKE PATIENTS}

Y.D. Zhang, X.J. Lin, Q.S. Guan, Q. Di, J.P. Shi, W.G. Liu

Nanjing Brain Hospital, Nanjing, China

Background: Resistin has been proposed to serve as a link between obesity and insulin resistin (IR), a prevalent risk factor for stroke. This study was aimed at exploring the plasma resistin level and its gene single-nucleotide polymorphisms (SNPs) in $5^{\prime}$ flanking region in stroke patients to analyze the role of resistin in IR. Methods: In 103 patients with atherothrombotic cerebral infartcion (ACI), 85 patietns with lacunar infarction (LI), 70 patietns with intracerebral hemorrhage (ICH) and 86 healthy controls, plasma resistin and insulin levels were measured by means of ELISA, resistin gene SNPs were detected by PCR and direct DNA sequencing. The subjects' blood pressure and fasting plasma glucose (GLU), triglyceride (TG), total cholesterol (CHO), low-density lipoprotein (LDL) and high-density lipoprotein (HDL) concentrations were also determined in the routine way. Every subject's body mass index (BMI) and quantitative insulin sensitivity check index (QUICKI) were calculated as well.

Results: QUICKI was significantly lower in the ACI and ICH patients than that in the controls, while plasma resistin level was significantly higher in the ACI and ICH patients than that in the controls, but was not in the LI patients. There was a negative correlation between plasma resistin level with QUICKI. The distribution of allele and genotype frequencies of resistin gene $-420 \mathrm{C}>\mathrm{G}$ and $-537 \mathrm{~A}>\mathrm{C}$ SNPs was shown to be not of significant difference among the different groups, and those SNPs were not correlated with other clincial and biochemical parameters.

Discussion: Plasma resistin is associated with stroke by participating in the development of IR. The SNPs in resistin gene 5' flanking region has no impact on the plasma resistin level.
15 Risk factors of stroke

BETA-FIBRINOGENE GENE -455 G/A POLYMORPHISM IN ISCHEMIC STROKE PATIENTS

K.H. Cho, M.S. Park, S.H. Lee, S.M. Choi, B.C. Kim, M.K. Kim, Y.S. Kim

Chonnam National University Hospital, South Korea

Background: Elevated plasma fibrinogen level is a major risk factor of ischemic stroke. Polymorphisms at the beta-fibrinogen locus have been shown to be associated with elevated plasma concentration of fibrinogen and ischemic stroke. We aimed to investigate the relationship between the $\beta$-fibrinogen gene $-455 \mathrm{G} / \mathrm{A}$ polymorphism and plasma fibrinogen level in ischemic stroke patients. Also we studied this polymorphism may affect the pathomechanism of ischemic stoke.

Methods: 267 consecutive patients with ischemic stroke(152 large artery atherosclerosis and 115 small artery occlusion) were enrolled in this study. Plasma fibrinogen, CRP levels and other risk factors of stoke were evaluated. -455G/A genotype were determined by polymerase chain reaction with restrictive enzyme Hae III (PCRrestriction fragment length polymorphism method) and automatic DNA sequencing. Results: -455G/A polymorphism was significantly associated with elevated plasma fibrinogen level in stroke patients $(\mathrm{p}<0.001)$. The $-455 \mathrm{~A}$ allele frequency in stroke patients was 0.17 . The frequencies of $-455 \mathrm{G} / \mathrm{A}$ polymorphism were not different between large artery disease and small artery occlusion.

Conclusion: Plasma fibrinogen level could be affected by the $\beta$-fibrinogen gene $-455 \mathrm{G} / \mathrm{A}$ polymorphism. However, genetic influences on stoke subtype by -455 G/A polymorphism between large artery disease and small artery occlusion were not detected.

\section{Risk factors of stroke}

\section{CENTRAL ARTERIAL STIFFNESS IS HIGHLY CORRELATED WITH} CEREBROVASCULAR RISK FACTORS AND BLOOD PRESSURE IN THE ACUTE STROKE PHASE

D. Eveson, N. Shah, T. Robinson, J. Potter

University of Leicester, Leicester, United Kingdom

Introduction: Central arterial stiffness is an independent risk factor for cardiovascular and cerebrovascular disease. Increases in central arterial stiffness have been demonstrated in acute stroke patients in comparison to control subjects, but it is unclear if this is due to cerebrovascular disease per se or the attrition of associated risk factors. We evaluated the influence of acute stroke on arterial stiffness by studying a group of ischaemic stroke patients and a carefully-matched control group.

Methods: 70 acute ischaemic stroke patients and 33 controls were recruited. Stroke severity, casual brachial BP, carotid-femoral pulse wave velocity (PWV) and cerebrovascular risk factors were recorded. Regression models were constructed with PWV as the dependent variable. The natural logarithm of PWV was used in statistical analyses to maintain linearity of residuals.

Results (see table): In univariate analysis of the stroke group there was no relationship between arterial stiffness and stroke severity. In multivariate analysis, PWV was not significantly related to stroke status $(r=0.07, p=0.25)$, with significan predictive variables being age $(\mathrm{r}=0.43, \mathrm{p}<0.001), \operatorname{MABP}(\mathrm{r}=0.51, \mathrm{p}<0.001)$ and diabetes $(\mathrm{r}=0.21, \mathrm{p}<0.01)$. Standardised correlation coefficients for all other measured variables were $\leq 0.1$ in the model. The combination of age, MABP and diabetes accounted for $45 \%$ of PWV variance.

Conclusion: Central arterial stiffness in acute ischaemic stroke patients is closely related to BP, age and diabetes but not necessarily to acute cerebrovascular disease An advantage of the current analysis is the very close matching of groups to an extent not previously achieved.

\begin{tabular}{lcc}
\hline Variable & Stroke & Control \\
\hline Age (yrs) & $66.9 \pm 9.2$ & $65.2 \pm 9.4$ \\
Mean arterial blood pressure - MABP (mmHg) & $107.6 \pm 14.5$ & $105.7 \pm 11.8$ \\
PWV (m/s) & $11.1 \pm 2.8^{*}$ & $9.9 \pm 1.9$ \\
Heart rate (bpm) & $69 \pm 12$ & $66 \pm 11$ \\
Gender & $48 \mathrm{M}: 22 \mathrm{~F}$ & $20 \mathrm{M}: 13 \mathrm{~F}$ \\
Diabetes mellitus & $10 \%$ & $6 \%$ \\
Hypercholesterolaemia & $26 \%$ & $18 \%$ \\
Ischaemic heart disease & $16 \%$ & $15 \%$ \\
Peripheral vascular disease & $7 \%$ & $6 \%$ \\
Current smoker & $31 \% *$ & $12 \%$ \\
Body mass index & $29 \pm 5$ & $29 \pm 5$ \\
\hline
\end{tabular}

$* \mathrm{p}<0.05$ between groups
Poster Session Second Visit

Risk factors of stroke 
17 Risk factors of stroke

IMPACT OF METABOLIC SYNDROME ON FIRST-EVER ISCHEMIC STROKE

G. Cucurella, A. Ois, A. Rodríguez-Campello, M. Gomis, J. Jiménez-Conde,

E. Munteis, J. Martinez, J. Roquer

Hospital del Mar, Barcelona, Spain

Purpose: To determine prospectively the presence of metabolic syndrome (MS) in patients with first-ever ischemic stroke. We evaluate the relationship between MS and clinical severity, evolution and prognostic after stroke.

Patients and Methods: Patients with first-ever ischemic stroke assessed in our hospital from 2003 to 2005 were included. We evaluated the presence of MS defined by the ATP-III criteria. The following variables were analysed: age, sex, current smoking, previous history of coronary heart disease $(\mathrm{CH})$, peripheral vascular disease (VP), atrial fibrillation, initial stroke severity measured by NIHss, previous ischemic lesions on brain Computer Tomography (CT) scan, significant carotid or intracranial atherosclerosis (SA) evaluated by ultrasonographic studies recurrent stroke, non-cerebrovascular events, and functional situation and mortality at three months after the stroke onset.

Results: A total of 743 patients were analyzed, mean age 73.27 (SD 12.23). 286 (38.5\%) patients fulfilled the criteria of MS. Univariate analyses showed no statistical differences in age, smoking, atrial fibrillation, recurrent stroke and three-month mortality. Regression model showed that: female $(\mathrm{p}=0.001 ; \mathrm{OR}=1.78,95 \% \mathrm{CI}$ $1.28-2.47)$, previous $\mathrm{CH}(\mathrm{p}=0.001$; $\mathrm{OR}=2.05,95 \% \mathrm{CI} 1.34-3.14)$ or $\mathrm{VP}(\mathrm{p}=0.023$; $\mathrm{OR}=1.83,95 \%$ CI $1.08-3.08), \mathrm{SA}(\mathrm{p}<0.001 ; \mathrm{OR}=2.38,95 \% \mathrm{CI} 1.62-3.50)$ and noncerebral vascular events at three months $(\mathrm{p}<0.019 ; \mathrm{OR}=2.33,95 \%$ CI $1.15-4.75)$ were independently related to MS.

Conclusion: In our series, the $38.5 \%$ of patients had MS. There was an independent association between MS and significant atherosclerosis, female sex, history of $\mathrm{CH}$ or VP and an increased risk of non-cerebrovascular events at three months after the stroke onset. However, MS was not related to initial stroke severity, mortality or stroke recurrence at three-month.

\section{Risk factors of stroke}

PATIENTS WITH CERVICAL ARTERY DISSECTION HAVE NO CLINICAL SIGNS OF A CONNECTIVE TISSUE DISORDER

R. Dittrich, A. Heidbreder, D. Rohsbach, I. Nassenstein, R. Bachmann,

G. Kuhlenbaeumer, E.B. Ringelstein, D.G. Nabavi

University of Muenster, Muenster, Germany

Background and Purpose: Electron microscopy investigations have reported on alterations of collagen fibrils in patients with cervical artery dissection (CAD) suggestive of so far unknown connective tissue disorders. We performed a prospective study to evaluate whether patients with $\mathrm{CAD}$ have clinical signs or stigmata of connective tissue disorders.

Methods: 43 consecutive patients with CAD and 43 consecutive patients with an ischemic stroke of other etiology (non-CAD) of similar age were investigated. All patients had underwent standardized MR-imaging to detect typical signs of CAD e.g. mural hematoma in the cervical arteries. The clinical investigation contained 24 items of clinical signs characteristic for classical connective tissue diseases e.g. Ehlers-Danlos syndrome type IV such as articular hypermobility (investigation included parts of the Beighton Score), cutis laxa, capillary fragility and typical visual stigmata. A sum score was built by counting any positive item with a maximum of 24 points per patient.

Results: In the CAD group, the incidence of stigmata like epicanthus, blue sclera, pectus excavatum and carinatum was slightly higher, but the difference was not significant $(\mathrm{p}=0.43)$. One patient in the CAD group suffered from an osteogenesis imperfecta as an example of a known hereditary disease with a sum score of 7 . None of the 86 patients had a facial dysmorphia. There was only a minor statistical trend towards a higher sum score in patients with CAD (mean 2.37, \pm 2.1 , median 2, min. 0 , max. 7) as compared with the non-CAD group (mean 1.95, \pm 1.9 , min. 0 , max.7, $\mathrm{p}=0,38)$.

Conclusion: According to our investigation, there is no clinically apparent evidence of an even subtle visible connective tissue disorder in patients with CAD.
19 Risk factors of stroke

THE FINDING OF PULMONARY ARTERIOVENOUS MALFORMATIONS IN YOUNG PATIENTS WITH NEUROLOGICAL EVENTS

B.S. Rana, B. Unsworth, S. Pashos, G. Clark, D.P. Francis, J. Mayet, I.S. Malik Cardiology Department, St Mary's Hospital, Praed Street, London, United Kingdom

Background: The presence of a patent foramen ovale (PFO) is established as a cause of stroke. However this remains a diagnosis by exclusion. What is not known is the prevalence of other shunts in this population, which might play a causative role. Hence, we assessed the presence of pulmonary arteriovenous malformations (PAVM) during echocardiography examination in young patients with cerebrovascular events.

Methods: 37 patients, below 55 years underwent transthoracic echocardiogram following an ischaemic event. A strict protocol to assess for PFO and PAVM, using agitated gelofusin bubble contrast was performed. The presence of bubbles in the left heart within 3 cardiac cycles after arrival in the right heart was deemed positive for a PFO, while a greater delay diagnosed a PAVM. The characteristic pattern of contrast appearance in the left heart helped differentiate between a PFO and PAVM. Results: Of 37 patients, 16 had a stroke and 21 transient ischaemic attacks. Mean age 41years, $62 \%$ male. 19 patients were diagnosed with a PFO $(51 \%)$, 11 with PAVM $(33 \%)$ and 6 had both (PFO and PAVM). 13 patients had neither. Of 11 patients with PAVM's 7 underwent further investigation including a high resolution CT chest. None were positive. Transoesophageal echocardiogram in these 7 patients confirmed the presence of an intrapulmonary shunt. Retrospective review of the notes did not suggest a diagnosis of Hereditary Haemorrhagic Telangectasia.

Conclusion: Agitated gelofusin contrast is a safe and effective test for detecting right to left shunting in the heart (PFO) and/or in the lung (PAVM). Contrast is detectable even when PAVMs are not large enough to visualise by chest CT. Our findings suggest the presence of such lesions is higher than thought in this population and might contribute to paradoxical embolic events not previously identified. This warrants further investigation.

\section{Risk factors of stroke}

\section{THE MANAGEMENT OF ELDERLY HYPERPTENSIVES IN ITALY: THE \\ G.A.I.A. STUDY}

A. Zaninelli, G.F. Gensini, M. Volpe, U. Senin, A.A. Bignamini, A. Zanchetti, the GAIA Study Investigators

University of Florence, University of Rome, University of Perugia, University of Milan, Italy

The G.A.I.A. (Gestione Ambulatoriale dell'Iperteso Anziano) project is an observational study in the setting of the Italian General Practice, to evaluate the management of elderly hypertensives with regards to blood pressure control and cognitive function. The study was designed to monitor over 2 years approximately 2,000 hypertensives over 65, during regular access to the GP's office. We present the baseline data on 1907 patients surveyed by 440 GPs representing fairly well the whole Italian territory. Results (mean \pm SD) are: age: $72.7 \pm 5.4$; male $45.5 \%$; weight $74.1 \pm 12.1 \mathrm{~kg}$; height $163.4 \pm 8.2 \mathrm{~cm}$; BMI $27.8 \pm 4.2 \mathrm{~kg} / \mathrm{m}^{2}$; waist $99.4 \pm 15.1$ $\mathrm{cm}$; SBP $145.6 \pm 17.1 \mathrm{mmHg}$; DBP $81.2 \pm 9.0 \mathrm{mmHg}$; heart rate $74.1 \pm 9.2 \mathrm{~b} / \mathrm{min}$. Additional risk factors or associate clinical conditions included: diabetes $22 \%$, high total cholesterol $45.8 \%$; current smoking $8.4 \%$; previous smoking $28.1 \%$; CHD $6.3 \%$, angina $7.3 \%$; history of stroke/TIA $7.9 \%$; CABG/PTCA/vascular surgery $5.1 \%$, PAD $3.5 \%$; heart failure $2.4 \%$, other $9.4 \%$. The MMSE yielded a mean score $( \pm \mathrm{SD})$ of $25.04 \pm 4.07$ (range $8-30)$. BP was found properly controlled $(<140$ and $<90 \mathrm{mmHg}$; $<130$ and $<80 \mathrm{mmHg}$ in diabetics) in 585 patients $(32.9 \%)$. In conclusion, the $\%$ of elderly subjects adequately treated appears higher compared to the results reported in the general population, although there is large room for improvement. The higher number of contacts of the elderly with their GPs and a greater awareness of the hypertensive risk in elderly patients and GPs alike might explain such somewhat better control. The cognitive performance seems to be independent of current blood pressure levels and control. The proportion of statin prescription is substantial but still lower than the prevalence of conditions for which it is indicated. Also in this study, in agreement with others, the control of SBP in the elderly appears to be the major issue requiring adequate control. 


\section{Risk factors of stroke}

\section{CAROTID STENOSIS: IS THE "RULER" DEAD?}

G.L. Lenzi, E. Vicenzini

University of Rome "La Sapienza", Rome, Italy

NASCET and ECST criteria have shown that symptomatic patients with severe carotid stenosis (haemodynamically significative stenosis, $>70 \%$ ) are adressed toward surgery or endovascular treatment. In fully asymptomatic patients, when the finding of a carotid stenosis is truly incidental, medical treatment is preferred when the degree of stenosis is below than $60 \%$. Surgery is to be preferred in stenosis greater than this threshold, if the risk score of the surgical team is below $3 \%$. Again, the decision if the patient has to be addressed to surgery depends on how the "ruler"is applied: much more importance is nowadays referred to the characteristics of the plaque: "stable" or "unstable"? The definition of all the features of an "unstable" plaque is still under review and many factors have to be taken into account, apart from the degree of stenosis. Therefore, in those patients who have a carotid stenosis inferior to $60 \%$ something may happen because unknown or undetected mechanisms, at the level of the arterial wall, producing the rupture of the plaque and provoking an important embolus and a devastating stroke. We report here two patients, showing a particular "plaque motion" possibly belonging to this group.

Case A: R.F., female, 64 year old, asymptomatic. Vascular risk factors: hypertension. ECST criteria: $54 \%$ internal carotid artery stenosis. MRI: multiple ischemic lacunes. Case B: L.G., male, 61 years old, asymptmatic. Vascular risk factor: hypertensions, diabetes. ECST: $64 \%$ internal carotid artery stenosis. MRI: multiple ischemic lacunes.

Carotid Duplex ultrasound with high resolution technique showed a different, but impressive, "plaque motion" In case 1, at every cardiac cycle, movement of the plaque along vessel course was of $0.6 \mathrm{~cm}$, and in case 2 of $0.06 \mathrm{~cm}$.

Which patients is then deputed to surgery?

\section{Risk factors of stroke}

\section{OBSTRUCTIVE SLEEP APNEA AS IMPORTANT RISK FACTOR FOR STENOTIC ARTERY DISEASE IN PATIENTS WITH ISCHEMIC STROKE R. Dziewas}

University Hospital Münster, Münster, Germany

Introduction: Obstructive sleep apnea (OSA) is suggested to be a risk factor for ischemic stroke. The mechanism by which OSA causes predisposition to stroke is still unclear. The aim of this study was to assess the association between OSA and stenotic artery disease in patients with ischemic stroke.

Patients and Methods: 214 stroke patients were included in this prospective observational study. The apnea-hypopnea index (AHI) was determined by cardiorespiratory polygraphy. OSA was defined as $\mathrm{AHI} \geq 10 / \mathrm{h}$. Duplex ultrasonography was used for estimating atherosclerosis of the internal carotid artery (ICA). Stenosis of the ICA was classified as "low grade $(<50 \%)$ ", "medium to high-grade $(50$ $99 \%$ )" and "occlusion" according to standard criteria. Stroke severity was assessed with the NIH stroke scale. All patients were examined for the presence of vascular risk factors.

Results: Stroke patients with OSA were significantly older and more obese, suffered more often from arterial hypertension and had more frequently atherosclerotic artery disease of the ICA than stroke patients without OSA (all p < 0.05). Conversely, multivariate logistic regression analysis revealed that the parameters $\mathrm{AHI} \geq 10 / \mathrm{h}$ (Odd's ratio $[\mathrm{OR}]=1.90)$, smoking $(\mathrm{OR}=2.81)$, and increasing age $(\mathrm{OR}=1.04)$ were independently associated with atherosclerosis.

Discussion: These results suggest that OSA is independently associated with atherosclerotic artery disease. OSA may contribute along this pathophysiological pathway to a predisposition for ischemic stroke.

\section{Risk factors of stroke}

\section{DOES SLEEP PROTECT AGAINST STROKE? SLEEP STROKE, LESS}

\section{FREQUENT AND MORE SEVERE}

J. Jiménez-Conde, A. Ois, A. Rodríguez-Campello, M. Gomis, J. Roquer Hospital del Mar, Barcelona, Spain

Background: to evaluate the incidence, etiologic distribution, severity, prognosis and associated risk factors of stroke with clinical onset during the night sleep time. Methods: Patients with ischemic stroke that were assessed consecutively in our hospital during the last two years were recorded with the time of clinical onset, pathological antecedents, severity with NIHSS clinical scale, clinical Oxford classification, etiologic TOAST classification and functional outcome at 3 months. Functional deterioration (FD) was defined as an increase of 2 or more points in $\mathrm{mRS}$

Poster Session Second Visit

Risk factors of stroke at 3 months with respect to the previous $\mathrm{mRS}$. When the clinical disturbance came up during the night sleep time it was considered as sleep stroke (SS). Differences between SS and nonSS were analysed by means of Chi-square, t-student and logistic regression tests.

Results: From a total of 813 patients included, 127 of them were SS (15.6\%). Incidence was fewer than expected for the corresponding interval of hours. After the univariant and posterior logistic regression analysis of the studied risk factors, obesity was the only factor associated with SS (OR: 1.6, $\mathrm{p}=0.035)$. After adjusting for age and gender, atrial fibrillation was less prevalent in the SS group (OR: 0.42 $\mathrm{p}=0.007$ ). Patients with SS had a greater initial clinical severity (NIHSS: 9.62-6.21, $\mathrm{p}<.001$ ) and a worse functional outcome at 3 months (FD: $36.6 \%-27.4 \%, \mathrm{p}<.001$ ). Conclusions and Discussion: Our results show that stroke incidence decreases during night sleep. Patients with SS had a greater severity and a worse functional prognosis than non-sleep stroke. Obesity was related independently with SS. Prevalence of atrial fibrillation was fewer in SS group than in nonSS group after adjusting by age and gender.

\section{Risk factors of stroke}

\section{PREVALENCE OF FABRY DISEASE IN SPANISH PATIENTS WITH} CRYPTOGENIC STROKE

T. Segura, M.A. Barba, O. Ayo, B. Rallo

Hospital General Universitario de Albacete, Spain

Background and Purpose: Fabry Disease (FD) is a rare X-linked genetic disorder resulting from a defect in the gene for the enzyme alpha-galactosidase A (A-GAL). FD causes an accumulation of glycosphingolipids in vascular endothelium and visceral tissues. Incidence of cardiovascular events is very high in Fabry's patients Stroke frequently complicates FD and usually involves small brain arteries. FD diagnosis could be overlooked if patients do not present with classical symptoms and stroke could be the first clinical manifestation. Recently a high prevalence of FD has been reported among German patients with cryptogenic stroke. We investigate the frequency of FD in our stroke patients.

Methods: Our stroke data bank (SDB) prospectively collected the data referred to the stroke patients admitted in the neurology department of our Hospital, located in the inland Spain, from June-00 to December-03. This SDB is included in the BADISEN project. The BADISEN uses a criteria for classification of subtypes of stroke very similar to the TOAST. We selected from our SDB all males among the ages of 18 and 55 with the final diagnosis of small-vessel disease or stroke of undetermined etiology, and carried out an enzymatic determination (ED) of A-GAL. Results: Out of 502 patients evaluated, $35(7 \%)$ fulfilled the criteria. Three had died, it was impossible to contact with other 3 and 2 refused to participate in the study. So, we did the ED in 27 patients. Mean age, 45 (22-55), 37\% were smokers, $40 \%$ hypertensives, $18 \%$ diabetics. None of them had angiokeratomas. Ten $(37 \%$ complaint about acroparesthesias, and 5 (18\%) about hypohydrosis. Two (11\%) had microalbuminuria and 2 had left ventricular hypertrophia at stroke time. The A-GAL activity was normal in all cases.

Discussion: We have not found cases of FD among our young male stroke patients of undetermined or small-vessel disease etiology. This may reflect a low prevalence of FD in our population's stroke patients.

\section{Risk factors of stroke}

\section{RELATION BETWEEN SERUM CREATININE LEVELS AND CREATININE} CLEARANCE IN PATIENTS WITH ISCHEMIC STROKE

G. Piñol, I. De la Puerta, I. Beltran, J.A. Mauri, E. Mostacero

Clincal Hospital of Zaragoza, Zaragoza, Spain

Introduction and Objectives: Serum creatinine is a product of muscular metabolism and it is a good index of renal function. However, it only increases when renal function decreased more than $50 \%$ and it is influenced by age, sex, body mass and weight. For this, we think that creatinine clearance $(\mathrm{CrC})$, which includes age and body weight, is better index to detect patients with mild and moderate renal failure (RF) than serum creatinine levels (SC). In many occasions aterosclerotic disease affects brain, heart and kidney. We know that RF is a risk factor for silent lacunar infarcts. We think that it is necessary to detect the patients with stroke and RF to treat them with the most appropiate drugs.

Method: During one year, we measured the SC and $\mathrm{CrC}$ in 273 consecutive patients with ischemic stroke. Patients were categorized according to $\mathrm{SC}$ : no $\mathrm{RF}$ ( $\mathrm{SC}<1.2$ $\mathrm{mg} / \mathrm{dl}$ ), mild (SC 1.2-2), moderate (SC 2-3) and severe (SC $>3$ ); and according to CrC calculated by Cokcroft-Gault formula: no $\mathrm{RF}\left(\mathrm{CrC}>90 \mathrm{ml} / \mathrm{min} / 1.73 \mathrm{~m}^{2}\right)$, mild (60-89), moderate (30-59), severe (15-29) and end-stage renal disease $(<15)$.

Results: $86.4 \%$ of the 273 patients have a normal renal function according to CS, however the percentage falls to $23.1 \%$ by using $\mathrm{CrC}$. Of the 231 patients with 
normal SC 46.8\% (108), 24.7\% (57) and 1.7\% (4) have mild, moderate and severe $\mathrm{RF}$ respectively according to $\mathrm{CrC}$; of the 34 patients with mild $\mathrm{RF} 73.5 \%$ (25) and $23.5 \%$ (8) have moderate and severe RF; and of the 7 patients with moderate RF $42.9 \%$ (3) and $14.3 \%$ (1) have severe and end-stage RF respectively.

Conclusions: Our findings provide support for the concept that serum creatinine levels are not sufficient to detect RF in stroke patients and it is necessary to calculate $\mathrm{CrC}$ to detect this patients and to treat them correctly.

\section{Risk factors of stroke}

\section{G/5G POLYMORPHISM OF PAI-1 IS ASSOCIATED WITH} ATHEROTHROMBOTIC ISCHEMIC STROKE

Y. Wang, M. Levi, M. Parsons, J. Attia, L. Lincz, C. Meldrum, J. Sturm, P. McGettigan, R. Scott, J. Macquire

John Hunter Hospital/Hunter Medical Research Institute, Australia

Background: Plasminogen activator inhibitor type 1 (PAI-1) and tissue-type plasminogen activator (t-PA) are key components in the fibrinolytic system. We aimed to determine whether the $4 \mathrm{G} / 5 \mathrm{G}$ polymorphism of PAI-1 and the $-7351 \mathrm{C} / \mathrm{T}$ polymorphism of t-PA are associated with atherothrombosis of ischemic stroke.

Methods: We conducted a case control study of 190 hospital cases of first-ever ischemic stroke and 185 community-based controls. T-PA and PAI-1 genotypes were determined using a TaqMan allelic-discrimination assay. Differences in the distribution of genotypes between cases and controls, and among subtypes of stroke were calculated. Associations between polymorphisms and stroke risk factors were also assessed.

Result: The 4G/4G polymorphism was more frequent in large artery artherothrombotic and lacunar strokes. When these two groups are combined, the association between 4G/4G PAI-1 genotypes and stroke is statistically significant (Odds Ratio $[\mathrm{OR}]: 2.2$; $95 \%$ Confidence Interval $[\mathrm{CI}]: 1.01-4.8 ; \mathrm{p}=0.047$ ) in a multivariate logistic regression model. A trend of increasing admission diastolic $(\mathrm{p}=0.01)$ and systolic blood pressure $(\mathrm{p}=0.05)$, and triglycerides $(\mathrm{p}=0.04)$ was found from $5 \mathrm{G} / 5 \mathrm{G}$, $4 \mathrm{G} / 5 \mathrm{G}$ to $4 \mathrm{G} / 4 \mathrm{G}$ of PAI-1 genotyping. The $4 \mathrm{G} / 4 \mathrm{G}$ genotyping was associated with admission hypertriglyceridemia (OR, 3.0, 95\% CI, 1.1-8.3, p=0.037) in the multivariate analysis. No significant association between the t-PA genotyping and stroke and its risk factors was found.

\section{Risk factors of stroke}

STROKE IN WOMEN: A HOSPITAL BASED STUDY IN TUZLA, BOSNIA AND HERZEGOVINA

D. Smajlovic, D. Salihovic, O. Ibrahimagic, A. Burina, O. Sinanovic,

M. Vidovic

University Clinical Centre Tuzla, Tuzla, Bosnia-Herzegovina

Background: The aim of this study was to analyze characteristics of acute stroke in women.

Methods: From January 2001 to December 2004, 2986 patients with first-ever acute stroke were admitted at the Department of Neurology Tuzla, Bosnia and Herzegovina. Of these $72 \%$ were ischemic strokes, $19 \%$ intracerebral hemorrhages, $4 \%$ subarachnoid hemorrhages and 7\% unknown strokes. Risk factors, stroke severity (Scandinavian Stroke Scale, SSS), stroke type, etiology, outcome at 1 month and predictors of death were assessed in all patients.

Results: Overall, there were $1569(52.5 \%)$ women with stroke. Mean age was higher in women $(67.8$ vs $65.0, \mathrm{p}<0.0001)$. Hypertension $(\mathrm{p}<0.0001)$, hearth disease $(p=0.02)$, atrial fibrillation $(p<0.0001)$ and diabetes mellitus $(p<0.0001)$ were factors related to women. Stroke severity at admission was higher in women than in men (SSS 27.0 vs 31.0, p<0.0001). There were no differences in ischemic and hemorrhagic strokes according to sex. Women suffered more cardioembolic stroke ( $22.6 \%$ vs $14.4 \%, \mathrm{p}<0.0001)$, men suffered more atherothrombotic $(40.5$ vs $32.7 \%$, $\mathrm{p}=0.0002)$. Mortality at 1 month was higher in women than in men $(34.6 \% \mathrm{vs}$ $28.7 \%, \mathrm{p}=0.0006)$ and also handicap (Rankin Scale, mean) (2.8 vs 2.5, $\mathrm{p}=0.002)$. Older age, atrial fibrillation, cardioembolic stroke and severe stroke at admission were predictors of death in women with first-ever stroke $(\mathrm{p}<0.005)$

Conclusion: Stroke in women is associated with specific patterns of a profile of risk factors and a different distribution of ischemic stroke subtypes. Mortality and handicap at 1 month in women with first-ever stroke is higher than in men.
FACTORS RELATED TO DEGREE OF PERIPHERAL ARTERIAL OCCLUSIVE DISEASE IN ACUTE STROKE PATIENTS (BY TOE-BRACHIAL INDEX)

J.H. Lee, Y.H. Jung, S.A. Choi, G.S. Kim, J.H. Cho

National Health Insurance Corporation Ilsan Hospital, Koyang-shi, Kyonggi-do, South Korea

Background: A low ankle-brachial index(ABI) is associated with an increased risk of peripheral artery disease, cardiovascular disease and stroke. Recently, the toe-brachial index(TBI) has been receiving attention for non-invasive method for measurement of atherosclerosis which is generally regarded as a risk factor for stroke. Limited data exist regarding the relation between a low TBI and stroke. In this study, we investigated the relation between a low TBI and stroke.

Method: We reviewed Ilsan Hospital stroke database of recent 3 years. Total 50 acute cerebral infarction patients with completed stroke database and TBI study was included. The relationship with TBI, TOAST classification, risk factors of stroke and degree of cerebral artery stenosis was investigated.

Results: According to degree of TBI, peripheral arterial occlusive disease(PAOD) was classified as normal (TBI $>0.9)$, moderate $(0.6<\mathrm{TBI}<0.9)$ and severe $(\mathrm{TBI}<0.6)$ Moderate and severe PAOD was more common in large artery atherosclerosis (LAA) patients than small vessel occlusion (SVO) and cardioembolism (CE) patients between subgroups by TOAST classification. PAOD was not significantly correlated with the number of stenotic vessels by the transcranial Doppler study(TCD).

Conclusion: Toe brachial index can be a useful marker which reflect the degree of peripheral atherosclerosis in stroke patients. PAOD was more common and severe in large artery atherosclerosis patients among cerebral infarction patients.

\section{Long-term outcome of stroke}

\section{Long-term outcome of stroke}

THREE-MONTHS OUTCOME IN ISCHEMIC STROKE PATIENTS WHO ARE NOT TREATED WITH TPA DUE TO MILD OR IMPROVING DEFICITS M. Nesi, P. Nencini, V. Palumbo, D. Inzitari Careggi Hospital, Florence, Firenze, Italy

Background: Patients with mild or improving ischemic stroke symptoms do not receive intravenous tissue plasminogen activator (tPA) according to SITS-MOST criteria. A substantial minority of these patients are unable to be discharged home. Information on long-term outcome in this subgroup is still limited.

Methods: We analysed data prospectively collected in the Careggi Hospital Acute Stroke Registry from February 2004 to August 2005. We retrospectively selected a subgroup of patients arrived within 3 hours who were clinically "too good to treat" and were admitted to Stroke Unit. We followed them up for three months.

Results: Of the 290 acute ischemic strokes arriving in Emergency Room within 3 hours from onset, $62(21 \%)$ were not given tPA because of mild or improving deficit. All had National Institute of Health Stroke Scale (NIHSS) $<5$. Thirty-two $(52 \%)$ were admitted to the Stroke Unit. No patients died during hospitalisation; 6/32 $(19 \%)$ were not discharged home because of neurological worsening; $25 \%(8 / 32)$ had some functional impairment at three month with a Modified Rankin Scale of 1 or more (mRS 1 in 4 patients, mRS 2 or more in 4 patients).

Conclusions: Almost $20 \%$ of patients excluded from treatment because of mild or rapidly improving symptoms suffered substantial in-hospital and 3 month disability. Predictors of outcome need to be identified for an appropriate selection of patients with mild symptoms who could benefit of thrombolytic treatment.

\section{Long-term outcome of stroke}

FALL EVENTS AMONG PEOPLE WITH STROKE IN THE COMMUNITY S. Psarakis, S. Voyaki, A. Efstratopoulos, E. Papakitsoy, K. Dretakis, E. Vasiliadis, P. Stavlas

Orthopedic Dept. of General Hospital of Elefsis “Thriasio”, Greece

Aim: Aim of the present study was the investigation of frequency and circumstances of falls among community patients with stroke and to compare characteristics of fallers and nonfallers.

Methods: We studied 123 patients living in the community with stroke (58M/65 $\mathrm{F}$, mean age, $74.5 \pm 11.5$ years) of which 45 had right-hemisphere infarction, 28 left-hemisphere infarction and 50 a brainstem lesion. Time since onset of stroke ranged from 6 to 220 months (mean 48 months). We used standardized tests to 
measure mobility, upper limber function, activities of daily living (ADL ability), MMS score. Information about fall events was collected by using a questionnaire.

Results: 60 patients $(48.7 \%)$ were classified as non fallers and 63 patients $(51.3 \%)$ as fallers of whom 30 had fallen repeatedly, mean 3.4 falls. No significant differences were found between fallers and non fallers on any of the measures used. However, those who had 2 or more falls $(\mathrm{N}=30)$ had significantly reduced ADL ability $(\mathrm{P}=0.020)$ and MMS score $(\mathrm{P}=0.045)$ in comparison with those who had not fallen or experienced one fall event.

Age was significantly correlated with the number of falls $(\mathrm{r}=0.42, \mathrm{P}=0.001)$.

Among 63 patients $35(55.6 \%)$ reported minor injuries and $28(44.4 \%)$ major fractures. The suspected causes of falls were loss of balance and foot weakness during walking.

Conclusions: The high risk of falling among people with stroke is evident even long period after the stroke event. Repeat fallers were older, had reduced ADL activity, and lower MMS score in comparison to non fallers.

\section{Long-term outcome of stroke}

LONGER-TERM OUTCOME OF YOUNG ADULT STROKE PATIENTS IN A MULTIETHNIC POPULATION - AN ANALYSIS FROM THE SOUTH LONDON STROKE REGISTER

M.A Busch, C. Coshall, I. Burger, C. Wolfe

King's College London, Division of Health and Social Care Research, London, United Kingdom

Background: Stroke is rare in young adults but can have a devastating effect on individual lives and a high public health impact due to long years of disability and lost productivity. This study aimed to determine the longer-term outcome of young adult stroke patients and to identify factors that influence outcome.

Methods: A prospective population-based register of all incident strokes with annual follow-up in a multi-ethnic population of 234533 , of whom $21 \%$ are black. Data from all patients aged 15 to 45 years registered between 1995 and 2004 were used. Outcomes were case fatality, long-term survival and disability (Barthel Index $<20$ ). Statistical analysis included multivariable analysis to examine associations between patient characteristics and poor outcome.

Results: Of 2764 registered incident strokes, $174(6.3 \%)$ were in young adults (median age 38.5 years, $52.3 \%$ men, $40.8 \%$ black ethnicity). Stroke subtype was ischaemic in $41.4 \%$, intracerebral haemorrhage in $23.6 \%$, and subarachnoid haemorrhage in $33.9 \%$ of cases. Case fatality was $17.8 \%$ at 30 days and $19.5 \%$ from 3 months to 1 year. Haemorrhagic stroke was associated with higher fatality (OR $2.3, \mathrm{p}<0.005$ ), adjusting for other clinical and socio-demographic factors. Some disability was found in $31.9 \%$ and $41.4 \%$ of interviewed survivors after one and three years, respectively. Kaplan-Meier analysis showed better survival for black than white people and for ischaemic than for haemorrhagic stroke but no survival difference in relation to these or other factors was found in the multivariable model. Conclusion: Young adult stroke patients had a high case fatality but good long-term survival after three months, though persistent disability was frequent. Haemorrhagic stroke was the most common subtype and had a much poorer prognosis in the short term but did not independently influence longer-term outcome.

\section{Long-term outcome of stroke}

\section{BASILAR ARTERY DIAMETERS AND 5 YEAR MORTALITY IN STROKE} PATIENTS

F. Pico, J. Labreuche, P. Amarenco, the GENICS Investigators

Versailles Hospital, Versailles, France

Background and Purpose: Few and conflicting data exist on the case fatality in stroke patients with basilar artery dolichoectasia. We analyzed basilar artery characteristics (diameter, height of bifurcation, transverse position) and 5 year mortality (i.e. all-causes, vascular stroke and nonstroke deaths) in stroke patients

Methods: 466 consecutively recruited patients with brain infarction confirmed by MRI had measurement of the basilar artery diameter with a 16-dioptre lens. The height of the bifurcation and transverse position of the basilar artery (BA) were assessed using semi quantitative scales. Patients were followed during a median of 5.3 (range, 1.5 to 6.6 ) years, and were classify into stroke, vascular nonstroke and non-vascular deaths using the French National registry of death certificates.

Results: 157 total and 88 vascular deaths including 54 strokes deaths occurred BA diameters were associated with increased 5-year stroke mortality but not with total and nonstroke vascular mortality. The adjusted hazard ratio (HR) of stroke mortality per one millimeter increase in BA diameter was 1.23 (95\% CI, 1.07-1.41). A higher risk of stroke death was associated with BA diameter at the 95th percentile

Poster Session Second Visit

Long-term outcome of stroke
(BA $>4.3 \mathrm{~mm}$ ) (adjusted HR, 3.69; 95\%CI, 1.63-8.38) and height of bifurcation (adjusted HR for score $>1,2.08$; 95\%CI, 0.93-4.68) but not with transverse position Conclusions: Basilar artery diameters were independently associated with cerebrovascular mortality. BA diameter $>4.3 \mathrm{~mm}$ may be a marker for a high risk of fatal stroke

\section{Long-term outcome of stroke}

\section{CLINICAL AND RADIOLOGICAL OUTCOME AFTER MCA INTRA-ARTERIAL THROMBOLYSIS: CLINICAL AND RADIOLOGICAL RISK FACTORS}

A. Costa, M. Cappellari, M. Pavia, R. Spezi, V. Vergani, R. Tortorella,

S. Filipponi, R. Gasparotti, M. Magoni

Vascular Neurology - Spedali Civili di Brescia; Neuroradiology - University of Brescia, Brescia, Italy

Intra-arterial thrombolysis (IAT) provides an alternative to endovenous thrombolysis in selected patients with acute middle cerebral artery (MCA) occlusion within 6 hours from stroke onset.

Negative recanalization and poor outcome are common findings.

Clinical and radiological risk factors can help in selecting patients.

We correlate a large number of risk factors with MCA recanalization and clinical outcome after IAT to find a predictive score.

We evaluated 30 patients with non lacunar MCA stroke treated with IAT prourokinase or rtPA. On arrival in hospital patients underwent ECG, blood chemistry and CT scan before thrombolysis.

Age, sex, history of hypertension, diabetes, atrial fibrillation and other vascular risk factors were collected.

NIHSS was assessed on admission and with the timing of SITS-MOST protocol until 36 hours after IAT. Another NIHSS was assessed at 15 days.

CT scan follow up was performed at 24-36 hours and 30 days.

Three months outcome was assessed with modified Rankin Scale (mRS).

An ANOVA analysis with Statwiew software was performed.

On admission mean NIHSS was $17.3 \pm 6.8$ and at 15 days $9.8 \pm 6.5$. Four patients died in acute phase and 2 during follow-up (mortality rate 20\%). At 90 days 13 (43\%) became functionally independent (mRS 0-2)

Partial or complete recanalization was obtained in $25(83 \%)$.

Age $>70$ years $(p=.01)$ and history of diabetes $(p=.04)$ were significantly associated with negative recanalization.

Age $>70$ years $(\mathrm{p}<.01)$, baseline and 15 days NIHSS $>17(\mathrm{p}<.001$ and $\mathrm{p}<.0001)$ and final ischemic area $(\mathrm{p}<.01)$ were significantly associated with $\mathrm{mRS}>2$ at 90 days. Atrial fibrillation had a trend with $m R S>2(p=.07)$.

The combination of clinical and radiological findings are well related with 90 days MCA stroke outcome after IAT. A score can be purposed.

\section{Long-term outcome of stroke}

\section{PROSPECTIVE FOLLOW-UP OF STROKE PATIENTS: ASSESSING THE UTILITY OF A STROKE NETWORK OF CARE (THE RUN-STROKE EXPERIMENT)}

D. Chavot, E. Medeiros-De Bustos, V.J. Cote, P. Ragionieri, M. Pellicier,

P. Kehoe, O. Retel, T. Moulin

CHU Besançon, Besançon, France

Background: Since 2003 stroke patients have been prospectively included in a follow-up cohort in the framework of a stroke network of care. The main objectives of the stroke network are to monitor risk factor management and compliance with treatment, stroke evaluation and sequelae of stroke.

Methods: Stroke patients are prospectively included after standardised stroke management in the Besançon Stoke unit. Follow-up is organised by 2 nurse coordinators; patients are contacted by telephone every 6 months following discharge. Using a semi-structured interview, cardiovascular risk factors and compliance with specific treatment, stroke evaluation (clinical and imaging examinations) sequelae (cognitive, depression, epilepsy, handicap) are recorded and entered into a database. Necessary actions are taken; patients are seen by the network private-practice based neurologist if required. GPs are systematically notified and given feedback of key data.

Results: The cohort at present includes 1,294 patients, (52\% male, $48 \%$ female). Mean age at event 70 y (19-101). Patients who refused follow-up and who are not residents in the region are excluded from the network. To date, 737/984 of included patients have received follow-up by telephone at 6 months. Approximately $30 \%$ $(n=218)$ of patients screened by telephone needed action to be taken regarding their post-stroke follow-up care. Of a total of 429 actions (i.e. about 2 actions per patient) $30 \%$ related to risk factor control and compliance with treatment $(n=127), 13 \%$ 
stroke evaluation $(n=54), 30 \%$ sequelae $(n=129)$. A further $27 \%(n=119)$ of actions related to minor symptoms and general follow-up have also been taken.

Discussion: The network structures and organises stroke care and has taken specific action for $30 \%$ of patients which will improve long-term follow-up and secondary prevention of stroke.

\section{Long-term outcome of stroke}

\section{RELATIONSHIP BETWEEN ENDOTHELIAL PROGENITOR CELLS (EPCS)} AND OUTCOME IN ISCHEMIC STROKE

T. Sobrino, J. Arenillas, D. Brea, M. Castellanos, R. Rodríguez-González,

O. Hurtado, M. Rodríguez-Yañez, E. Mosquera, R. Leira, J. Castillo

Hospital Clínico Universitario de Santiago, Santiago de Compostela, Spain

Objective: EPCs mediate processes of neovascularization, re-endothelialization and repair of vascular endothelium promoted by biochemical factors such as, VEGF, SDF-1alpha, and active MMP-9. The objective was to analyze the function of EPCs in the recovery of the cerebral infarction.

Patients and Methods: 24 patients (12 males, average $71.4 \pm 8.8$ years) with first episode of ischemic stroke ( $<12 \mathrm{~h}$ from onset) and without risk of atheromatosis, were prospectively included in the study. NIHSS was performed at admission, 24h, $72 \mathrm{~h}$, day 7 , and day 90). Outcome was evaluated by mRS at 3 months. Serum levels of VEGF, SDF-1 alpha, and active MMP-9 (determined by ELISA), and quantification of EPCs were performed at admission, 72h, day 7, and day 90 .

Results: There was no significant difference in EPC count on admission, at $72 \mathrm{~h}$ and at day 7. However, $46 \%$ of the patients showed an increment in the EPC number during the first week, which was more significant in patients with highest EPC number on admission. There was an inverse relationship between EPC levels on admission and functional outcome $(r=-0.735, \mathrm{p}<0.0001)$. This relationship was stronger for increment percentage of the EPCs between admission and day $7(\mathrm{r}=-0.875, \mathrm{p}<0.0001)$. VEGF $(\mathrm{r}=0.650)$, SDF-1alpha $(\mathrm{r}=0.699)$ and active MMP-9 $(\mathrm{r}=0.712)$ at baseline showed a correlation with the increased EPCs. Similar results were found for EPC concentration on admission. In a logistic model, including EPCs concentration on admission, outcome was associated to molecular markers at admission $(\mathrm{p}<0.01)$. In a second model we introduce the EPC increment, only VEGF levels on admission were associated with outcome $(\mathrm{p}=0.015)$. These data suggest other action mechanisms for VEGF.

Conclusion: Increment of the circulating concentration of EPCs in peripheral blood was associated to a better outcome in stroke, and was a strongly correlation with active MMP-9, VEGF and SDF-1 alpha.

\section{Long-term outcome of stroke}

\section{BARTHEL INDEX IN A MIDDLE EAST COUNTRY: TRANSLATION, VALIDITY AND RELIABILITY}

S. Oveisgharan

Isfahan Cardiovascular Research Center, a WHO Collaborating Center, Isfahan, Iran

Background: In Iran, a Middle East country, no disability scale has been translated and validated for use in stroke clinical trials. This study was designed to translate Barthel Index and make its Persian translated form valid and reliable.

Methods: All Barthel Indices' items were translated to Persian. Also, Modified Rankin Scale (MRS)'s item was also translated to Persian. Telephone interview was used as the method of data acquisition. 2 interviewers were chosen for this means, in order to accelerate data gathering and measure interrater agreement. Samples were selected from Isfahan Cardiovascular Research Center's Stroke Registry Unit, a WHO collaborating center in the center of Iran. All the patients were registered as stroke or intracerebral hemorrhage (ICH). These patients were inhabitants of Isfahan Province who had suffered from stroke or ICH between 12 to 24 months before data acquisition. Chronbach's alpha, test - retest reliability, concurrent validity with MRS, interrater agreement and item analysis were done for the translated questionnaire.

Results: Translated questionnaires were filled by interview from 459 stroke patients. Their mean age was $68.11 \pm 11.59 .243$ of them were male (52.9\%). Cronbach's alpha was 0.9354 . Spearman correlation coefficient between translated barthel index scores and Modified Rankin Scale scores was -0.912. Spearman correlation coefficient between 2 scores, to determine test - retest reliability was 0.989 . Concordance correlation, to determine interrater agreement was 0.994 . All corrected item - total correlations were greater than 0.5 .

Discussion: Persian translated version of Barthel index is a reliable and valid questionnaire for use in stroke clinical trials.
9 Long-term outcome of stroke

OUTCOME OF FIRST-EVER STROKE IN TARTU, ESTONIA. A POPULATION-BASED STUDY

R. Vibo, J. Kñrv, M. Roose

University of Tartu, Tartu, Estonia

Background: The aim of the current study was to evaluate the outcome of first-ever stroke at 6 months. The study was based on the Third Stroke Registry in Tartu, Estonia.

Methods: The outcome of stroke was assessed using the Barthel Index (BI) score on the 2nd and 7th day in hospital and 6 months after the stroke. The pre-stroke dependence was measured with the modified Rankin Score (mRS). The mRS scores were also evaluated on the 7th day in hospital and 6 months after the stroke.

Results: The data from 433 patients were used with mean age of $72.0 \pm 12.1$ years Fifty-six men and 110 women had died 6 months after the stroke and the overall probability of death was 0.43 (95\% CI $0.38-0.48)$. The changes in BI scores in individual surviving patients showed, that from day 7 to 6 months, the BI scores of $43 \%$ of patients improved, $33 \%$ experienced a decline and $24 \%$ of patients had an unchanged score $(\mathrm{p}=0.01)$. The $\mathrm{mRS}$ score 3 to 5 before the stroke significantly increased the odds of higher mRS 6 months following stroke occurrence $(\mathrm{OR}=11.9)$. In the multivariate analysis, the predictors for dependency were sex $(\mathrm{OR}=2.85)$ and age $(\mathrm{OR}=3.03)$

Conclusions: The comparison of stroke outcome is difficult due to lacking of uniform assessment criteria. The probability of death is somewhat higher than in other centers. The proportion of functionally independent patients 6 months following stroke is similar to other populations, but the percentage of functionally dependent patients in our study is somewhat lower compared to others. It could be related to poor rehabilitation facilities.

\section{Long-term outcome of stroke}

\section{HAND PALSY DUE TO CORTICAL STROKE HAS GOOD FUNCTIONAL} PROGNOSIS

L. Hadrane, M. Teichmann, P. Brugières, A. Gueguen, G. Fénelon, H. Hosseini Hôpital Henri Mondor, Créteil, France

Introduction: Isolated hand palsy due to stroke in a limited part of the precentral gyrus has previously been reported but little is known about the functional recovery in such patients. We report 3 cases with long-term follow-up.

Case Reports: Three men $(70,50$, and 82 yrs) presented with acute and isolated hand palsy yielding complete functional incapacity. MRI diffusion weighted imaging of the 3 patients showed a hyperintensity limited to an area previously referred to as the "precentral knob" and shown to correspond to the hand area of the precentral gyrus. Hand palsy was complete in 2 cases (1 and 2), predominant on finger and hand extensors in one case (3) and involved dominant side in 2 cases ( 1 and 3 ). Stroke was consecutive to a stenosis of the right internal carotid artery responsive of embolic migration (case 1), to cardiac embolism secondary to a patent foramen ovale associated with an atrial septal aneurysm (case 2), and of undetermined origin in patient 3.

Functional recovery was evaluated at 10 days and 12 months after stroke onset except for patient 3 who was evaluated at 10 days and 1 month. All patients had motor rehabilitation following stroke. We scored manual dexterity using a four-point scale (0: no function, 1: function severely impaired, 2 : mildly impaired, 3 : normal) Scores were for patient 1: 0 at 10 days and 2 at 12 months; patient 2: 0 at 10 days and 3 at 12 months; patient 3: 0 at 10 days and 2 at 1 month.

Discussion: Our study confirms that the motor hand area is located in a knob-shaped part of the precentral gyrus. Isolated hand palsy evokes damage to this particular structure. Data on the long term evolution of case 3 will be presented. Two patient regained normal or near normal function of their hand at one year, suggesting that.

\section{Long-term outcome of stroke}

\section{MALNUTRITION AFTER STROKE - FACTORS ASSOCIATED WITH POST-STROKE WEIGHT LOSS}

A.-C. Jonsson, A. Lindgren

Clinical Sciences,Lund University Hospital, Lund, Sweden

Background: Malnutrition is a risk factor for poor outcome after stroke. We examined prevalence of weight loss $\geq 3 \mathrm{~kg}$ in an unselected group of stroke survivors.

Methods: Body Mass Index (BMI) and NIH Stroke Scale (NIHSS) were assessed at baseline. After 4 and 16 months (follow-up I and II) we measured Barthel Index (BI), serum albumin (A) prealbumin (PA) and weight for 305 surviving patients 
from a population-based group of 416 patients with first-ever stroke. Variables associated with weight loss $\geq 3 \mathrm{~kg}$ were analysed using Mann-Whitney test. Multiple regression was used to find predictors of weight loss.

Results: Weight loss of $\geq 3 \mathrm{~kg}$ between baseline and follow up I was found in 74 patients, and between follow-up I and II in 55 patients. Weight loss at follow-up I was associated with: NIHSS score $(\mathrm{p}<0.001), \mathrm{HbA1c}(\mathrm{p}=0.02), \mathrm{BI}(\mathrm{p}<0.001)$, living situation $(\mathrm{p}<0.001)$, mental dysfunction $(\mathrm{p}=0.001)$, eating problems $(\mathrm{p}<0.001), \mathrm{A}$ $(\mathrm{p}<0.001)$, and PA $(\mathrm{p}=0.02)$. Baseline predictors of weight loss at follow-up I were: higher NIHSS $(\mathrm{p}=0.001)$, sex $(\mathrm{p}=0.004)$ and lower BMI $(\mathrm{p}<0.001)$. Weight loss between follow-up I and II, was associated with: BMI $(\mathrm{p}<0.05)$, A ( $\mathrm{p}=0.001)$, PA $(\mathrm{p}=0.006)$, and mental dysfunction $(\mathrm{p}=0.01)$. Multiple regression showed association between weight loss and the following factors: $\operatorname{sex}(p<0.03)$, living in nursing home $(\mathrm{p}<0.01)$ and lower $\mathrm{A}(\mathrm{p}=0.02)$.

Discussion: Weight is a simple but important measure to detect malnutrition that may occur especially in patients with more severe strokes. In the post-stroke care it is important to observe patients living alone or in nursing homes and screen for possible eating problems which may be a consequence of stroke and associated with mental dysfunction.

\section{Long-term outcome of stroke}

\section{THE APOE EPSILON4, GENDER, AND ONE-YEAR OUTCOME AFTER} ISCHEMIC STROKE

A. Czlonkowska, G. Gromadzka, M. Baranska-Gieruszczak,

I. Sarzyńska-Długosz, A. Ciesielska

Institute of Psychiatry and Neurology, Medical University, Warsaw, Poland

Background: During recent years, a strong evidence for association of the apolipoprotein E gene (APOE) e4 allele with increased risk, severity, and/or poor prognosis in various forms of acute or chronic central nervous system (CNS) impairments. Evidence is accumulated that gender influences APOE e4 effects in CNS impairments. We aimed to evaluate whether the APOE e4 allele status is associated with severity of an acute ischemic stroke (IS), and if it predicts 1-year outcomes indicated by severe functional disability, dependence in daily living or death, in men and women.

Methods: Six hundred sixty six IS patients ( 330 men, 336 women) were studied. Neurological condition of patients was measured with Scandinavian Stroke Scale, disability rate was assessed with the Barthel Index, the handicap level was measured with the use of Rankin Scale. Information on patients' survival status was also collected. APOE genotyping was performed by the PCR-RFLP method.

Results: Among women APOE e4 allele status was not related to stroke severity and outcome. Men carriers of the APOE e4 had more severely compromised clinical conditions at time of maximum impairment and greater neurological impairment on admission, than men not-carrying this allele. In men population, the APOE e4 was a significant predictor of death within 1-month $(\mathrm{OR}=3.00,95 \% \mathrm{CI}$ : 1.23-7.36), 3 -months ( $\mathrm{OR}=2.25,95 \% \mathrm{CI}: 1.02-4.94)$, and 1 -year $(\mathrm{OR}=2.30,95 \% \mathrm{CI}: 1.04-5.05)$ following stroke.

Conclusion: APOE e4 allele is a significant genetic factors modifying stroke severity and one-year mortality in men.

\section{Long-term outcome of stroke}

\section{LONG-TERM OUTCOME AND RISK PROFILE 4 YEARS AFTER STROKE OR} TRANSIENT ISCHEMIC ATTACKS

C. Haacke, Y. Epifanov, R. Dodel, A. Althaus, A. Spottke, T. Back

Klinikum Mannheim, University of Heidelberg, Mannheim, Germany

Objective: Despite declining acute mortality, long-term outcome of stroke is poor. In this study, long-term survival, outcome, and risk factors were assessed 4 years after stroke or transient ischemic attacks (TIA)

Methods and Patients: We included 151 consecutive patients admitted to the Dept. of Neurology, Marburg, between January 1 and March 31, 1999, with the diagnosis of TIA, ischemic stroke (IS) or intracerebral hemorrhage (ICH). After 12 and 48 months follow-up exams were performed using modified Rankin scale (mRS), Barthel index (BI), Short Form-36 (SF-36), and Hospital Anxiety and Depression Scale (HADS). Analysis of risk factors included hypertension, body mass index and depression scores.

Results: Seventy-seven patients completed the 4-year follow-up, 54 had died. Kaplan-Meier survival curves revealed a cumulative mortality of 50\% (ICH group), followed by IS (44\%) and TIA (18\%). Recurrent cerebrovascular events $(n=76)$ occurred in $46 \%(\mathrm{n}=35)$ of survivors with the highest rate in TIA patients $(51 \%)$. IS and ICH patients showed significant improvement in mRS and BI scores. TIA patients, in contrast, had reduced mean scores of $\mathrm{mRS}$ and $\mathrm{BI}(\mathrm{p}<0.05)$ in follow-up exams. SF-36 revealed decreased scores for physical and mental health among survivors indicating reduced quality of life. BMI did not correlate with clinical scores and was increased in $66 \%$ of patients, hypertension was present in $60 \%$ of stroke survivors, and pathological HADS depression scores were observed in 25\% of patients only $40 \%$ of which received antidepressive medication.

Conclusion: Cumulative mortality and morbidity of TIA approximates that of ischemic stroke on a long-term scale. TIA is a concerning disease with a high rate of subsequent cerebrovascular events resulting in increasing disability.

\section{Long-term outcome of stroke}

LONG-TERM MORTALITY IN YOUNG STROKE PATIENTS AND CONTROLSA STUDY IN WESTERN NORWAY, HORDALAND COUNTY U. Waje-Andreassen, H. Naess, L. Thomassen, G.E. Eide, C.A. Vedeler Haukeland University Hospital, Bergen, Norway

Background: Mortality in stroke patients is often reported higher than in controls. We wanted to identify long-term mortality as well as risk factors for mortality in young stroke patients.

Methods: We evaluated 232 patients aged 15-49 years with first-ever cerebral infarction in 1988-1997 and 453 birthday- and sex-matched controls with a median observation time of 11.1 years. Patients and controls were evaluated by KaplanMeier survival analysis. Kaplan-Meier method with log-rank testing was used for comparing categories of risk factor variables in regard to mortality in 192 patients who had participated actively in a former follow-up study. Cox proportional hazards model was applied to adjust for the effect of multiple risk factors on mortality.

Results: 45/232 (19\%) patients and 9/453 (2\%) controls died during follow-up (p $<0.00005)$. The following variables were risk factors for long-term mortality in the patients: active tumour disease $(\mathrm{p}<0.00005)$, alcohol (more than 12 alcohol units/week) $(\mathrm{p}<0.00005)$, coronary disease $(\mathrm{p}<0.00005)$, marital status $(\mathrm{p}=$ $0.0003)$ and smoking (more than 20 cigarettes/day in young adult age) $(\mathrm{p}=0.003)$. Conclusion: Our study shows a significant increased long-term mortality in young stroke patients compared to controls. Identified risk factors were high alcohol consumption, heavy smoking and living alone. Causes of deaths revealed several cases of pulmonary cancer, probably due to heavy smoking.

\section{Long-term outcome of stroke}

\section{STROKE IN HOSPITALISED PATIENTS VERSUS ADMITTED PATIENTS:} ARE THERE INEQUALITIES IN STROKE CARE

A. Bhalla, N. Smeeton, A. Rudd, C. Wolfe

King's College, London, Carshalton, Surrey, United Kingdom

Background: In order to improve outcome for all stroke patients requiring hospital treatment information is required to understand their access to stroke care. We investigated the process of care between patients admitted to hospital with stroke (admitted) and patients having had a stroke during hospitalisation (inpatient).

Methods: We used data from 2561 patients with first ever stroke collected by a population based register from 1995 to 2005. Using multivariate analyses controlled for sociodemographic and clinical factors, we investigated the access to stroke care for both inpatient and admitted patients. Mortality data at 3 months was also analysed.

Results: 2266 patients (88\%) were admitted to hospital compared with 295 (12\%) who were inpatients at the time of stroke. Inpatients were more likely to be older $(\mathrm{P}<0.001)$, incontinent $(\mathrm{P}<0.001)$, dysphagic $(\mathrm{P}=0.001)$ and have coma $(\mathrm{P}=0.001)$ than admitted patients. Being admitted at the time of stroke was associated with higher odds of stroke unit treatment $(\mathrm{OR}, 2.7 ; 95 \% \mathrm{CI}, 1.87$ to $3.94, \mathrm{P}<0.001)$ as well as a higher odds in receiving brain imaging (OR, 2.2; 95\% CI, 1.1 to 4.69 , $\mathrm{P}=0.03$ ). There was no significant difference in the interval between stroke onset and discharge from hospital (admitted 19 days vs. inpatient 21 days) $\mathrm{P}=0.28$. By 3 months, $458(20 \%)$ of admitted patients were dead compared with 129 (44\%) inpatients $(\mathrm{P}<0.001) .913(40 \%)$ of admitted patients were discharged home compared with $71(24 \%)$ of inpatient strokes $(\mathrm{P}<0.001)$ at 3 months.

Conclusion: There is inequity in the provision of stroke care to patients requiring hospital treatment with lower rates observed in inpatients after case mix adjustmen and poorer outcomes. Policies need to be developed to ensure all hospitalised patients receive evidence based stroke care.

Long-term outcome of stroke 
16

Long-term outcome of stroke

PERCEIVED QUALITY OF LIFE IN SURVIVORS OF BASILAR ARTERY OCCLUSION TREATED WITH INTRAVENOUS THROMBOLYSIS

L. Soinne, P.J. Lindsberg, T. Tatlisumak, R.O. Roine, M. Kallela, O. Häppölä,

M. Kaste

Helsinki University Central Hospital, Helsinki, Finland

Background: Occlusion of the basilar artery (BAO) has a dismal natural course. Even with interventions, mortality remains high and the survivors have a propensity to severe physical disability.

Methods: We treated 50 consecutive patients (aged 28 to 84 years, mean 64.4 years) with proven BAO with intravenous thrombolysis (alteplase). The subjective quality of life of the survivors were evaluated at the chronic stage using a perceived qualityof-life assessment (PQoL) rating the subject's present satisfaction with ten different aspects of life on a 10-point scale (100 as maximum). The functional outcome was assessed with modified Rankin Scale (mRS) and disability with Barthel Index (BI). Results: Twenty-seven patients (54\%) survived the extended follow-up, of them 23 ( $85 \%$ ) completed the PQoL scale. Median PQoL sum was 74 points (mean 71.7, range 20-99), the highest scores being given to social and communicative aspect and lowest to individual mobility aspect. On the mRS, 15 patients $(56 \%$ of the survivors) were scored as 0-2, categorized as good outcome, whereas $12(44 \%)$ were dependent (mRS 3-4). Mean BI was 81.5 (range 10-100). PQoL scores were strongly correlated to independence in daily life and outcome scoring ( $R=0.65$ and -0.78 , respectively, $\mathrm{p}<0.001$, Spearman rank correlation coefficient).

Conclusions: The favourable long-term outcome after successful treatment of basilar artery occlusion is associated with a high level of subjective wellbeing.

17 Long-term outcome of stroke

PERCEPTION OF QUALITY OF LIFE IN STROKE: A PRELIMINARY STUDY USING A STROKE-SPECIFIC SCALE

N. Rude, P. Decavel, M. Puyraveau, P. Kehoe, O. Retel, T. Moulin

University of Franche-Comté, Besançon, France

Background: Perception of quality of life (QoL) is important to determine the long-term outcome of stroke patients. Our objective was to study the feasibility of administering a French version of the Stroke Impact Scale (SIS) and compare it to a generic QoL scale (SF-12) and a specific semi-structured interview (SSSI).

Method: Stroke patients were selected from the Besançon Stroke Unit, examined at 6 months (outpatients living at home = "group H"; patients living in institutionalised care $=$ "group I") and given a neurological assessment (NIHSS, Barthel Index, mRS, MMS, MADRS). QoL was evaluated by SIS, SF-12 and SSSI (42 questions). Each patient assessment was associated with an interview (face-to-face or telephone) with the referent care giver (GP or hospital doctor) and the patient's relatives.

Results: Thirty patients were included, mean age: 60 (19-86), 30\% were working and $50 \%$ were married. All scales were successfully administered for $84 \%$ of patients. The failure rate ( $>2$ questions not answered) was higher in group I (40\%) compared to group H (5\%).SSSI highlighted the QoL issues important to patients and included in the SIS, such as communication with relatives, autonomy and fears, as well as those not taken into account, i.e. future perspectives, sleep and pain.Care givers and relatives were successfully interviewed, except for GPs ( $>80 \%$ failure rate).

Discussion: Our study showed that the different QoL dimensions must be adapted to the patient's health status in order to better analyse their perception of QoL and to improve the correlation between stroke type and QoL. In order to validate the SIS, a cohort of 500 patients will be evaluated using the same protocol but without the specific semi-structured interviews.

\section{Long-term outcome of stroke}

ADMISSION BLOOD GLUCOSE AND LONG-TERM MORTALITY AFTER INTRACEREBRAL HEMORRHAGE. THE ABBA TRIAL INVESTIGATORS S.H. Lee, H.J. Bae, K.S. Hong, K.H. Yu, B.C. Lee, Y.S. Lee, J.M. Park, K.H. Cho, B.J. Park, B.W. Yoon, the ABBA Trial Investigators

Seoul National University Hospital, Seoul, South Korea

Background: It is well understood that uncontrolled hyperglycemia after acute ischemic stroke is associated with poor prognosis. However, few studies have been conducted on the association between admission hyperglycemia $(\mathrm{AH})$ and prognosis of intracerebral hemorrhage (ICH).

Methods: Patients with supratentorial ICH were selected from the database of the Acute Brain Bleeding Analysis (ABBA) trial conducted between Oct 2002 and Mar 2004 in Korea. Effects of AH were examined in relation to long-term mortality using
Cox regression models. AH was coded as positive if glucose level was ${ }^{3} 7 \mathrm{mmol} / \mathrm{L}$. Mortality was confirmed using national death certificate data.

Results: During the follow-up, 194 (18.6\%) of 1,044 ICH patients died. The 30-day, 90-day, and 1-year mortalities were $5.9 \%, 8.8 \%$, and $16.0 \%$, respectively. Mortality was significantly higher in the patients with AH: the 30-day, 90-day, and 1-year mortalities were $7.9 \%, 10.8 \%$, and $20.0 \%$ for the patients with $\mathrm{AH}$, whereas the mortality figures were $3.2 \%, 6.0 \%$, and $10.3 \%$ ( $\mathrm{p}<0.001$ with log-rank test). After adjusted for sex, age, ICH volume, low Glasgow coma scale scores, and intraventricular hemorrhage, $\mathrm{AH}$ was an independent risk factor for long-term mortality (HR $1.40,95 \%$ CI 1.01 to 1.93 ). As the blood glucose level was used instead of the presence of $\mathrm{AH}, 1 \mathrm{mg} / \mathrm{dL}$ increment of glucose level increased the risk of death independently (HR $1.004,95 \%$ CI 1.002 to 1.007 ).

Discussion: Our study shows that $\mathrm{AH}$ and the increase of blood glucose level are associated with the long-term mortality after ICH. It may be postulated that hyperglycemia should be corrected in the acute stage of $\mathrm{ICH}$, regardless of presence of diabetes.

\section{Chronic conditions and recurrences}

\section{Chronic conditions and recurrences}

\section{FIVE CASES OF ISOLATED ANGIITIS OF THE CENTRAL NERVOUS} SYSTEM (IACNS)

A. González-Hernández, J.C. López-Fernández, O. Fabre-Pi, J. Hernández-Gállego, M.C. Pérez-Viéitez, V. Araña-Toledo, M.J. Alemany-Rodríguez, A. Cubero-González

Hospital Universitario de Gran Canaria Dr. Negrín, Las Palmas, Spain

Introduction: IACNS is an uncommon cause of stroke. Its diagnosis requires the exclusion of other causes of vasculitis and the support of pathological or radiological studies.

Methods: We reviewed the history of patients diagnosed of IACNS between 1996-2005, all attended in a tertiary hospital covering 400.000 inhabitants.

Results: We report five patients diagnosed of IACNS, four fulfilling diagnostic criteria and one with neither radiological nor pathological confirmation but with the exclusion of secondary vasculitis. They were three males and two females between 41-57 years old. The follow up varied from 1 to 9 years. Four patients had focal deficit; the other presented headache and encephalopathy. Four of them had seizures, well controlled by one antiepileptic drug. Cerebral MRI was abnormal in all cases Two patients showed ischemic lesions, two had ischemic and haemorrhagic strokes, and one presented haemorrhagic findings. The cerebral angiography was performed in four patients; only one showed multiple cerebral arterial segmental narrowing. The CSF had mild raised protein levels $(54.2-183 \mathrm{mg} / \mathrm{dL})$ in all cases, with normal cell amount in four of them and 20 cells in the other. Leptomeningeal and brain biopsies revealed mononuclear infiltration in the wall of small vessels in four cases, being unspecific in one. First, all patients received only steroid treatment, adding cyclophosphamyde in only one in the follow up, with a good response.

Conclusion: IACNS is a rare cause of stroke that may produce ischemic and haemorrhagic lesions, being necessary for diagnosis to exclude other causes of vasculitis. Diagnosis is based on angiographic and pathological findings, with limited specificity and sensibility. The overall prognosis may be good with a low rate of relapses.

\section{Chronic conditions and recurrences}

CLINICAL FEATURES AND CLINICAL EVOLUTION IN PARKINSONISM WITH "VASCULAR FEATURES"

E. Fabrizio, N. Vanacore, P. Stirpe, E. Cecconi, G.L. Lenzi, G. Meco

"La Sapienza" University Rome, Rome, Italy

Background: Parkinsonism due to cerebrovascular disease (vascular parkinsonism, VP) is a distinct clinicopathological entity. It accounts for $4.4-12 \%$ of all cases of parkinsonism. Typically, parkinsonism in slow-onset VP tends to be bilaterally symmetrical, affecting the lower limbs more than the upper limbs ('lower-body parkinsonism'), and resting tremor is usually absent. Vascular risk factors are commonly present. It is suggested that VP does not usually respond to dopaminergic treatment. MRI of the brain is a useful test to define vascular lesion load. Functional imaging with SPECT and PET helps in differentiating PD from VP. Aim of this study is to evaluate clinical features and clinical evolution between patients with and without "vascular features".

Design: We conducted a descriptive and prospective study on a consecutive series of outpatients. In each patients has been performed a medical history, a neurological 
examination and a TC or MRI scan. Patients were assigned in two groups: in the first group was enrolled patients with "vascular features" (risk factors and/or cerebrovascular lesions to neuroimaging), in the second group patients without "vascular features".

Results: Out of 123 patients, 84 of the first group and 39 of the second group. In patients with "vascular features" the age at the onset and the UPDRS motor score was significantly higher compared to the second group (respectively $\mathrm{p}=0.03$ and $\mathrm{p}=0.05$ ). At 1 year no statistical significance in clinical evolution and in UPDRS motor score were found between two groups.

Conclusions: Data of this study confirm that the age and the severity at the onset is higher in patient with "vascular features". This data confirm that VP is a common and important cause of parkinsonism in older people.

\section{Chronic conditions and recurrences}

\section{EFFICACY OF REANGIOPLASTY OF IN-STENT RESTENOSES AFTER}

\section{CAROTID ARTERY STENTING}

P.A. Ringleb, J.U. Regula, S. Külkens, M. Hartmann

Universityclinic Heidelberg, Heidelberg, Germany

Background: Rate of in-stent-restenosis (ISR) after carotid artery stenting (CAS) differ between 2.7 and $20.8 \%$ in the literature. The method of choice to treat ISR is a matter of debate.

Methods: All patients treated with CAS were prospectively followed up using ultrasound. Significant ISR was defined as peak systolic velocity (PSV) of at least $210 \mathrm{~cm} / \mathrm{s}$ at duplex. In this study we focused on patients with at least 6 months follow-up.

Results: For 143 patients with 146 interventions follow-up results of at least 6 months are available. During follow-up of 6 to 104 months (mean 27.7 months), 21 patients (14.4\%) developed a severe ISR. Median time interval to detection of the ISR was 6 months (range 2 to 47 months). Four patients (19\%) had temporary neurological symptoms (TIA) caused by the ISR. Angiography was performed on 14 patients to treat the ISR by re-angioplasty. Two types of stenosis were found: longrange stenosis and more circumscript stenosis. No neurological symptoms occurred during rechts-intervention. Follow-up after re-intervention of at least 12 months is available for 12 patients. After 12 months 5 patients treated with re-angioplasty have remained free of recurrence, 5 patients got minor restenoses up to $50 \%$. One patient needed another re-intervention because of another ISR, one developed an asymptomatic occlusion of the stent while pausing antiplatelet therapy.

Conclusion: In our patients we found an ISR-rate of $14.4 \%$ with a median time interval to detection of 6 months after intervention. Despite there is still no consensus on ultrasound measurement of ISR, it was possible to detect ISR by ultrasound and to confirm ISR by angiography in all patients. Our case series showed in contrast to others a relatively high rate of neurological symptoms caused by ISR. Best treatment of ISR is still unclear, but patients can be treated by rec-angioplasty with low complication rate and satisfying long-term results.

\section{Chronic conditions and recurrences}

\section{ASPIRIN IS NOT ASPIRIN - EVIDENCE OF DIFFERENCES IN STROKE} PROPHYLAXIS

A.K.-H. Grotemeyer

Klinikum Saarbruecken, Saarbruecken, Germany

Background: Different acetylsalicylic acid (ASA) application frequencies and dosages are never directly compared in clinical studies. Sacco et al. (Arch Neurol 62:403-408) presented the EPSS II data analysed according the stroke prognosis instrument II (SPI-II) criteria (Kernan et al., Stroke; 31:456-462). Based on these results is may be possible to compare patients with different stroke recurrence risk within different studies.

Methods: According to the SPI-II criteria the 563 included patients of the doubleblind Piracetam vs. ASA- study (J Neurol Sci.181:65-72.) were reclassified.

Results: 239 patients were at low recurrence risk ( $\leq 3$ points), 274 showed a medium risk (4-7 points) and 50 patients were classified with high recurrence risk ( $>8$ points). The recurrence of (only) stroke/year (only in the ASA-group) was $1.85 \%$ in the low risk group, $3.35 \%$ in the medium risk group and $2.14 \%$ in the high risk group. Sacco et al. found in the EPSS II reanalysis (only for the ASA-group) a stroke recurrence of $3.7 \%$ in the low, $9.6 \%$ in the medium and $13.2 \%$ high risk group/year. - If the patient classification according to an expected recurrence of stroke risk is accepted the mean difference of the studies remains the ASA-dosage and the application frequency given for stroke-prophylaxis $(2 \times 25 \mathrm{mg}$ ASA in the Aggrenox ${ }^{\circledR}$-study and $3 \times 200 \mathrm{mg}$ ASA in the Piracetam-ASA-study). In the medium risk group difference within the two studies is significant (fisher's exact test (two sided) $\mathrm{p}=0.015$ )
Discussion: The given score shows the expected difference of low and medium risk patients.(The group of high-risk patients is too small.) However the number of stroke recurrence is different. So it should be kept in mind that it is not to exclude that a daily dosage of ASA that minimizes ASA-nonresponder (Thromb.Res.63; 587-593) may be a relevant clinical point for the observed effectiveness of ASA in stroke prophylaxis-studies.

\section{Chronic conditions and recurrences}

\section{PERIPHERAL ARTERIAL DISEASE AMONG A HIGH RISK ASIAN} POPULATION WITH ISCHEMIC STROKE, HEART DISEASE OR DIABETES MELLITUS

N. Venketasubramanian, K.C. Loh, J. Tan, K.H. Mak

National Neuroscience Institute, Singapore, Singapore

Introduction: Peripheral arterial disease(PAD), an important manifestation of atherothrombosis, remains a largely under-diagnosed disease. The frequency of PAD among Asians is not well described. Aim: This study was performed to determine the frequency of PAD among Singaporean patients with ischemic stroke(IS), coronary artery disease(CAD) or diabetes mellitus(DM).

Methods: Patients were included if they had an acute IS, myocardial infarction/unstable angina, or DM, target of 150 subjects in each group. Exclusion criteria included age $<40 y$, amputation of both upper or both lower limbs. A standardised questionnaire was administered, collecting data on demographics, history of vascular risk factors, and history of claudication(Edinburgh questionnaire, EQ), and biochemical indices. The ankle-brachial index(ABI) was determined using a handheld Doppler, dividing the higher of the ankle systolic pressures by the higher of the brachial systolic pressures. PAD was diagnosed if $\mathrm{ABI}$ was $<0.9$. Data was analysed using SPSSv11.0.

Results: A total of 444 subjects were recruited, 148 each with IS, CAD, or DM Male:female ratio $1.3: 1$, mean age $62.1 \mathrm{y}$ (SD $10.24 \mathrm{y}$, range $40.0-90.0 \mathrm{y}) .59 .9 \%$ had hypertension, $42.1 \%$ were smokers/ex-smokers. $11.0 \%$ were claudicant. PAD was found among $22.3 \%$ of IS, $29.7 \%$ of $\mathrm{DM}$ and $30.4 \%$ of $\mathrm{CAD}(\mathrm{p}>0.05) ; 32 \%$ of nonlacunar and $17.3 \%$ of lacunar stroke $(\mathrm{p}=0.042)$ had PAD. PAD was found in $46.9 \%$ of claudicants and $25.1 \%$ of non-claudicants. PAD was significantly associated with advanced age, female gender, higher body-mass index(BMI) hyperlipidemia, abnormal electrocardiogram(ECG). By regression analysis, PAD was significantly associated with advanced age, abnormal ECG, and total cholesterol levels.

Conclusions: PAD is not uncommon among Asian patients with cerebrovascular or cardiac disease, and diabetics. The Edinburgh questionnaire is not an accurate screening test for PAD. PAD is associated with advanced age, abnormal ECG and hyperlipidemia.

\section{Chronic conditions and recurrences}

\section{EFFECT OF CLOPIDOGREL ON PLATELET PARAMETERS IN NEUROLOGICAL PATIENTS: STROKE AND TRANSIENT ISCHEMIC ATTACK DESPITE ASPIRIN}

A. Debliquis, B. Chatelain, C. Chatelain, P. Laloux

Department of Neurology and Laboratory of Haematology, Mont-Godinne University Hospital, Yvoir, Mulhouse, France

Background: Clopidogrel is recommended in patients who experience a stroke or transient ischemic attack despite aspirin treatment. Further relapses occur nevertheless under clopidogrel. It will be helpful to detect this resistance by biological parameters. The aim of this study is to measure the effect of clopidogrel on platelet parameters.

Methods: Three populations are defined. Group 1: 21 normal subjects without clopidogrel; Group 2: 7 healthy volunteers before and after clopidogrel administration (75 mg Q.D) for 24 days; Group 3: 26 patients treated with clopidogrel (75 mg Q.D). Platelet parameters studied are PFA, PRP platelet aggregation with ADP 5 $\mu \mathrm{M}$ with Arachidonic Acid 0,5 mg/ml and with collagen $190 \mu \mathrm{g} / \mathrm{ml}$, flow cytometry of platelet VASP phosphorylation, platelet expression of PAC-1 before and after ADP and Arachidonic Acid, molecular biology of P2Y12 ADP receptor.

Results: Clopidogrel induces modification of VASP phosphorylation, platelet aggregation with ADP and Arachidonic Acid, PAC-1 expression after ADP and Arachidonic Acid, but has no effect on aggregation with collagen, PFA, PAC-1 expression without ADP and Arachidonic Acid. Fifty percent of neurological patients show the same results for VASP phosphorylation as normal subject without clopidogrel. Lack of correlation between PRP platelet aggregation with ADP 5 $\mu \mathrm{M}$ and VASP phosphorylation is observed in neurological patients. These two parameters are actually well correlated on normal subjects. Concerning molecular biology, the two studied mutations (G52T and Ins801A) cannot predict the response of clopidro- 
gel on platelet parameters. One of the two neurological patients who relapsed on clopidogrel shows paradoxically a normal response of VASP phosphorylation.

Discussion: Some biological parameters are markedly influenced by clopidogrel treatment. Neurological patients had abnormal platelet parameters in comparison with healthy volunteers. Prospective study is needed to evaluate the significance of this biological resistance in order to determine the clinical resistance to clopidogrel.

\section{Chronic conditions and recurrences}

\section{DOES ASYMPTOMATIC MIDDLE CEREBRAL ARTERAL STENOSIS SHOW PLATELET HYPERACTIVATION? \\ J.-K. Cha, J.-H. Seo, S.-R. Kim \\ College of Medicine, Dong-A Unversity Hospital, Busan, South Korea}

Middle cerebral arterial Stenosis is a common occlusive disease in Asian patients. However, it has been little known about its detail pathophysiological mechanisms. Particularly, with increasing prescription of anti-platelet agents for prevention of progression of MCA stenosis, there has been little information about relationship between MCA stenosis and platelet activation.

Thirty-two patients (Mean age, 52.2 \pm 11.8 ) with asymptomatic MCA stenosis (Mean Flow Velocity $>100 \mathrm{~cm} / \mathrm{s}$ on Transcranial Doppler study) were enrolled in this study. The control group comprised of 42 normal subjects (Mean age, 54.1 \pm 11.8 ) who showed no MCA stenosis on TCD study, recruited as the control group. Platelet activation was measured ADP $(10 \mathrm{uM})$ induced aggregability by using whole blood aggregometer.

The extent of ADP induced platelet aggregation in asymptomatic MCA group $(13.1 \pm 5.5 \mathrm{ohm}, \mathrm{p}<0.01)$ was significantly increased than those in control group $(7.9 \pm 4.9 \mathrm{ohm})$. Also, Asymptomatic MCA stenosis showed considerable extent of platelet activation as much as those of acute ischemic stroke in acute ischemic stroke in our previous study.

Platelet hyperactivation might be an important pathological mechanism in asymptomatic MCA stenosis as like as acute ischemic stroke. In the future, we need the more detailed study about importance of changes of platelet activation on the progression of asymptomatic MCA stenosis.

\section{Recovery and rehabilitation}

\section{Recovery and rehabilitation}

\section{A 4-WEEK HOME-BASED REHABILITATION PROGRAM IMPROVED MOTOR RECOVERY IN SPASTIC HEMIPLEGIA: A RANDOMISED, PLACEBO-CONTROLLED STUDY \\ S.S. Ng, C.W. Hui-Chan \\ The Hong Kong Polytechnic University, Kowloon, Hong-kong}

Background and Purpose: TENS and task-related training (TRT) have been found to improve motor recovery in patients with chronic stroke in separate studies. However, no study has compared the relative effectiveness of TENS (a passive approach) and TRT (an active approach) in improving the motor function of patients with chronic stroke. The objective of this study was to evaluate the efficacy of a home-based TENS and TRT on ankle spasticity, muscle strength and gait in spastic hemiplegia.

Methods: Fifty-nine patients (51 males, 8 females) with moderate to severe spastic ankle plantarflexors were recruited into a randomized, single-blinded, placebocontrolled trial. Patients were randomly assigned to either one of the three 4-week home-based program: (1) TENS, or (2) placebo-TENS plus TRT, or (3) control. Outcome measures included Composite Spasticity Scale, peak torques generated during maximum voluntary contraction (MIVC) of ankle dorsiflexors and plantarflexors, and gait velocity at baseline, and bi-weekly during the 4 weeks of treatment.

Results: The TENS group demonstrated significantly earlier decreases of spasticity and earlier increase of peak dorsiflexor torque than the placebo-TENS plus TRT group $(\mathrm{P}<0.05)$, when compared with those of the control group. However, only the latter but not the former group showed a significant increase in the plantarflexor torque $(\mathrm{P}<0.01)$ when compared with that of the control group. Nevertheless, no significant difference was found among the 3 groups in gait velocity over the 4 -week treatment period.

Discussion: Our results thus suggest that TENS and TRT may improve different aspects of motor functions in patients with chronic stroke. The study also shows that this home-based rehabilitation program was feasible and acceptable by patients with chronic stroke.

\section{Recovery and rehabilitation}

\section{SHOULD WE CONSIDER AGE WHEN PLANNING AND DELIVERING STROKE REHABILITATION?}

J. Hammerton, S. Mawson, P. Enderby

Sheffield Hallam University, Sheffield, South Yorkshire, United Kingdom

Background: Increased age is recognised as a risk factor for stroke, so an aging population combined with reduced stroke mortality means costs of rehabilitation are rising. Time limits are one way to achieve effective and efficient services, based on evidence that most recovery occurs in the first 12 weeks following the event. However evidence also suggests that older people take more time to consolidate learning and utilise skills functionally. This paper describes the results of a study investigating the influence of age on treatment intensity, therapy referral at discharge from acute rehabilitation and on the pattern of recovery observed.

Methods: A cohort sample of consecutive referrals to a UK community stroke rehabilitation team (CRT) entered this prospective longitudinal study. Subjects were divided into 2 groups according to their age at stroke onset. Amount of CRT treatment, referral at discharge and proportion of change in disability were assessed at baseline, 6 weeks, 12 weeks and 6 months. T-test and t-test for proportions were used to analyse the data, a $95 \%$ confidence level was set.

Results: 102 subjects entered the study and 82 were accounted for at the 6 month assessment. 43 subjects were under 65 years, mean $56.8(41.1-64.6,6.7), 59$ subjects were over 65 years mean, $74.8(65.6-88.3,5.7)$. No significant difference was found in the amount of CRT treatment. The under 65 group were significantly more likely to be referred for further treatment at discharge from CRT and the over 65 group showed a significantly greater proportion of change in disability at 6 months.

Discussion: This study has provided evidence that older people respond to rehabilitation later in the recovery process. Delayed functional improvement may lower the therapists' expectations of older people and therefore result in reduced referral rates to further treatment. These factors should be considered when planning and delivering acute stroke rehabilitation to older people.

\section{Recovery and rehabilitation}

\section{CHARACTERISTIC OF VISUAL ATTENTION AMONG DIFFERENT} VISUOSPATIAL POSITIONS IN STROKE PATIENTS WITH EXPERIENCE OF FALLS

S. Shimizu, M. Maeda, Y. Ikeda

Kitasato Univ., International Univ. of Health and Welfare, Tokyo Metropolitan Univ., Sagamihara, Kanagawa, Japan

Background: Many studies have investigated falls and attention function among stroke patients; however, few studies have reported the relationship between visuospatial attention and falls in detail. Purpose: This study investigated the relationship between falls and visual attention characteristics among various visuospatial positions based on simple reaction times (RTs) to visual stimuli for stroke patients and healthy elderly.

Methods: Participants were 28 stroke patients (17 patients have experience of falls in the past year, and 11 patients have not) and 18 healthy elderly. All healthy elderly have not experience of falls in the past year. RTs were recorded using RT estimation software on the personal computer. Fixation point and reaction stimuli were presented on the screen. The stimulus was presented at one of the 16 sites, which were located on circles with the radius of $1 \mathrm{or} 11 \mathrm{~cm}$. Therefore, a visual angle of the stimuli was 2 (VA2) or $20 \mathrm{deg}$. (VA20). Stimuli were randomly presented five times each site, the total was 80 times. The delay from the stimulus to the pressing a key was recorded as RT. RTs longer than 1000 ms or trials without a reaction were considered to be errors.

Results: The number of errors among patients who experienced falls was significantly higher than the patients without experience of falls and healthy elderly. In addition, RTs among participants with experience of falls were significantly slower than RTs among participants without experience and healthy elderly at all stimulus positions for VA2 and VA20.

Conclusions: These results may indicate that stroke patients with experience of falls have decreased visual attention function in all visual fields in compared to stroke patients without experience of falls and healthy elderly. 


\section{Recovery and rehabilitation}

EFFECTS OF SOMATOSENSORY STIMULATION ON MOTOR FUNCTION IN CHRONIC STROKE PATIENTS

A.B Conforto, L.G. Cohen, R.L. Santos, M. Scaff, S.K.N. Marie

Hospital das Clínicas/São Paulo University, São Paulo, Brazil

Background: Somatosensory stimulation elicits improvements in motor function in patients with chronic subcortical stroke. The purpose of this study is to study the applicability of this adjuvant approach in patients with predominantly cortical lesions, an issue that is less well known.

Methods: Eleven patients with chronic corticosubcortical strokes and mild to moderate upper limb disability were randomized in a cross-over design to $2 \mathrm{~h}$ somatosensory stimulation in the form of median nerve stimulation above sensory threshold (MNS) or control stimulation (median nerve stimulation below sensory threshold, CS). Following stimulation, patients were submitted to motor training. Hand disability was evaluated with the Jebsen-Taylor test (JTT) before, after MNS or CS and after training.

Results: Baseline JTT scores were, on average $121.6 \pm 67$ s. No significant differences were found between JTT scores in CS and MNS sessions (Wilcoxon, $\mathrm{p}=0.168$ ). JTT scores were normalized according to baseline performance for statistical analysis. ANOVA with repeated measures with two factors (intervention: MNS or CS) and three levels (pre-stimulation, post-stimulation and post-training) revealed a significant effect of time $(\mathrm{p}<0.001)$ and interaction intervention $\mathrm{x}$ time $(\mathrm{p}=0.042)$ with no significant effect of intervention $(\mathrm{p}=0.180)$.

Conclusion: A single MNS session brings motor performance to a higher level prior to training in patients with chronic corticosubcortical strokes. Further studies should investigate whether single or repeated MNS sessions can be used to shorten duration or decrease intensity of training of relevant daily life activities and hence, decrease the need of assisted therapy in clinical stroke rehabilitation.

\section{Recovery and rehabilitation}

\section{DEVELOPING A PROTOCOL FOR 3D MOVEMENT ANALYSIS OF THE STROKE UPPER LIMB \\ J. Hammerton, S. Mawson, G. Mountain, M. Gittoes \\ Sheffield Hallam University, Sheffield, South Yorkshire, United Kingdom}

Background: Kinematic analysis of gait is used as an assessment and outcome tool in many areas of physiotherapy, however evidence for its use in the rehabilitation of the stroke upper limb is poor, despite the impact disability in this area has on personal care, balance and length hospitalisation. Currently no standardised protocol exists to collect and analyse functional and clinically relevant kinematic parameters. This study aimed to develop and pilot a protocol for collecting kinematic data for functional upper limb movement following stroke as part of an EPSRC funded study.

Methods: Computerised 3D Movement data, using one CODA cx1, was collected and analysed. A minimum of 3 markers were required on each segment to collect movement in the sagittal, coronal and horizontal planes. A variety of marker placements were trialed to establish an optimum location allowing sufficient visibility for accurate analysis. Age matched normal data was also collected in order to select the optimum number of markers to capture all abnormal movements. Functional movements were chosen based on key rehabilitation goals.

Results: 9 normal ( 3 male) and 8 stroke participants $(5$ male) were recruited to the study.Age range was 60-74(70.8, 6.77), upper limb disability was measured using DASH(range 32-75) and the length of time since stroke ranged from 3 months-5 years. The final protocol has 16 markers across 3 segments(trunk, upper and lower arm). Visibility was ensured through the use of wands. The protocol provides information on the kinematic parameters of range of movement, angle displacement and cycle duration. Clinically significant differences between normal and stroke participants were evident.

Discussion: The chosen marker protocol was simple to apply, did not impede functional movement and was able to collect and analyse abnormal movement from the defined planes. This newly developed protocol will enable further research in the area of stroke rehabilitation.

\section{Recovery and rehabilitation}

STROKE PATIENT REHABILITATION AFTER DISCHARGE FROM STROKE UNITS IN POLAND - EVALUATION OF NEEDS

I. Sarzyńska-Długosz, A. Czlonkowska, M. Krawczyk

Institute of Psychiatry and Neurology, Warsaw, Poland

Every stroke patient should undergo rehabilitation. We aimed to evaluate the accessibility and needs of comprehensive stroke in-patient rehabilitation in Poland. Materials and Methods: We have sent a questionnaire evaluating rehabilitation departments in Poland, where stroke patients undergo early rehabilitation. We divided them into 5 classes from A to F: class A - comprehensive rehabilitation: kinesitherapy minimum 60 minutes/day, speech therapy minimum 30 minutes 5 days a week, rehabilitation of other cognitive impairments, group kinesitherapy; B - possibility of all types of therapy, but done less frequently; C - kinesitherapy, speech therapy; D - kinesitherapy, cognitive rehabilitation; E - kinesitherapy. We estimated number of acute stroke patients in Poland. We evaluated number of patients requiring in-patient rehabilitation based on results from 2nd Department of Neurology, Institute of Psychiatry and Neurology, Warsaw - all stroke patients who need in-patient rehabilitation have such possibility. We estimated number of beds required on rehabilitation departments for stroke patient in Poland.

Results: We obtained response from 115 of $172(66,9 \%)$ rehabilitation departments: 11 class A, 31 class B, 28 class C, 4 class D and 41 class E. 159 beds on class A departments are for stroke patients. From 64,896 acute stroke patients 9,927 will need in-patient rehabilitation. We need 604 more beds on rehabilitation departments for stroke patients in Poland.

Conclusion: Only $10 \%$ of rehabilitation departments could provide comprehensive stroke rehabilitation in Poland. Actually there is $20,8 \%$ from needed beds on rehabilitation departments for stroke patients. Improvement of rehabilitation accessibility is necessary for lowering number of disabled post-stroke patients.

\section{Recovery and rehabilitation}

\section{HEMISPHERIC ASYMMETRIES AND CHANGES IN THE STANDING POSTURE INPATIENTS FOLLOWING RECENT STROKES OR OLDER STROKES}

I.V. Karmanova, A.N. Novoselsky, I.A. Sinitsky, A.S. Ocheretovy Ivanovo State Medical Academy, Ivanovo Regional Rehabilitation Centre, Ivanovo, Russian Federation

We introduce our method of computer kyphoscoliosography (CKSG) which is intended for the spatial definition of 3 dimensional coordinates of markers applied to observed bone protrusions (spine, scapula corners etc). Thus the spatial characteristics of the curves determined by these markers are identified by the CKSG computer programme. The aim of this study was to analyse the changes in standing posture of stroke patients

obtained with CKSG.

102 patients (median age 57,8 years) with residual motor defects due to remote ischemicstrokes were included in the study; 48 patients had right-hand hemiparesis (66\% within 9 weeks, 34\% within 12 months or more); 54 patients had a left-hand hemiparesis ( $70 \%$ within 9 weeks,

$30 \%$ within 12 months or more) and 26 persons in control group. Patients were examined clinically, by CKSG, by functional tests (Motor club assessment: functional movement activities, Standing Balance, FIM) through 4 and 8 weeks after brain damage.

The images received from carrying out CKSG and the graph curves constructed from the results revealed the most typical changes of pose to the right and left hand hemineglect. The changes of force and tonus of muscles in paretic extremities in right hand stroke patients does not influence the changes of posture reflected in parameters of CKSG and are comparable to that of normal subjects $(\mathrm{R}=0.21)$. Right hemisphere stroke subjects demonstrated the relationship between changes of force and tonus of muscles in the paralysed limbs and disorders of upright posture ascribed by parameters of $\mathrm{CKSG}(\mathrm{R}=0.42-0.74, \mathrm{P}<0.05)$.

The results are consistent with the dynamic dominance hypothesis, which links left hemisphere specialisation to movement trajectory control and right hemisphere specialisation to position control.

\section{Recovery and rehabilitation}

VALIDITY OF NORTHWICK PARK DEPENDENCY SCORE (NPDS) SWEDISH VERSION 6.0

S. Svensson, K.S. Sunnerhagen

Institute of Neuroscience and Physiology, Göteborg University, Göteborg, Sweden

Inclusion criteria: Inpatients from three different clinics in Sweden, between 16-65 years of age with stroke.

Background: During the last 10-15 years, the medical care for stroke victims has improved as well as the rehabilitation efforts resulting in that more persons with severe impairments survive. There is a need for outcome measure instruments that are sensitive to small changes in dependency among these patients.

Method: Data from fifty six persons with a stroke, thirty six men and twenty women 
with a mean age of fifty two years were gathered. The assessment were made with Functional Independence Measure (FIM ${ }^{\mathrm{TM}}$ ), to measure level of disability in different situations and BCN-section of Northwick Park Dependency Score (NPDS), to measure level of dependency.

Gamma correlation was used to measure the concurrent validity between the total score between the FIM ${ }^{\mathrm{TM}}$ and the BCN-section of NPDS. Agreement of measure was tested with the rank-transformable pattern of agreement (RTPA).

Results: Concordance was good with a gamma -0.837 , Std Error 0.030 and P-value 0.000 . The observed distribution was compared with the total agreement of the total score of each patient, which tendency showed that NPDS differentiate when the dependency is high and FIM ${ }^{\mathrm{TM}}$ differentiate when the dependency is low.

Conclusion: The correlation between total FIM ${ }^{\mathrm{TM}}$ and the BCN section of NPDS is high. FIM ${ }^{\mathrm{TM}}$ and NPDS might complement each other. NPDS can bee used as a measure for severe injured patients and are sensitive when needs of nursing time is high.

\section{Recovery and rehabilitation}

\section{PLEXUS ANAESTHESIA: IMPROVING HAND FUNCTION IN SUBACUTE} STROKE

M. Stollorz, T. Steinforth, K.M. Stephan, V. Hoemberg

St. Mauritius Therapieklinik Meerbusch, Meerbusch, Germany

Recently Muellbacher et al. (2003) demonstrated dramatically improved hand motor function including some activities of daily living after plexus anaesthesia in chronic stroke. We were interested to see, whether this procedure would also help patients in the subacute phase after stroke ( 4 to 8 weeks after stroke).

Until now, 20 patients were included They were divided into three groups depending on the severity of sensorimotor symptoms. A special daily training course was implemented for all participants lasting for two weeks. Motor testing was done by occupational therapists and physiotherapists before and after therapy. Routinely, neurophysiological parameters were collected (somatosensory evoked potentials and motor evoked potentials). In about half of the patients, plexus anaesthesia on the clinically affected side was performed by an experienced anesthetist. The others served as controls.

Preliminary results suggest, that plexus anaesthesia ipsilateral to the affected arm helps to improve arm motor function not only in the chronic but also in the subacute stage. Further analysis with a larger number of patients will have to show, whether those patients, who underwent plexus anaesthesia and motor training had a better sensorimotor outcome than those, who had structured physiotherapy only.

\section{Recovery and rehabilitation}

\section{THE BIOELECTRICAL ACTIVITY OF LOWER LIMBS EXTENSORS IN PATIENTS WITH POSTSTROKE HEMIPARESIS AND GAIT ASYMMETRY \\ E.A. Kovrazhkina, V.V. Gudkova, V.I. Skvortsova}

Russian State Medical University, Moscow, Russian Federation

Background: The aim of our study was to define specific aspects of the bioelectrical activity (BA) of lower limbs extensors in gait cycle (GC) of patients with poststroke hemiparesis and gait asymmetry.

Methods: The study was conducted on 50 patients with poststroke hemiparesis: 20 patients with medium ( 3 points) paresis (group 1) and 30 patients with light (4-5 points) paresis (group 2). All patients had gait asymmetry with incorrect contact between paretic foot and its support. Were used podometry and curved EMG of proximal and distal lower limbs extensors in GC.

Results: The differences in muscle strength were discovered between the two groups $(\mathrm{p}<0.05)$. The maximum BA was below the line in both groups, was reliable below the line in group $1(\mathrm{p}<0.05)$. There were discovered abnormalities of phase muscle activity in the form of simultaneous activation of proximal and distal extensors in GC with prolongation of their activity during the whole period of support for all patients. These differences were founded on paretic and intact sides. The reliable amplitude prevalence of BA maximums in muscles on the intact side was discovered only in patients with light paresis $(\mathrm{p}<0.05)$.

Discussion: The amplitude contraction of BA maximums relates with working motor units common diminution, with GC slowing down, with biomechanical walk conditions changing. The presence of invalid pattern of extensors activation on both sides aims at asymmetry compensation with compensatory strengthening of function of intact limb extensors in the case of light paresis. The registered EMG pattern resembles one of animals that move with four supports and small children, which defines the adequacy of phylogenic and ontogenetic approaches to studying the processes of rehabilitation.

\section{Recovery and rehabilitation}

INTRATHECAL BACLOFEN IN STROKE RELATED DISABILITY P. Abreu, N. Fontes, J. Correia, E. Azevedo Hospital de S. João, Porto, Portugal

Background: More than $75 \%$ of stroke patients have motor sequelae, including spasticity, often resulting in incapacity to perform many ADL. Intrathecal baclofen (ITB) has shown the advantage over oral medication of effectively decreasing diffuse spasticity without causing untoward effects on arousal and cognition. Authors present results of this treatment in a series of stroke patients.

Methods: Patients with residual deficits after stroke were evaluated before discharge from the acute event and at 3,6 and 12 months. When there was severe spasticity refractory to oral medication and physical rehabilitation, persisting for at least 6 months, associated with important incapacity (mRankin Scale $>2$ ), patient was selected to $50-100 \mathrm{mg}$ ITB test, and if positive a ITB pump implantation was performed. Evaluation pre and post treatment included Ashworth Scale (AS), Penn spasm frequency scale (PSF), OT-reflex evaluation, muscle strength (Medical Research Council - MRC) score, and informal evaluation of ADL. Assessment of morbidity was also made.

Results: Ten stroke patients, mean age 54 years, were selected for ITB pump implantation. All patients improved significantly in the AS, PSF and reflex scales. Improvement was noticed in MRC scale, but without statistical significance. Seven patients improved markedly in ADL. Average continuous ITB dose used was $282,5 \mathrm{mg}$. Transient headache and vomiting were noticed in three patients after pump implantation, and low transient fever and local catheter infection was registered in one patient.

Conclusions: ITB can be useful in some neurological diseases with refractory spasticity such as stroke. This treatment approach allows an improvement in moto functional status and quality of life, with a low morbidity, in properly selected stroke patients.

\section{Recovery and rehabilitation}

THE EFFECTS OF FUNCTIONAL STRENGTH TRAINING ON LOWER LIMB STRENGTH AND FUNCTION AFTER STROKE

E. Cooke, V.M. Pomeroy, S. Miller, R.C. Tallis

St. George's University of London, London, United Kingdom

Background: Stroke survivors are often left with permanent impairment, which impacts, on activities of daily living. This may be due to the fact that strength training is discouraged due to the incorrect belief that it will exacerbate abnormalities of movement control. The limited empirical evidence suggests that strength training might be beneficial. It is, however, too early to advocate resistive training from evidence of preliminary studies.

Hypothesis: Adding functional strength training to conventional therapy improves muscle function and gait more than either conventional therapy alone or increased intensity of conventional therapy Design A multi-centred, observer-blind randomised controlled trial.

Subjects: Patients within three months of either an infarction or haemorrhage in the anterior circulation with some ability to move their leg and/or foot. A "power calculation' has estimated the sample size.

Procedure: Patients who provide written informed consent are recruited to the study. Subjects then participate in a baseline measurement sessions before being allocated randomly to one of three intervention groups: 1 . conventional therapy provided as normal for the clinical setting (control); 2 . conventional therapy plus additional 'neuro-facilitation' physiotherapy (experimental 1); 3. conventional therapy plus additional functional strength training physiotherapy (experimental 2). All additional therapy is provided 1 hour a day, 4 times a week, for 6 weeks.

Outcome Measures: Outcome measures at 6 weeks and follow-up at 12 weeks. Primary outcomes are muscle strength (maximum torque) around the knee and walking speed. Secondary outcomes include normality of walking and health related quality of life (Euroqol) Progress In the 18 months since the study began 70 subjects have been recruited. 


\section{Recovery and rehabilitation}

TRANSCRANIAL DC STIMULATION (TDCS): A TOOL FOR DOUBLE-BLIND SHAM-CONTROLLED CLINICAL STUDIES IN NEUROREHABILITATION F. Hummel, P. Gandiga, C. Gerloff, L.G. Cohen

Clinical Brain Research, Eberhard-Karls University Tuebingen, Germany; Human Cortical Physiology Section, National Institute of Neurological Disorders and Stroke, National Institutes of Health, Bethesda, MD, USA

Background: Transcranial direct current stimulation (tDCS), a form of brain polarization, which influences motor function and learning processes, has been proposed as an adjuvant strategy to enhance functional recovery in Neurorehabilitation. Appropriate testing in neurorehabilitative studies requires double-blind sham-controlled study designs. In the present study, we determined, in more than 170 sessions, the effects of tDCS and sham stimulation (SHAM) on discomfort, duration of elicited sensations, attention and fatigue in a group of healthy volunteers and stroke patients.

Methods: During different experimental session, tDCS or SHAM was applied to the motor cortex. Attention, fatigue, and discomfort were self rated by study participants using visual analog scales. Duration of perceived sensations and the ability of the study participants to distinguish tDCS from Sham sessions were determined. Investigators questioning the patients were blind to the intervention type.

Results: In the present study, tDCS and SHAM elicited comparably minimal discomfort and duration of sensations in the absence of differences in attention or fatigue between the two conditions. The two interventional conditions (tDCS and Sham) could not be distinguished by study participants nor investigators.

Discussion: Comparable attention and fatigue, ease of application simultaneously with motor tasks and training protocols and successful blinding of subjects and investigators supports the feasibility of using tDCS in double-blind, sham-controlled randomized trials in clinical Neurorehabilitation. Thus, tDCS has the potential to evolve into a useful tool, in addition to TMS, to modulate cortical activity in Neurorehabilitation.

\section{Recovery and rehabilitation}

A NEW SCALE FOR POST STROKE FATIGUE-INITIAL VALIDATION

R.J. Mills, M. Koufali, A. Sharma, C.A. Young

The Walton Centre for Neurology and Neurosurgery, Liverpool, United Kingdom

Background: Fatigue can be defined as reversible, motor and cognitive impairment with reduced motivation and desire to rest, brought on separately by either mental or physical activity, humidity, acute infection, food ingestion or it may occur spontaneously. It is relieved by sleep or rest without sleep and while it may occur at any time it is usually worse in the afternoon. The objective was to design a new scale which would both fully capture the dimension of post stroke fatigue and generate data that would fit the Rasch measurement model. The Rasch measurement model is the only way to convert ordinal scale scores into interval level data, suitable for parametric analysis

Method: A pool of scale items was derived from parallel work in multiple sclerosis fatigue. A four point, Likert response option was used. The scale was posted to patients with a documented history of having suffered a stroke within the previous 3 years, of any age, sex, disability and stroke type. Initial analysis was made on 150 respondents. 'Non functioning' items were deleted until the data from the scale had acceptable fit to the Rasch model.

Results: The final scale had excellent fit to the Rasch model and was free of differential item functioning (DIF) for a wide variety of person factors. External validation was made by comparison to some commonly used, existing scales including the Fatigue Severity Scale, Hospital Anxiety and Depression Scale and the Stroke Impact Scale.

Conclusion: The resultant scale was shown to fit the Rasch model; the scale can therefore be assumed to both measure a unidimensional construct and generate data that can be transformed to an interval scale and is specific for stroke patients. Other qualities of the scale are discussed, as well as plans for further development.

\section{Recovery and rehabilitation}

COMBINED ELECTROPHYSIOLOGICAL STIMULATION AND VISUAL-EXPLORATION THERAPY IN UNILATERAL SPATIAL NEGLECT: THERAPEUTIC IMPLICATIONS

J. Rossmüller, V. Krause, S.M. Golaszewski, K.M. Stephan, V. Hömberg

St. Mauritius Therapieklinik Meerbusch, Meerbusch, Germany

Unilateral spatial neglect comprises a number of discrete symptoms including defective awareness of visual targets in the neglected sector of space. Therapeutic interventions include afferent electrophysiological stimulation via transcutaneous nerve stimulation (TENS), visual exploration training (VIS EX) and exposure to slowly moving stimuli that are thought to facilitate attentional directing responses to the left hemispace (optokinetc stimulation, OKS). Recent studies imply that respective interventions result in considerable reduction of neglect symptoms. However, the remission of neglect symptoms is rarely complete and residual deficits undermine independence in activities of daily living. Further, the application of TENS-stimulation is limited when the training candidate reports aversive sensations resulting from the above-threshold current intensity. The MESH-Glove may repre sent an alternative to overcome this problem: It allows the activation of the sensory cortex through application of subthreshold current intensities to the contralateral hand. Starting with the hypothesis that activation of perilesional tissue via MESHGlove may induce beneficial effects on neglect symptoms and that combination of therapeutic techniques may bear additional positive effects on visuo-spatial neglect, we contrasted combined electrophysiological and visual-explorative therapy using TENS+VIS EX+OKS $(n=10)$ vs. MESH+VIS EX+OKS $(n=10)$. Both therapeutic interventions significantly decreased inattention to the left hemispace in our patients suffering from right-hemisphere cerebrovascular accidents and the improvement in both experimental groups was higher than in our control patients who received VIS EX+OKS $(n=5)$ without additional electrophysiological stimulation. We thus feel encouraged to favour MESH-Glove-stimulation in the treatment of neglect because of the potentiality to stimulate with currents that lie under the threshold of subjective detectability.

\section{Recovery and rehabilitation}

\section{THE INFLUENCE OF WALKING SPEED ON THE CORRECTION OF} PATHOLOGICAL MOTOR STEREOTYPE

\section{E.A. Kovrazhkina, V.V. Gudkova, V.I. Skvortsova}

Russian State Medical University, Moscow, Russian Federation

Background: The aim of our study was to specify the influence of walking speed (WS) reduction on the abilities of pathological motor stereotype (PMS) correction in patients with poststroke motor dysfunctions.

Methods: The study was conducted on 50 patients with light (4-5 points) poststroke hemiparesis: 20 patients with normal symmetric gait (group 1) and 30 patients with gait asymmetry (GA), pathological positions in the joints of paretic leg and incorrect contact between foot and its support (group 2). Were used podometry and curved EMG of proximal and distal lower limbs extensors in gait cycle.

Results: The WS was below the line in both groups, was reliable below the line in group $2(\mathrm{p}<0.05)$. At first all patients were examined at their usual WS. Then the patients of group 1 were offered to increase WS at their will. GA with reduction of single support period on paretic side and with increasing of support periods on intact side, pathological positions in the joints of paretic leg with incorrect contact between foot and its support, pathological stereotype of extensors activity in the form of simultaneous activation of proximal and distal extensors in gait style were discovered in $60 \%$ of patients. The patients of group 2 were offered to reduce WS below the line with attempt to correct PMS at their will. The $50 \%$ of patients were able to correct the PMS at their will with GA reduction and normalization other biomechanical parameters.

Discussion: The present study makes it clear that the reduction of WS promotes possibilities of voluntary and involuntary correction of MPS and GA in the part of patients.

This data can be useful for evaluation of reduction value of motor dysfunction and for rehabilitation prognostication.

\section{Recovery and rehabilitation}

\section{ELECTRICAL UPPER EXTREMITY STIMULATION INFLUENCES SUSTAINED SENSORIMOTOR EXCITABILITY}

B. Jansen, K.M. Stephan, S.M. Golaszewski, C. Bütefisch, R.J. Seitz, V. Hoemberg

St. Mauritius Therapy Hospital, Meerbusch, Germany

Motor performance of stroke patients can be improved using electrical whole hand stimulation with a mesh glove (1). Modulation of cortical activity is a possible mechanism for these improvements. The aim of the present study was to investigate, whether such a stimulation led to sustained changes of corticomotoneuronal excitability.

We investigated five healthy subjects with transcranial magnetic stimulation (TMS) before and after electrical mesh glove stimulation. Single TMS pulses were delivered contralateral to the stimulated hand at the optimal site to evoke hand extension. Their intensity was increased stepwise. Surface EMG was recorded bilaterally from 
forearm extensor muscles and from first interosseus muscles (FDI). Motor threshold and amplitude of EMG responses were recorded.

Our results showed a consistent decrease of motor threshold in all five subjects and an increase in EMG amplitude after electrical whole hand stimulation on this side. This effect was most marked directly after the end of the stimulation period. It showed a gradual decrease over the next two hours.

The present study demonstrates, that subthreshold electrical stimulation can influence the excitability of cortical motoneurons. A recent fMRI study showed an increase in activity during a simple motor task after mesh glove stimulation within contralateral cortical primary sensorimotor, bilateral premotor and parietal areas (2). These findings suggest a modulation of cortical sensorimotor processing during and even after sensorimotor stimulation. This may help to explain the observed increase of effectiveness of rehabilitative interventions after mesh glove stimulation.

\section{Recovery and rehabilitation}

\section{GRANULOCYTE-COLONY STIMULATING FACTOR ENHANCES FUNCTIONAL RECOVERY AND INDUCES NEUROGENESIS AFTER CORTICAL STROKE}

W.R. Schäbitz, T. Steigleder, C. Krüger, R. Laage, H.G. Kuhn, A. Schneider

University of Münster, Münster, Germany

Granulocyte-colony stimulating factor (G-CSF) was recently shown to have potent neuroprotective effects after focal cerebral ischemia, and is currently investigated in a multicenter stroke trial for the treatment of acute ischemic stroke (AXIS, Phase II, see "Ongoing clinical trials"). Beside its efficient interaction within acute stroke pathology, we could demonstrate for the first time that this factor displays profound neurotrophic effects. Indeed, systemic treatment with G-CSF beginning $1 \mathrm{~h}$ after cortical stroke induced a profound and longlasting sensorimotor recovery, which could be reproduced when treatment initiation was delayed to day 3 after stroke. This significant improvement in functional outcome correlated to an enhanced generation of new neurons in the periphery of the infarction as well as in distant non-affected areas. Interestingly, neurogenesis was also significantly enhanced in healthy, non-ischemic animals. Due to this trophic effects and its good tolerability, G-CSF could be an ideal candidate to be developed for future recovery enhancing trial in humans.

\section{9}

Recovery and rehabilitation

COMPREHENSIVE SCREENING OF LIMB PRAXIS FUNCTIONS IN STROKE PATIENTS: CONSTRUCTION AND STANDARDISATION OF THE

DÜSSELDORFER APRAXIA TEST (DAXT)

J. Rossmüller, S. Pahl, V. Hömberg

St. Mauritius Therapieklinik Meerbusch, Meerbusch, Germany

We present a comprehensive test battery for the assessment of ideomotor and ideational apraxia that was standardised on a representative sample of 35 stroke and 35 elderly orthopaedic inpatients without known history of neurological disease. Following the neuropsychological model of Rothi, Ochipa and Heilman (1997), upper-limb apraxia was assessed along three different input-modalities, namely imitation, pantomime on visually presented objects and actual tool use. Aiming at an increase of the instructor's objectivity, we applied two additional tests: One requiring the imitation of movements that were applied via video-sequences and another test demanding pantomimed movements on photographs of objects. Since the model of Rothi, Ochipa and Heilman (1997) implies it to be relevant, errors were scored on two dimensions: One for content errors and one for spatiotemporal errors. Zero responses were singled out. Subsequent analyses of test criteria revealed satisfactory figures concerning interrater-reliability, test reliability and various aspects of test validity.

\section{Recovery and rehabilitation}

\section{USER INVOLVEMENT IN THE DEVELOPMENT OF TECHNOLOGICAL} APPLICATIONS FOR DOMICILIARY STROKE REHABILITATION

P. Ware, J. Hammerton, S. Mawson, G. Mountain, H. Zheng, R. Davies, N. Black

Sheffield Hallam University, Faculty of Health and Wellbeing, Sheffield, United Kingdom

Background: The SMART project aims to enable home rehabilitation for stroke patients by providing computer based feedback on performance of exercises alongside electronic links to physiotherapists. User involvement is a key element in the development of any technology and a major aspect of government policy in reforming the NHS. This paper describes the methods, process and results of involving users in the early stages of the development of remote monitoring, interactive feedback and rehabilitation equipment.

Method: Seven focus groups were conducted with a total of 32 patients and carers. Phase one investigated attitudes to rehabilitative technology prior to the development of prototypes. The resulting feedback informed the development of both the software interface of the decision platform and the movement sensors. In phase two, user and carers commented on the interactive information platform and the placement of sensors. Groups were video taped to capture the way patients were able to attach the sensors themselves.

Results: Requirements emerging from the initial focus groups were that any devices should be compact, simple to operate, usable by patients without the help of carers and able to give measurable feedback. In phase two, the team were able to further modify sensor attachment and refine the display of screen information ensuring an interactive decision making process.

Discussion: Phase two enabled us to develop an expert patient group who will participate in the clinical testing of the equipment currently being developed. Whilst user involvement is essential to product design and has benefited our study, we are aware that service users as participants are anxious to please and their feedback may be problematic.

\section{Recovery and rehabilitation}

\section{BOTULINUM TOXIN ADDED TO INTRATHECAL BACLOFEN IN STROKE}

\section{REHABILITATION}

P. Abreu, M.J. Rosas, M.J. Festas, J. Correia, E. Azevedo

Hospital de S. João, Porto, Portugal

Introduction: Disability from spasticity is frequent after stroke. Intrathecal baclofen (ITB) has shown the advantage over oral medication of effectively decreasing diffuse spasticity without causing untoward effects on arousal and cognition. However, residual severe upper-limb spasticity often impedes the use of the hand. Also botulinum toxin (BT) is known to be effective in reducing hypertonia, but its use must be restricted to a small number of muscles. Authors present the results of combined treatment with ITB and BT.

Methods: Patients with residual deficits after stroke were evaluated before discharge from the acute event and at 3,6 and 12 months. When there was severe spasticity refractory to oral medication and physical rehabilitation, associated with important incapacity (mRankin Scale $>2$ ), patient was selected to $50-100 \mathrm{mg}$ ITB test, and if positive a ITB pump implantation was performed. Cases with severe residual upper-limb spasticity were evaluated for local BT treatment. Evaluation pre and post treatment included Ashworth Scale (AS), Penn spasm frequency scale (PSF), OT-reflex evaluation, muscle strength (Medical Research Council - MRC) score, and informal evaluation of ADL. Assessment of morbidity was also made.

Results: The two first stroke patients treated with this combined therapy are 46 and 64 years old. Patients improved significantly in the AS, PSF, reflex scales, MRC and in activities of daily living. The average ITB dose used was $345 \mathrm{mg}$ and the BT dose was $100 \mathrm{U}$. No side effects were registered.

Conclusions: In these patients ITB plus BT therapeutic approach allowed a significant symptomatic and functional improvement with good tolerance. A multidisciplinary team remains a vital component in the overall management plan of stroke rehabilitation.

\section{Recovery and rehabilitation}

\section{APHASIAS IN STROKE UNIT}

Y.M. Zhang, X.Q. Zhao, Y.I. Wang, X.P. Gong

Beijing Tiantan Hospital, affiliated with Capital University of Medical Sciences, China

Background: Aphasia is present in $21-38 \%$ of acute stroke individuals, and is associated with high short-and long-term morbidity, mortality and expenditure, and can have a dramatic impact on a person's ability to communicate. In this study, we tried to evaluate aphasia characteristics at the acute stage in patients admitted to our stroke unit.

Methods: We selected 1198 in-patients in the Neurology Dempartment of Beijing Tiantan Hospital, who had been diagnosed with cerebral ischemic by CT between February 2002 and February 2005. The disease lesions for all patients were clarified with magnetic resonance imaging, we assessed aphasia types by the Western Aphasia Battery within one month, we also recorded the average age and sex of every aphasia patient, then we analyzed what effect the aphasia types in our stroke unit. Results: Of 1198 stroke patients, there were 325 patients with aphasia, and the lesion of most aphasia patients were located at language functional area, such as Broca's area and Wernicke's area, and about 30 present aphasia patients could 
not be given a certain aphasia type, namely non-classified aphasia, and globe and non-classified aphasia accounted for $52 \%$ of all aphasia, whereas Broca's aphasia accounted for $21 \%$ and Wernicke's aphasia accounted for $15 \%$ respectively, the other types accounted for $12 \%$. Age differed among aphasic syndromes in stroke unit, such as conduction aphasia patients were younger $(56.34 \pm 3.56)$ while Wernicke's aphasia patients were older $(69.47 \pm 3.93)$, and woman has a little chance to got aphasia than man $(\mathrm{p}=0.003)$.

Discussions: Our study shows: (1) that lesion location, age and sex are related with aphasia types; (2) that not all aphasia can be given a certain type;(3) that the main determinant of aphasia characteristics is lesion location

\section{Recovery and rehabilitation}

\section{REACTIVE AND ENDOREACTIVE POST-STROKE DEPRESSIONS}

V.A. Kontsevoy, M.A. Savina, O.S. Nazarov, E.A. Petrova, V.I. Skvortsova

Russian State Medical University, Moscow, Russian Federation

Background: The study of risk factors of post-stroke depression (PSD) is of great importance. A great number of risk factors of PSD were found, data concerning each of this factors remain inconsistent. It possibly deals with heterogeneity of PSD. Methods: 115 subjects with first stroke (57 males, 58 females, mean age 65 years) were included. The mental state of the subject was assessed by clinical descriptive method and the Hamilton Depression Scale. The degree of neurological impairment was assessed by the Orgogozo Scale and the Barthel Index. Depression was diagnosed using criteria of ICD-10.

Results: During one year after stroke depressions were observed in 33\%. In 21 patient were diagnosed reactive PSD. In 12 patients were diagnosed endoreactive depressions that had both reactive and endogenic features. Patients with reactive depression had lower score of Barthel Index in 14 days after stroke $(74,03 \pm 32,13)$ in comparison with patients with endoreactive PSD $(86,25 \pm 26,47)$ and subjects without depression $(82,13 \pm 27,33)(\mathrm{p}=0,063)$. Patients with endoreactive PSD less past history of mental disorders compared with subjects with reactive depression or without depression: they had more frequently a history of depressions $(p=0,003)$, dysmorphophobia $(\mathrm{p}=0,020)$, phobias $(\mathrm{p}=0,008)$, panic attacks $(\mathrm{p}=0,001)$, manias $(\mathrm{p}=0,024)$, premenstrual syndrome $(\mathrm{p}=0,015)$, hypochondric reactions $(\mathrm{p}=0.001)$.

Discussion: PSD seem to be a heterogeneous group of depressive disorders with different clinical features and different risk factors. Risk factor for reactive PSD is severe neurological impairment. Risk factor for endoreactive depressive states is history of mental disorders.

\section{Recovery and rehabilitation}

\section{MY HAND IS A LUMP OF MEAT}

K. Ikeda, K. Sakamoto, D. Miyagi, M. Kozono

Kochi School of Allied Health and Medical Profession, Kochi, Japan

Introduction: What is happening inside the brain during the course of recovery? We used the first-person(FP) concept of F.J. Varela aiming to monitor changes in the patient. The following outlines the neuro-cognitive rehabilitation.

Cases: The patient was a 75-year old male who had suffered a right MCA infarction. At the time of admission, the Brunnstrom Stage (BRS) was 1 for upper/lower $\operatorname{limb}(\mathrm{U} / \mathrm{L})$ and fingers. Hypesthesia were severe. Other significant findings included left homonymous hemianopsia and asomatognosia.

Courses: He was requested to draw his whole body and fingers. In the selfportrait(SP) of the whole body drawn on day 52, the fingers of the left were missing and the right had been combined so that the individual fingers could not be seen. In the FP description (FPD), the patient said his left hand was "heavy", "like horsemeat", that its flesh was "dead". Assignments were then focused on parts of his body where perception was significantly different from normal, with the aim of activating the parietal association region (PA). The patient was first given the task of touching U/L using a sponge and then a matching exercise involving imagery. On week 10, he began to draw fingers and comments about hand changed to "light", "my hand" and "feels like the right again". It is thought that his recognition and attention of parts of his body was aroused through perception experience, which suggests that the body image in PA had changed. U/L had improved to BRS 2 .

Discussion: Having the patient give FPD and draw SP was effective as a means of understanding the his perception and gaps in his image. It was useful as information for investigating hypotheses. Objective evaluation of changes in the human brain from observation is always difficult. However, whenever there is sign of improvement in paralysis, it will be reflected in FPD. Going forward, while focusing on such difficulties, we would like to become closer to patients through Romantic Science by A.R. Luria.
THE USE OF CLIENT CENTRED PRACTICE WITHIN FIRST THREE MONTHS POST STROKE

J. Jansa, Z. Sicherl, K. Angleitner, A. Grad

University Medical Centre Ljubljana, Ljubljana, Slovenia

Background: To implement client-centred occupational therapy (OT) assessment in the first-ever acute ischaemic stroke (IS) patients within early hospital stay. Methods: We included IS patients within early hospital stay and three months after stroke. We used following OT assessment tools: Extended Barthel index (EBI), Assessment of Motor and Process Skills (AMPS), Canadian Occupational Performance Measure (COPM) and EuroQol (EQ). The EBI was done at the beginning of OT, at discharge and follow up. COPM; AMPS and EQ were done during OT interventions and at follow up.

Results: There were 50 acute IS patients assessed during early OT intervention and followed in month three post stroke; 24 with R-hemiplegia, 23 with L- hemiplegia, 3 with brain steam stroke; 31 males, 19 females. The EBI, AMPS and EQ were assessed in the whole sample; we were able to perform COPM in 32 patients only. Differences between EBI scores were statistically important from the first assessment to both follow-ups ( $\mathrm{p}<0,05 ; \mathrm{p}<0,01)$. Differences in AMPS-motor and AMPS-process: $\mathrm{p}=0,0003, \mathrm{p}<0,0001$; differences in EQ score: $\mathrm{p}<0,0001$; differences in COPM-performance and satisfaction: $\mathrm{p}<0.0001, \mathrm{p}=0,00014$. Regarding the use of COPM, 32 patients $(64 \%)$ of our sample expressed in total 87 activities. 58 activities $(66 \%)$ were allocated to basic activities of daily living (ADL), $15(18 \%)$ to productivity issues and $14(16 \%)$ to leisure activities.

Discussion: The results showed that patients needs less help from another person as measured with the EBI. Accordingly the quality of performance has improved, as measured with AMPS. Also, the quality of life has improved, as measured with EQ. However, we were able to follow client priorities in terms of expressing their functional priorities in $64 \%$ of our sample. The main priorities were in terms of basic ADL, followed by productivity issues and leisure. 


\section{Brain imaging}

\section{Brain imaging}

NEW CEREBRAL MICROBLEEDS WITHIN A WEEK IN ACUTE ISCHEMIC STROKE PATIENTS

J. Sang-Beom, S.U. Kwon, K.H. Yu, A.-H. Cho, S.-H. Yoo, J.S. Kim,

D.W. Kang

Asan Medical Center, Seoul, South Korea

Background: Intracerebral microbleeds (MBs) are considered as remnants of old hemorrhages. However, it has never been studied whether new MBs occur during early post-stroke period.

Methods: We examined consecutive 237 acute ischemic stroke patients who underwent stroke MRI including diffusion-weighted imaging, gradient echo T2*-weighted imaging (GRE) and T2-weighted or fluid attenuation inversion recovery imaging within 24 hours and repeatedly within one week after stroke onset. The number and location of initial MBs and early new microbleeds (ENMBs), and the degree of leukoaraiosis were evaluated. The relationships of ENMBs with initial MBs, leukoaraiosis, risk factors and laboratory data were explored.

Results: Seventy-four (31.2\%) patients had MBs on initial GRE and $30(12.7 \%)$ patients developed ENMBs. The presence of MBs on initial GRE (odds ratio [95\% confidence interval], 6.34 [2.36 to 17.06]) and severe leukoaraiosis (odds ratio [95\% confidence interval], 3.26 [1.08 to 9.78]) independently predicted ENMBs within a week in multiple logistic regression analysis. The location of ENMBs was associated with the location of initial MBs $(\mathrm{p}<0.0001)$.

Conclusions: This study provides novel evidence that new MBs occur during early post-stroke period. Further studies are needed to identify the clinical implication and underlying pathomechanism of these findings.

\section{Brain imaging}

THE EFFECT OF BLOOD PRESSURE CHANGE ON ACUTE ISCHEMIC STROKE OUTCOME

Y. Yang, P. Ho, H.M. Chang, M.C. Wong, C. Chen

National Neuroscience Institute, Singapore General Hospital Campus, Singapore

Background and Purpose: The prognostic effects of different blood pressure (BP) indices during acute ischemic stroke is not well understood. We therefore investigated the relationships between changes in different BP indices during the acute phase of stroke with clinical and magnetic resonance image (MRI) parameters.

Methods: Consented patients with ischemic stroke underwent MRI scans within 12 hours of stroke onset and at day 90. Patients had their BP measured acutely, 12,24 and 48 hours later. Systolic BP (SBP), diastolic BP (DBP), Pulse pressure $(\mathrm{PP})$, mean arterial pressure (MAP), mean $\mathrm{BP}(\mathrm{MBP})$, and pulse pressure index (PPI) were measured. Rates of change in BP indices over 48 hours were calculated by regression analysis. Acutely acquired diffusion-weighted images (DWI) and T2 images at day 90 were used to measure infarct volume by planimetric methods. The National Institutes of Health Stroke Scale (NIHSS) was used to assess neurologic deficits acutely and at day 90 .

Results: 23 patients were studied. Percentage infarct growth showed significant negative correlations with SBP, MAP and MBP change rates $(r=-0.57,-0.46,-0.50$ respectively, all $\mathrm{p}<0.05$ ), but not with DBP, PP and PPI. NIHSS scores at day 90 also showed significant positive correlations with SBP, MAP and MBP change rates only $(\mathrm{r}=0.46,0.58,0.58$ respectively, all $\mathrm{p}<0.05)$.

Conclusion: Rates of change in SBP, MAP and MBP showed significant correlation with infarct growth and neurologic deficits. This suggests that failure to maintain perfusion of the ischemic penumbra may be deleterious in acute stroke. Further randomised studies of BP management in acute stroke, using MRI as a surrogate end point in addition to clinical outcomes, are needed.

\section{Brain imaging}

\section{PLASMINOGEN ACTIVATOR INHIBITOR-1 DOES NOT INFLUENCE} INFARCT EVOLUTION IN ACUTE STROKE: A SERIAL FIBRINOLYTIC AND MRI STUDY

L.E. Allport, A. Tuckfield, K.S. Butcher, L. MacGregor, B.M. Tress, S.M. Davis The Royal Melbourne Hospital and The University of Melbourne; Parkville, VIC, Australia

Background: Hypofibrinolysis due to elevated plasminogen activator inhibitor-1 (PAI-1) and tissue-type plasminogen activator (t-PA) has been observed during acute ischemic stroke. Experimental studies have shown that hyperglycemia, which worsens stroke outcome, stimulates PAI-1 production suppressing fibrinolysis. We aimed to establish whether PAI-1 and t-PA influence infarct evolution and if there is a relationship between hyperglycemia and PAI-1 at the time of acute stroke.

Methods: Admission glucose and serial (admission, day 1, day 90) PAI-1 activity and t-PA antigen were performed in 40 patients presenting within 24 hours of symptom onset. Serial MRI (baseline, day 3-5, day 90) was used to measure infarct expansion and final infarct volume.

Results: Median time from stroke onset to fibrinolytic sampling was 7.0 hours 23.3 hours and 89 days. Median PAI- 1 activity increased by $30 \%$ from admission to day 1 , with no evidence of further release at day 90 . There was no significant relationship between either larger baseline infarct volume $(\mathrm{P}=0.481)$ or greater stroke severity $(\mathrm{P}=0.646)$ with higher PAI-1. There was no evidence that PAI-1 $(\mathrm{OR}=0.88$ per doubling of PAI-1, 95\% CI $0.44,1.79 ; \mathrm{P}=0.733)$ or $\mathrm{t}-\mathrm{PA}(\mathrm{OR}=2.32$ per doubling of t-PA, 95\% CI $0.61,8.78 ; \mathrm{P}=0.217$ ) were associated with a greater probability of infarct expansion or larger final infarct volume. Multivariate analysis indicated admission glucose and PAI- 1 activity were inversely related (ratio $=0.69$ per doubling of admission glucose, 95\% CI 0.48,0.98; $\mathrm{P}=0.041$ ), after adjusting for triglycerides, body mass index and gender.

Conclusion: We found no evidence for a role of plasma PAI-1 activity or t-PA antigen in the determination of tissue fate following acute stroke. The adverse effect of poststroke hyperglycemia does not appear to be explained by systemic hypofibrinolysis. The finding of lower PAI-1 with hyperglycemia might explain the link between hyperglycemia and hemorrhagic transformation after rt-PA.

\section{Brain imaging}

MAGNETIC RESONANCE PERFUSION DIFFUSION MISMATCH IN ACUTE ISCHAEMIC STROKE: SYSTEMATIC REVIEW OF METHODS USED, INFLUENCE ON PROGNOSIS AND IMPACT ON RESPONSE TO THROMBOLYTIC THERAPY

I. Kane, P. Sandercock, J. Wardlaw

University of Edinburgh, Edinburgh, United Kingdom

Background: Imaging the mismatch between perfusion (PWI) and diffusion (DWI) lesions on MR may identify patients for thrombolysis. We assessed the evidence underlying this hypothesis.

Methods: We systematically reviewed all papers describing MR PWI/DWI findings in patients with acute ischaemic stroke, and their clinical and/or radiological outcome at one month, with or without thrombolysis.

Results: 11 papers fulfilled study inclusion criteria. Only 3 had useable data on mismatch, outcome and influence of thrombolysis, including 61 patients with, and 18 without mismatch. Mismatch was associated with a non-significant two-fold increase in the odds of infarct expansion (versus no mismatch) (OR 2.2, 95\% 0.34-14.1), which changed little with thrombolysis (OR 2.0, 95\% CI 0.37-10.9). Half the patients without mismatch had infarct growth. Data on functional outcome were not extractable. There were 5 different mismatch definitions and at least 7 different PWI methods amongst the 11 papers.

Conclusions: (1) Data are inadequate to use mismatch to guide thrombolysis. (2) The absence of mismatch does not mean that there is no 'tissue at risk'. (3) Patients without mismatch should not be excluded from trials. (4) Standardised definitions of mismatch and perfusion are required. 


\section{Brain imaging}

PROOF OF PRINCIPLE PHASE II MR IMAGING STUDIES IN STROKE: SAMPLE SIZE ESTIMATES USING DICHOTOMOUS AND CONTINUOUS DATA

A. None, the MRSTROKE Group

MRSTROKE Group, Clayton, VIC, Australia

Background and Purpose: Since the failure of a number of phase III trials of neuroprotection in ischemic stroke the need for smaller phase II studies using MR surrogates has emerged. There is, however, little information available about sample size requirements for such phase II trials and rarely enough patients in single studies to make robust estimates. We have formed an international collaborative group to asssemble larger datasets and from these generated sample size tables for MR based infarct expansion as the outcome measure.

Methods: Centers from Australia, Europe and North America contributed data from patients with hemispheric ischemic stroke. Infarct expansion was defined from initial diffusion-weighted image and later FLAIR or T2 images. Sample size estimates were calculated using data on infarct expansion ratios treated as dichotomous or continuous variables. A non-parametric approached was used since the distribution of infarct expansion was resistant to all forms of transformation.

Results: For a $20 \%$ absolute reduction, $80 \%$ power and alpha $0.05,99$ patients in each arm are required using dichotomous infarct expansion ratio $(\leq 1)$. To achieve an approximate equivalent effect size using a continuous approach requires 61 patients. Conclusions: These tables will be useful in planning phase II trials of therapy using MR outcome measures. For positive studies, biologically plausible surrogates such as these may provide a rationale for proceeding to phase III trials.

\section{Brain imaging}

IRREVERSIBLE ISCHEMIC BRAIN INJURY PREDICTED BY CEREBRAL BLOOD VOLUME IMAGING

C. Disque, G. Gahn, I. Dzialowski, C. Dannenberg, O. Wunderlich, R. von Kummer

Technical University Dresden, Dresden, Germany

Background: The extent of irreversible ischemic brain injury is associated with the response to thrombolytic treatment. Patients with an ASPECTS (Alberta Stroke Program Early CT Score) below 8 on native CT have an increased risk for cerebral hemorrhage after thrombolytic treatment within 3 hours of stroke onset. We transferred ASPECTS to CBV (cerebral blood volume) images to test the hypothesis that impaired $\mathrm{CBV}$ within the first $6 \mathrm{~h}$ of stroke onset is specific in predicting brain infarction.

Methods: Following a prospective protocol, we performed native CT, CT-perfusion and CT-angiography in patients presenting with middle cerebral artery (MCA) syndrome in a six hours time window. Patients' treatment followed the national stroke guidelines, a local study protocol, or compassionate use. NIHSS was assessed on day 0 and at discharge. We repeated CT or MRI at 24 hours \pm 12 hours after acute stroke to assess ischemic tissue damage. CT-CBV images fusion and follow-up CT/MRI were reviewed by one neurologist using the ASPECTS. The extent of pathological area in CBV images was estimated in comparison to the healthy hemisphere by viewing the colour coded images. Accordance of CBV and control-CT was defined as a difference in ASPECTS less than one.

Results: We included 90 acute stroke patients between 12/2003 and 12/2005. In 36 patients clinical or CT data were missing. In $41(76 \%)$ of the remaining 54 patients, the CBV-ASPECTS matched the infarct-ASPECTS on follow up images; in $13(24 \%)$ patients, the follow up infarct ASPECTS exceeded the initial CBVASPECTS by more than 1 point. Interestingly, the ASPECTS matched in all patients not treated with thrombolytics.

Conclusions: Confirming the observations of others, we found that impairment in $\mathrm{CBV}$ within the first 6 hours of stroke onset is specific in predicting ischemic damage. The semi-quantitative assessment of CBV on CT-perfusion images with ASPECTS appears as sufficient to use for this prediction.

\section{Brain imaging}

INSULAR CORTEX HYPOPERFUSION AND ACUTE POST-STROKE HYPERGLYCAEMIA: A PERFUSION CT STUDY

F. Moreton, M. McCormick, K.W. Muir

University of Glasgow, Glasgow, United Kingdom

Background: Post-stroke hyperglycaemia (PSH) occurs in 40-60\% of patients and adversely affects prognosis. The cause of PSH is not fully understood, but it may represent a physiological stress response. The insular cortex mediates autonomic function in animals and insular infarction has been associated with PSH and other dysautonomias in man. We studied the relationship between insular hypoperfusion and PSH using perfusion CT (pCT).

Methods: Patients having pCT within $24 \mathrm{~h}$ of ischemic stroke were reviewed retrospectively. Admission NIHSS and blood glucose (BG), and peak BG within $72 \mathrm{~h}$ of onset were recorded. Regions of interest (ROIs) in anterior and posterior insular cortices of both hemispheres on $\mathrm{pCT}$ perfusion maps were used to measure time to peak (TTP) and cerebral blood flow (CBF). Hypoperfusion was defined as relative TTP $($ rTTP) $>6$ s delayed, or $\mathrm{rCBF}<50 \%$. A logistic regression model including rTTP, rCBF, lateralisation and NIHSS was used to determine prediction of PSH $(>7 \mathrm{mmol} / \mathrm{l})$ on admission or within $72 \mathrm{~h}$, and a general linear model explored these variables in prediction of $\mathrm{BG}$ concentration.

Results: 35 subjects, median NIHSS 18, underwent pCT a median 180 mins post onset. PSH occurred in 11/35 on admission and 18/35 within $72 \mathrm{~h}$. Insular hypoperfusion affected 19/35 (rCBF definition) or 23/35 by rTTP. Neither PSH on admission, nor within $72 \mathrm{~h}$, was significantly associated with insular hypoperfusion (anterior or posterior, either definition), NIHSS, or lateralisation. Admission NIHSS, but not other variables, predicted BG peak within $72 \mathrm{~h}(\mathrm{p}=0.011)$, but not admission BG

Conclusions: Acute insular cortex hypoperfusion was not associated with acute or subacute PSH. Stroke severity was associated with peak glucose within the first $72 \mathrm{~h}$, but not on admission.

\section{Brain imaging}

\section{INFARCT PATTERNS PREDICT NEUROLOGICAL DETERIORATION IN PATIENTS WITH INFARCTION IN THE TERRITORY OF THE LENTICULOSTRIATE ARTERY}

S.W. Park, S.I. Sohn, S.S. Jang, S.H. Choi

Keimyung University, Sunlin Hospital, Dongkang Hospital, Daegu, South Korea

Background: Neurologic worsening after acute ischemic stroke is observed frequently in clinicians. Detection of acute brain infarcts by diffusion weighted magnetic resonance imaging (DWI) may provide information about this mechanism. This study was performed to identify infarct patterns in the lenticulostriate artery (LSA) territory and determine the predictors of neurological deterioration by DWI and laboratory parameters.

Methods: We evaluated 1,710 consecutive acute stroke patients, collected by a Keimyung Stroke Registry from March 2003 to December 2005. This study enrolled 211 patients with infarction in the LSA territory who showed normal MR angiogram (MRA). DWI was obtained with $5 \mathrm{~mm}$ of section thickness. The lesion type was divided into DWI type I (lacune, axial slice number(ASN) $<2$ ), type II (punctate), type III (lacune, ASN $\geq 3$ ) and type IV (large striatocapsular) by infarct pattern or ASN of DWI. Neurological deterioration was defined as worsening by more than 2 points on the National Institute of Health Stroke Scale (NIHSS) within 7 days after onset.

Results: Two hundred eleven patients showed DWI type I (39.8\%), type II (12.8\%), type III (29.8\%), and type IV (17.5\%). $55(26.1 \%)$ of 211 patients showed neurological deterioration. Compared to DWI type I, type III (ASN $\geq 3$ ) was more often found in patients with neurological deterioration $(16.7 \%$ Vs $67.3 \%)(\mathrm{p}<0.05)$. Patients with neurological deterioration showed higher initial NIHSS score. By analyzing parameters, initial higher blood pressure and lower level of serum albumin were more found in neurological deterioration group $(\mathrm{p}<0.05)$

Conclusions: DWI type I is most common infarct pattern in the LSA territory. Patients with neurological deterioration often showed DWI type III pattern, more extended lesion to the corona radiata. Predictors of neurologic worsening after stroke remain to be determined with proper management of these patients.

\section{Brain imaging}

SONOGRAPHIC MONITORING OF HEMORRHAGIC TRANSFORMATION IN ACUTE ISCHEMIC STROKE

G. Seidel, K. Meyer-Wiethe, H. Cangür, A. Burgemeister

University Hospital Schleswig-Holstein, Campus Lübeck, Lübeck, Germany

Background: We conducted this prospective study to evaluate the diagnostic potential of transcranial sonography (TCS) in depicting hemorrhagic transformation (HT) in the early phase of ischemic stroke. This non-invasive bedside method offers the possibility to perform a close monitoring of brain structures and hemorrhagic transformation of infarction.

Methods: 22 patients with acute ischemic hemispheric stroke were evaluated. A 2 $\mathrm{MHz}$ sector probe (S3, SONOS 5500) was used for the TCS evaluation $<36$ hours after symptom onset. A daily follow-up investigation was done up to 15 days. Lesion size and localization were determined by CCT or MRI. The evaluation of the TCS 
scans was performed without any knowledge of clinical or imaging information offline after the investigation by an experienced investigator.

Results: 7 out of 8 patients with HT displayed by CCT or MRI could be identified by TCS. In one patient TCS provided a wrong positive result and in another patient TCS was unable to detect the small cortical HT (sensitivity $88 \%$, specificity $99 \%$ ). The maximal area of HT increased between day 2 and 10. The brightness of the HT signal was constant between day 1 and 6 and decrease afterwards.

\section{Brain imaging}

PREDICTION OF THE VOLUME OF CEREBRAL INFARCTION. AN MRI-DWI AND SERUM S-100B PROTEIN STUDY

R. Herzig, P. Schneiderka, D. Horak, D. Sanak, I. Vlachova, A. Bartkova, J. Mares, B. Krupka, S. Burval, P. Kanovsky

Stroke Center, Depts. of Neurol., Clin. Biochem. and Radiol., Univ. Hosp., Olomouc, Olomouc, Czech Republic

Background: S-100B protein (S-100B) is an acidic calcium-binding protein found in the nervous system of vertebrates, where it is released by damaged brain tissue. The aim was to assess its role as a predictor of the volume of cerebral infarction (CI).

Methods: A prospective, hospital-based study was used. The set consisted of 48 patients ( 30 males, 18 females; aged $25-77$, mean $64.1 \pm 10.0$ years), treated at the Department of Neurology, University Hospital, Olomouc, Czech Republic. Serum S-100B levels were measured 24 (S-100B24), 48 (S-100B48), 72 (S-100B72), and 96 (S-100B96) hours following the $\mathrm{CI}$ onset. CI volume was assessed using diffusion-weighted images in magnetic resonance imaging (MRI-DWI), as a part of an acute MRI stroke protocol. Pearson's correlation analysis was applied when assessing the relationship between the particular and also maximal (S-100Bmax) values of S-100B, and CI volume in each patient

Results: Significant intermediate positive linear correlations were found between S-100B24, S-100B48, S-100B96 and S-100Bmax values, and CI volume $-r=0.60$, $\mathrm{r}=0.67, \mathrm{r}=0.51$ and $\mathrm{r}=0.66$, respectively $(\mathrm{p}<0.01$ in all cases). Significant modest positive linear correlation was found between S-100B72 and CI volume $-r$ $=0.39(\mathrm{p}<0.01)$

Discussion: Serum S-100B values measured at 24, 48 and 96 hours, as well as the maximum S-100B value obtained within the $24-96$ hour interval following the $\mathrm{CI}$ onset can be used as a prognostic marker of CI volume.

Acknowledgement: Supported by the IGA MH CR grant number NR/8579-3/2005.

\section{Brain imaging}

SIGNIFICANCE OF DIFFUSION-WEIGHTED IMAGING IN ACUTE MULTIPLE INFARCTIONS WITH INTERNAL CAROTID ARTERY DISEASE AND MIDDLE CEREBRAL ARTERY DISEASE

D.-K. Jung, J.-T. Lee, S.-P. Park, C.-K. Suh

Kuyngpook National University Hospital, Daegu, South Korea

Background and Purpose: Diffusion-weighted imaging (DWI) is a sensitive technique in identification of early ischemic lesions. To find out differentiating factor in acute multiple cerebral infarction patients with internal carotid artery disease (ICAD, stenosis $\geq 50 \%$ ) and middle cerebral artery disease (MCAD, stenosis $\geq$ $50 \%$ ), we analyzed acute multiple ischemic lesions (AMIL) on DWI.

Methods: We detected AMIL in 107 stroke patients with ICAD ( $n=43$ ) and MCAD $(\mathrm{n}=64)$ who underwent DWI and MRI/MR angiography within 3 days of stroke onset. Patients were classified into 1 of 5 observed lesion patterns: (1) multiple small $(<1 \mathrm{~cm})$ lesions, (2) multiple small and medium (1 to $3 \mathrm{~cm})$ lesions, (3) multiple small and large $(>3 \mathrm{~cm})$ lesions, (4) multiple lesions in hemodynamic risk zone, and (5) others. Evaluation was done on whether or not involvement was made on insula, periventricular white matter, basal ganglia, and cortex. Comparisons of all characteristics including DWI lesion size and location were made between ICAD and MCAD patients.

Results: Multiple small lesions was most commonly seen in patients with ICAD, and multiple small and medium lesions in patients with MCAD (34\% and $40 \%$, respectively). In terms of maximal lesion size: compared with ICAD, multiple large-sized lesion $(>3 \mathrm{~cm})$ was found to be more closely related with MCAD $(\mathrm{p}=0.018)$; on the other hand, when compared with MCAD, small-sized lesion $(<1 \mathrm{~cm})$ had significant relationship with ICAD $(\mathrm{p}=0.021)$. Periventricular white matter involvement had more to do with MCAD than ICAD $(\mathrm{p}=0.007)$.

Conclusions: DWI has utility in differentiating between MCAD and ICAD in patients with AMIL. Periventricular white matter involvement and maximal lesion size on DWI are useful DWI factors for the differentiation between MCAD and ICAD in acute multiple cerebral infarction patients with ICAD or MCAD.

\section{Brain imaging}

BIOMARKERS IN ACUTE STROKE: EARLY MMP-9 LEVELS PREDICT STROKE SIZE AS EVIDENCED BY DWI-MR

S.C. Bukow, M. Stroick, S. Kreisel, A. Gass, M. Fatar, M. Griebe, K. Szabo, R. Kern, B. Reuter, M.G. Hennerici

Klinikum Mannheim University of Heidelberg, Mannheim, Germany

Background: Two recent reports suggest that matrix metalloproteinases (MMP), in particular MMP-9 are upregulated after acute stroke and may be considered as quantitative indicators of the extent of cerebral ischemia as documented by MRI. We assessed the relationship of MRI characteristics in acute stroke patients and serum MMP-2 and MMP-9 proteins.

Methods: A total of 88 patients with hyper-acute stroke entered the study. Serum protein levels of MMP-2 and MMP-9 were determined and compared to MRI studies (including DWI, FLAIR, T2w-, T2*w and MRA) performed within 24h after symptom onset. Acute ischemic lesion volumes, the presence and extent of hemorrhage/hemorrhagic transformation (HT), and the extent of chronic white matter lesions were determined.

Results: Logistic regression analysis showed a high correlation of MR-DWI lesion volumes ( $\mathrm{p}=0.0001$ ) with MMP-9, but not with MMP-2. No correlations were found for chronic subcortical tissue changes and MMP-9 or MMP-2 respectively.

Conclusions: This is the largest series comparing putative biomarkers of acute stroke. The data confirm and extend previous observations of correlations of MMP-9 with MR-DWI lesion size. This is in line with the suspected mechanism and role of MMP-9 expression on damaged endothelial tissue. If administered in an emergency kit MMP-9 level assessments could be considered highly useful for a rapid tentative diagnosis of stroke and facilitate best management of these patients already outside the hospital.

\section{Brain imaging}

CHANGES OF ULTRASONIC BRAIN PERFUSION IMAGING PARAMETERS IN THE ACUTE COURSE OF ISCHEMIC STROKE

C. Krogias, T. Postert, S.H. Meves, J. Eyding

St. Josef Hospital, Ruhr University Bochum, Bochum, Germany

Background: Ultrasonic brain perfusion imaging has the ability of forecasting localization and size of acute brain infarction. It remains unclear how the onset-toexamination-time influences the sensitivity and specificity of this new diagnostic tool. Furthermore, it remains uncertain if parametric imaging analysis is as reliable as region-wise analysis.

Methods: Bilateral Phase Inversion Harmonic Imaging examinations (bolus kinetic, fitted model function) were performed twice (acutely and 28 hours later) in 10 patients with acute ischemic stroke ( $<12$ hours). Examinations were evaluated using a region-wise analysis of the time intensity curve and by parametric images of the time to peak intensity maps. Results were correlated in-between the ultrasound examinations and to follow-up CCT scans.

Results: Correlation between the early region-wise analysis and follow-up CCT was the strongest (Spearman correlation coefficient 0.76 , sensitivity $84 \%$, specificity $96 \%)$. Spearman coefficient between the late ultrasonic perfusion imaging and CCT was 0.51 ; sensitivity and specificity were $71 \%$ and $82 \%$. Values in-between the ultrasonic brain perfusion examinations were $57 \%$ and $88 \%$ with Spearman coefficient of 0.47 ( $\mathrm{p}$ for all $<0.001$ ). Values of the analysis of the parametric images were less strong. Concordance between both of the ultrasonic perfusion imaging methods was $65 \%$ in the early examination and $72 \%$ in the late examination.

Conclusions: Brain perfusion pathology is most correctly detected in the early course of stroke, when core of infarction can be differentiated from penumbra and viable tissue. Re-perfusion phenomena may impair the diagnostic impact in later examinations. Parametric imaging does not seem to be as accurate as region-wise analysis, yet.

\section{Brain imaging}

SIGNIFICANCES OF HYPOINTENSE TRANSCEREBRAL OR CORTICAL VEINS ON T2*-WEIGHTED GRADIENT ECHO MR IMAGES IN PATIENTS WITH ACUTE CEREBRAL ARTERY OCCLUSION

S.I. Sohn, S.W. Park, Y.W. Cho, H. Lee, J.G. Lim, S.D. Yi, C.H. Sohn, S.H. Lee Keimyung University, Masan Samsung Hospital, SunKyunkwan University, South Korea

Introduction: Hypointense transcerebral or cortical veins (HypoTCV) on T2* weighted gradient echo (GRE) MR images was presented in acute ischemic stroke 
with large perfusion defects. We investigate clinical significances and hematological or radiological factors for abnormal visualization of HypoTCV on GRE MR images. Methods: We evaluated 106 patients who had acute ischemic stroke with occlusion of the internal carotid or middle cerebral artery admitted within 12 hours of symptom onset. This study enrolled 86 patients and 20 patients excluded from the study due to general contraindication to MRI and movement artifact precluding MRI interpretation. Of 86 patients, $45(52.3 \%)$ were treated with intravenous or intraarterial thrombolytics, 41 (47.7\%) were not treated due to not included indication of acute thrombolytic treatment

Results: Fifty-nine (68.6\%) of 86 patients had abnormal visibility of HypoTCV on GRE images, all disappeared in GRE MR images at day 7 after symptom onset. In unpaired $\mathrm{t}$ tests, predictors of visibility of HypoTCV were higher hemoglobin $(\mathrm{p}<0.05)$, higher cholesterol level $(\mathrm{p}<0.01)$, and PWI/DWI mismatch $(\mathrm{p}<0.05)$. In the multivariate analysis, cholesterol $(\mathrm{OR} 0.97,95 \% \mathrm{CI} 0.95-1.0 \mathrm{P}=0.03)$ was independent predictor of visibility of HypoTCV. In patients received thrombolytic treatment, presence of HypoTCV was not associated with hemorhagic transformation.

Conclusion: Visibility of HypoTCV on GRE in patients with acute ischemic stroke may be related to larger perfusion defect, especially ischemic penumbra. But HypoTCV may be not presented in patients with lower serum cholesterol and hemoglobin. Also visibility of HypoTCV is not associated with hemorrhagic transformation after acute thrombolytic treatment.

\section{Brain imaging}

\section{DIFFUSION WEIGHTED MAGNETIC RESONANCE IMAGING (DWI) IN PATIENTS WITH MILD STROKES - IMPORTANT TO IDENTIFY STROKE SUBTYPE BUT MANY DWI ARE NEGATIVE}

F.N. Doubal, J.M. Wardlaw, M.S. Dennis

University of Edinburgh, Edinburgh, United Kingdom

Background: DWI identifies the acute lesion in mild ischaemic stroke but can produce false negative (-ve) scans. We aimed to determine the rate of -ve DWI scans and associated factors in patients presenting with mild strokes.

Methods: In an ongoing study of causes of mild strokes (lacunar (LACS) or partial anterior circulation stroke (PACS) we recruited patients prospectively up to 3 months post stroke from a neurovascular outpatient clinic and acute stroke unit. Each patient had a diagnosis and classification of stroke made by an experienced stroke physician. Patients had T2/DWI/Gradient Echo and Fluid Attenuated Inversion Recovery (FLAIR) sequences. Scans were read by an experienced neuroradiologist with access to the clinical signs to identify symptomatic lesions.

Results: Of 42 patients, 17 (40\%) did not have an acute stroke lesion on DWI, but $3 / 17$ had a recent infarct on FLAIR or T2, therefore 14/42 (33\%) had no definite acute lesion on any MRI sequence. The overall median time from stroke to MRI was 19 days (range 1-84); for -ve DWI 24 days (range 12-76) and +ve DWI 13 days (range $1-84 \mathrm{p}<0.01,2$ tailed Mann-Whitney $\mathrm{U}$ test). Of the 42 patients 23 were LACS and 19 PACS (same proportion in -ve and +ve DWI), but the stroke classification was changed by imaging from LACS to PACS or vice versa in $8 / 42$ $(19 \%)$.

Discussion: DWI is needed for accurate stroke subtyping of $1 / 5$ th of recent mild strokes, but a large proportion are -ve. Delays to presentation increase the proportion of -ve MRI scans. Further work is needed to ascertain for what time period DWI reliably indicates ischaemia post stroke. DWI is essential for accurate stroke subtyping in studies of lacunar stroke.

\section{Brain imaging}

DIFFERENCE IN MRI AND TRANSESOPHAGEAL ECHOCARDIOGRAPHY FINDINGS AMONG PATIENTS HAVING CARDIOGENIC, PARADOXICAL, AND AORTOGENIC EMBOLIC SOURCES

S. Fujimoto, K. Toyoda, M. Yasaka, S. Sadoshima, Y. Okada

National Hospital Organization Kyushu Medical Center \& Nippon Steel Yawata Memorial Hospital, Kitakyushu, Japan

Background: The purpose of the present study was to clarify the difference in the characteristics and diffusion weighted image (DWI) and transesophageal echocardiography (TEE) findings among patients having cardiogenic, paradoxical, and aortogenic embolic sources.

Methods: We studied 270 consecutive patients with acute stroke or transient ischemic attack who had DWI-documented cortical or subcortical ischemic lesions without significant stenotic lesions in the cerebral arteries and who underwent TEE for detection of embolic sources. Patients were classified into 5 groups: those only with cardiogenic embolic sources (C-Group), those only with paradoxical embolic sources such as atrial septal defect, patent foramen ovale, or pulmonary arteriovenous fistula (P-Group), those only with aortogenic embolic sources (aortic atherom $\geq 4.0 \mathrm{~mm}$ ) (A-Group), those having multiple embolic sources (M-Group), and those without any embolic sources (U-Group).

Results: Fifty patients belonged to C-Group, 42 to P-Group, 55 to A-Group, 78 to M-Group, and 45 to U-Group. Cardiogenic embolic sources were independently contributed to ischemic lesions of one-third of the parent cerebral arterial territory or more (OR, 5.59; $95 \% \mathrm{CI}, 2.64-11.83$ ), and aortic atheroma $\geq 4.0 \mathrm{~mm}$ was independently contributed to ischemic lesions in the vertebrobasilar system (OR, 2.87; 95\% CI, 1.29-6.41). Atrial septal aneurysm was an independent predictor for distinguishing P-Group patients from M-Group patients having paradoxical embolic sources (OR, 3.95; 95\% CI, 1.19-13.16). Aortic atheroma with any of mobile plaque, extension to branches, or ulcer formation was an independent predictor for distinguishing A-Group patients from M-Group patients having aortic atheroma $>4.0 \mathrm{~mm}$ (OR, 2.94; $95 \%$ CI, 1.14-7.56).

Discussions: DWI and TEE findings are useful for specifying the appropriate embolic sources.

\section{Brain imaging}

\section{THE USE OF FAST FLAIR FOR EARLY DIAGNOSIS OF THE BRAIN} INFARCTION

L.V. Gubskii, N.A. Shamalov, V.I. Skvortsova

Russian State Medical University, Moscow, Russian Federation

Background: The aim of this study was to assess the contrast of ischemic brain lesions (IBL) by FLAIR images on the low-field MR-scanner within the 72 hours after stroke onset.

Methods: 103 patients with hemispheric infarction (aged from 48 to 89 years old, 50 males and 53 females) have been studied, 82 of them have been studied twice during this period. The study was performed at the 0.15 Tesla MR-scanner. Used pulse sequences were: fast FLAIR $(\mathrm{TR}=4100, \mathrm{TE}=100, \mathrm{TI}=1155 \mathrm{~ms})$, TSE (T2-weighted), SE and GRE (T1- weighted). Contrast (C) of IBL was calculated in relation to the intact white matter $(w m)$ as: $\mathrm{C}=(\mathrm{SIibl}-\mathrm{SIwm}) / \mathrm{SIwm}$, where $\mathrm{SI}-$ signal intensity.

Results: The values of contrast on FLAIR images were positive and gradually increased from 4-th to the 24-th hours after stroke onset in 97 patients $(94,2 \%)$. Mos of IBL during the first 12 hours of stroke onset were found in gray matter (cortex or basal ganglia). During the 24-th - 36-th hours the contrast of IBL increased significantly (by $30 \%, \mathrm{p}<0.05$ in comparison with the 4 -th -8 -th hours) and at 48-th -60 -th hours this difference became more significant $(\mathrm{p}<0.01)$. At the end of the 3-d day (60-72 hours) the values of contrast increased by $40 \%$ in comparison with the 2 -d day after stroke onset $(\mathrm{p}<0.01)$. In $6(5.8 \%)$ patients infarction was not visible during the first day after stroke. FLAIR was more sensitive and reliable sequence in exposure and assessment of IBL in comparison with T1-WI and T2-WI. Discussion: Thus FLAIR used on 0.15 Tesla scanner is the helpful sequence for early diagnosis and assessment of brain infarction.

\section{8}

\section{Brain imaging}

THE RELATIONSHIP BETWEEN BRAIN TEMPERATURE AND LACTATE FORMATION IN SUB REGIONS OF ACUTE ISCHEMIC STROKE IN PATIENTS

B. Karaszewski, J.M. Wardlaw, I. Marshall, V. Cvoro, K. Wartolowska, P.A. Armitage, M.E. Bastin, K. Haga, K. Chwojnicki, M.S. Dennis Medical Univ. of Gdansk, Gdansk \& Univ. of Edinburgh, Edinburgh, Poland and United Kingdom

Background: Formation of lactate, the main product of anaerobic metabolism, is increased in ischemic brain tissue. Temperature $(\mathrm{T})$ lowering may reduce lactate formation. We compared lactate and brain $\mathrm{T}$ in sub-regions of acute ischaemic stroke. Methods: 10 acute ischemic stroke patients had MR DWI (tensor), PWI and single slice spectroscopic imaging $<23 \mathrm{~h}$ from stroke. From a $0.8 \mathrm{~cm}^{3}$ voxel grid placed on the DWI, voxels were coded "abnormal" (ie definitely hyperintense-DH), "possibly penumbral" (ie possibly hyperintense -PH or "lesion edge" -edge), or normal ("ipsi-" or "contra-lateral normal") brain, blind to all other data. We calculated voxel T $\left({ }^{\circ} \mathrm{C}\right)$ from water-to-NAA chemical shift. We compared Lactate and T by DWI-based voxel type.

Results: Lactate was highest in the most abnormal tissue (DH mean 41.7 $\pm 21.4 \mathrm{SD}$ $\mathrm{PH} 26.2 \pm 18.3$; edge 13.9 \pm 9.5 ; normal $11.1 \pm 16$; $\mathrm{p}<0.0001)$. Brain $\mathrm{T}^{\circ} \mathrm{C}$ was highest in less abnormal "possibly penumbral" tissue (edge $38.1 \pm 1.4$ and $\mathrm{PH} 37.5 \pm 1$; DH $37.0 \pm 1.4$; normal $37 \pm 1, \mathrm{p}<0.007)$. The association between lactate and $\mathrm{T}$ appeared to differ between normal/DH (positive assoc.) and PH/edge (penumbral; negative assoc.) tissue, but more data are needed to test this in more detail reliably.

Discussion: The relationship between LA and T is not direct - highest LA and 
highest $\mathrm{T}$ occur in different parts of the ischaemic stroke. Brain cooling may not influence lactate formation in ischemic brain in humans.

\section{Brain imaging}

\section{THE ASPECTS TEMPLATE IS WEIGHTED IN FAVOR OF THE} STRIATOCAPSULAR REGION

T.G. Phan, G.A. Donnan, M. Koga, L.A. Mitchell, M. Molan, G. Fitt, W. Chong, M. Holt, D.C. Reutens

Austin Health, Melbourne University, MIND, Monash University, Clayton, VIC, Australia

The extent of cerebral infarction correlates with increased risk of intracerebra hemorrhage $(\mathrm{ICH})$ following recombinant tissue plasminogen activator (rt-PA) administration. The Alberta Stroke Program Early CT Score (ASPECTS) is a widely-used, validated method which assesses involvement of 10 selected regions of the MCA territory. An ASPECTS score $>7$ is associated with a higher risk of ICH following thrombolysis than lower scores. To understand the internal structure of the ASPECTS template better, we estimated the infarct volume corresponding to each region. We hypothesized that in the ASPECTS scoring system, the striatocapsular region is weighted disproportionally. Four experienced radiologists rated individual ASPECTS regions on subacute CT images (day 5-day 10) of nineteen patients with MCA territory stroke. Infarct volume was determined from manual segmentation of infarcts on CT images. Linear regression was used to estimate the regional volume associated with each ASPECTS region. The ASPECTS regions are weighted unequally with the striatocapsular region accounting for $21 \%$ of the MCA territory infarct volume. Together, the 10 ASPECTS regions account for approximately $51 \%$ of the maximum MCA infarct territory volume. These findings should provide impetus for research to develop a scoring system explicitly based on regional hemorrhage risk as an aid to selecting patients for thrombolysis.

\section{Vascular imaging}

\section{Vascular imaging}

DIFFERENTIAL CLINICAL IMPLICATION OF MICROEMBOLIC SIGNALS IN ACUTE ISCHEMIC STROKE WITH ATHEROSCLEROTIC MIDDLE CEREBRAL ARTERY AND CAROTID ARTERY DISEASE

H.Y. Shin, D.J. Yoon, C.S. Chung, K.H. Lee, G.M. Kim

Samsung Medical Center, Sungkyunkwan University School of Medicine, Seoul, South Korea

Background: Microembolic signals (MES) have been implicated as an important mechanism of ischemic stroke in atherosclerotic large artery disease. The purpose of this study was to examine the relationship between lesion patterns on diffusionweighted MR imaging (DWI) and MES in acute ischemic stroke associated with atherosclerotic middle cerebral artery (MCA) and internal carotid artery (ICA) disease

Methods: A total of 211 consecutive patients with ischemic stroke within 24 hours of symptom onset were monitored for MES. Patients with MES, DWI lesion in MCA or ICA territory, and corresponding MCA/ICA stenosis or occlusion on MR angiography (MRA) were included. Patients with cardiogenic or other systemic embolic sources and who received thrombolytic therapy were excluded. MCA velocities on transcranial Doppler (TCD) examination, lesion patterns and multiplicity on DWI, and the degree of stenosis on MRA were compared between MCA and ICA disease groups.

Results: MES were detected in 18 patients (MCA: 9, ICA: 9) who fulfilled our inclusion criteria. The mean numbers of MES during 30 minutes monitoring was $8.44 \pm 9.4$ (range: $1-39$, MCA: $9.89 \pm 8.2$, ICA: $7.0 \pm 12.1$ ). Mean flow velocity of insonated ipsilateral MCA in MCA group was higher than that of ICA group $(118.56 \pm 80.3 \mathrm{~cm} / \mathrm{s}$ vs $59.3 \pm 27.0 \mathrm{~cm} / \mathrm{s}, \mathrm{p}=0.013)$. The vascular occlusion on MRA was more frequent in ICA group $(\mathrm{p}=0.014)$ than that of MCA group. There was a significantly higher frequency of lesion multiplicity on DWI in ICA group (22\% vs $100 \%, \mathrm{p}=0.003$ ). Patients with ICA disease showed a tendency to involve more cortical or cortical plus subcortical areas but the MCA group revealed mainly subcortical distribution of lesions on DWI.

Conclusions: Our results suggest that differential clinical implication of MES may exist between MCA and ICA disease. Further study is necessary to identify whether the appearance of MES implicates a predictor for recurrent embolic stroke or a sign of recanalization.
2 Vascular imaging

DISTRIBUTION AND OUTCOME OF SYMPTOMATIC STENOSES AND OCCLUSIONS IN PATIENTS WITH ACUTE CEREBRAL ISCHEMIA C. Weimar, M. Goertler, H.C. Diener, the German Stroke Study Collaboration University of Duisburg-Essen, Duisburg-Essen, Germany

Background: A cerebrovascular diagnostic examination is considered part of the standard work-up after cerebral ischemia. However, only scarce data exist on the distribution and prognosis of steno-occlusive disease in symptomatic patients. We therefore assessed the frequency and recurrence rate of defined extra- and intracranial locations in a large consecutive hospital-based Caucasian cohort. Methods: All patients with acute IS or TIA were prospectively documented in 11 German departments of Neurology with acute stroke unit. A complete cerebrovascular examination was performed in 4157 patients $(89.6 \%)$ and documented on standardized case report forms. A predominantly central follow-up assessed recurrent stroke and death up to 1 year after the event in $85.3 \%$ of these patients.

Results: Symptomatic vessel occlusions were found most often in the proxima internal carotid artery $(6.5 \%)$, the M1 segment of the middle cerebral artery $(3.7 \%)$, and the vertebral artery $(3.0 \%)$. Symptomatic stenosis $>50 \%-99 \%$ of the internal carotid artery was found in 308 patients $(7.4 \%)$ and 272 patients $(6.5 \%)$ had symptomatic intracranial stenosis. The highest rates of recurrent stroke during the first 3 days occurred in patients with symptomatic carotid and intracranial occlusions. Overall 82 patients $(9.0 \%)$ with symptomatic cerebrovascular disease suffered a recurrent stroke between day 4 and 1 year but no significant differences in recurrent stroke rates could be found when comparing different locations of steno-occlusive disease.

Discussion: Our study provides representative data on distribution and outcome of steno-occlusive disease in patients with acute cerebral ischemia. In contrast to prior studies in more selected populations, the risk of recurrent stroke in patients with symptomatic intracranial stenosis does not seem elevated compared to patients without steno-occlusive disease.

\section{Vascular imaging}

INTRACRANIAL OCCLUSION ON CT ANGIOGRAPHY PREDICTS POOR OUTCOME IN ACUTE ISCHEMIC STROKE

I. Dzialowski, A. Krol, J. Roy, M.D. Hill, P.A. Barber, J.M. Boulanger,

S.B. Coutts, S. Subramaniam, A.M. Demchuk

University of Calgary, Calgary, Canada

Background: CT angiography (CTA) has excellent diagnostic accuracy in detecting large intracranial vessel occlusions and is widely available and easily feasible. We studied the hypothesis that an intracranial arterial occlusion as detected by CTA is an independent predictor of outcome in acute ischemic stroke.

Methods: We retrospectively studied patients with acute ischemic stroke in whom a CTA of the circle of Willis was performed within $24 \mathrm{hrs}$ of symptom onset from 04/2002-04/2005. We assessed vessel status and recorded baseline NIHSS score. Discharge modified Rankin Scale (mRS) score was assessed from the patient chart. We reviewed all baseline non-contrast CT scans applying the Alberta Stroke Program Early CT Score (ASPECTS). We calculated risk ratios (RR) for good outcome $(\mathrm{mRS}$ score $\leq 2)$ and performed a multivariable analysis with previously determined important outcome predicting variables: age, baseline NIHSS and baseline mRS, onset-to-CTA-time, baseline ASPECTS and thrombolytic treatment. We also included intracranial occlusion on CTA

Results: We identified 254 patients with a median age of 72 (range 31-94) years, onset-to-CTA-time 190 min (range 30-1350), baseline NIHSS 7 (range 0-32) and baseline ASPECTS 10 (range 2-10). CTA revealed 114 patients with intracranial occlusion and 140 patients with no occlusion. Intracranial occlusion was associated with a reduced likelihood of good outcome $(R R=0.55$ CI95 0.43-0.74, $p<0.0001)$ Independent predictors for good outcome were the absence of intracranial occlusion $(\mathrm{p}=0.032)$, lower age $(\mathrm{p}<0.0001)$, lower baseline NIHSS $(\mathrm{p}<0.0001)$ and shorter onset-to-CTA-time $(\mathrm{p}=0.024)$.

Conclusion: The presence of an intracranial occlusion as demonstrated by CTA is an independent predictor of outcome in acute ischemic stroke. 


\section{Vascular imaging}

IMAGING OF MIDDLE CEREBRAL ARTERY (MCA) PLAQUES IN MAGNETIC RESONANCE IMAGING (MRI): DIAGNOSTIC ACCURACY OF ARTERY STENOSIS

X.Y. Chen, W.M. Lam, H.K. Ng, H.L. Zhao, K.S. Wong

Chinese University of Hong Kong, Hong-kong

Background: Magnetic resonance imaging (MRI) has been widely applied in detecting intrancranial large artry stenosis but there have been few validation studies to compare with histopathology. The aim of the post-mortem study is to assess the accuracy of MRI in identifying MCA stenosis and its correlation with clinical ischemic events.

Methods: We recruited consecutively Chinese patients (more than 45 years) who underwent post-mortem in our hospital during nineteen months. MRI was performed in the post-mortem brains, to scan the cross-sections of MCAs with barium expanding the artery lumen. The MCAs were then removed for histopathological studies. With histopathology as a reference standard, the accuracy of MRI was evaluated and the correlation between MCA stenosis identified by MRI and clinical ischemic events was investigated.

Results: Seventy-six consecutive autopsies were recruited. The sensitivity and specificity of MRI in detecting more than $30 \%$ MCA stenosis were $38.6 \%$ and $92.2 \%$, with a positive predictive value of $87.2 \%$ and negative predictive value of $52.2 \%$, and the corresponding values of MRI in identifying more than 50\% MCA stenosis were $57.1 \%, 90.8 \%, 50 \%$ and $83.0 \%$, respectively. Both more than $30 \%$ and more than $50 \%$ MCA stenosis identified by MRI were found to be associated with infarctions in corresponding MCA territory $(\mathrm{p}=0.001$, odds ratio $=4.365,95 \%$ CI: 1.684-11.313; and $\mathrm{p}=0.039$, odds ratio $=2.694,95 \% \mathrm{CI}: 1.139-6.377$ ).

Discussion: Our study demonstrates the agreement between ex vivo MRI and histopathology in identifying MCA stenosis, and the correlation between the MCA stenosis identified by MRI and clinical ischemic events. MRI may be a potential application to study MCA in clinical trials. Further study is needed to evaluate the accuracy of MRI in identifying the composition of atherosclerotic plaque in MCAs.

\section{Vascular imaging}

\section{NEUROANATOMIC CORRELATES OF THE POST-STROKE APHASIAS STUDIED WITH IMAGING}

Y. Yu-mei Zhang, Yong-jun Wang, Xing-quan Zhao, Chun-xue Wang,

Yi-long Wang, Xi-ping Gong

Tiantan hospital, affiliated with Capital University of Medical Sciences, Beijing, China

Background and Objective: Neuroanatomic correlates of the post-stroke aphasias is still a uncertain problem. In this study, we try to reveal the relationship between post-stroke aphasia types and lesions distribution by evaluating aphasia types and observing damaged lesions located.

Methods: We used imaging recorded the damaged lesions of 1198 inpatients who suffered from cerebral infarction and hospitalized in Neurology department, Beijing Tiantan Hospital from Feb, 2002 to Feb, 2005, then we selected the inpatients who meeted with our selected standard to carry out the Western Aphasia Battery, grade criterion of the Boston Diagnostic Aphasia Examination.

Results: Of which 1198 cases, there were 5 cases whose Broca's area and 4 cases whose Wernicke's area were damaged had no language disorders. Of which 325 aphasia cases, there were 83 Broca's aphasia cases, 48 Wernicke's aphasia cases, 12 conduction aphasia cases, 30 transcortical motor aphasia cases, 17 transcortical sensory aphasia cases, 19 transcortical mix aphasia cases, 58 global aphasia cases, 44 anomic aphasia cases, 6 alexia cases and 8 agraphia cases. Among all aphasia cases, 240 cases damaged lesions were located at classical language centers, such as Broca's area, Wernicke's area, arcuate fasciculus and angular gyrus, etc, and 85 cases damaged lesions were located at other sites besides language functional areas. Of 325 aphasia patients, the level of aphasia severiously were that grade $0,1,2,3$, 4 had $84,79,77,63,22$ cases respectively and many of 0 and 1 level aphasia cases were located at classical language centers.

Discussions: The damaged lesions of most aphasia cases are meeted with classical language functional areas, Others site damaged also can produce aphasia while language functional areas are damaged may not produce aphasia. The language disorder of patients whose lesion located at classical language functional areas are more serious.
6 Vascular imaging

PLASMA CONCENTRATION OF OSTEOPONTIN IS INCREASED IN PATIENTS WITH HETEROGENEOUS CAROTID PLAQUES

H. Kato, M. Watanabe, M. Okamoto, Y. Baba, D. Takahashi, M. Nukata, K. Imai, Y. Saeki, N. Kinoshita

Osaka University Graduate School of Medicine, Osaka, Japan

Background: Recent clinical studies suggest the possibility that osteopontin (OPN) plays an important role in vascular remodeling including dystrophic calcification, macrophage infiltration, and smooth muscle cell migration in atherosclerotic plaques. Although OPN is supposed to be correlated with histological characteristics of atherosclerotic plaques, the correlation between plasma OPN concentration and carotid plaque morphology has not been revealed yet. In present report, we investigated the correlation between ultrasonography (US)-assessed carotid plaque morphology and plasma concentration of OPN.

Method: Seventy-three patients (male\%: $59 \%$, age: $66 \pm 8.8$ yo) at our hospital underwent carotid US, and plaque volume and morphology were evaluated. As representation of plaque morphology, heterogeneity of plaque images was assessed visually by two physicians and automatically by newly introduced heterogeneity index based on computer assisted gray-scale analysis. For all the patients, plasma OPN concentration was also measured.

Results: By visual assessment of carotid plaques, the patients were classified into three groups; patients without any carotid plaque $(n=19)$, with solely homogeneous plaques $(n=28)$, and with heterogeneous plaques $(n=26)$. Visual and automatic assessment of plaque morphology showed good concordance $(\mathrm{P}<0.001)$. Plasma OPN concentration was significantly higher in patients with heterogeneous plaques than in others $(\mathrm{P}=0.001)$. Plasma OPN concentration and the heterogeneity index exhibited positive correlation $(\mathrm{P}<0.001)$.

Conclusion: It was revealed that plasma OPN concentration was positively correlated with plaque heterogeneity.

\section{New vascular imaging}

\section{New vascular imaging}

\section{VOXEL-GUIDED MORPHOMETRY (VGM) FOR INTRAINDIVIDUAL MRI} MORPHOMETRY AFTER STROKE

M. Kraemer, T. Schormann, O.W. Witte, R.J. Seitz

St. Mauritius Therapieklinik Meerbusch, Meerbusch, Germany

In our study, we examined volume alterations in brain areas adjacent to and remote from the lesion in 6 stroke patients with an incomplete MCA infarction. MRI scans were carried out 7 days and between 3 and 16 months after stroke onset. Patients were examined by a 3D FLASH MRI scan. Brains from the same patient at two different times were matched to each other using Voxel-Guided Morphometry [1] thus achieving sub-voxel accuracy between source and reference brain. This enabled exact reproducibility of results due to the completely automated procedure and the ability to determine the unique movement from source to the reference volume using the total gray-value information. The resulting deformation fields were visualized encoded in gray-values.

The investigated brains exhibited a volume reduction in widespread cortical areas even remote from the lesion site and in the underlying white matter. Atrophy was also detected in the ipsilateral thalamus and caudate nucleus that were both not affected by the initial ischemia.

These remote atrophic brain areas demonstrate, that secondary long-term brain volume alterations develop after acute cerebral ischemia probably due to degeneration of white matter projections. Remarkably, this secondary brain atrophy was accompanied by an improvement of the clinical state, marked by an improved European Stroke Scale in the follow-up examination. Obviously, secondary brain atrophy does not necessarily correlate with the clinical outcome.

In summary, Voxel-Guided Morphometry demonstrates a widespread and locally differential brain atrophy in brain areas adjacent to and remote from the stroke lesion site. These volume changes do not necessarily correlate with the improvement of clinical outcome.

References: [1] Voxel-guided morphometry ("VGM") and application to stroke. IEEE Trans Med Imaging. 2003, 22:62-74. 


\section{New vascular imaging}

3D MAGNETIC RESONANCE ANGIOGRAPHY USING COMBINED TIME-RESOLVED CONTRAST-ENHANCED IMAGING AND PARALLEL IMAGING IN THE ASSESSMENT OF INTRACRANIAL VASCULAR MALFORMATIONS

J.Y. Gauvrit, M. Petkova, D. Trystram, F. Nataf, C. Oppenheim, J.F. Meder

Hospital R Salengro, Lille; Ch St Anne, Paris, France

Purpose: To investigate the usefulness of a new 3D Magnetic Resonance Digital (3D MRA) technique combining time-resolved contrast-enhanced imaging and parallel imaging in the assessment of intracranial vascular malformations.

Method and Materials: 3D MRDSA time-resolved contrast-enhanced imaging in combination with parallel imaging was performed in 35 patients (arteriovenous malformations AVMs $n=28$; dural arteriovenous fistulas DAVFs $n=3$; no intracranial vascular malformation $n=2$; developmental venous anomaly $n=1$; temporosylvian anastomosis $\mathrm{n}=1$ ). The method was compared with 3D MRDSA using parallel imaging alone and with conventional X-ray Digital Subtraction Angiography (DSA). The type and number of feeders and drainers and the size of AVMs nidi were assessed. The temporal resolution was evaluated by a three-point grading scale and the spatial resolution by the ability to visualise the more distal arterial branches.

Results: 3D MRDSA using time-resolved and parallel imaging afforded superio temporal, 0,8 seconds versus to 1.7 seconds and spatial resolution (40\%) in comparison with the 3D MRDSA using parallel imaging alone. Thus, this technique allows better detectability of early venous filling in patients with AVM and DAVF. When compared with conventional X-ray DSA, it displays less exhaustive delineation of feeders.

Conclusion: Our study shows that 3D MRDSA using better temporal and spatial resolution can be employed as a reliable and less invasive dynamic angiographic tool for intracranial vascular malformations.

\section{New vascular imaging}

\section{PERFUSION-CT SHOWS HYPOPERFUSION DURING REVERSIBLE POSTERIOR LEUKOENCEPHALOPATHY SYNDROME}

P. Michel, M. Reichhart, M. Delgado, P. Browaeys, R. Meuli, J. Bogousslavsky

Centre Hospitalier Universitaire Vaudois, Lausanne, Switzerland

Background: Reversible posterior leukoencephalopathy syndrome (RPLS) is characterized by altered mental function, seizures, cortical visual symptoms and headaches. We present clinical, perfusion-CT (PCT) and MRI findings in three patients with RPLS.

Patients and Methods: Patient 1. A 61-year-old lady presented sudden cortical blindness and confusion, very high blood pressure (BP), and six hours later had two generalized seizures. Symptoms resolved with antihypertensive and antiepileptic drugs over 3 days. Patient 2. A 61-year-old hypertensive man with colon adenocarcinoma had a generalized seizure followed by cortical blindness at the end of a 5FU infusion. Symptoms resolved spontaneousely over 12 hours. Patient 3: An 87 year old hypertensive lady, anticoagulated for atrial fibrillation, was found with confusion, bilateral incomplete hemianopia and very high BP. Symptoms resolved with BP-treatment over 4 days.

Results: In patient 1, acute PCT showed bilateral occipital hypoperfusion. Diffusionweighed (DWI) MRI on day 4 was normal, T2 weighted (T2W) imaging showed bioccipital white matter changes. In patient 2, acute PCT showed widespread bilaterally decreased parieto-occipital flow. DWI and T2W on day 2 were normal. In patient 3, acute PCT showed patchy occipital hypoperfusion. DWI on day 4 was normal, $\mathrm{CT}$ and $\mathrm{T} 2 \mathrm{~W}$ showed diffuse leukoaraiosis. Acute CT-angiography performed was normal in all patients as were MR-angiographic studies later on Further work-up showed no sources of emboli in patients 1 and 2. In patient 3 with known atrial fibrillation, a carotid origin of both her posterior cerebral arteries was present. This and a normal DWI made cardioembolic stroke an unlikely alternative diagnosis for problems.

Conclusion: Perfusion CT in acute RPLS shows bilateral posterior hypoperfusion This hypoperfusion seems to be of a different nature than in ischemic stroke, as it does not result in DWI lesions nor does it respect vascular territories.
4 New vascular imaging

CE-MRA FOR THE RAPID DETECTION OF VASCULAR DISEASE FROM THE AORTIC ARCH TO THE CIRCLE OF WILLIS (MR-VAS STUDY): FINAL RESULTS

V.L. Wright, W. Olan, B. Dick, H. Yu, N. Alberts-Grill, S. Debrey, L.L. Latour, A.E. Baird

National Institutes of Health, Bethesda, MD, USA

Background: Contrast-enhanced magnetic resonance angiography (CE-MRA) using a combined head and neck coil permits non-invasive imaging of the vascular from the aortic arch through to the Circle of Willis in less than 2 minutes.

Methods: In a prospective study of 132 patients referred for digital subtraction angiography (DSA), CE-MRA and DSA studies were performed within 72 hours of each other. CE-MRA was performed on a 1.5 Tesla Scanner using a 5 channel neurovascular array coil. CE-MRAs and DSA films were read by two interventional neuroradiologists blinded to the clinical presentation of the patient. The degree of stenosis for each extracranial and intracranial vessel and graded using the North American Symptomatic Carotid Endarterectomy Trial criteria, and divided into $\leq 50 \%$ and $\geq 50 \%$ categories

Results: On DSA there were a total of 114 vascular stenoses $\geq 50 \%$ identified, 79 extracranial and 35 intracranial. The overall sensitivity of CE-MRA for the detection of vascular stenoses $\geq 50 \%$ was $60 \%$ (95\% CI: $51-69 \%$ ) with a specificity of $99 \%$ $(98-99 \%)$. The sensitivity for the detection of extracranial vascular stenoses $>50 \%$ was $82 \%$ (74\%-90\%) with a specificity of $96 \%$ (95-97\%). However, the sensitivity for the detection of intracranial vascular stenoses $\geq 50 \%$ was only $9 \%(0 \%-18 \%)$, with a specificity of $99 \%(98-100 \%)$.

Discussion: CE-MRA using a neurovascular coil shows promise as a rapid and non-invasive screening method for extracranial vascular disease. This method could complement current acute stroke MR imaging protocols for decisions regarding emergent thrombolysis, early surgical intervention and stroke prevention.

5 New vascular imaging

\section{SENSITIVITY OF AN AUTOMATED TRANSCRANIAL DOPPLER EMBOLI} DETECTION SYSTEM

J.M. Boulanger, E. Stewart, C. Stephenson, A.M. Demchuk University of Calgary, Calgary, Canada

Background: Microembolic signals (MES) visualized on a transcranial Doppler (TCD) are associated with an increased risk of subsequent stroke in patients with carotid stenosis. The standard TCD emboli detection method is time consuming and not easily applicable in routine practice. An automated emboli detection system (Spencer technologies, Seattle WA) exists but its sensitivity is unknown.

Methods: We compared two methods of TCD emboli detection in patients with a symptomatic carotid stenosis $(>50 \%)$ : a one hour digital recording analyzed by a sonographer and an automated detection system (Spencer technologies, Seattle WA) in which signals resembling MES were recordered by the TCD machine and later reviewed by a sonographer blinded to clinical data. International spectrogram MES diagnostic criteria were used.

Results: Twenty three patients with a symptomatic carotid stenosis had 33 TCD exams. The automated detection system recorded 662 signals, of which $76(11.5 \%)$ were diagnosed as MES by the sonographer. Eight $(8 / 84 ; 9.5 \%)$ MES were missed by the automated system.

Discussion: The TCD automated emboli detection system studied was sensitive $(90.5 \%)$ in detecting MES. Because of the high false positive rate $(88.5 \%)$, al recorded signals should be reviewed by a sonographer. This method of emboli detection would require a few minutes, thus being far less labor intensive than the standard TCD emboli detection process.

Conclusion: An automated TCD emboli detection system (Spencer technologies, Seattle WA) misses few MES and may be useful clinically.

\section{New vascular imaging}

QUANTITATIVE SELECTIVE ARTERIAL SPIN LABELING

M. Guenther, M. Griebe, K. Szabo, J. Hirsch, R. Kern, J. Binder, M.G. Hennerici, A. Gass

Universitätsklinikum Mannheim, University of Heidelberg, Mannheim, Germany

Introduction: Recently, several papers were published on selective arterial spin labeling (selective ASL) which describe the separation of blood flow (CBF) of different intracranial vascular territories. However, it is still very difficult to yield quantitative measures since the amount of labeling within a certain vessel 
is unknown. The goal of our study was to yield quantitative CBF of different vascular regions by combining quantitative standard ASL methods with selective ASL.

Methods: Ten young healthy normal controls were examined on a dedicated $3 \mathrm{~T}$ head MR scanner (Allegra, Siemens, Germany) starting with isotropic $1 \mathrm{~mm}$ anatomical T1- MRI (3D MPRAGE). A standard ASL time series with inflow times ranging from $200 \mathrm{~ms}$ to $2800 \mathrm{~ms}$ (14 steps, increment $200 \mathrm{~ms}$ ) with reduced in-plane resolution of $5.8 \mathrm{~mm}$ were used to determine regional $\mathrm{CBF}$ and bolus arrival time (BAT). In addition, selective ASL data at an inflow time of $2200 \mathrm{~ms}$ were acquired at higher resolution (3.5mm in-plane) which was used for vascular territory information. Labeling slabs were positioned on maximum intensity projection of the previously acquired anatomical data set. An efficient single-shot 3D readout module (ss3D-GRASE [M.Guenther et al. Magn Res Med 2005, 54(2): 491-498]) was employed which reduced the total measurement time for selective ASL and ASL time series acquisition to 8 minutes.

Results: Vascular territories could be clearly distinguished. Mean gray matter $\mathrm{CBF}$ was $71.3 \pm 19.0 \mathrm{ml} / 100 \mathrm{~g} / \mathrm{min}$ for anterior circulation while it was $85.3 \pm 13.0$ $\mathrm{ml} / 100 \mathrm{~g} / \mathrm{min}$ for posterior circulation. Mean BAT was $823 \pm 50 \mathrm{~ms}$ for both, anterior and posterior circulation. Watershed areas were typically filled $400 \mathrm{~ms}$ later.

Discussion: The combination of conventional ASL time series for quantification of $\mathrm{CBF}$ and selective ASL for determination of vascular territories increases the in-vivo knowledge of cortical physiology in a non-invasive manner. The relatively short acquisition time enables use in clinical routine protocols.

\section{New vascular imaging}

TRANSCRANIAL CONTRAST DIMINUTION AND REPLENISHMENT IMAGING FOR THE EVALUATION OF CEREBRAL HYPOPERFUSION IN ACUTE ISCHEMIC STROKE - A PILOT STUDY

K. Meyer-Wiethe, L.A. Schindler, C. Kier, H. Cangür, G. Seidel

University Hospital Schleswig-Holstein, Campus Lübeck, Lübeck, Germany

Background: Transcranial Contrast Diminution and Replenishment Harmonic Imaging, respectively (DHI, RHI) are new ultrasound tools to assess cerebral perfusion. In a pilot study, we demonstrate the potential of these methods to identify critically hypoperfused brain regions in acute ischemic stroke.

Methods: Eight patients with clinical signs of acute middle cerebral artery stroke were investigated with DHI and RHI (SONOS 5500, 1.8/3.6 MHz, MI 1.6) at a SonoVue ${ }^{\circledR}$ continuous infusion, no longer than $4 \mathrm{~h}$ after MRI (perfusion and diffusion weighted sequences; PWI/DWI). Normalized time-intensity data of the affected hemisphere were analyzed. For DHI-analysis, we applied a linear (amplitude: A), exponential decay (half life: $\mathrm{H}$ ), and an exponential destruction/reperfusion model (perfusion coefficient: P). Replenishment curves were analyzed using an exponential model (beta and F). After dichotomizing our data into normal vs. delayed perfusion of $>4 \mathrm{~s}$ as diagnosed by PWI, receiver operating characteristics analysis provided optimum cutoff values for each model. Parametric images were calculated for each curve parameter.

Results: For brain regions of delayed perfusion, we calculated the following cutoff-values: A: $9.8 \%, \mathrm{H}: 98 \mathrm{~ms}, \mathrm{P}: 0.042 \mathrm{~s}^{-1}$, beta: $8.08 \times 10^{-6}$, F: $4.81 \times 10^{-7}$. No significant thresholds were found for differentiation between normal brain regions and DWI lesions. The area of significantly reduced perfusion coefficient in the parametric image correlated with the area of delayed perfusion in PWI $(R=0.80, P$ $=0.02$ ).

Discussion: Both DHI and RHI can detect regions with altered brain perfusion in acute ischemic stroke. We defined thresholds of ultrasound parameters to differentiate between brain areas with normal and delayed perfusion. Further studies are justified to evaluate this fast bedside method of perfusion analysis.

\section{New vascular imaging}

EVALUATION OF A MICROCONVEX ARRAY TRANSDUCER FOR THE ULTRASONOGRAPHIC EXAMINATION OF THE INTRATHORACIC SEGMENTS OF THE SUPRAAORTIC ARTERIES

B. von Sarnowski, U. Schminke, C. Kessler

Universitätsklinikum Greifswald, Greifswald, Germany

Introduction: Due to the geometry of linear array transducers and the anatomy of the supraclavicular and jugular fossa it is difficult or even impossible to get an appropriate ultrasonic view of the most proximal segments of the supraaortic arteries and their origin from the aortic arch.

Methods: We examined 21 patients for the origin of the common carotid arteries (CCA), subclavian arteries (SA), vertebral arteries (VA), brachiocephalic (innominate) artery (IA) and the visibility of the aortic arch (AA) with a $5.7-10-\mathrm{MHz}$ linear array transducer and a 3,5-11,5-MHz microconvex array transducer. Our aim was

Poster Session First Visit

Brain imaging - new developments to find out if the microconvex probe is of advantage in imaging the vessels named above.

Results: The left CCA ( $0 \%$ vs $47.6 \%, p=0.0005)$, SA ( $0 \%$ vs. $23.8 \%, p=0.0478)$ and VA $(47.6 \%$ vs. $90.5 \%, \mathrm{p}=0.0063)$, the AA $(4.8 \%$ vs. $52.4 \%, \mathrm{p}=0.0014)$ and the IA $(14.2 \%$ vs. $61.9 \%, \mathrm{p}=0.0036)$ were significantly more often depictable by the means of the microconvex probe than by the linear probe. No statistically significant difference in the representation of the right CCA, SA and VA exists between the two probes. A subjective comparison by the examiner of which ultrasound image was of higher quality and gave a better view showed that the microconvex probe delivered better images in all examined vessels.

Conclusion: The microconvex array transducer revealed a better and further view of the intrathoracic segments of the supraaortic arteries and the aortic arch. This is mostly due to its much smaller contact surface, which improves the coupling between the transducer and the skin surface even in complicated areas as the supraclavicular or jugular fossa.

\section{New vascular imaging}

FETAL ORIGIN OF THE POSTERIOR CEREBRAL ARTERY IN PATIENTS WITH A TIA OR INFARCT IN THE TERRITORY OF THE POSTERIOR CEREBRAL ARTERY ASSESSED WITH 16-MULTIDETECTOR-ROW CT ANGIOGRAPHY

C. De Monyé, D.W.J. Dippel, T.A.M. Siepman, M. Dijkshoorn, H. Tanghe,

A. van der Lugt

Erasmus Medical Center Rotterdam, Rotterdam, The Netherlands

Purpose: An uncommon cause of cerebral ischemia in the territory of the posterior cerebral artery (PCA) is the combination of a fetal origin of the PCA and atherosclerotic disease in the internal carotid artery (ICA). This study assessed the frequency of a fetal origin of the PCA and the degree of stenosis of the ipsilateral ICA in patients with a recent TIA or infarct in the territory of the PCA with 16-multidetector-row CT angiography.

Materials and Methods: 82 patients (52 male; mean age $=64$; range 19-90yrs) with complaints of isolated homonymous hemianopia or a recent PCA infarct underwent CT angiography of the carotid artery from the aortic arch to the intracranial circulation. All patients received $80 \mathrm{~mL}$ contrast material i.v. at $4 \mathrm{~mL} / \mathrm{sec}$. A fetal origin of the PCA was defined as a posterior-communicating-artery-size greater or equal to the ipsilateral P1-segment.

Results: A fetal origin of the PCA was present ipsilateral to the symptomatic side in 14 patients $(17 \%)$ and contralateral in 18 patients $(22 \%)$. Degree of stenosis (NASCET-criteria) of the ipsilateral ICA was 0\%, 1-29\%, 30-49\% and $>50 \%$ in 32 , 40,2 and 8 patients, respectively. Number and frequency of a fetal origin in these groups were $6(19 \%), 6(15 \%), 0(0 \%)$ and $2(25 \%)$, respectively.

Conclusion: Some patients with a TIA or infarct of the PCA have a fetal origin of the PCA in combination with a high-grade stenosis of the ipsilateral ICA, but not more often than one would expect from a chance association. Still, these patients may benefit from carotid endarteriectomy.

\section{Brain imaging - new developments}

\section{Brain imaging - new developments}

\section{D GRASE ASL IS HIGHLY SENSITIVE TO DETECT HYPERPERFUSION} STATES IN PATIENTS WITH ACUTE STROKE

M. Griebe, R. Kern, M. Guenther, K. Szabo, F. Sallustio, M.G. Hennerici, A. Gass

Universitaetsklinikum Mannheim, University of Heidelberg, Mannheim, Germany

Background: In stroke, assessment of hyperperfusion may have therapeutic implications for stroke patients since thrombolysis may be needless or even harmful when vessel recanalization has already occurred. In the present study we compared novel non-invasive arterial spin labelling (ASL) perfusion maps to time-to-peak maps (TTP) of dynamic susceptibility contrast (DSC) perfusion MRI.

Methods: MRI on a $1.5 \mathrm{~T}$ scanner (Siemens Sonata) included TOF-MRA, DWI and DSC perfusion MRI. ASL perfusion maps were obtained using a single-shot 3D-GRASE read-out module from which CBF and bolus arrival time (BAT) were extracted. 36 patients with acute hemispheric stroke (31 MCA, 5 PCA territories) were examined within 48 hours after symptom onset. According to TTP maps und TOF-MRA, hypoperfusion was present in 28 patients, hyperperfusion in 7 patients and no perfusion deficit in 1 patient.

Results: ASL perfusion maps were obtained in good quality in $34 / 36$ patients. The 


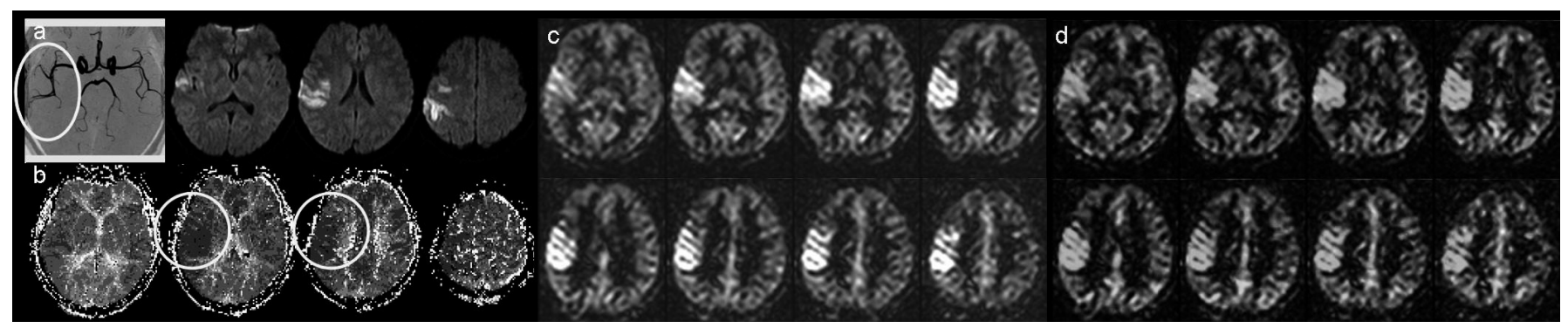

Abstract 1 - Figure

7 patients with hyperperfusion in TTP maps revealed a strong increase on ASL perfusion maps of CBF as shown below.

The figure illustrates a patient with an acute stroke in the right MCA territory according to DWI and stronger flow signal of the affected MCA on TOF-MRA, indicating vessel recanalization (a). TTP maps show an area of lower signal which reflects earlier contrast arrival in the ischemic tissue (b). This is displayed as an area of strong $\mathrm{CBF}$ increase in the ASL perfusion (c: grey scale CBF; d: colour-coded overlay of CBF and BAT).

Discussion: ASL MRI is sensitive to deviations from normal perfusion, both hypoand hyperperfusion as demonstrated in this study. It will serve in the characterisation of the pathophysiology and fine detail of cerebral ischemia and has the potential to extend current concepts.

\section{Brain imaging - new developments}

\section{STUDIES OF ACUTE ISCHEMIC STROKE WITH PERFUSION-WEIGHTED IMAGING}

Y.M. Zhang, Y.L. Wang, X.P. Gong, Y.J. Wang

Beijing Tiantan Hospital, affiliated with Capital University of Medical Sciences, China

Background: Perfusion-weighted imaging (PWI) can be used to study blood flow abnormalities in the brains of stroke patients. We have used it to examine the perfuse changes of damaged areas and evaluated cerebral blood flow whether or not can predict lesion growth in acute stroke patients.

Methods: Between February 2004 and December 2005, 120 in-patients in the Neurology department of Beijing Tiantan Hospital, who had been diagnosed with cerebral ischemic by MRI and 48 hours of symptom onset were selected for this study. We measured the volume of damaged areas and sought to measure the temporal evolution and spatial distribution of lesion blood flow, that is regional blood volume (rCBV) and regional cerebral blood flow (rCBF), mean transit time (MTT), and time to peak (TTP) of damaged areas. The results were compared with that of the contralateral hemisphere. After 10 days, we studied all patients by MRI and measured the volume of damaged areas again.

Results: Of 120 cases, there were 78 male and 42 female. The average was $62.9 \pm 4.23$ years. The results of PWI showed that there were statistically significant differences in the amount of $\mathrm{rCBF}, \mathrm{rCBV}$ and MTT between damaged areas and the mirror hemisphere $(\mathrm{P}=0.028, \mathrm{P}=0.012$ and $\mathrm{P}=0.010$, respectively), but there were no significant differences in TTP $(\mathrm{P}=0.534)$. Reduced $\mathrm{rCBF}$ and $\mathrm{rCBV}$ in the infarct within the first 48 hours was related to the lesion growth.

Discussions: The damaged areas of acute stroke patients exhibit hypoperfusion, the reduction in $\mathrm{rCBF}$ and $\mathrm{rCBV}$ in a damaged area was related to infarct extent, that is to say, cerebral blood flow changes can predict lesion growth in acute stroke patients.

\section{Brain imaging - new developments}

\section{MAGNETIC RESONANCE SPECTROSCOPY AND COGNITION IN} CEREBRAL SMALL VESSEL DISEASE

A. Nitkunan, R.A. Charlton, T.R. Barrick, D.J.O. McIntyre, Y. Shen, F.A. Howe, H.S. Markus

Centre for Clinical Neuroscience, St George's, University of London, London, United Kingdom

Background: Cerebral small vessel disease (SVD) is an important cause of cognitive impairment and dementia but the pathophysiological mechanisms remains unclear. We characterized brain metabolic differences between SVD patients and controls using magnetic resonance spectroscopy and correlated this with neuropsychology. Methods: 35 patients with SVD (lacunar stroke and radiological leukoaraiosis) and 35 controls underwent neuropsychology tests and chemical shift imaging (CSI) of white matter. Differences in the metabolite ratios for $\mathrm{N}$-acetylaspartate (NAA - a neuronal marker), creatine (Cr), choline (Cho) and myo-inositol (mI) were determined. In patients, cognitive scores were correlated with the ratios. A subset of 20 subjects underwent single voxel imaging to obtain absolute metabolite concentrations. The lesion load of each individual voxel was calculated.

Results: On CSI, NAA/Cr and NAA/Cho were significantly reduced in patients compared to controls (lower by $9.14 \%\left(\mathrm{p}=6 \times 10^{-5}\right)$ and $6.64 \%(\mathrm{p}=0.021)$ respectively). Single voxel analysis demonstrated significant absolute reductions in NAA choline and creatine in SVD patients. However, when lesion load (mean 32\% in SVD v $2 \%$ in controls, $\mathrm{p}=1 \times 10^{-15}$ ) was added as a co-variate, these differences were no longer significant, suggesting that the metabolite differences arose primarily from differences in lesioned regions. Neuropsychology did not correlate with CSI metabolite ratios.

Discussion: The principal change in SVD is a reduction in NAA - a marker of neuronal integrity. The lack of correlation with cognition does not support the use of MRS as a surrogate disease marker.

\section{Brain imaging - new developments}

\section{ISCHEMIC LESION RECURRENCE IN RELATION TO ARTERIAL} TERRITORY, TIME AND INITIAL VOLUME

M. Pavlovic, L. MacGregor, L.E. Allport, J. Prosser, P. Hand, B. Tress, P. Desmond, S. Davis

Royal Melbourne Hospital, Parkville, VIC, Australia

Background and Aims: One third of stroke patients demonstrate recurrent ischemic lesions (RIL) on diffusion-weighted (DWI) images in the first 90 days. However, little is known about recurrence related to vascular territory and initial lesion volumes. RIL within the initial perfusion deficit in the first days after stroke may reflect evolution of the initial ischemic lesion, rather than true recurrence. In the Oxfordshire study, smaller infarcts were associated with higher recurrence rates. We aimed to evaluate predictive factors for lesion recurrence with regard to time, vascular territory and initial lesion volume.

Methods: We enrolled 101 patients with anterior circulation ischemic stroke. Serial MRI scanning with both DWI and perfusion imaging was performed on Day 1, Days 3-5 (early RIL), and Day 90 (late RIL). Early RIL were restricted to new ischemic lesions outside the initial DWI lesion, while late RIL were new lesions occurring at any location. Multiple logistic regression analysis was used to evaluate the relationship between stroke pathogenesis, multiple acute lesions, age, initial DWI lesion volume and RIL.

Results: RIL were detected in 37/101 (36.6\%) patients. Early RIL were found in $25 / 101(24.8 \%)$ and late in $14 / 76(18.4 \%)$ patients. Two patients $(2.0 \%)$ had both early and late RIL. For early RIL, only the presence of multiple acute lesions on initial MRI scans was predictive. For combined early and late RIL, independent predictors included age (OR 1.04; 95\% CI 0.99-1.08; $\mathrm{p}=0.08$ ) and smaller baseline DWI volume (OR 0.64; 95\%CI 0.44-0.94; $\mathrm{p}=0.028$ ).

Discussion and Conclusions: RIL are common after acute ischemic stroke. Multiple acute lesions are associated with early recurrence. Smaller lesion volume and age are both associated with the overall risk of recurrent lesions. The association between lesion recurrence and smaller initial DWI volume is a novel finding and appears to define a higher risk group. 


\section{Brain imaging - new developments}

FUNCTIONAL MRI WITH PASSIVE MOVEMENT TASK OBTAINED BY NEUROMUSCULAR ELECTROSTIMULATION

R.M. Umarova, M.M. Tanashyan, L.A. Chernikova, M.V. Krotenkova

Institute of Neurology, Russian Federation

Background: Functional MRI (fMRI) examination is often impossible in acute stroke because of paresis or patient's neglect. Assessment of brain function at acute stroke is important for understanding mechanisms of neuroplasicity. Purpose of our study was to find the way for cortex reorganization assessment at acute stroke patients by using of neuromuscular electrostimulation (NES) as a passive task.

Methods: We examined 6 acute stroke patients. They were examined by MRI including fMRI at 29, 48 hours, 3, 5 days after stroke onset; two patients had the second fMRI in 10 days. At first as motor paradigm for fMRI we used the active wrist extension movement task, then the passive movement task which was obtained by neuromuscular electrostimulation (NES) of paretic wrist and fingers extensors. 5 healthy persons were examined by this way too (control group).

Results: While using active movements as motor task there were a lot of activation areas both the contra- and ipsilateral hemisphere. The attempts of active movements in acute stroke patients were accompanied by synergies. So the interpretation of the fMRI with active movements was impossible. NES paradigm revealed activation only in contralateral hemisphere at sensomotor area in patients with subcortical lesion and periinfarct area in cortical lesion. The analysis of dynamic fMRI revealed the reduction of activation areas quantity and size at active motor paradigm and passive movements obtained by NES; this fMRI changes were accompanied by motor recovery.

Discussion: The NES during fMRI can be used as a tool for precision localization of stimulated movements at acute stroke patients and cortex reorganization assessment.

\section{Management and economics}

\section{Management and economics}

THE COST OF STROKE IN POLAND FROM SOCIETAL PERSPECTIVE PRELIMINARY DATA FROM COST OF STROKE IN POLAND STUDY M.P. Niewada, I. Skibicka, B. Kamicski, A. Czlonkowki, A. Czlonkowska Warsaw Medical University, Warsaw, Poland

Background: To estimate total, direct and indirect, costs associated with stroke in Poland from societal perspective.

Methods: Study design: The prospective, bottom-up, naturalistic, multicenter cost of illness study. Patients: Primary consecutive stroke patients admitted to three hospital centres of different institution were followed for $2 \mathrm{yrs}$. Data on medical and non-medical resource use and patients clinical characteristics were collected using specially designed questionnaires. Unit costs for all resources used were estimated using participating centres accounting systems for initial hospitalisation costs, tariffs list for medical services founded by National Health Insurance, retail drug prices, minimal work payments for informal care and information on out-of-pocket patients' expenses. To quantify the indirect costs, the human capital approach was used. Values are expressed in $\mathrm{zl}(1 \mathrm{USD}=1,95 \mathrm{zl}$; 1 Euro=2,32 purchasing power parities).

Results: One hundred sixty four patients were enrolled (mean age:70; man: $41 \%$ ) Strokes were diagnosed as ischaemic in $85,8 \%$, hemorrhagic in $8,02 \%$ and unclassified in $6,17 \%$. During two year of follow-up 41 patients died. Cost were highest within first 3 month following initial hospitalisation, mainly because of inpatients rehabilitation costs as 33 patients were transferred to rehabilitation unit. Total direct two years costs amounted to $20554 \mathrm{zl}$. The costs structure encompassed: initial hospital stay $-10,86 \%$; subsequent hospitalisation costs $-22,37 \%$; nursing costs $28,91 \%$; rehabilitation costs $-16,94 \%$; drug costs $-14,54 \%$; diagnostic tests costs $-3,58 \%$; consultations costs $-2,11 \%$; medical aids and equipment costs $-0,69 \%$. Indirect costs were estimated at $13060 \mathrm{zł}$ per patients per two years.

Conclusions: Assuming the standardised incidence stroke of 177 per 100 thousand general population, the mean annual cost of stroke in Poland amounts to 675,18 million zł, i.e. $2,1 \%$ of total healthcare expenditures.
2 Management and economics

WHAT FACTORS AFFECT EARLY ADMISSION OF ACUTE STROKE PATIENTS?

A.R.F. Sztajzel, B.A.C. Griesser, C.L. Sekoranja, D.P. Temperli, E.F. Herrmann, F.F. Sarasin

University Hospital University, Geneva, Switzerland

Background: Despite the evidence that early admission to hospital followed by a rapid administration of thrombolytic therapy improves prognosis of acute stroke patients, only a minority of patients present at the emergency department (ED) within the accepted delay ( $<3$ hours).

Purpose: To determine the different factors of the pre-hospital process which affect early admission.

Patients and Methods: Time from onset of symptoms until arrival at the ED was evaluated in every stroke patient during a period of 6 months. The ability of the patients to recognize their symptoms as a stroke was also assessed.

Results: 329 patients were included in the study. The median and mean delays between onset of symptoms and time of arrival at the hospital were respectively $3 \mathrm{~h} 20$ min and $22 \mathrm{~h} 15 \mathrm{~min}$. When the transport was performed either by a "cardiomobile" type or by a normal ambulance, admission at the hospital was respectively 2.4 and 1.8 times quicker than the transportation done by the patient or family $(\mathrm{p}<0.0005)$. Recognition of the symptoms as a stroke $(\mathrm{p}<0.0005)$ and the severity $(\mathrm{NIH}>5)$ $(\mathrm{p}<0.002)$ also reduced the delay. Furthermore, the delay was also increased when the patient contacted his family physician $(\mathrm{p}<0.004)$.

Conclusions: Our results demonstrate the importance of educating the population and the different stakeholders of stroke care provision of the pre-hospital process, in order to improve the management and treatment of acute stroke patients.

\section{Management and economics}

HOW OFTEN DO PATIENTS DRIVE TO A TIA CLINIC?

M.O. McCarron, M.P. McNicholl

Altnagelvin Hospital, Derry, United Kingdom

Background: In the UK certain medical standards have to be attained for an individual to hold a driving licence. After a single transient ischaemic attack (TIA) or minor stroke the Driving and Vehicle Licensing Authority (DVLA) recommends a group 1 driving restriction for one month. We sought to determine how often the DVLA guidelines were applied following a TIA or minor stroke in patients prior to attending a TIA clinic.

Methods: Patients were recruited prospectively at a TIA clinic over a four month period. Age, sex, diagnosis and frequency of previous driving, recall of driving advice from referring doctor and whether the patient had driven to the clinic were recorded.

Results: Fifty two new patients (25 men and 27 women, mean age $58.3 \pm$ SD 14.6 years) were studied. Twenty-seven $(50 \%)$ were previous drivers. Sixteen $(31 \%)$ drove a car to the clinic. Seven $(13 \%)$ recalled driving advice from their referring doctor. Among 23 patients (44\% of total group) with a confirmed diagnosis of TIA or stroke, $15(65 \%)$ were previous drivers and six $(26 \%)$ drove a car to the clinic. Four patients $(17 \%)$ recalled previous driving advice, but none of these 4 patients had driven to the clinic.

Conclusions: Doctors referring patients to TIA clinics need to recognise their medicolegal obligation to inform such patients of the DVLA driving restriction.

\section{Management and economics}

GENDER-RELATED DIFFERENCES IN DIAGNOSTIC EVALUATION OF ISCHEMIC STROKE IN POLAND

W. Turaj, A. Slowik, M. Wnuk, I. Wietrzykowska, A. Szczudlik

Jagiellonian University College of Medicine, Krakow, Poland

Background: Gender-related differences in management of stroke were reported, but remain unexplained. Both biological and cultural factors may influence these findings, it is therefore important to search for them in various populations. The aim of the study was to compare the diagnostic evaluation of stroke between men and women with ischemic and undetermined stroke in large cohort of Polish patients.

Methods: We used the data of Cracow Stroke Registry (1999-2000) to compare clinical characteristics and diagnostic procedures [computed tomography (CT), carotid ultrasonography and echocardiography] in 938 subjects treated in hospitals of Cracow with the diagnosis of ischemic stroke $(n=532)$ or undetermined stroke $(\mathrm{n}=406)$.

Results: Women $(\mathrm{n}=477)$ were older than men $(\mathrm{n}=461)(74.4 \pm 11.1$ vs. $68.3 \pm 11.0$ years, $\mathrm{p}<0.001)$ and had atrial fibrillation more frequently $(30.0 \%$ vs. $17.6 \%$, 
$\mathrm{p}<0.001)$. Men were smokers more likely $(23.9 \%$ vs. $6.3 \%, \mathrm{p}<0.001)$ and more frequently had a history of myocardial infarction $(20.6 \%$ vs. $10.1 \%, \mathrm{p}<0.001)$ Neurological deficit on admission was greater in women [median SSS score: 36 $(20-47)$ vs. $42(29-47), \mathrm{p}<0.001]$. All major diagnostic procedures essential for the diagnosis of ischemic stroke were performed more often in men (CT: $65.5 \%$ vs. $53.6 \%$, carotid ultrasonography: $32.8 \%$ vs. $21.2 \%$, echocardiography: $18.4 \%$ vs. $12.2 \% ; \mathrm{p}<0.01$ for all differences). Regression models revealed that treatment in particular departments, younger age and smaller neurological deficit on admission, but not gender, were independently associated with having those diagnostic procedures performed.

Conclusions: Women are less likely to receive adequate evaluation of stroke Gender, however, does not seem to influence the performance of these studies as an independent factor.

\section{Management and economics}

\section{QUALITY OF CARE AND MEDICAL COST IN ATHEROSCLEROSIS} RELATED CO-MORBIDITY OF DEMENTIA PATIENTS

L. Chung-Hsiang, C. Chang-Ling, L. Tsai-Chung, C. Pai-Yi, T. Chon-Haw,

L. Cheng-Chieh

China Medical University Hospital, Taichung, Taiwan, ROC

Background: Many elderly patients suffer from co-morbidity. Due to the increasing medical care for the co-morbidity, healthcare insurance expenditure is expected to rise significantly. In this study we investigate the atherosclerosis related co-morbidity in patients with dementia in a medical center and the quality and cost of their care. Methods: Patients with dementia from a medical center were studied. The patients were selected between 1,1,2001 and 12,31, 2004 who were registered in the memory clinic. A total of 202 dementia patients who received more than twice cognitive evaluation 6 months later were identified in this study.

Results: Among 202 patients that we examined, 19\% had diabetes mellitus, $49 \%$ had hypertension, $15 \%$ had dyslipidemia, and $16 \%$ had cardiovascular diseases. $40 \%$ had cerebrovascular diseases. Among 39 patients with DM, 56\% had HbAlc below $8.25 \%$ with hypertension had SBP below 140 and $44 \%$ had DBP below 90. $26 \%$ with dyslipidemia had LDL below 130. The stepwise regression analysis $(\mathrm{R} 2=22.2 \%)$ indicated that factors affecting the variation of the medical cost is the patients had dyslipidemia or cardiovascular diseases. Under the control of other factors, the medical cost for dementia patients with dyslipidemia is $€ 12,683$ $(\mathrm{p}=0.023)$ higher than that for those without dyslipidemia. The medical cost for dementia patients with cardiovascular diseases is $€ 10,196.1(\mathrm{p}=0.025)$ higher than that for those without cardiovascular diseases.

Conclusions: The results showed that $78 \%$ of 202 dementia patients had at least one chronic disease and $40 \%$ had at least two. $44 \%$ had HbAlc below the therapeutic target. $75 \%$ had systolic pressure below the therapeutic target. 74\% had LDL below the therapeutic target. This indicates that atherosclerosis related co-morbidity of the dementia patients was not properly managed. Dementia patients with dyslipidemia or with cardiovascular diseases require more medical cost.

\section{Management and economics}

LONG-TERM DISABILITY AND RESOURCE USE AFTER STROKE - A FOLLOW-UP ANALYSIS FROM THE SOUTH LONDON STROKE REGISTER M.A. Busch, N. Fudge, C. Coshall, I. Burger, A. Rudd, C. McKevitt, C. Wolfe King's College London, Divison of Health and Social Care Research, London, United Kingdom

Background: Many stroke survivors suffer from long-term disability and require continuous support and input from health and social services. To guide allocation of resources and planning of services, information about patient needs and current resource provision is crucial. This study aimed to assess the need for and provision of resources and services in the long term after stroke.

Methods: A prospective population-based register of all incident strokes with annua follow-up in a multi-ethnic population of 234 533. Data from all patients registered between 1995 and 2003 were used. Outcomes were disability; institutionalised care; use of formal or informal domiciliary care, community support services, and community rehabilitation. Multivariable analysis was used to examine associations between patient characteristics and resource use.

Results: Over 9 years, 2563 patients with incident stroke were registered (median age 73.1 years (range 15-106), 49.2\% men, $17.8 \%$ black ethnicity, $54.9 \%$ manual occupational class). At 1, 3 and 5 years after their stroke, $26 \%, 35.6 \%$, and $23.8 \%$ of interviewed survivors had at least moderate (Barthel Index $<15$ ) and 12.9\%, 20\% and $10.9 \%$ severe $(<10)$ disability, respectively; $22.9 \%, 11.7 \%$ and $10.4 \%$ received community rehabilitation, with physiotherapy being the most common type; $12.7 \%$, $15 \%$ and $14.9 \%$ were living in institutionalised care. At 3 and 5 years post stroke,
$76.8 \%$ and $79.7 \%$ of those living at home had some support from informal, $24.1 \%$ and $21.8 \%$ from formal carers for everyday activities; $16.9 \%$ and $18.7 \%$ received community services such as meals on wheels or day centre care.

Conclusions: Stroke survivors in south London had high levels of persisting disability and support with everyday activities. Resource use remained comparatively stable after the first year post stroke except for community rehabilitation.

\section{Management and economics}

COMPONENTS OF STROKE CARE COSTS IN GERMANY

Y. Epifanov, R. Dodel, C. Haacke, M. Schaeg, M.G. Hennerici, T. Back Klinikum Mannheim, University of Heidelberg, Mannheim, Germany

Objective: Advances in stroke treatment possibly increase costs of inpatient care However, data on costs of stroke care are scarce. This study analyzed in great detail cost components for acute and postacute stroke care in Germany.

Methods and Patients: A total of 253 consecutive inpatients admitted on regular wards in the Dept. of Neurology, Marburg, between January 1 and June 30, 1998, with the diagnosis of ischemic stroke (IS), intracerebral hemorrhage (ICH) or transient ischemic attack (TIA) were included in the study. Acute care costs were calculated by means of a detailled "bottom-up" approach in prices of 2002 (Euro) for a variety of cost omponents (i.e., nursing care, physician charges, lab investigations etc). Costs for postacute inpatient treatment were estimated on the basis of daily hospital charges. Results were compared to those obtained for a stroke unit setting in a similar previous study.

Results: Cost of acute stroke care on regular wards was $€ 3270 \pm 2570$ (mean \pm SD) per patient. Mean daily costs were $€ 350 \pm 180$ for $\mathrm{ICH}, € 320 \pm 80$ for IS, and $€ 290 \pm 50$ for TIA $(\mathrm{p}<0.05)$. Resource use declined exponentially during the initial treatment days. Treatment of stroke patients with atrial fibrillation (AF) was approximately $32 \%$ more costly compared to non-AF patients $(p<0.05)$. In comparison to regular neurological wards, treatment on a stroke unit increased mean costs per patient by $7.0 \%$, mean costs per day by $16 \%$. The cost of postacute inpatient rehabilitation was $€ 10530 \pm 9120$ per patient $(6.5 \%$ higher compared to that following stroke unit care).

Conclusion: In patients who need inpatient rehabilitation, acute stroke care comprises $24 \%$ of total costs. In a stroke unit setting, acute care is more costly whereas poststroke treatment costs tend to decrease. Subgroups like AF patients need higher medical resources.

\section{Management and economics}

BETTER MEDICAL MANAGEMENT IN PATIENTS WITH PRIOR CAROTID ENDARTERECTOMY: RESULTS FROM THE REDUCTION OF ATHEROTHROMBOSIS FOR CONTINUED HEALTH REGISTRY

J.L. Mas, E. Touzé, M.J. Alberts, J. Röther, D.L. Bhatt, P.G. Steg, on behalf of the REACH Registry Investigators

Hôpital Saint-Anne, France

Background: The impact of a prior history of carotid endarterectomy (CE) on the quality of medical management in stroke/TIA patients is not well known. Methods: The REduction of Atherothrombosis for Continued Health (REACH) Registry enrolled 68,236 outpatients with documented atherothrombotic disease from 44 countries all over the world, including 18,450 patients with previous stroke and/or TIA.

Results: The Table shows the relationship between prior CE and medical management at baseline in stroke/TIA patients. The mean ages for the populations with and without prior $\mathrm{CE}$ are $71.5 \pm 9.3$ and $69.3 \pm 10.1(\mathrm{p}<0.0001)$ and the percentage male $68 \%$ and $59 \%(\mathrm{p}<0.0001)$, respectively.

Management of pts with and without $\mathrm{CE}$

\begin{tabular}{lccc}
\hline & With CE & Without CE & $p$ \\
& $\mathrm{~N}=1,474$ & $\mathrm{~N}=17,265$ & \\
\hline *Blood glucose, $<126 \mathrm{mg} / \mathrm{dL}, \%$ & 77 & 73 & 0.01 \\
Total cholest. $<200 \mathrm{mg} / \mathrm{dL}, \%$ & 53 & 65 & $<0.0001$ \\
$\mathrm{BP}<140 / 90 \mathrm{mmHg}, \%$ & 50 & 45 & 0.001 \\
**Smoking: F:C:N, \% & $56: 15: 29$ & $37: 14: 49$ & $<0.0001$ \\
$\geq 1$ AT/lipid-low. drug, \% & $96 / 78$ & $92 / 59$ & $<0.0001$ \\
\hline
\end{tabular}

*Fasting; ** F=Former, $\mathrm{C}=$ Current; $\mathrm{N}=$ Never

After adjustment for main risk factors and prior history of atherothrombotic disease in other territories, patients with prior $\mathrm{CE}$ were more likely to have blood pressure $<140 / 90 \mathrm{mmHg}(\mathrm{OR}=1.2 ; 95 \% \mathrm{CI}: 1.1-1.4)$ and cholesterol $<200 \mathrm{mg} / \mathrm{dL}(\mathrm{OR}=1.6$; 1.4-1.8) and to receive antithrombotic (AT) $(\mathrm{OR}=1.5 ; 1.1-2.0)$ and lipid-lowering $(\mathrm{OR}=1.8 ; 1.3-2.3)$ drugs. Results were similar when patients with prior history of 
surgery or endovascular treatment in other arterial territories, those with coronary artery disease or those with atrial fibrillation, were excluded.

Conclusion: Prior history of $\mathrm{CE}$ is associated with better medical management in stroke/TIA patients. Whether those differences can be explained by better patient compliance or better physician awareness remains to be investigated.

\section{Management and economics}

PROCESS INDICATORS FOR STROKE: GOOD OUTCOMES?

C. Sánchez, S. Lorenzo, J. Pardo, M. Barón, J.L. Dobato, L. Vela, A. Polo,

F.J. Barriga, J.A. Pareja

Fundacion Hospital Alcorcon, Alcorcon, Madrid, Spain

Background: Is important to stablish quality assessment for evaluating stroke care. The Joint Commission was defined some measures of process to evaluate this aspects $(*)$. We dont know his relationship with outcome indicators.

Objective: To compare process indicators and results indicators to evaluate stroke care in our hospital.

Methods: Retrospective study of clinical histories of consecutive stroke patients admitted from the comunity. We select five process indicators (Indicator 1 (IND1): discharged on antithrombotics; Indicator 2 (IND2): patients with atrial fibrillation receiving anticoagulation therapy; Indicator 3 (IND3): lipid profile; Indicator 4 (IND4): smoking cessation; Indicator 5 (IND5): plan of rehabilitation) and two results indicators (Indicador 6 (IND6): mortalitity in hospital; Indicator 7 (IND7): intrahospitalary complications. We calculated all of indicators in two periods: A (internal medicine'patients) and B (neurology 'patients). The indicators was refered at admission period.

Results: 129 patients with ischemic stroke were included (group A 79; group B 49). Mean age (A 75.06+14.05; B 73.84+13.48). The risk factor was similar in both groups. IND1 A:93\% B:73\%; IND2 A:43\% B:57\%; IND3 A: 80\% B:80\%; IND4 A:55\% B.0\%; IND5 A:29\% B:38\%; IND6 A:11\% B:8,2\%; IND7 A:26\% B:14\%. Only higer \% in anticoagulation in atrial fibrillation and rehabilitation is associated with decrease in mortality and complications in the hospital.

Conclusions: Our data suggest that good process indicators are not always related with good outcome at discharge. There are necessary news studies to clarified this question.

(*)www.jcaho.org/dscc/psc/guide.htm

\section{Management and economics}

\section{GEOGRAPHICAL VARIATION IN THE USE OF MODIFIED RELEASE DIPYRIDAMOLE IN ENGLAND \\ L.C. Bamber, R.J. Das-Gupta \\ Boehringer Ingelheim Ltd., United Kingdom}

Introduction: In May 2005, the National Institute for Health and Clinical Excellence (NICE) issued guidance that apply to practice in England and Wales that people who have had an ischaemic stroke or a TIA should be treated with a combination of modified-release (MR) dipyridamole and aspirin for 2 years except where they are intolerant of either aspirin or dipyridamole. In the light of these recommendations, this study aimed to estimate the extent to which practice followed this guidance at the time of issue by estimating for strategic health authorities (SHAs) in England the percentage of eligible patients receiving MR dipyridamole. In England there are 28 SHAs covering populations of 1.2-2.8 million. They provide the lead on the strategic development of the local health service and manage the performance of Primary care Trusts (PCTs) and NHS trusts against locally agreed targets.

Methods: The age and sex incidence rates for first stroke from the OXVASC study and information on recurrent stroke and TIA incidence from the OCSP were used to estimate an overall annual incidence rate for each SHA. Patient mean time on therapy was calculated from the ESPS-II trial. Available data on prescribing of MR dipyridamole from IMS was then benchmarked against NICE recommendations.

Results: The estimated uptake against NICE recommendations of MR dipyridamole for stroke/TIA patients by SHA ranges from $24 \%$ to $80 \%$ with an average for England of $47 \%$.

Conclusion: The results suggest a substantial geographical variation in the choice of antiplatelet treatment used to prevent recurrent stroke/TIA. It will be of interest to determine whether the issuing of guidance by NICE will reduce the level of divergence in treatment patterns given that one of the objectives of the Institute is to reduce the level of "post code prescribing". The results presented are part of an ongoing study to monitor the impact over time of this NICE guidance on geographical prescribing patterns.

\section{Management and economics}

EUROPEAN REGISTERS OF STROKE (EROS) PROJECT: PERCEPTIONS OF WHY LEVELS OF CARE VARY ACROSS EUROPE

I. Wellwood, P. Langhorne, I. Sayed, I. Burger, C. Wolfe

University of Glasgow, King's College London, United Kingdom

Background: Stroke services vary considerably across Europe. The EROS project is developing a tool to assess the quality of stroke care allowing us to better determine differences between countries. We also want to explore perceived barriers to providing "appropriate" care as defined by international guidelines and perceived reasons for variation in care.

Methods: Healthcare professionals from various backgrounds were selected in convenience samples from 7 European countries. We identified key elements of structure and planning of stroke care - what centres considered "appropriate" care, from published European and National stroke guidelines, and used these along with qualitative data from visits to the EROS sites, to determine the areas to be examined. Using a short questionnaire, subjects identified "barriers" to providing quality care and "incentives" to improve care in relation to key areas e.g. prevention, acute care and rehabilitation. They were asked to identify "good" points of their service and "areas for improvement". We were able to contrast these perceptions with data on actual service provision based on the stroke registers.

Results: Interim results show opinions and perceptions on "barriers" and "incentives" vary e.g. lack of qualified staff and funds. Many healthcare professionals perceived diagnostic procedures and acute care to be acceptable or good. Responses to questions on areas to be improved range from increasing family and carer support, to increasing availability of rehabilitation to introducing a thrombolysis service. The proportion of patients managed in a stroke unit varied from $0-85 \%$.

Conclusion: It is interesting to relate perceptions of care to actual provision and areas that could be targeted for development. Stroke unit care is far from universal, yet acute care was often perceived as adequate or good. The concerns and suggestions identified by the work, though limited, allow these themes to be developed in future research

\section{Management and economics}

\section{VARIATIONS AND PREDICTORS OF SURVIVAL IN STROKE PATIENTS ADMITTED TO SIX HOSPITALS IN CATALONIA AND THE BALEARIC ISLANDS, SPAIN}

O. Groene, D. Enric, A. Jordi, V. Montse

Health policies, Systems and Services, Barcelona, Spain

Background and Purpose: Previous studies reported significant variations in management and survival after stroke comparing hospitals between and within countries. We investigated if similar variations could be detected comparing hospitals in two geographically defined regions.

Methods: We established hospital-based stroke registers to identify first in a lifetime strokes between 1998 and 2003. Data collected included socio-demographic data, clinical and in-hospital resource use at the time of stroke and data on survival We used Kaplan Meier analysis and Cox proportional hazards regression models to compare unadjusted and adjusted survival at discharge, three and twelve months between hospitals.

Results: We registered 804 stroke patients and observed significant differences in case-mix and resource use. We detected significant differences in survival at discharge, three and twelve months between the six hospitals; however, part of these differences disappeared after adjusting for case-mix. After adjusting for case-mix and resource use, two of the six hospitals performed significantly worse then the reference hospital. At discharge, hospital 6 performed significantly worse (RR 3.26; CI 95 1.40-7.56); at three months, hospital 4 performed significantly worse (RR 1.88; CI 95 1.08-3.28) and the elevated risk of death in hospital 6 had become insignificant. At twelve months, hospital 4 remains significantly worse (RR 1.70; CI 95: 1.04-2.80)

Conclusions: We were able to identify variations in survival between six hospitals in two similar regions in Spain. The data suggests the difficulties that exist in identifying variations in outcome, as significant differences at one point in time may disappear compared to another point in time. 
VALIDITY OF THE APPROPRIATENESS EVALUATION PROTOCOL IN STROKE PATIENTS

J.M. Pons, A. Romero, A. Navarré, J. Juni, J. Sancho

Consorcio Hospital General Universitario de Valencia, Valencia, Spain

Background: Stroke importance makes necessary the development of strategies to optimize the hospital use. One of them could be the ambulatory attendance of patients who do not require hospital cares but a process fast diagnosis. The Appropriateness Evaluation Protocol (AEP) could help us to select those patients. The aim is to asses the inter-observer reliability and validity in respect of clinical appraisal given by AEP in Stroke patients.

Methods: prospective, descriptive study, A total of 117 admissions to Unit Stroke with definitive Stroke diagnosis were selected. The Spanish validated version of the AEP was used. Hospital Virgen del Rocío de Sevilla Stroke Unit's admission protocol (Ed. 2004) was used for clinical appraisal.

Results: The study shows a high degree of intra and inter-observer reliability (specific agreement $>100 \%$ and $98 \%$, Kappa $>1$ and 0.95 ) and a reasonable validity in comparison with clinical judgment (specific agreement $>84 \%$, Kappa $>0.57$ ). The AEP considered 31 inappropriate cases $(26,5 \%)$ as opposed to 26 cases of clinical appraisal $(22,2 \%)$ without significant differences among them $(\mathrm{P}>0,05)$. The AEP revealed a high degree of specify $(87 \%)$ and a moderate degree of sensitivity $(73 \%)$ in comparison with clinical judgment. Thus minimising the occurrence of false results when the stay was regarded as appropriated.

Discussion: The results showing high reliability and moderate validity regarding clinical assessment shows the AEP to be a useful tool in the sifting-out of inappropriate use of hospitalisation in Stroke patients. They could be studied in an inferior welfare level, as a specific external neurovascular consult. In spite of it, its design does not allow to select case by case.

\section{Management and economics}

DEVELOPMENT OF A STROKE SYSTEM IN COLORADO, USA: THE ROLE OF STROKE NEUROLOGISTS

R.L. Hughes, D.B. Smith

Denver Health Medical Center, Denver, CO, USA

Recent advances in the care of stroke victims require rapid response from the field into the hospital, advanced technologies and increasingly higher levels of specialization. Development of an adequate system requires coordination of local, state, regional and national organizations to achieve this goal in vastly diverse areas of the United States.

In Colorado, the emergency ambulance providers are separate private entities, rather than governmental, requiring the Colorado Department of Public Heath and Environment (CDPHE) to impliment ambulance routing. This required legislation (2002) and funding (2005) to create an initial data base to prepare the CDPHE to oversee a state Stroke System. A Stroke Advisory Committee has been created composed of CDPHE staff and professionals from around the state.

Nationally, Primary Stroke Center (PSC) began certification in 2004 in conjunction with the American Stroke Association (ASA) and the Joint Commission for Accreditation of Hospitals and Organizations (JCAHO). Comprehensive Stroke Centers (CSC) are expected to begin certification in two years, creating three hospital categories: CSC, PSC and uncertified hospitals. Stroke Neurologists began certification as Vascular Neurologists in 2005.

The CDPHE and the ASA/JCAHO initiatives must be coordinated with public and private hospitals, including existing hospital systems, and other less formal arrangements for transfer and referral.

To effectively develop a working statewide stroke system, the Colorado Vascular Neurologists who direct hospital or hospital system stroke programs must be involved at all levels. They must see that their institutions have sufficient lead time to prepare to be a CSC with the proper infrastructure and staff. Colorado Vascular Neurologist involvement in the national process further improves the ability of the CDPHE and Colorado CSC's to be prepared. This will help develop national initiatives which can be better implemented in large western states, such as Colorado.

\section{Meta-analysis and review papers}

\section{Meta-analysis and review papers}

\section{STROKE UNITS IN THEIR NATURAL HABITAT: A SYSTEMATIC REVIEW OF OBSERVATIONAL STUDIES OF ROUTINE STROKE UNIT CARE}

P. Seenan, P. Langhorne, M. Long

University of Glasgow, Glasgow, United Kingdom

Background: Systematic reviews have shown that, within clinical trials, stroke patients receiving organised (stroke unit) care are more likely to survive, regain independence and return home. However, there are concerns that the benefits seen in clinical trials may not be replicated in routine practice. We therefore carried out a systematic review of observational studies of stroke unit implementation.

Methods: We searched Medline, Embase, Cinahl, DARE, British Nursing Index, Cochrane Stroke Group register and recent conference abstracts for observational studies that compared outcomes of stroke patients managed in a stroke unit versus non stroke unit care. We excluded studies that did not describe matching for baseline prognostic factors or casemix adjustment. Two independent reviewers screened abstracts for selection and data extraction.

Data analysis used the Generic Inverse Variance method in Revman 4.2. Where raw data were provided, effect size and variance were calculated accordingly. We used a random effects model, exploring for sources of heterogeneity.

Results: We identified 73 papers on stroke unit outcomes; 24 were eligible for review, 14 provided data on case fatality or poor outcome (death/institutionalisation or death/dependency). Stroke unit care was associated with a significantly reduced odds of death within one year (odds ratio $0.79 ; 95 \%$ confidence interval 0.72 $0.87 ; \mathrm{p}<0.00001)$, and of poor outcome $(0.88 ; 0.80-0.97 ; \mathrm{p}=0.008)$. Results were complicated by significant heterogeneity $(\mathrm{p}<0.05)$, mainly in single centre studies. Discussion: These results are complicated by heterogeneity and potential bias However, the observed benefit of stroke unit care in routine practice is comparable to that in clinical trials.

\section{Meta-analysis and review papers}

META-ANALYSIS OF STROKE LIAISON WORKERS FOR PATIENTS AND CARERS: RESULTS BY INTERVENTION CHARACTERISTIC

G. Ellis, on behalf of the Stroke Liaison Workers Collaboration

Glasgow Royal Infirmary, Glasgow, United Kingdom

Background: The majority of people survive their stroke, but a third to a half remain functionally dependent after one year. This is associated with significant psycho-social problems for both patients and their carers. A number of different approaches have been evaluated to try to lessen these problems. We present an individual patient data meta-analysis of Stroke Liaison Worker trials.

Methods: We searched for randomised controlled trials comparing allocation to a stroke liaison worker with normal care. We searched the Cochrane Central Register of Controlled Trials, MEDLINE, EMBASE, CINAHL, ASSIA, PsychINFO and Social Science Citation Index. Two researchers independently selected trials for inclusion. Original patient data was sought where possible from the trialists.

Results: We found 16 randomised controlled trials (4, 916 participants). Overall there was no benefit on the primary outcomes of subjective health status or extended activities of daily living. No significant effect was seen for the outcomes of death, activities of daily living or dependency. There was a non-significant trend towards a reduction in depression scores $(\mathrm{p}<0.1)$. Pre-planned subgroup analysis suggested that interventions that employed nurses as opposed to other health professionals resulted in a significant reduction in depression score $(\mathrm{p}=0.007)$. Patients did report satisfaction that they felt someone had really listened to them $(p=0.006)$. Similarly for carers there was no significant difference in carer subjective health status, extended activities of daily living or mental health. Carers did however report satisfaction that they were not neglected $(\mathrm{p}=0.002)$, that they had received adequate information about the causes of stroke $(\mathrm{p}=0.03)$ and recovery from stroke $(\mathrm{p}=0.004)$ and that someone had really listened $(\mathrm{p}=0.0004)$.

Conclusions: Stroke Liaison Workers result in greater patient and carer satisfaction with certain aspects of stroke services. Pre-planned subgroup analyses suggest differences in intervention characteristics that may result in a significant reduction in depression. 


\section{Meta-analysis and review papers}

DAN SHEN AGENTS IN ACUTE ISCHEMIC STROKE: A SYSTEMATIC REVIEW

B. Bo Wu, M. Ming Liu, S. Shihong Zhang

West China Hospital, Sichuan University, Chengdu, China

Background and Purpose: Based mainly on experimental data which indicates improvement to the cerebral microcirculation, Dan Shen agents are widely used in the treatment of acute ischemic stroke in China. The aim of our study was to assess the evidence from randomised or quasi randomised controlled trials of their effects. Methods: Trials of Dan Shen agents were identified from searches of Cochrane Library, MEDLINE, China Biological Medicine Database and others and communication with the pharmaceutical company manufacturing Dan Shen. 10 Chinese journals were also handsearched. The primary outcome measure is deaths or dependent patients at the end of follow-up of at least three months and adverse events.

Results: Five trials (429 patients) were included. Numbers of deaths or dependent patients at the end of follow-up of at least three months were not reported in all included trials. Only two trials reported adverse events. Five trials measured the outcome "significant improvement in neurological deficit at the end of treatment". Dan Shen agents were associated with a significant increase in the number of patients with the outcome (Peto Odds Ratio (OR) 3.01, 95\% Confidence Interval (CI) 1.60 to 5.66). No deaths were reported within the first two weeks of treatment or during the whole follow-up period (14 to 28 days). The trials did not include any assessment of quality of life.

Conclusions: There were too few patients and outcome events to draw reliable conclusions from the present data. The methodological qualities of all included studies were poor. Further high-quality randomised controlled trials should be performed.

\section{Meta-analysis and review papers}

MANNITOL FOR ACUTE STROKE - AN UPDATE OF A COCHRANE REVIEW D. Bereczki, M. Liu, G.F. do Prado, I. Fekete

University of Debrecen, Health Science and Medical Center, Debrecen, Hungary

Background: Mannitol, an osmotic agent and a free radical scavenger is widely used to decrease edema and tissue damage in stroke. We performed a systematic review to test whether mannitol given in the acute phase of stroke decreases case fatality and disability.

Methods: Unconfounded randomized controlled trials were identified by searching the Cochrane Stroke Group Specialized Trials Register. Two reviewers independently selected the trials to be included in the review and performed data extraction. RevMan 4.2.8. was used for data analysis.

Results: The search resulted in 16 potential trials for inclusion. Of these only 3 were truly randomized unconfounded trials, including 226 patients. One trial with 77 subjects randomized patients with presumed ischemic stroke without CT verification, and the other 2 trials included patients with CT proven nontraumatic intracerebral hemorrhage. All trials used IV mannitol less than $1 \mathrm{~g} / \mathrm{kg} / \mathrm{dose}$. Based on the limited data available, short and long term case fatality, and the proportion of dependent and dead or dependent patients at the end of the follow up were not significantly different in mannitol treated patients and in controls.

Conclusions: Based on 3 small randomized trials there is currently not enough evidence to decide whether the routine use of mannitol in acute stroke would result in any beneficial or harmful effect. Therefore the routine use of mannitol in all patients with acute stroke is not supported by any evidence from the existing clinical trials

\section{Meta-analysis and review papers}

OPTIMISING THE ANALYSIS OF STROKE PREVENTION TRIALS (OAST-P): PILOT ASSESSMENT USING ORDERED RATHER THAN DICHOTOMOUS OUTCOMES

P.M.W. Bath, L.J. Gray, C. Geeganage, T. Collier, S. Pocock

Institute of Neuroscience, University of Nottingham, United Kingdom

Background: Vascular prevention trials mostly count dichotomous events, e.g stroke/no stroke. Ordered categorical outcomes based on stroke outcome (e.g. fatal/non-fatal/no stroke) could be more powerful statistically.

Methods: Published summary data from positive antithrombotic, lipid or blood pressure lowering, or carotid endarterectomy vascular prevention trials were identified for a pilot study. The efficiency of nominal and ordinal analyses were compared using Friedman 2 way ANOVA with multiple comparison procedures.

Results: Many trials did not publish sufficient event data to create ordered stroke outcomes. Data from 37 trials (153,346 patients) were analysed for stroke as fatal/non-fatal/no stroke. The analyses differed $(\mathrm{p}<0.0001)$ and were ranked $(\mathrm{mos}$ efficient first); bootstrap, Mann Whitney U test, robust rank test, ordinal regression, Cochran-Armitage (Chi-square for ordered data, $2 \times 3$ ), t-test, Chi-square $2 \times 2$ on stroke/no stroke, Chi-square $2 \times 3$ (non-ordered data), Chi-square $2 \times 2$ on death/alive, median test. When comparing the tests, bootstrap and MWU were more efficient than the other tests. Similar findings were obtained for analyses of myocardial infarction (fatal/non-fatal/no event), and combined vascular events. 4 level (stroke: fatal/severe non-fatal/mild/no event) and 5 level (stroke: fatal/severe non-fatal $/ \mathrm{mild} / \mathrm{TIA} / \mathrm{no}$ event) analyses appear to be even more efficient

Conclusions: Vascular prevention trials should use 3 or higher level ordered outcomes in preference to dichotomous events since these give additional information on treatment effects by severity of stroke, and will permit more efficient ordinal analyses thereby potentially resulting in smaller trials and the calculation of smaller numbers-needed-to-treat.

\section{Meta-analysis and review papers}

QUALITY OF ABSTRACTS IN PAPERS REPORTING THE RESULTS OF RANDOMISED CONTROLLED TRIALS IN ACUTE STROKE

P.A. Rashid, P.M.W. Bath

University of Nottingham, Nottingham, United Kingdom

Introduction: The quality of full publications reporting the results of acute stroke trials is variable thereby complicating trial interpretation. We assessed the quality of abstracts in these trial publications.

Method: Abstracts of randomised controlled trials in acute stroke and published by the end of 2003 were identified from searches of the Cochrane Library, Embase and Pubmed. Abstract quality was scored out of 46 points ( 22 from the CONSORT criteria) relating to the introduction, methods (design, assignment, blinding, statistical analysis), results (demographics, risk factors, outcome) and discussion. Trial quality related to the presence of true randomisation, placebo controlled and blinded, analysis by intention to treat, and sample size.

Results: 158 abstracts were identified from trials recruiting 79,816 patients (median 91); $40 \%$ of abstracts came from Stroke. The median abstract quality was 15.5 (range 2-23 out of 46) and trial quality 5.5 (2.5-9.5 out of 13). Abstract quality was associated with year of publication (Spearman's rs $=0.55, \mathrm{p}<0.001$ ), sample size ( $r s=0.47, \mathrm{p}<0.001)$, number of recruiting centres $(\mathrm{rs}=0.59, \mathrm{p}<0.001$ ), journal impact factor $(\mathrm{rs}=0.25, \mathrm{p}=0.003)$, and trial quality $(\mathrm{rs}=0.44, \mathrm{p}<0.001)$. When adjusted for sample size (partial correlation), abstract quality remained associated with publication year $(\mathrm{rs}=0.58, \mathrm{p}<0.001)$.

Discussion: The quality of abstracts in trial publications is generally poor but appears to be improving with time, in part due to increasing trial size and perhaps the introduction of structured abstracts in the 1990s. Stroke abstracts should be as informative as possible addressing key trial methodological and results criteria since they may be read in isolation in abstracting services and during electronic searches.

\section{Meta-analysis and review papers}

WHAT IS THE ROLE OF SOCIO-ECONOMIC STATUS ON STROKE INCIDENCE AND OUTCOMES? A LITERATURE REVIEW

A.M. Cox, C. McKevitt, A.G. Rudd, C.D.A. Wolfe

King's College London, London, United Kingdom

Background: There are significant variations in the impact of stroke around the world measured by incidence, case severity,mortality and survival. Numerous studies have investigated the role socio-economic status (SES) plays in stroke incidence and outcomes using routine and research generated data. We conducted a critical review of current evidence of the relationship between SES and stroke.

Methods: We searched MEDLINE for articles published in English between 1966 -2005 using the terms Cerebrovascular accident, Cerebrovascular disorders, Cerebral Haemorrhage, Subarachnoid Haemorrhage, Socioeconomic Factors, Social Class, Income, Education, Poverty, Inequality, Deprivation. Studies were included if they contained data that quantified the relationship between any measure of socio-economic status and stroke.

Results: Articles reviewed included studies on risk factors, incidence, mortality and survival, severity and stroke management. The studies varied widely in scope, design and measures of SES. Evidence of an association is strongest for incidence and mortality with higher rates of stroke in lower socio-economic groups consistently found. Lower socio-economic groups also have lower survival rates and more severe strokes although there are fewer studies available and the strength of the association is less robust.The mechanisms through which SES affects stroke risk and outcomes are not clear but some studies report that SES differences in risk factor prevalence may account for some of the variation. 
Conclusions: Comparisons across studies were difficult due to the heterogeneity of the studies. Studies using prospective population-based methods with improved control for confounding factors are required to confirm or refute the associations between SES and stroke. Understanding the causal relationships between SES and stroke will allow interventions to be appropriately targeted and evaluated.

\section{Meta-analysis and review papers}

\section{IMPROVING THE DEVELOPMENT AND EVALUATION OF COMPLEX INTERVENTIONS IN STROKE PREVENTION AND MANAGEMENT - BEYOND GUESSWORK \\ J. Redfern, C. McKevitt, C. Wolfe \\ Kings College London, London, United Kingdom}

Background: Stroke care is complex, requiring input from professionals, patients and carers. Identifying the most appropriate interventions is difficult. The MRC Framework for Developing and Evaluating Complex (non-pharmacological) Interventions aims to improve quality in intervention development. This study uses the framework to review the quality of complex interventions in stroke prevention and management.

Methods: Systematic review with 3 search strategies. Inclusion criteria: complex interventions; focus on stroke; evaluation studies; targeting health professionals, patients, carers or the general population. Criteria to assess the quality of these studies were developed using the MRC Framework.

Results: 66 complex intervention studies were included: 39 RCTs and 27 quasiexperimental/observational studies. Interventions targeted: healthcare professionals (16); those appropriate for primary stroke prevention (15); and secondary stroke prevention (5); patients and carers, to improve adjustment and recovery after stroke (30). Primary and secondary prevention studies were significantly less likely to have been evaluated in RCTs compared to recovery studies. RCTs had less success in influencing primary outcomes $(23 \%)$ than non-RCTs $(41 \%)$. 21/39 RCTs used 3 or more primary outcome measures to evaluate success. Of these, only 5 reported a power calculation and only 1 study considered power for all measures. Five interventions were judged to be theoretically well developed; 17 were moderately well developed; 16 had minimal/no theoretical basis.

Discussion: Inadequate development may explain failures of complex interventions in stroke care. Future intervention development needs greater attention to theory in intervention design and methodological quality in evaluation.

\section{New clinical trials}

\section{New clinical trials}

\section{SAINT II: INFLUENCE OF SAINT I ON DESIGN AND ANALYSIS The SAINT Trials Investigators}

NXY-059 is a novel free-radical trapping neuroprotectant in development for acute ischaemic stroke (AIS). SAINT I was a double-blind, multicentre, placebocontrolled trial in 1722 randomised AIS patients that demonstrated significant reduction of global disability assessed by mRS in patients treated with NXY-059 compared with placebo. Secondary outcomes, eg change of NIHSS from baseline, were not statistically significant but haemorrhages were fewer in rt-PA treated patients who received NXY-059. Other lessons were that a larger sample size is necessary to confirm the effect on global disability and that the NIHSS analysis method was inappropriate. As the twin trial, SAINT II, is still recruiting, SAINT I lessons have now been incorporated. Selection criteria and dose are unaltered. Primary outcome remains modified Rankin scale (mRS) distribution at 90 days analysed by the Cochran-Mantel-Haenszel test $(\mathrm{CMH})$, supplemented by dichotomisations and earlier ratings. The principal secondary (co-primary) outcome is now final NIHSS score, analysed by CMH. The hierarchy of endpoints is unchanged: mRS $>$ NIHSS $>$ Barthel index $>$ SIS, EQ-5D. Safety \& tolerability assessments include mortality, adverse events and, in patients receiving rt-PA, secondary ICH. Sample size increases from 1700 to 3200 patients, to deliver $>80 \%$ power to reproduce SAINT I findings on mRS (a common odds ratio of 1.2). Even with 3200 patients, power to detect benefit on the NIHSS remains $<60 \%$. Formal analysis of secondary ICH in rt-PA treated patients is added. SAINT II will complete recruitment in 2006 and report in 2007 . The pooled 4900 subjects from the SAINT programme will provide an important resource for examination of treatment effects in subgroups and will enhance power for all endpoints.

\section{New clinical trials}

INITIAL CLINICAL SAFETY AND PERFORMANCE OF THE PENUMBRA STROKE SYSTEM: A NOVEL DEVICE FOR THE TREATMENT OF ACUTE STROKE DUE TO LARGE VESSEL OCCLUSIVE DISEASE

The Penumbra Stroke Trial Investigators

Purpose: Substantial evidence supports the premise that in patients with acute stroke due to large vessel occlusive disease, early and safe recanalization may improve clinical outcome. In vitro and in vivo pre clinical data as well as early clinical feasibility data suggests the Penumbra Stroke System may allow safe recanalization in patients experiencing ischemic stroke secondary to large-vessel occlusive disease. Materials and Methods: A prospective, single arm, multi-center international trial is underway to assess the safety and performance of the Penumbra Stroke System. Up to 30 patients will be treated who meet the inclusion criteria of a deficit measurable on the National Institute of Health Stroke Scale (NIHSS) presenting within 8 hours of stroke onset and an angiographic occlusion (TIMI grade 0 or 1) of a treatable vessel. The primary endpoint for the trial is incidence of recanalization of the target vessel (TIMI grade 2 or 3 ). Secondary endpoints include the proportion of patients who achieve a modified Rankin Score $(\mathrm{mRS})$ of $<2$, or a 4 point improvement on the NIHSS at 30 days post treatment, and all cause mortality.

Initial Results: To date 14 patients have been treated within this multi-center trial. The occluded target vessels were: internal carotid artery (4), middle cerebral artery M1 segment (6), and basilar artery (4). All patients have had successful recanalization of the target vessel with no serious device-related adverse events. Average patient age was 60 years (range 29 to 84 ), and average pre-procedure NIHSS score was 20 (range 2 to 30). Post procedure, 7 of 14 patients experienced at least a 4-point improvement on the NIHSS measured at 24 hours.

Conclusion: Early experience suggests that the Penumbra Stroke System may offer a possible new treatment option for patients with acute ischemic stroke secondary to large-vessel occlusive disease.

\section{Acute stroke: clinical patterns and practise}

\section{Acute stroke: clinical patterns and practise}

\section{HYPOTHYROIDISM AS A PROTECTIVE FACTOR IN ACUTE STROKE} PATIENTS

K. Xynos, M. Synetou, S. Scalidi, J. Zafeiriou, E. Vavli-Vavlida, M. Alevizaki, K. Vemmos

Alexandra Hospital, University of Athens, Athens, Greece

Background: It has been reported that mildly elevated TSH may be associated with better survival in ambulatory elderly persons. We investigated possible associations of thyroid status with clinical severity and outcome in patients with acute stroke.

Patients: 761 patients who were consecutively admitted to hospital for first ever acute stroke were evaluated. Total T3, T4 \& TSH levels were assessed in the morning following admission. Severity of stroke was evaluated using the Scandinavian Stroke Scale (SSS) and the Glasgow Coma Score (GCS). One year stroke outcome was assessed using the modified Rankin Scale (mRS).

Results: Of 744 patients where thyroid function tests were available within the first 24 hrs of stroke, 13 had elevated TSH ( $\geq 10$, range $10-42 \mu \mathrm{U} / \mathrm{ml}$ ) (hypo-group), 51 had mildly elevated TSH $(3.3-9.9 \mu \mathrm{U} / \mathrm{ml})$ and 680 had normal TSH $(<3.3)$. Transient ischemic attacks (TIA's) were more prevalent in the hypo-group (46.2\%) compared to the groups of mildly elevated TSH $(11.8 \%)$ and normal TSH $(12.4 \%$, $\mathrm{p}<0.002$ ). Hypo-group had an adequate level of consciousness (GCS 14-15): $92.3 \%$ vs 74.5\% and 63.7\% ( $\mathrm{p}<0.033)$, and a milder neurological deficit (SSS score 45-58) $76.9 \%$ vs $39.2 \%$ and $38.7 \%$, ( $\mathrm{p}=0.02)$ compared to other two groups respectively. One year outcome tended to be better in hypo-group: mortality $(7.7 \%$ vs $26.2 \%$ and $27.6 \%$, ns by log-rank test) and $\mathrm{mRS}$ score $0-161.5 \%$ in hypo-group vs $42.9 \%$ and $47.5 \%$ respectively.

Discussion: Acute stroke patients with laboratory findings compatible with preexisting hypothyroidism on admission appear to have better clinical presentation and outcome; we speculate that reduced response to stress and previous TIA's, possibly related to endogenous "preconditioning", may contribute to this phenomenon. 


\section{Acute stroke: clinical patterns and practise}

\section{ISCHEMIA MODIFIED ALBUMIN, A NEW BIOMARKER OF ACUTE STROKE?}

H. Abboud, J. Labreuche, E. Meseguer, P. Lavallée, O. Simon, J.-M. Olivot,

M. Mazighi, J. Benessiano, P.G. Steg, P. Amarenco

Bichat hospital, Paris, France

Introduction: Ischemia Modified Albumin (IMA) is a new marker of ischemic tissue and has been found elevated in myocardial ischemia. We designed a pilot study to evaluate the clinical utility of IMA in patients with acute stroke.

Methods: We studied 118 consecutive patients presenting within 3 hours of acute neurological to the emergency department or to our stroke unit [84 brain infarctions (BI) (median age, 61 (22-90); men, 62\%), 18 brain hemorrhages( $\mathrm{ICH}$ ) (median age, 62 (44-82); men, 72\%), and 16 transient ischemic attacks lasting less than 1 hour or epileptic seizures (median age, 44 (26-75), men, 75\%)]. CT scan, 12-lead ECG and routine blood tests were performed in all patients. Serum samples for IMA were obtained for all patients at initial presentation and repeated only in patients with stroke at 6, 12 and 24 hours IMA was measured by the ACB test (Ischemia Technologies, Denver, Colorado, USA).

Results: The initial IMA levels were different between the three groups (BI, ICH and others diagnosis, $\mathrm{p}<0.01)$. Median IMA was $83 \mathrm{U} / \mathrm{ml}(79-86), 86 \mathrm{U} / \mathrm{ml}(75-90)$ in patients with BI and ICH respectively ( $\mathrm{p}=0.76)$, and was $73 \mathrm{U} / \mathrm{ml}(58-79)$ in others $(\mathrm{p}=0.003$ compared with $\mathrm{BI}$, and $\mathrm{p}=0.017$ with $\mathrm{ICH})$. Baseline IMA levels were correlated with NIHSS in BI $(\mathrm{p}=0.002)$ and in ICH $(\mathrm{p}=0.008)$.

During the first 24 hours, IMA levels increased in BI patients, whereas no change was observed in $\mathrm{ICH}$ patients.

Discussion: Although IMA levels do not permit to distinguish BI from $\mathrm{ICH}$, we found a continuous increase in IMA levels within the first 24 hours of stroke onset only in patients with BI. We also found a significant relationship between stroke severity (NIHSS) and baseline IMA values. These are encouraging results, and additional studies are needed to establish the validity of IMA in acute stroke.

\section{Acute stroke: clinical patterns and practise}

USE OF THE TRIAGE STROKE PANEL IN A NEUROLOGICAL EMERGENCY SERVICE

F. Rouanet, Y. Brouste, W. Meissner, J.M. Orgogozo

CHU Pellegrin, Bordeaux, Bordeaux, France

Objective: Evaluate test's additive utility to the triaging nurse for diagnosis of acute stroke.

Methods: Prospective, controlled, single-center study. All consecutive patients assessed for suspected stroke in the emergency department were included in the study during autumn 2005. The triage nurse considered a diagnosis of stroke based on sudden onset of weakness, difficulties with language, vision, mobility, balance, or when a physician referred the patient for stroke or TIA. The nurse assigned a probability that the patient had suffered a stroke (certain, very probable, probable, not likely, doubtful, other) then performed the blood test. The final diagnosis was made 1 day after admission based on clinical and imaging data by a senior neurologist blinded to the test results.

The Triage Stroke Panel is a rapid point-of-care immunoassay that simultaneously measures four biomarkers (BNP, D-dimer, MMP-9 and S100B) and presents a single composite result. The MultiMarker Index (MMX) result can range from 0 to 10 . If the result is lower than 1.3, the likelihood of a stroke is low; higher than 5.9, the likelihood of a stroke is high. A result between 1.3 and 5.9 indicates a risk of stroke or cerebral injury, warranting further investigation.

Results: 204 patients were evaluated in this manner. Confirmed strokes and TIAs represented $66,8 \%$, mimics $33,2 \%$. Sensitivity and specificity of the test at the 1.3 and 5.9 cutoffs were $93 \%-23 \%$ and $22 \%-83 \%$, respectively. Positive and negative predictive value at 1.3 were $71 \%-62 \%$. When considering a nurse assessment of "other", "doubtful" or "not likely" to be negative for stroke, the stroke panel MMX has identical accuracy $(66.2 \%)$ and equivalent sensitivity $(94 \%)$ and specificity (24\%) for stroke to the nurse probability. Also, $99 \%$ of strokes were positive if any positive nurse assessment or positive stroke panel MMX result is considered positive.

Conclusion: The Triage Stroke Panel appears to have utility for non-expert centers in improving the sensitivity of stroke diagnosis. It can reduce false negatives when taken together with triage nurse assessment.
4 Acute stroke: clinical patterns and practise

\section{POWER M-MODE TRANSCRANIAL DOPPLER EXAMINATION IN POSTERIOR CIRCULATION STROKES \\ M.R. Sivakumar, S. Sanjeev}

Cerebrovascular and Vasculitis Research Foundation, Chennai, India

Objective: We studied posterior circulation strokes using the Power M Mode Transcranial Doppler machine.

Background: Power Motion Mode Doppler TCD (PMD-TCD) is a new method that simultaneously displays flow signals at multiple depths. Any particular depth could be insonnated and Doppler spectrogram obtained from that depth.

Design/Methods: We evaluated patients with acute vertebro-basilar ischemia using the PMD-Transcranial Doppler machine from Spencer Technologies, Seattle, WA. Using a pre-defined set of TCD criteria, occlusion was localized to the proximal ( 80 $\mathrm{mm}$ depth), mid (90 mm depth) or distal (100 mm depth) Basilar Artery segments. The NIH Stroke Scale (NIHSS), Glasgow coma scale, Barthel index and Modified Rankin Scale were used to measure stroke severity, recovery and outcome in these patients. TCD flow in the vertebrobasilar and Carotid territories were graded using standard TIBI grades (0-5) as described by Qureshi et al.

Results: We evaluated 52 acute Vertebro-Basilar Strokes over a one year period. The mean age at onset was 58.6 years. The Median NIH Stroke Scale Score was 6.2. Basilar occlusion was present in 12 patients $(23.1 \%)$ with TIBI flow grades 0 - 3.Reversed BA (RBA) flow was noted in 5 patients $(9.6 \%)$. Baseline Median NIHSS scores in patients with RBA was 9 and Baseline Median NIHSS scores in patients without RBA was 11. Median discharge NIHSS scores was 5. Poor discharge outcome (NIHSS >13) was associated with no RBA.

Conclusions: 1. PMD display can be used for rapid identification of the level of vertebro-basilar occlusions.

2. PMD can show the presence of antegrade residual flow and retrograde collateral filling of the distal BA.

3. Reversed BA sign on PMD was associated with lower NIHSS scores and its absence was associated with bad outcomes including death, following BA occlusion.

\section{Acute stroke: clinical patterns and practise}

\section{PSEUDO-VESTIBULAR NEURITIS OF VASCULAR CAUSE: FREQUENCY,} CLINICAL FEATURES, AND VASCULAR TOPOGRAPHICAL PATTERNS H. Lee, S.-H. Lee, Y.-S. Min

Keimyung University School of Medicine, Masan Samsung Hospital, Masan Taebong Hospital, Daegu, South Korea

Background: While it is known that cerebellar infarction (CI) may simulate vestibular neuritis $(\mathrm{VN})$, the frequency and localization of this occurrence are still unclear. The aim of this study was to determine the frequency of CI mimicking VN, the pattern of clinical presentation, and the territory of the CI most commonly involved in this simulation.

Method: We studied 240 consecutive cases of isolated CI in the territories of the cerebellar arteries diagnosed by brain MRI from the acute stroke registry at the Keimyung University Dongsan Medical Center.

Results: We found 25 patients (10.4.\%) with isolated CI who had clinical features suggesting VN. Two types of CI simulating VN were found: isolated spontaneous prolonged vertigo as a sole manifestation of CI $(n=24)$ and isolated spontaneous prolonged vertigo as an initial manifestation of $\mathrm{CI}(\mathrm{n}=1)$ followed by delayed neurological deficits 2 days after the onset. The CI territory most commonly involved was the medial branch of the posterior inferior cerebellar artery (mPICA) territory $(24 / 25: 96 \%)$, followed by the anterior inferior cerebellar artery (AICA) territory $(1 / 25: 4 \%)$. None of patients with infarct(s) in either the territory of the superior cerebellar artery or multiple cerebellar arteries territories showed isolated spontaneous prolonged vertigo. All patients with isolated spontaneous prolonged vertigo as a sole manifestation of CI had mPICA territory CI whereas patient with isolated spontaneous prolonged vertigo prior to CI subsequently had AICA territory infarction.

Conclusions: CI simulating VN is more common than previously thought. Early recognition of the "pseudo- VN" of vascular cause may allow specific management. 
6 Acute stroke: clinical patterns and practise

AGREEMENT BETWEEN CLINICAL SYNDROME CLASSIFICATION AND BRAIN IMAGING IN THE INTRAVENOUS MAGNESIUM EFFICACY IN STROKE (IMAGES) TRIAL

K.W. Muir, E. Teasdale, K. Lees

University of Glasgow, Glasgow, United Kingdom

Background: The IMAGES trial was neutral overall, but prespecified sub-group analysis found significant benefit from magnesium in non-cortical stroke syndromes, a biologically plausible effect. We wished to establish the accuracy of clinical classification compared to brain imaging in IMAGES.

Methods: CT or MRI was allowed up to 7 days after trial entry. Structured scan review was undertaken centrally by a single neuroradiologist blind to all clinical data. Strokes were classified by Oxfordshire Community Stroke Project (OCSP) categories. Analyses compared lacunar syndromes (LACS) with partial or total anterior circulation syndromes (TACS or PACS) to define sensitivity and specificity of syndrome for imaging features. Posterior circulation syndromes were excluded. Associations were expressed as an odds ratios (OR) and 95\% confidence intervals. Results: Imaging was available for 2173/2386 (91\%) subjects from the efficacy population. Median onset-to-scan time was $15.5 \mathrm{~h}$. Imaging preceded randomisation in $36 \%$. Clinical diagnosis of LACS was associated with MCA perforator infarction (OR 1.75 [1.43-2.14], $\mathrm{p}<0.001$ ) and with leukoaraiosis (OR 1.47 [1.13-1.92], $\mathrm{p}<0.001$ ). Imaging was consistent with LACS (perforator infarct or normal) in $564 / 717$ subjects $(79 \%)$. LACS diagnosis was $43 \%$ sensitive and $70 \%$ specific for MCA perforator infarction in all subjects, and was $43 \%$ sensitive and $84 \%$ specific in 1562 subjects with acute lesions on imaging. PACS or TACS was $83 \%$ sensitive and $41 \%$ specific for a cortical infarct.

Conclusions: A clinical classification of LACS was specific for MCA perforator infarction, but had poor sensitivity, while clinical diagnosis of a cortical syndrome was sensitive for cortical infarction but not specific. Imaging was consistent with LACS in $79 \%$.

\section{Acute stroke: clinical patterns and practise}

NEUROLOGICAL SYNDROMES IN 20 PATIENTS WITH SPONTANEOUS CERVICAL ARTERY DISSECTION: MULTIMODAL MRI REVEALS MULTIFACTORIAL PATHOPHYSIOLOGY

D. Ulbricht, R.J. Metz, N.J. Diederich

Centre Hospitalier Emile Mayrisch, Luxemburg

Introduction: MRI facilitates to the diagnosis of spontaneous cervical artery dissection (SCAD) by showing wall-haematoma non-invasively. It has been postulated that it also demonstrates acute multiple brain infarction (AMBI) as the only mechanism of neurological deficits. We sought to verify the latter hypothesis.

Patients and Methods: 20 consecutive patients, 13 men and 7 women, with a mean age of 44.5 years and a total of 24 SCAD were prospectively studied. Multimoda MRI included at least cerebral diffusion weighted imaging and fat-saturated sequences of the neck. Cervical MRI and conventional angiography were performed as needed. Lesions were assessed according to standard vascular topography. AMBI was diagnosed when the lesion was distant from SCAD, local symptoms when best explained by direct effect of wall haematoma.

Results: In 11 patients the territory of the internal cerebral artery (ICA) and in the remainder the territory of the vertebral artery (VA) was affected. There was no SCAD in both territories, and four had bilateral SCAD (2 ICA, 2 VA). Lesions were directly visualized in $15 / 20$ patients. Five had SCAD and corresponding clinical symptoms without lesion of parenchyma. Lesions were situated in the corresponding arterial territory (ICA: distant $=5$, distant + local $=3$, local $=3$ ), $($ VA: distant $=4$, local $=4)$. The cause of neurological deficits was AMBI in 11 patients, local compression in 6 , and combined in 3 .

Discussion: The present series confirms the concept of AMBI as the main cause of neurological syndromes in SCAD. However, in situ compression by wall haematoma accounts for further deficits in about a third of the patients.

\section{Acute stroke: clinical patterns and practise}

A NEW SCALE FOR ASSESSING PATIENTS WITH VERTEBROBASILAR STROKE: THE ISRAELI VERTEBRO-BASILAR STROKE SCALE (IVBSS)

A. Gur, Y. Lampl, B. Gross, V. Royter, L. Shopin, N.M. Bornstein

Tel Aviv Sourasky Medical Center, Tel Aviv, Israel

Background: Most of existing stroke scoring systems have limited ability to evaluate patients with vertebrobasilar stroke. We devised a new scale, the Israeli Vertebrobasilar Stroke Scale (IVBSS) in order to directly and more accurately assess clinical deficits of patients with vertebrobasilar stroke. The present study measured the reliability and validity of the IVBSS.

Methods: 43 patients (mean age \pm SD $70.9 \pm 8.8$ years, 27 males) with vertebrobasilar stroke (18 in TASMC, 15 in WMC and 10 in WGH) were evaluated with the new IVBSS (11 items) and stroke (NIHSS) and disability (mRS) scales by independent examiners. Interobserver agreement was rated by weighted kappa statistics and Spearman rank coefficients (r) for each item and the total IVBSS score. Validity was examined with Spearman rank coefficients to compare the IVBSS with NIHSS and $\mathrm{mRS}$. The receiver operating characteristic (ROC) curve was used to determine the cutoff of the IVBSS score for distinguishing between mild-moderate and severe stroke.

Results: The interobserver agreement among the TASMC examiners for the total IVBSS score was: kappa $=0.71 ; \mathrm{r}=0.65(\mathrm{p}=0.002)$, for WMC kappa $=0.90 ; \mathrm{r}=$ 0.95 ( $\mathrm{p}<0.0001)$. Almost perfect reliability was also shown between the examiners for each item of the IVBSS (kappa $>0.80$ ). As a measure of concurrent validity, the total IVBSS score for TASMC was strongly associated with NIHSS and mRS $(\mathrm{r}=0.80$ and 0.76 , respectively; $\mathrm{p}<0.0002)$. The total IVBSS score for WGH was strongly associated with NIHSS $(r=0.91 ; p=0.002)$. Based on the ROC curve, we suggest a cutoff equal to 8 or more in the IVBSS score to demarcate severe stroke ( $87 \%$ sensitivity, $80 \%$ specificity).

Discussion: The IVBSS allows the assessment of vertebrobasilar stroke with high reliability. This new scale is valid and suitable for clinical practice as well as for clinical trials. Further observations are warranted to determine the predictive value of the IVBSS for stroke outcome.

\section{Acute stroke: clinical patterns and practise}

\section{ANTERIOR CHOROIDAL ARTERY INFARCTS. CLINICAL PROFILE,} ETIOLOGY AND OUTCOME

E. Palomeras, P. Fossas, M. Floriach, A. Cano, P. Sanz

Hospital de Mataró, Spain

Background: Isolated infarcts of anterior choroidal artery (AChA) are uncommon and less studied than infarcts involving other territories. Our aim is to study their clinical profile and severity, etiology and outcome.

Methods: From our prospective stroke registry (february 2002-december 2005, 1028 patients included), we've identified $41 \mathrm{AChA}$ infarcts, representing 5,23\% of all acute infarcts. We analyze their vascular risk factor profile, clinical subtype (OCSP), etiology (TOAST), NIHSS score at admission and discharge, and modified Rankin Scale (mRS) at discharge and after three months. Patients were independent for activities of daily life (ADL) if mRS score was 0-2.

Results: Mean age was 69 years, and $68.3 \%$ were male. Hypertension was the most common vascular risk factor $(75.6 \%)$. LACI syndromes were present in $83 \%$ of patients (sensory-motor syndrome in 20 , motor pure hemiparesis in 14), and PACI in $17 \%$. Motor deficit of at least one extremity was present in 40 of 41 patients. Etiology was atherothrombotic in $39 \%$, cardioembolic in $4.9 \%$, lacunar in $12.2 \%$ and cryptogenic in $43.9 \%$. Mean NIHSS at admission was 4,9 (it was 8 or more in 10 patients). At discharge, mean NIHSS was 4.3, only 22 patients $(53.6 \%)$ were independent for ADL and only $53.6 \%$ could go back to home from hospital. After three months, $63.4 \%$ of patients were independent for ADL. None of patients died during hospital stay or follow-up.

Conclusions: AChA infarcts usually appear as lacunar syndromes but most of them are atherothrombotic or cryptogenic infarcts. Almost all patients with AChA infarcts have some degree of motor deficit in extremities. Near half of patients are dependent for ADL at discharge and, most of them, also after 3 months.

\section{Acute stroke: clinical patterns and practise}

SCANDINAVIAN CANDESARTAN ACUTE STROKE TRIAL (SCAST)

E. Berge, R. Aakvik, A. Terént, G. Boysen

Ullevaal University Hospital, Oslo, Norway

Background: It has long been a controversy whether high blood pressure should be lowered in the acute phase of stroke. ACCESS (Stroke 2003; 34:1699) suggested a beneficial effect of the angiotensin receptor blocker candesartan in the acute phase of stroke, but these findings need to be confirmed in new, large trials.

Methods: SCAST is an international randomising, placebo-controlled, double blind trial of candesartan in acute stroke. Patients presenting within 30 hours of stroke (ischaemic or haemorrhagic) and with systolic blood pressure $\geq 140 \mathrm{~mm} \mathrm{Hg}$ are randomly assigned to candesartan or matching placebo for 7 days (doses increasing from 4 to $16 \mathrm{mg}$ once daily). The follow-up period is 6 months. Primary effect variables: i) Death or major disability; ii) Vascular events (vascular death, myocardial infarction or stroke). Target recruitment: 2,500 .

Status: 270 patients were included during the first 6 months (Sweden, Norway, 
Denmark and Belgium). Mean age 72 years; Blood pressure 172/89 mm Hg; Ischaemic stroke 85\%; Haemorrhagic stroke 15\%; Median SSS score 42 (IQR 31-51). Preliminary, blinded results will be presented at the meeting.

Funding: Basic funding from Norwegian authorities. Trial drugs and unconditional grants from AstraZeneca. Participating centres receive a limited economical compensation.

Conclusion: The start-up phase has been successful. Interested centres are welcome to join the trial.

\section{Acute stroke: clinical patterns and practise}

\section{S-100B AND NSE IDENTIFY SUBTYPES OF ISCHEMIC STROKE IN THE ACUTE PHASE}

G. Andsberg, B. Romner, J. Malm, K. Strandberg, E. Danielsson, B. Norrving, A. Lindgren

Clinical Sciences, Lund, Sweden

Background: One important task for the clinician is to design specific treatments for individual stroke patients. It is important to distinguish patients with cardiac embolic and carotid artery disease from patients with small vessel disease. Today there are no biochemical markers in clinical use to help the clinician to identify ischemic subtypes of stroke.

Method: We sampled serum, from 103 patients, daily for the first 5 days after the onset of ischemic stroke. Protein S-100B and neuron-specific enolase (NSE) levels were analysed by the use of immunoluminometric assays (Liason). Stroke subtypes were determined with the TOAST classification. Patients with small-vessel occlusion were defined as a small-vessel disease group (SVD; $n=34$ ), patients with large-artery disease or cardioembolism as a non-small-vessel disease group (NSVD; $\mathrm{n}=54$ ), and patients with stroke of undetermined etiology as a cryptogenic group $(\mathrm{CRY} ; \mathrm{n}=15)$. Values are presented as median, the 25 th and 75 th percentiles.

Results: The measurements of S-100B showed significantly higher levels in the NSVD as compared to the SVD group at each time point (day 1: $0,12(0,08-0,22)$ vs $0,08(0,06-0,12)$, day 2: $0,15(0,09-0,56)$ vs $0,10(0,07-0,14)$, day $3: 0,19(0,11-0,60)$ vs $0,10(0,08-0,14)$, day $4: 0,20(0,10-0,53)$ vs $0,09(0,07-0,13)$, day $5: 0,16(0,10-$ $0,45)$ vs $0,09(0,06-0,15))$, whereas for NSE the levels were significantly higher at day 4 and 5 in the NSVD as compared to SVD group (day 4: 11,9 (10,4-15,1) vs 9,2 $(7,9-10,7)$, day $5: 11,5(10,1-17,3)$ vs $9,2(7,4-10,7))$.

Discussion: This is one of the largest reported studies of multiple biochemical markers of brain damage in ischemic stroke. Measurments of S100B and NSE have a potential to distinguish subtypes of acute ischemic stroke.

\section{Acute stroke: clinical patterns and practise}

\section{DOES THE IMAGES STROKE SCALE PROVIDE MORE INFORMATION} THAN A SIMPLE CLINICAL CLASSIFICATION?

L. Govan, C.J. Weir, K.W. Muir, K.R. Lees, the IMAGES Study Group

University of Glasgow, Glasgow, United Kingdom

Rationale: In the analysis of the Intravenous Magnesium Efficacy in Stroke (IMAGES) trial a novel IMAGES Stroke Scale (ISS) was derived. Eight neurological features whose presence or absence define the Oxfordshire Community Stroke Project (OCSP) classification were combined with the Glasgow Coma Scale (GCS) components. We investigated whether the ISS predicted death or disability 90 days after stroke and whether it provided additional prognostic information to the OCSP. Methods: We analysed data from placebo patients in the IMAGES trial efficacy dataset. The ISS score assigned to each item is based on its weighting in the NIH Stroke Scale: unilateral weakness of face (1 point), arm (2), or leg (2), sensory loss (1), dysphasia (2), neglect (1), hemianopia (3), brainstem signs (1), any abnormality in the GCS eye (1), verbal (1) or motor (1) components. The maximum deficit was therefore 16 points. Outcomes, analysed using stepwise logistic regression, were expressed as odds ratios for death or disability, defined as Barthel Index $(\mathrm{BI})<95$ and modified Rankin Scale $(\mathrm{mRS})>1$.

Results: In the 1198 patients studied, OCSP and ISS were each associated significantly with both outcomes. For each 1 point increment of ISS the odds of $\mathrm{BI}<95$ increased by $1.31(95 \%$ CI $1.25-1.37)$ and the odds of $\mathrm{mRS}>1$ by $1.31(95 \%$ CI 1.25-1.38). ISS added significant prognostic information $(\mathrm{p}<0.0001$ for both outcomes) in addition to OCSP. However if the ISS is known, the OCSP does not add any additional prognostic information $(\mathrm{BI}<95$ : $\mathrm{p}=0.20$; $\mathrm{mRS}>1$ : $\mathrm{p}=0.57)$.

Discussion: The ISS can be derived from data routinely documented as present or absent in most stroke patients and provides prognostic information superior to the OCSP classification alone. The ISS merits comparison with more complex established stroke scales.
13 Acute stroke: clinical patterns and practise

ISCHAEMIC STROKE ONSET IN RELATION TO AGE AND GENDER - AN 8 YEARS SURVEY FROM THE BASEL STROKE REGISTRY

P. Lyrer, M. Gostynski, F. Fluri, L. Bonati, S. Papa, S. Engelter

University Hospital Basel, Basel, Switzerland

Background: The Basel Stroke registry was established in 1995 to systematically assess emergency admissions of acute stroke patients in the Basel Stroke Unit.

Patients and Methods: From March 1995 to February 2003 data from 2603 patients (men, $54.3 \%$, mean age 68.25 yrs, SD 13.2 , women $45.7 \%$, mean age 72.6 yrs, SD 16.6) with ischaemic stroke were prospectively registered. Stroke syndromes were classified according to the Oxfordshire Community Stroke Project and correlated to age and gender. Chi square statistics were applied.

Results: Lacunar strokes were more common in men $(32.9 \%)$ than in women $(28.8 \%)$, while partial anterior circulation infarcts were less frequent in men with $29.0 \%$ vs. $34.8 \%(\mathrm{p}<0.001)$ respectively. In women, the percentage of total anterior circulation infarcts increased with advancing age (i.e. $13.2 \%$ for women $<65$ years of age versus $25.6 \%$ for those aged over 85 years $(\mathrm{p}<0.001)$. In men the distribution of stroke syndromes did not change with advancing age. There were more men then women admitted within 3 hours from symptom onset (OR for early admission 1.35 CI 1.11-1.65)

Conclusions: 1. For women, the distribution of stroke syndromes changed with advancing age and total anterior circulation infarct became the most frequent. 2 . Male gender was associated with a higher likelihood for early hospitalisation. Both observations might be explained by the fact that women were older than men when they had an ischaemic stroke and were more likely to live alone at this crucial moment. These assumptions need to be analysed further.

\section{Acute stroke: clinical patterns and practise}

GSTP1 AND UFD1 AS EARLY BLOOD MARKERS OF ACUTE STROKE N. Turck, L. Allard, N. Walter, P.R. Burkhard, D.F. Hochstrasser, J. Montaner, J-C: Sanchez

Biomedical Proteomics Research Group, Geneva, Switzerland

Background: Gluthatione S-transferase P and Ubiquitin Fusion Degradation protein 1 were shown to be released in the CSF of deceased patients, a model of global brain ischemia. The aim of the present study was to evaluate whether the levels of GSTP and UFD1 in blood could rule-in ischemic stroke patients and complement clinical grounds and imaging $(\mathrm{CT})$

Methods: Consecutive patients with a final diagnosis of transient ischemic attack (TIA), ischemic stroke (IS) and intracerebral hemorrhage (ICH) from three different centers were included in the analysis. Patients with other causes mimicking stroke were also integrated. A homemade sandwich ELISA assay examined GSTP1 level in a Spanish cohort and UFD1 level in three independent cohorts (Spanish, Swiss and North American)

Results: GSTP1 appeared significantly elevated in the blood of Spanish stroke patients $(\mathrm{N}=39)$ compared to controls $(\mathrm{N}=29)$ with $72 \%$ sensitivity and $93 \%$ specificity (cut-off: $18.3 \mathrm{ng} / \mathrm{ml}, \mathrm{p}<0.0001$, Mann-Whitney test). A significant increase of UFD1 was also observed in the same stroke Spanish cohort with 95\% sensitivity and $76 \%$ specificity (cut-off: $2,2 \mathrm{ng} / \mathrm{ml}, \mathrm{p}<0.0001$, Mann-Whitney test). This result was confirmed in a Swiss cohort $(\mathrm{N}=31,87 \%$ sensitivity and $84 \%$ specificity, cut-off: $2,47 \mathrm{ng} / \mathrm{ml}, \mathrm{p}<0.0001$, Wilcoxon matched pairs test) and in a North American cohor $(\mathrm{N}=53,62,3 \%$ sensitivity and $90 \%$ specificity, cut-off at $0,445 \mathrm{ng} / \mathrm{ml}, \mathrm{p}<0.0001$, Mann-Whitney test)

Moreover, 13 conditions mimicking stroke symptoms were tested for both proteins Pathologies including demyelinizating disease and radial palsy displayed very low levels of both proteins, whereas some of the brain tumor patient displayed elevated levels. Moderate levels of GSTP1 and UFD1 were obtained for remaining groups (headache, vertigo, myelopathy)

Discussion: The present preliminary results established GSTP and UFD1 as early blood markers of ischemic stroke. Their combination with other markers including NDKA, PARK7 and caspase-3 will clearly improve the referral to treatment and management of stroke patients

\section{Acute stroke: clinical patterns and practise}

\section{CROSSED APHASIA}

Y. Yu-mei Zhang, Xi-ping Gong, Yi-long Wang, Xing-quan Zhao, Chun-xue Wang

Tiantan hospital, affiliated with Capital University of Medical Sciences, China

Backgrounds and Purposes: Crossed aphasia means that aphasia secondary to 
lesions in the right hemisphere in right-handed patients, its incidence is low. We reported five cases in order to analysis the type, mechanisms and prognosis of crossed aphasia.

Methods: Five right-handed patients with aphasia secondary to acute stroke in the right hemisphere were studied by means of oral fluent of Aphasia Battery of China, the Western Aphasia Battery. After rehabiliting thirty days, these cases were studied by the same ways, and the second results compared with that of the first.

Results: The diagnosis of crossed aphasia requires definite documentation of a language disorder in patients who are not only strongly right-handed but who also have no family history of left-handedness and have definite evidence of a lesion in the right hemisphere on CT or MRI scan, or autopsy. Our cases meeted with the diagnosis. Among five cases, there were four no-fluent oral and one fluent oral. According to crossed aphasia type, there were three mirror image aphasia, which mean if the same lesion locate at the left hemisphere, it would cause the same aphasia type, and there were two atypical crossed aphasia, which means if the same lesion located at left hemiphere, it would cause the other aphasia types. According to the results of Western Aphasia Battery, there were four Broca's aphasias and three Wernicke's aphasias. All cases got a well language function recovery after rehabilition.

Discussion: Crossed aphasia has two types: mirror image aphasia and atypical crossed aphasia. Proposed mechanisms of crossed aphasia: (1) A previously silent or unrecognized lesion in the left hemisphere that is somehow rendered symptomatic by the new lesion in the right hemisphere; (2) Ipsilateral control of the dominant hand; (3) Bilateral representation of linguistic functions and (4) An arrested developmental stage in the lateralization of language function. Prognosis of crossed aphasia is better.

\section{Acute stroke: clinical patterns and practise}

\section{PURE FACIAL PARESIS AS A STROKE SYNDROME}

K. Yonemura, Y. Hashimoto, M. Uchino

Kumamoto City Hospital, Kumamoto, Japan

Background: Isolated pure facial paresis (PFP) is rarely encountered clinical stroke syndromes, and has not yet been thoroughly characterized. We sought to clarify the clinical characteristics of PFP in patients with first-ever acute ischemic stroke.

Methods: We selected patients with PFP from 255 consecutive patients with first-ever acute ischemic stroke who presented with pure motor syndrome admitted between April 2000 and October 2005. Sex, age, vascular risk factors, emboligenic heart disease, occlusive cerebral artery disease, infarct size and location, and outcome data were evaluated. Acute infarcts were all identified by diffusion-weighted magnetic resonance imaging (MRI) within 7 days of stroke onset.

Results: 12 patients $(4.7 \%)$ presented with PFP. They consisted of 2 men and 10 women with age of $71 \pm 11$ years. Hypertension was the most frequent in the vascular risk factors (10 patients). Emboligenic heart disease and occlusive cerebral artery disease were detected in 2 and 1 patients, respectively. Conventional and diffusion-weighted MRI identified infarcts on the centrum ovale in 5 patients, cortical motor area in 2, corona radiata in 2, basal ganglia-corona radiata in 2 , and posterior limb of the internal capsule in 1.7 patients had infarcts $>15 \mathrm{~mm}$ in maximal diameter. Although 1 patient developed recurrent minor stroke during hospitalization, all patients were discharged with mild or no disability.

Conclusions: These results suggest that subcortical infarcts can predominantly cause PFP rather than cortical infarcts. Especially, the centrum ovale infarcts should be taken into consideration in patients with PFP.

\section{Acute stroke: clinical patterns and practise}

\section{THE SIGNIFICANCE OF ELEVATED PLASMA BRAIN NATRIURETIC PEPTIDE IN PATIENTS WITH ACUTE ISCHEMIC STROKE}

B. Yoo, M. Kim, J. Kim, E. Kim, Y. Kim

Kosin University College of Medicine, Kosin, South Korea

Background \& Purpose: BNP (brain natriuretic peptide) is vasoactive peptide hormone with natriuretic, diuretic, and vasodilator activity. The current information concerning the role of BNP in cerebrovascular disease is limited. We measured plasma BNP concentrations to determine the implications of BNP in patients with acute ischemic stroke.

Method: Seventy patients with acute ischemic stroke and 25 age- and sex-matched healthy volunteers were recruited in the study. Risk factors, laboratory results, and findings at physical and neurological examination, including the NIHSS and modified Rankin scale were collected. Plasma BNP was measured within 24 hours from symptoms onset. BNP levels were compared between ischemic subgroups categorized by TOAST classification. Correlation was investigated between BNP levels and various clinical and laboratory parameters.
Result: Men were $54.1 \%$, mean age was $64.0 \pm 11.1$ years. Mean NIHSS score was $6.1 \pm 5.9$. Mean BNP level was $144.5 \mathrm{pg} / \mathrm{ml}$. The plasma BNP levels were significantly higher in patients with cardioembolism than in patients with the other types $(\mathrm{P}<0.01)$. A significant correlation was found between neurologic status on admission and BNP levels $(\mathrm{P}<0.05)$; patients with worse neurologic status had higher BNP plasma values than those with better neurologic status. The BNP levels were correlated with mean arterial blood pressure on admission, lower hemoglobin and hematocrit, triglyceride, and stroke severity in acute stage.

Conclusion: These findings show that plasma levels of BNP are elevated in the acute phase of stroke and predict stroke severity. BNP levels may be also useful in detecting patients at higher risk for cardioembolic stroke.

\section{Acute stroke: clinical patterns and practise}

\section{ATHROMBOGENIC ABILITY OF VASCULAR ENDOTHELIUM IN ACUTE} ISCHEMIC STROKE

M.A. Domashenko, S.V. Orlov, M.V. Kostyreva, M.M. Tanashyan, V.G. Ionova, Z.A. Suslina

Institute of Neurology, RAMS, Moscow, Russian Federation

Background: The aim of the study was to evaluate the dynamics of the athrombogenic anticoagulant ability of vascular endothelium in acute ischemic stroke [IS].

Methods: Anticoagulant ability of vascular endothelium was investigated in 25 patients (age 67 [55; 79] years; 13 males, 12 females) with IS and was estimated as changes of serum von Willebrand factor [vWf] and antithrombin III [ÀÒ III] concentration before and after "cuff test" with the transient ischemia of the brachial vessels by the cuff of the manometer [CT] in the first 48 hours, on the 5-th and 21-st days of IS onset.

Results: The vWf concentration in the first 48 hours of IS was $172 \%[152 ; 192]$ before and $185 \%[176 ; 198]$ after CT. On the 5-th day of IS onset the vWf level decreased till 146\% [115; 188,5] before CT $(\mathrm{p}<0.05)$ and $170 \%[137 ; 197,5]$ after $\mathrm{CT}$. It may be possibly explained with the massive therapeutic action. On 21 -st day of IS the vWf concentration increased till $189 \%[151,5 ; 202,5]$ before CT $(p<0,05)$ and $194 \%[165,5 ; 214,5]$ after CT. This may be the evidence of the exhaustion of blood vessels' endothelium reserves by the end of the acute period of IS. The ÀO III concentration in the first 48 hours of IS was $104 \%$ [94; 125] before and $114 \%[97,9$; 129] after CT. On the 5-th day of IS the ÀO III level was $112 \%$ [88,2; 118], 117\% $[96,1 ; 126,5]$ after CT; and on 21 -st day $115 \%$ [103; 120] before and $120 \%[111,5$; 127] after CT. The ÀÒ III concentration on the 21-st day of IS was significantly higher then in the first 48 hours of IS ( $p<0,05)$.

Conclusion: Depression of the anticoagulant activity of vessel's endothelium occurs in IS patients. The slow increase of serum vWf and AT III concentration by the end of the acute period of IS was demonstrated.

\section{Acute stroke: clinical patterns and practise}

\section{OXIDATIVE STRESS IN ACUTE ISCHEMIC STROKE}

A. Ozkul, A. Akyol, C. Yenisey, C. Tataroglu, E. Arpaci, N. Kiylioglu Adnan Menderes University Medical Faculty, Aydin, Turkey

Objective: Oxidative stress plays an important role in acute phase of ischemic stroke pathogenesis. Free radical formation and subsequent oxidative damage may be a factor in stroke severity.

Method and Material: Serum levels of nitric oxide (NO), malondialdehyde (MDA) and glutathione (GSH) were measured within the first 48 hours of stroke in 70 stroke patients. The levels were also correlated with the clinical outcomes using Canadian Neurological Scale (CNS) scores. The results were compared with a control group consisted of 70 volunteers with similiar stroke risk factors.

Results: Serum NO, MDA and GSH levels were significantly elevated in acute stroke patients. CNS score was negatively correlated with both MDA and NO levels. However, there was no statistically significant correlation between GSH levels and CNS scores.

Conclusion: Our results suggest deleterious effects of oxidative stress on the clinical picture in acute ischemic stroke. The elevation of glutathione serum levels may be a part of the adaptive mechanism during this period. The further studies may help us to have a broader view on acute cerebral infarct pathophysiology. 


\section{Dementia and cognition}

\section{Dementia and cognition}

\section{BRAIN ATROPHY IN CHAGAS DISEASE CARDIOMYOPATHY:}

\section{A VOLUMETRIC STUDY}

R.M. Vieira-de-Melo, P.SO. Reis, I.SN. Ribeiro, C.CS. Santos, A.M. Lacerda,

L.C. Viana, D.F. Menezes, F.JFB. Reis, J. Oliveira-Filho

Federal University of Bahia, Stroke and Cardiomyopathy Clinics, Salvador, Brazil

Background: Cognitive dysfunction in Chagas disease has been attributed to cardiac disease, resulting in chronic brain ischemia due to hypoperfusion and/or multiple infarcts. Our objective was to investigate head computed tomography (CT) findings in patients with Chagas disease cardiomyopathy (CDC) in comparison with other cardiomyopathies (OC)

Methods: Patients with CDC and OC matched for age and gender were selected from a university-based cardiomyopathy clinic. A single investigator blinded to all clinical data evaluated head $\mathrm{CT}$ images for the presence of lacunar or territorial infarcts, as well as for measuring the total volumes of the brain, cerebellum and ventricles. Clinical data was extracted from a prospectively-collected database containing demographic information, cerebrovascular risk factors, electrocardiographic and echocardiographic data.

Results: We studied 79 patients, mean age $55 \pm 9$ years, 29 (37\%) male. CDC was the most frequent etiology $(52 \%)$, followed by hypertensive dilated cardiomyopathy $(21 \%)$ and idiopathic dilated cardiomyopathy $(9 \%)$. The frequency of cardiac arrhythmias was similar in both groups. Echocardiography showed a similar systolic function (mean ejection fraction of $42 \%$ in both groups), but a greater dilatation of cardiac chambers in OC patients when compared to CDC patients (left ventricle diastolic diameter $60 \pm 8$ vs. $66 \pm 9 \mathrm{~mm}, \mathrm{p}=0.021)$. Total brain volume was smaller in $\mathrm{CDC}$ as compared to OC patients ( $1135 \pm 150$ vs. $\left.1332 \pm 198 \mathrm{~cm}^{3}, \mathrm{p}<0.0001\right)$. Cerebellum and ventricle volumes did not differ between both groups. The proportion and number of brain infarcts was also similar in both groups.

Discussion: Total brain volume is significantly smaller in Chagas disease patients, despite a similar cardiac disease severity and number of brain infarcts. Active or previously active brain inflammation may predispose Chagas disease patients to cognitive dysfunction.

\section{Dementia and cognition}

\section{IS COGNITIVE DECLINE SO REVERSIBLE AFTER CAROTID} ANGIOPLASTY AND STENTING AS AFTER ENDARTERECTOMY?

A. Costa, E. Venturelli, V. Vergani, R. Spezi, E. Cottini, A. Pezzini, L. Rozzini,

R. Gasparotti, A. Padovani, M. Magoni

Vascular Neurology-Spedali Civili, Clinical Neurology and

Neuroradiology-University of Brescia, Brescia, Italy

Percutaneous transluminal angioplasty and stenting (CAS) is considered an alternative procedure to carotid endarterectomy (CEA) in the treatment of severe carotid stenosis (SCS); CAS had the advantage of avoiding general anesthesia, cranial nerve injury and the discomforts of surgery.

We evaluated if the neuropsychological decline associated to SCS could be considered reversible with CAS as with CEA.

Clinical and neuropsychological outcomes were prospectively and consecutively collected in patients with SCS before and after procedures (3 days, 3 and 9 months). CAS was performed without protection devices.

Neuropsychological test battery includes: Mini Mental State Examination (MMSE), Trail Making A, Short Story, Rey Copy (RC), Rey Recal and Geriatric Depression Scale (GDS); other outcomes scales were Scandinavian Stroke Scale, Barthel Index, Tinetti Scale and Individual Disease Severity.

We compared 20 patients undergoing CAS with 60 patients undergoing CEA of the same side with the same baseline MMSE.

Age, sex, education and baseline scales did not show any statistical difference between the two groups. No significative differences were found between the groups on any test at any time and in the rate of improvement at any of the assessment time. At 9 months, the 11 right sided stented patients significantly improved after CAS in two tests: RC $(-2.7$ vs +2.5 in CEA, $p=.03)$ and GDS $(-2.0$ vs 1.6 in CEA, $p=.01)$.

Our findings show that, in our patients, neurological and neuropsychological outcomes at 9 months are equivalent for CEA and CAS (also without protection devices).

Right sided SCS undergoing CAS significantly improved in depression and visuospatial functions.

The cognitive decline associated to SCS could be considered reversible with CAS as with CEA.

Poster Session First Visit

Cerebrovasc Dis 2006;21(suppl 4):1-151
PREVALENCE OF COGNITIVE IMPAIRMENT AND DEMENTIA AFTER STROKE IN SINGAPORE

S. Ang, S.Y. Tay, I. Akbar, T. Toh, C.H. Sim, H.M. Chang, M.C. Wong, C.P. Chen

Singapore General Hospital, Singapore

Background: The prevalence of post stroke cognitive impairment (CI) and dementia is variable, especially in Asian studies. We aim to investigate the prevalence and factors associated with $\mathrm{CI}$ and dementia in the acute phase and 3 months post stroke. Methods: Consecutive patients admitted for ischemic stroke from March to May 2005 were screened. Eligible patients were assessed within 3 days of admission with the Mini Mental State Examination (MMSE) and the Informant Questionnaire on Cognitive Decline in the Elderly. 3 months post stroke, patients impaired at baseline and a random sample of unimpaired patients underwent full cognitive reassessment Cognitive status was classified as Not Cognitively Impaired (NCI), Cognitively Impaired Not Demented (CIND) or Demented.

Results: Of the 293 patients screened, 169 patients were eligible (mean age $66 \pm 11$ years). The major reasons for exclusion were Transient Ischemic Attack (44\%), early discharge due to mild stroke (16\%), dysphasia $(10 \%)$ and pre-existing dementia (6\%). $25 \%$ of patients were cognitively impaired acutely: $77 \%$ were CIND and $12 \%$ demented at 3 months. Of the $26 \%$ of patients unimpaired acutely re-evaluated at 3 months, 39\% were CIND and none demented. The prevalence of CIND and dementia 3 months post stroke was $49 \%$ and 3\% respectively. Univariate analysis showed an association between age and acute MMSE score with cognitive status at month 3. Logistic regression analysis showed acute MMSE score as an independent predictor of cognitive status 3 months post stroke. An AUC of 0.89 was obtained by ROC analysis using an MMSE cutoff score $<22$.

Conclusion: MMSE scores in the acute phase of stroke is useful in identifying patients at risk of CI 3 months post stroke. Whilst CI is common after stroke, post stroke dementia at 3 months is less frequent and may be attributed to a relatively younger and less severe stroke casemix as well as host (cultural and genetic) factors.

\section{Dementia and cognition}

\section{COGNITIVE IMPAIRMENT AND DEPRESSION AFTER STROKE IN A MEXICAN POPULATION}

J. Coral, A. Arauz, G. Román, J. Rodriguez, A. Sosa, M. González, M. Chávez, F. Paz, C. Díaz

National Institute of Neurology and Neurosurgery "Manuel Velasco Suarez", Mexico DF, Mexico

Objectives: There is a dearth of information regarding frequency, clinical determinants and risk factors for post-stroke cognitive impairment and depression in Mexican patients. We aimed to evaluate the degree of cognitive impairment following stroke at the National Institute of Neurology in Mexico City, Mexico.

Methods: A group of 100 consecutive stroke patients admitted to our Institution underwent a comprehensive neuropsychological and detailed psychiatric examination 3 months after stroke. Basic demographic data, vascular risk factors, stroke subtype and stroke severity (NIH stroke scale and Rankin scale) were assessed. In addition, pre-stroke cognitive decline (Informant questionnaire on cognitive decline in the elderly; IQCODE), functional outcome and neuroimaging were obtained.

Severity of cognitive symptoms was graded according to the Clinical Dementia Rating Scale (CDR). Instrumental activities of daily living were evaluated by Lawton and Brody's scale and executive functions were assessed with the executive interview (EXIT). Depression was estimated with the Beck Depression Inventory.

Results: The mean age of the 100 patients was $57( \pm 18.4), 63 \%$ were men, and $93 \%$ had cognitive symptoms. According to the CDR: 7 patients (7\%) had normal cognitive function; 63 (63\%) had mild cognitive impairment; and 25 (25\%) moderate or severe cognitive impairment; (17\%) had mental decline assessed by IQCODE prior to the stroke. Executive deficits were found in $38 \%$; alterations of instrumenta activities of daily living were observed in 69\% and depression was diagnosed in 57 patients. The most frequent stroke risk factors were hypertension (50\%) and smoking (37\%).

Conclusions: Our results demonstrate that despite their younger age, this cohort of stroke patients in Mexico demonstrates high prevalence of cognitive impairment and depression.

Dementia and cognition 


\section{Dementia and cognition}

DO HYPOPERFUSION AND DIFFUSION CHANGES ASSOCIATED WITH A HIGH-GRADE CAROTID STENOSIS CAUSE A CORRECTABLE COGNITIVE DEFICIT?

L. Soinne, J. Helenius, I. Tikkala, E. Saimanen, O. Salonen, M. Hietanen, P.J. Lindsberg, M. Kaste

Helsinki University Central Hospital, Helsinki, Finland

Background: A high-grade carotid stenosis may impair ipsilateral cerebral perfusion in perfusion-weighted MR imaging (PWI) in part of the subjects and induce a tendency for higher ipsilateral apparent diffusion coefficient (ADC) levels in diffusion-weighted MR imaging (DWI). These findings are improved by carotid endarterectomy (CEA). We aimed to find out whether these features are associated with changes in cognitive performance.

Methods: Of the 44 patients (aged $64.3 \pm 8.8$ years) with a high-grade carotic stenosis (mean \pm SD $78.1 \pm 8.6 \%$ ), 23 were asymptomatic and 21 symptomatic. A neuropsychological battery as well as PWI and DWI were performed thrice: preoperatively, and 4 and 100 days after endarterectomy. Perfusion maps were created blindedly and evaluated by two independent observers, and ADC values representing selected regions of interest were determined for each hemisphere. Healthy volunteers $(n=22)$ matched for sex, age, and education underwent the same three-staged neuropsychological evaluation sequence.

Results: Preoperatively, in DWI the ipsilateral ADC values were higher (for white matter 0.69 vs. $0.66, \mathrm{p}<.001$, ANOVA) and in PWI the mean transit times longer ( 6.0 vs. $4.9 \mathrm{~s}, \mathrm{p}<.001$, ANOVA) than in subsequent measurements. Ten patients $(23 \%)$ had a visible ipsilateral perfusion deficit (six out of ten had left-hemisphere and four right-hemisphere deficits). Six patients had DWI deficits at baseline, and two new lesions $(4.5 \%)$ were detected in postoperative scans. Baseline cognitive performance of patients tended to be lower than in controls. The postoperative course of cognitive improvement ie. learning effect was comparable to controls regardless of preoperative deficits in PWI or change in ADC values.

Discussion: Despite a tendency for lower-level cognitive functioning in patients with a high-grade carotid stenosis, the postoperative course of cognition after CEA displays a comparable learning effect as in healthy controls regardless of changes in cerebral perfusion and diffusion.

\section{Dementia and cognition}

\section{PREVALENCE AND CHARACTERIZATION OF COGNITIVE IMPAIRMENT}

\section{IN SUBACUTE STROKE}

A. Jaillard, B. Naegele, S. Trabucco-Miguel, K. Garambois, O. Detante,

M. Hommel

UnCentre Hospitalier Universitaire de Grenoble, Grenoble, France

Background: Cognitive impairment (CI), outside dementia, is an important problem after stroke which may hinder functional recovery. Indeed, CI was reported in patients with apparent good recovery, leading to limitations in participation in previous social and professional activities. However the prevalence of CI may vary from 30 to $70 \%$. We aim to (1) To evaluate the prevalence of $\mathrm{CI}$ after subacute stroke (2) To determine impaired cognitive domains.

Methodology: Patients with first-ever infarction had prospectively neuropsychological evaluation within 1 month after ischemic stroke documented by diffusion MRI sequence if age $\geq 18$ years and Mini Mental state Evaluation (MMSE) was $\geq 23 / 30$. Evaluation included NIHSS and at day $1 \& 15$, and Rankin score at 6 months. Neuropsychological tests investigated depression, instrumental functions, memory, executive functions and explored the central executive of working memory (WM) using the PASAT and OWEN tests. Moderate CI was determined by scores $<1 \mathrm{SD}$ and severe CI by scores $<2 \mathrm{SD}$.

Results: We studied 177 patients and 81 controls. In patients, mean age was 50.6 years, mean MMSE 28.4, and mean Beck depression score was 8.4. Among the patients, $93.2 \%$ were impaired in at leat one cognitive test, $84.2 \%$ in WM, $82.2 \%$ in executive functions and $61.8 \%$ in memory. Patients were significantely impaired in comparison with the controls for all tests but short term memory. The cognitive profile of stroke versus control subjects was at best predicted by WM tests, i.e. the PASAT $(\mathrm{OR}=0.57$ per point; $\mathrm{p}=0.01)$ and the spatial WM Owen test $(\mathrm{OR}=1.245$ per error; $\mathrm{p}=0.003$ ) after adjsutment for age, sex, level of education and depression. Discussion: Most of patients had CI within 1 month after stroke, WM impairment being the outstanding feature.
7 Dementia and cognition

COGNITIVE FUNCTION CORRELATES WITH FRONTAL WHITE MATTER APPARENT DIFFUSION COEFFICIENTS (ADC) IN PATIENTS WITH AGE RELATED WHITE MATTER LESIONS (ARWML)

M. Viana-Baptista, P. Bugalho, C. Jordão, G. Horácio, O. Ribeiro, M.F. Secca, J.M. Ferro

Hospital Egas Moniz, Lisboa, Portugal

Background: Diffusion weighted imaging (DWI) higher sensitivity to white matter changes suggests stronger relation than conventional MRI with cognitive dysfunction. We aimed to relate ARWML and ADC (in lesions and in normal appearing white matter (NAWM)) with systolic, diastolic blood pressure (SBP,DBP), age and cognition.

Methods: 29 functional ARWML patients aged > 65 (mean72,6 $\pm 5,2$ ).Tests: Raven Progressive Matrices (R), Rey's Complex Figure, Digit Canceling (DC), Symbol-digit Substitution, Inverse Digit Repetition and Verbal Fluency (VF). ARWML were quantified globally (total score(T)), bilaterally in frontal ( $\mathrm{Fl}, \mathrm{Fr}$ ), temporal, parietoccipital(POr,POl), basal ganglia and sub-tentorial regions, with a visual rating scale(VRS). ADC was measured bilaterally on parietoccipital (DPOl, DPOr) and frontal (DFr,DFl) NAWM and on frontal periventricular white matter lesions(PVl,PVr)

Results: VRS: Significant correlations (controlling for T): age and $\mathrm{Fr}(\mathrm{r}=0.526$, $\mathrm{p}=0.003)$ and $\mathrm{Fl}(\mathrm{r}=0.492, \mathrm{p}=0.007) ; \mathrm{Fl}, \mathrm{Fr}$ and $\mathrm{DC}(\mathrm{r}=-0.494, \mathrm{p}=0.009, \mathrm{r}=-0.546$, $\mathrm{p}=0.003$ ).

DWI: Significant correlations (Spearman): age and $\mathrm{DFr}(\mathrm{r}=0.442, \mathrm{p}=0.016)$ and $\mathrm{DF}$ $(\mathrm{r}=0.403, \mathrm{p}=0.030) ; \mathrm{SBP}$ and $\mathrm{DFl}$ and $\mathrm{DFr}(\mathrm{r}=0.382, \mathrm{p}=0.049 ; \mathrm{r}=0.423, \mathrm{p}=0.028)$ DBP and: $\operatorname{DFl}(r=0.368, p=0.049), \operatorname{PVl}(r=0.370, p=0,048), D P O l(r=0.401, p=0.031)$ and DPOr $(r=0.415, p=0.025) ; R$ and DFl $(r=-0.389, p=0.037)$; $\mathrm{DC}$ and PVr $(r=-$ 0.490, $\mathrm{p}=0.008)$ and $\mathrm{PVl}(\mathrm{r}=-0.463, \mathrm{p}=0,013)$. Controlling for age: all $\mathrm{PVr}$ and PVl correlations remained significant; DFr and VF became significant $(r=-0.393$, $\mathrm{p}=0.047)$.

Conclusion: Both methods revealed correlation between frontal vascular changes, age and blood pressure and executive function, confirming higher susceptibility of frontal white matter and of frontal functions to vascular disease. DWI suggests this is true also for NAWM.

\section{Dementia and cognition}

INFLUENCE OF COGNITIVE IMPAIRMENT ON THE SOCIAL OUTCOME WITHIN 3 YEARS AFTER STROKE

M. Pasquini, D. Leys, M. Rousseaux, F. Pasquier, H. Hénon

Lille University Hospital, Lille, France

Background and Purpose: pre-existing and new-onset dementia are frequent in stroke patients, and are associated with an increased mortality. Whether cognitive impairment influences the social outcome remains unsettled. The aim of this study was to evaluate the influence of cognitive impairment on the social outcome within 3 years after stroke.

Method: in 165 consecutive acute stroke patients living at home before, we prospectively evaluated previous cognitive functions, with the Informant Questionnaire on Cognitive Decline in the Elderly (IQCODE), and dementia within 3 years after stroke onset (ICD10 criteria). We evaluated their influence on the social outcome.

Results: pre-existing dementia did not influence the proportion of patients discharged at home after the acute stage. Independent predictors at admission of institutionalization within 3 years were age (adjusted OR [adjOR] for 1 year increase: $1.08 ; 95 \%$ confidence interval [CI]: $1.03-1.15)$, severity of the clinical deficit (adjOR for 1 point increase in Orgogozo score: 0.98; 95\% CI: $0.96-0.99$ ), and IQCODE score (adjOR for 1 point increase: 1.03; 95\% CI: $1.0001-1.06$ ). Age (adjOR for 1 year increase $=1.17$; CI 95\%: 1.07-1.27), and any dementia, pre-existing or new (adjOR $=5.85$; CI 95\%: 1.59-21.59), was associated with institutionalization at year-3.

Conclusion: dementia (pre-existing or new), is associated with an increased rate of institutionalization within 3 years after stroke, but does not influence discharge at home after the acute stage. The cognitive evaluation at the acute stage of stroke, and during the follow-up, is crucial to predict social outcome and should be part of the diagnostic work-up of stroke patients. 


\section{Dementia and cognition}

PHONEMIC FLUENCY IMPAIRMENT AND SILENT BRAIN INFARCTIONS

C. Tejero, J.A. Mauri, L.F. Pascual, G. Piñol, M. Montori, S. Santos,

E. Mostacero

Hospital Clínico Universitario “Dr Lozano Blesa”, Spain

Background: Several studies indicate that Vascular Dementia (VaD) patients with primarily subcortical ischemic vascular dementia show a relatively greater deficit on measures of phonemic fluency compared with patients with Alzheimer Disease (AD) of similar severity. Silent brain infarctions (SBI) are common findings in advanced age, but their relationship to dementia is still uncertain. We investigate whether animal fluency and letter F scores impairment is associated to SBI in patients with $\mathrm{AD}$.

Methods: We compared two CT scan and cognitive test (Mini-Mental State Examination (MMSE) score and semantic and phonemic word generation tasks), with more of two years of difference, in patients with dementia $(n=50)$ with vascular risk factors.

All participants were administered standardized semantic and phonemic word generation. For semantic fluency, participants were asked to name as many different animals as they could within 60 seconds. Phonemic fluency was assessed using the number of correct words generated for the letter F. Difference scores between semantic and phonemic fluency were obtained by subtracting the number of letter $F$ words from the number of animal names generated (animal - letter F).

SBI were defined as CT evidence of infarcts not compatible with an acute event The patients were divided into two: control group $(\mathrm{CG} ; \mathrm{n}=29)$ and SBI group (SG; $\mathrm{n}=21$ ) according to $\mathrm{CT} / \mathrm{MRI}$ findings.

Results: No group differences were found for age, educational level or MMSE scores. SG patient scored significantly worse on the letter F fluency test than on the animals fluency test. MANCOVA controlling for age and years of education revealed overall group differences for letter $\mathrm{F}[\mathrm{F}(6,204)=17.91 ; \mathrm{p}<0.001]$,

Conclusions: Phonemic fluency impairment is associated to SBI in patients with $\mathrm{AD}$

\section{Behavior and mood}

\section{Behavior and mood}

\section{CHANGE OF DIALECT AFTER STROKE}

M. Kwon, J.S. Kim

Asan Medical Center, Asan, South Korea

Background: Although the accent may change after a brain damage, change in dialect after a stroke is exceedingly rare.

Method: We studied a patient who showed prominent change of accent from one to another Korean dialect. A 71-year-old Korean woman developed fluent aphasia and a change of dialect from Cholla-buk to Kangwon province dialect after an infarction occurring in the left temproparietal area. The Korean version of the Western Aphasia Battery was administered for assessing receptive and expressive language ability. For perceptual assessment of her speech, five independent listeners were made to assess her speech. For the tests of affective and linguistic prosody, 20 (10 for affective and 10 for linguistic) short sentences were prepared and tested. Narrow phonetic transcription was performed for the phonetic analyses and acoustic analyses using wavesurfer 1.6.2 software program were used to elucidate the prosodic pattern of her speech.

Results: Perceptual assessment revealed that the patient used Kangwon province dialect but not Cholla-buk dialect. On prosodic tests, the patient's linguistic prosody was impaired in both comprehension and expression. On acoustic analysis, she showed a large pitch excursion at the second syllable and at the end of sentences, a pattern which is not observed in Cholla-buk dialect but similar to Kangwon province dialect.

Conclusion: Based on these results, it appears that her prosodic alteration primarily made her speech perceived as a 'different dialect'. This unique observation broadens our concept of foreign accent syndrome.

\section{Behavior and mood}

\section{THE PREVALENCE OF DEPRESSION IN POST-STROKE RESIDENTS OF} OLD-AGE-HOMES

M. Siemiński, M. Karwacka, M. Potocka, A.W.M. Nyka

Medical University of Gdansk, Gdansk, Poland

Background: Post-stroke depression is well described clinical phenomenon but there is still a discussion on the etiology of the disease. At least three causative factors should be taken into account: the focal brain lesion, the level of patient's impairment and the amount of social support received by them. Performing studies clarifying the impact of each of the above factors is difficult due to heterogeneity of patients regarding the level of impairment and amount of social support. Eliminating the heterogeneity within at least one of those factors may help answering the question if the post-stroke depression is caused by brain lesion or by disturbed relations with the environment. Old-age home's $(\mathrm{OAH})$ residents constitute a model of population of subjects receiving the same amount of social support.

Aim of the study was to compare the prevalence of depression in post-stroke residents of OAHs and in controls (residents without stroke history).

Methods: We have performed a screening study in OAHs in Gdansk, Poland. We have used shortened Geriatric Depression Scale (GDS) and a structured neurologica interview.

Results: We have examined 164 residents of OAHs. Mean age of the examined group was $80.3 \pm 8.2$ years. 26 subjects $(15.8 \%)$ underwent stroke in the past. According to GDS results, depression was present in $73,1 \%$ of post-stroke patients and in $58.7 \%$ of controls $(\mathrm{p}<0,05)$. The mean severity of depression in the post-stroke group was 8,2 points and in controls it was $6.5(\mathrm{p}<0,05)$

Discussion: 1. Depression is more frequent among the post-stroke residents of old-age-homes. 2. The intensity of depression in the post-stroke population is higher. 3. These two findings may suggest that post-stroke depression is more likely to be a consequence of focal brain lesion than of social consequences of the stroke.

\section{Behavior and mood}

SELF-REPORTED DEPRESSIVE SYMPTOMS FOUR YEARS AFTER STROKE C.H. Nolte, G.J. Jungehulsing, J. Muller-Nordhorn, K. Rossnagel, S.N. Willich, S. Roll, A. Villringer

Universitätsmedizin Charite Berlin, Berlin, Germany

Background and Purpose: Data on depressive symptoms after stroke mainly refers to its short-term prevalence. We studied the frequency and therapy of self-reported depressive symptoms four years after stroke.

Methods: Data of patients with confirmed stroke consecutively recruited in the Berlin Acute Stroke Study were analysed. A follow-up questionnaire about resource use including the Beck Depression Inventory (BDI) was completed four years after acute hospital admission. Cutoff points of 10 and 17 on the BDI were used to group patients with mild and severe depressive symptoms, respectively. Multiple regression analysis, with stepwise backward variable selection, was used to determine factors associated with depression.

Results: Mean age of a total of 193 patients was 64 years (range 29-92) and $40 \%$ were female. Mild and severe depressive symptoms were reported by $21 \%$ and $15 \%$ of all patients, respectively. Of patients with mild and severe depressive symptoms, only 5\% and 32\% received antidepressants and $46 \%$ and $50 \%$ received specialized medical care, respectively. Factors associated with the frequency of severe depressive symptoms were younger age, living alone at time of stroke, a lower educational level, and non-professional care by family members.

Discussion: Depressive symptoms after stroke are frequent but undertreated. The setting of care has to be considered. Routine clinical practice to assess, diagnose and prompt treatment of depression after stroke appears to be still suboptimal.

\section{Behavior and mood}

POST-STROKE DEPRESSION: A 24-MONTH FOLLOW UP

M. Altieri, I. Maestrini, V. Di Piero, E. Sbardella, A. Mercurio, G.L. Lenzi University of Rome "La Sapienza", Rome, Italy

Introduction: Depression is a frequent psychiatric complication after stroke and is linked to a higher degree of disability and recurrences. Nevertheless, its time course and causes are still debated. During a 24-month period after stroke, we investigated the occurrence, time course and predictors of depressive symptoms.

Methods: all the consecutive acute stroke patients admitted to our ward were examined according to a standardized procedure. Patients with TIAs, severe disability (MRS score 5), serious comprehension difficulties or dementia and history of psychiatric disturbances were excluded. Depressive symptoms were assessed at 
2 weeks and 2, 6, 12, 18 and 24 months after stroke with the Beck Depression Inventory using a cut-off $\geq 10$. Depression was diagnosed according to the DSM-IV criteria.

Results: During a one-year period out of 116 stroke patients, 109 (age 64.5 \pm 10.2 years; $\mathrm{m} / \mathrm{f} 77 / 32)$ were enrolled. During the entire follow-up $34(31.2 \%)$ patient scored 10 or more in the BDI. Ten patients $(29.4 \%)$ developed depression at 2 weeks, $5(14.7 \%)$ at 2 months, $5(14.7 \%)$ at 6 months, $6(17.6 \%)$ at 12 months, $3(8.8 \%)$ at 18 months, and $5(14.7 \%)$ at 24 months. Depressive symptoms were associated with female sex $(\mathrm{p}=0.05)$, disability $(0.03)$, and lesion location in the anterior circulation $(\mathrm{p}=0.05)$. Of those who were depressed at 6 months, $61 \%$ were also depressed at the end of follow up.

Conclusion: Our data indicate that depressive symptoms are frequent after stroke, and often have a chronic course. The origin is multifactorial, as constitutional and clinical factors seem to be involved.

\section{Behavior and mood}

AWARENESS OF STROKE WARNING SIGNS IN TWO RURAL COUNTIES, MISSISSIPPI, USA

M. Shahbazi, Z. Sarraf, M. Sarraf

School of Public Health, Jackson State University; University of Shiraz Medical Sciences, Brandon, MS, USA

The soioeconomically influential citizens of the U.S. A. enjoy better health than do its minority and poorer citizens. The most striking health disparities involve shorter life expectancy among the poor, as well as higher rates of cancer, birth defects, infant mortality, asthma, diabetes, and cardiovascular disease - stroke in particular Mississippi has one of the highest stroke mortality rates in the nation. In preparation for a community stroke education intervention, baseline surveys were undertaken in the intervention and control counties.

Random-digit-dialed telephone surveys were conducted on adults 18 and olde sampled from the civilian, non-institutionalized populations of Warren (control) and Washington (intervention) counties, Mississippi. Using open-ended questions, respondents were asked about knowledge of stroke risk factors, stroke symptoms and signs, and actions to be taken in the event of a stroke. Data were analyzed (without weighting) using SPSS and SAS.

In both counties, approximately $80 \%$ of respondents stated that strokes could be prevented, but knowledge of specific stroke risk factors was poor. Less than $50 \%$ of respondents named hypertension, and knowledge of other risk factors was much poorer. Recognition of individual stroke warning signs was fair to poor: just fewer than $50 \%$ named weakness or numbness and fewer than $25 \%$ named others such as dizziness or loss of vision. When responses were analyzed in aggregate, the results were very poor: only $2-3 \%$ could name all 5 major warning signs of stroke, and only $1-2 \%$ could name all signs correctly and state the correct action to be taken (call 911).

Awareness of stroke warning signs and knowledge of correct action to be taken in the event of a stroke are very poor. A major public education campaign is in progress that would follow a post-survey to determine if stroke awareness education would impact stroke-related behaviors.

\section{Locomotion, gait and posture}

\section{Locomotion, gait and posture}

SPATIO-TEMPORAL EFFECTS OF 6-MONTHS TREADMILL TRAINING IN INDIVIDUALS WITH CHRONIC STROKE

L.W. Forrester, S.L. Patterson, M.M. Rodgers, R.F. Macko

University of Maryland School of Medicine/Baltimore VAMC, Baltimore, MD, USA

Background: Gait deficits due to chronic hemiparesis after stroke contribute to comorbidities related to reduced activity and consequent deconditioning. A recent randomized controlled trial indicates that 6-mos. treadmill aerobic exercise training (TAEX) can improve cardiovascular fitness and functional mobility in chronic stroke survivors, as measured by peak VO2 and timed walk tests. The purpose of this study was to determine changes in spatial and temporal gait parameters relative to observed changes in global walking function before and after 6 mos. TAEX.

Methods: Chronic stroke patients $(\mathrm{n}=39,>6$-mos. post stroke) were consented and medically cleared for TAEX. Baseline measures included VO2 peak, $30 \mathrm{ft}$ and 6-min walks with usual assistive device, and unassisted preferred-speed walks on an 8 meter instrumented walkway. Training was $3 \times$ weekly for 6 mos., with intensity progressed to achieve $60-70 \%$ heart rate reserve for up to $40 \mathrm{~min}$. Statistical comparisons used paired t-tests.

Results: TAEX improved peak VO2 (22\%), 30-ft (17\%) and 6-min (23\%) walks $(\mathrm{p}<0.01$ in all). Unassisted walking velocity $(\mathrm{p}<0.01)$, cadence $(\mathrm{p}<0.01)$ and stride length $(\mathrm{p}<0.01)$ increased. Paretic $(\mathrm{P})$ and nonparetic $(\mathrm{NP})$ step lengths increased (both: $\mathrm{p}<0.01$ ), absolute stance times (both: $\mathrm{p}<0.02$ ) and P-relative stance time $(\mathrm{p}<0.01)$ decreased. Absolute $(\mathrm{p}<0.02)$ and relative $(\mathrm{p}<0.02)$ double-stance times decreased. Paretic relative swing phase duration increased $(\mathrm{p}<0.01)$. Symmetry measures between limbs were unchanged.

Discussion: The TAEX intervention increased unassisted walking velocity in chronic hemiparetic stroke patients by improving step lengths and timing parameters in both P and NP legs, but it did not alter interlimb symmetry. The faster velocity also may be associated with improved dynamic postural stability as indexed by reduced double-stance times. Randomized studies are underway to further examine relationships between altered gait parameters and mobility function.

\section{Locomotion, gait and posture}

\section{THIGH-LEG COORDINATION IN THE UNAFFECTED LOWER EXTREMITY} IN CHRONIC HEMIPARESIS FOLLOWING STROKE

L. Rinaldi, M. Inzitari, M. Paci, M. Baccini, M. Di Bari, N. Marchionni, G. Masotti

Unit of Gerontology and Geriatric Medicine, University of Florence and Azienda Ospedaliero-Universitaria Careggi, Italy

Background: Movement abnormalities have been demonstrated in the unaffected upper extremity of chronic hemiparetic patients. Whether the lower extremity is also affected is unclear. This study investigated possible alterations of thigh-leg coordination in the unaffected lower extremity during treadmill walking, using computerized gait analysis.

Methods: Five patients with left hemiparesis (LH) (mean age \pm SD $69 \pm 8,60 \%$ men), and 5 with right hemiparesis (RH) (mean age $62 \pm 8,80 \%$ men) were enrolled 9 months after a stroke. Ten healthy volunteers (mean age $65 \pm 9,70 \%$ men, no significant difference compared to both $\mathrm{LH}$ and $\mathrm{RH}$ ) were recruited as a control group. Thigh-leg continuous relative phase (CRP) curves for each stride were obtained from 20 -second recordings, using a $5 \mathrm{TV}$ cameras $(100 \mathrm{~Hz})$ optoelectronic motion analysis system, while subjects were walking on a treadmill at two velocities $(0.38$ and $0.67 \mathrm{~m} / \mathrm{s}$ ). Each participant's individual curves were accepted for averaging after reliability analysis. Thigh-leg CRP curves of the paretic and unaffected extremity of the hemiparetic groups were compared to the mean curve of the two extremities in the control group: the differences were first tested using the ANOVA mixed linear model; the bootstrap 95\% confidence bands were then calculated, to identify the specific intervals of the gait cycle where the two sets of curves diverged.

Results: Thigh-leg CRP curves of the paretic extremity differed significantly at the two velocities in both RH and LH, compared to the control group ( $p<0.001$ for all the differences). In RH, the curves of the unaffected extremity were comparable to those in the control group, whereas they differed significantly in LH. Specifically, differences were observed, at lower velocity, during loading response (around 5\% of gait cycle), mid-stance $(25 \%)$, at the end of terminal stance $(50 \%)$ and at toe-off $(70 \%)(\mathrm{p}=0.001)$, whereas at higher velocity only at terminal stance $(\mathrm{p}=0.001)$.

Discussion: At computerized gait analysis, only patients with LH show abnormal thigh-leg coordination in the unaffected extremity.

\section{Locomotion, gait and posture}

\section{FACTOR OF DETERMINATION OF THE CENTER OF GRAVITY POINT ON STANDING IN HEMIPLEGICS FOLLOWING STROKE}

S. Morioka, A. Matsuo, M. Hiyamizu

Kio University, Kita-katsuragigun, Nara, Japan

Background and Purpose: In a standing position the center of gravity of hemiplegics following stroke is defined limited to the relative weight loading on paralyzed leg. The purpose of this study was to clarify whether or not the center of gravity shifting to the non-paralytic side in hemiplegics following stroke was influenced by relative distances of the voluntary shifted center of gravity from the paralytic side to the non-paralytic side and vice versa.

Methods: Sixty-five hemiplegics who could maintain a standing position for a long time participated in this study. Subjects were able to understand instructions about the experiment gave their informed consent. Bilateral points of the voluntary shifted center of gravity in a standing position were measured by means of the stabilometer (GS2000, ANIMA. CO., LTD.) for 10 seconds, each with the eyes open and closed, and also the voluntary shifting of the center of gravity to the non-paralytic side and the paralytic side. Mean of X-axis was employed as an index for the bilateral points of the center of gravity. The middle of the mean of $\mathrm{X}$ calculated from the center of 
gravity shifted between the non-paralytic side and the paralytic side was used as the middle point.

Results: A significant positive correlation between the spontaneous standing point and the middle point was obtained when the eyes were open $(r=0.61, p<0.01)$ and closed $(r=0.72, p<0.01)$.

Conclusion: The result suggests that the bilateral centers of gravity in the standing position of hemiplegics can be determined relatively by active shifted distances of the centers of gravity between the non-paralytic and paralytic sides.

\section{Epilepsy and stroke}

\section{Epilepsy and stroke}

CLINICALLY RELEVANT INCREASE OF INR IN PATIENTS WITH ISCHEMIC STROKE AND SYMPTOMATIC SEIZURES: DRUG INTERACTION BETWEEN PHENPROCOUMON AND VALPROATE

A. Felbecker, B. Weder, B. Tettenborn

Kantonsspital St. Gallen, St. Gallen, Switzerland

Background: Co-treatment with the oral anticoagulant phenprocoumon and valproate is common, especially in patients suffering from cardioembolic stroke and symptomatic seizures. There are no reports of clinically relevant interactions between these drugs.

Method: We reviewed the data of all in- and outpatients of our neurological department within the last 38 months. Two groups of patients were identified: group 1 was treated with valproate in addition to an established phenprocoumon therapy, whereas in group 2 phenprocoumon was started either at the same time or in addition to valproate. For group 1, the latest available International Normalized Ratio (INR) values were achieved, mean values calculated and correlated to the maximum INR values shortly after the initiation of valproate. Patients were excluded if the dosage of the drugs during the relevant time was unknown or compliance was insufficient. Results: 18 patients received a combined treatment with valproate and phenprocoumon. Three patients met exclusion criteria, the remaining patients were divided into group 1 (11 patients) and group 2 (4 patients). Means of INR values in group 1 before treatment with valproate ranged between 1.6 and 3.2 (SD 0.05-1.22) and raised significantly after initiation of valproate (mean $+77 \%$, range from +45 to $+138 \%$ ). In group 2 , the intended INR values were achieved with unexpectedly low doses of phenprocoumon.

Discussion: There is a clinically relevant interaction between valproate and phenprocoumon. Patients on valproate need unusually low doses of phenprocoumon for active anticoagulation. In patients receiving valproate with preexisting phenprocoumon a potentially hazardous rise of INR has to be observed.

\section{Infection and immunology}

\section{Infection and immunology}

PREDICTORS OF IN-HOSPITAL PNEUMONIA IN ACUTE ISCHEMIC STROKE PATIENTS ADMITTED TO A NEUROLOGICAL INTENSIVE CARE UNIT

A. Kloth, R. Knoblich, V. Steinhagen, R. Benecke, U. Walter

University of Rostock, Rostock, Germany

Background: Pneumonia is a frequent complication after acute stroke and causes approximately $30 \%$ of deaths within the first 30 days after stroke.

Methods: To determine initial clinical predictors of stroke associated pneumonia (SAP), we analyzed demographic and clinical data of 236 patients with complete ischemic stroke who were admitted to the neurological intensive care unit (NICU) of our Neurological Department over a one-year period.

Results: Pneumonia was diagnosed in $51(21.6 \%)$ patients. SAP+ patients had larger mean age compared to SAP- patients $(76.1 \pm 8.3$ vs. $68.1 \pm 13.8$ years; $\mathrm{p}<$ $0.001)$, larger NIHSS $(14.6 \pm 5.8$ vs. $7.6 \pm 5.7 ; \mathrm{p}<0.001)$ and dysphagia $(0.6 \pm 1.0$ vs. $2.0 \pm 1.2 ; \mathrm{p}<0.001)$ scores on admission. Univarate data analysis identified the following clinical predictors of SAP: dysphagia (relative risk $[R R]=9.92 ; p<$ $0.001)$, NIHSS $\geq 10(\mathrm{RR}=6.57 ; \mathrm{p}<0.001)$, combined cerebellar and brainstem infarction $(\mathrm{RR}=4.85 ; \mathrm{p}=0.001)$, infarction affecting $>66 \%$ of middle cerebral artery $(\mathrm{MCA})$ territory $(\mathrm{RR}=3.91 ; \mathrm{p}>0.001)$, infection present on admission $(\mathrm{RR}=3.78 ; \mathrm{p}<0.001)$, impaired vigilance $(\mathrm{RR}=3.47 ; \mathrm{p}<0.001)$, non-lacunar basal ganglia infarction $(\mathrm{RR}=3.10 ; \mathrm{p}=0.004)$, hemispheric stroke exceeding MCA territory $(\mathrm{RR}=2.79 ; \mathrm{p}=0.001)$, mechanical ventilation on admission $(\mathrm{RR}=2.78$; $\mathrm{p}=0.020)$, age $\geq 73$ years $(\mathrm{RR}=2.51 ; \mathrm{p}<0.001)$, current malignoma $(\mathrm{RR}=$ $2.43 ; \mathrm{p}=0.047)$ and cardioembolic stroke $(\mathrm{RR}=1.86 ; \mathrm{p}=0.012)$. Multivariable logistic regression analysis yielded the following independent risk factors: infection present on admission, dysphagia, non-lacunar infarction of basal ganglia, combined cerebellar and brainstem infarction, and NIHSS $\geq 10$. The logistic regression model reached a $82.4 \%$ sensitivity and a $87.5 \%$ specificity for individual SAP occurrence prediction.

Discussion: Independent predictors of SAP are dysphagia, non-lacunar basalganglia infarction, combined cerebellar and brainstem infarction, and NIHSS $\geq 10$. It needs to be further studied whether antibiotic treatment prior to evolution of SAP improves outcome in patients with these initial risk factors.

\section{Infection and immunology}

INITIAL CLINICAL PREDICTORS OF NON-RESPONSIVITY TO STANDARD ANTIBIOTIC TREATMENT IN ACUTE STROKE PATIENTS WITH EARLY-ONSET PNEUMONIA

U. Walter, A. Kloth, R. Knoblich, R. Benecke

University of Rostock, Rostock, Germany

Background: Pneumonia is a frequent complication after acute stroke and accounts for the highest attributable proportion of death in the stroke population. Failure of standard antibiotic treatment of early onset pneumonia (EOP; onset within 72 hours after admission) causes longer treatment duration on intensive care units and enhances probability of fatal outcome.

Methods: To determine clinical predictors, available on patient's admission, of failure of standard antibiotic regimen we analyzed demographic and clinical data of 30 patients with acute ischemic stroke who were admitted to the neurological intensive care unit (NICU) of our Neurological Department over a one-year period and deleloped EOP. EOP in these patients was initially treated with a combination of ceftriaxone and metronidazole.

Results: Seventeen (57\%) patients showed improvement, defined as decrease of CRP of at least $50 \%$ within 7 days and normalization of temperature, whereas $13(43 \%)$ did not respond to initial antibiotic treatment. Univariate data analysis identified occurrence of initial vomiting as a predictor of treatment non-responsivity (relative risk $[R R]=2.57 ; 95 \% \mathrm{CI}, 1.01-6.55 ; \mathrm{p}=0.03)$, whereas age $>74$ years $(\mathrm{RR}=0.37$ CI $0.17-0.79 ; p=0.013$ ) and presence of at least moderate dysphagia $(R R=0.35$; CI $0.28-0.58 ; \mathrm{p}=0.014$ ) were associated with responsivity. Stepwise multivariable logistic regression analysis yielded the following variables as independent predictors of non-responsivity: vomiting (increased risk) and dysphagia (decreased risk). The logistic regression model reached a $85 \%$ sensitivity and a $71 \%$ specificity for individual predicition of antibiotic treatment resonsivity. Microbiological monitoring revealed different pathogens in non-responsive EOP patients (Escherichia coli, aspergillus, candida) and responsive EOP patients (staphylococcus, enterococcus, corynebacterium)

Conclusion: We conclude that EOP patients without severe dysphagia but with initial vomiting should be set on different antibiotic treatment and broader microbiological monitoring.

\section{Infection and immunology}

ALLOPURINOL'S EFFECT ON INTERCELLULAR ADHESION MOLECULE 1 (ICAM) AND INFLAMMATORY CYTOKINES AFTER ISCHAEMIC STROKE: A PILOT STUDY

C.J. Harrow, S.W. Muir, K.R. Lees, M.R. Walters

University of Glasgow, Glasgow, United Kingdom

Background: Serum concentration of ICAM is elevated in stroke patients compared to controls, and elevated ICAM is associated with early neurological deterioration. Allopurinol, a xanthine oxidase inhibitor and free radical scavenger, may inhibit expression of ICAM-1 through modulation of regulatory cytokines. We investigated the effect of allopurinol on ICAM concentration in stroke patients.

Methods: We did a randomised, double blind placebo-controlled trial to investigate the effect of high (300mg od) and low (100mg od) doses of allopurinol on circulating ICAM-1 in patients within 3 days of ischaemic stroke. This was a substudy of a larger trial investigating the effect of allopurinol on urate. ICAM-1, CRP, IL-6 and NIHSS were measured at baseline, 1 and 6 weeks.

Results: 50 patients $(50 \%$ male) were recruited $(n=17,17$ and 16 for high $(H)$, low (L) and placebo (P) groups respectively). Mean (SD) age and baseline NIHSS were 70 (12)yrs and 3.2(1.7) respectively. Groups were well matched in clinical and biochemical characteristics. 2 (12\%) patients withdrew from L and P (1 GI upset and 1 potential drug interaction in each group). 1 patient withdrew from $\mathrm{H}$ (potential drug interaction). 4, 5 and 6 patients with concomitant infection $(\mathrm{CRP}>20)$ were excluded from the biochemical analysis in $\mathrm{H}, \mathrm{L}$ and $\mathrm{P}$ groups respectively. Median 
(IQR) \% change in ICAM at 6 weeks was $(-5.3(-19,+15) ;-3.2(-10,+9.7) ;+23$ $(+7,+38)$ for $\mathrm{H}, \mathrm{L}$ and P respectively $(\mathrm{p}=0.02$ and 0.01 by Mann-Whitney for HvsP and LvsP respectively). No between or within group difference was seen in ICAM at 1 week nor any difference in IL-6, NIHSS or CRP at any time.

Conclusion: Allopurinol attenuated the rise in ICAM seen in the placebo group. This effect was equivalent in both dose groups and took $>1$ week to occur. Manipulation of ICAM may be a future therapeutic target in stroke: further work is required to elucidate the potential clinical significance of this observation.

\section{Infection and immunology}

\section{SEASONAL VARIANCE OF INCIDENCE OF SPONTANEOUS CERVICAL} ARTERY DISSECTIONS

C. Lichy, M. Kloss, T. Brandt, M. Arnold, C. Grond-Ginsbach

University of Heidelberg, Heidelberg, Germany

Background: Recent infection and a proinflammatory diathesis are discussed to contribute to the risk for spontaneous cervical artery dissection (sCAD). Since morbidity due to respiratory infections is highest in the cold seasons, we tested the hypothesis of infection-triggered CAD by investigating the seasonal variance of sCAD incidence.

Methods: We retrospectively analysed the date of $\mathrm{SCAD}$ occurrence in all patients who were diagnosed and treated in our South-German university hospital during the seven years period from 01/1999 until 12/2005. Ninety six consecutive patients with sCAD of the internal carotid artery ( 80 strokes, 10 TIAs, and 6 with local signs only) and 54 with sCAD of the vertebral artery (47 strokes, 6 TIAs, and 1 with local signs) were identified. Mean age was $45.1 \pm 10$ years, $90 / 150$ patients were males. Results: Incidence of $\mathrm{SCAD}$ was highest during spring $(\mathrm{n}=46 / 150 ; 30.7 \%)$, whereas it was lowest during summer $(\mathrm{n}=31 / 150 ; 20.7 \%)$. None of the four seasons, however, showed a significant cluster of SCAD incidence as compared to the year's mean incidence rate.

Discussion: Our data do not support the hypothesis of a higher incidence of SCAD during the year's seasons prone to common respiratory infections.

\section{Infection and immunology}

\section{IMMUNOLOGIC PREDICTORS FOR THE DEVELOPMENT OF} ATHEROSCLEROTIC DISEASE IN PATIENTS WITH OR WITHOUT RISK FACTORS

S. Piconi, D. Trabattoni, A. Sparaco, G. Barozzi, A. d'Arminio-Monforte,

P. Danna, E. Perilli, D. Bray, M. Clerici

University of Milano, Milano, Italy

Background: Atherosclerosis, the underlying basis of myocardial infarction an stroke, can be considered an unusual form of chronic endothelial inflammation occurring within the artery wall. We analyzed the precursor immunological factors potentially associated with the development of atherosclerotic disease in patients with or without risk factors.

Materials and Methods: Observational study. Echo Doppler evaluation of the carotid and femoral arteries as well as immunologic analyses on peripheral blood mononuclear cells (PBMC) were performed on 58 age and sex-matched individuals divided into four groups: Group 1= risk factors (hypertension; smoke; high cholesterol blood concentration), symptoms (ischemic heart disease in various clinical stage and presentation), and plaques $(\mathrm{N}=10)$; Group $2=$ risk factors and plaques without symptoms $(\mathrm{N}=16)$; Group $3=$ risk factors without either plaques or symptoms ( $\mathrm{N}=27)$, and Group $4=$ healthy controls $(\mathrm{N}=5)$.

Results: A complex pattern of alterations was detected, that in most cases differentiated Group 4 from all other groups. Thus: 1) Adhesion markers: CD4/CD62L (L selectin) lymphocytes were significantly increased whereas CD4/CD49d (VLA-4) cells were significanly decreased in all groups compared to Gr 4 individuals; 2) Activation markers: CD4/CD25, CD4/DRII, and CD8/DRII cells were significantly increased in Gr 4 compared to all other patients; 3) Toll like receptors (TLR): TLR2 and TLR4-expressing CD14 cells were diminished in all groups compared to Gr 4 . In addition, a decrease of CD14/CD86 cells (TH2 cytokines-producing cells) was detected in all groups compared to $\mathrm{Gr} 4$ individuals.

Conclusions: Immunological alterations monitoring the rolling, adhesion, and diapedesis of immune cells within the endothelium and associated with the genesis of athero plaques can be detected even in individuals with risk factor alone in whom echo-Doppler does not suggest any abnormality of the vascular walls. Longitudinal analyses in a larger patient population will be necessary to assess whether such alterations have predictive clinical value.
6 Infection and immunology

\section{ASSOCIATION BETWEEN INFLUENZA VACCINATION AND ISCHEMIC} STROKE

G. Piñol, I. De la Puerta, S. Santos, E. Mostacero

Clinical Hospital of Zaragoza, Zaragoza, Spain

Introduction and Objectives: Prior studies had demostrated that infections might precipite ischemic stroke, but the role of infection as a risk factor remains unclear. The most accepted hypothesis is that infection events could cause a hypercoagulation state and arteriosclerotic plaque inestabilization, so that, avoiding influenza infection by vaccination it would protects the ateriosclerotic plaque from this inestabilization. If this is true, influenza vaccination only would protects atherotrombotic stroke and a $20 \%$ of lacunar syndromes, but it would not protects cardioembolic or haemodynamic strokes.

Method: During a year, we permorfed standardized interviews assessing vaccination status (last year, two last years and five last years), risk factors, chronic bronchitis, use of antibiotics in previous 3 month in 393 consecutive patients with ischemic stroke and 400 controls matched for age, sex, classical risk factors (blood pressure, diabetes mellitus, hypercholesterolemia), tabaquism, alcholism and body mass index. The patients with ischemic stroke were classificated in: atherotrombotic, lacunar, cardioembolic, haemodynamic and indeterminate.

Results: The percentage of influenza vaccination was similar in patients (56\%) and controls $(55 \%)(\mathrm{p}=0.8)$. Influenza vaccination does not protect any subtype of stroke $(p>0.05)$. However, chronic bronchitis and acute infections in the three previous month to stroke were more frequent in patients than controls $(\mathrm{p}>0.01$ and $\mathrm{p}<0,015$ respectively).

Conclusions: Our findings provide support for the concept that acute infections are associated with a transient increase in the risk of vascular events. Chronic bronchitis is a risk factor of stroke also. However, influenza vaccination does not protect to ischemic stroke.

\section{Infection and immunology}

ARE BLOOD MONOCYTES CD36 AND HLA-DR RELATED TO INTERNAL CAROTID ARTERY STENOSIS DEGREE?

A. Costa, L. Trainini, A. Caruso, R. Tortorella, V. Vergani, R. Spezi, M. Magoni Spedali Civili di Brescia - Vascular Neurology; University of Brescia Microbiology, Brescia, Italy

CD36 is a receptor for oxidized low density lipoprotein involved in angiogenesis, atherosclerosis, inflammation and lipid metabolism; a glycoprotein expressed on monocytes/macrophages and other cells.

HLA-DR is an index of cell activation and CD14 is selective in detecting monocytes. We attempted to correlate the degree of internal carotid artery (ICA) stenosis and blood monocytes CD36 and HLA-DR expression.

ICA stenosis was grouped with neurosonological criteria: minimal 0-30\% (CC), moderate $30-69 \%$ (MS), severe 70-99\% (SS) and occlusion (OC).

We selected 18 consecutive patients for each neurosonological group (only 7 in OC). With an immunofluorescence flow cytometric analysis with antiCD14-FITC we selected monocytes; we evaluated their CD36APC expression (CD36+) and HLADR-PE expression of CD36+ monocytes (DR+). All with mean fluorescence intensity (MFI), related to the number of markers expressed in a single cell.

An ANOVA analysis with Statwiew software was performed.

Inflammatory markers, vascular risk factors and therapy did not statiscally differ in the groups.

CD36 + in CC, MS, SS and OC were respectively 98.1, 96.3, 90.8 and 97.2\%; CD36 MFI was respectively $286,270,272$ and 254 .

DR+ were respectively $79.0,81.3,88.8$, and $86.5 \%$; HLA-DR MFI was respectively $69.9,79.6,96.0$ and 95.5

Significative differences were found in $\mathrm{DR}+$ group between CC and SS $(\mathrm{P}=.05)$ and between MS and SS $(\mathrm{p}=.05)$ and in HLA-DR MFI between CC and SS $(\mathrm{p}=.05)$.

CD36 expression has a negative correlation trend with ICA stenosis degree but not with occlusions. CD $36 \mathrm{MFI}$ has a negative correlation trend. HLA-DR expression and MFI has a positive correlation.

Blood CD36 low expression in more severe stenosis is probably related to the interaction with the ligand on the plaque, due to monocytes activation. 
Rare stroke conditions (experimental and clinical)

\author{
1 Rare stroke conditions (experimental and clinical)
}

A RE-APPRAISAL OF STROKE RISK FACTORS IN FABRY DISEASE

D. Hayoz, N. Brakch, F. Barbey

Centre Hospitalier Universitaire Vaudois, Lausanne, Lausanne, Switzerland

Background: Fabry disease (FD) is a life-threatening lysosomal storage disorder affecting both genders and is characterized by progressive small vessel heart, brain (TIA or stroke) and kidney disease.

Methods: We investigated possible common causes of cerebrovascular and cardiac lesions in FD by studying radial/carotid artery wall thickness, left ventricular (LV) mass and in vitro cellular proliferation.

Results: Radial artery wall thickness (high-resolution Doppler-echo) was increased in $16 / 17$ FD patients (pts.; $10 \mathrm{M} / 6 \mathrm{~F}, \mathrm{P}<0.001$ ) as compared to controls. In a larger cohort of FD pts. (24M/29F), mean (SD) common carotid artery intimamedia thickness (CCA IMT) was increased; M: 706 \pm 211 microm, F: 749 \pm 395 microm, controls: $614 \pm 113$ microm. Homogeneous circumferential thickening did not correlate with blood pressure. FD pts. (M: $45 \pm 1.7 \mathrm{yr}$; F: $55 \pm 2.2 \mathrm{yr}$ ) had no atherosclerotic lesions but carotid plaques were present in $44 \%$ of the controls $(\mathrm{M}$ : $\mathrm{n}=83,49 \pm 1.7 \mathrm{yr} ; \mathrm{F}: \mathrm{n}=37,52 \pm 1.5 \mathrm{yr}$ ). LV hypertrophy was found in $60 \%$ of men and $39 \%$ of women with FD. There was a strong positive correlation between LV mass and CCA IMT $(\mathrm{R} 2=0.27, \mathrm{P}<0.0001)$. In vitro vascular smooth muscle cell and neonatal cardiomyocyte proliferative response (plasma from 27 untreated FD pts. and 30 controls $)$ correlated with CCA IMT $(\mathrm{R} 2=0.48 ; \mathrm{P}<0.001)$ and LV mass index (R2=0.19, $\mathrm{P}=0.028)$.

Discussion: Most FD pts. showed arterial wall thickening without hypertension or atherosclerotic lesions. As correlations were found between LV mass, CCA IMT and proliferative response, the cardiac and cerebrovascular FD lesions may have a common cause (e.g. growth-promoting agents) other than substrate accumulation in vascular endothelial cells. The use of CCA IMT as a biomarker for cardiac involvement in FD has to be studied further. Cardiac complications such as atrial fibrillation and sustained arrhythmias add to the increased risk of stroke in FD - a disease for which enzyme replacement therapy is available.

\section{Rare stroke conditions (experimental and clinical)}

\section{PATHOLOGIC AND CLINICAL FEATURES OF WATERSHED INFARCTS IN THE BRAIN \\ F. Nagy}

Budai MÁV Hospital-Budapest, Hungary, Hungary

Background: Watershed infarcts are ischemic lesions situated along the border zones between the territories of two major arteries. Altogether, approximately $10-20 \%$ of all brain infarcts are watershed lesions.

Methods: Clinical and pathologic findings were reported in 75 patients. Three types of watershed infarcts were considered: 1) those between the superficial territories of the middle and anterior cerebral arteries, 2) those in the border zone between the superficial territories of the middle and posterior cerebral arteries, 3) those in the border zone between the superficial and deep territories of the middle cerebral artery.

Results: There were 48 men and 27 women. Watershed infarct was the first event in $55 \%$ of the patients. Clinical symptoms differed according to the site of infarct. Anterior infarcts (44\%) caused mainly lower limb hemiparesis sparing the face, associated with impaired sensation in a part of the cases; in dominant hemispheres there often occurred transcortical aphasia, preceded by mutism in a few cases. In posterior infarcts $(39 \%)$ hemianopia was the most common symptom; hemihypaesthesia was also common, but limb paresis was rare. In dominant hemisphere lesions, transcortical sensory aphasia or Wernicke- type aphasia was present. In subcortical infarcts $(17 \%)$ hemiparesis was seen in all cases, hemisensory loss was present in about one- half of the cases, aphasia was common in dominant hemispheric lesions. Discussion: The mechanism of the development of watershed infarcts is multifactorial, and distinguishing between watershed infarcts and other types of infarcts is important because the different pathogenic mechanisms require different therapeutic strategies.

\section{Acute stroke: early management and stroke units}

1 Acute stroke: early management and stroke units

MANAGEMENT OF STROKE IN AN EXTENDED STROKE UNIT COMBINED WITH A REHABILITATION DEPARTMENT AND IN GENERAL WARDS. WHICH ASPECTS MAKE THE BENEFIT?

P. Desfontaines, X. Masson, A. Debrun, P. Camelbeeck

Centre Hospitalier Chrétien - Liège, Montegnée, Belgium

Background: Several trials have shown that stroke unit (SU) care improves outcome for stroke patients. The aim of the study was to identify which aspects of the SU care were most responsible for the better outcome by comparison with the management in a general ward $(\mathrm{GW})$.

Method: 178 consecutive acute stroke patients were admitted in the SU during the first six months of 2005 and retrospectively compared with 134 consecutive acute stroke patients admitted in the GW within the last 6 months before the opening of the SU in 1998. We identified the differences in the investigation tools, medical and surgical treatments and rehabilitation cares given to the stroke patients. We analysed the association of these factors with the outcome, discharge to home or institutionalization within 3 months.

Results: At 3 months, $12.0 \%$ of the SU patients were in institutions versus (vs) $25.8 \%$ of the GW patients $(\mathrm{p}=0.003), 77.6 \%$ of the SU patients were at home vs $51.7 \%$ of the GW patients $(\mathrm{p}=0.001)$ and $10.4 \%$ of the SU patients were dead vs $22.5 \%$ of the GW patients $(\mathrm{p}=0.004) .18 \%$ of the $\mathrm{GW}$ patients died during the hospitalization vs $7.4 \%$ of the SU patients $(\mathrm{p}=0.05)$. At 3 months, $82.5 \%$ of the SU patients had a modified Rankin score $\leq 2$ vs $58.4 \%$ of the GW patients $(\mathrm{p}=0.01)$. Characteristic features of our SU were a standard protocol of management of stroke patients, an integrated physiotherapy and nursing, a teamwork and staff education program. Other significant differences with GW were an increased use of anticoagulation $(22.4 \%$ in the SU vs $4.5 \%$ in $\mathrm{GW}, \mathrm{p}=0.001)$ and fibrinolysis $(9.5 \%$ in the $\mathrm{SU}$ vs $1.2 \%$ in the $\mathrm{GW}, \mathrm{p}=0.004)$, and a shorter time to start the rehabilitation cares (more than 24 hours after admission in $60 \%$ of the cases in the GW vs $28 \%$ in the $\mathrm{SU}, \mathrm{p}=0.001$ ).

Conclusion: A shorter time to start the rehabilitation therapy and the increased use of fibrinolysis and anticoagulation were the most important factors distinguishing SU from GW. This was mainly due to the presence of a protocole of an integrated management of stroke patients from the emergency department to the rehabilitation service.

2 Acute stroke: early management and stroke units

PAROXYSMAL ATRIAL FIBRILLATION (PAF) IN PATIENTS WITH ISCHEMIC STROKE: DIAGNOSTIC IN STROKE UNIT

A. Rodríguez-Campello, A. Ois, M. Gomis, J. Jiménez-Conde, E. Cuadrado, A. Gálvez, N. Segura, J. Roquer

Stroke Unit, Hospital del Mar, Spain

Background: Atrial fibrillation (AF) is an independent risk factor for suffering stroke and it is an independent poor outcome predictor. Detection of this arrhythmia after stroke is essential due the therapeutic implications. The fibrillation pattern is intermittent in $30 \%$ of cases and it may not appear on a single recording. 24-hour ECG Holter allows the detection of previously unrecognized AF in $2 \%$ of stroke patients. Stroke Unit (SU) prolongs monitoring time and increases the probability of detecting PAF. The aim of our study is to evaluate the PAF incidence in SU and the characteristics of patients with unknown AF after suffering an acute ischemic stroke.

Methods: Consecutive patients with acute stroke admitted to SU between June to December 2005. All patients were monitorized during 48-72 hours. Diagnosis of PAF was confirmed if patient had no previous history of AF, the ECG performed on admission had sinus rhythm, and AF irregular register was greater than 30 seconds. Clinical data, stroke mechanism, antithrombotic treatment and outcome (modified Rankin Scale (mRS) at discharge) were evaluated.

Results: 117 patients with ischemic stroke were admitted in the Stroke Unit. In 12 patients $(10.3 \%)$ new diagnosis PAF was established during monitoring. Mean age was $78.8 \pm 5.8$ years. $50 \%$ of patients were women. Previous TOAST diagnostic was undetermined in all patients. After PAF detection, TOAST classification changed to cardioembolic stroke subtype. Initial antiplatelet treatment was continuated in 5 patients and anticoagulant therapy was started in 7 cases. Outcome was favourable: mRS $0-2$ in 8 patients $(66.7 \%)$. 1 patient died $(8.3 \%)$.

Conclusion: Stroke Unit ECG monitoring is useful and detects PAF in $10.3 \%$ of patients with normal standard ECG. This finding modified previous treatment in $58.3 \%$ cases. $66.7 \%$ of patients had good outcome. Large studies are necessary to stablish utility of monitoring in SU to detect new PAF.
Poster Session Second Visit Acute stroke: early management and stroke units 
3 Acute stroke: early management and stroke units

INSULAR INFARCTIONS ARE ASSOCIATED WITH STEEPER BLOOD PRESSURE CHANGES DURING THE ACUTE STAGE OF STROKE

E. Manios, K. Spengos, G. Tsivgoulis, E. Koroboki, K. Vemmos, N. Zakopoulos University of Athens, Athens, Greece

Background: The insular cortex is involved in the autonomic nervous system regulation and insular infarcts have been associated with increased norepinephrine levels, higher frequency of ECG abnormalities and pathological circadian blood pressure (BP) patterns. We aimed to assess the rate of BP changes in patients with ischemic insular vs. non-insular cortex infarcts using intra-arterial BP-monitoring (BPM).

Methods: A total of 32 patients with cortical infarcts underwent intra-arterial BPM within 3 hours of ictus. Demographics, risk factors, stroke severity and neuroimaging findings were documented. Patients on antihypertensive medication during the BPM were excluded. The rate of systolic (SBP) and diastolic (DBP) changes was assessed by computerized BPM data analysis as the first derivative of SBP and DBP values against time. Statistical analysis was performed by means of unpaired t-test and multiple linear regression models.

Results: The rate of SBP changes was significantly $(\mathrm{p}=0.036)$ higher in insular ( $\mathrm{n}=13$; 24h-rate: $0.015 \mathrm{mmHg} / \mathrm{sec}$, 95\%CI: $0.010-0.020 \mathrm{mmHg} / \mathrm{sec}$ ) than in non-insular cortical infarction $(n=19,24 \mathrm{~h}$-rate: $0.008 \mathrm{mmHg} / \mathrm{sec}, 95 \%$ CI: 0.004 $0.012 \mathrm{mmHg} / \mathrm{sec})$. We also documented significantly $(\mathrm{p}=0.021)$ steeper DBP changes in patients with insular (24h-rate: $0.010 \mathrm{mmHg} / \mathrm{sec}, 95 \% \mathrm{CI}: 0.007-0.013 \mathrm{mmHg} / \mathrm{sec}$ ) in comparison to the non-insular infarction group (24h-rate: $0.005 \mathrm{mmHg} / \mathrm{sec}$, 95\%CI: $0.002-0.008 \mathrm{mmHg} / \mathrm{sec})$. Involvement of the insular cortex was independently $(\mathrm{p}<0.05)$ associated with an increment of $0.007 \mathrm{mmHg} / \mathrm{sec}$ and of $0.005 \mathrm{mmHg} / \mathrm{sec}$ in the rate of SBP and DBP changes respectively even after adjusting for baseline characteristics, stroke severity and neuroimaging findings. Discussion: Steeper BP changes are associated with acute insular cortical infarction, underscoring the importance of insular cortex in the central autonomic network and its potential association with cardio-autonomic dysfunction during the acute stage of stroke.

\section{Acute stroke: early management and stroke units}

PRELIMINARY AUDIT RESULTS OF A CLINICIAN DRIVEN, GOVERNMENT FUNDED PROGRAM TO IMPLEMENT 19 'NETWORKED' STROKE CARE UNITS (SCUS) IN ONE AUSTRALIAN STATE

D.A. Cadilhac, D.C. Pearce, C. Levi, G.A. Donnan

National Stroke Research Institute, Heidelberg Heights, Australia

Background: About $20 \%$ of Australian public hospitals provide acute SCUs. In November 2001, the New South Wales (NSW) state government funded a large scale, health system re-design program that included the establishment of 19 new acute SCUs. This unique, clinician-led program aimed to improve patient access to evidence-based stroke services, with formalised networks between hospitals Time-series (pre-post intervention design), cross-sectional audits of medical records from 11 sites were conducted in 2005 to determine the effects of this initiative for improving access to organised stroke care and care quality.

Methods: 50 consecutive medical records for 2 time periods ( 12 months prior and a minimum 6-12 months following SCU implementation) were audited. Combined, intention-to-treat analysis for 8 sites are presented. Outcomes: process indicators and patient outcomes.

Results: Baseline cases ( $\mathrm{n}=415)$ (mean age 74 SD 13, male: $44 \%)$ and 2005 cases $(n=341)$ (mean age 74 SD 15, male: $45 \%)$ were comparable. The odds of admission to a SCU post GMCT was 31.9 (95\%CI 17.8-57.2). Significant improvements $(\mathrm{p}<0.05)$ across most process of care indicators were found, including aspirin with 24 hours $(57 \%$ versus $67 \%)$ and antithrombotic medication on discharge $(51 \%$ versus $74 \%$ ). Within 24 hours of admission, the odds of patients undergoing CT (OR 3.6 95\% CI 2.1-6.1) and swallowing assessment (OR 2.5 95\%CI 1.9-3.4) improved significantly over baseline. Despite sample size limitations, evidence for reduced mortality (OR 0.72 95\% CI 0.46-1.13) and greater independence (OR 1.06 95\% CI 0.76-1.49) were noted

Conclusions: This clinician-led, government funded stroke program in NSW, Australia has proved effective in improving access to SCUs and clinical performance given the indicators assessed.
5 Acute stroke: early management and stroke units

THE EFFECTS OF POSITIONING ON VITAL PARAMETERS IN ACUTE ISCHEMIC STROKE

J. Huzen, C. Steen, N. Maurits, J. De Keyser, G.J. Luijckx

University medical Centre Groningen, Groningen, The Netherlands

Background: prevention of secondary brain damage after acute ischemic stroke which can be caused hypoxemia and hypotension, is essential for outcome. In acute stroke care units vital parameters are monitored and corrected to minimize this secondary brain damage. Little is known what the effect is of routine positions on vital parameters in acute ischemic stroke patients. Therefore we investigated the effect of positioning on vital parameters in these patients.

Methods: Measurements of heart rate, oxygen saturation ( $\mathrm{SaO} 2)$, systolic, diastolic and mean blood pressure were recorded in 4 different positions (supine, half supine in bed, lying on left side and lying on right side). Each position was maintained for 1 hour whereby 4 measurements were recorded.

Results: 24 patients were recorded, of which 10 male, mean age was 73.4 years $(\mathrm{SD}=6.3)$, and 14 patients had a right sided brain infarction. Median time between start recording parameters and stroke onset was $14.2 \mathrm{~h}$ (2-24 h). Mean $\mathrm{SaO} 2$ was significant higher in supine position in comparison with the other 3 positions $\left(97.6 \%\right.$ vrs $\left.96.4 \%,{ }^{\prime} \mathrm{P}<0.05\right)$. There were no significant differences between the other 3 positions. Mean blood pressure in half supine differed significantly from the other positions $(105.5 \mathrm{~mm} \mathrm{Hg}$ vrs $99.4 \mathrm{~mm} \mathrm{Hg}, \mathrm{P}<0.05)$. Between the other 3 positions there were no significant differences in mean blood pressure. There were no significant differences found in mean heart rate between the 4 positions.

Discussion: Routine positioning in acute ischemic stroke patients may influence vital parameters. Sa02 was significantly higher in supine position and mean blood pressure in half supine position. Further study will need to see if positioning patients in acute ischemic stroke may improve outcome.

6 Acute stroke: early management and stroke units

\section{DIAGNOSTIC BENEFIT OF TRANSCRANIAL EMBOLI DETECTION IN} UNSELECTED STROKE PATIENTS

H. Poppert, S. Sadikovic, K. Sander, O. Wolf, L. Esposito, D. Sander

Technical University Munich, Munich, Germany

Background and Purpose: Embolic signals (ES) detected by transcranial ultrasound have been advocated as surrogate markers for future neurological events after first ischemic stroke.

We aimed to evaluate the feasibility and diagnostic benefit of the technique in clinical routine diagnostics.

Subjects and Methods: The records of 937 consecutive patients who underwent microembolic monitoring at the Neurovascular Laboratory of the Munich Technical University Hospital were reviewed. The TOAST criteria were utilized for classification of stroke etiology.

Results: ES were detected in $5.7 \%$ of patients. The presence of ES was significantly associated with embolic origin of stroke, short time from symptom onset and anticoagulation instead of antiplatelet treatment.

Conclusion: We found a high specificity of the technique aiming to clarify stroke etiology. The pharmacological efficacy of antithrombotic treatment can be assessed in ES-positive patients. However, with respect to the low overall prevalence of ES, the technique cannot generally be recommended as a routine examination in unselected stroke patients.

\section{Acute stroke: early management and stroke units}

MORE THAN HALF OF ALL STROKE PATIENTS ARE DEPENDENT OF SPOUSES/KINDRED THREE MONTH AFTER A STROKE. RESULTS FROM SWEDISH NATIONAL QUALITY REGISTER "RIKS-STROKE"

A.B. Stegmayr, for the Riks-Stroke Steering Committee

Department of Public Health and Clinical Medicine/Medicine, Sweden

Background: The burden of stroke is huge for the patients - but also for the spouses and other kindred to the patients.

Methods: Hospitalized stroke patients have been included in "Riks-Stroke", the Swedish national quality register for stroke care. Riks-Stroke has been ongoing for more than 10 years with the opportunity to evaluate routine stroke care. Riks-Stroke is established in all hospitals in Sweden caring for stroke patients $(n=80)$. In Sweden almost all stroke patients are treated in hospitals. Annually around 24,000 stroke events are included into the data bas. This report is based on data collection from 2004 and includes 24,017 events. A 3-month follow-up was performed in 88 per cent of all patients. 
Results: The mean age in men was 73.4 years and in women 77.9 years and $78 \%$ of the patients were treated in stroke units. Three month after the stroke event, $17 \%$ patients were completely dependent and $45 \%$ partly dependent of spouse/kindred. There were no differences if the patients had been treated in stroke units or in general ward. In patients $\geq 75$ years old, the proportion completely dependent were $21 \%$ and in younger, $<75$ yrs old stroke patients, $12.5 \%$ were completely dependent of a spouse or kindred with only minor differences between men and women.

Conclusions: Three month after a stroke, sixty per cent of the patients are completely or partly dependent of another person. The burden for the spouses or kindred is noticeable and need to be considered in the planning for the stroke patients.

\section{Acute stroke: early management and stroke units}

THROMBOLYSIS, THE MORE TIME LEFT, THE LESS SPEED

T. Schreuder, C. Franke

Atrium Medical Center, Heerlen, The Netherlands

Background: The time window for thrombolysis in acute ischemic stroke is three hours. This time window consisted of two parts: onset of stroke till arriving at hospital, the onset-to-door time, and from arriving at hospital till the start of the thrombolysis, the door-to-needle time. According to the NINDS-criteria the door-to-needle time may not exceed one hour. We investigated the door-to-needle time in relation to the onset-to-door time.

Methods: We registered data on onset-to-door and door-to-needle time prospectively as part of our thrombolysis registration in order to evaluate the quality of this therapy. The analysis centers on the door-to-needle time needed for thrombolytic therapy.

Results: 150 patients ( 80 men) with a mean age of 72 years (21-95) received treatment between June 2001 (first thrombolysis) and May 2005. The mean NIHSS-score at admittance was 11 (1-22), the mean onset-to-door time 57 minutes (10-130) and the mean door-to-needle time 78 (20-155). The mean onset-to-door time reduced from 69 minutes in 2001 to 48 in 2005 . The mean door-to-needle time shortened from 101 in 2001 to 76 minutes in 2003. After 2003 it did not shorten anymore. A short onset-to-door time led to an evidently longer door-to-needle time: of all patients the $25 \%$ with the shortest onset-to-door time (mean 29 minutes (10-40)) had a door-to-needle time of 88 minutes versus 68 minutes for the $25 \%$ with the longest onset-to-door time (mean 95 minutes (80-130)), a difference of 20 minutes. Discussion: The results of the study shows that we did not comply with the NINDScriteria for door-to-needle time. We should speed up pre-thrombolysis workup, not only for patients arriving late after stroke onset with less time left for thrombolysis, but especially for patients arriving early.

\section{Acute stroke: early management and stroke units}

\section{COMPARISON OF NON-INVASIVE OSCILLOMETRIC AND} INTRA-ARTERIAL BLOOD PRESSURE MEASUREMENTS IN HYPERACUTE STROKE

E. Manios, K. Xynos, M. Saliaris, C. Zafeiriou, K. Kostopoulos, E. Koroboki, A. Peppa, K. Vemmos

Acute Stroke Unit, Alexandra hospital, Athens University, Athens, Greece

Background: Blood pressure (BP) management in acute stroke remains a matter of little consensus. Aim of our study was to compare non-invasive automatic oscillometric (NIAO) BP recordings to simultaneous direct intra-arterial BP measurements in order to test the accuracy of oscillometric readings in our study population.

Methods: A total of 51 first-ever stroke patients underwent simultaneously NIAO and intra-arterial BP monitoring within 3 hours after symptom onset. Casual BP was measured in both arms using a standard mercury sphygmomanometer on hospital admission. Statistical analyses were performed using paired t-test and multivariate linear regression model.

Results: The estimation of systolic BP using NIAO recordings significantly underestimated direct radial artery systolic BP by $8.7 \mathrm{mmHg}$ (CI: $5.2-12.3$ ) and $12.0 \mathrm{mmHg}$ (CI: 4.3-19.7) in patients with IS and ICH respectively. In contrast, a significant upward bias of $6.1 \mathrm{mmHg}(\mathrm{CI}: 4.1-8.1)$ and $6.2 \mathrm{mmHg}$ (CI: $0.3-12.1)$ was documented when NIAO diastolic BP recordings were compared to intra-arterial diastolic $\mathrm{BP}$ recordings in IS and $\mathrm{ICH}$ patients respectively. An increment of $10-\mathrm{mmHg}$ in admission systolic BP was independently $(\mathrm{p}=0.001)$ associated with an increase of $2.35 \mathrm{mmHg}$ (CI: $0.97-3.37$ ) in the difference between intra-arterial and NIAO systolic BP (dSBP). History of diabetes correlated significantly $(\mathrm{p}=0.029)$ with an increase of $7.469 \mathrm{mmHg}$ (CI: 0.80-14.14) in dSBP.

Discussion: Non-invasive automatic oscillometric BP measurements underestimate direct SBP recordings and overestimate direct DBP readings in acute stroke. The magnitude of the discrepancy between intra-arterial and oscillometric SBP record- ings is even more prominent in patients with critically elevated SBP levels and diabetes.

10 Acute stroke: early management and stroke units

FEASIBILITY AND SAFETY OF A MULTICENTRE RANDOMISED CONTROLLED TRIAL OF VERY EARLY REHABILITATION (AVERT): PHASE II

J. Bernhardt, H.M. Dewey, J.M. Collier, A.G. Thrift, G.A. Donnan

National Stroke Research Institute, Heidelberg Heights, Melbourne, Australia

Background: Very early stroke rehabilitation (VER) with a focus on mobilisation, may play an important role in effective stroke unit care. In AVERT Phase II we aimed to establish the safety and feasibility of a single-blind, multicentre randomised controlled trial of VER.

Methods: We included non-comatose patients admitted within 24 hours of confirmed stroke presenting to 2 metropolitan stroke units. Randomisation was blocked and stratified for stroke severity. Patients with severe premorbid disability and comorbidities, or requiring rtPA and palliative care were excluded. Recruited patients were randomised into either VER or routine care. VER commences day 0 post stroke and continues for 14 days or until discharge, provided by a nurse/physiotherapist team. Assessments at days 7, 14 and 3 months were conducted by a blinded assessor. Primary outcome: Death at 3 months. Secondary outcomes: Complications, particularly falls.

Results: 71 patients (mean age 74.0) have been recruited since April 2004, with 2 drop outs. This represents $18 \%$ of all stroke patients admitted. Main reasons for ineligibility were: (1) admission $>24$ hours post stroke (40\%), (2) TIA or uncertain diagnosis (15\%) and (3) met trial exclusion criteria (23\%).

Demographics: There were $52 \%$ males, $90 \%$ were living at home prior to stroke and $75 \%$ had experienced their first stroke. Stroke type was classified with $23 \%$ LACI, $33 \%$ PACI, $16 \%$ POCI, $16 \%$ TACI and $12 \%$ haemorrhage. Sixty eight patients have completed 3 month follow up with a total of 12 deaths $(17.6 \%)$ over that time. There have been no serious falls during the intervention phase and no evidence of contamination of routine care.

Conclusion: The study is safe and feasible. The Phase II database will be closed Jan 14, 2006. Recently acquired funding of AUD \$2.8 million has prompted the Steering Committee to recommend early transition to the larger $(\mathrm{n}=2000)$ Phase III study.

11 Acute stroke: early management and stroke units

\section{SERUM PROTEIN S 100 B IN ACUTE STROKE PATIENTS ADMITTED TO NEUROINTENSIVE CARE UNIT}

V. Spatenkova, P. Skrabalek, P. Suchomel, J. Dienelt

Neurocentre ICU, Regional Hospital Liberec, Liberec, Czech Republic

Objective: Protein S 100B is a non-invasive marker of cerebral tissue damage. The aim of our study was to evaluate serum levels of protein S $100 \mathrm{~B}$ in patients with acute stroke admitted to the neurointensive care unit.

Methods: We prospectively measured serum protein S 100B in 45 patients after stroke (mean age 57 years, from 31 to 80 years; 19 male, 26 female) admitted to the neurointensive care unit. There were 11 patients with intracerebral haemorrhage (of whom 9 patients had brain operations), 19 patients with subarachnoid haemorrhage (of whom 13 patients had brain operations) and 15 patients with ischemic stroke (of whom 4 patients had operation). On admittance the patients' mean value of the Glasgow Coma Scale was 12 (ranging from 3 to 15 ) while their mean value on the Acute Physiology and Chronic Health Evaluation (APACHE) II score was 13 (ranging from 5 to 25 )

Results: Significant changes were found in the group of patients with intracerebral haemorrhage (mean value $0,488 \pm 0,286 \mathrm{ug} / \mathrm{l}, \mathrm{p}=0,03$ ), not in patients with subarachnoid haemorrhage (mean value $0,088 \pm 0,055 \mathrm{ug} / \mathrm{l}, \mathrm{p}=0,32$ ) or ischemic stroke (mean value $0,418 \pm 0,718 \mathrm{ug} / \mathrm{l}, \mathrm{p}=0,06)$.

Conclusion: Serum protein S 100B values significantly increased on admitted day only in group of patients with intracerebral haemorrhage. 
12 Acute stroke: early management and stroke units

CONTINUOUS ELECTROENCEPHALOGRAM IN ACUTE STROKE TO DETECT EPILEPTIC ACTIVITY

E. Carrera, P. Michel, P.A. Despland, M. Maeder-Ingvar, C. Ruffieux,

D. Debatisse, J. Ghika, G. Devuyst, J. Bogousslavsky

University Hospital, Lausanne, Switzerland

Background and Purpose: The role of continuous electroencephalogram (cEEG) in stroke unit is controversial. The purpose of this study is to determine the incidence and risk factors of electrical seizures and other electrical epileptic activity using cEEG in acute stroke patients.

Methods: One hundred consecutive acute stroke patients admitted to our stroke unit underwent cEEG using 10 electrodes. In addition to electrical seizures, the following EEG patterns were recorded: repetitive focal sharp waves (RSHW), repetitive focal spikes (RSP) and periodic lateralized epileptic discharges (PLEDS).

Results: Of the 100 patients cEEG was recorded for a mean duration of 17:34 hours (range 1:12-37:10). Epileptic activity occurred in 17 patients: RSHW in 5, RSP in 6 and PLEDS in 3 patients. Electrical seizures occurred in 2 patients. On univariate Cox regression analysis, predictors for electrical epileptic activity were stroke severity (high NIHSS) (hazard ratio (HR) 1.12; $\mathrm{p}=0.002$ ), cortical involvement (HR

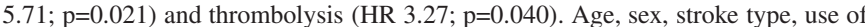
EEG-modifying medication, and cardiovascular risk factors were not predictor of electrical epileptic activity. On multivariate analysis, stroke severity was the only independent predictor (HR 1.09; $\mathrm{p}=0.016$ ).

Discussion: In acute stroke patients, electrical epileptic activity occurs more frequently than previously suspected. Because this activity may be associated with bad outcome or post stroke epilepsy, the usefulness of cEEG warrants further investigations especially in patients with severe strokes, cortical strokes and in those who benefit from thrombolysis.

13 Acute stroke: early management and stroke units

HYPERGLYCAEMIA HIGHER THAN 155 MG/DL AS THE THRESHOLD LEVEL FOR POOR OUTCOME IN ACUTE ISCHEMIC STROKE

B. Fuentes, E. Díez-Tejedor, J. Castillo, A. Dávalos, A. Gil-Nuñez, J. Vivancos, J. Egido

Cerebrovascular Diseases Study Group of the Spanish Society of Neurology, Madrid, Spain

Objective: To evaluate the influence of hyperglycaemia in outcome of acute ischemic stroke (IS) patients, adjusting for other prognostic factors and to establish the capillary glucose level associated to poor outcome.

Methods: Multicentre, observational study. We include acute IS patients $(<24 \mathrm{~h})$, excluding patients unconsciousness on admission, previous stroke with modified Rankin Scale $(\mathrm{mRS})>2$, other symptomatic intracranial lesion, or impossibility to complete a 3-months follow-up. Capillary glucose, blood pressure, body temperature, and neurological state (Canadian Stroke Scale, CSS) were determined on admission and 3 times a day during the first $48 \mathrm{~h}$. Outcome at 3 months was determined by $\mathrm{mRS}$. Statistical analysis: $x$-square, $t$-student, Spearman correlations, ROC-analysis, stepwise logistic regression, and Kaplan-Meyer survival analysis Results: 476 patients. Mean age $70.78 \pm 10.5$; time stroke-onset to inclusion: $6.8 \pm 5,7$ h. Mean glucose on admission (glu_adm): $137.2 \pm 57.2 \mathrm{mg} / \mathrm{dl}$. Both glu_adm and maximum glucose within the first $48 \mathrm{~h}$ (glumax_48h) significantly correlated to lesion volume ( $\mathrm{r} 0.204$ and 0.189$)$ and $\mathrm{mRS} \_3 \mathrm{~m}(\mathrm{r} 0.213$ y 0.282$)$. Glumax $48 \mathrm{~h}=$ $155 \mathrm{mg} / \mathrm{dl}$ was the point of maximum specificity and sensibility for outcome at 3 months in ROC analysis. Multivariate logistic analysis pointed out as independent prognostic factors for death or dependence at 3 months: glumax_48h $>155 \mathrm{mg} / \mathrm{dl}$ (OR 2.1; 95\%IC 1.2-3.7), age (OR 1.05; 95\%IC 1.01-1.08) and CSS on admission (OR $0.1 ; 95 \%$ IC 0.4-0.5). Known and unrecognised diabetic patients had poorer outcome than those non-diabetic $(\mathrm{p}<0.05)$. However, when analysing those patients with glumax_48h $>155 \mathrm{mg} / \mathrm{dl}$ there were no differences in outcome regarding the diagnosis of diabetes.

Conclusions: Hyperglycaemia $>155.5 \mathrm{mg} / \mathrm{dl}$ within the first $48 \mathrm{~h}$ is an independent predictive factor for death or dependence at 3 months. Acute stroke hyperglycaemia clearly determines poor outcome at 3 months in both diabetic and non diabetic patients.
14 Acute stroke: early management and stroke units

WELFARE MODELS FOR ACUTE STROKE:STROKE UNIT EFFICACY. DATA FROM EUROPEAN REGISTRY OF STROKE (E.R.O.S.) - FLORENCE UNITY A. Cramaro, A. Di Carlo, D. Inzitari

University of Florence, Florence, Italy

Background: To describe our stroke management and to show preliminar data collecting for the European Registry of Stroke and the differences between stroke unit and other wards.

Materials and Methods: Every acute first ischemic or haemorrhagic stroke admitted to Careggi Hospital has been evaluated by research team. For each patient, data about clinical status (including NIH scale, Glasgow Coma Scale and Barthel Index), therapy, imaging, rehabilitation were collected. 276 patients were enrolled: 122 were admitted to stroke unit, the others to general, neurology or neurosurgery ward. 23 out of 24 patients undergone to systemic or intra-arterial thrombolysis were admitted to stroke unit. Ischemic subtype of stroke was defined by TOAST classification; haemorrhagic stroke was defined as primary intracerebral haemorrhage or subarachnoidal haemorrhage. A 3 months follow up was performed.

Results: Patients admitted to stroke unit were younger $(\mathrm{p}<0,001)$ and more frequently independent before stroke $(\mathrm{p}=0,002)$. Patients admitted to stroke unit were more frequently treated by physiotherapist and logotherapist (respectively $\mathrm{p}=0,001$; $\mathrm{p}<0,001$ ) and, more frequently, diagnostic instruments (MRI EcocolorDoppler Transcranial Doppler) were used. No differences about therapy in acute phase in the two groups. While other wards accepted more patients with lacunar syndrome, stroke unit admitted more patients with a total anterior cerebral infarct $(\mathrm{p}=0,001)$ No differences between mortality at 1 and 3 months.

Discussion and Conclusion: Although patients recovered in stroke unit were younger, they had a more severe pathogenetic subtype. Data analysis showed a trend for the reduction of death and institutionalised care $(p=0,06)$ at three months follow up. The logistic regression analysis showed that the admission to a stroke unit reduces 3 months risk of death and institutionalised care $(\mathrm{OR}, 3,24 ; 95 \% \mathrm{CI}$ 1.19-8.79). This data was more strong in patients older than 80 (OR 4,64 95\% CI $1,12-17,68)$

15 Acute stroke: early management and stroke units

\section{FACTORS INFLUENCING DELAYS FROM ONSET TO ADMISSION TO A} STROKE UNIT

M. Koufali, E. Bacabac, K. Channell, J. Jones, A. Sharma

University Hospitals Aintree, Liverpool, United Kingdom

Background: Early admission and treatment are determinants of the level of acute stroke intervention and outcome. Our study aimed to examine the factors associated with delays from onset to admission following an acute stroke in North Liverpool. Methods: Data were prospectively and consecutively collected from patients who presented to the Acute Stroke Unit (ASU) during 2004. Delay from Onset to Arrival (DOA) was defined as time from symptom onset to arrival to a hospital emergency department. Patients were interviewed within 3 days of hospital admission, by means of a standardised structured interview. Univariate and multivariate regression analyses were conducted to analyse factors influencing DOA.

Results: A total of 517 patients who presented with symptoms and signs of acute stroke were studied. The median DOA was 26.6 hours while only $22.8 \%$ of the patients presented within 3.0 hours from symptom onset and $36.6 \%$ within 6.0 hours Delays in arrival time were significantly associated with age, sex, mode of referral, pre-morbid employment, cardiac failure and previous TIA/stroke risk factors, but not with the side affected by stroke or the OCSP classification of the incident.

Conclusions: Raising awareness of stroke symptoms and promoting immediate and direct access to stroke units should be a priority, since substantial delays still exist, resulting in significant numbers of patients missing out on appropriate treatment including thrombolysis.

\section{Acute stroke: early management and stroke units}

\section{LEUKOCYTE-PLATELET AGGREGATES AFTER ACUTE ISCHEMIC} STROKE

L. Marquardt, C. Anders, F. Buggle, F. Palm, A. Grau

Klinikum Ludwigshafen and Institute of Hemostaseology, Ludwigshafen,

Germany

Background: Leukocyte-platelet aggregates reflect platelet activation and a prothrombotic state. However, this parameter has not been investigated in the course of acute ischemic stroke.

Methods: We serially determined granulocyte-, lymphocyte- and monocyte-platelet 
aggregates, using flow cytometry at days $1,2,3,5,7,10$, and 90 in patients with ischemic stroke $(\mathrm{n}=21)$ and in age- and sex-matched healthy control subjects $(\mathrm{n}=10)$. Results: The percentage of granulocytes with $>1$ platelet on their surface (granulocyte-platelet aggregate) was higher on days 1 (median 4.4\%, 25\%-quantile $3.7 \%, 75 \%$-quantile $5.6 \%), 2$ and 3 after stroke than in control subjects $(2.8 \%$, $2.1 \%, 4.0 \% ; \mathrm{p}<0.05$, respectively). Values decreased towards day 5 but reincreased thereafter and were significantly higher than in control subjects on day $90(4.6 \%$, $3.8 \%, 5.6 \% ; \mathrm{p}=0.003$ ). Lymphocyte-platelet aggregates were not more common at any time-point after stroke than in control subjects. Monocyte-platelet aggregates showed a strong increase between day $1(2.3 \%, 1.7 \%, 3.3 \%)$ and day $2(4.0 \%, 1.9 \%$, $5.8 \% ; \mathrm{p}=0,002$ ) after stroke but rapidly declined thereafter (day $5: 1.8 \%, 1.2 \%$, $3.1 \%)$ and remained at a low level until day $90(2.2 \%, 1.8 \%, 4.3 \%)$. Compared to control subjects $(1.95 \%, 1.4 \%, 2.4 \%)$, monocyte-platelet aggregates were relatively more common at day 2 but not at other time points after stroke.

Discussion: The three leukocyte subpopulations showed different kinetics regarding aggregate formation with platelets after ischemic stroke. Monocyte-platelet aggregates may reflect an acute reaction to cerebral ischemia, whereas granulocyte-platelet aggregates could mirror a chronic prothrombotic state.

\section{Acute stroke: early management and stroke units}

VALUE OF ELECTROENCEPHALOGRAPHY AND TRANSCRANIAL DOPPLER MONITORING DURING AUTOLOGOUS BONE MARROW MONONUCLEAR CELLS TRANSPLANTATION IN ACUTE ISCHEMIC STROKE

M.L. Mendonca, D.C. Bezerra, G.R. de Freitas, S.A. Silva, R. Mendez-Otero, C. Andre, R. Borojevic, C. Gonzalez, C. Falcao, H.F. Dohmann Pro Cardiaco Hospital, Brazil

Objective: to assess the feasibility and usefulness of Transcranial Doppler (TCD) and electroencephalography (EEG) monitoring during autologous bone marrow mononuclear cells (BMMC) transplantation for acute stroke.

Methods: This phase I open label trial was designed assess the feasibility and safety of BMMC in 10 patients and 5 controls with an ischemic stroke including the territory of the middle cerebral artery (MCA) with spontaneous recanalization confirmed by Magnetic Resonance Angiography, and with a NIHSS score between 4 and 20. Between the third and tenth day after stroke onset, bone marrow cells were aspirated from the posterior iliac crest. On the same day, 30 million BMMC were injected in the MCA via catheter angiography. Brain perfusion with $99 \mathrm{mTc}-\mathrm{ECD}$ SPECT; PWI/DWI-MRI and brain PET-FDG were performed at baseline and after 7 days, 3 and 6 months of BMMC injection.

Results: We report the results of the first 5 treated patients. No EEG abnormalities were detected during BMMC injection. In 4 patients we could detect a mean of $85,5 \pm$ SD 94,9 microembolic signals (MES) during BMMC injection. In one of the patients a high number (228) of MES were detected at the start of the infusion, but after warming the cells at room temperature for 2 minutes the injection procedure could be resumed with no MES recording thereafter. Clinical and neuroimaging assessments at follow-up did not show any new abnormalities compared to baseline. Conclusion: TCD and EEG monitoring appear to be useful adjuncts to increase the safety of BMMC injection. If a high number of MES is detected during the procedure, warming the cells at room temperature seems to be helpful.

\section{Acute stroke: complications and early outcome}

\section{Acute stroke: complications and early outcome}

ONSET TO TREATMENT TIME, NIHSS ON ADMISSION AND MRI LESION VOLUMES DO NOT PREDICT PARENCHYMAL HEMORRHAGE AFTER IV-TPA WITHIN 6 HOURS IN MRI SELECTED STROKE PATIENTS G. Thomalla, C. Schwark, J. Sobesky, J.B. Fiebach, J. Fiehler, O. Zaro Weber, T. Kucinski, M. Rosenkranz, J. Röther, P. Schellinger, for the Stroke Imaging Net, Kompetenznetzwerk Schlaganfall

Stroke Imaging Net, Kompetenznetzwerk Schlaganfall, Hamburg, Germany

Background: It has been suggested, that the beneficial effect of intravenous thrombolysis in acute ischemic stroke is partially neutralized by an increased risk of intracerebral hemorrhage.

Methods: To find predictors of severe intracerebral hemorrhagic complications of intravenous thrombolysis, we analyzed data of $n=150$ patients treated with IV-tPA within $6 \mathrm{~h}$ of symptom onset in three German stroke centers. All patients were studied by perfusion and diffusion weighted imaging (PWI and DWI). Hemorrhagic transformation $(\mathrm{HT})$ and parenchymal hemorrhage $(\mathrm{PH})$ were rated on follow-up
MRI or CCT according to ECASS II-criteria. Neurological deficit on admission was assessed using the National Institutes of Health Stroke Scale (NIHSS). Logistic regression analysis was used to identify predictors of parenchymal hemorrhage. Results: Of $n=150$ patients treated with IV-tPA, $98(65 \%)$ were treated $<3 \mathrm{~h}$ and $52(35 \%)$ within 3-6 h. HT was found in $77(40.0 \%)$ and PH in $13(8.7 \%)$ patients. HT was more frequent in patients treated after $3-6 \mathrm{~h}(59.6 \%$ vs. $29.6 \%)$, whereas the rate of $\mathrm{PH}(7.7 \%$ vs. 9.2\%) was similar for patients treated after $3-6 \mathrm{~h}$ and within $3 \mathrm{~h}$. While age showed a tendency to predict $\mathrm{PH}(\mathrm{p}=0.063)$, NIHSS on admission ( $\mathrm{p}=0.551)$, onset to treatment time $(\mathrm{p}=0.945)$, PWI lesion volume $(\mathrm{p}=0.748)$ and DWI lesion volume $(\mathrm{p}=0.563)$ did not serve as predictors of $\mathrm{PH}$.

Discussion: Neither severity of neurological deficit, nor time to treatment and acute diffusion or perfusion lesion volumes predict severe intracerebral hemorrhage after intravenous thrombolysis in MRI selected patients. Further studies are needed, to identify possible risk factors for hemorrhagic complications after thrombolysis.

\section{Acute stroke: complications and early outcome}

INCREASE OF NEUROLOGICAL DETERIORATION AFTER ADMISSION ON A STROKE UNIT DUE TO TIA OR STROKE WITH MILD SYMPTOMS -FIRST DATA FROM THE AUSTRIAN STROKE UNIT REGISTRY

J. Ferrari, W. Lalouschek, W. Lang

Hospital Barmherzige Brueder, Vienna, Austria

Backgound: Former studies have shown that the clinical status deteriorates within the first seven days in one to $9 \%$ of patients with TIA (transient ischemic attack) and that the severity of stroke is a predictor for worsening. Little is known about the underlying mechanisms leading to deterioration. We report the results of our analysis concerning this matter based on data from the Austrian Stroke Unit Registry.

Methods: 3936 of 10356 patients from the Austrian Stroke Unit Registry (conducted by the Austrian Health Institution and the Austrian Stroke Society) fullfilled the following criteria: age more than 18 years, admission to a stroke unit due to a TIA or a stroke with mild symptoms (NIHSS $\leq 5$ ).

Results: The median duration of stay of the 3936 patients on the stroke unit was 2 days (interquartile range 1-4). 218 (5.5\%) of these patients (median age 73, IQR 63-78) showed a worsening of symptoms ( $\geq 2$ points on NIHSS). A multivariate analysis indicated that the following predictors were independently associated with worsening: known diabetes [OR1.9(1.4-2.7), $\mathrm{p}<0.001]$; cardiac source of embolism [OR 2.3 (1.2-4.5), $\mathrm{p}<0.017$ ]; reinfarction [OR $5.5(2.5-12.5), \mathrm{p}<0.001]$; oedema [OR 16.4 (6.0-45.5), $<<0.001$ ]; pneumonia [OR 4.8 (2.3-10.3), $\mathrm{p}<0.001$ ]; urinary infection [OR 2.9 (1.5-5.6), $\mathrm{p}<0.002$ ] and cardiac failure [OR 5.6 (2.2-14.0), $\mathrm{p}<$ $0.001)]$.

Discussion: In our data 5 to 6 of 100 patients with a TIA or an ischemic stroke with mild symptoms worsened during their treatment on the stroke unit. A major part of these patients deteriorated because of neurological causes. These results again underline the importance of immediate treatment and diagnostic workup of a TIA Furthermore, we urgently require concepts to prevent neurological deterioration in stroke patients.

\section{Acute stroke: complications and early outcome}

\section{POST-STROKE HYPERGLYCEMIA DIFFERENTIALLY AFFECTS} COGNITION AFTER CORTICAL OR SUBCORTICAL STROKE N.D. Kruyt, G.MS. Nys, H.B. Van der Worp, M.JE. Van Zandvoort, G.J. Biessels, L.J. Kappelle

University Medical Centre Utrecht, Utrecht, The Netherlands

Background and Purpose: Cognitive impairments (CI) after stroke are frequent and disabling. Predictors of CI after stroke are not well known. Diabetes mellitus (DM) and post-stroke hyperglycaemia (HG) are associated with poor physical recovery, in particular in cortical stroke. Aims of this study were: a) to examine the association of DM and $\mathrm{HG}$ with (i) $\mathrm{CI}$ at baseline and follow up (ii) stroke severity and (iii) lesion volume; b) to determine these associations for cortical and subcortical stroke separately.

Methods: Data from a prospective consecutive cohort of patients with supratentorial infarction were used. Cognitive assessments covering six cognitive domains were performed in the acute stage ( $<3 \mathrm{wks})$ and at follow up (6-10 months). Normative data were obtained from healthy controls $(\mathrm{N}=75)$. Stroke severity was assessed with the National Institutes of Health Stroke Scale (NIHSS). Lesion volume was assessed blind to clinical data. Associations were analysed using regression analyses. HG was defined as serum glucose $>7.0 \mathrm{mmol} / \mathrm{L}$.

Results: We included 114 patients, 55 had cortical infarcts (48\%), 16 had DM $(14 \%)$ and 43 had HG (38\%). Follow up was assessed in $77(68 \%)$ patients. At baseline $\mathrm{HG}$ was associated with poor executive function $(\mathrm{B}=-0.65 ; 95 \%$ c.i: -1.3 $0.01 ; \mathrm{p}<0.05)$ and $\mathrm{DM}$ with poor visual perception $(\mathrm{r}=-1.83 ; 95 \% \mathrm{c}$.i: $-3.58--0.08$; 
$\mathrm{p}<0.05$ ) only in patients with cortical infarcts. In this group HG was associated with stroke severity (NHISS $>7, \mathrm{r}=5.40 ; 95 \%$ c.i: $1.52-19.2 ; \mathrm{p}<0.05$ ) and lesion volume $(\mathrm{r}=0.39 ; 95 \%$ c.i: $0.10-0.68 ; \mathrm{p}<0.05)$. No association was found with follow up assessments.

Discussion: In parallel with previous studies on determinants of physical function, HG and DM are also associated with CI in cortical, but not in subcortical infarcts.

\section{Acute stroke: complications and early outcome}

RELEASE OF GLIAL FIBRILLARY ACIDIC PROTEIN (GFAP) IS RELATED TO THE NEUROVASCULAR STATUS IN ACUTE ISCHEMIC STROKE

M.T. Wunderlich, A. Oldag, M. Goertler, C.W. Wallesch

University Hospital Magdeburg, Magdeburg, Germany

Background: This study aimed at an analysis of Glial Fibrillary Acidic Protein (GFAP) in acute ischemic stroke, its association with the neurovascular status and its potential value as monitoring parameter.

Methods: In 53 consecutive patients, serial venous blood samples were taken on admission, $6,12,18,24,48,72,96$, and 120 hours after stroke onset. The neurovascular status was assessed by repetitive extracranial and transcranial duplex sonography. Neurological deficits were quantified by the NIH stroke scale, and functional outcome was assessed with the modified Rankin Scale.

Results: Mean GFAP values were elevated from admission on with highest level 48 hours after stroke onset. GFAP release was highly correlated with severity of neurological deficits and infarct volume. In patients with persistent middle cerebral artery occlusion, GFAP increased significantly compared to patients with normal sonographic findings $(\mathrm{P}=0.019)$ and recanalisation after thrombolysis resulted in a significant reduced increase $(\mathrm{P}=0.038)$. GFAP concentrations were associated with the functional outcome after 3 months.

Conclusion: Release kinetics of GFAP are associated with patients clinical deficits and infarct volume, depend on the neurovascular status on admission and on early recanalisation after thrombolysis, and may be used as an additional predictor of the early course and functional outcome.

\section{Acute stroke: complications and early outcome}

\section{PREDICTIVE FACTORS OF POOR NEUROLOGICAL EVOLUTION IN} PATIENTS WITH INITIAL MINOR STROKE

A. Ois, E. Cuadrado, N. Segura, A. Gálvez, C. Pont, J. Jiménez, J. Martínez, J. Roquer

Hospital del Mar, Barcelona, Spain

Background and Objective: An important proportion of patients with acute ischemic stroke have mild or no disabling symptoms at clinical presentation. The aim of our study was to analyze related factors with a poor neurological evolution (early progression or recurrence, EPR) in a non-selected series of patients with initial minor stroke.

Methods: Patients with acute ischemic stroke were prospectively assessed in our Hospital from June 2002 to October 2005. From a total of 910 patients, we selected 407 patients with an initial NIH Stroke Scale (NIHSS) at admission of 1 to 4 (minor stroke) in the first 6 hours from stroke onset. We evaluate the following factors related with the EPR: sex, age dichotomised in less or higher than 70 years, initial motor weakness, initial cortical symptoms, initial arterial blood pressure higher than $140 / 90 \mathrm{~mm} \mathrm{Hg}$, initial glucose levels $>200 \mathrm{mg} / \mathrm{mL}$, previous history of arterial hypertension, dyslipidemia, diabetes mellitus, obesity, prior stroke, coronary artery disease, peripheral arterial disease, carotid stenosis $(<50 \%, 50-70 \%$ and $>70 \%)$ and intracranial stenosis. The end point was considered either early clinical progression or recurrence within the follow-up period of three months.

Results: 74 patients $(18,2 \%)$ presented ERP. 30 patients $(7.4 \%)$ had early progression and 46 patients $(11.3 \%)$ presented recurrence ( 2 of them also with progression). Regression analysis showed that initial weakness $(\mathrm{p}=0.029, \mathrm{OR}=1.8895 \%$ CI 1.06 $3.31)$, Blood pressure $>140 / 90 \quad(\mathrm{p}=0.007, \mathrm{OR}=2.1595 \% \mathrm{CI} 1.23-3.74)$, previous TIA $(\mathrm{p}<0.001, \mathrm{OR}=3.3695 \%$ CI $2.24-15.04)$, obesity $(\mathrm{p}=0.009, \mathrm{OR}=2.0895 \% \mathrm{CI}$ $1.20-3.60)$, extracranial stenosis $>70 \%(\mathrm{p}<0.001, \mathrm{OR}=4.091 .97-8.48)$ and intracranial atheromatosis $>50 \%(\mathrm{p}=0.033, \mathrm{OR}=2.4795 \%$ CI 1.07-5.71) were associated with ERP.

Conclusion: In patients with minor stroke, combination of clinical, biological and ultrasonographic findings may allow to identify those patients with a higher risk of poor clinical evolution.
6 Acute stroke: complications and early outcome

\section{EARLY LESION RECURRENCE OF HYPERACUTE ISCHEMIC STROKE ON} MRI

J. Lee, S.J. Lee, J.S. Haa, M.Y. Baik, H.W. Jang, W.M. Byun

Yeungnam University Medical Center, Yeungnam, South Korea

Background: A high rate of ischemic lesion recurrence on brain MRI within several days after an acute ischemic stroke was suggested. Hemodynamic instability is associated with growth in lesion size and new lesion recurrence. We aim to investigate clinical and radiological predictors of the early lesion recurrence in a hyperacute ischemic stroke.

Method: We analyzed ischemic stroke patients who successively arrived at emergency room within 6 hours after symptoms onset. The patients who had initial MRI including diffusion weighted image(DWI) within 6 hours and follow-up scans between 5 and 14 days and were not treated by thrombolysis were eligible. Early lesion recurrence was defined as any new lesion on the follow-up DWI outside the regions of the acutely symptomatic lesion. Clinical and laboratory data were obtained. We estimated the independent risk of the recurrence.

Results: We included 35 patients. Early lesion recurrence occurred in 10 patiens $(28.5 \%)$. Clinical deteriorations according to the early lesion recurrence were founded in 4. Among the clinical and laboratory data, we found no effect for sex, diabetes, hypertension, hyperlipidemia, smoking, prior stroke, serum blood sugar level, C-reactive protein, initial NIHSS score, the degree of the arterial recanalization, the time latency between symptoms onset and the first DWI or follow-up DWI. A significant effect was found for cardioembolic causes, serum fibrinogen level, and multiple ischemic lesions on the first DWI $(\mathrm{p}<0.05)$. Multiple ischemic lesions on the first DWI(OR $10.2 ; 95 \%$ CI 1.1 to $89.5 ; \mathrm{p}<0.05)$ were independent predictor of the early lesion recurrence after adjustment for demographic variables.

Discussion: Early lesion recurrence was frequent and could be resulted in clinical deteriorations in hyperacute ischemic stroke. Attention to the clinicoradiologic features in a hyperacute ischemic stroke may guide future therapeutic intervention to prevent early recurrence.

\section{Acute stroke: complications and early outcome}

\section{TEMPORAL PROFILE AND CLINICAL SIGNIFICANCE OF SERUM NEURON-SPECIFIC ENOLASE (NSE) AND S-100 IN ISCHEMIC AND HEMORRHAGIC STROKE}

M. Blanco, T. Sobrino, M.A. Moro, E. Mosquera, J. Mallolas, M. Rodríguez-Yañez, J. Vivancos, J. Serena, A. Dávalos, J. Castillo Hospital Universitario, Santiago de Compostela, Spain

Objective: NSE (a neuronal-derived protein) and S-100 (a glial-derived protein) participate in multiple intracellular process, and functions as neurotrophic factors. They have been implicated in several brain injuries, such as ischemic stroke. The objective of this study was to analyze the molecular profile of NSE and S-100 in acute ischemic (IS) and hemorrhagic stroke (ICH).

Patients and Methods: 224 patients (mean age 70.2 y, mean time from onset 7.2 h) with IS; and 44 patients (mean age $61.5 \mathrm{y}$, mean time from onset $4.7 \mathrm{~h}$ ) with ICH were prospectively included. NIHSS on admission, $24 \mathrm{~h}$, and $72 \mathrm{~h}$ and CT on admission were performed in all patients. Early neurological deterioration (END) was defined as a fall $\geq 4$ points in NIHSS between admission and $72 \mathrm{~h}$. Functional outcome was evaluated by modified Rankin Scale at 3 months. Serum levels of NSE and S-100 (determined by Elecsys 2010 Analyzer, Roche Diagnostics) were measured on admission, 24 and $72 \mathrm{~h}$.

Results: END was observed in $18.7 \%$ and $29.5 \%$ of IS and ICH, while poor outcome $(\mathrm{mRS}>2)$ was found in $39.3 \%$ and $59.1 \%$. In IS, peak values were found at $72 \mathrm{~h}$ for NSE and at $24 \mathrm{~h}$ for S-100, whereas in ICH peak values were found at $24 \mathrm{~h}$ for both NSE and S-100. At these time intervals, but not at baseline, S-100 and NSE levels correlated with the NIHSS score $(\mathrm{p}<0.001)$. S-100 at 24h (OR 2.1, CI95\% 1.03 to 4.4 ) in IS, and NSE (OR 3.2, CI95\% 1.5 to 11.2 ) and S-100 at $24 \mathrm{~h}$ (OR 6.2, CI95\% 1.3 to 29.4 ) in ICH were independently associated with END. NSE at $72 \mathrm{~h}$ (OR 2.9, CI95\% 1.3 to 8.5) and S-100 at 24h (OR 4.7, CI95\% 2.5 to 7.6) in IS, and NSE at $24 \mathrm{~h}$ (OR 2.6, CI95\% 1.9 to 15.6 ) in ICH were independent markers of poor outcome.

Conclusions: Neuronal and glial proteins at baseline do not predict outcome. However at $24 \mathrm{~h}$ they are associated with END and poor outcome. 
8 Acute stroke: complications and early outcome

MECHANISMS OF EARLY DETERIORATION AFTER INITIAL IMPROVEMENT IN PATIENTS TREATED WITH INTRAVENOUS RT-PA M.G. Delgado, M. Reichhart, P. Browaeys, M. Wintermark, J. Bogousslavsky, P. Michel

Centre Hospitalier Universitaire Vaudois, Lausanne, Switzerland

Background: Little is known about the ischemic aetiology of early neurological deterioration after initial improvement following intravenous thrombolytic therapy (IVTT).

Objectives: To determine the possible mechanisms of early worsening after initial recovery following IVTT.

Methods: We selected all consecutive patients receiving IVTT within 3 hours after stroke onset in our stroke unit (May 2000- November 2005) who initially improved ( $>4$ points NIHSS) and then worsened ( $>4$ points NIHSS) within the first 48 hours after stroke onset. Perfusion-CT (P-CT) and CT-Angiography (CT-A) study at admission and control neuroimaging and neurovascular study were performed in all patients. Patients with symptomatic intracranial hemorrhage were excluded.

Results: 136 patients were identified. 10 patients ( 5 females, 5 males) aged between 52 and 85 years (mean 68 y) fulfilled the inclusion criteria. Stroke etiology was cardioembolic in $70 \%$, carotid dissection in $10 \%$, lacunar in $10 \%$ and unknown in $10 \%$. Mean NIHSS on admission was $18(8-23)$, during improvement was 10 (4 - 15), after worsening was $21(15-30)$ and at discharge was 13 (1 - 27). Most patients improved again after worsening, but two patients died. Causes of worsening were persistent occlusion in $40 \%$, extension of thrombus or progression of stenosis to occlusion in $20 \%$, distal embolus migration in $20 \%$, hemodynamic in $10 \%$ and early stroke recurrence in $10 \%$.

Conclusions: Early non-hemorragic deterioration after initial recovery following rtPA treatment has diverse arterial causes, including progression of occlusion, thrombus migration, and early cardioembolic stroke recurrence. Treatments to dissolve clots completely and to maintain vessel patency after recanalisation could potentially avoid secondary deterioration.

\section{Acute stroke: complications and early outcome}

ACUTE HEMORRHAGIC STROKE CARE AND OUTCOMES IN POLAND -PRELIMINARY DATA FROM POLKARD (NATIONAL CARDIOVASCULAR DISEASE PREVENTION AND TREATMENT PROGRAM FOR 2003-2005) STROKE REGISTRY

M.P. Niewada, M. Skowrońska, I. Sarzyńska-Długosz, B. Kamiński,

D. Ryglewicz, A. Czlonkowska

Medical University of Warsaw, Warsaw, Poland

Background: To estimate clinical presentation, the acute care and outcomes in stroke centers participating in POLKARD (National Cardiovascular Disease Prevention and Treatment Program for 2003-2005) STROKE Registry.

Methods: WHO Step Stroke and Swedish Stroke Registry web-based questionnaire was used to collect data on hemorrhagic stroke patients admitted to participating centers between June the 1st 2004 and May 31st 2005. To ensure the quality only centers reported at least 100 patients were analyzed. Patients characteristic, in-hospital care and early outcomes were analyzed for hemorrhagic stroke (I61 coding in International Classification of Diseases, 10th revision).

Results: There were 73 centers that met inclusion criteria and 2478 patients $(47.6 \%$ women, mean age: $67.3 \mathrm{yrs}$ ) were included in the final analyses. Mean time from onset to admission: 581 minutes. 1022 (41.2\%), 617 (24.9\%), 314 (12.7\%), 444 $(17.9 \%)$ patients were alert, drowsy, stupor and in coma at admission, respectively. Risk factors distribution: history of stroke -348 (14.0\%), history of TIA -48 $(1.9 \%)$, hypertension $-1831(73.9 \%)$, atrial fibrillation $-290(11.7 \%)$, history of myocardial infarction - $138(5.6 \%)$, diabetes - $373(15.1 \%)$, smoking $-295(11.9 \%)$, hiperlipidemia - 339 (13.7\%), alcohol abuse - 234 (9.4\%). Mean modified Rankin score following stroke was calculated on 4.22 .915 patients $(36.9 \%)$ died and for $74,4 \%$ death was directly and $22.4 \%$ indirectly associated with stroke.

Conclusions: Hemorrhagic stroke represents significant clinical matter and needs further monitoring in Poland.

\section{Acute stroke: complications and early outcome}

\section{BETA-BLOCKERS COULD REDUCE THE RISK OF EARLY DEATH IN} ISCHEMIC STROKE PATIENTS

T. Dziedzic, J. Pera, A. Klimkowicz, A. Slowik, A. Szczudlik

Jagiellonian University, Poland

Background: Beta-blockers (BB) reduce mortality in patients after myocardial in- farction. Experimental studies suggest that BB have also neuroprotective properties. The aim of our study was to assess if use of BB is associated with reduced risk of early death in ischemic stroke patients.

Methods: Retrospective data analysis of 833 consecutive patients with acute ischemic stroke (mean age: $69.6 \pm 12.6 ; 46.7 \%$ men) admitted to the stroke unit within 24 hours after stroke onset and not treated with thrombolysis.

Results: $10.6 \%$ of patients received BB during hospitalization; $94.3 \%$ of them were treated with BB before admission. The patients treated with BB were significantly younger and more often suffered from hypertension and ischemic heart disease and had history of myocardial infarction than those who did not receive BB. Thirty day case fatality was significantly lower in patients treated with BB than in those not treated with $\mathrm{BB}(6.8 \%$ vs $19.0 \%, \mathrm{P}<0.01)$. In the Cox proportional hazard model, Scandinavian Stroke Scale score on admission (HR: 0.94, 95\%CI: 0.93 $0.95)$, fasting glucose (HR: 1.08, 95\%CI: $1.03-1.14)$, total cholesterol level (HR: 0.83 , 95\%CI: $0.73-0.95)$, pneumonia (HR: $1.63,95 \% \mathrm{CI}: 1.13-2.37$ ) and use of BB (HR: $0.37,95 \% \mathrm{CI}: 0.16-0.84$ ), but not therapy with angiotensin converting enzyme-inhibitors were independent predictors of early death. When patients who died of cardiovascular causes (myocardial infarction, heart failure, sudden death) were excluded from the analysis, the use of $\mathrm{BB}$ was not longer significantly associated with risk of death $(\mathrm{P}=0.12)$.

Discussion: The results of our study suggest that BB decrease the risk of early death in stroke patients by reducing number of deaths due to cardiovascular events.

\section{Acute stroke: complications and early outcome}

\section{EVALUATION OF SERUM S100B AS A MARKER OF BLOOD BRAIN BARRIER DAMAGE FOR THE PREDICTION OF HEMORRHAGIC TRANSFORMATION AFTER THROMBOLYTIC THERAPY IN ACUTE} STROKE

C. Foerch, M. Wunderlich, F. Dvorak, T. Neumann-Haefelin, M. Sitzer, J. Montaner

Johann Wolfgang Goethe-University, Frankfurt am Main, Germany

Background: Intracerebral hemorrhage is an often fatal complication of thrombolytic therapy in patients with ischemic stroke. Experimental studies identified early blood brain barrier (BBB) disruption as a determinant of hemorrhagic transformation (HT) after thrombolysis. Astroglial protein S100B is known to be an indicator of BBB damage. We investigated whether elevated pre-treatment serum levels of S100B predict hemorrhagic complications in thrombolysed patients.

Methods: We retrospectively included 275 ischemic stroke patients (mean age $69 \pm 13$ years; $46 \%$ female) who received thrombolytic therapy within $6 \mathrm{~h}$ after symptom onset. All patients had previously been enrolled in prospective biomarker studies in three university hospitals. Blood samples were obtained at hospital admission, and S100B levels were determined using a commercially available analyser. Follow up brain scans were obtained $24 \mathrm{~h}$ after admission, and HT was rated as either hemorrhagic infarction (HI) or parenchymal hemorrhage (PH)

Results: Hemorrhagic transformation was present in 80 patients (45 HI, $35 \mathrm{PH}$ ). Serum S100B values were significantly higher in patients with HT compared to the remaining patients (median 0.14 [25th-75th percentile 0.07-0.27] vs. 0.11 [0.07-0.16]; $\mathrm{p}=0.017)$. Using univariate logistic regression, $\mathrm{S} 100 \mathrm{~B}$ was identified as a predictor of HT (OR 22.1 [95\% CI 3.7-131.9], p=0.001). After adjusting for age, sex, severity of symptoms, time span from symptom onset to hospital admission and vascular risk factors, $\mathrm{S} 100 \mathrm{~B}(\mathrm{p}=0.002)$ as well as diabetes mellitus $(\mathrm{p}=0.036)$ and NIHSS ( $\mathrm{p}=0.038$ ) remained as independent predictors of HT after thrombolytic therapy.

Discussion: In acute stroke, serum levels of protein S100B predict HT after thrombolytic therapy. Thus, in routine clinical practice, S100B may be a valuable supplement to clinical findings and may help to optimize patient identification for this potentially harmful treatment.

\section{Acute stroke: complications and early outcome}

\section{C-REACTIVE PROTEIN AND PROGNOSIS IN SINGAPOREAN ACUTE} ISCHEMIC STROKE PATIENTS

K. Kasiman, G. Ho, C. Teo, H.M. Chang, M.C. Wong, C.P. Chen Singapore General Hospital, Singapore

Background: Several studies have described the predictive value of increased plasma C-reactive protein levels (CRP) for future vascular events and disability following stroke. However, few studies are from non-Western populations. The aim of this study was to determine whether baseline CRP levels measured following ischemic stroke predicts for clinical outcome in Singaporean stroke patients.

Methods: 554 consenting patients with recent ischemic stroke or TIAs were examined for CRP levels (61\% male, 79\% Chinese, 10\% Malay, 10\% Indian and $1 \%$

Poster Session Second Visit 
Eurasian). 16 patients could not be contacted for follow-up at 6 months. Data was collected on outcome events and modified Rankin score (mRS).

Results: CRP concentration was significantly higher in patients with total or partial anterior circulation infarcts, greater age and who were not hyperlipedemic. There was no significant correlation of CRP with sampling delay. Univariate analysis showed that age, CRP, hyperlipedemia, stroke severity and atrial fibrillation were significantly associated with poor clinical outcome (mRS 3-6) at 6 months. Logistic regression showed that age, stroke subtype and CRP were independent predictors of poor outcome at 6 months. The highest CRP quartile had an increased risk of poor outcome at 6 months of 3.4 (95\% CI 2.5-4.0). Patients with recurrent vascular events or death at 6 months also had significantly elevated CRP levels.

Conclusions: Elevated level of hsCRP is an independent predictor for poor outcome 6 months following ischemic stroke in Singaporean stroke patients. Nevertheless, it remains to be determined whether CRP measurement is of therapeutic value.

\section{Acute stroke: complications and early outcome}

\section{DYSPHAGIA IN PURE BRAINSTEM INFARCTION: DIFFERENT CHARACTERISTICS ACCORDING TO THE LESION LOCATION M. Kwon, J.H. Lee, J.S. Kim}

Asan Medical Center, Asan, South Korea

Background: Brainstem strokes may produce dysphagia by the involvement of the swallowing center (SC), the adjacent structures, or the corticobulbar tract innervating the SC. Therefore, the characteristics of dyshagia may be different according to the location of strokes. The purpose of this study was to identify the characteristics of dysphagia to make clinical-anatomical correlation in brainstem infarction.

Method: Eighty-eight consecutive patients with unilateral brainstem infarction were divided into three groups: 1) ventral pontine (VPI, $n=42), 2$ ) medial medulla (MMI, n=9), and 3) lateral medulla infarction (LMI, n=37). Each of the VPI and medulla infarction (MI) was classified rostro-caudally as 'upper' and 'lower' lesion. Video-fluoroscopic examination was conducted, and the 8-point scale was used for assessing the severity of dysphagia. The characteristics of dysphagia were classified as problems on timing (PT) or the range (PR) of hyo-laryngeal excursion.

Results: There was no difference in the severity of dysphagia among the three groups. In MI group, dysphagia was significantly more severe in the upper $(\mathrm{p}<0.01)$ than in the lower group while, in VPI group, there was no difference between the upper and lower groups. The characteristics of dysphagia was significantly different $(\mathrm{p}<0.001)$ in that PR was more often observed in LMI $(58 \%)$ whereas PT was more often observed in MMI + VPI groups (97\%). Between the MMI and VPI groups, while five MMI patients ( $71 \%$ ) showed no responses to penetration/aspiration, silent dysphagia was observed in only 3 VPI patients $(7 \%)(\mathrm{p}<0.05)$

Conclusion: The severity of dysphagia is not different among the VPI, MMI and LMI patients. However, the types and characteristics of dysphagia are different suggesting different pathophysiologic mechanism of dysphagia according to the location of the brainstem stroke. Our results also have implications in the evaluation and treatment of dysphagia in patients with brainstem stroke.

\section{Acute stroke: complications and early outcome}

\section{PREVALENCE AND PROGNOSTIC SIGNIFICANCE OF ELECTROLYTE} IMBALANCE AT STROKE ONSET

L. Fofi, S. Lorenzano, L. Durastanti, M. Prencipe, D. Toni

University of Rome "La Sapienza”, IRCCS S Raffaele, Rome, Italy

Background: Identifying early predictors of poor outcome after stroke may be useful for improving patient treatment and outcome.Objective: We conducted a study on a continuous series of patients admitted to our Stroke Unit to evaluate the prevalence and influence of baseline electrolyte imbalance on the outcome of ischemic and hemorragic strokes.

Methods: 615 patients [544 $(88,4 \%)$ with ischemic and $71(11,5 \%)$ with hemorrhagic stroke] were enrolled. Baseline electrolyte values were obtained to evaluate the relationship with short term outcome, where a clinically significant change was a difference between discharge and baseline NIHSS scores more than 4 points or death. Prevalence of cerebrovascular risk factors and pre-stroke therapy were also taken into account.

Results: Hemorrhagic patients had lower potassium and sodium levels than ischemic patients, with respectively 3,9 vs $4,2 \mathrm{mEq} / \mathrm{L}$ and $138,9 \mathrm{vs} 139,7 \mathrm{mEq} / \mathrm{L}$ $(\mathrm{p}<.001$ and $=.045$, respectively $)$. After adjusting for gender, females showed the same difference, while hemorrhagic males showed only lower potassium levels. At univariate analysis, in ischemic stroke patients abnormal natriemia (16\%vs7,5\%, $\mathrm{p}=0,016)$, atrial fibrillation $(33 \% \mathrm{vs} 14,8 \%, \mathrm{p}<0,0001)$ and history of previous stroke $(7,2 \%$ vs $1,5 \%, p=0,003)$ were more prevalent in the poor outcome group, while in hemorrhagic patients no statistically significant correlation was found. Multiple correspondence analysis evidenced that predictors of bad short term outcome were abnormal sodium and urea levels and smoke in ischemic patients, and abnormal potassium and urea levels in hemorrhagic patients.

Conclusion: This study shows that electrolyte alterations might be a potential predictor of poor outcome in stroke, particulaly in ischemic patients.

\section{Acute stroke: complications and early outcome}

\section{INCIDENCE AND PREDICTORS OF SEVERE MEDICAL AND} NEUROLOGICAL COMPLICATIONS IN THE VERY ACUTE STAGE AFTER STROKE

M. Ritter, P. Kimmeyer, B. Heise, B. Kröger, F. Kleynmans, E.B. Ringelstein, P.U. Heuschmann, D.G. Nabavi

University of Münster, Münster, Germany

Background: Stroke Units are established institutions for acute stroke care, however, it is unclear which patients really need close monitoring. Medical and neurological complications influence outcome after stroke. Possibly, Stroke Unit treatment is superior to standard care because complications are detected earlier and with a higher sensitivity and can be treated more effectively. Data are lacking about frequency and predictors of early complications. We determined predictors of complications in the very acute phase after stroke.

Methods: 444 consecutive stroke patients admitted to our Stroke Unit were included. Frequency and type of medical and neurological complications (need for intubation, stroke progression, recurrent stroke, pneumonia, myocardial infarction, cardiac arrhythmias, other infection, other complication, and death) were documented. We investigated the impact of age, stroke severity (determined by the NIH stroke scale (NIHSS)), and stroke subtype (ischemic stroke according to TOAST criteria; intracerebral haemorrhage $(\mathrm{ICH})$ ) on the patients' risk to develop severe complications.

Results: Complications were observed $32 \%$ of the patients. Complication rates increased with increasing stroke severity. $85 \%$ of patients with NIHSS $0-1$ did not develop any severe complication compared with only $13 \%$ of patients with NIHSS $>10(\mathrm{p}<0.0001)$. Age showed no influence in the frequency of early complications, but patients with large artery embolism, cardiac embolism, or ICH were more likely to develop severe complications compared with other patients (each $\mathrm{p}<0.05)$.

Conclusions: Algorithms defining subgroups of stroke patients with a high likelihood to suffer complications should take stroke etiology into account.

\section{Acute stroke: complications and early outcome}

EARLY ANTIHYPERTENSIVE MEDICATION USE WORSENS OUTCOME OF ISCHEMIC STROKE

M. Moonis, L. Busekroos, K. Kane, S. Nanjundaswamy, B.A. Husaini, M. Fisher

University of Massachusetts Medical School, Worcester, MA, USA

Background: Early use of antihypertensive medications after acute ischemic stroke (AIS) is debatable and little is known about their use in affecting outcome after AIS. We performed a retrospective analysis of the phase 3 trial of citicoline database containing patients on antihypertensive medications where validated stroke outcomes of NIHSS and MRS were available 90 days.

Method: Patients with AIS and hypertension were divided into 2 groups. 1) on antihypertensive medications (AHM) for $>1$ week after AIS 2) not on AHM. Outcomes: Percentage achieving NIHSS and MRS scores $<2$. Univariate analyses for both outcome variables age, gender, diabetes, cardiac disease, previous stroke, hypertension, hypercholesterolemia, smoking, obesity, stroke type, severity and treatment with citicoline and AHM. Variables $\mathrm{P}<0.1$ in the univariate model were included in stepwise logistic regression analysis.

Results: Negative predictors of MRS $<2$ : diabetes $(\mathrm{p}<0.001)$, hypertension $(\mathrm{p}=0.003)$, history of cardiac disease $(\mathrm{p}<0.001)$, previous stroke or TIA $(\mathrm{p}=.013)$, stroke type $(\mathrm{p}=0.011)$, age $(\mathrm{p}<0.001)$, baseline NIHSS $(\mathrm{p}<0.001)$ and antihypertensive use $(\mathrm{p}<0.001)$. Similar results occurred in the univariate analysis of NIHSS $<2$.In multivariate model baseline NIHSS $(\mathrm{p}<0.001, \mathrm{OR}=0.756)$, age $(\mathrm{p}<0.001$, $\mathrm{OR}=0.960)$, diabetes $(\mathrm{p}<0.001, \mathrm{OR}=0.490)$, and antihypertensive medications $(\mathrm{P}=0.030, \mathrm{OR}=0.662)$ were negative predictors of $\mathrm{MRS}<2$. Compared to Angiotensin Converting Enzyme Inhibitor (ACEI) other AHM $(\mathrm{P}=0.022$, OR $=0.657)$ remained predictive of poor outcome on MRS with similar results in for NIHSS. Conclusions: Early use of antihypertensive medication post AIS is predictive of poor neurological and functional outcome with exception of ACEI. 
17 Acute stroke: complications and early outcome

PLATELET ACTIVATION AND EARLY OUTCOME OF ACUTE ISCHEMIC STROKE

S.H. Kim, J.-H. Seo, M.-J. Park, S.-R. Kim

Dong-A University, South Korea

Background: Platelet activation is an important pathophysiology in atherosclerosis and acute ischemic stroke. However, there was relatively little attempt to study the relationship between the extent of platelet activation and clinical severity in ischemic stroke. We investigated the hypothesis that the extent of platelet activation might be a useful maker to reveal the clinical severity of acute ischemic stroke. Methods: We measured the platelet aggregability for adenosine diphosphate (ADP) or collagen and surface expression of P-selectin in ischemic stroke patients in acute phase $(<24$ hrs). National Institutes of Health Stroke Scale (NIHSS) score was evaluated at admission day. Also, we scored the Barthel index (BI) after 90 days of ischemic events.

Results: The platelet aggregability for ADP or collagen and the extent of P-selectin expression were significantly higher in acute ischemic stroke than in normal subjects. The extent of platelet P-selectin expression significantly correlated with NIHSS $(\mathrm{r}=0.359, \mathrm{p}=0.004)$ at admission day and the Barthel index $(\mathrm{r}=0.412, \mathrm{p}<0.001)$ at 90 days after ischemic events.

\section{Acute stroke: complications and early outcome}

\section{PROBABILITY OF IN-HOSPITAL MORTALITY FOR ACUTE STROKE} PATIENTS AT THE TIME OF INITIAL DIAGNOSIS

N. Artemis, I. Heliopoulos, M. Papaioakim, K. Vadikolias, H. Piperidou,

D. Karakostas, I. Milonas

Aristotelion University of Thessaloniki, AHEPA University Hospital, Greece

Background: Most developed models for the prognosis of acute stroke attacks use complicated predictive equations and extended clinical or laboratory evaluation. Using data from the stroke database of North Greece Registry, we tried to develop a simple model for predicting in-hospital mortality using easily obtained acute stage information.

Patients and Methods: We examined 1773 consecutive acute stroke patients, 1184 male 589 female, age $58.05 \pm 10.38(10-86)$. The cause of acute stroke was: Hemorrhage $147(8.42 \%)$, infarct $891(51.06 \%)$ and transient ischemic attack (TIA) 707 $(40.52 \%) .45$ patients ( 22 with hemorrhagic stroke, 18 with infarct, 4 with TIA) died during their hospitalization $(2.54 \%)$.

Results: The only predictors, having at least marginal association with death, were cause of stroke, presence of atrial fibrillation (AF) and hypertension, history of previous stroke and use of antiplatelet medication. We did not find association between fatal outcome and gender, age, family history, obesity, heavy alcohol use, smoke habits, hyperlipidemia, diabetes mellitus, CAD, peripheral vasculopathy, valvular disease, arrhythmias other than AF, anticoagulant use or coagulopathy. In the multivariable logistic regression model we excluded antiplatelet medication and hypertension as those two predictors were strongly (collinearly) associated with the cause of stroke. The model with just three predictors (cause of stroke $p<0.001$, previous stroke $\mathrm{p}=0.001$ and $\mathrm{AF} \mathrm{p}=0.002$ ) had the best performance and correctly classified $81.7 \%$ of patients (sensitivity: $80 \%$, specificity: $82 \%$, AUC: 0.848 ).

Predicted probability of in-hospital mortality $\%$

\begin{tabular}{lcccc}
\hline & None & Previous stroke & Atrial fibrillation & Both \\
\hline Hemorrhagic & $12.2 \%(7.9,18.3)$ & $31.3 \%(17.6,49.4)$ & $0.6 \%(0.2,1.5)$ & $0.6 \%(0.2,1.5)$ \\
Infarct & $1.5 \%(0.1,2.5)$ & $4.9 \%(2.6,9.0)$ & $0.6 \%(0.2,1.5)$ & $17.0 \%(7.4,34.2)$ \\
TIA & $0.2 \%(0.1,0.5)$ & $0.6 \%(0.2,1.5)$ & $0.6 \%(0.2,1.5)$ & $17.0 \%(7.4,34.2)$ \\
\hline
\end{tabular}

Percentage (low and upper 95\% CI limits)

Conclusion: Using just two history elements, previous stroke and AF, we could easily and with adequate accuracy assess an individual's in-hospital mortality probability after an acute stroke attac.

19 Acute stroke: complications and early outcome

ATRIAL FIBRILATION AS A PROGNOSTIC FACTOR FOR ACUTE STROKE

S. Voyaki, P. Tsiodra, V. Dalaklidou, T. Loukopoulos, A. Haralampopoulos,

A. Stavrianou, S. Pagoni, C. Keramidas

General Hospital of Athens "G.Gennimatas", Athens, Greece

Aim of the study was the investigation of outcome in patients with atrial fibrillation (AF) and acute stroke.

Methods: We studied patients with acute ischaemic stroke hospitalized for at least one week in our Dept. All underwent a brain CT-scan at entry or within 48 hours after acute stroke. An ECG and an Echocardiography if necessary were performed in the emergency room. A specialist neurologist examined and recorded the Scandinavian Scale score (SSs) appropriately in admission and at days 3 and 7 during hospitalization.

Results: We included 489 patients (202M/287W, aged 61-98 years old). At entry, among 489 patients $156(31.9 \%)$ had atrial fibrillation, $160(32.7 \%)$ diabetes mellitus, $220(45 \%)$ hypertension and $179(36.6 \%)$ reported previous cardiovascular (CV) complications. At baseline patients with AF had lower SS scores in comparison to those without $\mathrm{AF}(30.3 \pm 18$ vs $36.2 \pm 17.6, \mathrm{P}=0.009)$ Among 489 patients $320(65.4 \%)$ improved their neurological deficit, $95(19.4 \%)$ remained stable and $68(13.9 \%)$ died during their hospitalization. At discharge, patients with $\mathrm{AF}$ had significantly lower neurological status in comparison to those without AF $(31.9 \pm 23.2$ vs $40.1 \pm 21.7, \mathrm{P}=0.003)$ and significantly more deaths were reported in the group of patients with $\mathrm{AF}$ in comparison to those without $\mathrm{AF}(22.6 \%$ vs $10.8 \%$, $\mathrm{P}=0.008$ ). In a forward multiple regression analysis, after correction for age, sex, previous cardiovascular complications and other risk factors, AF remained the more significant risk factor for poor outcome $(\mathrm{P}=0.003)$

Conclusions: Atrial fibrillation is associated with poor prognosis in patients with acute ischaemic stroke.

20 Acute stroke: complications and early outcome

EFFECTIVENESS OF STROKE THROMBOLYSIS: A CASE-CONTROL STUDY OF NINE YEARS' EXPERIENCE WITH INTRAVENOUS TISSUE-PLASMINOGEN ACTIVATOR (IV T-PA) AT A CANADIAN TEACHING HOSPITAL

J.M. Reid, S.J. Phillips, G.J. Gubitz, J. Jarrett, C. Christian

Dalhousie University, Halifax Infirmary, Halifax, Nova Scotia, Canada

Background: Clinical trials have shown that treatment with IV t-PA improves outcome after acute ischemic stroke. Uncertainty persists around the effectiveness of stroke thrombolysis in routine clinical practice.

Methods: Using a prospective registry of all patients admitted to our in-patient stroke service, we performed a case-control study of IV t-PA treatment. 151 patients treated with IV t-PA between May 1996 and May 2005 were matched 1:1 (with blinding to outcome) to controls based on age, sex, pre-stroke functional status, stroke severity, and Oxford Community Stroke Project (OCSP) subtype. The primary outcomes were in-hospital death, symptomatic intracranial hemorrhage (SICH), length-of-stay (LOS), and functional status at discharge (Barthel Index [BI] and Oxford Handicap Score [OHS])

Results: In hospital mortality was $23 \%$ for cases and $24 \%$ for controls $(\mathrm{p}=0.24)$. Median time from stroke onset to death was 4 days (interquartile range 2-12) for cases and 7 days $(4-14)$ for controls $(\mathrm{p}=0.24)$. SICH occurred in $7.9 \%$ of cases and $0.7 \%$ of controls. Overall, median LOS for survivors was non-significantly shorter for cases $(16$ [10-33] vs 21 [11-34] days, $\mathrm{p}=0.24)$. There was a trend toward reduced LOS in t-PA treated vs. non-treated women $(15$ [9-29] vs 23 [13-37] days, $\mathrm{p}=0.08)$; this was not evident for men ( 17 [10-34] vs 16 [9-34] days, $\mathrm{p}=0.95)$. Median BI and OHS scores at discharge were not significantly different although $10 \%$ of cases and $3 \%$ of controls had a discharge OHS of $<2(\mathrm{p}=0.02)$

Conclusion: The trajectory of early recovery after stroke seems to have been improved by IV t-PA. Overall the impact of t-PA on outcomes in our hospital has not been striking. Further research should target improved early stroke care delivery and possible gender differences in response to t-PA.

\section{Acute stroke: complications and early outcome}

SAFETY AND EFFICACY OF THROMBOLYSIS IN ACUTE ISCHEMIC STROKE: EXPERIENCE FROM THREE STROKE CENTERS IN BRAZIL J. Oliveira-Filho, S. Martins, P.AP. Jesus, R. Brondani, B.B. Pedreira, M. Araujo, A.S. Bacellar, M. Friedrich

Hospital Sao Rafael, Pontificia Universidade Catolica do Rio Grande do Sul and Hospital Mae de Deus, Salvador, Brazil

Background: Thrombolysis for acute ischemic stroke (AIS) was approved in Brazil in 2001, but only applied in few centers, partially because of lack of local data regarding safety and efficacy. We aimed to determine outcome predictors in AIS in three stroke centers with experience in thrombolysis.

Methods: Consecutive patients admitted with a diagnosis of AIS within 24 hours of symptom onset were studied in Salvador, and compared with consecutive patients who were thrombolysed in two centers in Porto Alegre. Data collected on admission included NIH Stroke Scale (NIHSS), TOAST classification scale, demographic information, previous cerebrovascular risk factors and vital signs. Proportion of "good outcome" was assessed at 3 months and defined as a Rankin score of 0 to

Poster Session Second Visit

Acute stroke: complications and early

outcome 
2. Significant $(\mathrm{p}<0.05)$ predictors of good outcome on univariable analyses were included in a multivariable logistic regression model.

Results: In Salvador the thrombolysis rate was 3\%, while in Porto Alegre the thrombolysis rate was $16 \%$. We compared 103 patients who received thrombolysis with 279 who did not. Mean age was $67 \pm 14$ years, $54 \%$ male. Stroke severity was greater in patients submitted to thrombolysis (median NIHSS score 5 vs. $11, \mathrm{p}<0.0001$ ). In multivariable analysis, thrombolysis was associated with an increased odds of good outcome $(\mathrm{OR}=2.53 ; 95 \% \mathrm{CI}=1.38-4.64, \mathrm{p}=0.003)$, but not death $(\mathrm{OR}=0.65 ; 95 \%$ $\mathrm{CI}=0.30-1.40, \mathrm{p}=0.276)$. Symptomatic hemorrhage occurred in $6(5.8 \%)$ patients and was associated with an increased odds of death $(\mathrm{OR}=15.4 ; 95 \% \mathrm{CI}=2.1-110.6$, $\mathrm{p}=0.007$ ).

Discussion: Acute stroke thrombolysis increases the odds of good outcome more than two-fold, without affecting overall mortality. Symptomatic hemorrhage was within accepted safety rates in comparison to previous multicenter trials. Widespread use of thrombolysis in specialized stroke centers should be stimulated in Brazil.

\section{Acute stroke: complications and early outcome}

LEUKOCYTOSES IN THE ACUTE PHASE OF THE SUBARACHNOIDAL HEMORRHAGE AS A RISK FACTOR FOR ARTERIAL VASOSPASM

J. Izquierdo, L. Soler, E. Vivas, M. Bosque, E. Balaguer, T. Sola, M. Leon Hospital General de Catalunya, Spain

Background: Actually, and with the apparition of the early endovascular treatment, vasospasm has become the most usual neurological complication in the subarachnoidal hemorrhage (SAH). The aetiology of vasospasm involves diverse inflammatory phenomenon. We studied the relationship between total number of leukocytes in the admission and the risk of vasospasm in patients with non traumatic SAH.

Material and Methods: We consecutively included the patients that arrived in our emergency area after suffering an acute SAH (between 0 and 24 hours of symptomatology). We studied age, gender, Hunt \& Hess and leukocytes in peripheral blood at the admission. Vasospasm was detected with medical examination, transcraneal Doppler and confirmed with cerebral angiography.

Results: A total of 61 patients were admitted with an acute SAH from January to October of 2005; 37 women $(61,7 \%)$ and 17 men $(28,3 \%)$. Symptomatic vasospasm was confirmed in 17 patients $(28,3 \%)$. Median age was 53,9 for vasospasm and 53,1 for patients without vasospasm. (p: NS). Hunt \& Hess in the admission was 2,18 in patients with vasospasm and 2,05 in patients without. (p: NS). Level of leukocytes in admission was $15,152 \times 103 / \mu 1$ in patients that developed vasospasm and 10,713 $\times 103 / \mu 1$ in patients that not developed $(\mathrm{p}<.001)$.

Conclusions: 1 . Symptomatic vasospasm appears in $28,3 \%$ of patients with non traumatic $\mathrm{SAH}$

2. High levels of leukocytes at admission were significantly higher in patients that developed symptomatic vasospasm.

3. Other conditions like age or intensity of symptoms were not related with the apparition of vasospasm

\section{Acute stroke: complications and early outcome}

\section{BIOMARKERS OF OXIDATIVE STRESS FOLLOWING T-PA INDUCED} REPERFUSION IN HUMAN STROKE

J. Montaner, C. Dominguez, P. Delgado, M. Ribó, E. Santamarina, C. Molina,

P. Martín, I. Fernández-Cadenas, A. Rosell, J. Alvarez-Sabín

Neurovascular Research Laboratory, Stroke Unit, Vall d'Hebron Hospital,

Barcelona, Spain

Background: Animal models of transient ischemia suggest that oxygen-derived free radicals produced upon reperfusion of ischemic brain represent the predominant cause of reperfusion injury. Since this hypothesis has not been tested in humans, we aimed to determine the presence and role of lipid peroxidation and protein oxidation related molecules following tPA-induced recanalization.

Methods: A total of 100 patients with strokes involving middle cerebral artery (MCA) territory that received $\mathrm{PA}<3 \mathrm{~h}$ from stroke onset and 40 healthy controls were included. Blood samples, TCD recordings and NIHSS scores were obtained at baseline (pretreatment), $1 \mathrm{~h}$ and $2 \mathrm{~h}$ following tPA bolus, and $12 \mathrm{~h}$ and $24 \mathrm{~h}$ from stroke onset. A lipid peroxidation product (malondialdehyde, MDA), oxidative damage to proteins quantified by advanced oxidation protein products (AOPPs) and myeloperoxidase (MPO), that mediates oxidation of plasma amino acids, were determined

Results: At baseline MDA $(\mathrm{p}<0.001)$ and MPO $(\mathrm{p}=0.002)$, but not AOPPs level $(\mathrm{p}=0.614)$, were elevated compared to controls. Temporal profiles showed highest level of protein oxidation biomarkers at baseline $(<3 \mathrm{~h})$ measurement $(\mathrm{MPO}=31.53$ and AOPPS=124.43). At the whole study population, an early reduction of both
MPO and AOPPs $(\mathrm{p}<0.05)$ appeared $2 \mathrm{~h}$ after tPA infusion $(\mathrm{MPO}=20.99$ and AOPPS=106.12). Lipid peroxidation remained elevated longer (MDA: baseline $=1.11,1 \mathrm{~h}=1.08,2 \mathrm{~h}=1.14$ and $12 \mathrm{~h}=1.02$ ). Among those with MCA recanalization by the end of tPA infusion (44\%), we could not identify an over expression or peaking of the studied molecules, except for a trend to higher level of MDA at $12 \mathrm{~h}$ ( 0.94 for no recanalization vs 1.11 for recanalization, $\mathrm{p}=0.06$ ). Clinical implications will be discussed.

Discussion: Although several oxidative stress related molecules are over expressed in the hyperacute phase of ischemic stroke, their role in reperfusion injury seems limited and restricted to lipid peroxidation products after human stroke.

\section{Acute stroke: complications and early outcome}

DEEP VENOUS THROMBOSIS IN AN ASIAN ACUTE STROKE POPULATION N. Venketasubramanian, S. Tan, T. Lim

National Neuroscience Institute, Singapore, Singapore

Introduction: The little published data suggests that deep venous thrombosis(DVT) is uncommon among Asian stroke patients. More information is needed about this entity, as DVT has serious consequences such as pulmonary embolism. Aim: This prospective study investigated the incidence of leg DVT in a Singapore population of patients immobilised by stroke.

Methods: Subjects were included in the study if they were evaluable within 48 hours of onset after an acute stroke, had lower limb weakness with proximal power in at least 1 lower limb being less than Grade 3 on the MRC rating scale. Study patients were reassessed after 1 week and 1 month for limb power and signs of DVT. An initial duplex ultrasound of both legs was performed within 48 hours of admission, with a follow-up ultrasound 1 week later. The radiologist was blinded to any clinical suspicion of DVT. Data was analysed using SPSS for Windows v11.0. Results: We planned to study 100 patients, but due to the relocation of neurology patients to a new hospital site, only 44 patients could be recruited. male:female $=$ 1:1, mean age $65 \mathrm{yr}$, range 35.0 to 92.0 years. Racial distribution was 36 Chinese, 5 Malay, and 2 Indian patients. Brain computed tomography $(C T)$ Scan showed cerebral haemorrhage in 14 , cerebral infarcts in 22 , and 7 with normal looking brain scans, presumed ischemic stroke. No subject had signs or symptoms of DVT on admission. All initial ultrasound scans were negative for DVT. No patient received DVT prophylaxis using anticoagulation. Repeat ultrasound at 1 week detected DVT in 1 of 44 patients. Another patient developed clinical DVT 3 weeks after admission in the contralateral leg, confirmed on ultrasound. DVT in both patients resolved after short-term anticoagulation Conclusion: This study lends further support to the hypothesis that DVT is an uncommon complication among Asian stroke patients.

\section{Acute stroke: complications and early outcome}

\section{OCCULT CARDIAC FUNCTION IMPAIRMENT AND OUTCOME OF ACUTE STROKE: SIGNIFICANCE OF NT-PRO BNP AND A TARGET FOR} INTERVENTION

J.C. Sharma, I.N. Ross, K. Ananda, R. Hill, M. Vassallo

Kings Mill Hospital, United Kingdom

Objective: To investigate the significance of NT -ProBNP to predict outcome of acute stroke in relation to other cardiovascular variables and stroke severity.

Design: Prospective study of previously independent acute ischaemic and haemorrhagic stroke patients admitted to the acute stroke ward.

Methods: Measurement of NT-ProBNP in acute phase in addition to standard assessment of cardiovascular and neurological details.

Main Outcome Measures: Concentrations and significance of NT-proBNP in patients who were dead versus alive at 120 days after acute stroke and cox regression analysis with Scandinavian stroke scale and other cardiovascular disease to predict mortality.

Results: One hundred fourteen patients were recruited, median age 74 years. At 120 days 13 patients had died. Dead patients had a significantly higher concentration of NT-proBNP as compared to alive patients, $\mathrm{p}<0.001$. All patients who had died had NT-proBNP concentration above the median for the whole group. Cox regression analysis revealed that NT-proBNP was the most significant independent variable predicting mortality (Odds ratio $2.4,95 \%$ CI 1.35 to $4.41, \mathrm{p}=0.003$ ) followed by the Scandinavian stroke scale - OR $0.95,95 \%$ CI 0.90 to $0.98, \mathrm{p}=0.005$ ). Highe NT-proBNP and lower SSS predicted mortality. There was a significant negative correlation of NT-proBNP with SSS score, $r=-0.24, \mathrm{p}=0.02$. There were no significant difference in NT-proBNP concentrations between ischaemic and haemorrhagic strokes $\mathrm{p}<0.346$

Conclusion: NT-proBNP, an index of cardiac impairment, has an independent prognostic value in acute stroke patients over other cardiovascular variables and stroke severity. This may provide a channel for interventional therapy in acute stroke. 
THE ASSOCIATION OF HYPERGLYCAEMIA WITH BRAIN EDEMA IN ISCHEMIC STROKE

V. Melnyk, S. Vinychuk

National Medicfl University, Ukraina

Background: In most studies even moderately elevated glucose levels are associated with both a 3-fold higher risk of short-term mortality and increased risk poor functional recovery compared with lower glucose levels.

Design: A retrospective review of stroke patients admitted to our hospital revealed 231 patients diagnosed as suffering an acute completed ischemic stroke presenting with mild to moderate HG and sequential computer tomography (CT) studies. The patients were divided into three groups: on the basis of mean serum glucose leve (SGL): group $1(\mathrm{n}=76)$ mean SGL at admission was between 13,0 to $16,0 \mathrm{mmol} / \mathrm{l}$; group $2(\mathrm{n}=81)$ mean SGL $-10,0$ to $12,9 \mathrm{mmol} / \mathrm{l}$; and group $3(\mathrm{n}=74)$ mean SGL

- 7,0 to $9,9 \mathrm{mmol} / \mathrm{l}$. The three groups were comparable with respect to mean age, average mean arterial blood pressure and initial infarct size.

Results: Our results show that in group $1-62,5 \%$ of the patients died from fatal brain swelling within the first week with clinical evidence of transtentorial herniation. In contrast, none of the group 3 patients died and only one showed radiological evidence for CE. Group 2 patients showed intermediate mortality and evidence of $\mathrm{CE}$. These trends were statistically significant at $\mathrm{p}<0.005$. In addition, the combined moderate HG group (1 and 2) had a significantly higher rate of development of hypodensity on CT $(\mathrm{p}<0.05)$ than the patients with mild HG

Conclusions: Our findings suggest that patients with moderate HG at admission are at high risk for developing fatal brain swelling.

\section{Acute stroke: complications and early outcome}

\section{DELIRIUM WITHIN 3 DAYS OF STROKE IN A COHORT OF ELDERLY PATIENTS \\ S. Sheng, Q. Shen, D. Cordato, Y. Zhang, D. Chan}

Bankstown-Lidcombe Hospital, Sydney, Bankstown/Sydney, Australia

Background: There is limited data on the long-term outcomes and prognosis of delirium in elderly stroke patients. The purpose of this study was to evaluate the incidence, risk factors and outcomes in elderly stroke patients with delirium.

Methods: This is a cohort study with 12-month follow-up, conducted at BankstownLidcombe Hospital, a 450-bed teaching hospital of the University of New South Wales, Australia. 156 stroke patients age ${ }^{3} 65$ were recruited over one year. Data collected including incidence of delirium within three days post-stroke, length of hospital stay, discharge destination, short- and long-term mortality, functional independence measurement (FIM) scores, and mini-mental state examination (MMSE) scores.

Results: 39(25\%) elderly stroke patients had delirium within 3 days after stroke. Logistic Regression analysis found that increasing age, hemorrhagic stroke, metabolic disorders, dementia pre-stroke, Glasgow Coma Scale (GCS) Score $<15$ and inability to lift both arms on admission were independent predisposing factors for delirium ( $\mathrm{p}<0.05$ for all above variables). Patients with a cardio-embolic stroke $(\mathrm{OR}=5.6)$ or total anterior circulation infarction $(\mathrm{OR}=3.4)$ were also more likely to develop delirium. Patients with delirium were associated with a higher 6 and 12 month mortality $(\mathrm{P}<0.05)$, lower 12 month FIM and MMSE scores, and a higher 12 months institutionalization rate.

Conclusion: Delirium occurred frequently in elderly acute stroke patients. Factors independently associated with delirium included old age, intracerebral hemorrhage, metabolic factors, pre-stroke dementia, initial GCS $<15$, and inability to lift both arms on admission. Patients with delirium had higher long-term mortality rate and a worse functional outcome.

28 Acute stroke: complications and early outcome

MANY DISORDERS OF SWALLOWING AFTER STROKE BUT ANY SPECIFIC AND SENSITIVE METHOD FOR DETECTING THEM

M. Vandemeulebroecke, G. Lawson, P. Laloux, J. Jamart, T. Deltombe, P. Hanson

UCL Mont Godinne, Frasnes-lez-Anvaing, Belgium

Dysphagia is a frequent problem after stroke. Dysphagia is responsible for complications such as aspirations, airway obstruction, pneumonia which increases morbidity and mortality in stroke patients.

The aim of the retrospective study was to evaluate the frequency of the respiratory complications related to dysphagia, to identify the risk factors leading to such complications (mental status, neglect, apraxia, tonus), to evaluate the efficacy of

Poster Session Second Visit

Cerebrovasc Dis 2006;21(suppl 4):1-151

the fiber optic endoscopic examination of swallowing (FEES) to predict respiratory complications.

This retrospective study included 105 acute stroke patients with neurological persistent deficit and admitted into the physical medicine and rehabilitation service. FEES was performed in 61 cases (there were 63 cases of dysphagia) $(58.1 \%$ ) and modified barium swallowing video fluoroscopic evaluation (VFS) in 4 cases. FEES was abnormal in $25(41 \%)$ and VFS in all the 4 cases.

Even with dietary secure decisions 9 of the 25 patients with abnormal FEES developed pneumonia; 8 of the 36 patients with normal FEES developed pneumonia (silent aspirations and some false negative results with FEES).

Five patients with strict enteral feeding developed pneumonia.

Four patients died consecutively of there respiratory complications.

Median time for resumption of normal feeding was 30 days for patients with normal FEES, and 35 days for patients with abnormal FEES.

There is no specific and sensitive method for detecting every dysphagia. We must use association of different methods: clinical examination, sensitive validated tests for aspiration and its complications ( $90 \mathrm{ml}$ water swallow test and Burke dysphagia screening test (De Pippo)), and FEES.

If there is high suspicion of clinical dysphagia with normal FEES, we must complete the investigation with VFS

For all stroke patients, we must do multidisciplinary dietary management to reduce aspiration, optimize nutritional status and hydration.

\section{Acute stroke: treatment concepts}

\section{Acute stroke: treatment concepts}

MRI-BASED THROMBOLYSIS WITHIN AND BEYOND THE 3 HOUR TIME WINDOW HAS AN IMPROVED SAFETY PROFILE AND IS AT LEAST AS EFFECTIVE AS STANDARD CT-BASED TREATMENT

M. Köhrmann, E. Jüttler, C. Schwark, J.B. Fiebach, H.B. Huttner, S. Siebert, P.A. Ringleb, W. Hacke, P.D. Schellinger University of Heidelberg, Heidelberg, Germany

Background and Purpose: Intravenous rtPA is approved within $3 \mathrm{~h}$ after stroke onset. Thus only a small percentage of patients can be treated. Meta-analyses and more recent studies suggest a benefit for a subset of patients even beyond $3 \mathrm{~h}$. We examined safety and efficacy of a stroke-MRI selection protocol within and beyond 3 hours compared to standard CT-based treatment

Methods: At our institution patients eligible for thrombolysis within $3 \mathrm{~h}$ are selected either by CT- or MR-imaging and beyond $3 \mathrm{~h}$ only by MRI. We assessed clinical outcome and occurrence of symptomatic ICH $(\mathrm{sICH})$ in 400 consecutive patients treated wit rtPA. 18 patients were excluded from analysis because of violation of the algorithm (CT-based treatment beyond $3 \mathrm{~h}$ ). The remaining 382 patients were divided into three groups (1) $n=209$ CT-based treatment $<3 \mathrm{~h}(2) \mathrm{n}=103 \mathrm{MRI}$-based $<3 \mathrm{~h}$ and (3) $\mathrm{n}=70 \mathrm{MRI}$-based $>3 \mathrm{~h}$.

Results: Stroke severity was similar in all 3 groups (median NIHSS score 13). Patients in group 3 had the best $90 \mathrm{~d}$ outcome $(41.4 \%$ favourable outcome vs $37.8 \%$ in group 1 and $33 \%$ in group 2; $55.7 \%$ independent outcome vs $47.8 \%$ and $50.5 \%)$ without an increased risk for $\operatorname{siCH}(5.7 \%$ vs $9.1 \%$ and $1 \%)$ or mortality (11.4\% vs $21 \%$ and $12.6 \%$ ). MRI selected patients overall had a significantly lower risk for $\operatorname{sICH}(2.8 \%$ vs $9.1 \%$ CT-based; $\mathrm{p}=0.018)$ and mortality $(12.1 \%$ vs $21 \%$; $\mathrm{p}=0.021$ ). Time to treatment proved to be irrelevant for all outcomes in univariate and multivariate analyses.

Discussion: Our data suggest that beyond $3 \mathrm{~h}$ and maybe even within $3 \mathrm{~h}$ patient selection is more important than time to treatment. Furthermore, MRI-based thrombolysis regardless of the time-window shows an improved safety profile regarding incidence of sICH and mortality, while being at least as effective as standard CT-based treatment within $3 \mathrm{~h}$.

\section{Acute stroke: treatment concepts}

\section{A SHORT-TERM ASSESSMENT OF RECOMBINANT HUMAN GRANULOCYTE COLONY-STIMULATING FACTOR (RHG-CSF) IN} TREATMENT OF ACUTE CEREBRAL INFARCTION

J. Jun Zhang, Min Deng, Yuanjin Zhang, Wei Sui, Liping Wang, Aping Sun, Hongsong Song, Ming Lu, Dongsheng Fan

Peking University Third Hospital, Beijing, China

Background: In humans, bone marrow stem cells can be mobilized into the circulation using recombinant human granulocyte colony-stimulating factor (rhG- 
CSF), an approach that is effective in experimental stroke. And a randomized placebo-controlled pilot trial (STEMS) are performing by Sprigg et al.

Objective: To assess the safety and efficacy of rhG-CSF in treatment of acute cerebral infarction by a randomized and placebo-controlled clinical trial.

Method: 45 cases with acute cerebral infarction were randomly allocated to receive treatment by either rhG-CSF or placebo within 1 week of stroke onset. Among them, 15 cases were consecutively given rhG-CSF $2 \mathrm{~g} / \mathrm{kg}$ for 5 days by subcutaneous injection. The other 30 cases were treated with placebo. The end points included NIH Stroke Scale and adverse reaction.

Results: On the 5th day in rhG-CSF treatment, there was markedly increase of WBC count, and CD34+ cell proportion ( $>1 \%$, i.e. $>106)$. For the treatment group, there was no significant difference between NIHSS score on the 10th day and that before treatment, but there was on the 20th day $(\mathrm{P}=0.004)$. For parallel group, no significant differences in NIHSS scores on both the 10th and 20th day compared with those before treatment. There were no significant differences between the two groups in terms of their respective NIHSS scores on the 10th and 20th day. No definite side effects were found in the treatment group during the 20 days.

Conclusion: There was a statistical difference on the 20th day during the treatment of acute cerebral infarction with rhG-CSF, showing that rhG-CSF is an effective drug in the treatment of acute cerebral infarction. Finding no statistical difference on the 10th day means that the efficacy of rhG-CSF can only be seen in the long run. Although there was no statistical difference between the two groups, the gap has widening tendency with time until the 20th day.

\section{Acute stroke: treatment concepts}

\section{EARLY DECREASE IN BLOOD PRESSURE PREDICTS DWI LESION GROWTH IN STROKE PATIENTS TREATED WITH INTRAVENOUS TPA \\ E. Santamarina, R. Delgado-Mederos, M. Ribo, M. Rubiera, P. Delgado, J. Montaner, O. Maisterre, J. Alvarez-Sabín, C.A. Molina \\ Hospital Vall d'Hebron, Barcelona, Spain}

We aimed to evaluate the impact of BP changes in the evolution of acute DWI lesion volume in tPA-treated stroke patients. We studied 35 stroke patients due to an intracranial artery occlusion treated with iv tPA who were imaged with multiparametric MRI before and 24-36 hours after tPA bolus. DWI volume change ( $\triangle$ DWI: DWI final-initial) was measured. Recanalization (RE) was assessed by TCD during the first 6 hours of treatment. Maximum, minimum, mean, variability $(\triangle \mathrm{BP})$ values of systolic (SBP), diastolic (DBP) and mean (MBP) BP were obtained on admission and serially monitored during 12 hours after tPA bolus. BP drop was defined as the maximal BP decrease between baseline and minimal BP values during 12-hour monitoring. Clinical improvement was defined as a decrease of $\geq 4$ points on the NIHSS score at 48 hours from baseline. 18 (51.4\%) patients had previous history of hypertension. Mean SBP, DBP and MBP on admission were $155 \pm 32.4$, $82.5 \pm 18.7$ and $106.7 \pm 21 \mathrm{~mm} \mathrm{Hg}$. Mean $\triangle \mathrm{SBP}$ and $\triangle \mathrm{DBP}$ were $58.8 \pm 27$ and $36.3 \pm 17.3 \mathrm{~mm} \mathrm{Hg}$, respectively. RE on TCD occurred in $20(57.1 \%)$ patients. DWI lesion growth was strongly correlated with the decrease of $\mathrm{SBP}(\mathrm{r}=0.520, \mathrm{p}=0.002)$ and DBP $(r=0.562, p=0.001)$ during the first $12 \mathrm{~h}$. For every $10 \mathrm{mmHg}$ decrease of SBP and DBP, DWI increased in 6.95 and $12.01 \mathrm{cc}$, respectively. The impact of BP drop on DWI lesion growth varied depending on the occurrence of RE: SBP $(r=0.545, p=0.044)$ and DBP $(r=0.653, p=0.011)$ decrease were significantly correlated with DWI growth in non-reperfused, but not in reperfused tPA- treated patients. Similarly, clinical improvement at $48 \mathrm{~h}$ was associated with a lesser degree of SBP drop only in patients who did not recanalized $(\mathrm{p}=0.039)$. BP decrease during stroke thrombolysis is associated with greater DWI lesion growth and worse clinical course. The deleterious role of BP drop is higher in patients who did not achieve RE. Avoiding BP drop may limit infarct expansion and improve outcome after thrombolysis.

\section{Acute stroke: treatment concepts}

\section{BLOOD PRESSURE IN PATIENTS WITH ACUTE STROKE: A CHINESE HOSPITAL BASED STUDY \\ M. Liu, Y. Tan, Q.F. Wang, Q.W. Yang, S. Tan, B. Wu \\ West China Hospital, Sichuan University, Chengdu, China}

Background and Purpose: The influence of blood pressure (BP) to outcomes of Chinese acute stroke patients is unclear. This study aimed to observe the blood pressure and relationship between BP and outcomes in hospitalized Chinese patients with acute stroke.

Methods: We registered prospectively acute stroke patients admitted consecutively to the ward of Department of Neurology, West China Hospital, Sichuan University from March 2002 to March 2004. The patients with ischaemic stroke or cerebral hemorrhage within 24 hours of stroke onset were included. Blood pressure was recorded from day 1 to day 10 of hospitalization. Outcome measures were death, dependency (Barthel Index $\leq 60$ ) at 1 month and 6 month after stroke onset. Logistic regression model was used to estimate the relationship between BP and outcomes. Results: 592 patients were included. 53\% of patients had a history of hypertension and half of them did not receive treatment for their hypertension. The mean blood pressure at admission was $151 \pm 29 / 90 \pm 18 \mathrm{mmHg}$. $1.4 \%$ of patients had systolic blood pressure (SBP) $\leq 220 \mathrm{mmHg}$ and $5.6 \%$ of the patients had diastolic blood pressure (DBP) $\geq 120 \mathrm{mmHg} .11 .8 \%$ of patients had SBP $<120 \mathrm{~mm} \mathrm{Hg}$. An U-shaped relationship was observed between BP and outcomes. The cutoff levels were set at $120 / 90 \mathrm{mmHg}$ at admission, $140 / 90 \mathrm{mmHg}$ on day $1-7$. This relationship was observed in both ischaemic stroke and cerebral haemorrhage when analyzed by stroke types. The rapid reduction of BP within 24 hours and levels of BP on day 3-7 influenced independently death at 1 month (OR 1.450,95\% CI 1.049 2.003) and death/dependency at 6 months respectively.

Discussion: BP levels in acute stage influenced independently the outcomes of patients with acute stroke. Management of BP in acute stroke needs to consider this point and further randomized conrolled trials are required to provide more reliable evidence for BP management.

\section{Acute stroke: treatment concepts}

CONTINUING PRIOR ANTIHYPERTENSIVE MEDICATION IN ACUTE STROKE LOWERS BLOOD PRESSURE: DATA FROM THE CONTINUE VS STOP ARM OF THE 'EFFICACY OF NITRIC OXIDE IN STROKE' (ENOS) TRIAL

N. Sprigg, L.J. Gray, P.M.W. Bath, the ENOS Investigators Institute of Neuroscience, University of Nottingham, Nottingham, United Kingdom

Background: Half of patients with acute stroke are taking antihypertensive therapy prior to the event. It is not known whether this should be continued or stopped temporarily, and what effect this has on the course of BP during the acute phase. Methods: ENOS is a collaborative, international, randomised controlled trial in 5,000 patients with acute ischaemic or haemorrhagic stroke which is investigating the safety and efficacy of lowering BP with transdermal glyceryl trinitrate, a nitric oxide donor. Patients taking prior antihypertensive therapy are also randomised in a factorial design to continue or temporarily stop this. Systolic and diastolic BP are measured daily for 7 days using validated semi-automatic monitors (Omron $705 \mathrm{CP}$ ). Differences in BP between the continue and stop groups were analysed by ANCOVA with adjustment for baseline values.

Results: As of 31 May 2005, 168 patients (21 centres, 6 countries) had been recruited into the continue vs. stop arm of ENOS. At baseline, mean age 70 years, male 54\%, severity (Scandinavian Stroke Scale) 41, BP 167/90 mmHg. The mean differences in BP between patients randomised to continue vs. stop prior antihypertensive medication was $(\mathrm{mmHg})$ : day $1,-0.2 /+2.5$; day $2,-9.4 /-4.4$; day $3,-11.3 /-2.1$; day $4,-7.6 /-2.0$; day $5,-9.4 /-1.8$; day $6,-12.7 /-5.4$; and day 7 , $-8.6 /-2.3$ (SBP, $\mathrm{p}=0.002$; DBP, $\mathrm{p}=0.047$ ).

Conclusions: During the acute phase of stroke, blood pressure differs significantly between patients randomised to continue or stop prior antihypertensive therapy. Whether this difference in management alters functional outcome after stroke is being studied within ENOS.

\section{Acute stroke: treatment concepts}

\section{DIFFERENT THERAPEUTIC APPROACHES IN ACUTE BASILAR ARTERY} OCCLUSION: COMPLICATIONS AND OUTCOME

U. Becker, G. Urban, D. Mucha, A. Mueller, R. von Kummer, G. Gahn

University of Technology Dresden, Dresden, Germany

Background and Purpose: Acute basilar artery occlusion (ABAO) is a disease with high mortality and morbidity. We analyzed the rate of recanalization, complications and outcome in patients with different therapeutic approaches.

Methods: Since 1996, we treated 52 patients with ABAO with different therapeutic approaches: 15 patients were treated with the combination of Abciximab IV and Alteplase IA following a prospective protocol (Group A), 12 with Urokinase IA (Group B), 5 with Alteplase IA (Group C), and 20 patients received exclusive IV therapy (Group D), either Alteplase (6) or Abciximab (8) or the combination (6). To assess differences in those groups, we investigated the rate of recanalization and complications by studying the medical records, and the clinical course by telephone interview. Furthermore we studied the association between age, Glasgow Coma Scale (GCS) at admission, and the period from stroke to treatment to assess independent variables affecting good outcome (modified Rankin scale 0-2).

Results: Age, therapeutic time-window, recanalization rate and GCS were not different among the groups. Symptomatic intracranial haemorrhage occurred only 
in groups A and B. Survival and good outcome after 6 months was achieved more frequently in the IV treated group. All in all 11 patients survived with good outcome. All of them were men and all of them had had a GCS greater than 7 on admission. In contrast, none of the women or patients with an initial GCS less than 8 achieved a good outcome. Time to treatment and age did not affect clinical outcome.

Conclusion: We learned from these observations that a GCS less than 8 predicts poor clinical outcome in stroke caused by basilar artery occlusion. Systemic thrombolysis was safe and effective compared to other approaches.

\section{Acute stroke: treatment concepts}

\section{RELATIONSHIP BETWEEN BLOOD PRESSURE AND HEMATOMA} ENLARGEMENT IN ACUTE SPONTANEOUS INTRACEREBRAL

\section{HEMORRHAGE}

J. Martí-Fàbregas, D. Cocho, Y. Bravo, J. Díaz-Manera, L. Molina-Porcel,

M. Martínez, S. Martínez, E. Guardia, E. Martínez, J.L. Martí-Vilalta

Hospital de la Santa Creu i Sant Pau, Spain

Background: The predictors of hematoma enlargement (HE) in patients with intracerebral hemorrhage (ICH) are unknown. We examined the relationship between blood pressure (BP) and HE in the acute stage of ICH.

Methods: We prospectively studied consecutive patients with spontaneous supratentorial ICH, within the first 6 hours after symptom onset. HE was defined as an increase $>33 \%$ in the volume of hematoma on the CT obtained 24-48 hours after the onset of symptoms, in comparison with the admission CT. The volume was calculated using the formula $\mathrm{AxBxC} / 2$. Patients were treated when their MBP reached values above $110 \mathrm{mmHg}$. The following $\mathrm{BP}$ parameters were analyzed: systolic (SBP), diastolic (DBP) and mean (MBP) BP at admission, and at 6, 12, 18 and 24 hours after symptom onset; Maximum SBP, DBP and MBP between intervals (admission-6 hours, 6-12 hours, 12-18 hours, 18-24 hours); maximum and mean SBP and DBP within the first 24 hours. Statistics: t-test, Chi-square, 2-way repeated-measures ANOVA.

Results: We studied 43 patients with a mean age of $71.6 \pm 11.1$ years, and $60.5 \%$ were men. Admission CT was obtained $145.5 \pm 72.2$ minutes after symptom onset, while control CT was obtained $30.8 \pm 10.2$ hours after onset. HE was observed in 20 patients (46.5\%). Admission SBP/DBP were equivalent in the HE and non-HE groups. We observed a progressive reduction in SBP $(\mathrm{p}=0.005)$, DBP $(\mathrm{p}<0.0001)$ and MBP $(p<0.0001)$ in successive measurements within the first 24 hours, but without differences at any time point when comparing the HE and non-HE groups. Maximum BP between intervals was also equivalent between groups. We found no statistically significant in maximum and mean BP values when comparing groups. Discussion: There is no relationship between blood pressure and hematoma enlargement.

\section{Acute stroke: treatment concepts}

PRIOR USE OF ASPIRIN AND BASELINE STROKE SEVERITY: AN ANALYSIS OF 17,850 PATIENTS IN THE INTERNATIONAL STROKE TRIAL (IST)

S. Ricci, S. Lewis, P. Sandercock

UOSD Neurologia e Ictus, USL 2 dell'Umbria, Perugia, Italy

Background and Purpose: Some studies suggest that, taking aspirin regularly at the time of the onset of stroke reduces stroke severity. Other studies suggest the converse, i.e. that prior aspirin therapy is associated with greater stroke severity. We sought to examine this question among the patients enrolled in the International Stroke Trial (IST).

Methods: Analysis of the associations of reported use of aspirin in the 3 days prior to randomisation in IST with: baseline stroke severity (as assessed by stroke clinica syndrome, predicted outcome at six months and observed outcome at six months). We adjusted analyses for confounding factors.

Results: We excluded those patients who were first scanned after trial entry and were found to have an intracerebral haemorrhage as the cause of the stroke leading to randomisation. We performed analyses for all treatment groups combined.' For the 17,850 patients with ischaemic stroke, data at baseline and follow-up were available for $100 \%$ and $99 \%$ respectively. Among these patients, 3,820 (21.4\%) reported prior aspirin use. Prior aspirin use appeared, in univariate analyses, to be associated with greater baseline stroke severity, more severe stroke syndrome and - in control subjects - worse observed outcome at 6 months. However, after adjustment, these associations were no longer significant.

Conclusions: In this large prospective and complete data set, we found no evidence of any association of prior aspirin use with baseline stroke severity. The analyses suggest that previously reported positive and negative associations may well have

Poster Session Second Visit

Acute stroke: treatment concepts been due to the play of chance in small samples, confounding or other biases, rather than a biological effect of aspirin.

9 Acute stroke: treatment concepts

INHIBITION OF MULTIDRUG RESISTANCE TRANSPORTER-1 FACILITATES NEUROPROTECTIVE THERAPIES AFTER FOCAL CEREBRAL ISCHEMIA

A. Spudich, E. Kilic, Ü. Kilic, H. Wunderli-Allenspach, C.L. Bassetti, D.M. Hermann

University Hospital, Zürich, Switzerland

The blood-brain barrier possesses transporters on its luminal surface, which carry back xenobiotics into the vasculature against concentration gradients. To define the role of one of them, multidrug resistance transporter (Mdr)-1, which is physiologically expressed in the brain and made responsible for pharmacoresistance in epilepsy, we subjected mice to middle cerebral artery occlusions. In histochemical studies we show that Mdr-1 is upregulated on endothelial cells after ischemia. Following Mdr-1 deactivation by its pharmacological inhibitor, tariquidar, tissue concentrations of the neuroprotectant and known Mdr-1 substrate FK506 were selectively increased in the ischemic brain by 14 -fold. Inhibition of Mdr-1 by tariquidar or mdr-1(a/b)-knockout potentiated the neuroprotective activity of two Mdr-1 substrates (FK506, rifampicin), providing tissue rescue at doses otherwise subtherapeutic. Our results demonstrate the in vivo relevance of Mdr- 1 after stroke. As up to $50 \%$ of pharmaceutical compounds newly developed are thought to be Mdr-1 substrates, Mdr-1 inhibition may greatly facilitate stroke therapy.

\section{Acute stroke: treatment concepts}

INTRAVENOUS INSULIN-BASED GLUCOSE CONTROL PROTOCOL REDUCES IN-HOSPITAL ACUTE STROKE MORTALITY

T.G. Fukuda, J.MM. Teles, J.LB. Pereira, J.C. Seixas, T.M. Godinho, A.M. Almeida, A. Farias, S. Agareno, O. Messeder, J. Oliveira-Filho Stroke Clinic, Federal University of Bahia and Intensive Care Unit, Hospital Portugues, Salvador, Brazil

Background: Hyperglycemia is associated with bad outcome in acute stroke. In general intensive care unit (ICU) patients, rigid glucose control has been demonstrated to improve outcome. However, this strategy has not been tested in acute stroke patients. Our aim was to evaluate the safety and clinical impact of a rigid insulin-based glucose control protocol (GCP) in stroke outcome.

Methods: We studied consecutive acute stroke patients admitted to a general ICU between May 2004 and January 2005. A rigid GCP was implemented in August 2004, consisting of intravenous insulin titrated for a goal blood glucose of $100-150 \mathrm{mg} / \mathrm{dl}$. Patients in the GCP group were compared to those admitted before August 2004 (controls). Data collected included demographic variables, mean blood glucose, disease severity scales, in-hospital complications and mortality.

Results: We admitted 116 patients, $71(61 \%)$ in the GCP group. Baseline characteristics were similar between GCP and controls: mean age $71 \pm 12$ vs. $69 \pm 15$, NIH Stroke Scale score $9.1 \pm 3.9$ vs. $9.5 \pm 4.6$, APACHE II score $13.4 \pm 6.5$ vs. $15.4 \pm 7.8$, Glasgow Coma Score $13.1 \pm 3.2$ vs. $12.6 \pm 3.5$, and proportion of hemorrhagic strokes $27 \%$ vs. $17 \%$ ( $p>0.1$ for all analyses). Mean blood glucose was $127.17 \pm 24.99$ $\mathrm{mg} / \mathrm{dl}$ in GCP group and $145.35 \pm 27.93 \mathrm{mg} / \mathrm{dl}$ in controls, $\mathrm{p}=0.001$. GCP group had a lower frequency of sepsis $(16.9 \%$ vs $33.3 \%, \mathrm{p}=0.046)$ and required less vasoactive drugs $(16.9 \%$ vs. $40.0 \%, \mathrm{p}=0.009)$ when compared to controls. In-hospital mortality was lower in GCP group (5.6\% vs. $17.4 \%, \mathrm{p}=0.041)$. Hypoglycemic episodes were rare, short-lived and did not affect outcome.

Discussion: A rigid GCP based on intravenous insulin therapy is safe, feasible and was associated with reduced mortality in acute stroke patients when compared to historical controls. A randomized controlled study of rigid blood glucose control in acute stroke is warranted.

\section{Acute stroke: treatment concepts}

\section{IMPROVING DOOR-TO-NEEDLE TIME FOR ACUTE ISCHEMIC STROKE PATIENTS TREATED WITH INTRAVENOUS THROMBOLYTIC} MEDICATION

T.J. Ingall, B.M. Demaerschalk, C.L. Stemper-Bartkus, N.F. Lendzion, C. Stewart, B.J. Bobrow, D. Dodick

Mayo Clinic, Scottsdale, AZ, USA

Background: The management of acute ischemic stroke (AIS) in the Emergency Department (ED) focuses on assessing the patient's eligibility for thrombolytic 
therapy with intravenous tissue plasminogen activator (tPA) within 3 hours of the onset of symptoms. Improving the number of eligible AIS patients who receive tPA by decreasing the time from symptom onset to delivery of the drug is a major treatment priority. Objective: To determine if the implementation of a number of measures in the ED would influence the speed with which the stroke team acts.

Methods: In 2003, the following measures were implemented in the ED at Mayo Clinic Hospital. Nursing and medical staff were provided with extensive education related to the administration of tPA. An order set was created to expedite the acquisition of the initial required diagnostic tests. - A stroke "Clot Box" was assembled which stores everything necessary for the administration of tPA. The ED nurses were instructed to immediately notify the stroke neurologist and ED staff physician when patients with possible AIS arrived within 3 hours of symptom onset. An acute stroke algorithm and target times were posted in the ED triage area. A prospective hospital registry of all thrombolysed AIS patients was maintained to allow pre- and post- target comparison.

Results: Thirty patients were thrombolysed prior to initiating the tPA related measures and 30 were thrombolysed after. On average, the Door-to-CT time improved by 10 minutes and the CT-to-Needle times improved by 5 minutes, resulting in a Door-to-Needle time improvement of 15 minutes.

Conclusion: A comprehensive educational program aimed at ED staff, combined with other measures including the posting of recommended target times in the ED, was associated with significantly faster stroke team care and a mean improvement of 15 minutes in Door-to-Needle time.

\section{Acute stroke: treatment concepts}

\section{DOES ANTICOAGULATION HARM IN ACUTE CERVICAL ARTERY DISSECTION? \\ F. Perren, L. Bocquet, F. Saulnier, T. Landis, R. Sztajzel}

HUG, University Hospital and Medical School of Geneva, Geneva, Switzerland

Background: Cervical artery dissection (CAD) is one of the major cause of cerebral infarction in the young adult. Prognosis is highly variable and intravenous followed by oral anticoagulation is the treatment of choice in most medical centres, although this treatment is based on rather incomplete evidence. Since clinical observations suggested even a worsening after initiation of anticoagulation, our objective was to study the dynamics of residual flow and clinical development in a larger series of CAD.

Methods: We studied over 4 years all sequentially admitted patients suffering from acute "spontaneous" CAD who were treated by anticoagulation. All have been examined with color-coded duplex flow imaging (CDFI) and either by CT-, MR-, or RX-angiography at admission. Follow-up CDFI and neurological examinations have been performed in all patients.

Results: 33 patients (21 men; mean age: 46.4 years) have been diagnosed with acute CAD. $23(70 \%)$ of them were admitted with an acute ischemic stroke. Involved were: in 16: the internal carotid artery (ICA) alone, in 2: both ICA, in 9: the vertebral artery (VA) alone, in 5: both VA and in 1 combined ICA-VA. All patients were anticoagulated in the acute phase (0-3 days). Residual flow on CDFI in the dissected vessel worsened in 17/33 patients (51.5\%). 9/33 (27\%) showed clinical deterioration. 4/33 (12\%) suffered from secondary bleeding. 3/33 (9\%) developed pseudoaneurysms.

Discussion: These findings show that worsening either hemodynamic or clinic is not rare $(>50 \%)$ and could be due to anticoagulation. Whether anticoagulation should not be used more carefully or even delayed in patients with acute CAD should be investigated in a further prospective randomised trial

\section{Acute stroke: treatment concepts}

\section{NEUROLOGISTS' PRACTICES AND BELIEFS ABOUT TREATMENT AND} SECONDARY STROKE PREVENTION FOLLOWING ACUTE ISCHEMIC

\section{STROKE}

M. Moonis, L. Busekroos, S. Nanjundaswamy, K. Kane, B.A. Husaini,

M. Fisher

University of Massachusetts Medical School, Worcester, MA, USA

Introduction: The practice of stroke prevention is undergoing a rapid change with increasing options for treatment being made available to the neurologist. This study was designed to assess the methods and rationale of treatment and stroke prevention in the United States by neurologists.

Method: A questionnaire based on a stroke scenario was mailed to 1200 neurologists across the United States. Individual questions regarded use of: 1. Antithrombotic therapy after acute ischemic stroke (AIS), 2.a) AIS with peripheral vascular disease b) Ischemic heart, ejection fraction $>50 \%$, normal sinus rhythm 3. lipid-lowering therapy 4. Alternate or concurrent antihypertensive. For each scenario, neurologists were asked to select the basis of their response from five choices: Published literature, personal experience, defensive medicine, individualized therapy, and others. Neurologists were also asked to indicate their years in clinical practice after residency and whether stroke was their sub-specialty.

Results: Most neurologists indicated using Aggrenox as antithrombotic therapy $(34.8 \%)$, with (2a and $\mathrm{b}$ ) more prescribing aspirin+Plavix with peripheral vascular disease and ischemic heart disease. (3) $87.9 \%$ would use a lipid lowering drug, $71.8 \%$ a statin. (4) $74.3 \%$ would consider an antihypertensive $(78.3 \%$ prefer an angiotensinconverting enzyme inhibitor). $63 \%$ based their choices on published literature, $24 \%$ on their experience, and $10 \%$ on individualized therapy. Few responders believed they were practicing defensive medicine $10 \%$ were stroke neurologists. Were more likely $(72.7 \%$ vs. $38.9 \%)$ would switch medication to Plavix for peripheral vascular disease.

Conclusions: Neurologists in general seem to be well informed, though more education of the recent literature is warranted.

\section{Acute stroke: treatment concepts}

\section{THE EFFECT OF STATINS ON INFARCT SIZE AND PROGNOSIS}

H. Dib, H. Horozoglu, N. Afşar, G. Ekinci, S. Aktan

Marmara University Hospital, Istanbul, Turkey

Background: Although statins are indicated for secondary prevention of ischemic stroke, they may be used in the treatment of acute ischemic stroke due to their pleotropic effect as recently shown in experimental data.

Methods: The present study is a pilot, randomized, controled study. Consecutive patients with ischemic stroke of less than 48 hours duration, with an NIHSS above 10 and classified according to OCSP as TACI/PACI/POCI were included. Patients with history of previous stroke or already on statin treatment were excluded from this trial. Patients were randomized to two groups (simvastatin $40 \mathrm{mg} /$ no treatment). At admission diffusion weighted MRI and C-reactive protein were obtained for each patient. NIHSS, modified Rankin score, stroke risk factors, demographic data and medication history were recorded. At fifth day diffusion MRI, CRP and stroke scales were renewed. At nineteeth day $\mathrm{m}$ RS and barthel index were measured. MRI scans were analyzed by two different blinded radiologist using a standard software. Data between both groups was compared using student $t$ test.

Results: Sixteen patients ( 8 statins, 8 without treatment) were enrolled in the trial. The mean age was 79.7 in the statin group and 71.4 in the non statin group. Clinical classifications did not show significant differences between the two groups. There was no difference between both group according to neurological outcome during the acute and chronic stage. Bazal diffusion MRI, bazal CRP, 5th day diffusion MRI, 5th day CRP also showed no difference between both groups.

Conclusions: Simvastatin initiated in the first 48 hours after ischemic stroke does not decrease the infarct size, CRP and does not affect neurological outcome. However a large scale trial with early initiation of a statin within the first few hours of stroke onset may be necessary to demonstrate the potential effect of statins.

\section{5}

Acute stroke: treatment concepts

INCREASED BODY IRON STORES ARE ASSOCIATED WITH POOR OUTCOME AFTER THROMBOLYTIC TREATMENT IN ACUTE STROKE

M. Millán, T. Sobrino, M. Castellanos, F. Nombela, E. Riva, I. Cristobo, J. Arenillas, J. Castillo, A. Dávalos

Hospital Universitari Germans Trias i Pujol, Badalona, Spain

Background: Iron overload has been associated with greater brain injury in experimental and human stroke. In ischemia/reperfusion, the iron-catalyzed radical hydroxyl may have a role in disrupting the blood brain barrier and promoting brain oedema. Our aim was to investigate whether high serum ferritin levels, as an index of iron overload, were associated with poor functional outcome, hemorrhagic transformation (HT) and brain oedema after tPA treatment in patients with acute ischemic stroke.

Methods: Baseline ferritin levels, at 24 and 72 hours after treatment were determined in 134 patients (67y, TTT $145 \mathrm{~min}$, NIHSS score 13.9) prospectively registered in the SITS-MOST study in 4 university hospitals. Patients with known cancer, infectious or inflammatory diseases at the time of treatment were excluded. Results: At 24h, neurologic improvement ( $>3$ points in the NIHSS) was found in $43 \%$ and worsening in 6\%. At 24-36 hours, HT was observed in 27 patients (HI in 15 , and PH in 12; symptomatic(sHT) in 4), while brain swelling with midline shift was found in 15 . Good functional outcome (Rankin $\leq 2$ ) at 90 days was recorded in $43.8 \%$ of patients. Ferritin levels were stable within the first $24 \mathrm{~h}$ and showed a moderate increase from 24 to 72 hours. Median [quartiles] ferritin levels at baseline were significantly higher in patients who developed $\mathrm{PH}(\mathrm{p}=0.006)$, sHT $(\mathrm{p}=0.008)$, severe brain swelling $(\mathrm{p}<0.001)$, early neurologic worsening $(\mathrm{p}=0.01)$, and poor 
outcome at 90 days $(192[109,313]$ versus $17.5[11.9,36.8] \mathrm{ng} / \mathrm{mL} ; \mathrm{p}<0.001)$, while levels were lower in patients with early improvement $(\mathrm{p}<0.001)$. Ferritin levels higher than $79 \mathrm{ng} / \mathrm{mL}$ (median value) had an odds of 300 [95\% CI, 41-2198] of poor outcome after adjusting for age, baseline NIHSS score, and unbalanced variables (history of atrial fibrillation and diabetes).

Conclusions: Iron overoald is associated with poor outcome, sHT and substantial edema in patients treated with tPA after ischemic stroke.

\section{Acute stroke: treatment concepts}

\section{THROMBOLYSIS AT A STROKE UNIT IN A RURAL AREA - A 5-YEAR REVIEW \\ C. Rückert, T. Staudacher \\ Oberschwabenklinik, Krankenhaus St. Elisabeth, Ravensburg, Germany}

Introduction: Rapid administration of recombinant tissue plasminogen activator (rt-PA) in stroke patients improved outcomes in many previous trials. We aimed to analyze 5-year data from our stroke unit to confirm the feasibility of rapid treatment serving a rural area of approximately 1300 square miles with around 600.000 inhabitants.

Methods: We analyzed data of 195 patients treated with intravenous thrombolytic therapy at our clinic between December 2000 and May 2005.

Results: Median age was 71 years, $47,2 \%$ were women, $52,8 \%$ were men. While the median time from onset of symptoms to arrival at our clinic was 73,5 minutes, the median door-to-needle-time was 50 minutes. Median baseline National Institute of Health Stroke Scale (NIHSS) was 16 at admission and 10 at discharge. Median Modified Rankin Scale (mRS) was 5 at 24 hours, 4 at discharge, and 3 after 3 months. $23,2 \%$ scored $2 / 3$ points more on mRS in the time period between 24 hours of stroke onset and discharge, another $50,3 \%$ had an increase of $2 / 3$ points on mRS after 3 months after discharge. 19,5\% had reached Rankin 0/1 after 3 months, mortality at 3 months was $12,3 \%$. 57,9\% had a cardio-embolic etiology, $15,9 \%$ an arteriosclerotic origin, and in $18,5 \%$ no etiology could be found. Symptomatic intracranial haemorrhage was seen in 9 patients $(=4,7 \%)$, overall incidence of intracranial hemorrhage was $15,4 \%$.

Conclusion: As in previous studies our data prove the possibility of

administering intravenous thrombolysis in a routine setting with a complication rate and outcome comparable to the results in NINDS (National Institute of Neurological Disorders and Stroke). Furthermore it confirms the feasibilitiy of rapid thrombolytic treatment in rural areas by organizing an efficient pre-clinical care system so that thrombolytic therapy can be provided for patients in an area the extent of at least 70 miles in diameter.

\section{Acute stroke: treatment concepts}

COMBINED INTRAVENOUS AND INTRA-ARTERIAL THROMBOLYTIC THERAPY WITH OPTIONAL ADDITIONAL ENDOVASCULAR TREATMENT IN 10 PATIENTS WITH INTERNAL CAROTID ARTERY T OCCLUSION J. Brich, A. Berlis, A. Hetzel, M. Schumacher, C. Weiller, W.D. Niesen University of Freiburg, Freiburg, Germany

Background: A retrospective analysis was performed on 10 consecutive patients who presented with severe acute ischemic stroke due to carotid-T occlusion (CTO). They were evaluated for a combined intravenous (IV) and local intra-arterial (IA) thrombolytic approach with rtPA and optional additional endovascular treatment within 3 hours of onset.

Methods: Ten consecutive patients with CTO were evaluated over a 22-month period $(03 / 2003-12 / 2005)$. Total-rtPA $(0,9 \mathrm{mg} / \mathrm{kg})$ was calculated, and a IV bolus of $10 \%$ of total-rtPA was given. One to maximal two thirds of total-rtPA was given intravenously, depending on the time of onset of IA-thrombolysis. IA-thrombolysis was allowed to use the remaining rtPA, but at least 1/3 of the total-rtPA. Extracranial (ESI) and intracranial stent implantation (ISI) as well as percutaneous transluminal angioplasty (PTA) was allowed if deemed necessary. IV treatment was initiated within 3 hours in all 10 patients, IA treatment was allowed up to 6 hours.

Results: The median baseline NIHSS was 16 (range from 9 to 22). The median time from stroke onset to IV treatment was $2 \mathrm{~h} 14 \mathrm{~min}$, and median time to initiation of IA treatment was $3 \mathrm{~h} 20 \mathrm{~min}$. EIS was performed in 4 patients, ISI in 4 patients with one patient receiving both extra- and intracranial stents. A total of 8 patients underwent additional PTA. 5 patients $(50 \%)$ had a favourable (mRS $\leq 2)$ and 4 patients $(40 \%)$ a poor outcome (mRS 3 to 5 ) after rehabilitation. 2 patients developed space-occupying infarction, one of them underwent decompressive craniectomy, the other patient died due to cerebral herniation.

Discussion: Thrombolytic therapy using a combination of IV and IA routes and additional endovascular treatment may be effective in improving outcome for patients suffering from CTO.

Poster Session Second Visit
18 Acute stroke: treatment concepts

PERCUTANEOUS TRANSLUMINAL ANGIOPLASTY AND STENTING OF POSTERIOR CIRCULATION SYMPTOMATIC STENOSIS

W.J. Schonewille, S. vd Boogaard, Th.C. Overtoom, J.A. Vos, M.J. Suttorp, J.P.M. de Vries, R.G.A. Ackerstaff

St. Antonius Ziekenhuis, Nieuwegein, The Netherlands

Background: Approximately 25\% of strokes occur in the region of the brain supplied by the posterior circulation. While angioplasty with stenting is well studied in the carotid or anterior circulation, the data available on endovascular treatment of posterior circulation stenosis is limited.

Methods: We retrospectively collected data on all patients treated with angioplasty and/or stenting of the vertebral and basilar arteries in our institution.

Results: From March 2003 to November 2005, 23 consecutive patients with a total of 27 stenoses were treated. Stenoses were located in the proximal vertebral artery $(\mathrm{n}=15)$, intracranial vertebral artery $(\mathrm{n}=6)$ and basilar artery $(\mathrm{n}=6)$. Three patients who were asymptomatic were treated in preparation of coronary bypass grafting, all others were symptomatic despite maximum medical therapy. All lesions in the proximal vertebral artery were treated with percutaneous transluminal angioplasty (PTA) and stenting except one which was not stented. One patient had a worsening of his prior stroke. None had recurrent symptoms. Of the 6 patients with an intracranial vertebral artery stenosis two patients were stented in addition to their PTA, and 3 were treated with PTA alone. One patient treated with PTA alone had further hemodynamic symptoms after an initial period of improvement. One patient who had a progressive brainstem stroke prior to treatment and a contra-lateral vertebral occlusion died shortly after the procedure in which the pinpoint stenosis occluded during the procedure. Of the 6 patients with basilar artery stenosis 4 were treated with PTA alone, two had additional stenting. Five patients became symptom free after an uncomplicated procedure. One patient died because of her initial stroke after a technically successful PTA.

Conclusion: PTA and stenting of posterior circulation stenosis appears to be an effective and feasible therapy. The more urgent the treatment indication the higher the complication risk.

\section{Acute stroke: treatment concepts}

\section{SAFE IMPLEMENTATION OF INTRAVENOUS THROMBOLYSIS IN} TURKEY: RESULTS OF A NATION-WIDE PHASE I SURVEY

Y. Krespi, B. Bir, N. Afşar, D. Kaya, B. Dora, for the Turkish Neurological

Florence Nightingale Hospital, Istanbul, Turkey

Background: rtPA has recently been approved for intravenous (IV) thrombolysis in acute ischemic stroke in Turkey.

The purpose of this study was to determine the background stroke care map of the country in order to to build-up a professional network system for the safe implementation of this therapy.

Methods: A nation-wide, two-phase survey was planed by The Turkish Neurological Society "Stroke Working Group". The first phase of the survey was conducted in cities of Turkey having a medical faculty. The first phase had two parts; the screening part, held on November 2004 and carried out by a structured telephone interviewing technique included all teaching and non teaching hospitals to find out target hospitals having the minimum infrastructure for IV thrombolytic therapy. In the second part, held in March 2005, all the target institutions were reevaluated with

Results: A total of 354 institutions were contacted in 35 provinces of Turkey. A response was obtained from 312 hospitals. 141 institutions were classified as target hospital and $101(73 \%)$ are situated in 3 of the 7 geographical regions of the country, mainly in 16 cities $(45 \%)$. In only $5 \%$ of the hospitals stroke care is delivered in a specialized unit, and in the remaining this takes place in a general or neurology ward.

Sixty six percent of the centers have no previous experience with thrombolysis but only $8 \%$ declared themselves reluctant to this form of therapy. Ninety four and $96 \%$ of the hospitals were willing to participate to a national thrombolysis data-base and to follow national guidelines, respectively.

Discussion: Many centers stated their will to use IV thrombolysis in acute stroke care and to join local organisations. This data encouraged the wide spread implementation of this therapy in our country. Association Stroke Working Group a face-to-face interview technique.

Acute stroke: treatment concepts 
ANGIOPLASTY OF SYMPTOMATIC INTRACRANIAL ATHEROSCLEROTIC STENOSIS IN PATIENTS WITH HEMODYNAMIC FAILURE

V. Puetz, D. Mucha, A. Mueller, J. Gerber, U. Becker, R. von Kummer, G. Gahn University Clinics Dresden, Dresden, Germany

Background: Percutaneous transluminal angioplasty (PTA) and stenting for intracranial stenosis are mainly reserved for patients who have ongoing ischemic events despite standard medical therapy. In patients with hypoperfusion related to symptomatic intracranial stenosis endovascular treatment might be indicated before antithrombotic therapy fails.

Methods: We reviewed our endovacular revascularization procedures performed between 01/1998 and 12/2005. Inclusion criteria were symptomatic intracranial stenosis interventionally treated because of hypoperfusion as shown by magnetic resonance perfusion imaging, abolished cerebrovascular reserve capacity as determined by Doppler breathholding test, border-zone infarction or hemodynamic transitoric ischemic attacks while not on antithrombotic therapy. We analysed the technical success, complication rate and long-term outcome.

Results: We performed 11 angioplasties in the anterior and 3 in the posterior circulation which fulfilled the inclusion criteria. Thirteen of 14 procedures were technically successful. We performed PTA in 5 and stenting in 8 patients. The 30 -day periprocedural complication rate was $14.3 \%$ (1 intracranial hemorrhage, 1 in-stent thrombosis which ocurred 3 days after the procedure). Follow-up data were available for 9 of 13 procedures for a mean period of 24.6 months (2-72 months). No patient had further cerebrovascular events related to the index vessel.

Discussion: Considering the high risk of the disease, patients with symptomatic intracranial stenosis and hypoperfusion may benefit from interventional therapies.

\section{Acute stroke: treatment concepts}

\section{ESTIMATION OF PATIENT ELIGIBILITY FOR TROMBOLYSIS IN ACUTE ISCHEMIC STROKE BASED ON A HOSPITAL STROKE REGISTRY IN} WARSAW

A. Kobayashi, I. Sarzyńska-Długosz, M. Niewada, M. Skowrońska,

A. Czlonkowska

Institute of Psychiatry and Neurology, Warsaw, Poland

Background: Trombolysis in acute ischemic stroke was introduced in Poland in 2003. The aim of the study is to evaluate eligibility of patients for intravenous recombinant tissue plasminogen activator (rt-PA) and comparison with the number of treated patients. We also were to find out if the information about stroke symptoms provided for patients and general doctors, as well as changes in the mode of action introduced in 2nd Department of Neurology in 2003 influenced the eligibility and the number of treated patients.

Methods: A retrospective analysis of the 2nd Department of Neurology database of ischemic stroke patients admitted in the years 1995-2003 and 2003-2005 (before and after introduction of trombolysis) has been performed. Eligibility for rt-PA treatment was assessed using the criteria outlined by the Safe Implementation of Trombolysis (SITS) Protocol. Number of patients eligible for trombolysis has been estimated and than compared with the number of treated patients.

Results: 1533 patients with ischemic stroke were admitted from June 1st 1995 to November 1st 2003, 18.7\% within 2 hours from onset, $78 \%$ aged under $80.4 .6 \%$ of ischemic stroke patients were potentially eligible for rt-PA.

552 ischemic stroke patients were admitted from November 1st 2003 to September 30th 2005, 19,2\% within 2 hours from onset, $71,6 \%$ under 80 years old. $6,9 \%$ of ischemic stroke patients were eligible for rt-PA. $8,6 \%$ were treated.

Discussion: Age and time from onset to admission were the most common exclusion criteria. The number of treated patients was higher then estimated. After providing the information about stroke symptoms there was a trend in increasing the number of patients admitted within 2 hours from onset. Changes in the mode of action increased the number of treated patients.

\section{Acute stroke: treatment concepts}

THE EFFECT OF STATINS WITHDRAWAL ON STROKE OUTCOME DEPENDS ON STROKE SUBTYPE

F. Nombela, M. Blanco, L. Lopez-Manzanares, M. Rodríguez-Yañez, Y. Silva, M. Millán, M.A. Moro, A. Dávalos, J. Vivancos, J. Castillo

Hospital Universitario de La Princesa, Madrid, Spain

A controlled study of our group confirmed that statins withdrawal (SW) in acute cerebral infarction (CI) is associated with bad outcome. We sought to investigate this effect by stroke subtype.
Materials and Methods: Prospective, open-labeled, randomized study of 215 patients with CI, 91 (42.3\%) patients had atherothrombotic origin (ACI), and 124 $(57.7 \%)$ non atherothrombotic (NACI). In the ACI group, 50 patients $(55 \%)$, previously on statin treatment, were randomized to SW (27) (Group ACI-1), or to keep on statins (23) (Group ACI-2), 41 (45\%) patients who were not on statins, were the control group (Group ACI-3). Likewise, in the NACI group, 39 (31.4\%) patients previously on statins, were randomized to SW (19) (Group NACI-1), or to keep on statins (20) (Group NACI-2) and $85(68.6 \%)$ patients who were not on statins, were the control group (Group NACI-3). Outcome variables were early neurological deterioration (END), defined as a fall $\geq 4$ points in NIHSS between admission and 48 hours, infarct volume on CT at day 4-7, and poor neurological outcome, defined as a modified Rankin scale score $>2$ at 3 month. Results are expressed as median [quartiles].

Results: Baseline clinical characteristics were well balanced between groups. Group ACI-1 had the poorest outcome (mRS ACI-1, 4[2-6]; ACI-2, 3[1-3]; ACI-3, 2[1-4] $\mathrm{p}=0.001$ ), the greater infarct volume (ACI-1, 107 [72-167]; ACI-2, 55.4 [18-106]; ACI-3, 74 [21-130.9] p= 0.033), and the higher frecuency of END (ACI-1, 81.5\%; ACI-2, 34.8\%; ACI-3, 51.2\%. p=0.002). After adjustment for baseline stroke severity and other potential clinical confounders, the odds ratios of END and poor outcome for SW in ACI were 25.9 [4.0-166.1] and 6.6 [1.6-28.1] respectively. Only END showed significant differences between NACI groups (NACI-1, 42.1\% NACI-2, 5.0\%; NACI-3, 16.5\%. p=0.008)

Conclusion: SW in the acute phase of $\mathrm{CI}$ is associated with poor outcome and larger brain injury particularly in cerebral infarctions of atherothrombotic origin

\section{Acute stroke: treatment concepts}

\section{LOADING CLOPIDOGREL IN THE MANGEMENT OF ACUTE ISCHEMIC} STROKE: CASE PRESENTATIONS

H.M. Aref, N.M. Nahas, M. Awad

Ain Shams University, Cairo, Egypt

Background: Starting experience with loading high doses of clopidogrel, was first explored by cardiologists. Recent trials in cardiology had showed that higher doses are more potent, yet with similar safety profile among patients treated with loading doses of $300 \mathrm{mg}, 600 \mathrm{mg}$ and $900 \mathrm{mg}$ of clopidogrel: ARMYDA-2 study, ISARCHOICE study and ALBION trial. The intense anti-platelet suppression represents our rationale for giving loading clopidogrel in the management of acute ischemic stroke.

Methods: We report six cases treated with loading clopidogrel in acute ischemic stroke, with different dosages 600 and $900 \mathrm{mg}$, when IV rTPA was not indicated contraindicated or couldn't be afforded. The drug was given in the first 12 hours from stroke onset The patients were assessed by the NIH-SS on admission and for follow up after 3 days and 7 days. All patients had a CT scan on admission to exclude hemorrhage. MRI with T1WI, T2WI, diffusion study, T2* and MRA was done on admission or next day.

Results: Regression of the NIH-SS ranged from 5 to 25 points after 7 days Bleeding whether intra or extra-cerebral occurred only in cases co-medicated with anticoagulants and was under control with discontinuation of anticoagulant. Clinical improvement correlated with high dose of clopidogrel.

Discussion: These case reports demonstrate the efficacy of high dose clopidogrel, without any major side effects. Despite the significant and rapid improvement in these cases there are a number of issues to be further investigated: First, the safety of high loading doses of clopidogrel in ischemic stroke. Despite the proved safety in cardiologic studies, the condition might be different with acute cerebral ischemia due to the possible tendency of infarct brain tissue for hemorrhagic transformation. Second, if there is a therapeutic difference between $600 \mathrm{mg}$ and $900 \mathrm{mg}$ loading doses in acute stroke patients. All those issues need to be fully investigated by more detailed studies.

\section{4}

Acute stroke: treatment concepts

SEVERITY OF BRAIN DAMAGE IN SALVAGEABLE PATIENTS WITH ACUTE CEREBRAL INFARCTION IS REFLECTED BY PLASMA BIOMARKERS, WHICH MIRROR THE THERAPEUTIC EFFICACY

K.T. Kitazato, M. Uno, A. Suzue, K. Nishi, K. Matsuzaki, H. Itabe, S. Nagahiro The University of Tokushima, Tokushima, Japan

Background: To obtain a worthwhile treatment effect, an exact and early diagnosis is required. Current imaging techniques are often not useful for the rapid identification of patients who are mostly to benefit from neuronal rescue therapy. To assess the availability of plasma biomarkers for the diagnosis and therapeutic monitoring in acute cerebral infarct and to examine whether the severity of brain damage and the therapeutic efficacy are reflected in their plasma levels, we compared the 
plasma levels of biomarkers in ischemic stroke patients who did, or did not, receive edaravone.

Subjects and Methods: Fifty one patients with ischemic cerebral infarcts were divided into 2 groups; GI $(n=24)$ had cortical lesions, GII $(n=27)$ had lesions in the basal ganglia or brain stem. Edaravone was administered to 27 randomly selected patients (GIa, $\mathrm{n}=13$; GIIa, $\mathrm{n}=14$ ) and their plasma OxLDL-, S-100B-, and MnSOD levels as biomarkers of brain damage and NIH stroke scale were compared with those in patients without edaravone treatment (GIb, n=11, GIIb, n=13).

Results: In patients with acute cerebral infarction, plasma OxLDL levels were significantly increased after the insult and edaravone significantly reduced them at 3 days after the start of treatment (GIa; $0.219 \pm 0.026$ vs GIb; $0.177 \pm 0.024, p<0.05$ ) Their plasma S-100B and MnSOD levels in patients treated with edaravone were decreased as well. Corresponding to these changes, the NIHSS at discharge had recovered in the GI patients (from $10.9 \pm 2.5$ to $7.0 \pm 1.2, \mathrm{p}<0.05$ ). In GII patients, preand post-treatment plasma biomarkers and NIHSS were not significantly different. Conclusions: Here we first demonstrate that the brain damage in early stage and the therapeutic efficacy of edaravone can be monitored by measuring plasma biomarkers that detect oxidative and astrocyte damage in acute cerebral infarction, thereby limiting the degree of brain damage.

\section{Acute stroke: treatment concepts}

INFLUENCE OF DECOMPRESSIVE HEMICRANIECTOMY IN MALIGNANT MIDDLE CEREBRAL ARTERY TERRITORY INFARCTION ON MORTALITY AND FUNCTIONAL OUTCOME

C. Maris, C. Popa

Institute of Cerebro-Vascular Diseases, Bucharest, Bucharest, Romania

Background and Purpose: Hemicraniectomy have been proposed to relieve intracranial hypertension and tissue shifts in patients with large hemispheric infarcts. Our objective was to determine the value of decompressive craniectomy in patients with malignant MCA territory infarction and to compare functional outcome related to the patients' age.

Methods: Patients with malignant MCA territory infarction treated in our hospital between January 2002 and October 2005 were prospectively studied. Twenty out of them underwent decompressive craniectomy, and the 31 were treated conservatively. All survivors were reexamined after 3 months with the clinical evaluation graded according to the modified Rankin Scale and Barthel Index. We analyzed the influence of early decompressive surgery ( $<24$ hours after symptom onset) versus late surgery, and conservative treatment on mortality and functional outcome.

Results: 14 patients $(70 \%)$ from the surgical group survived versus 8 patients $(25.8 \%)$ in nonsurgical group. In surgical group, 1 patient $(5 \%)$ was functionally independent, $8(40 \%)$ were mildly to moderately disabled, and $11(55 \%)$ died or were severely disabled. Conversely, in nonsurgical group, only $8(26 \%)$ patients survived, 5 being moderately disabled and 3 severe disabled. The mortality rate was also significantly higher $(64.3 \%)$ for those $>50$ years of age compared with $47.7 \%$ for those $\leq 50(\mathrm{P}<0.01)$. Of the 7 patients who underwent hemicraniectomy within 24 hours 5 had a good outcome, and 2 were severely disabled or dead at follow-up. Discussion: Outcome of patients treated with hemicraniectomy in severe hemispheric infarction was good, early surgery appearing to supplementary improve outcome. Age appears to be a determining factor in predicting functional outcome after hemicraniectomy

\section{Acute stroke: treatment concepts}

\section{SYSTEMIC THROMBOLYSIS IN ISCHEMIC STROKE IN THE} NEUROLOGICAL DEPARTMENT OF THE MEDICAL UNIVERSITY OF GDANSK

D. Gasecki, G. Kozera, M. Miastkowska-Swierkocka, K. Chwojnicki,

S. Szczerba, A.W.M. Nyka

Medical University of Gdansk, Gdansk, Poland

Background: Based on several large controlled randomized trials rt-PA has been approved for clinical use in the USA, Canada (1996) and the European Community (2003) for the therapy within three hours after onset of an acute ischemic stroke.

Materials and Methods: In our institution we perform this therapy according to the criteria of the NINDS-trial. In this study we have reviewed patients treated with systemic rt-PA in our center. Between 01.11.2003 and 31.12.2005 we have been treating 440 patients presenting with ischemic stroke. $40(9,1 \%)$ patients have been treated with systemic rt-PA. 35 patients with 90-day clinical follow up - $12(34,3 \%)$ women and $23(65,7 \%)$ men (average age $67 \pm 14$ years, range 19-81 years) were analysed.

Results: The average baseline NIHSS score was 13,9 $\pm 6,2$ (range, 6-24). The mean time from onset to rtPA treatment was $169 \pm 25,5$ minutes (range, 130 to 180 minutes). At the follow up after 3 months the ratio of patients with none or mild disability (Rankin $0-2$ ) was $65,7 \%$ (23 patients), in this group 13 patients $(37,1 \%)$ had none or minimal disability (Rankin $0-1)$. 10 patients $(28,6 \%$ ) were dependent in daily living activities (Rankin 3-5). The rate of intracranial bleeding was 8,6\% (3 patients), only in $1(2,9 \%)$ patient it was a symptomatic bleeding. Mortality on day 90 was $5,7 \%$

Conclusions: Our data proof that systemic thrombolysis with rtPA to three hours after onset of symptoms of stroke is efficient and safe.

\section{Vascular surgery and PTCA}

\section{Vascular surgery and PTCA}

IS STEREOTACTIC HEMATOMA EVACUATION VALUABLE TO MOTOR FUNCTION RECOVERY AND BETTER OUTCOME OF PATIENTS WITH SPONTANEOUS PUTAMINAL HEMORRHAGE? A RANDOMIZED TRIAL

J. Moroi, A. Suzuki, N. Kobayashi, N. Yasui

Research Institute for Brain and Blood Vessels, Akita, Japan

Objective: The present prospective trial was undertaken to evaluate the effectiveness of stereotactic hematoma evacuation following spontaneous putaminal hemorrhage $(\mathrm{SPH})$

Methods: Patients who satisfy the following conditions are included this study; 1) admitted to our institute within 24 hours after onset, 2) with SPH of $20 \mathrm{ml}$ or more hematoma volume, 3 ) with neurological grade of somnolence or better on admission, 4) with motor weakness of active movement against gravity or worse. Patients were randomized by means of sealed envelopes to stereotactic hematoma evacuation or initial conservative treatment. Surgery was performed between 12 to 72 hours after onset. We evaluated motor weakness at 6 months after onset. We used the Barthel index (BI) and modified Rankin scale (mRS) at 6 months follow-up as the primary outcome measure. Between 1999 and 2005, 30 patients (6 women, 24 men, 43-74 years old) were randomized: 14 to surgery (Group 1) and 16 to initial conservative treatment (Group 2). Motor weakness was improved at 6 months in both groups, and there was no significant difference between two groups. Compared with group 2, group 1 resulted better BI and mRS but again these differences were not significant.

Conclusion: Patients with SPH, whose neurological grade is somnolence or better on admission and weakness are equal or worse than active movement against gravity, show little benefit from stereotactic hematoma evacuation.

\section{Vascular surgery and PTCA}

CAROTID ARTERY STENTING USING FILTERWIRE IN CONSECUTIVE SYMPTOMATIC PATIENTS WITH SEVERE CAROTID STENOSIS

S.M. Sung, T.H. Lee, K.P. Park, S.W. Lee, H.J. Kim

Medical Reserch Institute, Pusan National University Hospital, Pusan, South Korea

Background \& Purpose: The most important acute complications during Carotid artery stenting (CAS) are procedure-related strokes, which are mostly caused by distal embolization of particles. Here, the authors evaluated the performance of the FilterWire protection device (Boston Scientific) for the capture of debris and prevention of embolic events during the CAS

Methods: Between June 2004 and December 2005, a total of 60 cases of 56 symptomatic patients who had $>70 \%$ diameter stenosis of the internal carotid artery underwent CAS with FilterWire. Thirty-three cases had near-complete occlusion $(>90 \%)$ and twenty-seven had severe stenosis $(>70 \%)$. Before and after the procedure, we evaluated the cerebral ischemic lesions of embolic origin by diffusion-weighted image (DWI). Satisfactory performance of the FilterWire was defined as effectiveness in preventing embolic events shown by visible debris in the filter and unchanged neurological status. Visible debris within the FilterWire was sent for histological/cytological analysis.

Results: Protected CAS was successfully performed in all 60 cases. The mean post-procedural stenosis was nearly $0 \%$. The 30 -day mortality/morbidity rates were as follows: death, $0 \%$; major strokes, $1.7 \%$ ( 1 case); minor strokes, $1.7 \%$ ( 1 case) One major stroke was not related to the intervention because the stroke occurred in the contralateral MCA territories at the 3rd post-procedural days. One minor stroke achieved complete recovery. Twenty-six (43.3\%) cases had visible debris within the filter. Maximum diameter of the debris was $3 \mathrm{~mm}$. Histological analysis revealed fibrin meshwork, RBC and other blood components. On post-procedural diffusionweighted images performed in 43 patients, asymptomatic embolic infarctions were seen in 9 patients $(9 / 43,20.9 \%)$ on post-procedural DWI. 
Conclusion: CAS with distal protection by FilterWire seems a feasible, safe and effective means of preventing thromboembolic complication during the procedure.

\section{Vascular surgery and PTCA}

SUBSTANTIAL UNDER-INVESTIGATION AND TREATMENT OF CAROTID DISEASE IN OLDER PATIENTS WITH TIA AND STROKE

J.F. Fairhead, S.T. Pendlebury, P.M. Rothwell

Stroke Prevention Research Unit, University Department of Clinical Neurology, Oxford, United Kingdom

Background: Incidence of stroke increases with age, as generally does absolute benefit from preventative treatments. To identify any evidence of inappropriate under-investigation or treatment of older patients, we compared age-specific rates of carotid imaging, $\geq 50 \%$ symptomatic carotid stenosis, and subsequent endarterectomy, in patients with recent TIA or stroke in a nested population-based incidence study (Oxford Vascular Study - OXVASC) with rates in a population-based audit of routine clinical practice in the rest of Oxfordshire.

Methods: We identified all patients undergoing carotid imaging for ischaemic TIA or stroke from $01 / 04 / 02-31 / 03 / 05$ in the OXVASC population $(n=91,106)$ and from $01 / 04 / 02-31 / 03 / 03$ in routine clinical practice in the non-OXVASC Oxfordshire Primary Care Trust (NOPCT) population $(n=589,899)$ and determined age-specific rates of carotid imaging, diagnosed $\geq 50 \%$ symptomatic carotid stenosis, and subsequent endarterectomy.

Results: 833 patients with TIA or ischaemic stroke had carotid imaging in NOPCT and 693 in OXVASC, with similar rates up to age 80 years. However, although incidence of $\geq 50 \%$ symptomatic stenosis increased steeply with age in OXVASC, particularly at age $\geq 80$ years, the rates of carotid imaging $(0.36,0.27-0.48$, $\mathrm{p}<0.001)$, diagnosis of $\geq 50 \%$ symptomatic stenosis $(0.21,0.09-0.49, \mathrm{p}<0.001)$, and surgery $(0.14,0.04-0.57, \mathrm{p}=0.001)$ in this age group in NOPCT were all substantially lower than in OXVASC.

Conclusions: Incidence of symptomatic carotid stenosis increases steeply with age, but despite evidence of major benefit from endarterectomy in older age groups, there is substantial under-investigation after TIA or ischaemic stroke in routine clinical practice in those aged $\geq 80$ years.

\section{Vascular surgery and PTCA}

\section{CAROTID STENTING IN ELDERLY}

C. Tejero, M.A. De Gregorio, M.R. Rengel, J. Medrano, A. Mainar,

M. Montori, J.A. Mauri, E. Mostacero

Hospital Clínico Universitario "Dr Lozano Blesa”, Spain

BACKGROUND AND PURPOSE: Epidemiological studies indicate that the risk of first ever stroke is strongly related to age. An analysis of data from the NASCET group, clearly demonstrates that elderly patients (age $>75$ years) with symptomatic ICA stenosis benefit more from CEA compared to younger patients. Carotid angioplasty and stenting (CAS) is being evaluated as an alternative to carotid endarterectomy (CEA) for treatment of severe carotid artery stenosis. Because CAS does not require general anesthesia and is less traumatic, it might be especially advantageous in older patients.

Data concerning CAS treatment method in older patients are scarce.

Methods: The periprocedural complication rate in 33 patients aged 75 years or older who had undergone CAS between 1999 and 2004 were compared with those in a group of 60 patients aged 75 years or less. All patients were evaluated by a neurologist both before and after surgery. According to the criteria set forth by the large trials the occurrence of minor, major, or fatal stroke, and myocardial infarction within 30 days was determined.

Results: The demographic characteristics and indications for an intervention were similar in both treatment groups. In neither group was there any fatal stroke or myocardial infarction. The 30 -day stroke rate was similar in the older group (1 minor, 1 major strokes; $6.06 \%$ ) than in the younger group (1 minor, 2 major strokes; $5 \% ; \mathrm{P}=0.65)$.

Conclusion: The neurological complication rate in patients aged 75 years and older associated with CAS was similar than in $<75$ years old patients.

\section{Vascular surgery and PTCA}

EFFECT OF CAROTID STENTING ON THE HEMORHEOLOGICAL PARAMETERS AND PLATELET AGGREGATION

L. Szapary, G. Feher, F. Kover, E. Onodi, S. Komoly, K. Toth, T. Doczi Medical Univ. of Pecs, Pecs, Hungary

Introduction: Carotid artery stenting (CAS) has emerged as an acceptable treatment alternative in high-risk patients with carotid stenosis. Pathologic hemorheological properties and increased platelet aggregation are risk factors of subacute stent thrombosis.

Methods: 18 patients (11 males, mean age:68 \pm 9 years, 7 females, mean age: $62 \pm 8$ years) with significant carotid artery stenosis and undergoing CAS were involved in our study (adatok, ffi, n?, átlagéletkor?). Hemorheological parameters (hematocrit, plasma fibrinogen level, plasma and whole blood viscosity, red blood cell aggregation and deformability), and epinephrine-, ADP- and collagen-induced platelet aggregation were measured before and immediately after the procedure, at 1, 2, 5 days, and 1 month. Before stenting all patients received loading-dose of clopidogrel (300mg), following combined (clopidogrel $75 \mathrm{mg}+$ aspirin $100 \mathrm{mg}$ dayly) antiplatelet treatment.

Results: Hematocrit (HTC), plasma fibrinogen (PF), whole blood viscosity (WBV) and plasma viscosity (PV) decreased significantly immediately after stenting procedure compared to the baseline values $(\mathrm{p}<0,001)$. On the fifth day WBV, PF, PV, red blood cell (RBC) and ADP-dependent platelet aggregation significantly increased $(\mathrm{p}<0,0001)$ compared to the values immediately after stenting. The parameters except WBV significantly decreased at the first month compared to the fifth day.

Conclusion: Hemorheological parameters and platelet aggregation showed a specific reaction after carotid stenting. Deterioration of the flow characteristic of the blood, activation of platelets are predominant within the first week, and may induce the thrombotic occlusion of the stent.

\section{Vascular surgery and PTCA}

\section{TIME DELAYS FOR CAROTID ENDARTERECTOMY IN A U.K. TEACHING HOSPITAL \\ N. Altaf, S. Goode, S.T. MacSweeney \\ Queen's Medical Centre, Nottingham, United Kingdom}

Background: The benefit of carotid surgery in symptomatic patients is established More recently, the importance of minimising delay to surgery has been recognised. Maximum benefit is achieved when carotid endarterectomy (CEA) is performed within two weeks after the initial event. The aim of this study was to review the delays for CEA in our institution, a UK teaching hospital covering a population of 665000 .

Methods: We performed a retrospective review of all patients who had CEA performed for symptomatic carotid disease over a two year period from November 2003 to November 2005. Time delays were examined from the initial presentation to CEA.

Results: Of the 106 CEAs' that were performed, it was not possible to acquire the initial symptom event date on 13 patients. The median delay from the initial event to the CEA was 17.7 weeks (IQR 9.9-28.9). During this period $35.6 \%$ of all patients had further symptoms.

The median delay from the initial event to the initial review by any clinician was 0.15 weeks (0-0.86); delay between the initial clinical reviews to referral to a surgeon was 2 weeks (0-8.4); and the delay between the referral and the review by a surgeon was 4 weeks (2-5.8). The median time from the initial event to a Duplex scan was 6.4 weeks (3.6-13.9)

The median time from the surgical review to the operation was 4.7 weeks (2.9-8.7). Further imaging and cardiac examinations additionally added to the delay.

Conclusion: There a substantial delays occurring between the initial event and CEA in our own institution and we suspect that this is widespread. The potential benefits of CEA are not being maximised and some patients will suffer preventable strokes. We believe that patients with transient ischaemic attacks should be managed more aggressively using a multidisciplinary approach. Easy access to imaging, early surgical review and urgent CEA are all necessary. We plan to introduce this shortly. 


\section{Vascular surgery and PTCA}

HEMODYNAMIC EFFICACY ASSESSMENT OF ENDOVASCULAR STENT PLACEMENT FOR MIDDLE CEREBRAL ARTERY STENOSIS USING STATISTICAL ANALYSES OF BASAL AND ACETAZOLAMIDE TC-99M ECD BRAIN SPECT

T.H. Lee, S.J. Kim, S.W, Lee, S.M. Sung, H.J. Kim

Medical Research Institute, Pusan National University, Pusan, South Korea

Background \& Purpose: Statistical parametric mapping (SPM) and Statistical probabilistic anatomical mapping (SPAM) were applied to basal/acetazolamide Tc-99m ECD brain perfusion SPECT images in patients with middle cerebral artery (MCA) stenosis to assess the efficacy of endovascular stent of MCA.

Materials \& Methods: Eleven patients ( 8 men and 3 women, the mean age of $54.2 \pm 6.2$ year) who were undergone endovascular stent placement for MCA stenosis were enrolled. Using SPM and SPAM analyses, we compared the significant number of voxels and cerebral counts between pre- and post-stent basal/acetazolamide SPECT, and assessed the perfusion changes and cerebral vascular reserve index (CVRI).

Results: The numbers of voxels of brain SPECT were decreased from 6,321 $\pm 6,738$ to $2,411 \pm 2,321(\mathrm{p}=0.0674)$ in basal images and from $7,677 \pm 11,288$ to $2,203 \pm 2,876$ $(\mathrm{p}=0.042)$ in acetazolamide images. On SPAM analysis, the increases in cerebral counts were significant in the acetazolamide images $(90.9 \pm 2.2$ to $93.5 \pm 2.3$, $\mathrm{p}=0.0098$ ) but not in basal images ( $91 \pm 2.7$ to $92 \pm 2.6, \mathrm{p}=0.1602)$. The CVRI showed also statistically significant increase from pre-stent (median $0.32 ; 95 \% \mathrm{CI}$, $-2.19-2.37$ ) to post-stent (median 1.59 ; $95 \%$ CI, $-0.85-4.16)(\mathrm{p}=0.0068)$.

Conclusion: The optimum role of SPM and SPAM analyses in patients with cerebrovascular disease is still not clearly defined. This feasibility study suggests the possible role of voxel based analysis of basal/acetazolamide brain perfusion SPECT after MCA stent placement and these techniques need more refining before they can be valid in a range of diseases affecting cerebral perfusion.

\section{Vascular surgery and PTCA}

LOCAL ANAESTHESIA VERSUS GENERAL ANAESTHESIA FOR CAROTID ENDARTERECTOMY: THE NOTTINGHAM EXPERIENCE

K. Wadhwa, A. Oluwale, N. Altaf, S. Goode, S.N. Chandrasekar,

S.T. MacSweeney, W.G. Tennant

Queen's Medical Centre, Nottingham, United Kingdom

Background: The best anaesthetic mode during carotid endarterectomy (CEA) is still controversial. Interest and the use of local anaesthesia (LA) for CEA has increased considerably in the UK as it is thought to confer significant benefits over the use of general anaesthesia (GA). The aim of this study was to compare perioperative complication rates in patients undergoing CEA under LA and GA.

Methods: A retrospective review of 359 consecutive patients who underwent unilateral CEA for symptomatic carotid disease between 1995 and 2004 at our institution was conducted. The effect of the mode of anaesthesia upon operative technique as well as perioperative and postoperative complications was assessed.

Results: LA was used in 158 operations (44.0\%) and GA in 201 operations (56.0\%). There were no significant differences in sex between the two groups or in the indication for surgery; however patients were significantly younger in the GA group (mean age $67.6 \pm 8.8$ yrs GA vs. $70.05 \pm 8.5$ yrs.-LA, $\mathrm{p}<0.05$ ) CEA was significantly longer in the GA group (mean time $163.1 \pm 40.2$ mins.-GA vs. $146.9 \pm 27.7$ mins.LA, $\mathrm{p}<0.05)$ and a carotid shunt (used selectively) was used in $51(25.3 \%)$ patients in the GA group and in $23(14.6 \%)$ patients in the LA group.

There was no difference in risk of stroke and death (3.48\%-GA vs. $2.53 \%$ LA) between the 2 groups. There were no significant differences in the pulmonary complication rate (0\%- GA vs. $0.63 \%$-LA), MI (1.49\%-GA vs. $1.27 \%$-LA) and immediate cranial nerve injuries (4.48\%-GA vs. $3.80 \%$-LA).

Conclusions: Local anaesthesia yields similar perioperative results to general anaesthesia and allows more reliable cerebral monitoring with lesser shunt rates and shortening the operative times. Although the advantages over GA are relatively small, the safety and versatility of the technique has made it increasingly popular in the United Kingdom. 
Cerebrovascular Diseases

Aakvik, R., 124

Abboud, D., 77

Abboud, H., 123

Abboud, S., 44

AbESTT-II Investigators, 59

Abilleira, S., 80

Abreu, P., 104, 106

Acciarresi, M., 18

Ackerstaff, R.G.A., 147

Adler, Y., 90

Afşar, N., 146, 147

Agal, A., 83

Agareno, S., 145

Agelidakis, P., 69

Agnelli, G., 18

Ágoston, V.A., 52

Ahmed, N., 36

Ahn, M.Y., 64, 69

Aichner, F.T., 12

Aigbirhio, F.I., 33, 51, 81

Airian, N.Y., 26

Ajdacic-Gross, V., 26

Akbar, I., 127

Akhmetov, V.V., 13

Aktan, S., 146

Akyol, A., 126

Albers, G.W., 37

Alberti, A., 18

Alberts, M.J., 25, 55, 118

Alberts-Grill, N., 114

Albrecht, K.W., 54

Alcolea, D., 48

Alemany-Rodríguez, M.J., 100

Alessandri, B., 79, 82

Alevizaki, M., 122

Alexandrov, AV., 20

Alexeeva, G.S., 13

Aliseda, D., 68

Allard, L., 125

Allemann, E., 50, 79

Allport, L.E., 108, 116

Almeida, A.M., 145

Alonso, A., 50, 78, 79

Alperovitch, A., 8

AlQteishat, A., 84

Altaf, N., 14, 63, 150, 151

Althaus, A., 99

Altieri, M., 3, 16, 129

Alvarez, B., 12

Alvarez-Sabín, J., 12, 21, 22, 32 , $37,38,39,62,80,142,144$

Álvaro, L.-C., 24

Alves, M.M., 72, 88

Amarenco, P., 14, 23, 73, 97, 123

Amberger, N., 92

Ameri, A., 72

Amouyel, P., 8

\section{Author Index}

The number refers to the page number
Ananda, K., 142

Anders, C., 136

Andersen, K.K., 29, 40

Anderson, C.S., 20

Anderson, P., 49

Andre, C., 36, 137

Andrikopoulou, A., 69, 73

Andsberg, G., 125

Ang, S., 127

Angleitner, K., 107

Annoni, J.M., 21

Anslow, P., 34

Antonc, I., 87

Antrian, V., 73

Apfalter, P., 42

Aping Sun, 143

Aposporos, G., 74

Araña-Toledo, V., 100

Araujo, M., 141

Arauz, A., 41, 54, 84, 92, 127

Arboix, A., 65

Aref, H.M., 148

Arenillas, J., 98, 146

Arenillas, J.F., 63

Argentiero, V., 74

Arias-Rivas, S., 28

Armani, M., 74

Armitage, P.A., 35, 36, 111

Arnold, M., 54, 63, 132

Aronis, S., 77

Arpaci, E., 126

Arquizan, C., 29

Artemis, N., 141

Aslanyan, S., 38

Attia, J., 11, 96

Audebert, H.J., 27, 57

Auer, D.P., 14, 63

Autret, A., 66, 69

Awad, M., 148

Ayo, O., 95

Azevedo, E., 78, 104, 106

Baba, Y., 113

Bacabac, E., 136

Baccini, M., 130

Bacellar, A.S., 141

Bachmann, R., 94

Back, T., 99, 118

Badaut, J., 52

Badimon, L., 7, 10

Bae, H.J., 65, 100

Baert, I., 5

Baezner, H., 17

Baik, M.Y., 138

Baird, A.E., 114

Bakker, S.L.M., 46

Balaguer, E., 142
Bamber, L.C., 119

Bamford, J., 76

Bammer, R., 37

Banfi, C., 52

Baranska-Gieruszczak, M., 99

Barba, M.A., 95

Barber, P.A., 26, 45, 112

Barbey, F., 133

Barbolini, M., 58

Barinagarrementeria, F., 39, 54, 84, 92

Barkhof, F., 16

Baron, J.C., 2, 33, 51, 81

Barón, M., 119

Barozzi, G., 132

Barrick, T.R., 116

Barriga, F.J., 9, 119

Bartels, P., 84

Bartkova, A., 110

Bartolini, L., 43

Bartrés-Faz, D., 65

Basile, A.M., 74

Bassetti, C.L., 145

Bastin, M.E., 35, 36, 111

Bateman, G.A., 32

Bath, P.M.W., 4, 6, 42, 121, 144

Baumgartner, R.W., 63, 87

Bayley, M., 3

Bäzner, H., 17, 68

Becker, U., 144, 148

Becker, W.P., 50

Beech, A., 14

Beech, J.S., 51, 81

Beleyev, L., 81

Belinchón, J.C., 9

Beltran, I., 95

Bembenek, J., 33, 35

Ben-Assayag, E., 54

Benavente, L., 41, 69

Bendel, O., 52

Benecke, R., 131

Benessiano, J., 123

Benninger, D., 63

Bereczki, D., 71, 88, 121

Berge, E., 55, 124

Beridze, I., 25

Berkefeld, J., 28

Berliner, S., 54

Berlis, A., 147

Bernhardt, J., 3, 135

Berry, E., 58

Besa, M., 65

Best, J,J., 58

Betensky, R., 64

Bevan, S., 43, 78

Bezerra, D.C., 36, 137

Bhalla, A., 21, 99
Bhatia, R., 46

Bhatt, D.L., 25, 55, 118

Biagini, S., 18

Bianchi, S., 43, 77

Biessels, G.J., 137

Bignamini, A.A., 94

Binder, J., 26, 114

Biousse, V., 72

Bir, B., 147

Birns, J., 8

Black, N., 106

Blacquiere, D., 30

Bladin, C., 27

Blahak, C., 17, 68

Bla?ina, K., 87

Blanc-Guillemaud, V., 52

Blanco, M., 28, 138, 148

Blecic, S., 8, 44, 77

Blecic, S.A., 23, 70, 88

Blomstrand, C., 91

Bobrow, B.J., 145

Bocquet, L., 146

Bogdanovic, M., 4

Bogousslavsky, J., 12, 13, 20, 21, $25,26,31,49,52,67,70,73$, $87,114,136,139$

Boissier, N., 47

Bombois, S., 16, 17

Bonaffini, N., 3

Bonati, L., 19, 41, 42, 125

Bonita, R., 85

Bonnans, V., 31

Bonnaud, I., 66, 69

Bonny, C., 49

Bordet, R., 28

Bori, Z., 80, 82, 84

Born, C., 26

Bornstein, N.M., 25, 54, 124

Borojevic, R., 137

Borratynska, A., 70

Borrell, M., 48

Bosak, P., 29, 72

Bosque, M., 142

Boukobza, M., 39

Boulanger, J.M., 27, 45, 112, 114

Bourg, V., 47

Bousser, M.G., 20, 39, 43, 72

Boutron, C., 43

Bova, I., 54

Bo Wu, B., 121

Boysen, G., 52, 53, 124

Brainin, M., 29, 72

Brakch, N., 133

Brandt, T., 132

Bravo, Y., 37, 41, 48, 145

Bray, D., 132

Bray, J., 27 
Brcic, I., 76

Brea, D., 98

Brekenfeld, C., 54

Brenner, D., 81

Brich, J., 147

Briesenick, C., 90

Briley, D., 18, 34

Brioschi, M., 52

Brisc, C., 62

Brisc, C.M., 62

Briscoe, C., 43

Broderick, J.P., 23, 48

Brondani, R., 141

Brouste, Y., 123

Browaeys, P., 114, 139

Brown, J., 47

Brown, M.M., 42

Bruandet, A., 16, 17

Brückmann, H., 49

Brugières, P., 98

Bruins Slot, K., 55

Brun, N.C., 23, 48

Bruzzone, M.G., 68

Brzozowski, K., 75

Buc, M., 87

Buchan, A.M., 26, 27

Budišc, M., 89

Bueters, T., 52

Buffon, F., 20, 43

Bugalho, P., 128

Bugert, P., 78

Buggle, F., 136

Bukow, S., 18, 78

Bukow, S.C., 110

Buonanno, F., 64

Burduladze, I., 25

Burgemeister, A., 109

Burger, I., 8, 97, 118, 119

Burina, A., 96

Burkhard, P.R., 125

Burval, S., 67, 110

Busch, M.A, 97

Busch, M.A., 118

Busekroos, L., 140, 146

Buser, P., 41

Busson, L., 74

Butcher, K.S., 108

Bütefisch, C., 105

Byrne, J.V., 34

Byun, W.M., 138

Cadilhac, D.A., 57, 134

Cadioli, M., 14

Calleja, J., 41

Calleja, S., 41, 69

Calvet, D., 32

Camelbeeck, P., 133

Canbolat, A., 51

Cangür, H., 109, 115

Canhão, P., 39

Cano, A., 124

Cansever, M.T., 51

Cantú, C., 54

Cantu, C., 84, 92

Capele, C., 16

Cappellari, M., 97

Caputi, L., 68

Cardenas, A., 82
Cardona, P., 15, 64, 65

Carod-Artal, F.J., 71

Carolei, A., 10, 11, 48, 87

Carota, A., 21, 67, 70

Carpenter, T., 34

Carpenter, T.A., 2

Carpenter, T.K., 35

Carrera, E., 25, 136

Carreras, F., 41

Carriero, M.R., 68

Carruzzo, A., 20, 31

Carter, R.C., 57

Caruso, A., 132

Carvajal, A., 50, 64

Carvajal Collado, A., 7, 10

Caso, V., 18

Castellanos, M., 28, 37, 98, 146

Castillo, J., 28, 82, 98, 136, 138, 146, 148

Cattin, F., 40

Cecconi, E., 100

Celani, M.G., 21

Celnik, P., 4

Cendrós, V., 65

Cerone, D., 11, 48

Cha, J.-K., 102

Chabriat, H., 20, 39, 43

Chacón, P., 21

Chan, D., 143

Chan, J., 3

Chan, L.C., 77

Chan, P.H., 77

Chandrasekar, S.N., 151

Chang, H.M., 53, 55, 108, 127, 139

Chang-Ling, C., 118

Channell, K., 136

CHANT Investigators, 1

Chappell, F., 42, 58

Charchat-Fichman, H., 16

CHARISMA Investigators, 1

Charlton, R.A., 116

Chatel, M., 71

Chatelain, B., 101

Chatelain, C., 101

Chau, Y., 47, 71

Chávez, M., 127

Chavot, D., 97

Chayer, C., 75

Cheah, T.S., 85

Chen, C., 108

Chen, C.P., 53, 55, 127, 139

Chen, X.Y., 113

Cheng-Chieh, L., 118

Chernikova, L.A., 117

Cho, A.-H., 108

Cho, A.H., 48

Cho, J.H., 96

Cho, J.Y., 80

Cho, K.H., 93, 100

Cho, Y.J., 80

Cho, Y.W., 110

Choi, C.G., 48

Choi, E.S., 80

Choi, J.H., 46, 47

Choi, S.A., 96

Choi, S.H., 109

Choi, S.M., 93

Chong, W., 112
Chon-Haw, T., 118

Chou, S., 64

Christensen, M.C., 47, 71

Christian, C., 141

Chun, J.-U., 65

Chung, C.S., 112

Chung-Hsiang, L., 118

Chun-xue Wang, 113, 125

Chwojnicki, A.K., 76

Chwojnicki, K., 89, 111, 149

Ciesielska, A., 99

Clark, G., 94

Clark, J.C., 51, 81

Cleij, M., 81

Clerici, M., 132

Cloud, G., 5

Cocho, D., 37, 41, 48, 145

CODICIA Study Group, 1

Cohen, L.G., 4, 103, 105

Collet-Sassere, E., 29

Collett, J., 4

Collier, A., 61

Collier, J.M., 3, 135

Collier, T., 121

Colombo, E., 68

Comi, G., 14

Conforto, A.B, 103

Conrad, B., 7

Cooke, E., 104

Coral, J., 127

Cordato, D., 143

Cordonnier, C., 16

Corea, F., 14

Corfini, A., 74

Cornil, V., 91

Correia, J., 104, 106

Coshall, C., 97, 118

Costa, A., 97, 127, 132

Costa, L., 74

Cote, V.J., 97

Cottini, E., 127

Coughlan, K., 27

Coull, A.J., 34

Coull, B., 81

Coutts, S.B., 35, 45, 112

Covickovic-Sternic, N., 92

Cox, A.M., 121

Craig, A., 92

Cramaro, A., 136

Cramer, S.C., 81

Crassard, I., 39, 72

Cristobo, I., 28, 146

Croquelois, A., 25

Crossland, L., 89

Csiba, L., 71, 88

Csonka, E., 82

Cuadrado, E., 37, 65, 133, 138

Cubero-González, A., 100

Cucurella, G., 94

Cumurcuic, R., 43

Cupples, M.E., 7

Cuvelier, C., 8

Cvoro, V., 35, 36, 111

Czlonkowki, A., 117

Czlonkowska, A., 28, 33, 35, 89, $99,103,117,139,148$

Czosnyka, M., 74
d'Arminio-Monforte, A., 132

D'Incerti, L., 68

Daenekindt, T., 71

Dahl, M., 70

Dalabie, A.L., 70

Dalaklidou, V., 141

Daniels, L., 14, 63

Danielsson, E., 125

Danna, P., 132

Dannenberg, C., 45, 109

Darius, H., 9

Dartigues, J.F., 8

Das-Gupta, R.J., 119

Dávalos, A., 28, 63, 82, 136, 138, 146,148

Davey, R.J., 76

Davidoff, A.W., 81

Davies, R., 106

Davis, S., 23, 47, 48, 116

Davis, S.M., 108

Dawes, H., 4

Day, D.J., 2, 33

Debatisse, D., 136

Debette, S., 8, 16, 17

Debiais, S., 66, 69

Debliquis, A., 101

de Bray, J.M., 22

Debrey, S., 114

Debrun, A., 133

Decavel, P., 100

Decisso, B., 64

de Freitas, G.R., 36, 137

De Gregorio, M.A., 150

De Keyser, J., 27, 134

De la Puerta, I., 95, 132

Delbeuck, X., 16, 17

Deleu, D., 74

Delgado, M., 26, 114

Delgado, M.G., 139

Delgado, P., 21, 37, 39, 62, 80, 142, 144

Delgado-Mederos, R., 20, 32, 37, $38,39,144$

Della Martina, A., 50, 79

Delmaire, C., 16, 17

Deltombe, T., 143

De Luca, A., 58

Demaerschalk, B.M., 145

Demarin, V., 89

Demchuk, A.M., 26, 35, 45, 112, 114

Demeter, K., 52

De Monyé, C., 115

Dempfle, C.-E., 50

Dénes, L., 80, 82, 83, 84

De Nigris, E., 58

Dennis, M., 4, 89

Dennis, M.S., 35, 36, 111

Deplanque, D., 28

Deschaintre, Y., 16

Desfontaines, P., 57, 77, 133

Desmond, P., 116

Despland, P.A., 136

DESTINY Study Group (Germany), 59

Detante, O., 17, 128

Dethy, S., 70

De Toffol, B., 69 
de Vries, J.P.M., 147

Devuyst, G., 12, 13, 25, 26, 31, 136

De Weerdt, W., 5

Dewey, H., 19

Dewey, H.M., 57, 86, 135

Dewindt, A., 23, 70

De Wit, L., 5

Di, Q., 93

Diabl, E., 42

Diamant, N.E., 3, 5

Dias, J.S., 16

Díaz, C., 127

Díaz-Manera, J., 48, 145

Dib, H., 146

Di Bari, M., 130

Di Carlo, A., 136

Dichgans, M., 27, 43, 44, 76, 79

Dick, B., 114

Di Dionisio, L., 10, 11

Diederich, N.J., 124

Dieguez, S., 67, 70

Dienelt, J., 135

Diener, H.C., 9, 38, 112

Dieterich, M., 68, 82

Díez-Tejedor, E., 29, 66, 136

Dijkshoorn, M., 115

Dimitrijeski, B., 30

Di Piero, V., 3, 16, 129

Dippel, D.W.J., 9, 61, 115

Diringer, M.N., 23, 48

Disque, C., 45, 109

Dittrich, R., 14, 94

Divjak, I., 66

Djibuti, M., 25

Dobato, J.L., 119

Doczi, T., 150

Dodel, R., 99, 118

Dodick, D., 145

Doerfler, A., 50

Dohmann, H.F., 137

Dohmen, C., 33

Domashenko, M.A., 126

Domigo, V., 29

Dominguez, C., 142

Donaldson, C., 5

Dongsheng Fan, 143

Donnan, G.A., 86, 112, 134, 135

Donnellan, C., 20

Doppler, E., 83

do Prado, G.F., 121

Dora, B., 147

Dornbach, F., 59

Dotti, M.T., 43, 77

Doubal, F.N., 111

Down, C., 31, 57

Drambarean, E., 62

Dreier, J.P., 46

Drenckhahn, C., 46

Dretakis, K., 96

Dubaniewicz, C.M., 76

Dubas, F., 22

Ducimetière, P., 8

Duda, E., 84

Dunac, A., 47, 71

Dunatov, S., 87

Dupere, D., 30

Dupont, P., 15

Durastanti, L., 140
Dvorak, F., 139

Dzialowski, I., 26, 45, 109, 112

Dziedzic, T., 44, 139

Dziewas, R., 95

Eckert, B., 11

Eckhardt, R., 29, 72

Eeckhout, E., 73

Efstratopoulos, A., 96

Egido, J., 136

Eicke, B.M., 82

Eicke, M., 68

Eide, G.E., 99

Ekinci, G., 146

Eliasziw, M., 35

Ellis, G., 92, 120

Emery, P., 76

Enderby, P., 102

Endler, G., 70

Engelhorn, T., 50

Engelter, S., 19, 26, 42, 87, 125

Engelter, S.T., 41

Engström, G., 91

Enric, D., 119

Enzinger, C., 4

Epifanov, Y., 99, 118

Erdö, F., 82

Erntz, L., 82

Erro, M.E., 68

Esposito, L., 15, 134

ESPRIT Study Group, 60

Evans, A., 8

EVAS-3S Investigators (France), 60

Eveson, D., 93

Eyding, J., 34, 110

Fabre-Pi, O., 100

Fabricius, M., 46

Fabrizio, E., 100

Fairhead, J.F., 150

Falcao, C., 137

Falcao, CH., 36

Fan, Y.H., 77

Farias, A., 145

Farrall, A.J., 35

Fatar, M., 50, 78, 79, 110

Fazekas, F., 4

Federico, A., 43, 77

Feher, G., 150

Fekete, I., 71, 88, 121

Felbecker, A., 131

Felber, S., 37

Fénelon, G., 98

Fernandez, J.A., 84

Fernández, J.M., 69

Fernández, S., 64, 65

Fernández-Cadenas, I., 21, 37, 80, 142

Ferrari, J., 137

Ferreira, S., 78

Ferri, M., 58

Ferrieres, J., 7

Ferro, J.M., 39, 128

Fesl, G., 49

Festas, M.J., 106

Feys, H., 5

Fiebach, J.B., 137, 143

Fiehler, J., 11, 28, 137
Filipponi, S., 97

Finet, P., 8, 23

Finklestein, S.P., 83

Fiorot, J., 72

Fischer, T., 14

Fischer, U., 54

Fisher, M., 140, 146

Fitt, G., 112

Fitzmaurice, D., 6

Flach, H., 46

Floel, A., 4

Floriach, M., 124

Flossmann, E., 6, 18, 45

Fluri, F., 19, 125

Foerch, C., 46, 139

Fofi, L., 140

Foglieni, C, 14

Font, M.A, 50

Fontcuberta, J., 48

Fontes, N., 104

Font Padros, M.A., 10

Ford, G., 36

Ford, G.A., 19

Forrester, L.W., 130

Förster, A., 18

Forsting, M., 50

Fortmann, S.P., 87

Fossas, P., 124

Frackowiak, M., 33

Francia, C., 58

Francis, D.P., 94

Franke, C., 135

Frei, K., 72

Frei, M., 26

Freilinger, T., 76

Friedrich, M., 141

Fryer, T.D., 33, 51, 81

Fudge, N., 59, 118

Fuentes, B., 29, 66, 136

Fujimoto, S., 111

Fukuda, T.G., 145

Fulesdi, B., 71

Fulton, A., 22

Funk, G., 29, 72

Funk, M., 70

Furie, K., 64

Furtner, M., 37

Gadelha, M., 72

Gaffney, J., 84

Gahn, G., 45, 109, 144, 148

Gál, A., 80, 83

Galea, M., 3

Gállego, J., 68

Gálvez, A., 133, 138

Gan, H., 53

Gandiga, P., 105

Gandjour, J., 63

Garambois, K., 17, 128

Garcia, E., 31, 84

García, J., 28

García-García, J., 41

Gariépy, J., 8

Garrett, C., 78

Garro, C., 65

Gletterasecki, A.D., 76

Gasecki, D., 149

Gasparini, M., 16
Gasparotti, R., 97, 127

Gass, A., 18, 26, 28, 110, 114, 115

Gauvrit, J.Y., 15, 114

Gazagnes, M.D., 77

Geana, I., 73

Geeganage, C., 121

Gelosa, P., 52

Genius, J., 72

Gensini, G.F., 94

Georgiadis, D., 63, 87

Geppina, E., 14

Gerber, J., 148

Gerloff, C., 4, 105

Gesztelyi, R., 88

Ghika, J., 136

Ghosh, A.K., 61

Ghosh, S.K., 61

Gianella, A., 52

Giazzon, A., 14

Giesel, F., 79

Giles, M., 18, 58

Giles, M.F., 6, 24

Gillard, J., 42, 58

Gilligan, A.K., 86

Gil-Nuñez, A., 136

Giordano, G.P., 77

Giovagnoli, A.R., 68

Giraux, P., 4

Gittoes, M., 103

Gladman, J.R., 14, 63

Gnahn, H., 7

Godinho, T.M., 145

Goericke, S.L., 50

Goertler, M., 9, 40, 112, 138

Golaszewski, S.M., 105

Goldbourt, U., 25, 86, 90

Goldhagen, T., 45

Golovko, O., 33, 81

Gomes, D.L., 72

Gomis, M., 65, 94, 95, 133

Gong, X.P., 106, 116

Gongora-Rivera, F., 73

Gonin, C., 12

Gonzalez, C., 137

Gonzalez, C.S., 36

González, M., 127

González-Hernández, A., 100

Goode, S., 150, 151

Gormley, K., 78

Gorossman, E., 25

Gospodaru, N., 91

Gostynski, M., 19, 26, 125

Goto, S., 55

Gotwald, T., 37

Gouw, A.A., 17

Govan, L., 30, 125

Grad, A., 107

Grau, A., 136

Grau-Olivares, M., 65

Gray, C.S., 11

Gray, L.J., 6, 42, 121, 144

Greber, C., 12, 13

Greg, L., 6

Greil, O., 15

Greisenegger, S., 70

Griebe, M., 18, 50, 78, 79, 110, 114,115

Grieshofer, P., 6 
Griesmacher, A., 9

Griesser, B.A.C., 117

Grivé, E., 22, 32

Groene, O., 119

Gromadzka, G., 99

Grønbæk, M., 53

Grond-Ginsbach, C., 132

Gross, B., 124

Grotemeyer, A.K.-H., 101

Grotta, J.C., 20

Gschwendtner, A., 43, 44, 76

Guadagno, J.V., 33

Guan, Q.S., 93

Guardia, E., 145

Guasticchi, G., 58

Gubitz, G., 30

Gubitz, G.J., 141

Gubskii, L.V., 111

Gudkova, V.V., 104, 105

Gueguen, A., 98

Guenther, M., 114, 115

Guerrini, U., 52

Guichard, J.-P., 43

Guijarro, C., 9

Guimarães, J., 78

Gur, A., 124

Gusev, E.I., 26

Guseva, O.I., 13

Gutiérrez, E., 24

Gutsaluk, A.G., 13

Gutzwiller, F., 26

Guyennet, H., 31

Haa, J.S., 138

Haacke, C., 99, 118

Hacke, W., 33, 61, 69, 143

Hackett, M.L., 20

Haddad, A.F., 36

Hadrane, L., 98

Haefeli, T., 54

Haga, K., 111

Hagan, N., 64

Hakjae, R., 69

Hakkinen, U., 56

Hallström, B., 22

Hamad, A.A., 74

Hamann, G.F., 79

Hammer, M., 30

Hammerton, J., 102, 103, 106

Han, J.H., 31

Han, M.-K., 65

Han, S.W., 67

Hand, P., 47, 116

Hand, P.J., 36

Hanson, P., 143

Häppölä, O., 100

Haralampopoulos, A., 141

Haring, H.-P., 12

Harms, L., 92

Harrow, C.J., 75, 131

Hart, N.D., 7

Hart, W.M., 71

Hartmann, A., 30

Hartmann, M., 101

Hashimoto, Y., 126

Hassan, A., 22

Hassanpour, H., 53

Hauw, J.J., 73
Hayoz, D., 133

Hedblad, B., 91

Heidbreder, A., 94

Heider, P., 15

Heidrich, J., 8

Heimann, A., 40, 79, 82

Heise, B., 140

Heiss, W.D., 33

Helenius, J., 128

Heliopoulos, I., 141

Heng, B.H., 85

Hennerici, M.G., 17, 18, 26, 45, 50, $68,78,79,110,114,115,118$

Hénon, H., 128

Hentschel, H., 45

Herm, J., 49

Herman, M., 67

Hermann, D.M., 145

Hermans, S., 15

Hernández-Gállego, J., 100

Herrera, M., 68

Herreros, B., 9

Herrmann, A., 12, 13

Herrmann, E.F., 117

Herrmann, O., 51

Herweh, C., 33

Herzig, R., 67, 110

Herzog, J., 44

Hetzel, A., 147

Heuschmann, P.U., 8, 56, 57, 59, $85,85,140$

Hevey, D., 20

Hickey, A., 20

Hietanen, M., 128

Hildreth, A.J., 11

Hill, M.D., 20, 25, 27, 45, 112

Hill, R., 142

Hilton, A., 53

Hirsch, A.T., 55

Hirsch, J., 114

Hirt, L., 49, 52

Hiyamizu, M., 130

Hlustik, P., 67

Ho, G., 139

Ho, P., 108

Hobbs, R., 6

Hochstrasser, D.F., 125

Hoemberg, V., 104, 105

Hoffman, A., 31, 57

Hofmeister, C., 46

Höglund, P., 22

Hölig, S., 28

Holmberg, P., 83

Holt, M., 112

Holtmannspötter, M., 43, 44

Hömberg, V., 105, 106

Hommel, M., 17, 128

Hommet, C., 66

Hong, K.S., 100

Hongsong Song, 143

Horácio, G., 128

Horak, D., 110

Horner, S., 58, 76

Horozoglu, H., 146

Hosseini, H., 98

Howe, F.A., 116

Howells, E., 57

Hoyos, L., 41
Hug, A., 47

Hughes, J.L., 51, 81

Hughes, R.L., 120

Hui-Chan, C.W., 102

Hummel, F., 4, 105

Humpich, M., 28

Hunink, M.G.M., 46

Hurtado, O., 82, 98

Husaini, B.A., 140, 146

Huth, C., 40

Huttner, H.B., 61, 143

Huzen, J., 134

Hwang, S.Y., 69

Hyun Sook Kim, H., 78

Iborra, E., 7

Ibrahimagic, O., 96

Idbaih, A., 39

Igase, K., 51

Ijäs, P., 64

Ikeda, K., 107

Ikeda, Y., 102

Il Hyung Lee, I., 78

Imai, K., 113

Ingall, T.J., 145

Ingemann, A., 84

Inglin, M., 13

Inzitari, D., 17, 43, 77, 86, 96, 136

Inzitari, M., 130

Ionova, V.G., 126

Isoviita, P.M., 64

Itabe, H., 148

Iuhasz, S.A., 62

Izquierdo, J., 142

Jackson, C., 89

Jacobi, C., 69

Jacobs, A.H., 33

Jaillard, A., 17, 128

Jamart, J., 91, 143

Jamous, M.A., 93

Jang, H.W., 138

Jang, S.S., 109

Jansa, J., 107

Jansen, B., 105

Januszewicz, A., 89

Jaramillo, A., 73

Jarrett, J., 141

Jayavel, S., 55

Jeangette, S., 8, 23, 70, 77

Jenni, W., 5

Jeon, S.B., 48

Jeong, S.W, 80

Jern, C., 90, 91

Jerrard-Dunne, P., 8

Jesus, P.AP., 16, 141

Jiménez, J., 9, 65, 138

Jiménez-Conde, J., 94, 95, 133

Johansen-Berg, H., 4

Johansson, L., 90

Johansson, S.E., 10, 24

Johnsen, S.P., 84

Jones, J., 136

Jones, P.S., 2, 33, 51

Jönsson, A-C., 22

Jonsson, A.-C., 98

Jood, K., 91

Jordão, C., 128
Jordi, A., 119

Jotic, A., 92

Jougla, E., 23

Jovanovic, A., 66

Jovanovic, M., 84

Jovanovic, Z., 92

Jovicevic, M., 66

Jowett, S., 6

Juan-Babot, O., 7

Jung, D.-K., 110

Jung, Y.H., 96

Jungehulsing, G.J., 129

Juni, J., 120

Junqué, C., 65

Jun Zhang, J., 143

Jurjevic, A., 87

Jüttler, E., 33, 61, 143

Kablau, M., 26, 34, 45

Kakuda, W., 37

Kallela, M., 100

Kalra, L., 8

Kamicski, B., 117

Kamiński, B., 28, 139

Kammersgaard, L.P., 24, 29

Kamran, S., 74

Kane, I., 33, 34, 35, 108

Kane, K., 140, 146

Kanematsu, Y., 78

Kang, D.W., 48, 108

Kanoksri, S., 87

Kanovsky, P., 67, 110

Kappeler, L., 54

Kappelle, L.J., 137

Kapsimali, Z., 77

Karahalios, G., 66

Karakostas, D., 141

Karapanayiotides, T., 12, 13, 74

Karasu, A., 51

Karaszewski, B., 111

Karavasili, E., 73

Karhunen, P., 44, 77

Karmanova, I.V., 103

Karwacka, M., 129

Kasiman, K., 139

Ka Sing, W., 31

Kaste, M., 38, 56, 64, 100, 128

Kastrup, A., 28

Kato, H., 113

Kaya, D., 147

Kaye, A., 47

Kehoe, P., 97, 100

Keil, U., 8

Keller, E., 72

Kémény, V., 12, 13

Kemp, S., 37

Kempski, O., 40, 79, 82

Kennedy, B.S., 87

Kentroti, D., 69

Keramidas, C., 141

Kern, C., 79

Kern, R., 18, 34, 45, 50, 110, 114, 115

Kesic, M.J., 89

Kessel-Schaefer, A., 41

Kessler, C., 115

Khan, U., 8, 22

Kharaishvili, Z., 25 
Khoutorova, L., 81

Kiechl, S., 9, 37

Kier, C., 115

Kiessling, F., 79

Kilic, E., 145

Kilic, Ü., 145

Kim, B.C., 93

Kim, D.W., 80

Kim, E., 126

Kim, E.Y., 80

Kim, G.M., 112

Kim, G.S., 96

Kim, H.J., 149, 151

Kim, I.-H., 9

Kim, J., 126

Kim, J.S., 48, 108, 129, 140

Kim, J.Y., 67

Kim, M., 126

Kim, M.K., 93

Kim, S.H., 80, 141

Kim, S.J., 151

Kim, S.-R., 102, 141

Kim, Y., 126

Kim, Y.S., 93

Kim Kyoung Keun, K., 78

Kimmeyer, P., 140

Kimura, R., 40

Kinoshita, N., 113

Kioulahidis, K., 69

Kiris, T., 51

Kischka, U., 4

Kitazato, K.T, 78

Kitazato, K.T., 51, 81, 93, 148

Kiylioglu, N., 126

Klein, G.E., 76

Klein, I.F., 14

Kleynmans, F., 140

Klimkowicz, A., 139

Klimkowicz-Mrowiec, A., 70

Kloska, S.P., 14

Kloss, M., 72, 132

Kloth, A., 131

Knoblich, R., 131

Knoflach, M., 9, 37

Kñrv, J., 98

Kobayashi, A., 33, 35, 148

Kobayashi, N., 149

Koffijberg, H., 48

Koga, M., 112

Köhrmann, M., 61, 143

Kolev, K., 84

Kolominsky-Rabas, P., 56

Komitopoulos, N., 77

Komitopoulou, A., 77

Komoly, S., 150

Kontsevoy, V.A., 107

Kool, H., 54

Koren-Morag, N., 25, 90

Koroboki, E., 90, 91, 134, 135

Koska, P., 84

Kostic, V., 92

Kostopoulos, K., 135

Kostyreva, M.V., 126

Koton, S., 25

Kotowicz, J., 75

Kotsi, V., 74

Koudstaal, P.J., 9, 46, 61

Koufali, M., 105, 136
Kouperberg, E., 88

Koutsis, G., 66

Kovanen, P.T., 64

Kover, F., 150

Kovrazhkina, E.A., 104, 105

Kowgier, M., 5

Kozera, A.G., 76

Kozera, G., 89, 149

Kozono, M., 107

Kraemer, M., 113

Krause, V., 105

Krawczyk, M., 103

Kraywinkel, K., 8

Kreisel, S., 50, 110

Krespi, Y., 147

Kress, B., 69

Kristoffersson, U., 22

Krobot, K., 56

Kröger, B., 140

Krogias, C., 34, 110

Krol, A., 45, 112

Krotenkova, M.V., 117

Kroumova, M., 28

Krüger, C., 106

Krupinski, J., 7, 10, 15, 50, 64, 65 , 84

Krupka, B., 110

Krützelmann, A., 11

Kruyt, N.D., 137

Kucinski, T., 11, 137

Kuhlenbaeumer, G., 94

Kuhn, H.G., 106

Kuker, W., 34

Külkens, S., 101

Kumar, P., 84

Kumar, S., 84

Kunte, H., 92

Kuruttukulam, G.V., 88

Kwon, M., 129, 140

Kwon, S.U., 108

Kwon, S.W., 48

Laage, R., 106

Laaksonen, R., 44, 77

Labreuche, J., 23, 73, 97, 123

Lacerda, A.M., 16, 127

Ladenvall, P., 90

Lahoz, C.H., 41, 69

Lalic, N., 92

Lalouschek, W., 70, 137

Laloux, P., 44, 57, 91, 101, 143

Lam, C.W., 77

Lam, W.M., 113

Lambert, J.C., 8

Lampl, Y., 124

Lamy, C., 32

Landis, T., 36, 146

Lang, W., 70, 137

Langhorne, P., 30, 92, 119, 120

Lansberg, M., 37

Lanthier, S., 75

Lapayre, J.C., 31

Lara, N., 22

Larrue, V., 7, 36, 74

Latour, L.L., 114

Laurent, C., 28

Lauritzen, M., 46

Lavallée, P., 123
Lavallée, P.C., 14, 23

Lawson, G., 143

Leal, R., 92

Leclerc, X., 15, 28

Lederer, M., 64

Lee, B.C., 100

Lee, H., 110, 123

Lee, J., 138

Lee, J.H., 96, 140

Lee, J.S., 80

Lee, J.-T., 110

Lee, K.B., 64, 69

Lee, K.H., 112

Lee, M., 53

Lee, S.-H., 123

Lee, S.H., 65, 93, 100, 110

Lee, S.J., 138

Lee, S.W, 151

Lee, S.W., 149

Lee, T.H., 149, 151

Lee, Y.S., 100

Lees, K., 47, 124

Lees, K.R., 38, 125, 131

Lehmann, T.-A., 46

Lehnhardt, F.G., 33

Lehtimaki, T., 44, 77

Leira, R., 28, 98

Lemenev, V.L., 13

Lendzion, N.F., 145

Lenzi, D., 3

Lenzi, G.L., 3, 16, 95, 100, 129

Leon, M., 142

Lerond, L., 52

Leta, R., 41

Leung, T., 31

Leung, T.W., 77

Levi, C., 134

Levi, C.R., 11, 32

Levi, M., 96

Lewis, S., 33, 35, 44, 55, 145

Leys, D., 16, 28, 128

Leyva, A., 41, 54

Li, X., 10, 24

Lianos, K., 74

Lichy, C., 72, 132

Liebeskind, D.S., 28

Liljequist, S., 83

Lim, J.G., 110

Lim, T., 142

Lin, X.J., 93

Lincz, L., 96

Lindgren, A., 22, 98, 125

Lindsberg, P.J., 64, 100, 128

Linka, A.Z., 41

Linn, J., 49

Linna, M., 56

Liodou, A.A., 49

Liping Wang, 143

Liski, A., 56

Liu, H., 51, 78

Liu, M., 121, 144

Liu, W.G., 93

Lizasoain, I., 28, 82

Lloyd, A., 49

Lobato, R., 29

Lodder, J., 53, 63

Loh, K.C., 101

Lomidze, G., 25
Long, M., 120

Lopes, A.A., 16

Lopez, J., 71

López, M., 54, 92

López-Arbeloa, P., 24

Lopez Bescos, L., 9

López-Fernández, J.C., 100

Lopez-Manzanares, L., 148

Lorenzano, S., 140

Lorenzo, S., 119

Loukopoulos, T., 141

Loulidi, J., 36

Lovelock, C.E., 6, 24, 34

Lovett, E.C., 11

Lovett, J., 10

Lövkvist, H., 22

Lovrenc Huzjan, A., 89

Lowe, D., 31, 57

Lowe, J., 11

Lubahn, W., 40

Lucas, B., 66

Lucchesi, C., 12, 13

Luetjohann, D., 44, 77

Luijckx, G.J., 27, 134

Luthman, H., 22

Lye, J., 27

Lyrer, P., 19, 26, 41, 42, 87, 125

Macdonell, R.A., 86

MacGregor, L., 108, 116

Macko, R.F., 130

Macleod, D., 30

Macleod, M., 42, 43

Macleod, M.M., 4

Macquire, J., 96

MacSweeney, S.T., 14, 63, 150, 151

Madarász, E., 52

Madej-Dmochowska, B.A., 76

Madero, R., 66

Maeda, M., 102

Maeder, P., 20, 21

Maeder-Ingvar, M., 25, 136

Maestre, J., 37

Maestrini, I., 16, 129

Magoni, M., 97, 127, 132

Mahagne, M.H., 47, 71

Maillard, G., 21

Mainar, A., 150

Mainz, J., 84

Maisterra, O., 32, 38, 39

Maisterre, O., 144

Major, S., 46

Mak, K.H., 101

Malcolm, L., 75

Malecki, M., 44

Malik, I.S., 94

Malik, O., 67

Malkoff, M., 20

Mallolas, J., 63, 138

Malm, J., 125

Mamantas, A., 74

Manawadu, D., 67

Manios, E., 67, 90, 91, 134, 135

Mannhalter, C., 70

Mant, J., 6

Maramattom, B.V., 88

Marc, G., 22

Marchionni, N., 130 
Mares, J., 67, 110

Margherita, A., 74

Marie, S.K.N., 103

Marini, C., 10, 11, 48, 87

Maris, C., 149

Markus, H., 8, 22, 43

Markus, H.S., 78, 116

Marquardt, L., 136

Marschall, D., 56

Marshall, I., 35, 111

Marti, H.H., 51

Martí-Fàbregas, J., 37, 41, 48, 145

Martín, P., 142

Martin, S., 29

Martínez, E., 48, 145

Martínez, J., 65, 138

Martinez, J., 50, 94

Martínez, M., 145

Martinez, P., 29

Martínez, S., 145

Martinez Gonzalez, J., 7

Martinez-Gonzalez, N.A, 44

Martini, M., 86

Martino, R., 3, 5

Martins, S., 141

Martí-Vilalta, J.L., 37, 41, 48, 145

Mas, J.L., 25, 29, 32, 55, 118

Masotti, G., 130

Massaro, A.M., 88

Massaro, A.R., 72

Massaro, L., 30

Masse, I., 28

Masson, X., 133

Mast, H., 46, 47

Matas, M., 12

Matcau, L., 62

Mathew, O.P., 80

Matikas, N., 69, 73

Matsuo, A., 130

Matsuzaki, K., 148

Matte, G., 75

Matthews, P.M., 4

Mattle, H.P., 54

Matz, K., 29, 72

Mau, J., 38, 55

Mauri, J.A., 95, 129, 150

Maurits, N., 134

Mawson, S., 102, 103, 106

Mayer, S.A., 23, 48

Mayer, T., 27

Mayet, J., 94

Mäyränpää, M., 64

Mazighi, M., 123

Mazzucco, S., 45

McAlpine, C., 92

McCarron, M.O., 117

McCormick, M., 109

McCormick, M.T., 62

McDonagh, P., 81

McGettigan, P., 96

McGovern, R., 8

McGregor, L., 47

McGuire, A., 47, 56

McIntyre, D.J.O., 116

McKevitt, C., 59, 118, 121, 122

McManus, J.A., 92

McNicholl, M.P., 117

Meagher, T., 34
Meairs, S., 14, 34, 45, 50, 79

Meco, G., 100

Medeiros-De Bustos, E., 97

Meder, J.-F., 32

Meder, J.F., 15, 114

Medrano, J., 150

Meissner, W., 123

Meldrum, C., 96

Melnyk, V., 143

Mendez-Otero, R., 36, 137

Mendonca, M.L., 36, 137

Menezes, D.F., 127

Menon, D.K., 81

Mercurio, A., 129

Meretoja, A., 56

Meseguer, E., 123

Messeder, O., 145

Metz, R.J., 124

Meuli, R., 114

Meves, S., 34

Meves, S.H., 110

Meyer-Wiethe, K., 34, 109, 115

Miastkowska-Swierkocka, M., 149

Michael, D., 6

Michel, P., 67, 73, 87, 114, 136, 139

Mielczarek, B.M., 76

Miguel, M., 15, 50, 64

Miguel Turu, M., 7, 10

Mihálka, L., 88

Mihancea, P., 62

Mijajlovic, M., 92

Miklya, I., 80, 82

Mikula, I., 89

Mikulik, R., 20

Milia, P., 18

Millán, M., 28, 146, 148

Millan, M., 63

Miller, K., 3

Miller, S., 5, 104

Mills, R.J., 105

Milonas, I., 141

Min, Y.-S., 123

Min Deng, 143

Ming Liu, M., 121

Ming Lu, 143

Misselwitz, B., 46

Mitchell, L.A., 112

Mitsios, N., 84

Miyagi, D., 107

Moeller Hartmann, W., 33

Moessler, H., 83

Mohd Nor, A., 19

Mohn, B., 59

Mohr, J.P., 46, 47

Molan, M., 112

Molina, C., 12, 20, 22, 32, 80, 142

Molina, C.A., 21, 32, 37, 38, 39, 62, 144

Molina-Porcel, L., 48, 145

Molyneux, A.J., 24, 34

Mondon, K., 69

Montaner, J., 21, 37, 39, 62, 80, $125,139,142,144$

Montori, M., 129, 150

Montse, V., 119

Moody, A.R., 14, 63

Moonis, M., 140, 146

Moore, A.F., 81
Moreton, F., 109

Moretti, M., 77

Morgan, P.S., 14, 63

Morioka, S., 130

Moro, M.A, 63

Moro, M.A., 82, 138, 148

Moroi, J., 149

Moroney, J., 22

Morovic, S., 89

Moseley, M., 37

Mosquera, E., 98, 138

Mostacero, E., 95, 129, 132, 150

Moulin, T., 31, 40, 97, 100

Mountain, G., 103, 106

Mrsulja, B., 84

Mucha, D., 144, 148

Mueller, A., 144, 148

Muir, K.W., 38, 62, 109, 124, 125

Muir, S.W., 131

Müller, R., 27

Muller-Nordhorn, J., 129

Muñoz, R., 68

Munteis, E., 65, 94

Munuera, J., 32

Murillo, L., 84, 92

Muroi, C., 72

Murray, E., 6

Nabavi, D.G., 14, 94, 140

Naegele, B., 17, 128

Naess, H., 99

Nagafuji, T., 49

Nagahiro, S., 51, 78, 81, 93, 148

Nagy, F., 133

Nagy, Z., 52, 80, 82, 83, 84

Nahas, N.M., 148

Nakase, H., 40

Nam, E., 14

Nam Keun Kim, N., 78

Nanjundaswamy, S., 140, 146

Nardi, K., 18

Nasr, N., 74

Nassenstein, I., 94

Nataf, F., 15, 114

Navarré, A., 120

Navarrete, P., 71

Nayak, V., 5

Nazarov, O.S., 107

Nazir, F., 19

Nedelmann, M., 82

Nedeltchev, K., 54

Nencini, P., 86, 96

Nesi, M., 86, 96

Neumann-Haefelin, T., 28, 46, 139

Neveling, M., 33

Ng, H.K., 113

Ng, S.S., 102

Nguyen, D.K., 75

Nicholson, G., 3

Nicola, I., 3

Nicoletta, A., 14

Niederkorn, K., 76

Niesen, W., 11

Niesen, W.D., 147

Nieuwboer, A., 5

Niewada, M., 148

Niewada, M.P., 28, 117, 139

Ninomiya, M., 49
Nishi, K., 81, 148

Nishioka, T., 40

Nitkunan, A., 116

Nitrini, R., 16

Nobili, E., 52

Nolte, C.H., 129

Nombela, F., 28, 146, 148

None, A., 109

Norrving, B., 22, 56, 125

Novoselsky, A.N., 103

Novotny, M., 29, 72

Novy, J., 20

Nukata, M., 113

Nuotio, K., 64

Nussbaumer, K., 12

Nyka, A.W.M., 76, 89, 129, 149

Nys, G.MS., 137

O'Brien, R.E, 11

O'Connell, J.E., 11

O'Neill, D., 20

Obach, V,, 37

Ocheretovy, A.S., 103

Odier, C., 75

Ofek, K., 54

Oh, G.T., 80

Ohman, E.M., 55

Oh-Sang, K., 75

Ois, A., 65, 94, 95, 133, 138

Okada, Y., 111

Okamoto, M., 113

Ok Joon Kim, O., 78

Olan, W., 114

Oldag, A., 138

Oldroyd, K., 75

Oliveira, J.P., 78

Oliveira-Filho, J., 16, 127, 141, 145

Olivieri, L., 48, 87

Olivot, J.-M., 123

Olsen, T.S., 24, 29, 40, 58

Oluwale, A., 151

Onodi, E., 150

Opherk, C., 44, 76

Oppenheim, C., 15, 32, 114

Orgogozo, J.M., 123

Orlov, S.V., 126

Ortega, L., 80

Ortega-Aznar, A., 37

Ortega-Casarrubios, M.A., 29, 66

Ossemann, M., 91

Oveisgharan, S., 98

Overgaard, K., 52

Overtoom, Th.C., 147

Ozkul, A., 126

Paci, M., 130

Paciaroni, M., 18

Padovani, A., 127

Pagoni, S., 141

Pahl, S., 106

Paik, J.S., 67

Pai-Yi, C., 118

Pakola, S., 52

Palacios, M.E., 21

Palasí, A., 15, 65

Palm, F., 136

Palmerini, F., 18

Palnum, K.D., 84 
Palomeras, E., 124

Palumbo, V., 27, 96

Pandolfo, M., 8, 23, 44, 77

Pantano, P., 3

Pantoni, L., 17, 43, 77

Papa, S., 19, 26, 125

Papadopoulou, M., 67, 90, 91

Papaioakim, M., 141

Papakitsoy, E., 96

Papapostolou, A., 67, 90, 91

Pappa, T., 49

Parati, E.A., 68

Pardo, J., 119

Pareja, J.A., 119

Park, B.J., 100

Park, E.M., 80

Park, J.H., 67

Park, J.M., 100

Park, J.Y., 69

Park, K.P., 149

Park, M.-J., 141

Park, M.S., 93

Park, S.-H., 65

Park, S.-P., 110

Park, S.W., 109, 110

Parsons, M., 96

Parsons, M.W., 11, 32

Partyka, D., 44, 70

Pasco, A., 22

Pasco, A.K., 21

Pascual, J.L., 53, 55

Pascual, L.F., 129

Pashos, S., 94

Pasquier, F., 16, 17, 128

Pasquini, M., 3, 28, 128

Patak, P., 43

Paternoster, L., 44

Patterson, S.L., 130

Pautot, V., 22

Pavia, M., 97

Pavlovic, A., 92

Pavlovic, M., 116

Pavy-Le Traon, A., 74

Payne, J., 81

Paz, F., 127

Pearce, D.C., 134

Pearson, M., 31

Pedreira, B.B., 141

Peeters, A., 57

Pellicier, M., 97

Pendlebury, S.T., 150

Pengo, V., 74

Penumbra Stroke Trial Investigators, 122

Penz, D., 9

Peppa, A., 135

Pepper, E.M., 32

Peppes, V., 38

Pera, J., 44, 139

Pereira, J.LB., 145

Pérez de la Ossa, N., 37

Pérez-Viéitez, M.C., 100

Pergantou, H., 77

Perilli, E., 132

Perren, F., 36, 146

Perret, B., 7

Pescini, F., 43, 77

Pessah-Rasmussen, H., 91
Peteers, A., 44

Peters, N., 44, 76

Petersen, B., 22

Petersen, P., 84

Petit, R., 7

Petkova, M., 114

Petre, I., 19

Petro-ex Investigators, 2

Petrova, E.A., 107

Pexman, J.H., 26

Pezzini, A., 127

Pfefferkorn, T., 27, 76

Phan, T.G., 112

Phillips, B., 3

Phillips, S., 30

Phillips, S.J., 141

Pico, F., 97

Piconi, S., 132

Piechowski-Jozwiak, B., 12, 13, 31

Pieri, A., 72, 88

Piñol, G., 95, 129, 132

Piperidou, H., 141

Pistoia, F., 10

Pitkäniemi, J., 64

Platokouki, H., 77

Pocock, S., 121

Poglia, D., 36

Polo, A., 119

Pomeroy, V., 5

Pomeroy, V.M., 104

Pons, G., 41

Pons, J.M., 120

Pont, C., 138

Popa, A.R., 62

Popa, C., 73, 149

Popescu, A., 73

Popowski, P., 89

Poppert, H., 15, 134

Porcher, R., 39

Postert, T., 110

Potocka, M., 129

Potter, J., 93

Pracucci, G., 86

Prencipe, M., 58, 140

Pretorius, P.M., 34

Price, M., 52

Prosser, J., 116

Prugger, C., 57

Psarakis, S., 96

Puca, E., 58

Puetz, V., 148

Pujadas, F., 22

Puras, E., 9

Purroy, F., 62

Puumalainen, A., 56

Puyraveau, M., 100

Qiu, S.M., 83

Quintana, M., 62, 80

Quintero, L., 41

Radenovic, L., 84

Radue, E.W., 41

Radzwill, N., 79

Ragionieri, P., 97

Raicevic, R., 84

Rallo, B., 95

Ram, D., 6
Rana, B.S., 94

Randall, M., 42

Ranganna, K., 80

Rangel, R., 84, 92

Rashid, P.A., 121

Rasmussen, B.H., 24

Rasmussen, R.S., 52

Ray, A., 57

Redfern, J., 122

Redfors, P., 91

Redgrave, J., 10, 34

Redgrave, J.N., 18, 45

Redondo, P., 23

Reeves, R., 57

Regula, J., 69

Regula, J.U., 101

Reichhart, M., 114, 139

Reid, J.M., 141

Reis, F.JFB., 16, 127

Reis, J., 78

Reis, P.SO., 127

Remiche, G.A., 23

Remonda, L., 54

Ren, J.M., 83

Renault, G., 34

Rengel, M.R., 150

Retel, O., 97, 100

Reuner, K., 72

Reutens, D.C., 112

Reuter, B., 110

Revela, J., 38

Reverte, I., 12, 13

Reyes, S., 92

Ribeiro, I.SN., 127

Ribeiro, O., 128

Ribó, M., 21, 37, 142

Ribo, M., 12, 20, 32, 38, 39, 62, 144

Ricci, S., 21, 145

Richard, F., 16

Ricolfi, F., 40

Rigal, M., 7

Righetti, E., 21

Rinaldi, L., 130

Ringelstein, E.B., 9, 14, 94, 140

Ringleb, P.A., 61, 69, 101, 143

Rinkel, G.J.E., 54

Ritchie, K., 8

Ritter, M., 59, 140

Riva, E., 146

Rival, G., 66

Rivers, C.S., 34, 35, 36

Robatscher, P., 9

Robinson, T., 93

Rocha, M.S., 88

Rodgers, M.M., 130

Roditi, G., 42, 58

Rodríguez, A., 65

Rodriguez, J., 127

Rodríguez, S., 41, 69

Rodríguez-Campello, A., 94, 95, 133

Rodriguez de Rivera, F.J., 29

Rodríguez-González, R., 98

Rodríguez-Yañez, M., 28, 37, 63, 98, 138, 148

Roebers, S., 59

Roether, J., 9

Roger, H., 6
Roh, H., 64

Rohsbach, D., 94

Roine, R.O., 100

Roine, S., 56

Roine, S.O., 56

Roje Bedekovic, M., 89

Roll, S., 129

Roman, A., 84

Román, G., 127

Romani, I., 86

Romero, A., 120

Romner, B., 125

Rønning, O.M., 70

Roose, M., 98

Ropele, S., 4

Roquer, J., 37, 65, 94, 95, 133, 138

Rosas, M.J., 106

Rosell, A., 21, 37, 80, 142

Rosengren, A., 91

Rosenkranz, M., 11, 137

Ross, I.N., 142

Rossmann, A., 9

Rossmüller, J., 105, 106

Rossnagel, K., 129

Röther, J., 118, 137

Rother, J., 25, 55

Rothwell, P., 10, 18, 34, 53, 58

Rothwell, P.M., 6, 18, 24, 34, 42, $45,58,150$

Rouanet, F., 123

Rouhl, R., 63

Rousseaux, M., 128

Rovira, A., 22, 32

Rovira, R., 22

Roy, J., 45, 112

Royter, V., 124

Rozzini, L., 127

Rubiera, M., 12, 20, 21, 22, 32, 38, $39,62,144$

Rubio, F., 7, 10, 15, 50, 64, 65, 84

Ruchat, P., 12, 13, 73

Rückert, C., 147

Rückert, R.-I., 92

Rudd, A., 8, 99, 118

Rudd, A.G., 31, 57, 121

Rude, N., 100

Ruffieux, C., 136

Ruidavets, J.B., 7

Ruiz-Sandoval, J.L., 84, 92

Russell, N., 4

Russo, T., 10, 11

Ryglewicz, D., 28, 139

Sacchetti, M.L., 58

Sacco, S., 48, 87

Sádaba, F., 24

Sadikovic, S., 134

Sadoshima, S., 111

Saeki, Y., 113

Sahraie, A., 4

Saimanen, E., 128

SAINT Trials Investigators, 122

Saka, Ö., 56

Sakaki, T., 40

Sakamoto, K., 107

Saksi, J., 64

Saliaris, M., 38, 135

Salihovic, D., 96 
Sallustio, F., 34, 45, 115

Salonen, O., 128

Salvadori, E., 77

Salzman, F.M., 81

Sämann, P., 44

Sameli, S., 66

Samson, Y., 28

Sanak, D., 110

Sánchez, C., 9, 119

Sanchez, J-C:, 125

Sancho, J., 120

Sander, D., 7, 15, 27, 90, 134

Sander, K., 7, 15, 90, 134

Sandercock, P., 33, 35, 42, 43, 55, 108,145

Sandin, J., 52

Sang-Beom, J., 108

Sanjeev, S., 123

San José, B., 66

Santamarina, E., 38, 39, 62, 80, 142,144

Santos, C.CS., 127

Santos, R.L., 103

Santos, S., 129, 132

Sanz, P., 124

Sarasin, F.F., 117

Sarraf, M., 130

Sarraf, Z., 130

Sarrafzadeh, A., 46

Sarti, C., 43, 77

Sartor, K., 33

Sarzyńska-Długosz, I., 28, 99, 103 , 139, 148

Sasaran, Al., 73

SaTIS Investigators, 60

Sato, K., 93

Saudeau, D., 69

Saulnier, F., 146

Savina, M.A., 107

Sayed, I., 119

Sbardella, E., 16, 129

Scaff, M., 103

Scalidi, S., 122

Scandalidi, S., 49

Scarpatetti, M., 6

Schäbitz, W.R., 106

Schaeg, M., 118

Schellinger, P., 137

Schellinger, P.D., 33, 61, 143

Scheltens, P., 16, 17

Schenkel, J., 57

Scherer, R., 6

Schindler, L.A., 115

Schlaug, G., 37

Schlindwein, P., 68

Schmidauer, C., 9

Schmidt, E., 74

Schminke, U., 115

Schneider, A., 106

Schneider, F., 82

Schneiderka, P., 110

Schöffski, O., 56

Scholle, T., 45

Schonewille, W.J., 147

Schoof, J., 40

Schormann, T., 113

Schouman-Claeys, E., 14

Schramm, P., 33
Schreuder, T., 135

Schroth, G., 54

Schuback, B., 5

Schuler, R., 12

Schulz, U., 34, 45

Schulz, U.G., 18

Schulze-Horn, C., 7, 90

Schumacher, M., 147

Schupp, W., 5

Schwammenthal, Y., 25

Schwaninger, M., 51

Schwark, C., 61, 137, 143

Schwarz, R., 25

Sciacca, R.R., 47

Scott, R., 96

Scotti, G., 14

Secades, J.J., 82

Secca, M.F., 128

Sedat, J., 47, 71

Seenan, P., 120

Segura, N., 133, 138

Segura, T., 95

Seidel, G., 34, 109, 115

Seitz, R.J., 105, 113

Seixas, J.C., 145

Sekoranja, C.L., 117

Selakovic, V., 84

Sena, E., 42, 43

Sencer, A., 51

Senin, U., 94

Seo, J.-H., 102, 141

Serena, J., 28, 63, 82, 138

Sessa, M., 14

Settakis, J., 71

Sgoifo, A., 21

Shah, I.M., 61

Shah, N., 93

Shahbazi, M., 130

Shakarishvili, R., 25

Shamalov, N.A., 111

Shapira, I., 54

Sharma, A., 105, 136

Sharma, J.C., 142

Sharma, N., 2

Shen, Q., 143

Shen, Y., 116

Sheng, S., 143

Shenhar-Tsarfaty, S., 54

Shi, J.P., 93

Shihong Zhang, S., 121

Shimizu, S., 102

Shin, H.Y., 112

Shopin, L., 54, 124

Sicherl, Z., 107

Siebert, S., 143

Siemiński, M., 129

Siepman, T.A.M., 115

Sietsma, D., 83

Sievers, M., 15

Silva, G.S., 72, 88

Silva, S.A., 36, 137

Silva, Y., 28, 63, 148

Silver, F., 3, 5

Sim, C.H., 127

Simmons, L.H., 2

Simon, O., 123

Sinanovic, O., 96

Sinitsky, I.A., 103
Sirimarco, G., 3

Sironi, L., 52

SITS Collaborators, 2

Sitzer, M., 43, 46, 139

Sivakumar, M.R., 123

Skalabrin, E., 37

Skibicka, I., 117

Skolnick, B.E., 23, 48

Skoufi, M., 49

Skowrońska, M., 28, 33, 35, 139, 148

Skrabalek, P., 135

Skvortsova, V.I., 13, 26, 104, 105, 107, 111

Slevin, M., 50, 84

Slevin, M.A., 7

Sliwka, U., 11

Slowik, A., 44, 70, 117, 139

Sluimer, J.D., 16

Smajlovic, D., 96

Smeeton, N., 99

Smith, D.B., 120

Smith, R., 51, 81

Smolders, B., 7

Sobesky, J., 33, 137

Sobrino, T., 28, 63, 82, 98, 138, 146

Sohn, C.H., 110

Sohn, S.I., 65, 109, 110

Soinne, L., 100, 128

Sola, T., 142

Soler, L., 142

Sonnberger, M., 12

Soo-Hyun, P., 75

Sørensen, T.K., 58

Soreq, H., 54

Sosa, A., 127

SPACE-Study Group (Germany), 59

Sparaco, A., 132

SPARCL Investigators, 1

Spatenkova, V., 135

Speck, U., 50

Spengos, K., 66, 67, 90, 91, 134

Spezi, R., 97, 127, 132

Spiegel, M., 37

Spieler, J-F., 23

Spottke, A., 99

Sprigg, N., 4, 6, 144

Spudich, A., 145

Staals, J., 53

Stafford, R.S., 87

Stakhovskaya, L.V., 13, 26

Stam, J., 39

Stanton, A., 22

Stapf, C., 20, 46, 47

Staszewski, J., 75

Staub, F., 70

Staudacher, T., 147

Stavlas, P., 96

Stavrianou, A., 141

Steen, C., 134

Steffens, M., 78

Steg, P.G., 25, 55, 73, 118, 123

Stegmayr, A.B., 31, 90, 134

Steigleder, T., 106

Stein, D., 64

Steiner, T., 23, 48

Steinforth, T., 104

Steinhagen, V., 131
Stemper-Bartkus, C.L., 145

Stephan, K.M., 104, 105

Stephenson, C., 114

Stepien, A., 75

Stewart, C., 145

Stewart, E., 114

Stibrant Sunnerhagen, K., 5

Stirpe, P., 100

Stoeter, P., 68

Stollorz, M., 104

Strandberg, K., 125

Strenja-Linic, I., 87

Stroick, M., 50, 78, 79, 110

Strong, A., 49

Strong, A.J., 46

Studer, L., 12, 13

Stuparu, A., 73

Sturm, J., 96

Sturm, J.W., 86

Subramaniam, S., 35, 45, 112

Subsai, K., 87

Sucapane, P., 87

Suchomel, P., 135

Sudlow, C., 44, 89

Suh, C.-K., 110

Sun, L., 51

Sundquist, J., 10, 24

Sundquist, K., 10, 24

Sung, S.M., 149, 151

Sunnerhagen, K.S., 103

Suslina, Z.A., 126

Suttorp, M.J., 147

Suzue, A., 51, 78, 81, 148

Suzuki, A., 149

Svensson, S., 103

Swistak, J., 75

Sylaja, P.N., 35

Synetou, M., 38, 122

Szabo, K., 18, 26, 110, 114, 115

Szapary, L., 150

Szczerba, S., 149

Szczudlik, A., 44, 70, 117, 139

Szilágyi, G., 80, 82, 83

Sztajzel, A.R.F., 117

Sztajzel, R., 36, 87, 146

Taccone, F., 23

TACS Study Group, 2

Tai Hwan, P., 75

Takahashi, D., 113

Takasawa, M., 51

Takis, K., 38, 49

Tallis, R., 5

Tallis, R.C., 104

Tamaki, R., 40, 79

Tamaki, T., 78

Tamura, K., 40, 79

Tamura, T., 93

Tan, J., 101

Tan, S., 142, 144

Tan, Y., 144

Tanashyan, M.M., 117, 126

Tanghe, H., 115

Tanghe, H.L.J., 46

Tanne, D., 25, 86, 90

Tataroglu, C., 126

Tatlisumak, T., 100

Tatschl, C., 29, 72 
Tavernarakis, A., 73

Tavolato, B., 74

Tay, S.Y., 127

Teasdale, E., 124

Teasell, R., 3

Teddy, P., 47

Teichmann, M., 98

Tejero, C., 129, 150

Teles, J.MM., 145

Telman, G., 88

Temperli, D.P., 117

Tennant, W.G., 151

Teo, C., 53, 55, 139

Terént, A., 124

Tettenborn, B., 87, 131

Thaler, D., 76

Thevenet, J., 52

Thijs, V., 5, 7, 15, 32, 37, 57, 71, 88

Thiran, J.P., 31

Thomalla, G., 11, 137

Thomas, S., 58

Thomassen, L., 99

Thrift, A.G., 57, 135

Tiberge, M., 74

Tikkala, I., 128

Tjärnlund Wolf, A., 90

Toh, M., 85

Toh, T., 127

Toledo, M., 22

Tombari, D., 3

Tomlinson, G., 5

Tonello, S., 74

Toni, D., 58, 140

Topakian, R., 12

Tortorella, R., 97, 132

Toth, K., 150

Touboul, PJ., 14

Tournier Lasserve, E., 66

Touzé, E., 29, 32, 118

Touze, E., 6, 53, 55

Toyoda, K., 111

Trabattoni, D., 132

Trabucco-Miguel, S., 17, 128

Trainini, L., 132

Tremoli, E., 52

Trenkler, J., 12

Tress, B., 47, 116

Tress, B.M., 108

Trevethan, C., 4

Trianti, M.S., 49

Trinkl, A., 79

Tröster, H., 79

Truelsen, T., 53, 85

Trystram, D., 114

Tsai-Chung, L., 118

Tsiodra, P., 141

Tsiskaridze, A., 25

Tsivgoulis, G., 66, 67, 90, 91, 134

Tuche, F.A., 36

Tucker, J.T., 81

Tuckfield, A., 108

Tufi, A., 21

Turaj, W., 44, 117

Turck, N., 125

Tuškan-Mohar, L., 87

Tzourio, C., 8

Uchino, K., 30
Uchino, M., 126

Ulbricht, D., 124

Umarova, R.M., 117

Uno, M., 51, 78, 81, 93, 148

Unsworth, B., 94

Urban, G., 144

Uyttenboogaart, M., 27

Vadikolias, K., 141

Valenti, M., 29

Valiente, R.A., 88

Vallance, P., 22

Vallejo, E., 41

Vanacore, N., 100

Van Calenbergh, F., 71

Vandemeulebroecke, M., 143

van der Flier, W.M., 16

van der Jagt, M., 46

van der Lugt, A., 46, 115

van der Schaaf, I.C., 48

Van der Worp, H.B., 137

van Oostenbrugge, R., 63

van Raak, L., 53

Van Zandvoort, M.JE., 137

Vashadze, T., 25

Vasiliadis, E., 96

Vassallo, M., 142

Vassilopoulos, D., 66, 90, 91

Vatankhah, B., 27

Vatca, L.A., 62

Vavli-Vavlida, E., 122

vd Boogaard, S., 147

Vedeler, C.A., 99

Vela, L., 119

Veltkamp, A.R., 51

Veltkamp, R., 69

Vemmos, K., 38, 49, 122, 134, 135

Venables, G., 42

Venketasubramanian, N., 85, 101, 142

Venti, M., 18

Venturelli, E., 127

Vergani, V., 97, 127, 132

Verheyden, G., 5

Vermeer, S.E., 61

Vesin, J.-M., 12, 13

Viana, L.C., 16, 127

Viana-Baptista, M., 128

Vibo, R., 98

Vicenzini, E., 95

Vidovic, M., 96

Vieira-de-Melo, R.M., 16, 127

Viguier, A., 7

Viiri, L., 44

Vila, R., 15

Villafani, J., 41, 69

Villarreal, J., 84, 92

Villringer, A., 30, 129

Vinychuk, S., 143

Virley, D., 49

Viswanathan, A., 20, 43

Vitali, P., 68

Vivancos, J., 28, 63, 82, 136, 138, 148

Vivas, E., 142

Vlachova, I., 110

Voller, B., 4

Volpe, M., 94 von Euler, A., 52

von Euler, G., 52

von Kummer, R., 45, 109, 144, 148

von Sarnowski, B., 115

Vos, J.A., 147

Vosko, M., 79

Voyaki, S., 96, 141

Vroomen, P.C., 27

Vuillier, F., 40

Vukovic, V., 89

Wade, D., 4

Wadhwa, K., 151

Wagner, A., 83

Wagner, M., 59

Wahle, K., 59

Wahlgren, N.G., 36

Waje-Andreassen, U., 99

Walker, M., 4

Wallesch, C.W., 40, 138

Walter, N., 125

Walter, U., 131

Walters, M., 75

Walters, M.R., 131

Wang, D., 33

Wang, L., 53

Wang, Q.F., 144

Wang, Y., 96

Wang, Y.I., 106

Wang, Y.J., 116

Wang, Y.L., 116

Wappler, E., 82, 83

Warburton, E.A., 2, 33

Wardlaw, J., 33, 34, 35, 89, 108

Wardlaw, J.M., 35, 36, 42, 58, 111

Ware, P., 106

Warlow, C., 53

Wartolowska, K., 35, 111

Watanabe, M., 113

Watson, T., 45

Watts, H., 49

Weber, U.J., 24

Wechsler, L., 37

Weder, B., 131

Weiller, C., 147

Weimar, C., 9, 112

Weir, C., 30

Weir, C.J., 38, 125

Wei Sui, 143

Wellmann, J., 8

Wellwood, I., 119

Wermer, M.J.H., 48, 54

Werner, A., 45

West, D., 84

Wetzel, S.G., 41

Wick, G., 9

Wiegler, K., 49

Wienker, T., 78

Wierucki, Ł., 89

Wiesmann, M., 49

Wietrzykowska, I., 117

Wiggam, M.I., 7

Wijnhoud, A.D., 9

Wiklund, P.G., 90

Wilhelm-Schwenkmezger, T., 82

Willeit, J., 9, 37

Willheim, K., 87

Willich, S.N., 129
Willmot, M., 4

Wilms, G., 71

Wilson, P.W.F., 55

Winkleby, M.A., 87

Wintermark, M., 139

Witte, O.W., 113

Wittkugel, O., 11

Wnuk, M., 117

Wöhrle, J., 68

Woimant, F., 40

Woitzik, J., 46

Wojczal, D.J., 76

Wolf, O., 15, 134

Wolfe, C., 8, 56, 97, 99, 118, 119, 122

Wolfe, C.D.A., 59, 121

Wolferts, G., 51

Wolff, V., 72

Wolkow, P., 44

Won-Chan Kim, W., 78

Wong, K.S., 77, 113

Wong, L.Y., 85

Wong, M.C., 53, 55, 108, 127, 139

Wright, V.L., 114

Wu, B., 144

Wunderli-Allenspach, H., 145

Wunderlich, M., 139

Wunderlich, M.T., 138

Wunderlich, N., 79

Wunderlich, O., 109

Wynnie, L., 31

Wyrzykowski, B., 89

Wyrzykowski, B.B., 76

Xie, X., 53

Xing-quan Zhao, 35, 113, 125

Xi-ping Gong, 35, 113, 125

Xydakis, I., 73

Xynos, K., 38, 122, 135

Yang, Q.W., 144

Yang, Y., 108

Yannie, S., 31

Yarnell, J.W., 7

Yarnitsky, D., 88

Yasaka, M., 111

Yasui, N., 149

Yatsu, F.M., 80

Ybot, I., 66

Yenisey, C., 126

Yi, S.D., 110

Yi-long Wang, 113, 125

Yin, A., 85

Yip, S.F., 77

Yonekawa, Y., 72

Yonemura, K., 126

Yong, M., 38, 55

Yong-jun Wang, 35, 113

Yoo, B., 126

Yoo, S.-H., 108

Yoon, B.W., 100

Yoon, D.J., 112

Young, C.A., 105

Young, G., 42, 58

Young-Chul, Y., 75

Young Ho Ku, Y., 78

Yu, H., 114

Yu, K.H., 100, 108 
Yuanjin Zhang, 143

Yu-mei Zhang, Y., 35, 113, 125

Zádori, A., 52

Zafeiriou, C., 38, 135

Zafeiriou, J., 49, 122

Zaheg, K., 12, 13

Zakopoulos, N., 66, 67, 90, 91, 134

Zaloni, I., 74

Zanchetti, A., 94

Zandio, B., 68

Zangerle, A., 9, 37

Zaninelli, A., 94

Zaro Weber, O., 33, 137

Zarzeczna-Baran, M., 89

Zdrojewski, B.T., 76, 89

Zechmann, S., 79

Zeumer, H., 11

Zhang, Y., 143

Zhang, Y.D., 93

Zhang, Y.M., 106, 116

Zhao, H.L., 113

Zhao, K.L., 81

Zhao, L., 4

Zhao, X.Q., 106

Zheng, H., 106

Zia, E., 91

Zidverc-Trajkovic, J., 92

Zis, V., 67

Zsuga, J., 88

Zureik, M., 8 